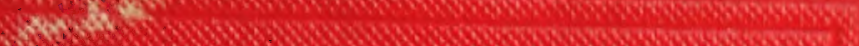

4.
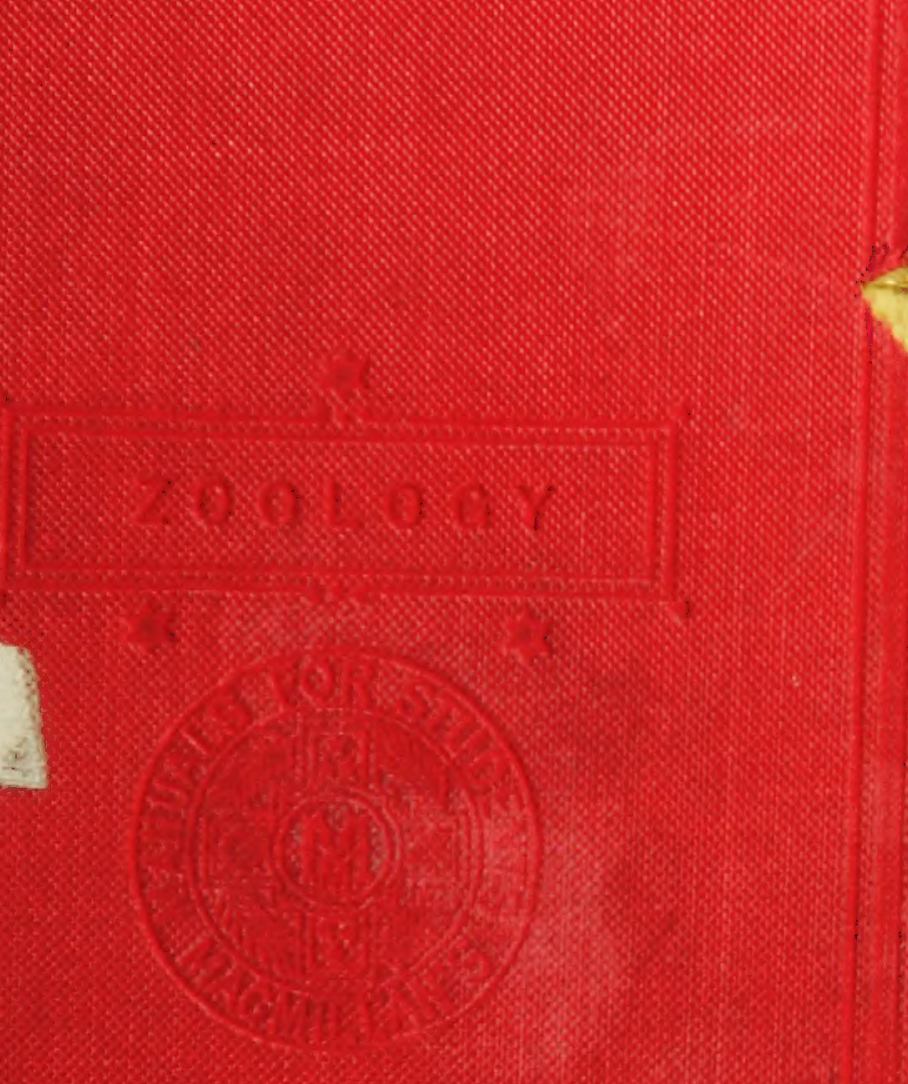


\section{Cowmell alluwersity 道ibraty}

BOUGHT WITH THE INCOME, FROM THE

SAGE ENDOWMENT FUND THE GIFT OF

Henry fll. Sage 1891

A. 152296 
arV17488

Cornell University Library

An elementary course of practical zoolog

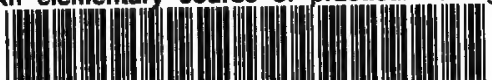

$\begin{array}{lllll}3 & 1924 & 031 & 272 & 291\end{array}$

olin, anx 


\section{Cornell University Library}

The original of this book is in the Cornell University Library.

There are no known copyright restrictions in the United States on the use of the text.

http://www.archive.org/details/cu31924031272291 


\section{AN ELEMENTARY COURSE}

$$
\text { OF }
$$

\section{PRACTICAL ZOOLOGY}





\section{AN ELEMENTARY COURSE}

\section{OF \\ PRACTICAL ZOOLOGY}

BY THE LATE

T. JEFFERY PARKER, D.Sc., F.R.S. PROFESSOR OF BIOLOGY IN THE UNIVERSITY OF OTAGO DUKEDIN, NEW ZEALAND

$\because$ AND

\section{W. N. PARKER, Ph.D.}

PROFESSOR OF ZOOLOGY AT THE UNIVERSITY COLLEGE OF SOUTH WALES AND MONMOUTHSHIRE, IN THE ÚNIVERSIT' OF WALES

With One Hundred and Fifty-six Illustrations

\section{些 oñon}

\section{MACMILLAN AND CO., Limited NEW YORK: THE MACMILLAN COMPANY}


Richasd Clay and Sons, Limited, LONDON AND BUNGAY. 


\section{PREFACE}

IN the early part of 1897 , my brother and I had arranged to collaborate in writing a practical text-book of Elementary Zoology, adapted more particularly to the requirements of Students pursuing courses in the subject as laid down by various examining bodies. We had, however, only reached the stage of deciding on a general plan at the time of my brother's death in November of the same year.

The following are the chief points on which we had agreed :-

r. To adopt the method pursued in Huxley and Martin's Elementary Biology of giving a connected account of each example. 2. To give brief practical directions which should serve mainly as a guide, the student being able to refer, in case of difficulty, to the descriptive accounts preceding them. 3. In the larger animals, to arrange for as much work as possible to be done on one specimen: there is much to be said in favour of this plan apart from the fact that the average student cannot give sufficient time to the subject to dissect a fresh specimen for each system of organs. 4. To begin the course of instruction by an introductory study of one of the higher animals; to include in this introduction the elements of Histology and Physiology; and 
to select the Frog for the purpose: after trying various methods, I have found this plan to be the most satisfactory in practice. 5. To give drawings and diagrams of difficult dissections, and of details which the beginner cannot as a rule make out satisfactorily for himself; but otherwise to limit the number of illustrations so as not to tempt the student to neglect observing the things themselves. 6. To include a short account of methods and technique, limited to the barest essential outlines, sufficient for a student working by himself to make out the things described, but not going into such details as would naturally be learnt in a properly organised laboratory.

In the meantime, my brother had in preparation a Biology for Beginners, in which he intended to carry out the plan, suggested in the preface to his Elementary Biology, of giving a simple account, with practical directions, of one of the higher animals and one of the higher plants, as an introduction to the study of Biology. The animal he selected was the Frog, and the manuscript of the greater part of this section of the book was already finished and the rest in rough draft. He had previously suggested that some of this work might be utilised for our proposed Practical Zoology; and I found that, with certain additions and with modifications in the arrangement, the whole of it was exactly the kind of introduction $I$ had in view for the first part of our book. Some of the illustrations that my brother had intended to insert in the Biology for Beginners I have found it advisable to omit, and even now the figures in the introductory part are purposely nearly as numerous as those in the rest of the book. But apart from these various minor 
modifications, Chapters I--XII in Part I are almost entirely taken from my brother's manuscript.

We felt that there could be no object in entirely rewriting the descriptions of several familiar animals already given in my brother's published works; and in Part II. I have intentionally made use of these descriptions, borrowing very freely from the Elementary Biology, as well as (with Professor Haswell's permission) from the Text-book of Zoology; and, to a less extent, from the Zootomy.

The practical directions are mainly based on a series of Laboratory-instructions I drew up some years ago for the use of my junior classes, which consist principally of students preparing for the Intermediate Science examination of the University of Wales, the Preliminary Scientific examination of the London University, and the first examination of the Conjoint Board of the Royal Colleges of Surgeons and Physicians. The time such students can devote to an elementary course in the subject is limited; and throughout the book I have borne in mind that the main object of teaching Zoology "as a part of a liberal education is to familiarise the student not so much with the facts as with the ideas of the science," but at the same time that he should be provided with a sound basis of facts so arranged, selected, and compared as to carry out this principle.

Our original intention was to include one or more examples of each of the larger phyla, and also to add a practical exercise after each type, giving general directions for the examination of an allied form for comparison. But I found that this would be impossible within the space of a 
single volume, and it was therefore necessary to limit the descriptions mainly to those animals to which the students for whom the book is chiefly intended have to give special attention. This has resulted in rather a heavy balance on the side of Vertebrates; but on the whole, I think that if sufficient work is done on the lower animals to illustrate certain main facts and generalisations, a comparative study of several Vertebrates forms as good a training as any for beginners-more especially in the case of medical students.

I am indebted to Mr. H. Spencer Harrison, B.Sc., Demonstrator of Biology in this College, for much assistance in testing and improving the practical instructions, as well as for various suggestions and for help while the work was passing through the press. The new figures were re-drawn from the originals by Mr. M. P. Parker.

$$
\text { W. N. PARKER. }
$$

University College, CardifF,

November, 1899. 


\section{CONTENTS}

PREFACE . . . . . . . . . . . $v$

\section{PART I}

CHAPTER I

SCOPE OF THE SCIENCE OF BIOLOGY-THE FROG : PRELIMINARY SKETCH OF ITS STRUCTURE, LIFE-HISTORY, AND VITAL FUNC'TLNS . . . . . . . . . . . . . . . . I HINTS ON DISSECTION . . . . . . . . . . . . . I2

\section{CHAPTER II}

THE FROG (continued): GENERAL INTERNAL STRUCTURE . . I6. PRACTICAL DIRECTIONS . . . . . . . . . . . . . 3 I

CHAPTER III

THE FROG (continued): THE SKELETON . . . . . . . 35

PRACTICAL DIRECTIONS . . . . . . . . . . . 53

\section{CHAPTER IV}

- THE FROG (continued): THE JOINTS AND MUSClES . . . . 55 PRACTICAL DIRECTIONS . . . . . . . . . . . . 64

\section{CHAPTER V}

THE FROG (continued): WASTE AND REPAIR OF SUBSTANCE 一THE DIGESTIVE ORgans-NuTRITION . . . . . . . 66 PRACTICAL DIRECTIONS . . . . . . . . . . . . . 76 


\section{CHAPTER VI}

PAGE

THE FROG (continued): THE VASCULAR SYSTEM--THE CIRCU-

LATION OF THE BLOOD . . . . . . . . . . 78

PRACTICAL DIRECTIONS . . . . . 98

\section{CHAPTER VII}

THE FROG (continued): 'IHE MICROSCOPICAL EXAMINATION OF THE SIMPLE TISSUES . . . . 104 PRACTICAL DIRECTIONS . . . . . II9

CHAPTER VIII

THE FROG (continued): THE MICROSCOPICAL EXAMINATION OF THE COMPOUND TISSUES-GLANDS-SECRETION AND ABSORPIOX . . . . . . . . . 126 PRACTICAL DIRECTIONS . 135

CHAPTER IX

THE FROG (continued): RESPIRATION AND EXCRETION . . . I4I PRACTICAL DIRECTIONS... . . . . . . . . I I52

CHAPTER X

THE FROG (contialual): THE NERYOUS SYSTEM. . . I54 PRACTICAL DIRECTIONS . . . . . . I75

\section{CHAPTER XI}

THE FROG (continued) : THE ORGAX'S OF SPECIAL SENSE . 179 PRACTICAL DIRECTIONS . . . . . . . I9I

\section{CHAPTER XII}

THE FROG (continued): REPRODUCTION AND DEVELOPMENT. 193 PRACTICAL IJIRECTIONS . . . . .

\section{CHAPTER XIII}

THE FROG (conlinued) : MEANING OF THE TERM SPECIES-THE PRINCIPLES OF CLASSIFICATION-EVOLUTION-ONTOGENY AND PHYLOGENY-HEREDITY AND VARIATION-STRUGGLE FOR EXISTEXCE-SELECTION-ORIGIN OF SPECIES 


\section{PART II}

CHAPTER I

AMGBA-UNICELLULAR AND MUITICELLULAR ANIMALS . . $\begin{array}{r}\text { Page } \\ 229\end{array}$ PRACTICAL DIRECTIONS . . . . . 238

\section{CHAPTER II}

HAMATOCOCCUS AND EUGLENA-MONADS AND BACTERIA-DIFFERENCES BETWEEN ANIMALS AND PLANIS-SAPROPHYTES 240 PRACTICAL DIRECTIONS . . . . . 259

\section{CHAPTER III}

PARAMGEIUM, OPALINA, VORTICELLA AND ITS AILIES-PARASITIC AND COLONIAL ORGANISMS-BIOGENESIS AND ABIOGENESIS-CLASSIFICATION OF THE UNICELLULAR ORGANISMS EXAMINED . . . . . . . . 26I PRACTICAL DIRECTIONS . . . . . . . 286

\section{CHAPTER IV.}

HYIRA : BOUGAINVILLEA-ALTERNATION OF GENERATIONS-

CHARACTERS OF THE PHYLUM CELENTERAT. . . . . $\quad 289$ PRACTICAL DIRECTIONS . . . . 314.

\section{CHAPTER V}

THE EARTHIVORM-CHARACTERS OF THE PHYLUM ANNUI,ATA 318 PRACTICAL DIRECTIONS

CHAPTER VI

THE CRAYTISH-CHARACTERS OF THE PHYLUM ARTHROPODA - 346 PRACTICAL DIRECTIONS . . . . . . . . . . . . . 372

\section{CHAPTER VII}

THE FRESH-WATER MUSSEL-CHARACTERS OF THE PHYLUM MOLLUSCA-ENUMERATION OF THE CHIEF PHYLA OF THE ANIMAL KINGDOM . . . . . . . . . . . . . $38 \mathrm{I}$ PRACTICAL DIRECTIONS . . . . . . . . . . 397 


\section{CHAPTER VIII}

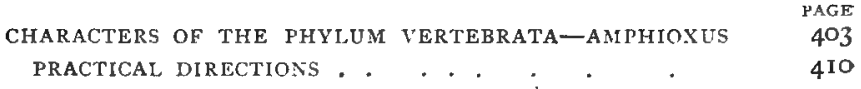

\section{CHAPTER IX}

CHARAC'TERS OF THE CLASS PISCES-THE DOGFISH

\section{CHAPTER X}

CHARACTERS OF THE CLASS MAMMALIA-THE RARBIT . . . 466 PRACTICAL DIRECTIONS. . . . . . 525

\section{CHAPTER XI}

THE MINUTE STRUCTURE OF CELLS-CELL DIVISION-OOGENESIS AND SPERMATOGENESIS-MATURATION AND FERTILIZATION OF THE OVUM-EFFECT OF FOOD-YOLK ON DFTELOPMENT-FORMATION OF THE CHIEF ORGANS OF THE ADULT IN VERTEBRATES, AND OF THE AMNION, ALLANTOIS AND PLACEYTA . . . . . . . . 54 I PRACTICAL DIRECTIONS * . . $\quad 585$

INDEX . . 


\title{
AN ELEMENTARY COURSE OF PRACTICAL ZOOLOGY
}

\author{
PART I
}

\section{CHAPTER 1}

SCOPE OF THE SCIENCE OF BIOLOGY-THE FROG: PRELIMINARY SKETCH OF ITS STRUCTURE, LIFE-HISTORY, AND VITAL FUNCTIONS-HINTS ON DISSECTION

Biology, Zoology and Botany.-There is a good deal of misconception as to the scope of the science of Biology. One often meets with students who think that while the study of animals as a whole is Zoology and the study of plants as a whole Botany, Biology is the study of a limited number of animals and plants, treated as if they had no connection with anything else,--even with one another.

This is quite wrong. Biology is the master-science which deals with all living things, whether animals or plants, under whatever aspect they may be studied. Physiology, treated for practical purposes as a separate subject, is a branch of biology; so is anatomy, to which the medical student PRact. Zool. 
devotes so much time; so are botany and zoology, in the ordinary sense of the words, i.e. the study of the structure, the mutual relations, and the arrangement or classification of plants and animals. But biology may also be pursued, and very profitably pursued too, quite independently of teachers, class-rooms, and examinations. The country boy who knows the song of every bird, its nesting place, the number of its eggs, the nature of its food, the lurking. place of the trout in the stream or the frogs in the marsh; who has watched the ants with their burden of grain, or the bees with their loads of honey or pollen; has begun the study of biology in one of its most important branches. The intelligent gardener who observes the habits of plants, their individual tastes as to soil, moisture, sunshine and the like, is also something of a biologist without knowing it. So also is the collector of eggs, shells, or insects, provided he honestly tries to learn all he can about the things he collects, and does not consider them merely as a hoard or as objects for barter. Indeed, all that is often spoken of as natural history, so far as it deals with living things-plants and animals -and not with lifeless natural objects, such as rocks and minerals, is included under the head of biology.

What then is the connection between biology in this wide sense and the kind of thing you are expected to learn in a limited number of lessons ? Simply this :-In the class-room nature cannot be studied under her broader aspects : indeed, much out-door natural history cannot be taught at all, but must be picked up by those who have a love of the subject, a keen eye, and patience. But there is one thing we can do within the narrow limits of the class-room: we can confine ourselves to some department of biology small enough to be manageable: we can take, for instance, one or more familiar animals and plants, and, by studying them in some 
detail, get some kind of conception of animals and plants as a whole. This book deals with the zoological side of biology only; and what we have now to do is, in fact, what you have often done in the study of English: you take a single verse of a poem at a time, analyse it, parse it, criticise its construction, try to get at its exact meaning. If you have any real love of literature this detailed study of the part will not blind you to the beauty of the whole. And so if you have any real love of nature, the somewhat dry and detailed study we have now to enter upon should serve to awaken your interests in the broader aspects of biology by showing you, in a few instances, what wonderful and complex things animals are.

One word of warning before we begin work. You must at the outset disabuse your mind of the fatal error that zoology or any other branch of natural science can be learnt from books alone. In the study of languages the subject matter is furnished by the words, phrases, and sentences of the language; in mathematics, by the figures or other symbols. All these are found in books, and, as languages and mathematics are commonly the chief subjects studied at school, they tend to produce the habit of looking upon books as authorities to which a final appeal may be made in disputed questions. But in natural science the subject-matter is furnished by the facts and phenomena of nature; and the chief educational benefit of the study of science is that it sends the student direct to nature, and teaches him that a statement is to be tested, not by an appeal to the authority of a teacher or of a book, but by careful and repeated observation and experiment.

The object of this book, therefore, is not only to give you some idea of what animals are, but also to induce you to verify the statements contained in it for your- 
self. The description of each animal you should follow with the animal before you; and if you find the account in the book does not agree with what you see, you must conclude, not that there is something wrong with your subject, but either that the description is imperfect or erroneous, or that your observation is at fault and that the matter must be looked into again. In a word, zoology must be learnt by the personal examination of animals : a text-book is merely a guide-post, and all doubtful points must be decided by an appeal to the facts of nature.

It matters very little what animal we choose as a startingpoint-a rabbit, a sparrow, or an earthworm-one will serve almost as well as another to bring out the essential nature of an animal, how it grows, how it is nourished, how it multiplies. On the whole, one of the best subjects to begin with is a frog: partly because it is easily obtained, partly because its examination presents no difficulties which an intelligent student may not be expected to surmount by due exercise of patience.

Let us therefore begin our studies by catching a frog and placing it in a convenient position for examination, as, for instance, under an inverted glass bell-jar or even a large tumbler.

External Characters.-Notice, first of all, the short, broad trunk, passing insensibly in front into the flattened head - there being no trace of a neck-and ending behind without the least vestige of a tail : these constitute the axial parts of the animal. In the ordinary squatting position the back has a bend near the middle, producing a peculiar humped appearance. The head ends in front in a nearly semicircular snout, round the whole edge of which extends the huge slit-like mouth. On the top of the fore-end of the 
snout are the two small nostrils, one on each side of the middle line; and, some distance behind them, the large, bright, prominent eyes, in which we can distinguish, as in our own eyes, a coloured ring or iris, surrounding a roundish black space or pupil. The eyelids, however, are rather different from our own: the upper is fairly well developed, but the lower is a mere fold of skin, incapable itself of being folded over the eye, but produced into a thin transparent skin, the nictitating membrane, which can be drawn upwards over the eye. The entire absence of eyebrows and eyelashes is a point worthy of notice.

Extending backwards from the eye is a large black patch, in the middle part of which is a circular area of tightly stretched skin, reminding one of the parchment of a tambourine: this is the drum-membrane, or tympanic membrane, a part of the ear. Here again we see a striking difference from our own organs: in ourselves the drummembrane, instead of being flush with the surface of the head, is placed at the inner end of a deep passage or tunnel, the entrance to which is guarded by the large external ear. Of the latter there is no trace in the frog.

Attached to the trunk are two pairs of offshoots or appendages, the arms and legs, or fore-and hind-limbs, in which the resemblance to our own limbs will be at once obvious. The arms are very short: each consists of an upper arm, a fore-arm, and a hand, the latter provided with four fingers, which are slender and tapering and have no nails. The legs, on the other hand, are very long : each consists of a stout thigh, a long shank, with a well-marked "calf," and a very curious foot. The ankle-region is long-almost like a second shank-and has no heel: it is followed by five toes, the first or innermost short, the second of moderate length, the third longer, the fourth longer still, and the fifth 
of about the same length as the third. All the toes are joined together by thin transparent webs, and, like the fingers, have no nails. The name digit is conveniently applied both to fingers and toes. Between the bases of the thighs, at the hinder end of the trunk, is a small aperture, the vent or anus.

In the squatting posture the body is raised upon the arms, which are kept slightly bent at the elbows, with the fingers spread out and directed forwards. In this position the innermost of the four fingers corresponds with our own index finger, the frog having no thumb. The hind-limb, under similar circumstances, is bent into a sort of $Z$, the knee being directed forwards and the ankle-joint backwards. The toes are turned forwards, and the inner one, which is the smallest of all, corresponds with our own great toe.

Owing to the bent position of the limbs, we cannot very well, as in our own arms and legs, speak of their upper and lower ends. It is therefore customary to call the end of a limb, or of any division of a limb, which is nearest to the trunk, the proximal end, that which is furthest away the distal end. Thus the proximal end of the fore-arm is the elbow region, the distal end of a digit is its tip.

The whole body, including head, trunk, and limbs, is covered with a soft, slimy skin, of a brown colour, irregularly spotted with brown or black on the upper or dorsal surface, and whitish on the under or ventral surface. The colouring is, however, not constant; in a frog kept in the dark the black spots increase to such an extent that the whole animal becomes almost black, while if kept in full daylight a corresponding brightening of the tints takes place. Moreover, the spots and patches of brighter colour are very variable: if you examine a dozen specimens you will see at once that no two are alike in this respect The 
large black patch situated behind the eye and containing the tympanic membrane, is, however, always present, and is one of the chief distinguishing marks of the common British frog as compared with other kinds, such as the "edible frog." of the Continent.

Sexual Characters.-As in so many of the more familiar animals there are two sexes of frogs, easily distinguished from one another. If you examine several of them you will find that a certain number have on the palm of the hand, towards the inner side, a large swelling, rather like the ball of our own thumb, but much more prominent and of a black colour. Frogs having this structure are males; there is no trace of it in the females.

Actions performed by the Living Frog.-Kept under suitable conditions a frog very soon shows evidences of life. If touched or otherwise alarmed it attempts to escape by making a series of vigorous leaps - suddenly extending the hind-legs and jumping to a considerable height. Thrown into water it swims by powerful strokes of the hind-limbs. It has thus, like so many living things with which we are familiar, the power of voluntary movement.

If kept under observation for a sufficient time-weeks or months-it will be found that frogs grow until they reach a certain limit of size. Growth, in the case of the frog, is an increase in size and weight affecting all parts of the body, so that the proportions remain practically unaltered, and no new parts are added.

Careful observation shows that the throat is constantly rising and falling, and the nostrils opening and shutting. These movements, like the expansion and contraction of the human chest, are respiratory or breathing movements, and serve to pump air into and out of the lungs.

It requires frequent watching and sharp observation to see 
a frog feed. It lives upon insects, worms, slugs, and the like. Opening its mouth it suddenly darts out a tolerably long, nearly colourless, and very sticky tongue; if the prey is a small insect, such as a fly, it adheres to the end, and the tongue is quickly drawn back into the mouth, the whole operation being performed with almost inconceivable rapidity.

Like other animals the frog discharges zeaste matters from its body. Its droppings or foeces, discharged from the vent, are black and semi-solid. From the same aperture, it expels periodically a quantity of clear fluid, the urine, which is perfectly clear and colourless, and contains little beyond water.

Sometimes a frog will escape from confinement, leaving its damp box or vivarium for the warm, dry atmosphere of an ordinary room. When this happens the animal is usually found next morning dead and shrunken, and with its naturally moist skin dry and hard. From this it may be inferred that there is a constant evaporation of zeater from the skin, which, under ordinary circumstances, is checked by a damp atmosphere or by occasional immersion in water.

Hibernation.-In winter frogs bury themselves in damp places and become sluggish, all manifestations of life becoming hardly apparent until the following spring, when they emerge from their holes. In this way they escape the dangers of frost which would otherwise be fatal to them. This suspension of activity during winter is known as hibernation, or the winter sleep.

Reproduction and Development.-If you examine a number of frogs towards the end of winter-about February in England-you will find that the full-grown females are distinguished from the males, not only by the absence of 
the pad on the hand, but by the swollen condition of the trunk, due to the interior being distended with eggs. After a time the eggs are laid, being passed out of the vent by hundreds; each is a little globular body about $\frac{1}{12}$ th inch in diameter, half black and half white, and surrounded by a sphere of clear jelly, by means of which the eggs adhere together in large irregular masses, the well-known "frogspawn." As the eggs are laid the male passes out of his body, also by the vent, a milky fluid, the milt or spermatic fuid, which gets access to the eggs and impregnates or fertilises them. Without impregnation they are incapable of developing.

Neither male nor female takes the slightest care of the eggs when once they are deposited and fertilised. They are simply left in the water unprotected in any way; and, naturally enough, the mortality among them during the course of development is very great, the majority being eaten or otherwise destroyed, and only a very small percentage coming to maturity.

The first noticeable change in the spawn is that the sphere of jelly surrounding each egg swells up so as to acquire several times the diameter of the enclosed egg. The egg itself, or embryo, as it must now be called, gradually becomes entirely black, then elongates, and takes on the form of a little creature (Fig. I, I), with a large head, a short tail, and no limbs; which, after wriggling about for a time, escapes from the jelly and fixes itself, by means of a sucker on the under side of its head, to a water-weed. Great numbers of these tadpoles, as the freeliving immature young or larva of the frog are called, may be seen attached in this way. At first they are sluggish and do not feed, but, before long, they begin to swim actively by lashing movements of their tails, and to browse on the 
weeds. They are thus in the main vegetable-feeders, not carnivorous, like the adult frog. On each side of the head appear three little branched tufts or gills, which serve as

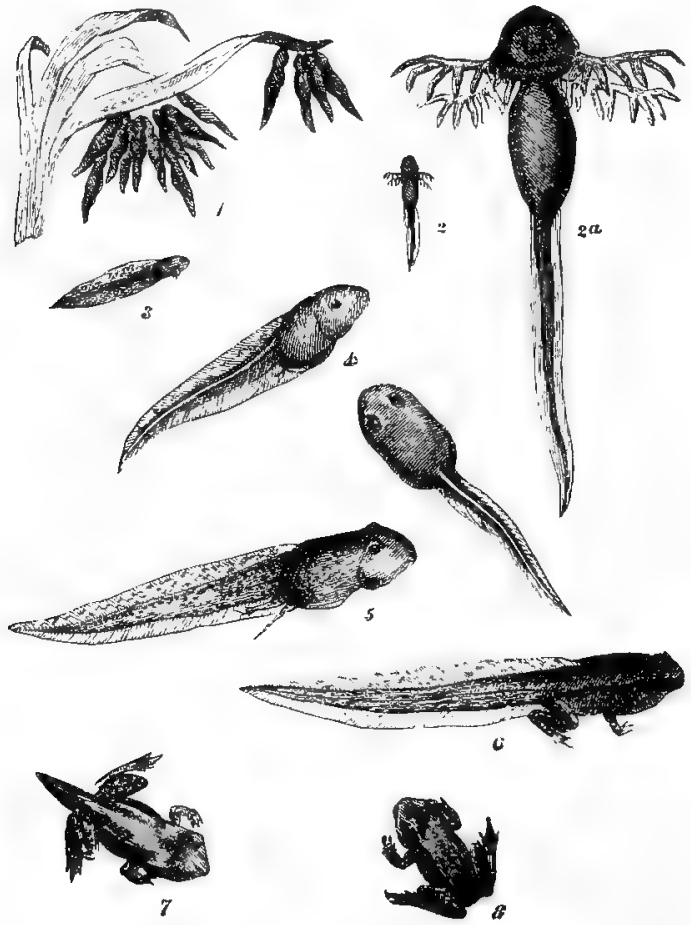

FIG, 1,-Stages in the life-history of the Common Frog, from the newly-hatched tadpoles ( $I$ ) to the young frog ( 8 ); $2 a$ is a magnified view of 2 . (After Mivart).

respiratory organs $\left(2,2^{a}\right)$, the tadpole, like a fish, breathing air which is dissolved in the water. After a while the gills shrivel up and the tadpole then comes periodically to the surface to breathe, lungs having in the meantime made 
their appearance. A little pair of hind-limbs appears at the root of the tail, and a pair of fore-limbs behind the head $(5,6)$. As these increase in size the tail slowly dwindles, the head and trunk assume the characteristic frog-form, and the little animal now comes on land and hops about as a small, tailed frog (7). As growth goes on the tail further diminishes and finally disappears altogether, the transformation or metamorphosis being thus completed (8).

Death and Decomposition.-Frogs may live for many years, but, sooner or later, either in the ordinary course of nature or by accident, they die. The heart stops beating, the flesh undergoes what is called "death-stiffening," becoming hard and rigid, and all vital manifestations cease. Before long the process of decomposition ensues, the flesh, viscera, etc., soften and emit a bad smell, and in course of time rot away completely, leaving only the bones.

Summary of Chapter.-The very brief and cursory study we have made so far shows us (I) that a frog has certain definite parts arranged in a particular way; (2) that it performs characteristic movements, some of them, such as leaping and swimming, voluntary; others, such as breathing, involuntary ; (3) that it takes in solid food, consisting mainly of vegetable matter in the tadpole, of living animals in the adult; (4) that it gives off waste matters; (5) that it reproduces its kind by laying eggs, which develop only if impregnated; (6) that it undergoes a transformation or metamorphosis, the egg giving rise to a larva, the tadpole, which, after living for a time the life of a fish, gradually changes into a frog. 


\section{HINTS ON DISSECTION}

\section{Instruments and other Requisites for Dissection. ${ }^{-}$-In} order to carry out the dissection of the frog and other animals successfully it is necessary to be provided with proper tooIs. The most important are-

I. Three or four sharp dissecting knives, or scalpels, of different sizes.

2. A large and a small pair of straight dissecting forceps; the small pair should have a peg on one leg fitting into a hole on the other, to prevent the points crossing; the points should be roughened.

3. A large and a small, fine-pointed pair of dissecting scissors; the small pair for the more delicate work, and the large pair for coarser work and for cutting through bones. For the latter purpose a pair of bone-forceps is useful, but is not necessary in the case of such a small animal as the frog.

4. A seeker, i.e., a blunt needle mounted in a handle.

5. Three or four probes: a seeker or knitting needle, or a thin slip of whalebone will answer for some purposes, but the most generally useful form of probe is made by sticking the end of a hog's bristle into melted sealing-wax, and immediately withdrawing it so as to affix a little knob or guard.

6. An anatomical blorepipe, or, failing this, a piece of glass-tubing, 6 or 8 inches long, with one end drawn out in the flame until it is not more than $\frac{1}{1} \frac{1}{6}$ th to $\frac{1}{2} \delta$ th of an inch in diameter.

7. An ordinary " medicine-dropper," or "feeder" of a self-feeding pen (see Fig. 25), made of a piece of glass-tubing about 3 inches long, drawn out in the flame at one end, and thickened at the other, so as to form a collar, over which an india-rubber cap-an ordinary nonperforated teat-is fixed. This is useful for washing fine dissections, as well as for injecting.

8. A dissecting dish. Get a common white pie-dish, about 6 or 8 inches long, with rather low sides. Cut out a piece of self-coloured (brown) cork-carpet or thick linoleum the size of the bottom of the dish, and a piece of sheet lead of the same size, and fasten the two together by three or four ties of copper wire or strong thread. Place this in the

${ }^{1}$ A suitable box of dissecting instruments can be bought from most scientific instrument makers for about $£ \mathrm{I}$. (For further apparatus required in connection with injection and microscopical work, see pp. 99, I 19 , and 135 .) 
dish with the lead (which is simply to keep the cork-board from floating in water) downwards. Or, place a few strips of sheet lead in the bottom of the dish, and then pou in some melted paraffin-wax into which a little lamp-black has been stirred, so as to make a layer half an inch or more in thickness.

For larger animals than the frog, in addition to a larger dish, a dissecting board will also be required. Get a piece of soft deal or pine about 18 inches $\times 11$ inches and 8 inch thick, and nail round its edge a strip of wood about $\frac{3}{4}$ inch $\times \frac{1}{4}$ inch, so as to form a projecting rim.

9. A magnifying glass. Any good pocket lens or a common watchmaker's glass will answer the purpose. As it is often desirable to have both hands free while using the lens, a stand of some kind is useful. One of the simplest is made by fixing a piece of thick flexible wire, 6 or 8 inches long, into a heavy block of wood, 3 or 4 inches in diameter; the free end of the wire is bent into a loop to carry the lens, which can thus, by bending the wire, be raised or lowered as required. Or, get a piece of narrow clock-spring, about I 3 inches long, and rivet one end of it to the outside of the rim of a watchmaker's glass, and the other to a small piece of zinc or brass; on passing the spring round the head, the lens is kept in place at the eye without exertion.

10. Medium and small-sized pins. Large blanket pins are useful for fixing down larger animals.

II. A small sponge and a duster.

12. One or more wide-mouthed bottles or jars, containing a preserzative in which to place your subjects after each day's work. The most convenient preservative for the purpose in most cases is the fluid sold as formaline, ${ }^{1}$ which can be diluted with water as it is wanted. For preserving your dissections from day to day, a I per cent. solution of formaline is strong enough in many cases-i.e., I cubic centimetre of formaline to 99 c.c. of water, or three-quarters of a dram of formaline to half a pint of water. For permanent preservation, a stronger solution -2 to 4 per cent., according to circumstances-should be used, or methylated spirit. If formaline is not available, use strong methylated spirit (i.e., about 90 per cent.) diluted , with one-third of its bulk of water.

13. A plentiful supply of clean water.

14. An ounce or two of chloroform.

1 A 40 per cent. solution of the gas formic aldehyde. 
Rules to be observed in Dissection.-Many of the parts and organs of animals are bound together by means of a substance known as "connective-tissue," and the main object of dissection is to tear away and remove this substance so as to separate the parts from one another.

The subject should be firmly fixed down in the dissecting-dish or on the dissecting-board by means of pins, inserted obliquely, so that they do not interfere with the dissection. The dissecting-dish must always be used for finer dissections, which should be done under water; only just enough water being put into the dish to cover the dissection, which should be washed under the tap from time to time.

When dissecting a part keep it on the stretch, and avoid fingering it or damaging it with the forceps.

Never remove anything until you know what you are removing. Dissect along, and not across, such structures as blood-vessels and nerves.

See that your instruments are kept clean and sharp, and never use the smaller scissors and scalpels for coarse work.

Drawing. - Iou should make a point of drawing as many of your preparations, as well as of the living animals, as possible : an accurate skétch, taken fronı Nature, no matter how rough, is of more value in teaching observation and in impressing the facts on your memory than the examination and copying of more perfect drawings made by others. Any one can soon learn to make sketches of this kind, even without having any previous knowledge of drawing.

Each sketch should be made to scale, and small objects should be enlarged several times; it is much easier to insert details in a large drawing than in a small one. Mark the scale against each drawing-e.g., $\times 2$, $\times \frac{1}{2}$.

Using a rule and compasses, first sketch in an outline of the principal parts with a hard pencil; if your object is bilaterally symmetrical, draw a faint line down the middle of the paper, and then sketch in one side first. When you have sketched in all the outlines correctly, go over them again with a softer pencil, so as to make them clear and distinct. Do not attempt any shading unless you have some knowledge of drawing.

Then tint the various parts in different colours, using very light tints except for such structures as vessels and nerves. It is as well to keep to the same colours for the corresponding organs or tissues in all the animals you examine : thus you might in all cases colour the alimentary 
canal yellow, the arteries red, the veins blue, glands brown, cartilage green, and so on.

Make your drawings on one side of the page only ; the opposite side can then be used for explanations of the figures.

Never insert on your original sketches anything you have not actually seen; you can copy as many other figures as you like from various sources, but these should be kept apart from your own original drawings.

Directions for the examination of the external characters of the adult frog, as described in this chapter, will be given at the end of Chapter II. ; and of the eggs and tadpoles in Chapter XII. 


\section{CHAPTER II}

THE FROG (continued): GENERAL INTERNAL STRUCTURE

You have now seen that a frog can perform a number of very complicated actions; and, if you have any curiosity in these matters, you will probably want to know something of the mechanism by which these actions are brought about. Now, the best way to understand the construction of a machine, such as a clock or a steamengine, is to begin by taking it to pieces; and, in the same way, you can find out the parts of which the living machine we call a frog is made, and the way they are related to one another, only by taking it to pieces, or dissecting it.

First notice, in addition to the external characters described in the last chapter, that the various parts of the body are strengthened or stiffened, as in ourselves, by a number of bones, which together form the greater part of the skeleton. It is quite easy to ascertain by feeling that the head contains a hard skull; the lower jaw, a lower-jaw-bone or mandible; that running through the back is a jointed vertebral column, or back-bone; that the region of the chest is protected by a sternum, or breast-bone; and that each division of the limbs has its own bone or bones.

The Mouth-Cavity.--There are also several points to 
be observed in the interior of the mouth. All round the edge of the upper jaw is a row of small conical teeth (Fig. 7). There are no teeth in the lower jaw; but on the roof of the mouth, a short distance behind the snout, are two little patches of teeth, called the vomerine teeth (vo. $t$ ). Just behind these are two apertures, called the internal nostrils ( $p, n a)$ : a guarded bristle passed into one of the external nostrils and pushed gently backwards and downwards, will be found to enter the mouth by the corresponding internal nostril.

Behind the internal nostrils are two large hemispherical projections, due to the roof of the mouth being bulged out by the huge eyes, as can be readily made out by pushing the eyes from outside.

On the floor of the mouth is the large, flat tongue $(t n g)$, remarkable for the fact that it is attached at its front end, its hinder end being free and double-pointed. When the frog uses it to catch insects it is suddenly thrown forwards, almost like a released spring. Just behind the backwardly-turned tip of the longue is an oval elevation, having on its surface a longitudinal slit, called the glottis $(g l)$, which leads, as we shall see afterwards, into the lungs.

The back of the mouth narrows considerably, and the soft skin or mucous membrane lining it is here thrown into folds. A probe gently pushed backwards passes, as we shall see, into the stomach. The narrowed region of the mouth is the throat, or pharynx. On its upper wall, near the angles of the mouth, are two pits: a guarded bristle passed into one of these will be found to come into contact with the corresponding tympanic membrane, which will be pierced if sufficient force is used. The pits are known as the Eustachian recesses or tubes (eus. $t$ ).

Dissection of the Frog: Skin and Muscles.-If a slit is

Pract. Zoor, 
made in the skin of the belly, and a probe pushed in under it, it will be seen that the skin, instead of being firmly attached to the underlying flesh, as in a rabbit or a sheep, is for the most part quite loose, a spacious cavity lying between it and the flesh. Not, however, a single continuous cavity for the whole body: the probe, gently pushed in various directions, is stopped, in front, at about the level of the arms; behind, at the junction of the thighs with the trunk; and at each side, along an oblique line joining the armpit with the thigh. Moreover, by opening the skin of the back, throat, and limbs, and inserting the probe as before, similar cavities will be found in these regions, all separated from one another by partitions, along which the skin is firmly united to the underlying flesh. It will be noticed also that the probe, when withdrawn from any of these cavities, is wet. The cavities contain a watery fluid, called lymph, and are hence known as subcutaneous lymph sinuses (Fig. $7, d$. ly. s, v. ly.s).

When the skin is removed it will be seen that under the skin and separated from it by the lymph-sinuses is a nearly colourless, semi-transparent, fibrous substance, the flesh. At first this appears to be continuous over the whole body, but, by careful dissection with a sharp scalpel, a very delicate, transparent skin, called the fascia, can be separated from the flesh, which is then seen to consist of a number of separate bands (Fig. 2, pct, my. hy, etc.; see also Fig. 16), corered as aforesaid by the fascia, and separated from one another by a kind of packing substance, also very delicate and transparent, and known as connective-tissue. These bands or sheets are the muscles, and the whole of the flesh is made up of distinct muscles, readily separated from one another when once the requisite anatomical skill is attained. Here and there-for instance 
on the top of the head and the front of the shanks-there are no muscles, and the bones are covered only by skin and connective tissue.

Passing along the middle line of the belly is a dark longitudinal streak (Fig. 2, $a b d . v$ ) : this is a blood-vessel, the

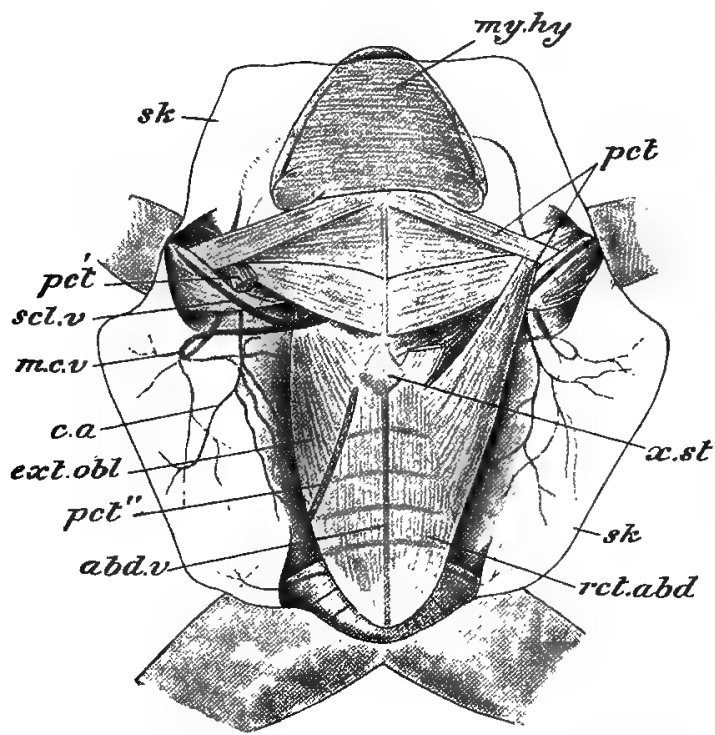

FIG. 2. $\rightarrow$ A frog with the skin $(s k)$ of the ventral surface cut through and turned back right and left, so as to expose the muscles. Of these the mylohyoid (my. hy), pectoralis ( $p c t)$, external oblique $(e x t . o b l$ ), and rectus abdominis ( $r c t . a b a)$ are lettered. On the right side (left in the figure), the posterior portion of the pectoral muscle is cut away, its two ends $\left(p c t^{\prime} p c t^{\prime \prime}\right)$ only being left. The cartilaginous extremity of the breast-bone (xiphisternum, $x$. st) is shown, as well as the abdominal $(a b d . v)$, musculo-cutaneous $\left(m, c_{*} v\right)$ and subclavian (scl. v) veins, and the cutaneous artery $(c, a)$.

abdominal vein. On each side of the body another vein $(m$. c. $v$ ) is seen forming a loop, one limb of which is on the turned-back flap of skin, while the other passes between the muscles not far from the armpit: this vessel is the musculo-cutaneous vein. Both these veins, and many others 
which will be seen in the course of the dissection, are thin. walled tubes full of blood, as will be proved if you should happen to cut one of them, when the blood will escape in considerable quantity.

Between the right and the left fore-limbs the ventral region of the trunk is protected by certain bones which form part of the shoulder-girdle: projecting backwards from this in the middle line is a flat, heart-shaped plate of a softer, gristle-like substance, known as cartilage (compare Fig. I2). Immediately between the thighs a cartilage called the pubis, part of the hip-girdle (Fig. 14), can be felt. Between the shoulder and hip-girdles the ventral body-wall is soft, being formed only of muscle and connective tissue.

The Abdomen and its Contents.-By cutting through the muscles of the belly or abdomen, a large cavity, the bodycavity or calome, is exposed, in which are contained numerous structures presently to be described. In order, however, to open the whole of the cavity the ventral part of the shoulder-girdle must be removed.

In the middle line, between the fore-limbs, and therefore covered in the entire animal by the shoulder-girdle, is a pink conical body (Figs. 3 and 4, v) connected in front with a thin-walled bag, $(r, a u, l$. au) of a purplish colour. The whole thing is the heart: the pink posterior portion is called the ventricle; the purple anterior part consists of two chambers, the auricles. The heart is enclosed in a transparent, membranous bag, the pericardium ( $p c d)$.

Just behind or posterior to the heart are two large masses ( $l r$ ), usually of a dark reddish-brown colour; these are the right and left lobes of the liver. They extend forwards, one on each side of the heart: between them is a globular bag of a greenish colour (Fig. 3, 


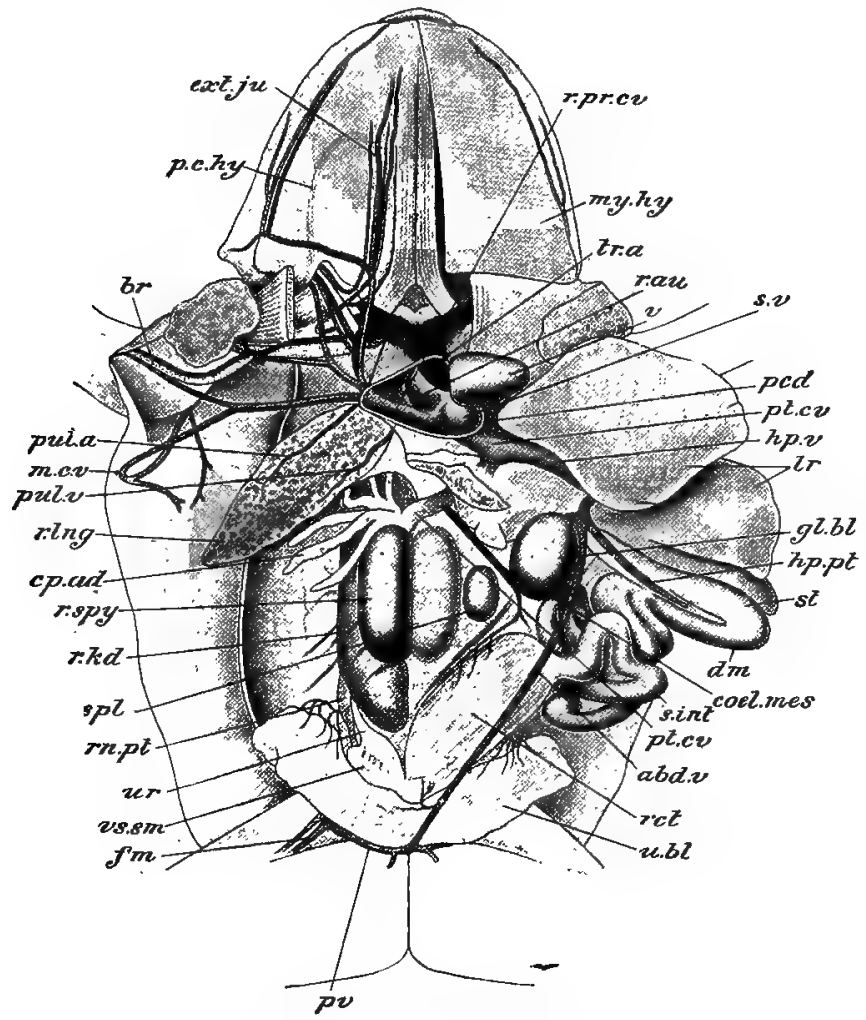

FIG. 3.-Dissection of a Male Frog. The enteric canal and liver are dispiacer to the animal's left, and part of the liver is cut away. Some of the muscles are cut away and certain nerves and blood-vessels traced into the bead and limbs.

$a b d . v$. abdominal vein; $b r$. brachial artery, vein, and nerve; cal. mes. splanchnic or coliaco-mesenteric artery; cp. ad. right fat-body; dm. duodenum; ext.ju. external jugular vein; $f m$. femoral vein; $g l . b l$ gall-bladder; $h p . p t$. hepatic portal vein; $h p . v$. hepatic vein; $l r$. liver; $n . c . v$. musculo-cutaneous vein; my. $h y$. mylohyoid muscle; pcd. pericardium; $p . c . h y$. anterior cornu of hyoid; $p t . c z$. postcaval vein; $\not u l . a$. pulmonary artery; $\not u l . z$. pulmonary vein; $p v$. pelvic vein; $r$. au. right auricle; rct. rectum; $r . k d$. right kidney; $r$. lng. right lung; $r$. pr. $c v$. right precaval vein; $m$. pt. right renal portal vein ; $r$. spy. right spermary; s. int. íleum; $s p l$. spleen; st. stomach; s. $v$. sinus venosus; $t r . a$. conus arteriosus; $u$. $b l$. wrinary bladder; $u r$. ureter ; $v$. ventricle; $v s$. sm. seminal vesicle. 
gl. bl), the gall-bladder. In front of the liver and left and right of the heart are two thin-walled, transparent sacs $(r . \ln g, l . \ln g)$ with a honeycombed surface, the lungs. Their appearance varies very much according to their state of distension. When full of air they are an inch or more in length in a full-sized frog, and protrude freely as soon as the abdomen is opened: when empty they hardly show unless the liver is turned aside.

Emerging from beneath the left lobe of the liver (beneath in the present position of the animai, actually above) is a wide, whitish tube (Fig. 3, st) which almost immediately turns to the right (the frog's right, not yours), so as to form a $\mathrm{U}$-shaped bend $(s t, d m)$. This is the stomach, which is connected with the pharynx by a short tube called the gullet or exsophagus (compare Fig. 7, gul, st), and which varies considerably in size according to whether it is empty or distended with food. The stomach becomes continuous with a narrower tube, the first part of which $(d m)$ passes forwards parallel with the stomach, thus forming the narrow limb of the $U$, while the rest of it (s.int) is thrown into a rather complex coil. This tube is the small intestine; the part in immediate connection with the stomach $(d m)$ is distinguished as the duodenum and the coiled part as the ileum. Between the stomach and duodenum, in the bend of the $\mathrm{U}$, is a small yellowish-white body of irregular form, the pancreas (Figs. $7, p n$, and $18, P$ ).

The stomach and intestine are kept in place and suspended to the dorsal wall of the body-cavity by a delicate membrane, the mesentery (Fig. 5, mes), which is folded in correspondence with the various coils. As we shall see, the mesentery is really a portion of a thin, moist membrane, the peritoneum, with which the body-cavity is lined. 
The small intestine becomes continuous, posteriorly, with a much wider tube (Figs. 3 and $7, r c t$ ), lying against the dorsal wall of the abdomen, and called the large intestine or rectum. It is continued into a short tube, the cloaca $(\mathrm{cl})$, which passes backwards, between the backbone above and the pubis below, to open externally by the vent. Thus the mouth-cavity, pharynx, gullet, stomach, small intestine, rectum, and cloaca form a continuous tube, opening externally at each end, by mouth and anus respectively, and, for the greater part of its extent, contained within the body-cavity. The whole tube is known as the enteric or alimentary canal.

Attached to the mesentery, close to the anterior end of the rectum, is a rounded body of a deep-red colour, the spleen (Figs. 3 and $7, s p l$ ). Quite at the posterior end of the abdominal cavity a very thin-walled and very transparent $\operatorname{sac}(u . b l)$ will be seen, connected with the ventral surface of the cloaca, and varying very much in size according to its state of distension. This is the urinary bladder, which communicates by an aperture (Fig. $7, b l^{\prime}$ ) with the cloaca, and when distended will be seen to be a bilobed sac of considerable size.

If your specimen should be an adult female, and the time of year approaching the breeding season, you will already have observed, as the most prominent organs in the body, two large, lobed structures of a dark colour, protruding one on each side, and partly obscuring the view of the other organs. Each (Fig. 4, l. ovy) contains an immense number of small globular bodies, half black and half white, and is suspended to the roof of the body-cavity by a sheet of peritoneum. 'These bodies are the ovaries, or organs for the manufacture of the eggs; the rounded bodies of which they are largely composed are the eggs themselves. To each ovary is attached a curious structure $(c p, a d)$ of a 


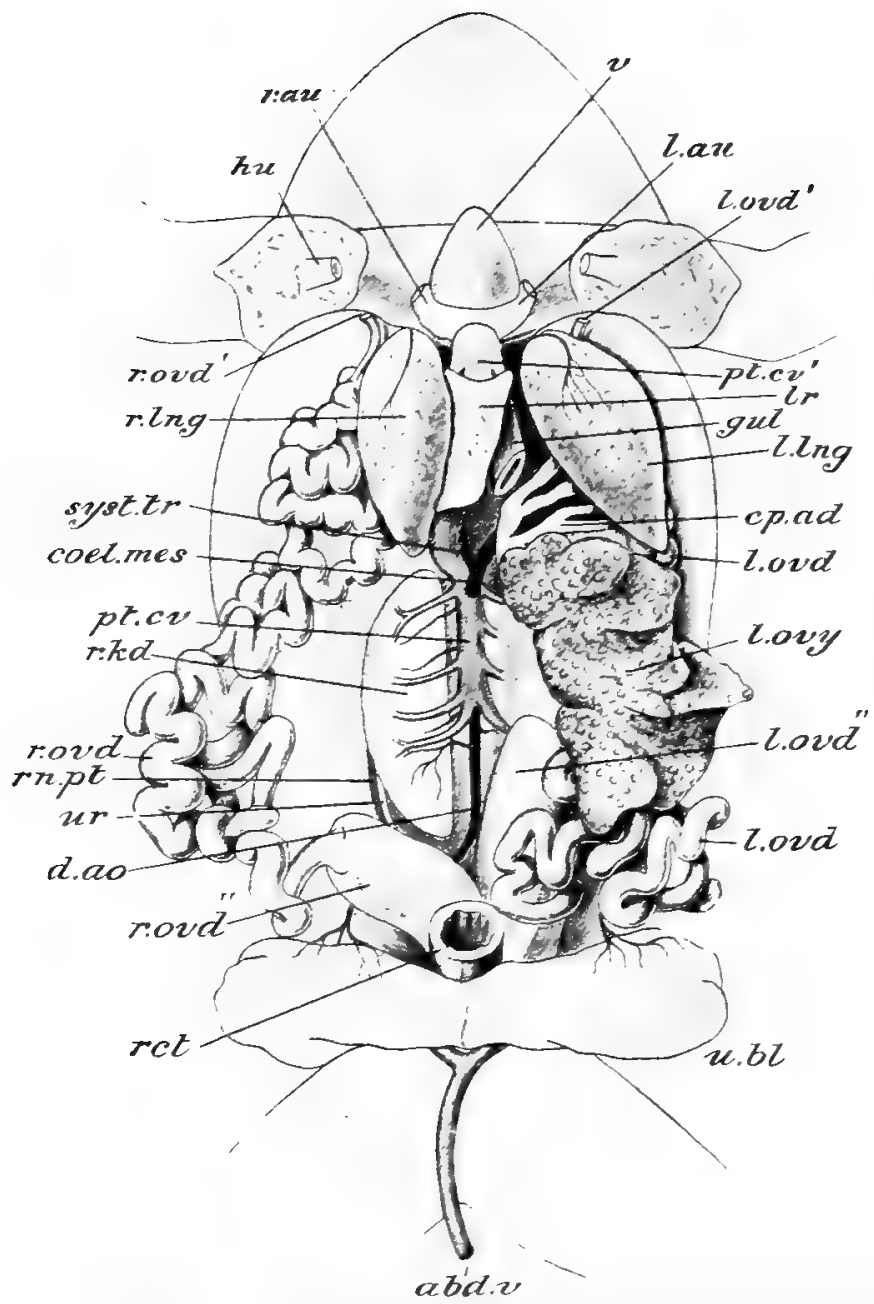

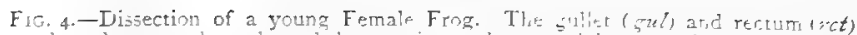
have been cut through, and the enterit ranal remosed between the-e tho prirt-. The liver is removed. with the exception of a small portion (/r) surrounding the pustcaval vein $\left(\not t+\left(c^{\prime}\right)\right.$. The vertricle of the heart $\left(\tau^{\prime}\right)$ is turned forwards. and 
the abdominal vein ( $a b d . z)$ is severed and turned backwards. The right ovary and fat-body are removed, and the right oviduct $\left(r . o v d^{2}\right)$ is slightly displaced outwards.

$a b d . v$. abdominal vein; cal. mes. splanchnic or coliaco-mesenteric artery; $c p$. ad. corpus adiposum, or fat-body; $d$. ao. dorsal aorta; gul. gullet; $h u$. cut end of humerus or upper-arm bone; $l$. $2 u$. left auricle; $l$. ling. left lung; $l .0 v d$. left oviduct; $l$.ovd". its opening "into the body cavity; $l$.ovd". its posteriur dilatation; $l$. ovy. left ovary; $l r$. portion of liver; $p t$. $c v$. postcaval vein ; pt. $c v^{\prime}$. its anterior portion passing between the liver and the heart; $r, a u$. right auricle; rct. rectum; $r$. kd. right kidney; r.lng. right lung; $m$. pt. renal portal vein; $r . o v d$. right oviduct; $r . o z d^{\prime}$. its opening into the body cavity ; $r$. ovd" its posterior dilatation; syst. $t r$. systemic trunks at their point of union; $u . b l$. urinary bladder; $u r$. ureter; $v$. ventricle.

bright yellow colour, and produced into a number of streamer-like lobes; this is the fat-body.

By lifting up either of the ovaries there is seen beneath it -in the natural position of the parts above or dorsal to ita greatly convoluted colourless tube $(l . o v d, r . o v d)$ of about the same diameter as the intestine. This is the oviduct, through which the eggs pass from the ovary to the cloaca. If the specimen is allowed to remain long in water the oviducts will be found to swell and finally to become disintegrated; this is due to the fact that in them is formed the jelly in which the laid eggs are enclosed, and which, as we have seen, swells in water.

In the male there is seen, on turning the intestines aside, a pair of yellow ovoidal bodies (Fig. 3, r.spy) about half an inch long, attached by peritoneum to the dorsal wall of the body-cavity. These are the spermaries or testes ; they manufacture the spermatic fluid or milt by which the eggs are impregnated. To the anterior end of each is attached a fat-body ( $c p . a d)$, like that of the female. In young specimens of both sexes the reproductive organs-spermaries, ovaries, and oviducts-are very small.

When the intestine is turned aside there will also be seen, in both sexes, a pair of flattened, irregularly-oval bodies (Figs. 3 and $4, r . k d$ ) lying in the posterior part of the abdominal cavity just above or dorsal to the ovaries or spermaries. 
These are the kidneys. With the outer edge of each is connected a tube, the ureter $\left(u{ }^{\circ}\right)$, by which the urine, formed in the kidneys, is carried to the cloaca (Fig. 7).

It has been pointed out that the abdomen is lined by peritoneum, and that the various organs are suspended by folds of the same membrane, called, in the case of the enteric canal, the mesentery. The relations of this membrane are best seen in a diagrammatic transverse section of

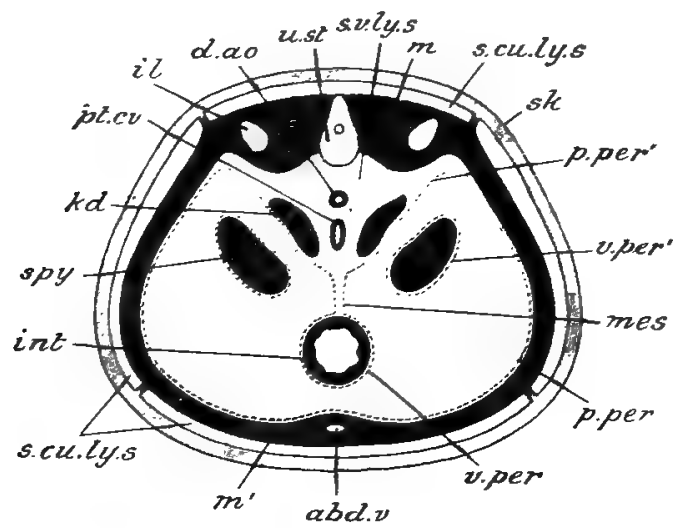

FIG. 5,-Diagrammatic transverse seztion through the trunk of a frog, to show the relations of the peritoneum.

$a b d$. $z$. abdominal vein; $d$. ao. dorsal aorta; il. ilium; int. intestine; $k d$. kidney; $m$. muscles of back; $m^{s}$. muscles of abdomen; mes. mesentery; $p$.per. parietal layer of peritoneum; $p . p e r$. the same, turning down to cover the kidney; $p t . c v$. postcaval vein; sk. skin; s.cu. ly.s. sub-cutaneous lymphsinuses; spy. spermary; s. $v$. ly. s. sub-vertebral lymph smus; $u$. st. urostyle (part of the vertebral column); $v$. per. visceral layer of peritoneum, investing intestine; $v$. per'. the same, investing spermary.

the body (Fig. 5), though many points can be perfectly well made out from the actual specimen. The body-cavity is lined by what is called the parietal layer of the peritoneum ( $p$. per), which adheres closely except in the middle dorsal region, where it leaves the body-wall and becomes closely applied to the ventral surface of the 
kidneys and reproductive organs. Leaving these, the peritoneum of the right approaches that of the left side, and the two, coming into contact, form a double vertical sheet, the mesentery (mes), which extends ventrally toward's the enteric canal. On reaching the latter, the two layers diverge again and surround the canal, forming the visceral layer of peritoneum (v. per). The liver, oviducts, etc., are suspended and covered in the same way. Thus the lining of the bodycavity, the investment of the various organs contained in it, and the folds by which they are suspended, are all parts of one continuous membrane. The space left between the two diverging layers of peritoneum, in the mid-dorsal region, contains lymph, and is known as the sub-vertebral lymph sinus (s. v. ly.s).

We have already noticed the abdominal and musculocutaneous veins. Other veins of greater or less size will be seen everywhere, passing, for instance, to the head and limbs (Fig. 3), and in the mesentery. Running parallel with many of the veins are smaller vessels, many of which have pigment in their walls, and which are of distinctly stouter texture. These are the arteries. They contain little blood in the dead animal, and, owing to the stoutness and elasticity of their walls, do not collapse when empty. Hence they are quite easy to see in a frog from which all the blood has been drained, while the thin-walled veins are almost invisible under like circumstances. Finally; there will be seen in many parts of the body, often lying parallel to an artery and a vein, white cords, the nerves.

The Neural Cavity and its Contents.-By turning the frog with its back upwards and cutting through the muscles of the back and the arches of the vertebræ (see Fig. 6), as well as, in front, the roof of the skull, you will see that the backbone contains a distinct cavity, the neural canal, in 
which lies a white rod, made of the same soft, pulpy substance as the nerves, and called the spinal cord (Fig. 6, $s p .(d)$, which ends behind in a thread-like prolongation $(f . t)$, some distance in front of the thighs. It will also be found that the neural canal is continued, with a. slightly

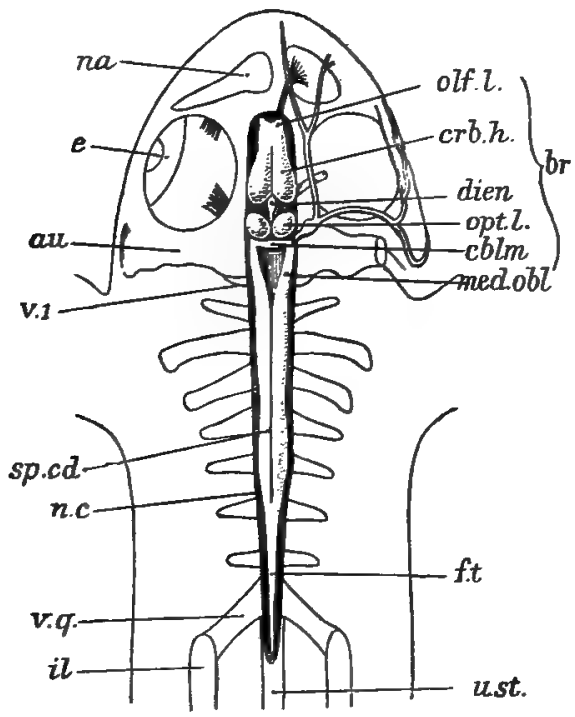

FIG. 6.-Dissection of a Frog in which the entire neural canal has been opened from above, and the brain (br) and spinal cord $(s p . c d)$ laid bare. The brain consists of olfactory lobes (olf.l), cerebral hemispheres (crb.h), diencephalon (dien), optic lobes (opt. l), cerebellum ( $(b l m)$, and medulla oblongata (med.obl), which will be referred to in Chapter $X$. The spinal cord ends in a delicate prolongation, the filum terminale $(f . t)$. The nasal bones $(n a)$, eyes (e), auditory region of the skull $(a u)$, transverse processes of the nine vertebra $(i, f-v, g)$, urostyle (u.st) and ilia (il) are indicated in outline, and serve as landmarks. (After Howes, slightly altered.)

increased diameter, into the skull, and that the spinal cord becomes continuous with the brain (br), a complex organ formed of several parts, which will be referred to hereafter.

General Structure of the Limbs.-A transverse section 
cut across one of the legs, at about the middle of the thigh, will show in the middle of the cut surface the thigh-bone, around it the flesh or muscle, and around this again the skin. Similar cuts through various parts of both fore- and hind-limbs show that these appendages of the body are solid,

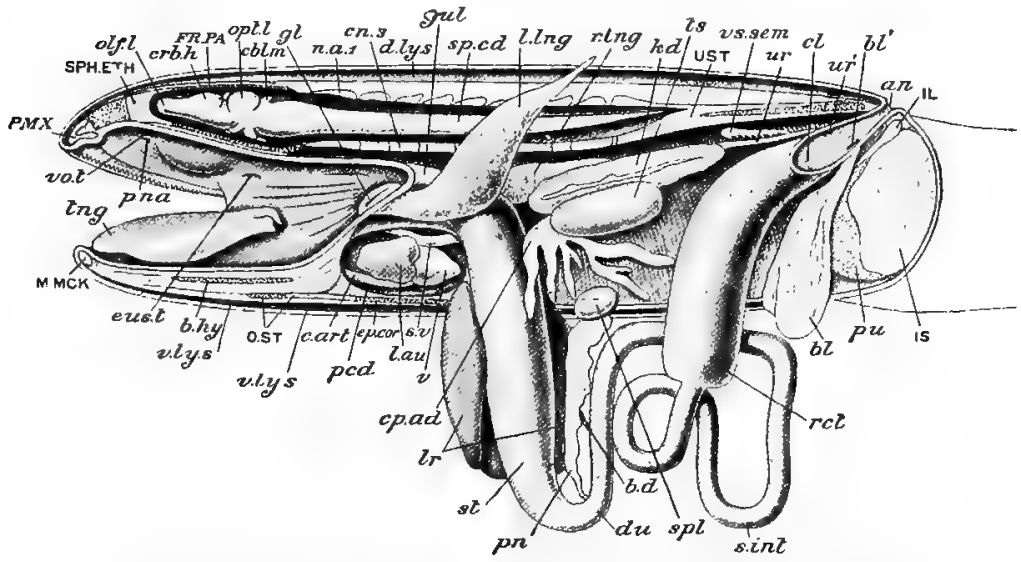

FIG. 7,-Dissection of a Male Frog from the left side. The left fore- and hind-limbs and the left sides of the head and trunk have been cut away, the enteric canal and liver are displaced downwards, and the mouth, pharynx, and cloaca laid open.

$a n$. anus; $b . d$. bile-duct; $b, k y$. body of hyoid ; $b l$. urinary bladder; $b z^{\prime}$, its opening into the cloaca; $c$. art. conus arteriosus; cblm. cerebellum; $c l$. cloaca; $c n .3$, centrum of third vertebra; $c \hat{p} . \alpha a^{\prime}$. corpus adiposum; $c r b . h$. cerebral hemisphere; d. $l y$. s. dorsal lymph sinus; $d u$. duodenum; ep. cor. epi-coracoid; eus. $t$. Eustachian recess; $F R$. $\mu A$. fronto-parietal; $g l$. glottis ; gzh. gullet ; IL. ilium; IS. ischium ; $k d$. kidney; $l$. au. left auricle; $l$. lng. left lung; $l \%$. liver ; M.MCK. mento-meckelian bone; $n . a . I$, arch of first vertebra; olf. $l$. olfactory lobe; opt. 2. optic lobe; O. ST. epi- and omo-sternum; pcd. pericardium; $P M T X$. premaxilla ; $p n$. pancreas; $p$. na. internal nostril; $p u$. pubis ; rct. rectum ; $r$. lng. right lung; s. int. ileum; sp. cd. spinal cord; SPH.ETH. sphenethmoid; spl. spleen; st. stomach; s. $v$. sinus venosus; thg. tongue; ts. spermary; $u r$. ureter; $u \gamma^{\prime}$. its aperture into the cloaca; UST. urostyle: $v$. ventricle; $v . l y . s$. ventral lymph sinus; zo.t. vomerine teeth; zs. sem. seminal vesicle. (From Parker and Haswell's Zoology.)

containing no cavities, except the sub-cutaneous lymphsinuses previously observed.

Summary.-We thus get a notion of the general plan of construction of a frog as follows. It consists of a central or 
axial portion, the head and trunk, and of two pairs of lateral offshoots or appendages, the fore- and hind-limbs. The trunk is hollowed out into two cavities: the abdominal or bodycavity (calome) below, and the neural canal above; of these the neural cavity alone is continued into the head. The abdominal cavity contains the greater part of the enteric canal, the liver, gall-bladder, pancreas, spleen, lungs, heart, kidneys, urinary bladder, and reproductive organs. The neural canal contains the brain and spinal cord. The anterior end of the enteric canal is continued forwards into the head, forming the mouth-cavity, and opens externally by the mouth aperture; its posterior end opens externally by the anus. The enteric canal passes through the containing body-cavity, having no communication with it. The lungs open into the pharynx, and thus communicate with the exterior not only by the mouth but also by the nostrils. The kidneys, bladder, and oviducts communicate with the cloaca, and thus with the exterior through the anus. Neither the neural nor the abdominal cavity has any communi. cation with the exterior. The walls of the head and trunk consist largely of muscles and bones covered with skin. The limbs are solid outgrowths of the trunk, formed also mainly of muscle, with bony supports and a covering of skin.

Organs. - Notice that the body consists of various definite structures, or organs as they are technically termed, which have various purposes or functions to perform. The enteric canal, together with the liver and pancreas, are organs of digestion; the lungs and skin, organs of respiration or breathing ; the heart and blood-vessels organs of circulation, serving as they do to propel and conduct the blood through the body; the kidneys, aided by the skin, organs of excretion, for getting rid of waste matters; the ovaries and spermaries, organs of 
reproduction; the muscles, organs of movement; the brain and spinal cord, together with the nerves, organs of control, serving to direct or control the actions of the body; the skin, nose, eye, and ear, sensory organs, by which communications are kept up with the external world.

Tissues.-Notice also that the various parts of the body are built up of different materials, or tissues as they are called. We have already distinguished muscle, bone, cartilage, connective-tissue and nervous 'tissue. Other tissues we shall meet with in the course of a more careful examination.

\section{PRACTICAL DIRECTIONS.}

To kill a Frog for Dissection.-Place a frog on a plate, and cover it with a tumbler, or put it into a stoppered bottle. Soak a little bit of cotton-wool or sponge in chloroform, and push it under the edge of the tumbler, or drop it into the bottle. In a few minutes the vapour will make the animal quite insensible, and a somewhat longer exposure will kill it painlessly.

External Characters. - Observe the voluntary and the involuntary respiratory movements of the living animal, and compare with a dead frog when making out the external characters (pp. 4-8) and the position of the various parts of the skeleton ( $p, 16)$.

Sketch the entire animal from the side or from above.

The Cavity of the Mouth.-Gently open the mouth of a dead frog as wide as possible, and make out the points described on Pp. I6 and 17 . Sketch.

The Body-wall. - Lay the frog on its back in the dissecting-dish, and fix it firmly by sticking pins through the skin of the arms and legs. With the forceps, held in the left hand, pinch up the skin of the abdomen near the middle line between the thighs, and make a nick in it with the points of the scissors. Then, holding the edge of the hole thus made with the forceps, pass in a probe and push it forwards as far as it will go without opposition. Note:-

The sub-cutaneous lymph-sinuses, and the underlying muscle.

With the scissors extend the incision made in the skin of the belly forwards, in a straight line, to the chin, Holding up the edge of the 
skin with a forceps, cut through, with a scalpel, the partitions between adjacent lymph-sinuses, so as to separate the whole of the skin of the ventral surface from the muscle, and, having done so, pin back the flaps, right and left (see Fig. 2). Similar cuts should be made in the skin of the limbs and back. Observe-

The fascia, the muscles of the body-wall, the abdominal and musculo-cutaneous veins, and the shoulder-girdle and pubic region of the hip-girdle.

The Abdomen and its Contents.-Pinch up the muscles on one side of the abdominal vein with the forceps, and make an incision in them by a single snip of the scissors. Then, holding the edge of the wound with the forceps, extend the cut forwards to the shoulder-girdle and backwards to the pubis. Keep the cut parallel to the abdominal vein, and be careful not to wound the latter. You will find that the incision thus made opens a large body-cavity or colome, in which a number of structures, the abdominal viscera, are contained. Note that the bodywall consists of three layers : (1) skin, (2) muscles, with their fascia, and (3) peritonezm.

So far, however, the cavity is not thoroughly opened. Lift up the side of the abdominal wall to which the abdominal vein is attached, and very carefully separate the vein by tearing through, with a needle or the point of a scalpel, the connective tissue by which it is attached to the inner face of the muscles : or, in order to prevent the possibility of injuring the vein, cut through the muscles of the body-wall longitudinally on the other side of the abdominal vein, so as to leave a narrow strip of muscle attached to it. Then make two cross-cuts, starting from the anterior end of the longitudinal incision, and extending outwards towards the fore-limbs: take care not to injure the musculo-cutaneous veins, and pin back the two flaps into which the soft abdominal wall is now divided (Figs. 3 and 4). Next dissect away the muscles covering the shoulder-girdle, so as to expose the bones: identify the bones called coracoid and clavicle (compare a skeleton and Fig. 12). With the strong scissors cut through both these bones as near as possible to the shoulder-joint: then lift up the middle portion of the shoulder-girdle thus separated, and carefully dissect it away from the underlying parts.

Having thus exposed the whole of the abdominal cavity, pour just enough water into the dissecting-dish to cover the animal, first washing away any blood which may have escaped from cut vessels. If your specimen is a female, dissolve a little common salt in the proportion of 
I per cent. in the water, or mix it with about one-third of its bulk of methylated spirit, in order to prevent the excessive swelling of the oviducts. If, however, you wish to make out the blood-vessels in this specimen without injecting them, it is as well to defer putting water into the dish until a later stage of the dissection.

Note-

I. The peritoneum-parietal and visceral layers.

2. The pericardium, containing the heart. If not already opened, the pericardium should be slit through, so that the auricles and ventricles can be plainly seen. If the frog has been killed quite recently, you will be able to observe the pulsation of the heart.

3. The right and left lobes of the liver, and the gall-bladder.

4. The two lungs: if contracted, inflate with a blowpipe through the glottis.

5. The enteric or alimentary canal, consisting of gullet or cesophagus, stomach, small intestine (duodenum and ileum), and large intestine or rectum communicating with the cloaca, which will be seen at a later stage, and which opens to the exterior by the vent.

6. The mesentery.

7. The pancreas.

8. The spleen.

9. The urinary bladder. If collapsed, insert a blowpipe into the vent and inflate. (You will very likely find some small parasitic flatworms, called Polystomum integerrinum, in the bladder; each worm has a ring of suckers round the hinder end.)

Io. In the male the spermaries and fat-bodies, and in the fenale the ovaries, fat-bodies, and oviducts.

II. The kidneys and ureters.

12. The mode of suspension of all these organs ( $p, 26$ ), and the position of the sub-vertebral lymph-sinus. In order to clearly understand the relations of these parts, a thick transverse section should be made through another frog in the region of the kidneys and examined under water (compare Fig. 5).

Sketch the contents of the abdomen in situ.

The Neural Cavity and its Contents. - Now turn the frog with its back upwards, and pin it firmly to the bottom of the dissecting-dish or to the dissecting-board as before. Pinch up the skin, make a longitudinal cut through it from the snout to within a short distance of the vent, and turn the flaps right arid left. The muscles of the back

Pract. ZoOr. 
will be exposed, and, in front, the roof of the skull, which lies just beneath the skin. Carefully dissect away the muscles along the middle line of the back until the vertebral column is seen. Compare a prepared skeleton and Fig 8, and make out the arches of the vertebra. Between the first of these and the back of the skull is a slight space : insert one blade of the strong scissors into this, directing the points backwards, and cut through the arch of the first vertebra, first on one, then on the other side, and finally detach and remove the little piece of bone. The neural canal will then be exposed, in which lies the spinal cord (compare Fig. 6).

Work backwards, cutting away the arches of the remaining vertebræ, and you will find that the spinal cord ends behind in a thread-like prolongation.

Next, using the scissors in the same manner, cut away, bit by bit, the roof of the skull: two large bones-the fronto-parietals (see Figs. 8 and 9), forming a considerable part of the roof, can be more easily removed by raising them up with the edge of a scalpel. Note the cavity of the skull and its contained brain.

General Structure of the Limbs.-With a strong knife, cut across one of the legs at about the middle of the thigh. Notice the thigh-bone, muscles, and skin. Sketch.

Now preserve your specimen in formaline ( $\mathrm{I}$ or 2 per cent.), or spirit (70 per cent.). 


\section{CHAPTER III}

THE FROG (continued): THE SKELETON

IF you have followed the description given in the preceding chapter with a frog before you, testing every statement as you proceeded by reference to the specimen, you will now have a very fair notion of the general build of the animal. The next thing to do is to study its various parts in somewhat greater detail.

As the bones and cartilages form the framework on which all the other parts are supported, it is convenient to begin with them. You may study them on a prepared skeleton, but a far better plan is to make a skeleton for yourself as directed on p. 53 .

Parts of the Skeleton.-The skeleton consists of the following regions :-

. I. The skull (Figs. 8 and 9): a complex mass of mingled bone and cartilage, enclosing the brain and the organs of smell and hearing, and supporting the upper jaw. Connected loosely with the skull, but really forming part of it, are the lower jaw and the tongue cartilage.

2. The vertebral column or backbone, consisting of nine movably united pieces, the vertebre (Fig. 8 v.I-v.9), and of a long bony rod, the urostyle (UST). 
3. The shoulder-girdle or pectoral arch, an inverted arch of bone and cartilage nearly encircling the anterior part of the trunk and giving attachment to-

4. The bones of the fore-limbs.

5. The hip-girdle or peliic arch, an apparatus shaped somewhat like a bird's "merrythought": it is attached in front to the ninth vertebra and behind gives attachment to-

6. The bones of the hind-limbs.

The Vertebral Column.-The essential structure of a vertebra may be best studied by examining any of the nine from the second to the seventh : the first, eighth, and ninth present certain peculiarities, and are best left till last.

The whole vertebra (Fig. 8, B) has something the form of a signet-ring with its sides produced into two outstanding projections. The part corresponding with the stone of the ring is ventral in position, and is called the body or centrum $(c n)$, the form of which is procalous, i.e., its anterior face is concave, its posterior face convex, and both faces are covered with a thin layer of cartilage. The part corresponding with the circle of the ring is the neural arch $(p d, l m)$ : it arches over the spinal cord and is produced in the middle line above into a blunt projection, the neurat spine (n. $s p)$. From the arch is given off, on each side, the large outstanding projection already referred to, the transverse process (tr. pr), which is tipped with cartilage in the second, third, and fourth vertebræ.

The neural arch gives off from its anterior face, just above the origin of the transverse processes, a pair of small shelflike projections, the articular processes or zygapophyses (a. zyg). Each has its upper surface flat and smooth, and covered with a thin layer of cartilage. A similar pair of processes spring from the posterior face of the arch, but have the smooth, cartilage-covered surface looking downwards. 


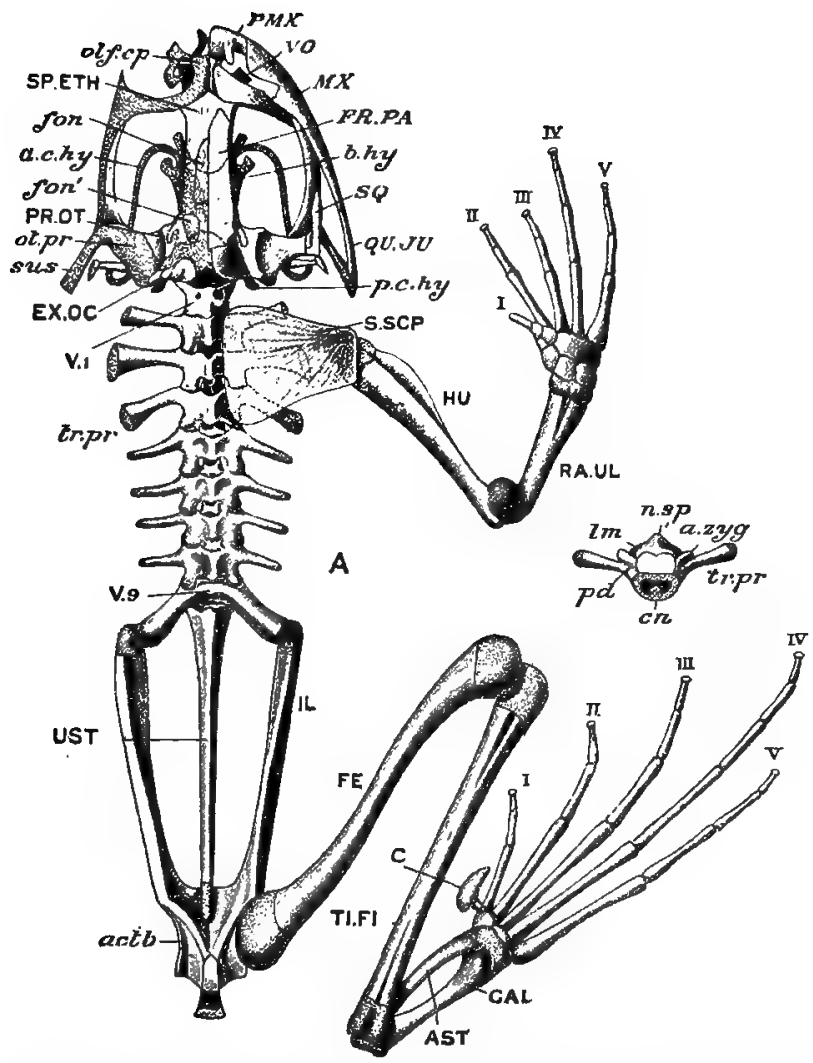

Fig. 8.-A, skeleton of Frog from the dorsal aspect; B, anterior face of the fourth vertebra. In $A$ the left half of the shoulder-girdle and the left fore- and hindlimbs are removed, as also are the membrane-bones of the left side of the skull. Cartilaginous parts are distinguished by dotting. The names of cartilage bones are in thick capital letters, those of membrane bones in italic capitals, other references in small italics.

a. $c . h y$. anterior horn of hyoid; $a c t b$. acetabulum; AST. astragalus; a.zyg. anterior articular processes, or zygapophysis ; b.hy. basi-hyal; C. calcar; CAL. calcaneum ; cn. centrum ; E.X. OC. exoccipital ; FE. femur ; fon, fon.' fontanelles; FR. PA. fronto-parietal; HU. humerus; IL. ilium; lin. lamina of neural arch; $M X$. maxilla; $n . s p$. neural spine; olf. cp. olfactory capsule: ot.pr. otic process; p.c.hy. posterior horn of hyoid; $p d$. pedicle of neural arch ; $P M X$. premaxilla : PR. OT. pro-otic; $Q U . J U$, quadrato-jugal ; RA. UL. radio-ulna; SP. ETH, sphenethmoid; $S Q$. squamosal; S. SCP. supra-scapula; sus. suspensorium; TI. FI. tibio-fibula ; tr. pr. transverse process ; UST, urostyle; $\nabla$. 1 , cervical vertebra ; $\mathbf{\nabla} .9$, sacral vertebra; $V O$. vomer ; $I-V$ digits. (From Parker and Haswell's Zoology, after Howes, slightly altered.) 
When two vertebræ are placed in position, the convex posterior face of the foremost centrum fits into the concave anterior face of its successor, like a cup and ball, and at the same time the posterior articular processes of the first fit over the anterior articular processes of the second. All the touching surface is, as we have seen, capped with cartilage, and the vertebræ can be moved upon one another, either up and down or from side to side.

The centra and articular processes are the only parts of the vertebræ which are in contact when the bones are in their natural positions. Large gaps or notches are left between the dorsal portions of the arches (see Fig. 8, A) to allow of the requisite amount of up and down movement, and there are similar gaps between the sides of the arches, bounded by the articular processes above and the centra below. These are called the intervertebral foramina: through them the nerves pass from the spinal cord.

The only differences of importance between the vertebræ now under consideration is in the form of their transverse processes, which are specially large in the third, short and devoid of cartilaginous tips in the fifth, sixth, and seventh.

The first vertebra (v. I) has no transverse processes, and its anterior face bears, instead of the ordinary articular processes, a pair of obliquely placed, oval, slightly concave surfaces or facets, covered with cartilage, and serving for the articulation of the condyles of the skull, presently to be described. The transverse processes of the ninth vertebra are very long and strong, directed backwards, and tipped with cartilage: to them the arms of the pelvic-girdle are articulated.

The eighth vertebra differs from its predecessors in having its body concave behind as well as in front. Corresponding with this, the ninth 
(v.9.) has its centrum convex in front, while behind it presents two little rounded elevations placed side by side.

It will be seen that the vertebræ are all corresponding structures, following one another in a regular series from before backwards. A correspondence of this kind, in which there is a repetition of similar parts along the body, is termed a serial homology, and thus not only the vertebræ as a whole, but also their various parts are serially homologous, each to each, the correspondence being disturbed only by the first vertebra, in which the transverse processes are absent and the anterior face modified for articulation with the skull.

The urostyle (UST) is a long bone, the anterior part of which has somewhat the appearance of a small vertebra with no transverse processes, and having the posterior face of its centrum produced backwards into a long projection. On its front face it has a double concavity for articulation with the double convexity on the ninth vertebra. Near its anterior end there is on each side a small aperture for the last spinal nerve.

The skull is a very complex structure, consisting partly of bone, partly of cartilage. . It is divided into the following regions :

r. The brain-case or cranium, a sort of oblong box containing the brain (Figs. 8 and 9): it forms the middle portion of the skull and is a direct forward continuation of the vertebral column.

2. The auditory capsules, a pair of outstanding masses arising, right and left, from the posterior end of the braincase. They lodge the organs of hearing.

3. The olfactory capsules (olf. $c p$ ), smaller masses proceeding from the anterior part of the brain-case and united with one another in the middle line. They lodge the organs of smell. 
4. The suspensoria (sus), a pair of outstanding rods, springing from the outer and upper portions of the auditory capsules, and directed downwards, outwards and backwards. To them the ends of the lower jaw are attached.

5. The upper jaze, a half-circle of bone and cartilage, united in front to the olfactory capsules and behind to the auditory capsules and suspensoria. On each side of the skull, between the cranium and upper jaw, is a large space, the orbit, in which the eye is contained.

6. The lower jaw, a roughly semicircular bar of bone and cartilage, articulated at each end with the corresponding suspensorium.

7. The tongue-cartilage or hyoid $(b . h y)$, a shield-shaped cartilage connected by delicate curved rods $(a . c . h y)$ with the auditory capsules.

On the posterior surface of the brain-case is a large hole, the foramen magnum (fig. 9, c, for. mag), on each side of the lower edge of which is an oval elevation covered with cartilage, the occipital condyle (oc.cn). The foramen magnum leads into the cavity in which the brain is contained. If the first vertebra is placed in its natural position with regard to the skull it will be seen that the foramen magnum corresponds with the neural canal of the vertebra, and that the condyles fit into its articular surfaces. Thus the skull readily moves up and down upon the vertebra, the condyles acting as rockers ; a space between the neural arch and the dorsal edge of the foramen magnum covered by membrane in the fresh state, allows of the requisite amount of play.

The discrimination of the separate bones of the skill is rather difficult, and may very well be omitted by the beginner at the present stage.

The occipital condyles are borne on a pair of irregular bones (EX.OC) which bound the sides of the foramen magnum, nearly uniting 
above and below it, and extending over a considerable portion of the posterior surface of the auditory capsule. These bones are the exoccipitals. In front of each exoccipital is another irregular bone (PR.OT) forming the front part of the autlitory capsule, and called the pro-otic.
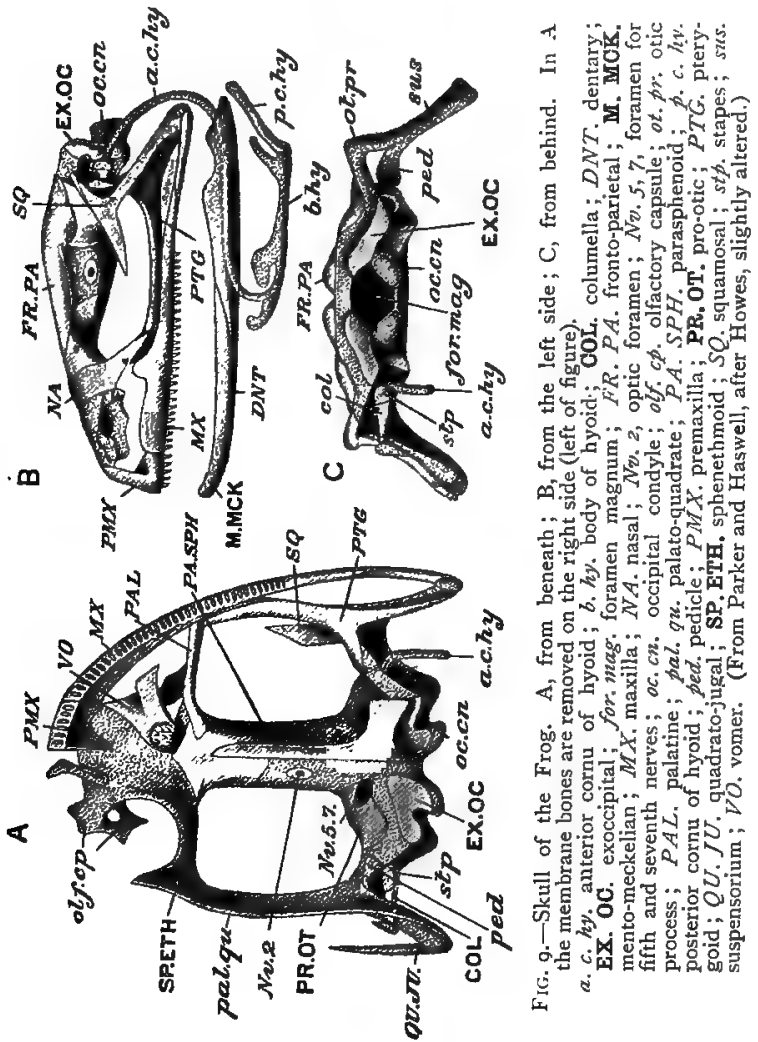

Each pro-otic is separated from the corresponding exoccipital, in young frogs, by a band of cartilage, but in old specimens the two bones are more or less completely united.

In the disarticulated skull it can be made out that the exoccipital and 
pro-otic of each side enclose a cavity; in this the organ of hearing is contained. The exoccipital is perforated, just in front of the condyle, by a large aperture through which two nerves, the glossopharyngeal and the vagus, pass on their way from the brain. The pro-otic is similarly perforated or notched for the trigeminal and facial nerves $(N v .5,7)$.

The dorsal surface of the brain-case is covered by two longish, flat bones (FR.PA). In the young condition each of these consists of two distinct bones, the front one the frontal, the hinder the parietal. As the young frog grows the frontal and parietal of each side becomes completely fused, forming a single fronto-parietal. On the upper surface of each olfactory capsule is a roughly triangular bone, the nasal $(N A)$, in front of which is the corresponding nostril.

The ventral surface of the brain-case is covered by a single bone $(P A . S P H)$ having the shape of a $\mathrm{T}$. The stem extends forwards in the middle line as far as the olfactory capsules, while the arms stretch outwards beneath the auditory capsules. This very characteristic bone is the parasphenoid. On the under surface of the olfactory capsules, corresponding to the nasals above, are a pair of irregular bones, the vomers $(V O)$. Their outer edges are notched and help to bound the internal nostrils; their posterior ends bear the vomerine teeth.

The anterior end of the brain-case is surrounded by a bone (SP.ETH) which extends forwards into the region of the olfactory capsules, and is partly covered by the fronto-parietals above and by the parasphenoid below. This is the girdle-bone or sphenethmoid. In the disarticulated skull it is seen to have a very peculiar shape. Its posterior half encloses a single cavity in which the fore-end of the brain (Fig. 6, olf. $l$ ) is lodged. Its anterior half encloses two cavities, right and left, separated from one another by a vertical partition, and serving to lodge the posterior ends of the olfactory sacs or organs of smell. Each of these cavities communicates with the single posterior cavity by a small hole through which the nerve of smell passes.

Between the girdle-bone in front and the pro-otic behind, the sidewalls of the skull are formed of cartilage perforated by a rounded aperture, the optic foramen $(N v, z)$ for the nerve of sight.

Forming the outer part of the suspensorium is a hammer-shaped bone, the squamosal $(S Q)$; its head is applied to the auditory capsule and projects forwards into the orbit.

The upper jaw is formed of three bones on each side. In front is the premaxilla $(P M I X)$, a short bone, sending off an upward process 
towards the nostril. Next follows the maxilla $(M X)$, a long, curved bone, forming the greater part of the upper jaw, and joined at its posterior end to a small, slender bone, the quadrato-jugal ( $Q U . J U$ ), which is firmly connected with the lower end of the suspensorium. Both premaxilla and maxilla are produced below into a prominent edge from which spring a number of small conical teeth, arranged in a single row.

Besickes these three bones there are two others which seem, as it were, to brace the upper jaw to the brain-case and suspensorium. The palatine $(P A L)$ is a narrow, rod-like bone, placed transversely behind the olfactory capsule. The pterygoid $(P T G)$ is a large, three-rayed bone; one ray is directed forward and connected with the outer end of the palatine and with the inner face of the maxilla; another passes backwards and inwards and is connected with the auditory capsule; the third extends backwards and outwards and forms the inner and ventral portion of the suspensorium. The main mass or core of the suspensorium, between the squamosal outside and the pterygoid within, is a rod of cartilage (sus), which is continued forwards by a bar (pal. qu) supporting the pterygoid and palatine.

There is an important distinction to be drawn between the bones of the skull which can be made out only by the exercise of a good deal of care and patience. By softening the connective-tissue which binds the bones together it is possible to remove the majority of them without injuring the underlying cartilage (compare the right and left sides of the skull in Figs. 8 and 9 , A and C), provided, of course, that the operation is skilfully performed : these bones are the nasals, vomers, frontoparietals, parasphenoid, premaxillæe, maxillæ, quadrato-jugals, palatines, pterygoids, and squamosals. A sort of foundation or groundwork (left side of figure in Figs. 8 and 9, A ; right side in Fig. 9, C) is then left behind, consisting mainly of cartilage, but containing the exoccipitals, pro-otics, and girdle-bone. These five bones cannot be removed without pulling the cartilaginous groundwork or chondrocranium to pieces. We thus get a distinction between cartilage-bones which are actually continuous with the cartilage and form part of the chondro. cranium, and membrane-bones which lie outside the chondrocranium, united to it only by connective tissue.

The chondrocranium has a cartilaginous roof, underlying the frontoparietals ; it is pierced by one large (Fig. 8, fon) and two small ( $f \circ n^{\prime}$ ) spaces, called fontanelles, covered by membrane. It has also a cartila- 
ginous fioor (Fig. 9, A) underlaid by the parasphenoid. The olfactory capsules (olf. $c p$ ) also have a cartilaginous roof and floor of irregular form, with the posterior end of which is united the cartilaginous palato-quadrate bar (pal. qu), with which the palatine and pterygoid bones are connected. Posteriorly this bar is joined to the cartilaginous groundwork or core of the suspensorium (sus), which unites above with the auditory capsule by two processes (Fig. 9, C, ot.pr, ped) and below furnishes an articular surface for the lower jaw.

Notice that in describing the vertebral column no distinction was drawn between cartilage-and membrane-bones. As a matter of fact the vertebræ and the urostyle are all cartilage-bones; each consists, in the tadpole, of cartilage which subsequently undergoes ossification, i.e., is replaced by bone in which a deposition of lime salts takes place.

The lower jaw (Fig. 9, B) consists of two halves, or rami, united with one another in front by ligament. At its posterior end each half bears on its upper surface a shallow pit, by which it articulates with the suspensorium, and a little in advance of this pit is an elevation of the dorsal edge of the jaw, called the coronary process.

Each half of the lower jaw consists of a cartilaginous core called Meckel's cartilage, which furnishes the articular surface referred to, and in front is ossified as a small cartilage-bone, the mento-meckelian (M.MCK). Outside the cartilage are two membrane-bones. One, the angulo-splenial, extends along the inner surface and lower edge of the jaw and forms the coronary process, while the dentary $(D N T)$ forms the outer surface of the anterior half of the jaw.

The hyoid is a thin, shield.shaped plate of cartilage (Figs. 8 and 9, b. hy) produced, both in front and behind, into a pair of processes or horns, as well as into less important offshoots. The anterior horns (Fig. 9, a.c.hy) are long, delicate, cartilaginous rods which curve backwards and then upwards, finally uniting with the auditory capsules. The posterior horns (p.c.hy) are short bony rods which pass backwards, diverging as they go, one on each side of the glottis. 
Two apparently insignificant structures connected with the skull must be described because of their connection with the organ of hearing. Behind the suspensorium is a recess, roofed over by the squamosal, and, in the entire frog, converted by muscle and other tissues into a chamber, the tympanic cavity (Fig. Io, tymp. cav), bounded externally by the tympanic membrane, and communicating with the mouth

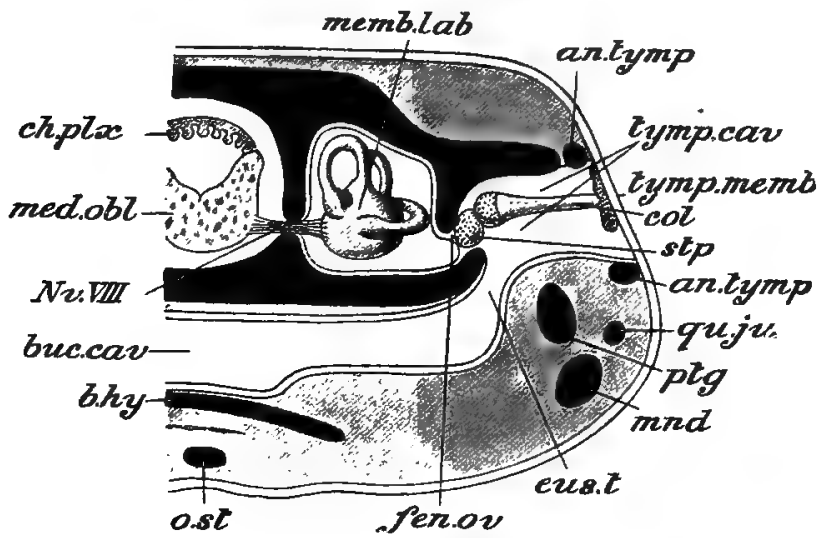

FIG. Io.-Transverse section (diagrammatic) through the nead of a Frog at the level of the tympanic cavity. The various parts of the skull shown in section are black, the muscle, \&c., grey, and the skin and mucous membrane white.

an. tymp. tympanic ring; $b$. hy. body of hyoid; buc. car. buccal cavity; $c h$. $p l x$. choroid plexus; col. columella; eus. t. Eustachian tube; fen. ov. fenestra ovalis; med. obl. medulla oblongata; memb. lab. membranous labyrinth; mnd. mandible; $N v$. VIII. auditory nerve; o. st. omosternum; ptg. pterygoid; gu. ju. quadrato-jugal ; stp. stapes; tymp. cav. tympanic cavity; tymp. memb. tympanic membrane. (From Parker and Haswell's Zoology.)

by the Eustachian tube. Supporting the tympanic membrane, as the frame of a tambourine supports the parchment, is a cartilaginous ring, the tympanic ring (shown in section in Fig. 1o, an. $t y m p)$. Stretching across the tympanic cavity from the outer wall of the auditory capsule to the tympanic membrane is a small, hammer-shaped rod, the columella (col), having a bony handle and a cartilaginous 
head, the latter firmly fixed to the inner face of the tympanic membrane. The inner end of the handle is tipped with cartilage, and is attached to a small cartilaginous nodule, the stapes (st), which is inserted into an aperture in the auditory capsule known as the fenestra ovalis (fen.ov). With care the columella can easily be removed with small forceps, in a wet skull, and examined under a magnifying glass.

The shoulder-girdle has the form of an inverted arch encircling the anterior region of the trunk, and having its dorsal ends turned inwards so as partly to cover the second to the fourth vertebræ (Figs. 8 and II). The dorsal region,

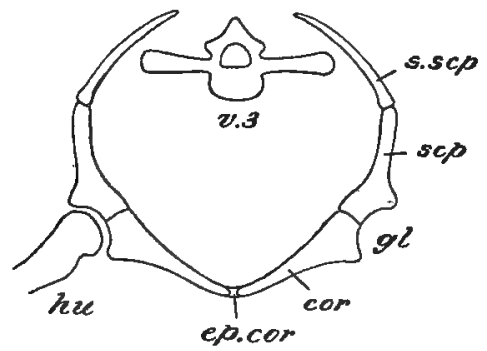

FIG. II-Diagrammatic transverse section through the shoulder-girdle. cor. coracoid ; ef. cor. epicoracoid ; $g l$. glenoid cavity ; hu. humerus; scp. scapula ; s. scp. supra-scapula; $v .3$, third vertebra.

on each side, is formed by a broad plate, the supra-scapula (s. scp), or upper blade-bone. It is mostly formed of bone, but its free edge consists of cartilage which, when dried, is seen to be impregnated with a granular deposit of lime-salts. This rough, brittle tissue is called calcified cartilage, and is distinguishable from true bone, which has usually a smooth, enamelled surface.

Connected with the ventral end of the supra-scapula and passing vertically downwards is a flat bone, broadened at each end, the scapula or blade-bone (Fig. I 1, scp: Fig. I2, S). 
From its lower end two bones (Fig. I2, Cl, Co; Fig. I I, cor) pass directly inwards, parallel with one another, to end in a plate of cartilage $\left(\mathrm{Co}^{1}\right)$, which meets with its fellow of the opposite side in the middle line of the chest $(m)$. The more anterior of these $(\mathrm{Cl})$ is a narrow bone and is called the clavicle or collar bone, the posterior one is

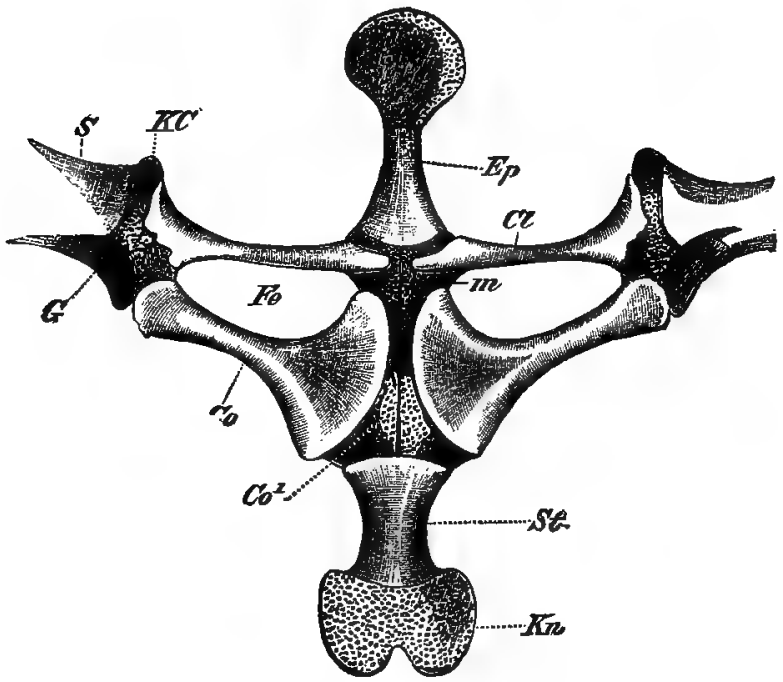

FIG. 12.-The shoulder-girdle of the Frog from the ventral aspect.

$C o$. coracoid ; $C o^{\prime}$. epicoracoid ; $C$. clavicle; $E p$. epi- and omo-sternum ; $G$. glenoid cavity; $\mathrm{Fe}$. fenestra between procoracoid and coracoid ; $K C$. cartilage separating

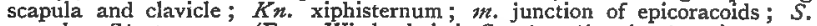
scapula ; St. sternum. (From Wiedersheim's Comparative Anatony.)

broader and is known as the coracoid (Co). Between the scapula on the one hand and the clavicle and coracoid on the other, there is a cartilaginous interval $(K C)$, the posterior edge of which is scooped out into a depression, the glenoid cavity (Fig. I2, $G$; Fig. I I, $g l$ ), for the articulation of the upper-arm bone. 
Connected with the median ventral portion of the shouldergirdle is the sternum, or breast-bone, which consists of two separate parts, one extending forwards, the other backwards, in the middle line, and each formed of a flattened bony rod $(E p, S t)$, tipped with a flat plate of cartilage.

The anterior bony rod is called the episternum ( $E p)$, its terminal cartilage the omosternum ; the posterior bony rod is the sternum (St.), the bilobed cartilage at its end the xiphisternum $(K n)$. The cartilages uniting the inner or ventral ends of the clavicles and coracoids are distinguished as the epicoracoids $\left(\mathrm{CO}^{1}\right)$.

All the bones of the shoulder-girdle and sternum are cartilage-bones except the clavicle. This can be removed, and is seen partly to surround a bar of cartilage, the procoracoid, which stretches between the scapula and the epicoracoid and is ordinarily completely concealed by the clavicle.

The Fore-limb. - The upper arm is supported by a single borre, the humerus (Fig. 8, HU), the first example we have had of what is conveniently called a long bone. It consists of a roughly cylindrical shaft, formed of dense bone, and of two extremities - the proximal of partially calcified cartilage, the distal of spongy or cancellated bone. The proximal extremity or head is convex, and fits into the glenoid cavity of the shoulder-girdle (Fig. I I); the distal extremity. or condyle is almost globular, and is articulated with the bone of the fore-arm.

In a longitudinal section of a humerus which has not been allowed to dry you will see that the shaft (Fig. $\mathrm{I} 3, s h$ ) is not a solid rod, but a tube, containing a cavity, the marrow-cavity. In this way the weight of the bone is diminished without its strength being impaired. The marrow-cavity contains a substance called bone-marrow', composed chiefly of connective tissue and fat, with blood-vessels. The proximal end of the hollow shaft is, as it were, plugged by the cartilaginous extremity. 
The fore-arm is also supported by a single bone, the radioulna (Fig. 8, RA. UL): Its proximal end is concave and articulates with the almost globular condyle of the humerus : the outer or posterior edge of the concavity is produced into a short process, the olecranon or elbow. The distal end is
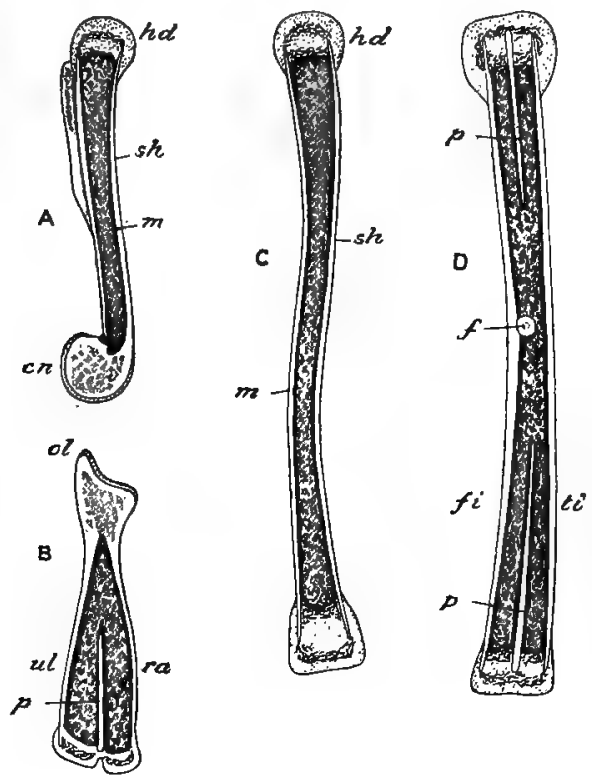

FIG. 13.-Longitudinal sections of the principal long bones of the Frog.

$A$, humerus; $B$, radio-ulna ; $C$, femur; $D$, tibio-fibula. $c n$. condyle; $f$. foramen for artery; $f$. fibula; $h d$. head ; $m$. marrow ; ol. olecranon process ; $p$. bony partition; ra. radius; sh. shaft; $t i$. tibia ; $u$. ulna.

incompletely divided into two articular surfaces, and between these is a groove passing for some distance towards the proximal end of the bone. A section shows that at this end there are two distinct marrow-cavities, indicating that the bone is really double: That this is the case is proved by the

Pract. Zool. 
examination of a very young frog, in which the single forearm bone is represented by two distinct cartilages, the radius on the inner or thumb-side, and the ulna on the outer or little-finger side. The olecranon is a process of the ulna.

The skeleton of the hand is divisible into three regions: the carpus or wrist, the metacarpus or mid-hand, and the phalanges or finger-bones. The carpus consists of six small irregular bones, arranged in two rows (Fig. 8). The proximal row articulates with the radio-ulna, while to the distal row are attached the metacarpals, which together constitute the metacarpus. Four of these are long rod-like bones and support the bases of the four fingers or digits : to them are attached the phalanges, of which the first or innermost digit (II), has two, the next two, and the remaining two digits three apiece. A very small metacarpal, with a single phalanx (I), occurs on the radial side and is concealed by the skin in the entire frog: it corresponds with our own thumb, so that the apparent first digit of the frog is really the second or index-finger.

The Hip-girdle-This, as we have seen, has somewhat the form of a bird's merrythought. It consists of two long arms (Fig. 8, IL: Fig. 14, Il), which are articulated with the transverse processes of the ninth vertebra, and sweeping backwards, unite in a disc-shaped mass, having on each side of it a deep, hemispherical cavity, the acetabulum (Fig. 8, $a c t b$; Fig. 14, $G$ ), for the articulation of the thigh bone.

Two sutures, or lines of separation, nearly at right angles to one another, divide the disc-shaped portion into three parts. One of these, dorsal and anterior in position, is continued into one of the arms of the hip-girdle and forms half of the acetabulum; this is the ilium (Fig. I4, Il, P). The second, posterior in position, is the ischium $(I s)$; like the ilium it is made of true bone. The third, or pubis (Kn), 
is ventral, and is formed of calcified cartilage. Originally each of these elements is paired, i.e., there is an ilium, an ischium, and a pubis on each side, the three together forming the innominate, but in the adult the right and left ischia and pubes become united in the median plane, the ilia only remaining free.

The Hind-limb.-The thigh, like the upper arm, is supported by a single long bone, the femur (Fig. 8, $\mathrm{FE}$ ), having a gently curved shaft and extremities of calcified cartilage. Its rounded proximal extremity, or head, fits into the acetabulum: its distal end articulates with the single bone of the shank, the tibio-fibula (TI. FI). This, the longest bone in the body, also has a shaft and extremities, and is further distinguished by grooves running from each end towards the middle of the

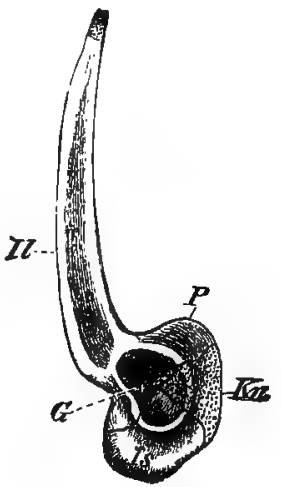

FrG. I4.-The pelvic girdle of the Frog seen from the right side.

$G$. acetabulum ; $K r$. pubis ; $I l, P$. ilium; $I s$, ischium. (From Wiedersheim.) shaft. Sections show that the grooved portions of the bone contain a double marrow-cavity (Fig. 1 3, D), and in the young animal there are found two shank-bones which afterwards unite, the tibia on the inner side, the fibula on the outer side.

The foot, like the hand, is divisible into three regions: the tarsus or ankle, the metatarsus or mid-foot, and the phalanges or toe-bones. The tarsus, like the carpus, consists of two rows, but with only two bones in each. Those of the proximal row (astragalus and calcaneum) are greatly elongated (AST., CAL.), and furnish an additional segment to the limb, thus increasing the frog's leaping powers : those of the distal row are very small.

The metatarsals are five in number: those of the first 
and second digits (I, II) bear two phalanges each, those of the third and fifth, three each, and that of the fourth, four. Attached to the inner side of the tarsus is a little claw-like structure (C) composed of two or three bones and called the calcar or spur.

Notice the striking correspondence in structure between the fore- and hind-limbs, a correspondence which extends also, though less obviously, to the limb-girdles. The humerus corresponds or is serially homologous (p. 39) with the femur, the radius with the tibia, the ulna with the fibula, the carpals with the tarsals, the metacarpals with the metatarsals, and the phalanges of the fingers with those of the toes. Then in the limb-girdles the glenoid cavity corresponds with the acetabulum, the scapula and supra-scapula (being above the articular cavity) with the ilium, the procoracoid and clavicle (being ventral and anterior in position) with the pubis, and the coracoid with the ischium. Thus not only are the limbs and limbgirdles serially homologous structures, but their several parts are also serially homologous, each to each.

Nature of Bone.-It is a mistake to suppose that bones are made exclusively of hard mineral matter, like rocks or stones. If one of the long bones, for example, is put into weak acid, bubbles of gas will rise from the bone, showing that the phosphate and carbonate of lime, of which it is partly composed, is being decomposed with the liberation of carbonic acid. When the liberation of bubbles is over; the bone will be found to be unaltered in form, but to be quite flexible instead of hard and rigid. It can be bent in any direction, and a bone of sufficient length, such as a sheep's rib, can be tied in a knot. This shows that the bone contains a large amount of organic or animal matter. On the other hand, a bone. may be 
completely calcined or heated to redness in a closed vessel, when its animal matter is completely consumed and its mineral matter left. Under these circumstances it becomes very brittle, falling to pieces at a touch, and its appearance is far more altered than by the removal of the mineral matter.

\section{PRACTICAL DIRECTIONS.}

Preparation of the Skeleton.-Kill a frog with chloroform (p. 31), open the abdomen according to the directions on p. 32, but without cutting the shoulder-girdle, and remove the contained organs. Then, the frog being firmly pinned down, remove the skin and gradually cut away the flesh from the bones with scalpel and scissors. In the case of the long bones of the limbs, it is best to cut through the muscles near one end of the bone and then gradually to strip them back towards the other end until the bone is exposed. The process is facilitated by dipping the frog occasionally into boiling water : this softens the connective tissue by which the bones and muscles are bound together, and thus allows them to be more readily separated. While at work keep Fig. 8 before you, and be particularly careful not to injure those parts of the skeleton which are made of cartilage (dotted in the figure), and are therefore easily cut. The most important of these cartilaginous parts are the hyoid or tongue-cartilage $(b . h y)$, lying in the floor of the mouth, the onosternum (Fig. I2, in front of Eph), which projects in the middle line in front of the shoulder-girdle, and the xiphisternum $(K z)$, which extends backwards from the same region. Great care will also be required in cleaning the bones of the hands and feet, since the fine cords or terrdons which pass to them from the muscles'are very strong, and if pulled upon with much force are sure to bring away the small toe-bones with them: they should be separated as far as possible and then cut off, close to the bones, with scissors.

Keep all the parts of the skeleton together, avoiding separation of the various bones until the general characteristics of the entixe skeleton have been made out : the only part which cannot be kept in connection with the rest is the shoulder-girdle, which will come apart, together with the fore-limbs.

Examination of the Skeleton.--You should have two skeletons to examine-one dried, after it has been thoroughly cleaned, and one 
which has been kept from the first in spirit or formaline: the latter is the more instructive. An additional skull should be carefully cleaned, and then boiled until the numerous bones become separated from one another or disarticulated.

After observing the form and relations of different parts of the skeleton as described on pp 35 and $3^{6}$ (Fig. 8, A), they may be separated from one another for mose detailed examination. The individual vertebræe should be strung on a piece of wire or string so as to prevent their being lost or misplaced.

With the specimen before you, work through the characters of the axial skeleton (pp. $36-46$ ) : if you omit the details given in small type (pp. 40-44) at the present stage, do not forget to examine them subsequently. Make sketches of $-a$. Any one of the vertebre from the first to the seventh, from the side and from the front and back.t; $b$. the first vertebra; $c$ the urostyle; $d$. the skull from above and from below; and $e$. the hyoid.

It requires considerable skill to make a satisfactory preparation of the chondrocranium, but if you wish to attempt it, procure a large skull which has not been dried, and boil it in water. Carefully separate, by means of a scalpel, most of the membrane-bones ( $p .43$ ); the palatines, pterygoids, and quadratojugals, and the dentaries and angulosplenials cannot well be disarticulated without destroying the soft cartilaginous parts beneath them.

Make out-I. The brain-case and its fontanelles and nerve apertures. 2. The olfactory capsules. 3. The auditory capsules. 4. The palatoquadrate bar (to which the palatine, pterygoid, and quadratojugal bones have been left attached). 5. The mandibulas" or Meckel's cartilage (to which the angulosplenial and dentary have been left attached). 6. The cartilage-bones (exoccipitals, pro-otics, sphenethmoid, and mento. meckelians). 7. The columella, stapes, and fenestra ovalis. Sketch from above and from below.

Now proceed to examine the appendicular skeleton (pp. 46-52), and sketch the shoulder-girdle and fore-limb, and the hip-girdle and hind-limb.

Split one of the longer limb-bones (e.g., humerus or femur) longitudinally with a krife, and note the marroze-cavity in the shaft (Fig. 13). Place another of the long bones in Io per cent. hydrochloric acid for an hour or two; wash thoroughly in water and examine. 


\section{CHAPTER IV}

THE FROG (continued): THE JOINTS AND MUSCLES

IN the previous chapter the bones-more than $\mathrm{r}_{50} \mathrm{O}$ in number-which together constitute the greater part of the skeleton of the frog, have been considered as so many separate parts, fitting into or against one another in certain ways. We must now see how they are joined together in the entire animal so as to afford the requisite support, and, at the same time, to allow of free movement.

The IIip-joint.-Let us begin by a study of the hip-joint (Fig. 15).

The acetabulum (actb), as you have already seen (p. 50), is a hemispherical depression on the outer surface of the hip-girdle. It is formed of cartilage, continued into a projecting rim round the edge of the cavity. The head of the femur $(h d)$ is also formed of cartilage, and fits accurately but rather loosely in the acetabulum.

The acetabulum is lined, and the head of the femur is covered, by a thin skin of connective tissue, the perichondrium ( $p . c h d)$, which, in both cases, is continued on to the adjacent bone, where it receives the name of periosteum (p.ost).

Attached all round the rim of the acetabulum is a strong sheet of connective tissue called the capsular ligament 
(cps. $l g$ ), forming a short, fibrous tube. The other end of this tube is fixed to the femur, just below the head, the ligament being continuous, in each case, with the perichondrium. There is thus a space between the head of the thigh bone and the acetabulum, closed all round by the capsular ligament. This space is filled with a delicate, fibrous, closed bag, the synovial capsule (sy.cps), one side of which

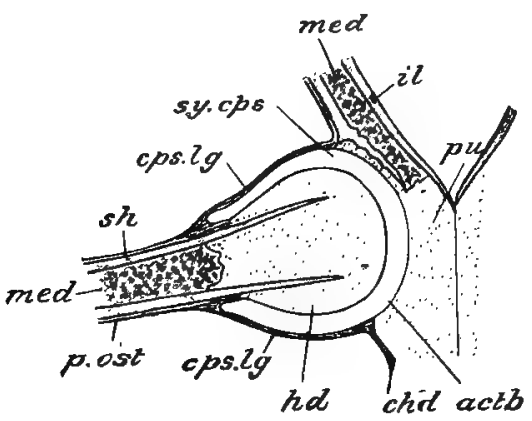

FIG. I5.-Horizontal section of the Frog's hip-joint.

$a c t b$. acetabulum : cps. $\mathrm{gg}$. capsular ligament; hd. head of femur; il. ilium; med. marrow; $p . c h d$. perichondrium; $\not$. ost. periosteum; fu. pubis; sh. shaft of femur; sy.cps. synovial capsule.

fits closely into the acetabulum, while the other as closely invests the head of the femur. The capsule is filled with a watery fluid, the synovia, and thus forms a buffer or watercushion between the adjacent bones. Thus the synovial capsule keeps the two bones slightly apart and prevents friction, while the capsular ligament keeps them together and prevents dislocation.

It is obvious that, in such a joint as this, movement is possible in all directions. The femur can be inclined either upwards, downwards, or sideways, and is capable of a certain amount of rotation. The joint is, in fact, a cup-and-ball 
joint, and is capable of movement in any plane. A similar but less perfect cup-and-ball joint is that of the shoulder, in which the cup is furnished by the glenoid cavity, the ball by the head of the humerus.

0ther Joints.-The elbow- and knee-joints are constructed on the same general plan, but, owing partly to the form of the adjacent surfaces, partly to the mode of attachment of the ligaments, they are capable of movement in one plane only, i.e., up and down, but not from side to side. They are therefore distinguished as hinge-joints.

The vertebræ are connected with one another in a similar way. Between the convex hinder face of one centrum and the concave front face of its successor is a synovial capsule, and the two centra are bound together by ligament, a shallow cup-and-ball joint, with a very limited range of movement, being produced. There are also synovial capsules between the articular processes, which, being in contact with one another by flat surfaces and working mainly from side to side, form gliding-joints. There are also strong ligaments connecting the neural arches with one another and joining the first vertebra to the skull.

In all cases where free movement is necessary the joints are formed in the same way; the bones are bound together by ligaments, and a synovial capsule is interposed between their adjacent surfaces. When little or no movement is required, as between the bones of the shoulder- and hip-girdles, the union is effected by cartilage or ligament only, and there is no synovial capsule. Such joints are therefore distinguished as immoveable or imperfect joints.

The Muscles.-We see then that the bones of the skeleton are attached to or articulated with one another by means of ligaments, so arranged, in most cases, as to allow of more or less frèe movement between the bones. We must now 


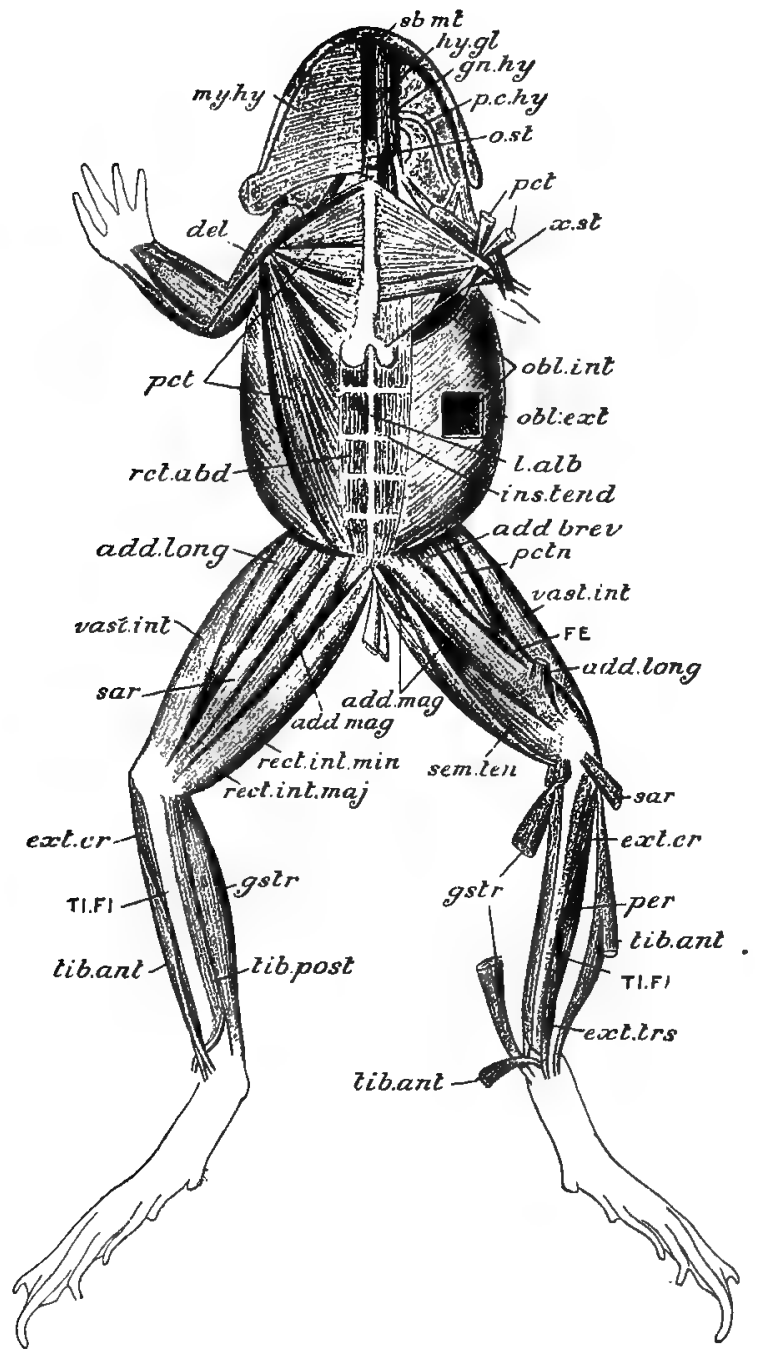

F1G. 16.-The muscles of the Frog from the ventral aspect. On the left side (right of figure) many of the superficial muscles have been cut and reflected to show the deep layer.

add. brev. adductor brevis; add. long. adductor longus ; add. mag. adductor magnus; del. deltoid ; ext.cr. extensor cruris; ext.trs. extensor tarsi ; FE. femur; 
gn. hy. genio-hyoid; gstr. gastrocnemius; $h y . g l$. hyoglossus; ins. ten. tendinous inscription; $l$.alb. linea alba; $m y$. $h y$. mylo-hyoid; obl. int. obliquus internus; obl. ext. obliquus externus; $0 . s t$. omosternum; $p$. $c . l y y$. posterior cornu of hyoid ; pct. pectoralis; pctn. pectineus; per. peronzus; rct. abd. rectus abdominis; rect. int. maj, rectus internus major; sar. sartorius; sb. mt. submentalis; sem.ten. semi-tendinosus; tib. ant. tibialis anticus; tib. post. tibialis posticus ; TI. FI. tibio-fibula ; vast. znt. vastus internus; $x$. st. xiphisternum. (From Parker and Haswell's Zoology.)

try to find out how the movements are effected in the living frog.

It was pointed out in the second chapter that the flesh is made up of distinct bands or sheets, the muscles, some of which came under your notice in your first dissection. It is quite easy to convince yourself that the whole of the flesh has this character by skinning a frog and carefully removing the fascia (p. I8) which covers the muscles and the more delicate web of connective tissue which forms a sort of packing substance between them. After noticing some of the muscles shown in Fig. r6, especially those of the leg, concentrate your attention on the muscle marked gstr, a prominent spindle-shaped mass of flesh forming the calf of the leg, and known as the gastrocnemius (gstr).

The spindle shaped, fleshy mass or belly of this muscle is continued at either end into a band of strong, tough connective tissue, the tendon (Fig. 17). The tendon at the proximal end is flat and is attached to the distal end of the femur and to the proximal end of the tibio-fibula, in each case becoming continuous with the periosteum of the bone. The tendon at the distal end has the form of a stout cord and is distinguished as the tendo Achillis; it corresponds with the strong tendon just above the heel of the human foot. At its distal end it is continued into a broad sheet of connective tissue, the plantar fascia, which spreads over the whole sole or plantar surface of the foot.

If the foot is bent upon the shank as in the ordinary sitting position of the frog, and the gastrocnemius pulled 
upwards or towards the thigh, the foot will instantly be bent backwards, so as to come into a straight line with the shank, the action being one of those performed by the living frog when leaping. It will be seen that the proximal tendon is attached to a relatively fixed point: it is distinguished as the tendon of origin, or the muscle is said to arise from the femur and tibio-fibula. The distal. tendon is attached to a relatively moveable part, the foot, and is called the tendon of insertion, the muscle being said to be inserted into the plantar fascia.

Muscular Contraction.-.Obviously, however, there is. nothing to pull upon the muscle from outside in the living. frog. We must, therefore, try to form some idea as to how the action of bending the foot, roughly imitated in the dead subject, is performed during life. If the gastrocnemius be exposed in a recently killed frog, the foot bent up as before, and a smart pinch be given to the belly of the gastrocnemius, the foot will be bent back, although no pull has been exerted on the muscle. The same thing will happen if you drop on the gastrocnemius a single drop of weak acid or of a strong solution of common salt, or if you touch it with a hot wire, or if you apply to it the electrodes from an induction coil so as to pass an electric current through it.

Careful observation shows that what happens under either of these circumstances is, that the belly of the muscle decreases in length and at the same time increases in breadth, so as to become shorter and thicker (Fig. I7). The result of this must necessarily be to cause its two ends to approach one another. As the tendon of origin is attached to the femur, which we suppose to be fixed, it is unable to. move, and the insertion is therefore drawn upwards, bringing with it the moveably articulated foot. In fact exactly the same 
thing takes place as when we raise our own forearm. This action is performed by means of the biceps muscle which arises from the scapula and is inserted into the fore-arm. When the latter is raised we feel a lump rise on the front of the upper arm due to the thickening of the biceps.

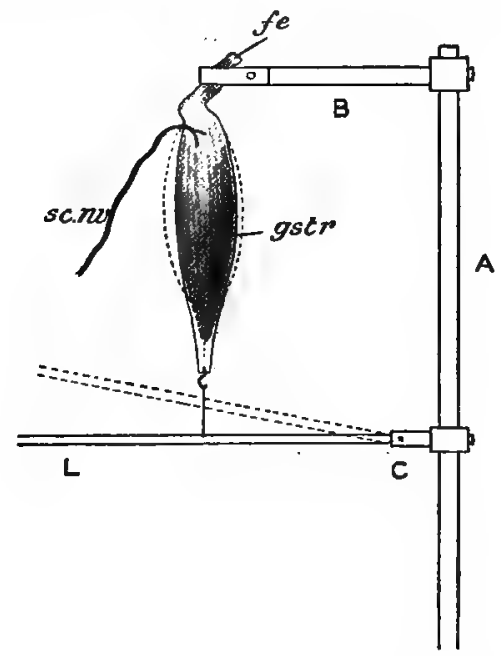

Fig. 17.-Diagram of apparatus for demonstrating the contraction of the gastrocnemius muscle.

$A$, upright bearing two adjustable horizontal arms. To the upper of these(B) is fixed by a clamp, the femur $(j e)$, having the gastrocnemius (gstr) in connection with it. To the lower arm (C) is fixed a light lever (L) moveable in a vertical plane, and having the tendon of insertion of the muscle attached to it by a thread. The dotted lines show the form of the gastrocnemius and the position of the lever $\therefore \quad$ The dotted lines show the form of the gastrocnemius and the

This shortening and thickening of the muscle is termed a contraction. Do not fail to notice that this word is used in a special sense. When we say that a red-hot bar of iron contracts on cooling we mean that it becomes smaller in all dimensions-undergoes an actual decrease in bulk. But in muscular contraction there is no alteration in bulk: the 
decrease in length is balanced by an increase in thickness, as when a stretched piece of india-rubber is relaxed.

The external influence by which a contraction is induced is called a stimulus. As we have seen, a stimulus may be produced by actual contact of some external object (mechanical stimulus), or by chemical action (chemical stimulus), or by heat (thermal stimulus), or by an electrical current (electrical stimulus).

Relation of Muscle and Nerve,-Evidently, however, we have by no means got to the bottom of the matter. In the living frog movements are always going on, and are all due to the contraction of muscles, and yet no stimuli of the kind enumerated are applied to any of them. As the muscles retain the power of contraction for some little time after the death of the animal, it is easy to make such experiments as that described in the next paragraph.

Running longitudinally between the muscles on the dorsal side of the thigh is a shining white cord, the sciatic nerve (Fig. I $7, s c . n v$ ), accompanied by a vein: it gives off branches to the muscles and skin, and, amongst others, one to the gastrocnemius. If this nerve be carefully separated as it traverses the thigh and pinched with the forceps, the gastrocnemius will contract just as if the stimulus had been applied to it directly, and the same will happen if a chemical, thermal, or electrical stimulus be applied.

Thus a stimulus applied to the nerve of a muscle has the same effect as if applied to the muscle directly : it gives rise to a nervous impulse, which, travelling along the nerve, induces contraction of the muscle.

Once more, however, external stimuli are not applied to the frog's nerves during life, and it is obvious that we must carry our inquiry a little further. The sciatic nerve if traced upwards will be found to pass into the trunk (Fig. 5I, Sci), 
and finally to join the spinal cord, which, as we have seen, is in connection with the brain. In the living frog nervous impulses originate in one or other of these organs, without the direct intervention of an external stimulus, and are conducted along the nerves to the muscles. But further consideration of this subject must be deferred until we have made a special study of the nervous system.

The Muscular System in General.-All over the body the muscles, though varying greatly in form-some being elongated and band-like (Fig. I6, sar), others spindle-shaped (gastr), others in the form of broad flat sheets (my. hy, obl. $e x t$ ) - have the same general relation to the skeleton as in the case of the gastrocnemius. Each muscle arises or has its origin in a relatively fixed part, and is inserted into a relatively moveable part. As each muscle contracts in one direction only, it follows that the more complex the movements any part is capable of performing, the more numerous must be its muscles. For instance, the femur, which, as we have seen, is capable of universal movement, has no fewer than nine muscles, arising from various parts of the hip-girdle, inserted into it. Even the minute phalanges of the fingers and toes all have their little slips of muscle by which the various movements of grasping and relaxing, approximating and separating the digits are effected.

There are certain terms applied to muscles which it is useful to know. A muscle which raises a part, e.g., the lower jaw, is called a levator, one which lowers a part a depressor. A muscle which serves to straighten one part upon another, e.g., to bring the shank into line with the thigh, is an extensor, one which bends one part on another is a flexor. A muscle which draws a limb towards the trunk is an adductor, one which draws it away an abductor, one which rotates one part upon another, e.g., the femur on the pelvis, a rotator.

Thus all the complex and accurately adjusted movements of the frog are performed by the contraction of its numerous 
muscles, acting either singly or in concert. The contractions of these muscles are brought about by nervous impulses sent from the brain or spinal cord along nerves which branch out and are distributed to the muscles, thus bringing the whole of the complex machinery which affects the movements of the animal under the direct control of its will.

\section{PRACTICAL DIRECTIONS.}

\section{The Joints and Ligaments.}

I. The hip-joint. Strip off most of the muscles from the thigh and the adjacent parts of the pelvis; decalcify in weak acid and then wash thoroughly. Cut the femur through lengthwise and continue the section through the pelvic girdle (Fig. I5).

Note: $a$. The cartilage of the acetabulum and head of the femur; the perichondrium and periosteum. (b) The capsular ligament and its relations to the synovial capsule. Observe that the hip.joint is a cup and ball joint. Sketch.

In a prepared wet skeleton-

2. Examine and compare a hinge-joint (e.g., elbow or knee).

3. Examine the cartilaginous union between the bones of the shoulderor hip-girdle (immoveable or imperfect joints).

4. Examine the joints and ligaments of the vertebral column.

The Muscles.

I. Remove the skin from part of the body and legs of a preserved frog (the one you have already dissected will do). Then clear away the fascia here and there and separate some of the muscles by dissecting away the connective tissue which binds them together. Notice the different forms of the muscles in different parts. (Compare Fig. 16 and p. 63).

2. In the hind-leg carefully dissect away the connective tissue investing the gastrocnemius muscle (Figs. 16 and 17), and trace it upwards towards the thigh, and downwards towards the foot, cutting away any of the other muscles which obscure the view. Notice the belly, and the tendons of origin and insertion (p. 60). Sketch.

Bend the foot upon the shank as in the ordinary sitting position of the frog. Hold the thigh firmly with one hand, and with the other take hold 
of the gastrocnemius and pull it upwards or towards the thigh. Note the result.

3. In a recently killed frog (see p. I03) expose the gastrocnemius as directed above, and with the small forceps give a sharp pinch to the belly of the muscle. Note the contraction following the simnulus.

Then remove the skin on the dorsal side of the thigh, and separate the muscles in this region so as to expose the sciatic nerve (Figs. 17 and 5I). Trace this towards the shank and notice its branch going to the gastrocnemius. Carefully separate the nerve as it traverses the thigh and pinch it with the forceps, noting again the contraction following the stimulus. 


\section{CHAPTER V.}

THE FROG (continued): WASTE AND REPAIR OF SUBSTANCETHE DIGESTIVE ORGANS-NUTRITION.

Waste and Repair--The effects of prolonged muscular exertion are familiar to every one. Sooner or later sensations of fatigue, hunger, and thirst are produced, accompanied by a loss of weight. Indeed, however little exertion we make and however often we feed, our weight always goes down between meals and rises again when we take food. The loss of substance, of which the diminution in weight is the index, takes place largely in the form of perspiration, a fluid consisting of water, with certain organic and inorganic matters in solution. A further loss is due to the air breathed out from the lungs; this is always moist, i.e., contains a good deal of water, and is further distinguished by containing a considerable volume of the gas carbon dioxide, or carbonic acid $\left(\mathrm{CO}_{2}\right)$. Besides these two constant sources of loss, there is an intermittent loss in the urine, which consists of water, containing certain matters in solution, the most characteristic of which are two complex substances called urea $\left(\mathrm{CON}_{2} \mathrm{H}_{4}\right)$ and uric acid $\left(\mathrm{C}_{5} \mathrm{H}_{4} \mathrm{~N}_{4} \mathrm{O}_{3}\right)$. Both of these, as well as carbon dioxide, act as poisons if 
allowed to remain in the system. Lastly there is an intermittent source of loss in the waste matters or fæces which are passed out from the intestine.

These losses are made good in two ways. Firstly, by breathing, in which process we constantly inhale pure air and replace the poisonous carbonic acid by oxygen. Secondly by eating and drinking, by which, at intervals, we make good the loss of solids and liquids. Just as a clock is constantly running down and has to be wound up in order to keep it going, so our bodies run down by loss of substance between meals, and require to be wound up by the repair of substance which results from food and drink.

The same thing is true of the frog. Every one of its numerous and often vigorous movements is done at the expense of a certain waste of substance. The various tissues of the body are constantly undergoing a process of wear and tear, expressed, not as in machines of human construction, by a wearing away of surfaces and a loosening of bolts and screws, but by a slow and almost imperceptible dwindling, the lost material being carried off principally in the form of water, carbon dioxide, and urea or some allied compound containing nitrogen.

Food of the Frog : general characteristics of the Digestive Process. - As we have seen, the food of the frog consists of worms, slugs, insects, and the like. These it catches and swallows whole, the stomach often becoming immensely distended with numbers of captured animals. After remaining for some time in the stomach the carcases are found to have undergone a marked change. Their soft parts become softer and slimy and finally semi-fluid, and in this way the food undergoes gradual disintegration. The quantity of food in the stomach decreases, some of it is . passed into the intestine, which it traverses from duodenum 
to rectum, and certain portions of it are finally ejected from the vent in the form of fæces.

It is not difficult to assure one's self that the weight of the fæces passed during a certain time is very much less than that of the food swallowed during the same time. Obviously some constituents of the food have disappeared during its progress through the enteric canal. The character of the fæcal matter, moreover, is very different from that of the food; the only portions of the swallowed animals discoverable in the rectum are bits of their hard parts ; for the rest, the fæces form a pulpy, black mass. That this change is due to certain definite chemical processes taking place in the enteric canal may be inferred from the fact that the contents of the stomach, as well as the walls of that organ, have an acid reaction, and turn blue litmus paper red. On the other hand the contents of the small intestine are, to a greater or less extent, alkaline, restoring reddened litmus paper to its original blue colour.

It is also obvious that there must be some definite mechanism for propelling the food from one end of the enteric canal to the other; its passage through so long, narrow, and coiled a tube can certainly not be accounted for by supposing it to be merely pushed onwards as fresh food is swallowed.

In order to understand the various processes connected with digestion we must make a renewed and more careful examination of the organs concerned, after removing them from the body.

The Digestive Organs.-Arising from the gall-bladder and passing backwards to the duodenum is an extremely delicate tube (Fig. I $8, D c, D c^{1}$ ), the common bile-duct, which opens into the duodenum. By gently squeezing the gallbladder a drop of greenish fluid may be made to ooze out 
of the end of the duct $\left(D c^{2}\right)$ into the intestine; this fluid is the bile.

Very careful dissection shows that the common bile-duct

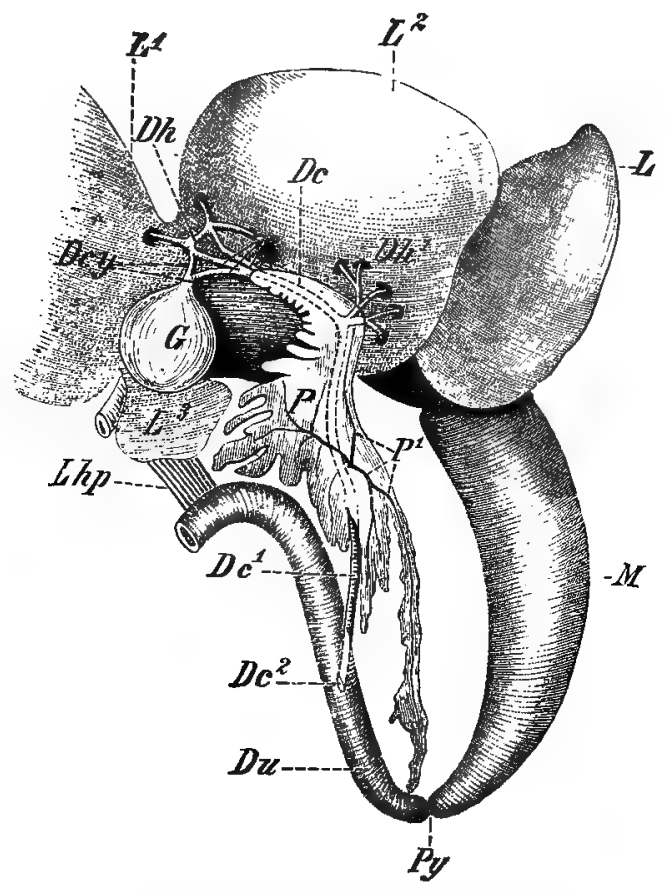

FIG. 18.-Stomach and duodenum of Frog with liver and pancreas.

$D c, D c .1$ common bile duct; $D c^{1}{ }^{2}$ its opening into the duodenum; $D$. cy. cystic ducts ; $D h, D h .{ }^{I}$ hepatic ducts; $D u$. duodenum ; G. gall-bladder; $L, L 1, L^{2}, L^{3}$, lobes of liver, turned forwards; $L h p$. duodeno-hepatic omentum, a sheet of peritoneum connecting the liver with the duodenum ; $M$. stomach ; $P$. pancreas; $P^{1}$, pancreatic duct; $P_{y}$. pylorus. (From Wiedersheim's Comparative Anatomy.)

is joined by several other tubes which are traceable into the liver and, are called hepatic ducts $\left(D h, D h^{1}\right)$. The tubes going to the gall-bladder are called cystic ducts (D.cy); by 
their union with the hepatic ducts the common bile duct is formed.

The bile is manufactured in the liver, and the gall-bladder is merely a reservoir in which it is stored up, to be discharged into the intestine when required for digestion.

It requires still more accurate observation to show that the pancreas also discharges a fluid, the pancreatic juice, into the intestine. A very delicate branching tube, the pancreatic duct $\left(P^{1}\right)$, joins the bile-duct, into which it discharges the pancreatic juice, the two fluids entering the intestine together. Both fluids are digestive juices, i.e., liquids which, as we shall see, act in a particular way upon the food.

By cutting open the enteric canal and examining its inner surface under water with a magnifying glass it is seen that the wall of the canal consists of two layers, easily separable from one another. The outer or muscular layer (Fig. I9 A, musc), is tough and strong, the inner layer or mucous membrane $(m . m)$, is soft and slimy. Between the two is very loose connective tissue, the submucosa-really a part of the mucous membrane-which, being easily torn, allows of the ready separation of the muscular and mucous layers.

In the stomach the mucous membrane is raised into longitudinal folds $(r)$, in order to allow of distension; in an empty stomach they are well marked, and give the cavity a star-like cross-section (Fig. 40); in one full of food they are entirely obliterated and the walls of the organ so stretched as to be almost transparent. Anteriorly the ridges thin out and disappear at the cardia or junction between the gullet and stomach ; posteriorly they converge, as the stomach narrows, towards the pylorus (Fig. I9 A, py), or junction with the duodenum. Here the muscular coat is greatly thickened in a ring-like form, forming the pyloric valve ( $p y . v)$, by which the aperture of communication between the stomach and 
intestine is greatly narrowed and only small particles can pass through: In the duodenum $(d u)$ the mucous membrane is raised into little tuft-like elevations $\left(r^{\prime \prime}\right)$; in the ileum the ridges $\left(\mathrm{B}, r^{\prime \prime}\right)$ become longitudinal again; in the rectum $(r c t)$ they are absent.

When food is taken into the stomach, a fluid, the gastric juice, oozes from the mucous membrane. It is this fluid
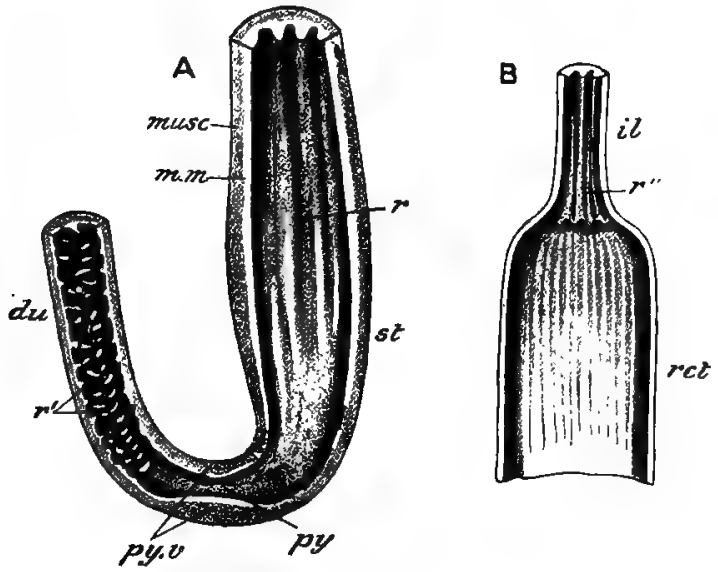

FIG. Ig.-Portions of the enteric canal of the Frog in longitudinal section.

$A$, stomach and duodenum; $B$, part of ileum and rectum. $d u$. duodenum ; il. ileum ; $m . m$. mucous membrane; musc. muscular layer; $b y$. pylorus; $p y, z$ : pyloric valve; $r$. longitudinal ridges (rugæ) of stomach ; $r$ '; transverse ridges of duodenum; $\gamma^{\prime \prime}$. longitudinal ridges of ileum; rct. rectum; st. stomach.

which reduces the slugs, insects, etc., to the pulpy condition referred to above : it is, like the bile and pancreatic fluid, a digestive juice.

General Properties of Food.-We must now devote a little attention to the characters of the food itself and to the precise nature of the changes brought about by the digestive process. 
As we have seen, the frog is a carnivorous animal. Now the digestible part of the substance of animals consists mainly of two classes of chemical compounds, called proteids and fats. The most familiar example of a proteid is white of egg : other proteids, of varying composition, are found in muscle, in blood, and in other animal tissues. All are composed of the five chemical elements-carbon, oxygen, hydrogen, nitrogen, and sulphur--the five elements being combined in the following proportions :-

\begin{tabular}{|c|c|c|c|c|}
\hline Carbon & from & $5 \mathrm{I}^{\circ} 5 \mathrm{t}$ & o 54.5 & er cent \\
\hline Hydrogen & ", & 6.9 &, 7.3 & $"$ \\
\hline Oxygen & " & 20.9, & , 23.5 & ", \\
\hline Nitrogen & ", & $15^{2}$, & , $17^{\circ} 0$ & ", \\
\hline Sulphur & ", & 0.3 & , 20 & ", \\
\hline
\end{tabular}

Fats differ from proteids in containing no nitrogen or sulphur : they are formed of carbon, oxygen, and hydrogen, the number of atoms of hydrogen being always more than twice as great as the number of atoms of oxygen.

It will be noticed that two important articles of diet are absent from the above list, namely sugar and starch-the latter the largest constituent of flour, oatmeal, rice, \&c. The vegetable substances used as food by animals, such as corn and grass, contain these bodies in varying proportions in addition to vegetable proteids, and there is no doubt that the frog must eat a small quantity of such vegetable food, if only in the stomachs of the herbivorous animals upon which it preys. Now, starch and sugar belong to a group of compounds called carbohydrates, composed of carbon, oxygen, and hydrogen, but differing from fats in that the number of atoms of hydrogen is always exactly double that of the atoms of oxygen, as in water. Lastly, the food always contains a certain quantity of saline or mineral matters, as well as water. 
Diffusible and Non-diffusible Foods.-These four classes of food materials-proteids, fats, carbohydrates, and minerals-may be arranged in two groups according to a certain physical peculiarity. If a solution of common salt is placed in a vessel with a bottom made of bladder, called a dialyser, which is floated in a larger vessel of pure water, it is found that, after a certain lapse of time, the water in the outer vessel has become salt. The sodium chloride has, in fact, passed by diffusion through the bladder. The same thing will happen if a solution of sugar is placed in the inner vessel : salt and sugar are both diffusible substances, capable of passing through an animal membrane.

On the other hand, if the inner vessel contains white of egg, or oil, or starch well boiled in water, no diffusion takes place. Hence proteids, fats, and starch are non-diffusible foods, and are thus sharply distinguished from salt and sugar, which are diffusible.

The mucous membrane of the stomach and intestine are animal membranes having the same physical properties as bladder. We may consequently infer that any salt or sugar contained in the enteric canal will diffuse through the mucous membrane and make its way, as we shall see more particularly hereafter, into the blood, thus serving to nourish the whole body, Proteids, fats, and starch, on the other hand, will be incapable of diffusing, and will, therefore, unless some change happens to them, be absolutely useless as nutriment. For, since the enteric canal communicates with the auter world at both ends, the food, paradoxical as it may sound, is practically outside the body as long as it remains in the canal: it is only when it is absorbed into the blood or lymph that it is actually, in the strict sense, taken into the body. Thus, if proteids, fats, and starch are to be of any use to the 
frog, they must, in some way, be rendered capable of being absorbed.

Action of Digestive Juices.-This is exactly what is done by the digestive juices. If white of egg or any other proteid is mixed. with gastric juice and kept at a suitable temperature, it is converted into a form of proteid called peptone, which is capable of diffusing through an animal membrane. The change is effected by means of a substance called pepsin, contained in the gastric juice, in which there is also a certain proportion of hydrochloric acid. To this the acid reaction of the gastric juice already alluded to (p. 68) is due.

By means of the gastric juice the bodies of the animals swallowed by the frog have their proteids largely converted into peptones, which, being diffusible, pass through the mucous membrane as readily as sugar or salt. Hence the great diminution in the bulk of the food during its sojourn in the stomach : a large proportion of it is absorbed there and then, and only a comparatively small quantity is passed through the pyloric valve into the intestine, where it becomes alkaline, owing to the action of the fluid which enters the duodenum through the bile duct, and which, as we have seen (p. 70 ), consists of bile and pancreatic juice.

Pancreatic juice has a similar effect on proteids, the change being effected by a substance called trypsin, which, however, acts in a alkaline solution. It also has the property of converting starch into sugar, and of splitting up fats into fatty acids and glycerine, both of which are diffusible. The substances by which these changes are effected are called by the general name of ferments: pepsin and trypsin are proteolytic or proteid-converting ferments, and the pancreatic juice also contains an amylolytic 
ferment, which converts starch into sugar, and a fatdecomposing ferment.

The exact mode of absorption of the fats is not thoroughly understood. It is usually supposed that only a small proportion of them are decomposed into fatty acids and glycerine, and that the greater part is merely broken up into particles so small that they can be taken up by the epithelial cells of the intestine. This emulsification of fat is effected by the combined action of the pancreatic juice and bile, the fats being reduced to the condition in which they exist in milk and in the emulsions of cod-liver oil so much used in place of the natural form of that medicine.

Thus during the passage of the food through the intestine the remainder of the proteids, the whole of the fats, and any starch which may be present, are rendered capable of being absorbed: they pass through the mucous membrane into the blood, and by the time the rectum is reached all the nutriment is extracted from the food, and there remains only a small quantity of indigestible matter, which is passed out in the form of freces.

Peristaltic Movements. - The passage of the food through the enteric canal is effected by the contraction of its muscular layer, which is really double, and which is composed of muscular fibres (see Chapter vii., Figs. 39 and 40). In the inner layer these fibres have a transverse direction, encircling the tube, and by their contraction narrowing it; the outer layer consists of longitudinal fibres, which by their contraction shorten it. . By the alternate contraction and relaxation of the two layers are produced a series of peristaltic movements, not unlike those by which an earthworm makes its way over the ground : they can be seen in a freshly-killed frog, and still better in a rabbit or rat.

Summary of Chapter--The body is constantly under- 
going waste, and in consequence needs continual repair. The waste-matters chiefly take the form of carbon dioxide, water, and urea. Repair is effected partly by breathing (see Chapter ix), partly by feeding. Food, consisting of proteids, fats, carbohydrates, inorganic substances, as well as water, is taken into the enteric canal, where, by the action of the three digestive juices-gastric juice, pancreatic juice, and bile-it is converted partly into a solution (peptones, sugar, fatty acids, glycerine), partly into an emulsion (fats). As it is driven along the canal, from stomach to rectum, by the action of the muscular coat, the dissolved or emulsified substances gradually disappear from the canal, and are absorbed into the system. Finally, the indigestible constituents are expelled as fæces.

Our next task must be to learn something of the process of absorption, and of the means by which the digested food is conveyed to the various parts of the body, so as to supply them with the means of repairing the waste they are constantly undergoing. For this purpose we must now study what is called the vascular system, i.e. the heart, the blood-vessels, and the various cavities containing lymph.

\section{PRACTICAL DIRECTIONS.}

The Digestive Organs.-Pin down under water, with the ventral side uppermost, as before, the specimen already dissected, or another in which the body-cavity has been opened in the same way. Note the positions of the postcaval vein (Figs. 3 and $4, p t . c v$ ), the hepatic portal vein (Fig. 3, $h p . p t$ ), the aorta (Fig. 4, d. ao), and the splanchnic or coliaco-mesenteric arlery (Figs. 3 and 4, cor. mes). Then, taking care not to injure the aorta, remove the greater part of the digestive organs, including the liver, by cutting through the gullet and rectum and severing the mesentery, cutting through the postcaval also where it enters the liver. (The cloaca will be examined at a later stage). Pin the organs in the dissecting dish as nearly as possible in their natural position. Turn the 
lobes of the liver forwards (i.e., towards the head) and after making out the relations of the parts already examined in situ (pp. 20-23), note-

The common bile-duct, formed by the union of the hepatic and cystic ducts, and the point at which it enters the duodenum (Fig. I8). Make a small slit in the duodenum just opposite its entrance, and gently squeeze the gall-bladder between your finger and thumb, so as to force a drop of bile into the intestine. (The pancreatic duct and its communication with the bile-duct cannot easily be made out by dissection). Sketch the whole dissection.

Now remove the liver, unravel the intestine by tearing through the mesentery, and lay open the enteric canal by inserting one blade of the scissors into the gullet, and cutting through the whole tube in a longitudinal direction. Test the contents of the stomach and intestine with litmus paper-(for this experiment a freshly killed frog is of course necessary)-and then pin out your dissection with the inner surface upwards, wash it under the tap, and examine under water with a magnifying glass. Make out-

I. The cardia, pylorus, and pyloric valve.

2. The mucous membrane, and its different appearances in the stomach, small intestine, and large intestine.

3. The muscular layer, covered externally by the peritoneum.

Make a simple dialyser (p. 73) by tying a piece of wet bladder firmly over one end of a wide glass tube about six inches long. Into this put a solution of sugar or salt, and immerse the tube up to the level of the solution in a rather larger vessel of distilled water, and leave it for a short time: taste the water in the outer vessel. Then place some white of egg in the dialyser, and test for albumen by heating some of the water in the outer vessel over a flame: if albumen is present, it will become coagulated and form a cloud in the water. 


\section{CHAPTER VI.}

THE FROG (continued): THE VASCULAR SYSTEM-THE CIRCULATION OF THE BLOOD.

IN our preliminary examination of the frog (Chap. II) we learned one or two facts about the vascular system. We found that there is a heart within a pericardium, two sets of vessels, arteries and veins, containing red blood, and a set of irregular cavities or sinuses, containing lymph. We must now try to get some more accurate and detailed information on these matters.

General Characteristics of Blood and Lymph.-It will be convenient to begin by studying certain easily verified characteristics of the blood. Frog's blood may be used, or as it is as well to have a considerable quantity, that of some larger, freshly-killed, red-blooded animal, such as a rat or rabbit.

When first drawn from the heart or vessels the blood will be seen to be a fluid, nearly as mobile as water or milk; it "finds its level," like any other liquid, and can be readily poured from one vessel to another. In a few minutes, however, it undergoes a change; it ceases to be fluid, and coagulates, or "sets" into a jelly, which if turned out of the vessel, retains the shape of the latter. Before long a further change takes place; the jelly begins to shrink, drops of 
yellowish fluid appear on its surface and gradually run together into larger and larger drops. The jelly contracts still further, and finally draws itself away from the walls of the vessel and floats in the accumulated fluid, still retaining the form of the vessel, but being greatly reduced in size. The process of coagulation of the blood is now complete; the red, jelly-like substance is called the clot, the yellowish fluid the serum.

When first drawn from a vein the blood is deep purple in colour, and the clot retains for a time the same hue. But before long, all parts of it which are fully exposed to the air take on a bright scarlet colour. We may therefore distinguish between red, or aërated, and purple, or non-aërated blood.

Lymph also coagulates on standing, producing a colourless clot. It is practically blood minus its peculiar red colouring matter, the properties of which, as well as the real nature of coagulation, will be discussed in the next chapter.

The Heart : external characters.-Some of the divisions of the heart have already been noticed (p. 20 ). The ventricle (Figs. 3, 4, 7, 20, $2 \mathrm{I}$ and 22, v), is a conical body of a pinkish colour, having its bluntly-pointed apex directed backwards. To its broad base is attached the dark-coloured, thin-walled auricular division, actually consisting of two chambers, the right and left auricles (r.au, l. au), but appearing single in the entire heart. Passing obliquely across the auricles is a cylindrical structure, the conus arteriosus $(c$. art $)$; it starts from the right side of the base of the ventricle, and passes forwards and to the left, finally dividing near the anterior boundary of the auricles into two branches, which extend respectively right and left.

By lifting up the ventricle, or turning it to one side (Figs. 3 and 4 ), there is seen in the dorsal part of the pericardial cavity a thin-walled chamber $(s, v)$ of a dark colour, con- 
nected with the right side of the auricular division. This is the sinus venosus.

The Arteries. - The two branches of the comus arteriosus just referred to soon branch again. Each divides into three vessels, often spoken of as arterial arches, called respectively the carotid trunk (Fig. 20, car. tr.) the systemic trunk (syst.tr) and the pulmo-cutaneous trunk (pul.cu.tr). All these conform to the definition of an artery given on p. 27, i.e., they are stout, elastic vessels, containing little blood after death, and not collapsing when empty.

The carotid trunk divides immediately into two, a lingual $\operatorname{artery}(\mathrm{l} g$ ) , which can be traced to the tongue, and a carotid artery (car), which branches repeatedly, its ultimate ramifications going to various parts of the head. At the origin of the carotid is a little rounded mass with a spongelike interior, the carotid plexus (car. gl).

The systemic or aortic trunk extends outwards, in contact with the gullet, then sweeps upwards, backwards, and inwards -i.e., towards the middle line-and finally joins with its fellow of the opposite side to form a single median vessel, the dorsal aorta (Figs. 4, 5, and 20, d. ao), which passes backwards, just beneath the vertebral column and between the kidneys.

As it sweeps round the gullet, the systemic trunk gives off a vertebral artery (Fig. 20, vert) to the vertebral column and part of the head, a subclavian artery to the fore-limb, and an asophageal artery to the gullet.

From the point of union of the two aortic trunks springs a single splanchnic or caliaco-mesenteric artery (cal. mes); it divides into several branches, which are traceable to the liver $(h p)$ stomach $(g s)$, duodenum $(d u)$, spleen $(s p l)$ and ileum (int).

The dorsal aorta gives off on each side four renal arteries 


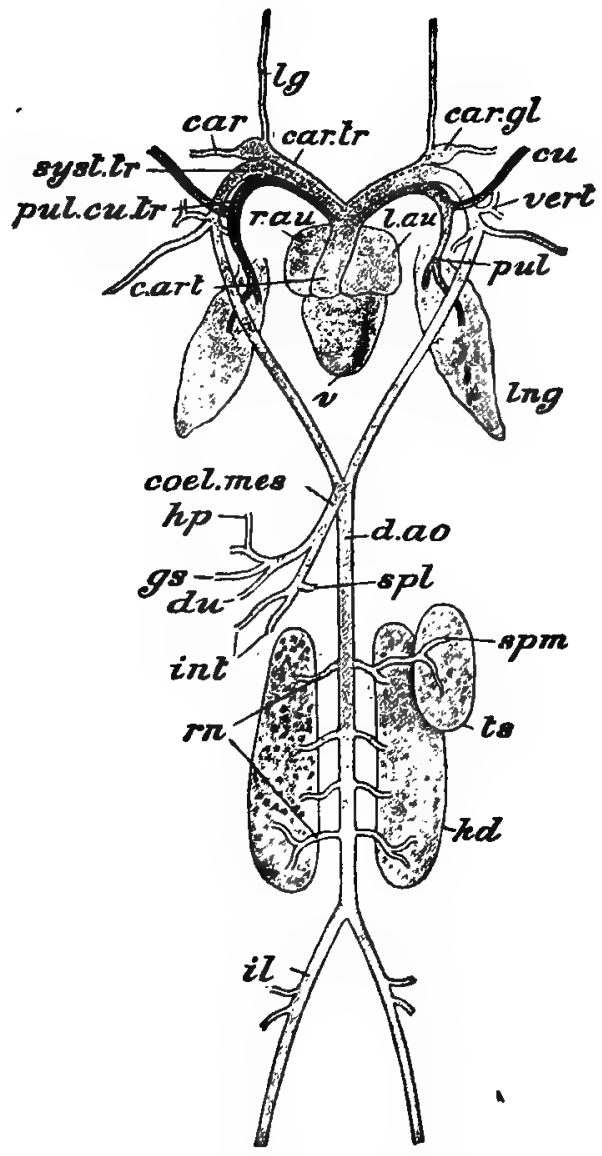

FIG. 20.-The arterial system of the frog, with the heart, lungs, kidneys and left testis, supposed to be removed from the body and viewed from the ventral aspect.

car. carotid artery; car.gl. carotid plexus; c. art. conus arteriosus ; car. tr. carotid trunk ; ceel. mes. splanchnic or coliaco mesenteric artery ; cu. cutaneous artery ; $a$. ao. dorsal aorta ; dz. duodenal artery ; $g s$. gastric artery; hp. hepatic artery; il. iliac artery; int. intestinal arteries; $k d$. kidney; 2 . au. left auricle; lg. lingual artery ; lng. lung ; pul. pulmonary artery; $p u l$. cu. tr. pulmo-cutaneous trunk; ur. au. right auricle; $r n$. renal arteries; spl. splenic artery; syst. $t r$. systemic trunk; spm. spermatic artery; $t$ s. testis; $v$. ventricle; vert. vertebral artery, just behind which are seen the subclavian and osophageal arteries. (From Parker and Haswell's Zoology.)

PrACT, ZOOL. 
$(r n)$ to the kidneys, and spermatic arteries $(s p m)$ in the male, or ovarian in the female, to the reproductive organs, and at the posterior end of the abdominal cavity divides into the right and left iliac arteries (il) which go to the hind limbs.

The pulmo-cutaneous trunk divides into two main branches, the pulmonary artery ( $p u t)$ which goes to the lung, and the cutaneous artery (cu) which forms an extensive system of branches over the skin.

With proper care all these arteries can be traced into the various organs to which they are distributed, when they will be found to branch repeatedly, sending ramifications to all parts. The iliac artery, for instance, may be followed along the whole length of the leg, giving off branches to all the muscles, to the skin, and to the digits, with their intervening web.

The Veins.-Since every part of the body has its vein as well as its artery, there is a rough correspondence between the two kinds of blood-vessel. The arrangement of the principal trunks is, however, very different in the two cases.

On each side of the base of the heart is a large vein called the precaval or vena cava anterior (Fig. $2 \mathrm{I}$, pr. $v$ ) : by turning the ventricle aside, the two precaval veins can be seen to join the anterior end of the sinus venosus (s.v). Each precaval is formed by the confluence of several veins, of which the most important are the external jugular (ext.ju) from the lower jaw and tongue, the internal jugular (int. ju) from the brain, eye, etc., the subclavian (br), from the fore-limb, and the musculo-cutaneous (mu. cu), already noticed, from the superficial parts of the head and part of the skin and muscles of the abdomen.

With the posterior end of the sinus venosus is connected a single large vein, the postcaval or vena cava posterior 


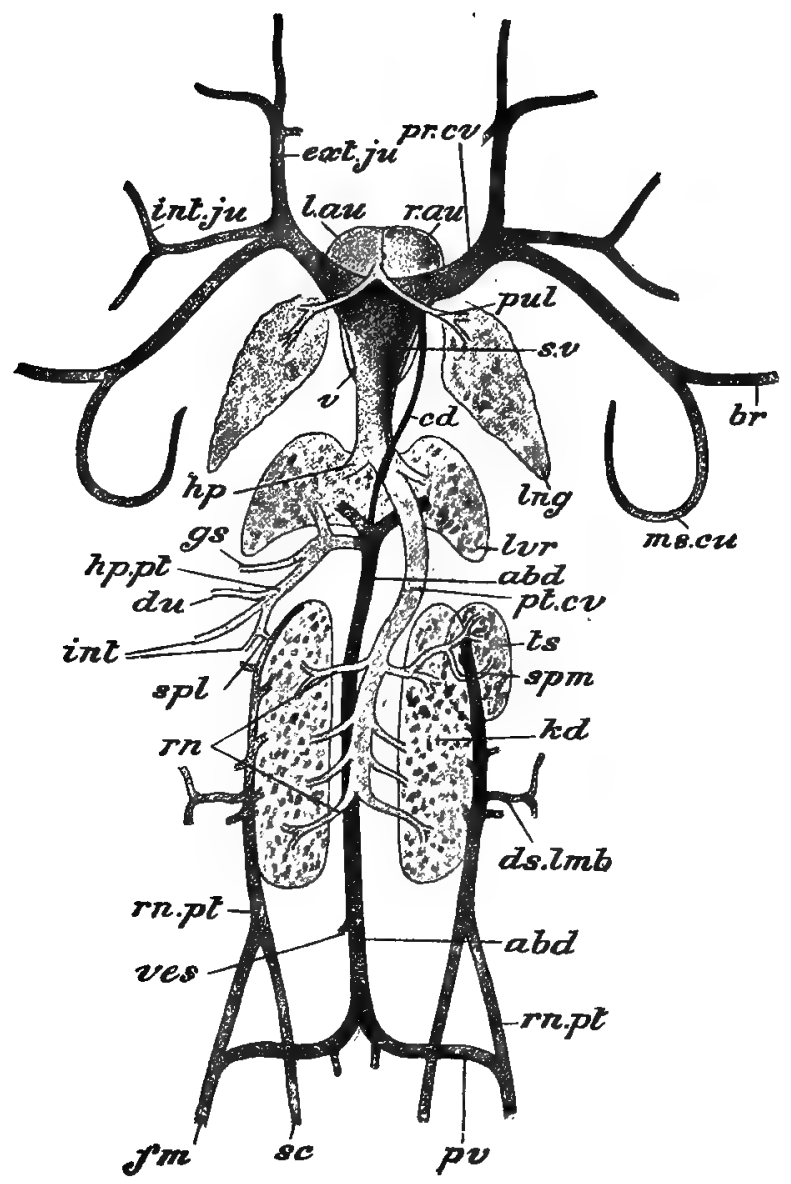

FIG. 2x.-The venous system of the frog, with the heart, lungs, liver, kidneys and right testis, supposed to be removed from the body and viewed from the dorsal aspect.

abd. abdominal vein; br. subclavian vein ; $c d$. cardiac vein; $d s$. lmb. dorso lumbar vein; dzt. duodenal vein; ext.ju. external jugular vein; fm. femoral vein; $g s$. gastric vein; $h p$. hepatic vein; $h p . p t$. hepatic portal vein ; int. intestinal veins : int.ju. internal jugular veirf; kd. kidney; l.au. left auricle; lng. lung; lur. liver; $m s . c u$. musculo-cutaneous vein; $p r . c v$. precaval vein; $p t . c v$. postcaval vein; pul. pulmonary vein; $p v$. pelvic vein; $r$. au. right auricle; $r n$. renal veins; m. pt. renal portal vein; sc. sciatic vein; spl. splenic vein; spm. spermatic vein; s. $v^{\prime}$. sinus venosus; $t s$, testis; ves. vesícal veins (from bladder). (From Parker and Haswell's Zoology.) 
( $p t . c v)$, a wide vessel lying between the kidneys and extending forwards to the liver (Fig. 4). It runs parallel with and beneath; i.e, ventral to, the dorsal aorta (Fig. 5), from which it is at once distinguished by its greater diameter. Posteriorily it is formed by the confluence of four renal veins (Fig. $21, m$ ) from each kidney, and it also receives in the male, spermatic veins (spm) from the spermaries, and in the female, ovarian veins from the ovaries. Anteriorly it perforates the liver (lvr), receiving two hepatic veins $(h p)$ from that organ, and finally enters the sinus venosus.

We have now to consider a striking want of correspondence between the arterial and venous systems of the frog. As you will remember, the dorsal aorta, after giving off the renal arteries, passes backwards and divides into the two iliac arteries for the legs. You might naturally expect a somewhat similar arrangement with the veins, especially if you have studied human physiology and learnt how the posterior (or inferior) vena cava of man is formed by the confluence of the veins from the legs, and receives higher up those from the kidneys. In the frog, as we have just seen, the postcaval does not reach to the hinder boundary of the kidneys, and the renal veins are the only vessels entering its posterior end.

In the frog, as a matter of fact, the connections of the veins of the legs are very peculiar. You remember the abdominal vein seen in our preliminary dissection (Figs. 2, 3 and $4, a b d . v$ ). This vessel, if traced backwards, is found to fork at the posterior end of the abdomen-or more accurately, the single abdominal vein is found to be formed by the confluence of two pelvic veins (Fig. $2 \mathrm{I}, p v$ ), which can be traced along the base of the leg (compare Fig. 3). Towards the front of the thigh is the principal vein of the leg, the femoral vein (Fig. $2 \mathrm{I}, f . m$ ), which on emerging from the leg, divides 
into two branches. One of these is the pelvic vein already seen ; it unites with the corresponding vessel of the opposite side to form the abdominal vein $(a b d)$. The other branch of the femoral is called the renalportal vein $(r n . p t)$; it passes directly forwards, receiving the sciatic vein (sc) from the back of the leg, and then goes along the outer border of the kidney, finally branching out in that organ. It also receives a large vein $(d s . \operatorname{lm} b)$ from the muscles of the back.

The abdominal vein receives, near its posterior end, small veins (ves) from the urinary bladder. It passes forwards, as already seen, receiving veins from the abdominal muscles, and, quite at its anterior end, a small vein $(c d)$ from the heart. It then divides into two branches which enter the liver and branch out in that gland.

The veins from the stomach $(g s)$, the intestine $(d u, i n t)$, spleen $(s p l)$, and pancreas run in the mesentery alongside the corresponding arteries. Near the liver they all unite to form a large vessel, the hepatic portal vein ( $h p . p t)$, which enters and branches out into the liver, first sending off a branch which joins the abdominal.

Thus the veins from all parts of the body, except the lungs, ultimately discharge into the sinus venosus. The veins from the lungs are quite singular in their course ; they unite, in each lung, to form a single pulmonary vein ( $p u l$ ), which passes behind, or dorsal to, the sinus venosus, and discharges, with its fellow of the opposite side, into the left auricle.

Character of the Blood in the Arteries and Veins.--There are certain differences between the arteries and veins in respect of the blood they contain. As a rule, the veins contain purple or non-aërated blood, the arteries more or less thoroughly aërated or scarlet blood. But there are certain exceptions. As we shall see in a later chapter, the 
blood is aërated in the lungs and skin; hence the blood returned from those organs by the pulmonary and musculocutaneous veins is aërated. On the other hand, the blood in the pulmo-cutaneous artery is non-aërated.

Flow of Blood.-We must now try to understand the function of this complicated blood-system and the reason why every part of the body has two vessels, an artery and a vein. That there is some kind of movement of the blood has been hinted in the foregoing description, in which arteries have been described as branching out to various parts, veins as formed by the confluence of smaller veins from various, parts.

Were an artery to be cut in a living frog, the blood would be found to flow out in a series of jerks corresponding with the beats of the heart. Moreover the blood would flow from the side of the cut nearest to the heart, and the flow might be stopped by tying or compressing the artery on that side, i.e., between the heart and the cut. Evidently, then, the blood in the living animal flows from the heart along the arteries to the various parts of the body, and is propelled by the pulsation of the heart.

If a vein were cut the result would be very different. The blood would flow in a comparatively slow stream and without jerks; it would flow, moreover, from the side of the cut furthest from the heart, so that, in order to stop the bleeding, the vein must be.tied or compressed on the far side of the cut. The blood in the veins flows, therefore, towards the heart in an even stream, unaffected by the heart's pulsations.

Thus the blood is driven by the heart to the various parts of the body through the arteries, and is returned from the various parts of the body to the heart by the veins. Two questions thus naturally arise: how is it that the blood 
takes this direction and not the other, and how does it make its way from the artery of a given organ into the vein?

Internal Structure of the Heart.-To answer the first question - why the blood leaves the heart by the arteries

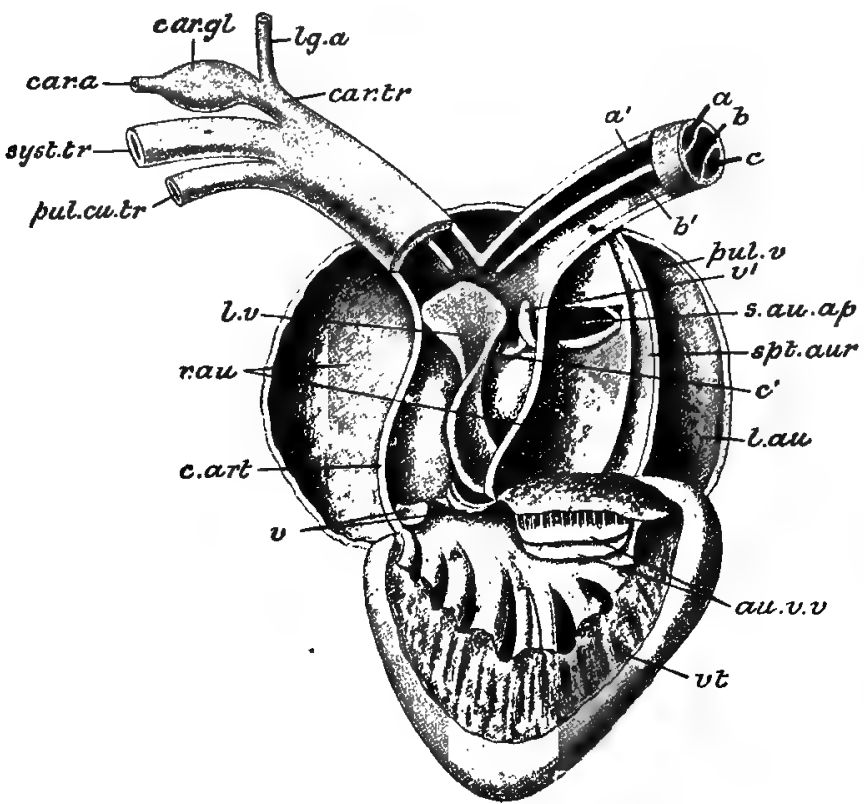

FIG. 22. - The heart of the frog from the ventral aspect, with the cavities laid open. $a, a,{ }^{\prime}$ bristle passed into left carotid trunk; $\alpha \pi, v, v$, auriculo-ventricular valves; b. $b$. $^{\prime}$ bristle in left systemic trunk; $c, c$, bristle in left pulmo-cutaneous trunk; car. a. carotid artery; cor. gl. carotid plexus; c. art. conus arteriosus ; car. tr. carotid trunk; $l . a u$. left auricle; $l g$. $a$. lingual artery; $l . v$. longitudinal valve; pzol. cu. tr. pulmo-cutaneous trunk; $p u l, v$. aperture of pulmonary veins; $r$, au. right auricle; s. au. ap. sinu-auricular aperture; spt. aur. septum auricularum; $z \prime, v$.' valves; $7 t$. ventricle. (Fron Parker and Haswell's Zoology.)

and returns to it by the veins, and not vice versâ-we must examine the heart itself in some detail.

The ventricle is a hollow structure with thick spongy walls and a small cavity (Fig. 22,vt), and there are two perfectly 
distinct auricles, the right ( $r . a u)$, considerably larger than the left $(l \cdot a u)$, separated from one another by a vertical partition (spt. aur).

You have already seen that the conus arises from the right side-the frog's right, not yours-of the base of the ventricle. A little to the left of this point there is an aperture through which a bristle can be passed from the ventricle into either of the auricles. Both auricles, then, communicate with the ventricle by a single auriculoventricular aperture. This is guarded by two little membranous flaps $(a u . v . v)$, which spring, one from the dorsal, one from the ventral edge of the aperture, and hang down into the ventricle, to the walls of which they are attached by little tendinous cords, represented in the figure by white streaks. Thus the flaps have the character of folding doors or valves opening only one way ; they readily flap backwards, i.e., into the ventricle, but are prevented from flapping forwards or into the auricles by the tendinous cords attached to their backs. The two flaps are the auriculoventricular valves. Their mode of action is easily understood. If the auricles, being full of blood, contract and squeeze themselves together, the pressure will force aside the valves and allow the blood a free passage into the ventricle. On the other hand, if the ventricle contracts, the blood, getting behind the valves, will force them together and close the aperture, the tendinous cords preventing their being driven into the auricles by undue pressure.

In the interior of the conus is a longitudinal fold or valve $(l . v)$, which traverses it obliquely, attached to its dorsal wall and free ventrally; and there are also three little semilunar or watch-pocket shaped valves $(v)$ guarding the aperture between the ventricle and the conus : they are arranged with their edges turned forwards or towards the cavity of the 
conus, so that any pressure of fluid from the side of the ventricle must force them back and allow ready ingress into the conus, while pressure in the opposite direction must fill them, bringing their edges together, and so barring the passage.

Anteriorly the longitudinal valve projects in the form of a free flap, and at the same level is a semilunar valve $\left(v^{\prime}\right)$ : the two together separate the conus proper from a small chamber, the bulbus aorte, from which the right and left carotid $\left(a, a^{\prime}\right)$ and systemic $\left(b, b^{\prime}\right)$ trunks arise. The pulmocutaneous trunks $(c)$ spring from the conus by an aperture (c) situated just posteriorly to the valve $v^{\prime}$, and itself guarded by a small valve.

In the dorsal wall of the right auricle is a large transverse aperture (s.au.ap). This leads into the sinus venosus : it is therefore called the sinu-auricular aperture; its two edges are produced into flaps, the sinu-auricular valves, which allow free passage from the sinus to the right auricle, but prevent any flow in the opposite direction.

Valves of the Veins.-In addition to the valves of the heart, many of the veins contain small watch-pocket valves, all arranged with their concavities directed towards the heart, so as to allow of a free passage in that direction. Any attempt to flow in the opposite direction, i.e., from the larger to the smaller veins, will result in filling the valves, bringing their edges into contact with the opposite wall of the vein, and thus effectually blocking the passage.

Circulation of the Blood.-We see then that an investigation of the structure of the heart shows that fluid can traverse it in one direction only, viz., from the sinus to the right auricles, from the auricle to the ventricle, from the ventricle to the conus, and from the conus to the bulbus aortæ, and so to the arteries. The valves in the veins are so 
arranged as to allow the blood in these vessels to flow only towards the heart. The experiment of cutting the vessels shows that the blood in the arteries does actually flow from the heart, that in the veins towards the heart. We thus demonstrate that there is not merely a movement but a true circulation of the blood, the current starting from the heart, passing by the arteries to all parts of the body, and being returned to the heart by the veins.

Action of the Heart.-The circulation of the blood is effected by the pulsation of the heart. This organ is made of muscle; each of its cavities is to be considered as a bag, the walls of which are formed of muscular fibres crossing one another in various directions and encircling the cavity. We have seen that when an ordinary spindleshaped muscle contracts, its two ends are brought nearer together. When a hollow muscular bag contracts the effect will be to squeeze the walls together and so diminish the cavity. Hence when any chamber of the heart contracts it must expel a part or the whole of its contained blood. The contraction of the chambers of the heart takes place in regular order: first the sinus, then the two auricles together, then the ventricle, and lastly the conus. The contraction in each case is visible as a sort of throb and is followed by a period of rest, during which the chamber regains its former dimensions.

The course of the blood through the heart will now be clear. When the sinus (Fig. 23, s.v) contracts, the contained blood, which, coming by the precavals and postcaval, is non-aërated, is acted upon in all directions and might therefore be forced either into the three great veins ( $p$ r.cv.v, $p t . c v . v)$ or into the right auricle $(r . a u)$. But the veins are full of blood steadily flowing towards the heart, and any regurgitation is further prevented by their valves: the right 


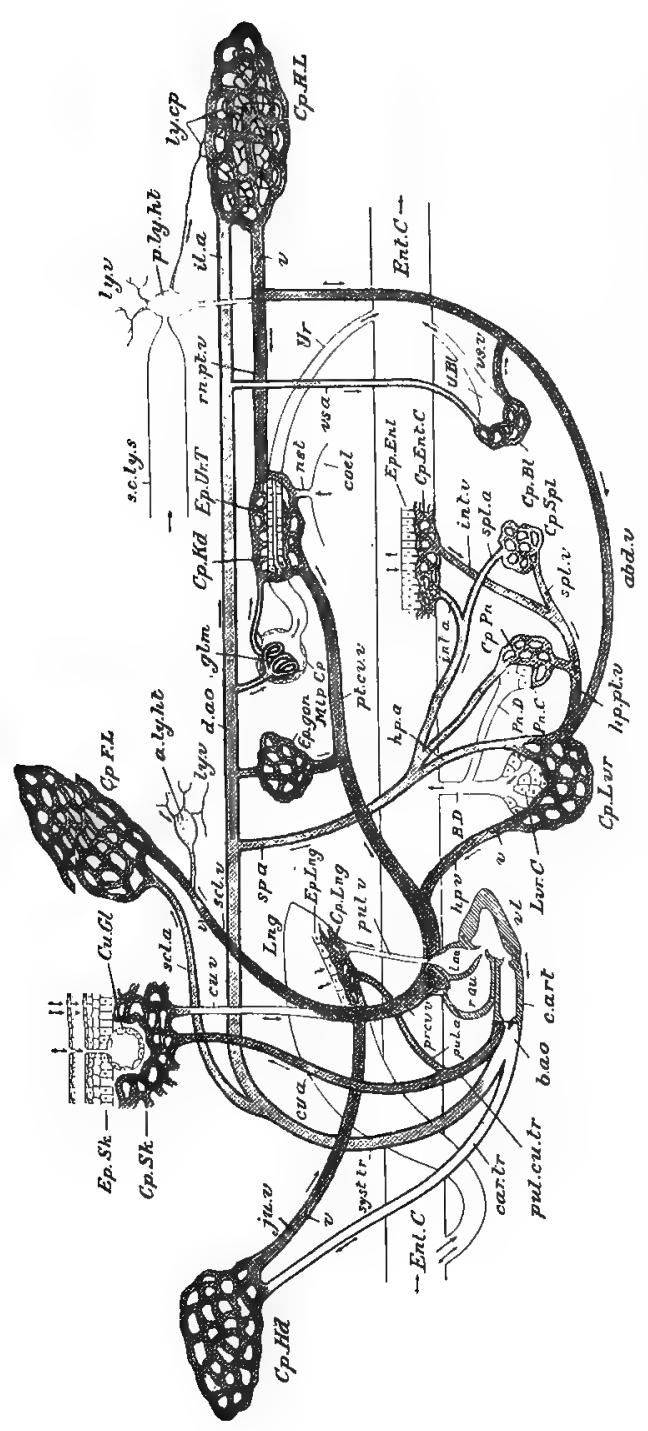

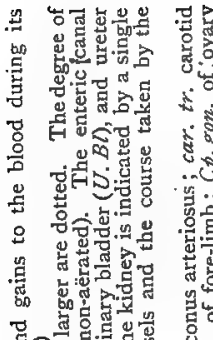

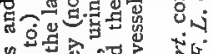

युँ

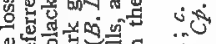

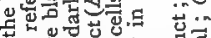
oo. 뎐

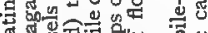
ฮึ.

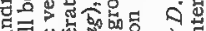

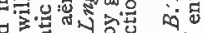
类出

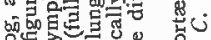
的 4 5. ज次 五出白

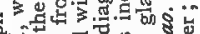

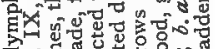

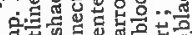

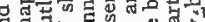

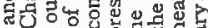

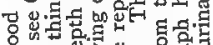
8 o 政

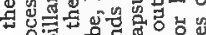

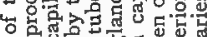
\& 0 b च y 8.

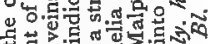
to 0.0 y ह 舟

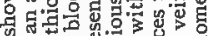

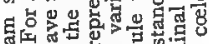

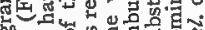
50 y. คै.

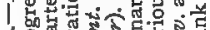
ผ่ํ⿺ㄹ की 至 
or testis; $C p . H d$. of head; $C p . H . L$. of hind-limb; $C p . K d$. of kidney; $C p$. Lng. of lung; $C p . L v r$. of liver; $C p$. $P n$. of pancreas; $C p$. Sk, of skin; $C p$. Spl. of spleen ; $c u$. a. cutaneous artery; $c u . v$. cutaneous vein; $C u$. $G l$. cutaneous gland; d. ao. dorsal aorta; Ent. C. enteric canal; Ep. Ent. epithelium of enteric canal; Ep. Lng. of lung; Ep.Sk. of skin; Ep.Ur.T. of urinary tubule; glm. glomerulus; il. $a$. iliac artery; int, $a_{\text {. artery to }}$ stomach and intestine; int. $v$. vein from stomach and intestine; $j u$. $v$. jugular vein; $h p . a$. hepatic artery (to liver); $h p . p t . v$. hepatic portal vein ; $h p . v$. hepatic vein; $l$. au. left auricle; $L n g$. lung; $L v r$. C. liver cells; $l y . c p$. lymph capillaries; $l y$. $v$. lymphatic vessels; $M l p . C p$. Malpighian capsule; nst. nephrostome ; $p . l y . h t$. posterior lymph-heart ; $P_{n}$. C. cells of pancreas; $P_{n} . D$. pancreatic duct; $\not r_{0}, c z, v$. precaval vein; $p t, c z, v$. post caval vein; $p u l, \alpha$. pul. monary artery; pul.cu. $t r$. pulmo-cutaneous trunk; pul.v. pulmonary vein; $r . a u$. right auricle; m. $p t . v$. renal portal vein; scl. $a$. subclavian artery; $s . c . l y . s$. sub-cutaneous lymph-sinus; $s c l . v$. subclavian vein; $s p$. $a$. splanchnic artery; spl. $v$. splenic artery; $s p l$. $v$. splenic vein; s. $v$. sinus venosus; syst.tr. systemic trunk; $U, B l$. urinary bladder; $U r$. ureter; $v$. valve in vein; $v s, a$. vesical artery (to bladder); vs. $v$. vesical vein; $v l$. ventricle.

auricle, on the other hand, has finished its contraction and is now relaxing; it is therefore empty. Thus, on the principle of least resistance, the contraction of the sinus fills the right auricle with blood from the great veins, and the sinus itself is refilled from the same source as soon as it begins to relax.

Immediately after the sinus has ceased to contract the two auricles contract together: the right, as we have seen, has just been filled from the sinus, the left $(l \cdot a u)$ is full of aërated blood brought to it by the pulmonary vein (pul.v). The presence of the sinu-auricular valves prevents the blood in the right auricle from being forced back into the sinus : that in the left auricle is prevented from being forced back into the pulmonary veins by the steady onward flow in the latter. On the other hand the ventricle is beginning to relax and is empty. Consequently the auriculo-ventricular valves are forced back into the ventricle $(v l)$ and the blood from both auricles flows into and fills that chamber, the right half of which becomes filled with non-aërated, the left with aërated blood, the two taking an appreciable time to mingle.

The instant it is thus filled the contraction of the ventricle begins. As it does so the blood, getting behind the auriculo- 
ventricular valves, forces them together, and thus prevents any backward flow into the auricle. At the same time the semilunar valves at the entrance of the conus $(c . a r t)$ are pushed aside and the blood flows into that chamber. Since the conus opens from the right side of the ventricle, the blood first entering it will be non-aërated ; there will then follow a certain amount of mixed blood; and finally, as the ventricle reaches the limit of its contraction, the aërated blood from its left side will be forced into the conus. (Compare Fig. 22.)

Last of all the chambers of the heart, the conus begins its contraction. The semilunar valves are immediately filled with blood, and, closing together, stop all backward flow into the ventricle. Two alternative courses are now open to the blood: it can pass either directly from the conus into the pulmo-cutaneous trunk ( $p u l$. $c u$. tr), or make its way into the bulbus aortæ $(b . a o)$. As a matter of fact it takes the former course, owing to the circumstance that there is little resistance in the limited blood-system of the lungs, while that in the systemic and carotid trunks is very great. Hence the blood just received into the conus from the ventricle, which, as we have seen, is non-aërated, goes immediately to the lungs and skin to be aërated.

Before long-in a fraction of a second-the flow of blood into them increases the pressure in the pulmonary vessels, and at the same time the blood is continually flowing onwards-i.e., away from the heart-in the systemic and carotid trunks. Consequently the pressure in these vessels rapidly diminishes, and the blood soon forces aside the valves between the conus and the bulbus and fills the latter. Here again the question of pressure comes in. It is easier for the blood to make its way into the wide systemic trunks (syst. tr) uniting immediately into the long dorsal aorta 
(d.ao) than into the comparatively narrow carotid trunks (car. $t r$ ), obstructed by the carotid plexuses. Hence, the non-aërated blood having been mostly driven into the pulmo-cutaneous trunk, the mixed blood, from the middle part of the ventricle, goes into the systemic trunk, and thence to the various arteries supplying the limbs $(s c l, a, i l . a)$ and the viscera $(s p . a$, etc.). Finally, when the pressure is sufficiently raised in the systemic trunks the remaining blood, which, coming from the left side of the heart, is aërated, is pumped into the carotid trunks (car. tr) and thence to the head.

Thus, owing to the arrangement of the valves, and to the varying pressures in different parts of the vascular system, the non-aërated blood returned from the various parts of the body to the heart is mostly sent to the lungs and skin to be aërated. Mixed blood is sent to the trunk, limbs, and viscera, while for the head with its contained brain-the directing and controlling organ of the whole animal-a special supply of pure, aërated blood is reserved.

We see then that the course of the circulation may be proved, as a simple matter of induction, from the structure of the heart and its valves, the direct observation of its beat, and the manner in which the flow from cut vessels takes place. It was by observation and experiments of this kind that thec irculation of the blood in the higher animals was demonstrated by William Harvey in the seventeenth century. But the final and most conclusive proof of the circulation-from directly observing the flow-became possible only after the invention of the microscope. This instrument, by furnishing a sufficiently high magnifying power, allows us to see for ourselvest he actual movement of the blood in an animal or organ of sufficient transparency; and, at the same time, clears up the question, previously 
insoluble, of how the blood, having reached a given part or organ by the arteries, finds its way into the veins to begin its return journey.

The Circulation in the Frog's Web.-There are three parts in the frog transparent enough to allow of the bloodflow being seen in them-the web of the foot, the tongue, and the mesentery. Of these the web is the most convenient, and can be examined under the microscope without any injury to the animal.

The Capillaries.-If you have the makings of a naturalist, you will acknowledge the sight to be one of the most wonderful you ever saw. In the thickness of the web is an irregular network of minute blood-vessels, called capillaries (Fig. 24), and through them the blood is seen to flow with great rapidity, its course being made especially evident by the minute particles or corpuscles it contains, the structure of which we shall study later on. You will also notice much larger vessels, the smallest arteries and veins. The arteries (a) are distinguished by the fact that the blood in them flows in the direction from the leg towards the margin of the web, while in the veins (v) it takes the opposite direction. You must remember, however, that under the microscope everything is reversed; right appears left and left right, and a current actually flowing towards the observer appears to go in the opposite direction.

By careful examination you will see that both arteries and veins are in connection, by minute branches, with the capillary network, and will be able to trace the blood from an artery, through the capillaries, into a vein.

The same thing can be seen in other transparent organs; and by injecting the vascular system with a fluid injectionmass, such as gelatine suitably coloured, it can be proved that all parts of the body are permeated with a capillary 
CHAP.

network into which the blood is passed by the arteries, and from which it is received into the veins.

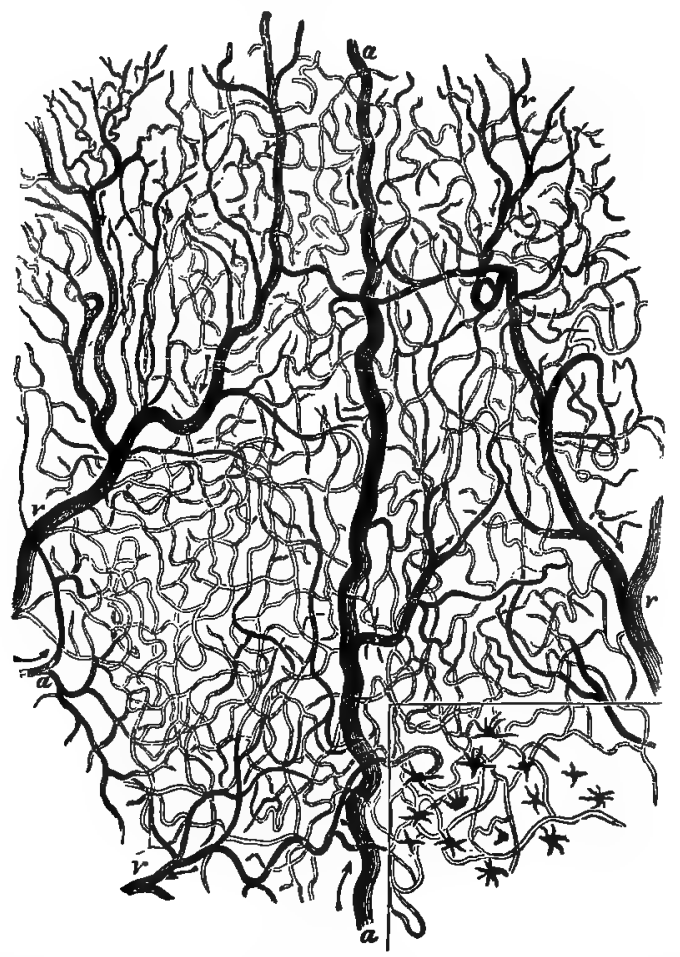

Fig. 24.-Blood-vessels of the web of a frog's foot seen under a low magnifying power. $a$. small arteries; $v$. small veins. The minute tubes joining the arteries to the veins are the capillaries. The arrows show the direction of the circulation. In the small portion marked off, the pigment cells, which occur throughout the web, are also represented. (From Huxley's Physiology.)

Thus by means of the microscope we are able to take the final step in demonstrating the circulation. The fact that 
the blood can flow in one direction only is proved by the disposition of the valves of the heart and of the veins, but the passage of the blood from the smallest arteries to the smallest vein by a connecting system of minute tubes or capillaries can be proved only by the employment of considerable magnifying powers. We see that the vascular system of the frog is a closed system of vessels : the blood is everywhere confined within definite tubes through which it flows in a definite direction, never escaping, as in some of the lower animals, into large irregular spaces among the tissues.

The Lymphatic System. - Included in the vascularsystem are certain cavities and vessels containing lymph, and together constituting the lymphatic system. We have already noticed the subcutaneous lymph sinuses (p. I 8, Fig. 23, s.c. ly.s) and the sub-vertebral lymph sinus (p. 27, Fig. 5, s.v. $l y . s)$. There are also found in nearly all parts of the body, delicate, thin-walled, branching tubes, the lymphatic vessels (Fig. 23, ly.v). Unlike the blood-vessels, the lymphatics are all of one kind, there being no distinction into anything of the nature of arteries and veins. They arise in lymphcapillaries (ly. $c p$ ), which are, as it were, interwoven with the blood-capillaries, but have no connection with them. By the lymph-capillaries the fluid which has exuded from the blood in its passage through the tissues is taken up and passed into the lymphatic vessels or sinuses, and these in their turn finally communicate with certain transparent muscular organs called lymph-hearts. Of these there are two pairs. The anterior lymph-hearts $(a . l y . h t)$ lie, one on either side, beneath the scapula and just behind the transverse process of the third vertebra: the posterior pair $(p . l y . h t)$ are situated one on each side of the posterior end of the urostyle. These organs pulsate regularly, like miniature hearts, and pump the lymph into the veins, the anterior pair communiPRACT, ZOOL, 
cating with the subclavian, the posterior with-the renal portal vein.

The lymphatics of the enteric canal have an important function to perform in that they absorb the fatty portions of the food (p. 75). The fluid they contain has a milky appearance, owing to the presence of minute suspended fat-globules, and for this reason they receive the name of lasteals.

The colome (Fig. 23, cal) is really a great lymph-sinus. It communicates with the veins of the kidneys through certain microscopic apertures called nephrostomes (nst).

The spleen (p. 23, and Fig. 3, spl) has important relations with the blood- and lymph-vessels, and probably acts as a blood-filter, removing particles in the blood which are no longer wanted.

\section{PRACTICAL DIRECTIONS.}

\section{The Vascular System.}

u. Let some blood from a frog-or better from the veins of some larger, freshly-killed, warm-blooded animal, such as a rat or a rabbit-flow directly into a white cup or porcelain capsule. Note that it soon coagulates, and soon afterwards separates into clot and serum. Notice also the difference in colour between the blood freshly drawn from a vein, and that soon assumed by exposed portions of the clot.

b. Pin a freshly-killed frog to the dissecting board, dorsal side upwards, and cut through the skin of the back along the middle line. The posterior lymph-hearts (p. 97, Fig. 23) will then be seen. To make out the anterior lymph-kearts, carefully separate the supra-scapulæ from the vertebral column. Some of the chief lymph-sinuses have already been seen : special methods are required to trace the lymph-vessels.

c. Now turn the frog the other way upwards, pin it down in the dissecting dish, and open the body-cavity as before (p. 32), taking great care not to cut the abdominal, musculo-cutaneous, and other veins. Slit open the pericardium and remove as much of it as possible, so as to expose the entire heart. The structure of the heart and the course of many of the blood-vessels can also be made out in the specimen from 
which you have already removed the alimentary canal. In the following dissections, use a dissecting lens whenever necessary.

I. In the heart (Figs. 3, 4, 7, 20 and 21 ), notice again the ventricle, and the right and left auricles (appearing single in the entire heart), and make out also the conus arteriosus, dividing into two distally, and the sinus venoszes (dorsal).

If the heart is still beating, notice the order of contraction of its different divisions (p. 9o).

Injection of the Arteries.-The tracing of the arteries is greatly facilitated by filling them with some coloured substance. The operation requires, therefore, a coloured fluid or injection-niass capable of traversing the arteries, and some contrivance by which it can be injected into them.

The most convenient injection-mass is made as follows:-

1. Grind up in a mortar 4 grammes of "French blue" (to be had at the oilman's), with 4 cubic centimetres of glycerine and the same quantity of methylated spirit.

2. Grind up 50 grammes of common laundry starch, with 5o cubic centimetres of water and 25 of methylated spirit, and add to the mixture the colour as prepared in ( 1 ). Mix thoroughly and strain through muslin.

This injection-mass will keep for an indefinite period in a stoppered bottle, requiring only to be stirred up when used. If it is considered too troublesome to make, a simpler but less satisfactory mass may be made by simply stirring up some French blue in water in the proportion of a teaspoonful to a tumbler.

For injecting the mass into the blood-vessels, the most satisfactory instrument is a brass injecting syringe, holding about one ounce, provided with nozzles of various sizes. This is, however, expensive, and an ordinary glass syringe, to be had of any druggist, will answer the purpose very fairly if provicled with a proper nozzle or cannula. This latter is made by drawing out one end of a piece of glass tubing about two inches long until it is fine enough to pass into the conus arteriosus : a piece of india-rubber tubing is then used to connect it with the fixed nozzle of the syringe. A still simpler injecting apparatus is furnished by a common "medicine dropper" (see p. 12, and Fig. 25). By alternative squeezing and releasing the cap, fluid is drawn into or expelled from the tube.

Having provided these requisites proceed as follows. Open the 
abdomen of a freshly-killed frog in the usual way, taking great care not to injure the blood-vessels. Remove the middle portion of the

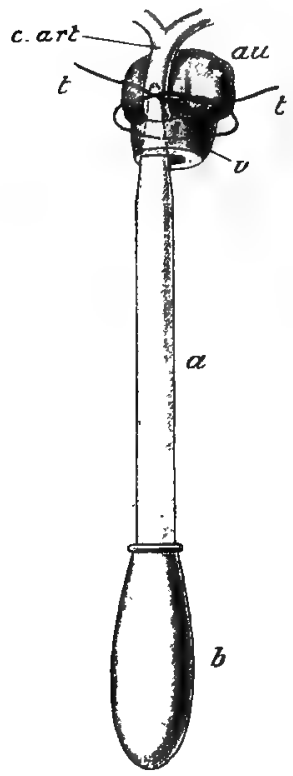

FIG, 25. -Sketch showing the method of injecting the frog's arteries.

u. glass "medicine-dropper" with india-rubber cap (b); its pointed end (dotted) is passed through the cut end of the ventricle $(v)$ into the anus (c.art); an. the auricular division; $t$. thread. shoulder-girdle so as to expose the heart, lay open the pericardium, and with a single snip of the scissors cut off the posterior half of the ventricle, allowing the blood to escape freely. Pass a piece of thread (not cotton), about six inches long, round the heart, at about the junction of the auricles and ventricle, and give it a single loose tie, as shown in Fig. 25. When the bleeding has ceased, fill the medicine dropper, or syringe. with injection-mass and pass the narrow end of the former, or the nozzle of the latter, through the cut end of the ventricle into the conus - take care not to push it into one of the auricles instead-and tighten the thread so as to keep it in place. Then squeeze the cap of the medicine dropper, or push in the piston of the syringe, and if the operation is successful, you will see the blue injection pass from the conus into the arterial trunks, and thence into the various arteries of the body. The contrast between the arteries, filled with the blue mass, and the veins, filled with blood, is then very striking, particularly in the mesentery. When the arteries are well-filled, withdraw the nozzle from the heart and instantly draw the thread tight and knot it so as to prevent escape of the injection. Then place the whole frog in spirit (methylated spirit 3 parts, water I part), for a few hours, after which time the injection mass will be found to have set hard enough to allow of the arteries being conveniently traced.

Injection of the Veins.-The veins are much more difficult to inject than the arteries, but if you wish to make a double injection on the same specimen, colour the injection-masss with vermilion or carmine 
in the case of the arteries instead of with French blue, using the latter for the veins. The operation is best performed by inserting the nozzle into an incision in the abdominal vein: by directing the nozzle forwards, the portal vein will be injected: by directing it backwards the pelvic and renal portal veins. But for a really satisfactory preparation, it is best to inject from the heart through the conus, as directed above, with a weak, warm solution of gelatine (in the proportion of one part of gelatine to two parts of water), coloured with precipitaled carmine. In this case the injection mass, containing only microscopic particles, passes from the arteries through the capillaries into the veins, keeping throughout to the course taken by the blood during life, and therefore unimpeded by the valves of the veins. A syringe must be used, since the medicine-dropper will not give sufficient pressure, and the animal should be placed in warm water during the process.

II. Now make out the course of the chief veins (p. 82, Fig. 21): (if not injected, put no water into the dissecting-dish at present):-

I. The two precavals, and the external jugular, internal jugular, sub-clavian, and mutsculo-cutaneous.

2. The postiaval, to see which turn the viscera on one side (Figs. 3 and 4 ). Note the renal, spermatic or ovarian, and hepatic veins.

3. The hepatic portal vein and its factors.

4. The abdominal vein and pelvic veins.

5. The veins from the hind legs can be more easily seen at a later stage, after the alimentary canal is removed, and so their examination is best left until certain of the arteries have been traced (or use the specimen you have dissected previously for this purpose). Remove the slin from the thigh, place the frog on its side, and make out the fenoral, pelvic (already seen), renal portal, and sciatic vein, as well as a large vein from the muscles of the back.

6. The two pulmonary veins.

Make a sketch of the heart and as many of the veins as you have followed out up to this point, inśerting the others after removing the alimentary canal (see p. IO2).

III. The chief arteries may now be followed out (p. 8o, Fig. 20) :-

Note the carotid, the systemic, and the pulmo-cutaneous trunk, arising from the conus arteriosus, and then trace each of these out as follows :-

I. The carotid trunk gives off a lingual artery, and is continued into the head as the carotid arterv, having at its origin the carotid plexus. 
2. The systemic trunk unites with its fellow to form the dorsa aorta, first giving off vertebral, subilavian, and asophageal arteries. From the point of union of the two systemic trunks arises the splanchnic or caliaco-mesenteric artery. After following this out to its distribution, remove the alimentary canal as directed on p. 76 , when the following branches of the dorsal aorta will be more plainly seen:--the renal, spermatic or orsalian, and iliac arteries.

3. The pulmo-cutaneous trunk divides into a pulmonary artery, passing along the outer side of the corresponding lung, and a cutaneous artery.

Sketch the heart and chief arteries, and then make out and sketch the renal portal system (p. IOI), if you have not already done so.

IV. ${ }^{1}$ Cut out the heart of a frog preserved in formaline, taking great care not to injure it. Fasten it out in a dissecting-dish with the ventral surface upwards, by sticking very small pins through the arteries and veins-not through the heart itself. Pinch up the ventricle with fine forceps, and with small scissors gradually snip away its ventral wall, noting that it is a hollow structure with thick, spongy walls and a small cavity, which will probably be full of clotted blood. Wash this out, and then proceed to open the auricles in a similar way, and to wash out the blood they contain. Observe the right and left auricles, separated by a partition. Slit open the conus arteriosus, and continue the cut forwards to the origin of the main arteries. Examine with a lens and make out (p. 87, Fig. 22):-

1. The auriculo-ventricular aperture and its valves.

2. The longitudinal valve and the three small semilunar valves in the conus arteriosus.

3. The origins of the carotid and systemic trunks from the bullus aorte, and the small aperture leading into the pulmo-cutaneous trunks.

4. The sinu-auriczlar aperture and its valves.

5. The aperture in the left auricle leading into the pulmonary veins.

Sketch.

Turn over the heart, so that its dorsal surface is upwards, and cut away enough of the dorsal wall of the sinus venosus to show the sinuauricular aperture from the other side.

1 On account of its small size, the examination of the structure of the frog's heart is somewhat difficult, and the student is advised to dissect first the heart of $\bar{x}$ larger animal, such as a dogfish or a rabbit. (See Part II.) 
V. Get a piece of thin board-e.g., the side of a cigar box-about six inches long by three wide. At the middle of one of the narrow sides make a round hole about half an inch in diameter, and about half an inch from each end of the same side make a notch or rather slit, with a penknife. This is called a "frog-board."

Next get as light coloured a frog as possible. Chloroform it as directed on p. $3 \mathrm{I}$, but remove it from the influence of the anæsthetic as soon as it is insensible, when the brain may be destroyed by the operation of pithing, so that there may be no suspicion of the frog feeling any inconvenience from this harmless experiment. To pith the frog, feel with the finger the joint between the skull and first vertebra on the dorsal side, and with a sharp scalpel make a small cut through the skin and underlying tissue, so as to expose the spinal cord in this region; then rapidly insert a blunt instrument, such as a seeker or a small piece of wood, into the cranial cavity, and move it about until the brain is completely destroyed. Lay the frog on the frog-board with a piece of wet rag wrapped loosely round the body, and take one or two turns around both frog and board with a piece of tapeyou must avoid tying it tightly or the circulation will be impeded. Stretch out one leg, and selecting the most transparent web, tie a piece of thick soft silk round each of the two toes by which it is bounded. Adjust the leg so that the web comes just over the hole in the frogboard, and bring the two pieces of silk through the slits, regulating them until the web is evenly stretched out over the hole. Lastly, place the frog-board on the stage of the microscope, ${ }^{1}$ with the hole over the aperture in the stage, and either fix it with the clips or rest the opposite end on some support: adjust the mirror so as to illuminate the web from beneath, and examine it with the low power. Note the network of capillaries and the circulation of the blood through the arteries, capillaries and veins (Fig. 24).

1 A brief description of the compound microscope will be given at the end of the next chapter. 


\section{CHAPTER VII}

THE FROG (continued): THE MICROSCOPICAL EXAMINATION OF THE SIMPLE TISSUES.

BEFORE carrying our enquiries any further into the anatomy and physiology of the frog it will be necessary to devote some consideration to its microscopic structure or histology, since there are many matters in connection with the various organs which can be further elucidated only by the examination of the minute structure of the organs as revealed by the microscope (see p. I I9).

Let us, first of all, examine a drop of the frog's blood under the low power of the microscope. It will at once be seen that the blood is not a simple homogeneous fluid, but that it contains a large number of minute solid bodies floating in it. These are called by the general name of blood-corpuscles: the fluid part of the blood in which they float is called the plasma. At first, owing to currents in the fluid, the corpuscles will be found to move to and fro, but after a time they come to rest. Under the high power you will notice that the corpuscles are of two kinds. The greater number of them are regularly oval in form (Fig. 26, C), and of a yellow colour. If the drop of blood is thick enough in one part for the corpuscles to lie over one another, so 
that the light passes through two or three layers of them to reach the eye, they will appear red : they are hence called red corpuscles. Frequently they are seen turned on edge (D), and their appearance in this position shows them to be flat oval discs with a swelling in the centre. They are about $\frac{1}{10}$ th of a millimetre (about $\frac{1}{1100}$ th inch) in long diameter.

Among the red corpuscles are found, in much smaller numbers, bodies (Fig. 26, A) not more than half the long diameter of the red corpuscles in size, quite colourless, distinctly granular-so as to have the appearance of ground glass-
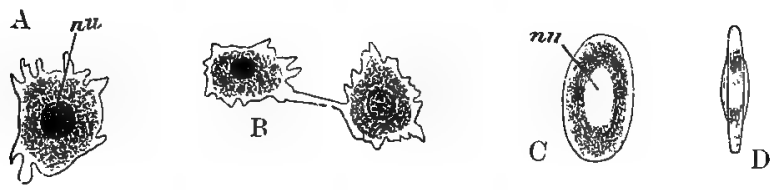

F1G. 26.-Blood corpuscles of the frog, highly magnified.

A, colourless corpuscle; $\mathrm{B}$, the same in process of divicion ; $\mathrm{C}_{\text {, }}$ red corpuscle, surface view; D, the same, edge view. nu. nucleus. (From Parker's Biology.

and with a slightly irregular outline. These are the colourless corpuscles or leucocytes. They are not flat, like the red corpuscles, but have the form of irregular lumps.

The plasma, like the leucocytes, is quite colourless, so that the colour of the blood is seen to be due entirely to the large number of red corpuscles it contains.

If the drop of blood has been prepared and examined under the high power with sufficient rapidity, a remarkable phenomenon can be made out with regard to the colourless corpuscles. This can be most easily demonstrated by making a series of outline sketches of the same leucocyte at intervals of a minute or two. You will then notice that the sketches all differ from one another: in one there will perhaps be a little projection going off to the right; in the next this will have disappeared and a similar projection will have appeared 
on the left, and so on. As a matter of fact, as long as the blood is quite fresh, the leucocytes are in constant movement, sending out and withdrawing little processes of their substance called pseudopods or "false feet," by means of which they can crawl slowly along like independent living things. These very peculiar and characteristic movements are called amceboid movements. Occasionally a leucocyte may be seen to elongate itself and divide into two (Fig. 26, B): this is a case of what is called simple fission. The red corpuscles neither move nor divide.

If a drop of some dye or staining fluid be run in under the cover-glass, the corpuscles will be seen to become rather faint in outline, very transparent, and lightly tinted ; but the most obvious effect is that in the middle of each is seen a rounded or oval granular body $(n u)$ deeply stained by the dye, so as to make a very well-defined coloured area in the interior of the corpuscle. This body is called the nucleus: it is present both in the red and the colourless corpuscles.

By adding to a fresh drop of blood, in the same manner, a drop of weak acetic acid, the nucleus again becomes distinct, while the body of the corpuscle is rendered very transparent and almost invisible : indeed it finally disappears altogether. It is thus proved that the corpuscles, both red and colourless, consist of two substances, an internal nucleus, stained by dyes, and unaffected by weak acids; and an external substance, called protoplasm, but slightly affected by dyes, and soluble in weak acids. Both nucleus and protoplasm consist mainly of proteids (p. 72 ), together with water and a small proportion of mineral matters.

When distilled water is added to a drop of blood on the slide, the corpuscles are seen to swell up and become partly dissolved, and the red colouring matter of the red corpuscles is dissolved out, the plasma becoming tinged with yellow. 
Thus the colouring matter is evidently a distinct substance from the protoplasm, and is called haemoglobin. It is characterised, among other things, by a strong attraction for oxygen: in combination with that gas it assumes a bright scarlet colour: when deprived of oxygen, it becomes purple. This affinity for oxygen accounts for the change undergone by the blood when exposed to the air, as described on p. 79.

Coagulated blood, as seen under the microscope, is characterised by the plasma being traversed by extremely delicate threads, forming a sort of network in which the corpuscles are entangled. These threads are formed of a substance called fibrin, which is separated from the plasma during coagulation, the remaining or fluid portion of the plasma constituting the serum. We may therefore express the coagulation of the blood in a diagrammatic form as follows :-

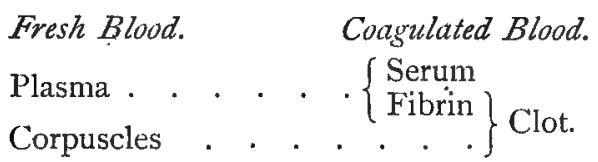

Having observed the microscopic characters of a drop of blood, let us examine once more the circulation in the web, this time under the high power (Fig. 27). The red corpuscles $(F)$ can be seen streaming through the vessels, those in the capillaries in single file, those in the arteries and veins two or more abreast: as they pass through narrow capillaries or round corners, they become bent or squeezed $(G, H)$. The leucocytes $(I)$ travel more slowly and often stick to the sides of the vessels.

Columnar Epithelium.-By carefully teasing out a small piece of the inner surface of the mucous membrane of the 


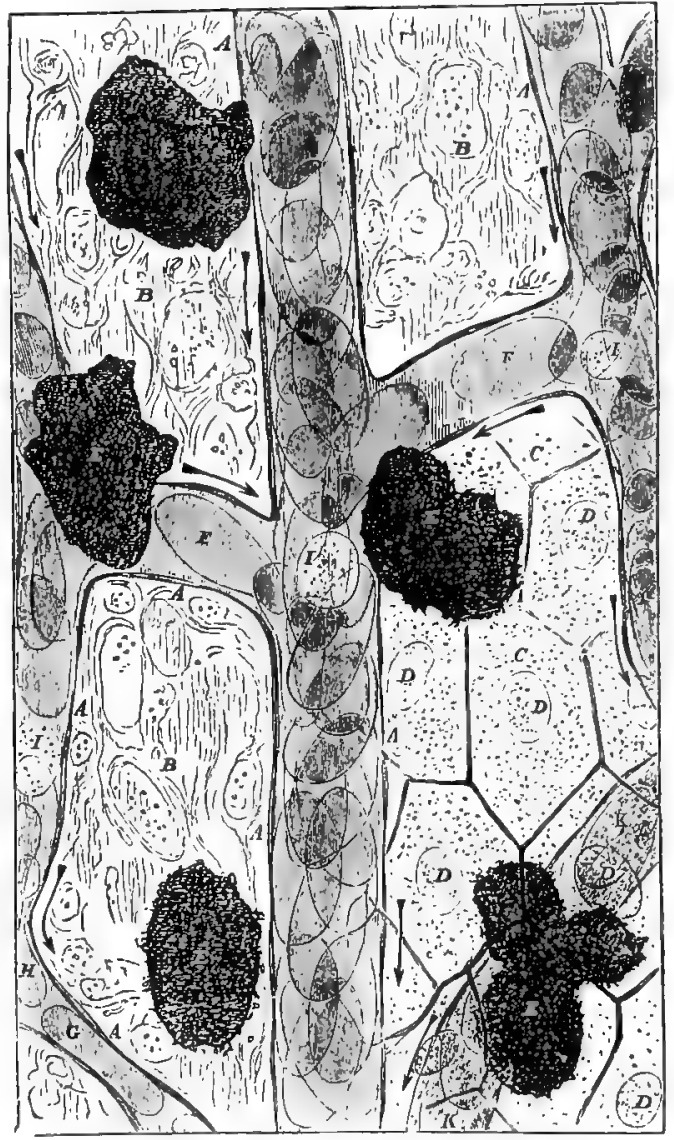

Fici, 27.-The circulation in the frog's wel, under a high power.

$A$, wall of capillaries; $B$, tissue of the web in which the capillaries lie; $C$, epiderm cells; $D$, their nuclei ; $E$, pigment cells ; F, red corpusclen ; $G, H$, red corpuscles being spueezed through a narrow capillary; $K$, capillary seen through the epiderm ; 1 , colourless corpuscles, (From Huxley's Pliysiology.) 
intestine into the smallest possible particles it will be found that the process has detached numerous, minute, conical bodies, about $\frac{1}{30} \mathrm{~mm} .\left(\frac{1}{7} \frac{1}{50}\right.$ in.) in length, polygonal in transverse section, and having one end flat and the other pointed (Fig. 28). These bodies are called epithelial cells: in the natural position they lie closely applied to one another, like the blocks of a wood pavement, their flattened ends facing the cavity of the intestine, while

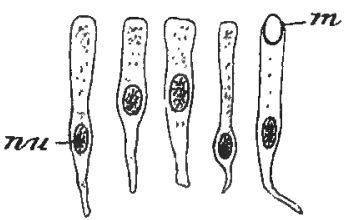

FIG. 28.-Columnar epithelial cells from the frog's intestine. m2. droplet of mucus exuding from cell ; $n u$. nucleus. their pointed ends abut against the submucosa (p. 70). Thus the epithelial cells together form an epithelium or epithelial layer of the mucous membrane directly bounding the cavity of the enteric canal.

Each cell consists of protoplasm and contains a rounded, granular nucleus $(n u)$, which is made very conspicuous by staining, and in which are one or more small bodies or nucleoli.

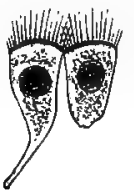

FIG. 29.-Ciliated epithelial cells from the mucous membrane of the frog's mouth.

(From Parker's Biology, after Howes.)

Certain of these cells have a space towards their free ends containing slime or mucus, and thus have the form of little cups or goblets: they are known as goblet-cells (see right hand cell in Fig. 28).

Ciliated Epithelium.-By the same method the mucous membrane of the mouth is also seen to be lined by an epithelium, but the cells comprising it (Fig. 29) are shorter in proportion to their length, and each is produced on its free surface into a number of delicate, transparent threads of protoplasm called citia, which, in the living condition are in constant movement, lashing backwards and forwards like minute whip-lashes, or, more 
accurately, like the blades of grass in a field when acted upon by a strong wind. If you happen to get under the microscope a good-sized bit of mucous membrane with the cells in position, you will see that the cilia produce a strong current by which small particles are swept along, while detached cells swim about, like little independent animals, by the action of their own cilia. These ciliated epithelial cells, like the columnar cells previously described, are made of protoplasm and each contains a nucleus with one or two nucleoli clearly brought into view by staining.

The action of the cilia can be demonstrated, on a large scale, by placing a freshly-killed frog on its back, turning back or cutting away the lower jaw, and placing a very small cube of cork on the roof of the mouth near to the projection due to the eyes. The cork will be slowly swept back towards the throat.

Squamous or Pavement Epithelium.-By scraping the outer surface of a piece of skin with a sharp knife, and

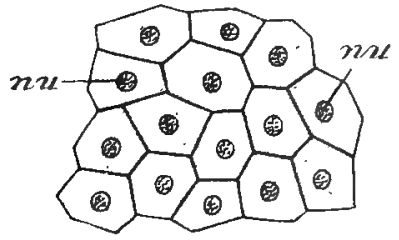

Fig. 30.-Squamous epithelial cells from the frog's skin. nu. nuclei. examining the scrapings in a drop of water, after staining them, the superficial layer of the skin will be found to be made up of flattened, roughly hexagonal plates (Fig. $3 \circ$ and Fig. $\left.{ }_{27}, \mathrm{C}, \mathrm{D}\right)$ set closely together, like the tiles of a mosaic pavement. Each plate has a nucleus, and, from its flattened form, is distinguished as a squamous or scale-like epithelial cell.

Meaning of the word "Cell."-We see thus that the body of the frog is partly made up of distinct elements, which, under a considerable diversity of form, exhibit the same essential structure. Each consists of a mass of living proto- 
plasm, containing in its interior a rounded body, the nucleus, specially distinguished by the affinity of parts of its substance for colouring matters. To a body having this essential structure, whatever its form, the name cell is applied.

Unstriped Muscle.-Examination of a teased preparation of the muscular coat of the intestine, stomach, or urinary bladder will show that it is composed of delicate fibres (Fig. 3r) tapering at both ends, and with a nucleus in the middle. These are called smooth or unstriped muscular fibres: they are obviously cells which have undergone a great elongation in length.

During the peristaltic movements of the intestine (p. 75) each fibre alternately contracts and relaxes, becoming shorter and thicker during the former process, like the large muscles of the body (p. 6o). The movements, in this case, however, are not under the control of the will, and unstriped muscular tissue is therefore often spoken of as involuntary muscle.

Contractility of Protoplasm. -We have now studied three different kinds of movement in

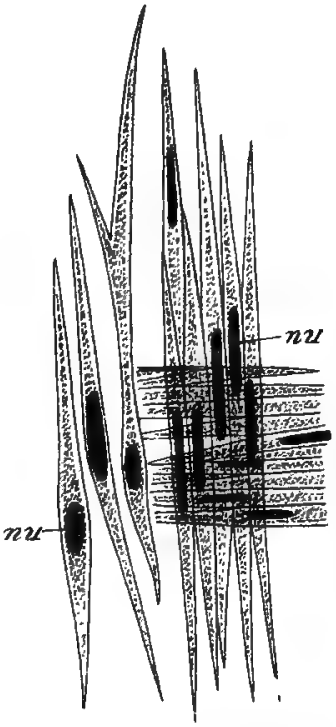

FIG. 3r. - Unstriped muscular fibres from the frog's intestine. To the right are shown fibres from the longitudinal and circular layers (see Chap. VIII) cross. ing one another; to the left isolated fibres. (After Howes.) cells:-muscular movement in the unstriped muscle-fibres, ciliary movement in the ciliated epithelial cells, and amoboid movement in the colourless blood-corpuscles. Muscular 
movement is due to the fibre undergoing a sudden shortening in a particular direction and a consequent approximation of its two ends. Ciliary movement is due to the alternate bending and straightening of the cilia; and the bending of a cilium in a particular direction is caused by the protoplasm of which it is composed shortening or contracting on the side towards which it bends. Amœboid movement is the protrusion and withdrawal of irregular processes of the cell: this results from the protoplasm undergoing a contraction or squeezing in a given direction, as a consequence of which one part of its substance is drawn in and another pushed out. Hence all three kinds of movement are movements of contraction; and contractility, or the power of contraction, may be considered as a general property of protoplasm.

Striped Muscle.-If a small piece of any of the body muscles is carefully teased out with the grain, i.e., in the direction of the length of the fibres, so as to break away the connective tissue binding them together, the fibres will readily separate from one another, and they will be seen to be long and cylindrical. Under the microscope each fibre shows a delicate transverse striation (Fig. 32), being made up of alternate bright (b) and $\operatorname{dim}(d)$ bands-or more accurately discs, the fibre being cylindrical--set at right angles to its length. Hence the ordinary body-muscles or voluntary muscles are composed of striped muscular fibres. ${ }^{\text {I }}$ In addition to the transverse striation a fainter longitudinal striation is more or less distinctly visible.

Each fibre is covered by a delicate membrane $(s)$ called sarcolemma, beneath which nuclei $(n)$ occur at intervals.

1 The muscles of the heart, although not under the control of the will, are transversely striated; but their structure differs from that of crdinary striped voluntary muscle. 
It will be seen that striped muscle, unlike the tissues previously considered, does not appear to be composed of cells, although the occurrence of nuclei seems to hint at their presence. In the embryo, however, the muscle is formed of ordinary nucleated cells, which, as growth goes
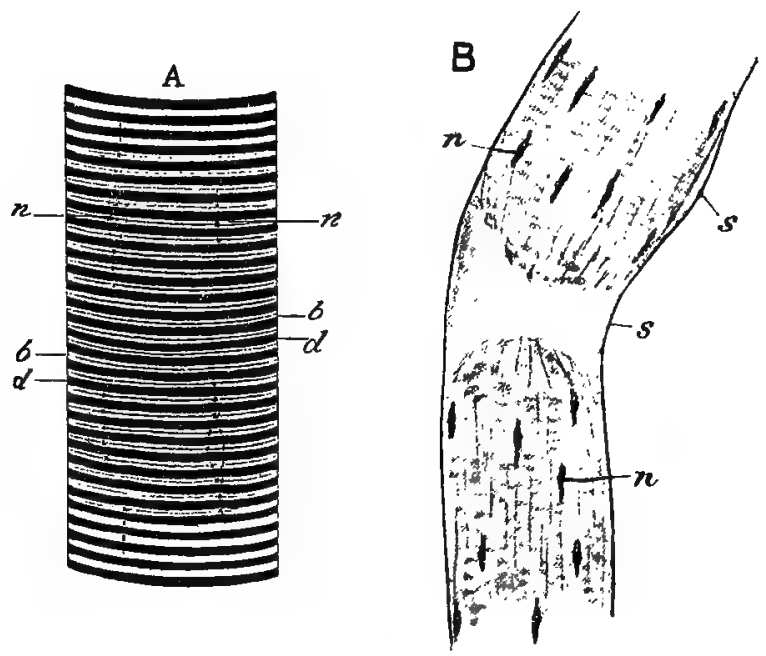

FIG. 32,-A, part on a fresh muscular fibre of a frog. $B$, the same after treatment with distilled water followed by methyl green.

$b$. bright bands; $d$. dim bands; $\pi$. nuclei $; s, s^{\prime}$. sarcolemma, rendered visible as a minute blister $\left(s^{\prime}\right)$ by absorption of water and by the rupture of the musclefibre at si (A, from Huxley's Physiology.)

on, increase in length while their nuclei multiply by fission, each enormously elongated cell thus containing a considerable number.

Connective Tissue.-We will next examine a piece of the delicate web of connective tissue which binds the muscles together.

Under the high power, connective tissue is seen to be PRACT, ZOOL. 
composed of a sort of irregular network of delicate bundles of wavy fibres called white connective tissue fibres (Fig. 33, w), which cross one another in all directions. Amongst them are found single fibres sweeping across the field in bold

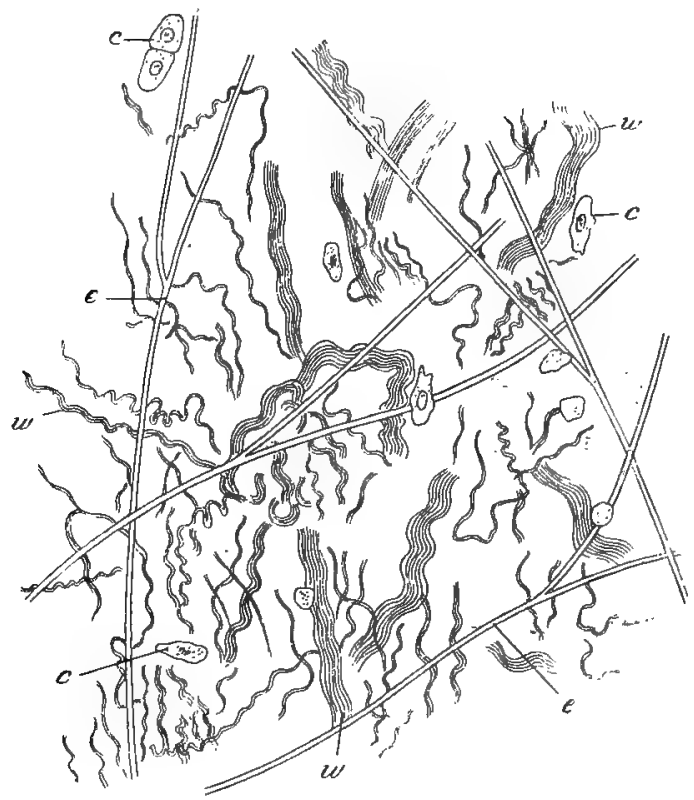

Fig. 33--Connective tissue from between muscles of frog's leg.

c. cells ; $\epsilon$. elastic fibres; $w$. white fibres : - all of which are imbedded in a delicate matrix.

curves and called elastic fibres (e): it is owing to their elasticity that the tissue cannot be spread out when wet. Scattered among the fibres are numerous nucleated cells $(c)$ of very varied and irregular form: these are the connective tissue cells. The fibres, as well as the cells, are imbedded in a soft, homogeneous ground-substance or matrix. 
Thus connective tissue consists partly of cells, but between these and forming the main substance of the tissue, is a matrix or intercellular substance, enclosing fibres. In the embryo the tissue consists of closely packed cells, but, as development proceeds, these separate from one another, and the ground-substance is formed between them.

Cartilage.-The ordinary clear or kyaline variety of this tissue is conveniently studied by examining a piece of the thin edge of the omo- or xiphi-sternum; or by taking a thin section with a razor of the head of the humerus or femur.

Cartilage consists of a tough, elastic, transparent, homogeneous matrix (Fig. 34, $m$ ) containing numerous

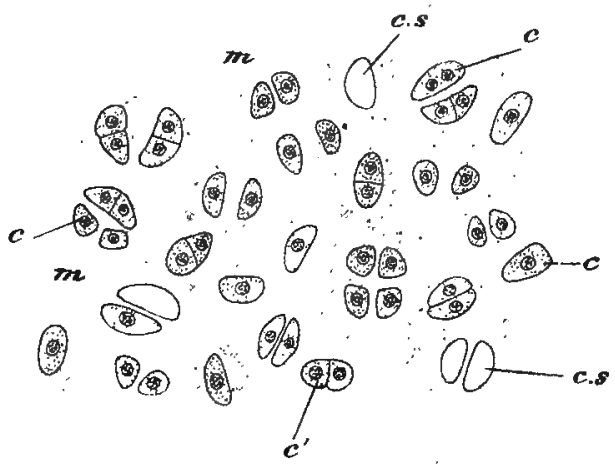

Fig. 34.-Section of cartilage, from the head of the frog's femur.

$c$. cells; $c^{\prime}$. cells undergoing fission; $c . s$, empty cell-space; m. matrix.

cavities or cell-spaces (c.s), in each of which is a nucleated cell $(c)$. The cell-spaces are in many cases arranged in groups of two or four, sometimes close together, sometimes with a narrow space of matrix or intercellular substance between them. This is due to the fact that cartilage grows by the cells undergoing binary fission, so that two cells are formed in one cell-space: the two then gradually separate 
from one another and intercellular-substance is formed between them. In the embryo, this tissue consists entirely of closely packed cells which gradually separate and form a structureless matrix which is firm and elastic, and which in some parts (pp. 46 and 48 ) may become calcified.

Bone.-As we have already seen (p. 52) bone is formed of two constituents, a basis of animal matter in which mineral matter-calcium phosphate and carbonate-is deposited. In microscopic examination we may therefore investigate

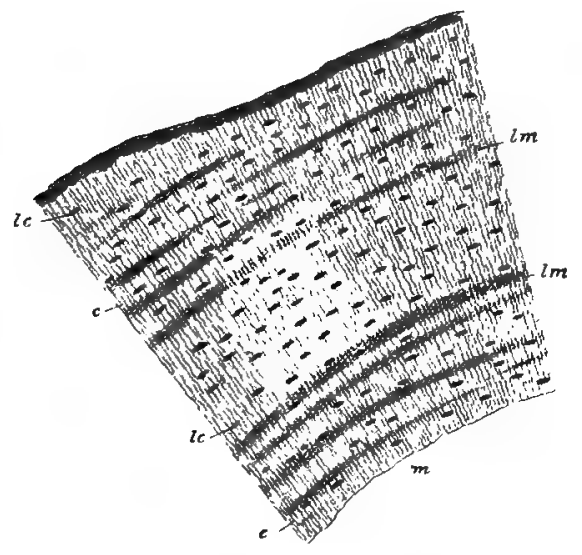

Fig. 35.-'lransverse section of dry femur of frog. c. canaliculi ; lc. lacunæ; $l m$. lamella; $m$. marrow cavity.

either the mineral matter by examining dried bone, or the animal matter by examining decalcified bone.

A thin section of a dried long bone, such as the femur, shows that it is formed of very numerous thin layers or lamella (Fig. 35, lm), surrounding and concentric with the marrow cavity. The lamellæ contain numerous cavities, the lacuna (lc), with delicate, branching tubes, the canaliculi 
(c), radiating from them in all directions, Both lacunæ and canaliculi commonly appear black, owing to their being filled either with air or with bone-dust produced in grinding the section.

In a section of decalcified bone (Fig. 36 ) the marrow is seen to be surrounded by lamellæ of a delicate fibrous substance, arranged in two layers, an outer $(b)$, having the periosteum $(p)$ closely investing it, and an inner $\left(b^{\prime}\right)$, in contact with the marrow. In the fibrous substance of the lamellæ are cell-spaces, corresponding with the lacunæ of the
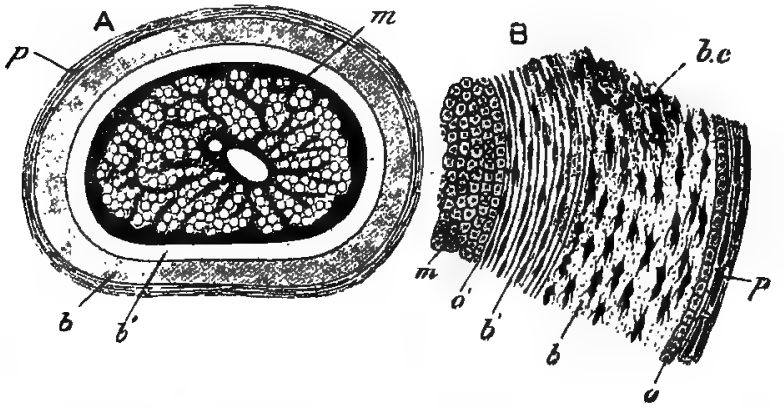

FIG. 36. - Transverse section of decalcified frog's femur under a low power, B, portion of the same under a high power.

$b$. outer, and $b^{\prime}$. inner layer of bone; $b . c$. bone cells; $m$. marrow; 0 . layer of osteoblasts in connection with periosteum; $\sigma^{\prime}$, layer of osteoblasts in connection with marrow ; $p$. periosteum. (After Howes.)

dried bone, and each containing a bone-iell (b.c), which sends off delicate branched processes of its protoplasm into the canaliculi. Thus the bone, like connective tissue and cartilage, consists of cells with an intercellular substance: the latter is in the form of concentric layers and is impregnated with lime-salts.

The long bones of the frog grow in two directions. Between the periosteum and the bone is a layer of cells, the 
asteollasts (o), by which new lamellæe of bone are formed on the outside of that already existing : thus the outer layer of bone (b) grows from within outwards. Between the marrow and the inner surface of the bone is another layer of osteoblasts $\left(o^{\prime}\right)$ which forms new lamella on the inner side of the existing bone, so that the inner layer $\left(b^{\prime}\right)$ grows from without inwards.

Summary.-The various simple tissues studied in the present chapter consist either entirely of cells, or of cells separated by an intercellular substance. Formed entirely of cells are the various kinds of epithelium-columnar, ciliated, and squamous, and unstriped muscle. In striped muscle the cells have elongated into fibres and their nuclei have multiplied. Of tissues consisting of cells with intercellular substance, connective tissue has the matrix soft and homogeneous, with fibres imbedded in it;-in hyaline cartilage it is structureless and tough, though elastic; and in bone laminated and calcified. In the blood, the plasma may be looked upon as a kind of liquid intercellular substance.

Cells, wherever they occur, have the same essential structure, being formed of protoplasm with a nucleus. In nearly all cases they increase by binary fission, first the nucleus and then the protoplasm dividing into two.

The distribution of the various tissues throughout the body is worth noting. Epithelium always bounds a free surface-covering the outer surface of the body or lining the inner surface of the enteric canal. Striped muscle forms the "flesh," unstriped muscle the outer layer of the enteric canal (p. 7o). Bone and cartilage form the framework of the body, while connective tissue is the packing between the other tissues, 


\section{PRACTICAL DIRECTIONS.}

General Structure of the Compound Microscope--The compound microscope, with which you must now become acquainted, consists of a strong stand (Fig. 37, a) from which rises a vertical pillar (b). To the latter are attached-a horizontal plate or stage $(c)$, perforated in the centre with an aperture $(d)$, the size of which can be varied by means of a diaphragm: an adjustable mirror (e), placed below the stage : and a vertical tabe $(f)$ attached above the stage by a horizontal arm. Two combinations ot lenses are usecl : an objcctive or objectglass $(h)$, consisting of a metal tube with two or more lenses fixed into it, which screws into the lower end of the tube : and an ocular or eye-piece (i), consisting of a metal cylinder with a lens at each end, which slides into the upper end of the tube. It is this arrangement of lenses which forms the essential feature of the compound microscope : the object, placed on the stage, is magnified by the objective, and the magnified image, thrown into the interior of the tube, is further enlarged by the ocular.

The object is brought into jocus-i.e., placed at such a distance from the objective that a perfectly clear and welldefined inage is obtained-in one ot two ways. The tube can be raised or lowered either by sliding it up and down in an outer tube or collar $(g)$, or, in the more expensive instruments, by a rack and pinion: this movement forms the coarse adjizstment. In addition, all good microscopes have a fine adjustment, usually consisting of a spring concealed in the 
pillar, and acting upon the horizontal arm which carries the tube : it is worked by a screw $(k)$, and by means of it the tube can be adjusted to within

When the object is transparent-as in most cases with which we shall have to deal-it is placed over the hole in the stage on a glass slide, and illuminated from below by adjusting the mirror until a beam of light from the window or lamp is reflected vertically upwards : a small hole in the diaphragm should be used with the high power. The object is thus said to be viewed by transmitted light. In the case of opaque substances the mirror is not used, and the object is illuminated by the light falling upon it directly: it is then said to be viewed by reflected light.

A student's microscope should have two objectives, one-the low porver-magnifying about 80 , the other-the high power-about 300 to 400 diameters. One eye-piece is quite sufficient, and a sliding coarse adjustment is nearly as convenient as a rack and pinion, besides being cheaper and less likely to get out of order. The mistake often made in choosing a microscope is to get one of elaborate construction, the money going largely in brass-work. The proper thing is to get the simplest form of stand consistent with perfect rigidity, fitted with the best possible fine adjustment and lenses : to save on either of the latter is false economy.

Reauisites for Microscopic Work.-In addition to the microscope, the following will be required before starting work :-

I. A few slides or slips of glass, 3 inches long by I inch wide, which can be obtained from an optician. They must be thoroughly cleaned before being used.

2. A supply (about $\frac{1}{2}$ oz.) of cover-glasses, small pieces v. very thin glass, to be had at the optician's. The most convenient size is $\frac{3}{4}$ inch square. They are best cleaned by being soaked for a few minutes in strong nitric acid, and then thoroughly washed under the tap, after which they should be dried by being placed flat on a clean surface and rubbed with a handkerchief : if held between the finger and thumb, they are very liable to be broken.

3. One or two thin glass rods, about 6 inches long and $\frac{1}{8}$ th inch in diameter : and one or two dipping-tubes, or pieces of glass tubing about 6 inches long and ${ }_{10}^{3}$ th inch in diameter. The ends both of rods and tubes should be rounded off in the flame of a blow-pipe.

4. Half a dozen dissecting needles, made by sticking a fine sewing 
needle into the end of a wooden penholder, allowing the point to project about half an inch.

5. A few reagent-bottles for holding the various fluids used for applying what are called micro-chemical tests to the tissues. Special bottles can be bought for the purpose, but sufficiently convenient ones can be made from ordinary one-ounce phials, fitted with sound corks. Bore a hole lengthwise through the cork, and insert into the hole a piece of narrow glass rod pointed at the end just long enough to reach nearly to the bottom of the bottle when the cork is inserted. This arrangement allows of the ready application of a single drop of fluid to the object under examination.

6. The following micro-chemical reagents :-

a. Salt solution. Dissolve 0"75 gramme of sodium chloride in roo c.c. of distilled water, so as to make a s per cent: solution.

b. Acetic acid, r per cent. One c.c. of strong acetic acid to 99 c.c. of distilled water.

c. Distilled zuater.

d. Solutions of one or two aniline dyes. For fresh tissues, dissolve enough methyl green in distilled water to make a deep bluish-green solution, and add I per cent. of strong acetic acid. For preserved tissues, make a saturated solution of magenta or safranin in strong alcohol, and dilute with an equal bulk of water.

e. Glycerine, 5o per cent. Equal parts of pure glycerine and distilled water.

\section{Microscopical examination of the simple tissues.}

For the following work, a freshly-killed frog must be used.

(If you wish to measure each kind of tissue element, you must learn to use a micronneter, which consists of a circular piece of glass, marked at regular intervals with lines or squares, the distance between which can be calculated by comparing them with a scale engraved on a slide known as a stage-micrometer.)

I. The blood.-Have ready a clean dry slide and cover-glass. In a freshly-killed frog open a vein or make an incision in the heart, and with a clean glass rod remove a drop of blood to the middle of a slide. Take hold of the edge of the cover-glass with small forceps, and supporting it with a mounted needle, gently lower it on to the drop of blood until the latter is spread out into an even, transparent, yellowish film. This operation of covering the drop of blood requires a little practice: if not done quickly, there is danger o. the blood coagulating before it is covered, in which case it will not spread out into a 
transparent layer: is the cover is lowered too suddenly, bubbles of air are commonly included.

Examine your preparation, first of all, under the low power, and learn to recognise the appearance of air-bubbles. Note the colourless plasma and the numerous minute blood-cells or corpuscles.

Now replace the low by the high power. Bear in mind that the higher the power, the shorter the focal distance. With the low power you will probably find that the object is in focus when about half an inch from the bottom lens of the objective. The high power, on the other hand, has to, be brought to within about $\frac{1}{2}$ th of an inch of the cover-glass, which is therefore liable to be broken and the lens to be injured by careless focussing. The safest plan is to lower the tube, keeping your eye at the level of the stage, until the objective almost touches the cover-glass: then, looking through the microscope, very slowly raise the tube by means of the coarse adjustment, until the object comes into view. Note (Fig. 26):-

u. The numerous flat, oval, red corpastles, each with a central swelling in which the nucleus is contained. Sketch.

b. The colourless corpuscles or leucocytes, much less numerous, having a granular appearance, and an irregular or rounded outline.

Focus a colourless corpuscle under the high power, and note its amoboid movements: sketch its outline rapidly but accurately, and after a minute or two make another sketch, and then another, until some halfdozen outline drawings of the corpuscle have been obtained: then compare your sketches.

Now place on the slide, against one edge of the cover-glass, a drop of methyl-green, and against the opposite edge a small strip of blotting paper. If the blood is sufficiently fresh-and if it has coagulated you must get another drop-the blotting paper will slowly absorb the blood on one side, and the methyl-green will be drawn in and will gradually mingle with the blood. When this has taken place, put a drop of salt solution in the place of the methyl-green, and allow it to be drawn across so as to remove the superfluous stain: then remove the blotting paper, and examine the blood once more uncler the high power. Notice the mucleus present in each "kind of corpuscle, and the surrounding protoplasm. Sketch.

To a fresh drop of blood add in the same manner a drop of I per cent. acetic acid. Note that the body of the corpuscles becomes transparent, while the nucleus is rendered distinct. 
To another drop of blood add distilled water. The corpuscles become swollen up and partly dissolved, and the colouring matter (homoglobin) of the red corpuscles is dissolved out into the plasma.

Examine some coagulated blood under the microscope, and note the threads of fibrin in which the red corpuscles are entangled, and the serum.

Examine once more the circulation in the web (p. 103), using the high power, and follow the course of both red and colourless corpuscles through the vessels and capillaries. By focussing to the surface of the web the flattened epithelial cells of the epidermis or outer skin can be seen, and at a deeper level the black pigment cells (see p. I28, and compare Figs. 24 and 27 ).

2. Columnar epithelium.-Take a small piece of frog's intestine, and place it for $\mathbf{2 4}$ hours in a mixture called Ranvier's alcohol, consisting of one part of methylated spirit and two parts of water. With fine scissors snip off a very small piece-not larger than a pin's head-from the inner surface of the mucous membrane, place it on a slide in a drop of water, and with two dissecting needles tease it out by tearing it into the smallest possible particles. The operation is best done under a lens. Then put on a cover-glass, and examine first with the low and then with the high power. (Remember that a cover-glass must always be used with the high power.)

Note the minute, more or less conical cells of columnar epithelium (Fig. 28), each containing a nucleus. Observe the goblet-cells amongst the ordinary columnar cells. Stain with magenta, which is more effective than methyl-green in specimens previously treated with alcohol, and wash with water. (Salt-solution need only be employed in the case of fresh or living tissues.) Sketch.

3. Ciliated epithelium.-Snip off a very small bit of mucous membrane from the mouth of a recently killed frog, and tease it out in salt-solution (Fig. 29).

Note the form of the cells and their nuclei : they are relatively shorter than the columnar cells just examined, and each bears a number of delicate vibratile cilia at its free end. Observe the movements of the cilia. Sketch.

Treat with methyl-green, magenta, or acetic acid, when the nucleus will become more apparent.

Perform experiment descrihed on $p$. I ro to show the action of the cilia as a whole in the entire animal. 
4. Pavement or squamous epithelium.-Take a bit of frog's skin which has been kept for a day or two in Ranvier's alcohol, scrape the outer surface with a sharp knife, and examine the scrapings in a clrop of water, afterwards staining with magenta. (Fig. 30.)

Note the flattened cells fitting together like tiles in a pavement, each one with its nucleus. Sketch.

5. Unstriped Muscle.-Snip off a small piece from an inflated urinary bladder of a frog which has been preserved in formaline, and wash with water. Or, snip off a very small piece-not bigger than a pin's head-from the muscular coat of the intestine or stomach, or from a urinary bladder, which has been in Ranvier's alcohol for at least twenty-four hours. Then tease out in a drop of water very thoroughly. Note the elongated unstriped muscular fibres tapering at both ends, and each containing a nucleus (Fig. 3I). Stain with magenta. Sketch.

6. Striped Muscle.-Snip off a small piece-about $\frac{1}{8}$ th inch long -from any of the body-muscles of a freshly-killed frog, put it on a slide in a drop of salt solution, and tease it out, with the grain, i.e., in the direction of the length of the fibres. The fibres will readily separate from one another: the teasing process must be stopped as soon as they are apart, and care must be taken not to tear or crush the individual fibres, which are large enough to be readily distin. guishable with a magnifying-glass.

Observe under the low power of the microscope the long cylindrical fibres (Fig. 32, A), bound together by connective tissue, and showing a distinct transverse striation and a less distinct longitudinal striation. Examine a single fibre under the high power (Fig. 32, B), and make out the sarcolemma and the numerous naclei, which will be rendered more distinct by the addition of methyl-green or acetic acid. Sketch.

7. Connective-tissue.-Carefully separate two of the muscles of the leg in a fresh frog, and note the delicate web of connective tissue between them: or, note the fine strands of connective tissue between the skin and the muscles of the body wall. With fine forceps lift up a small shred of this, snip it off with scissors, and place it on a dry slide. Then, with two needles, spread it out into a thin, even layer, breathing on it occasionally to prevent drying. Lastly, place a drop of salt solution on a cover-glass, and quickly lower it on the preparation. The reason for this procedure is that if connective tissue is placed in fluid, it contracts into a little lump, which is too opaque for examination and cannot be readily spreat out. 
Examıne first with the low and then with the high power, and note the bundles of while connective-tissue fobres, and the elasic fibres. (Fig. 33). Sketch.

Add acetic acid : the white fibres will be dissolved, the elastic fibres more readily distinguished, and the connective-tissue cells seen; the latter and the delicate ground-substance will be rendered more distinct by staining with methyl-green. Sketch.

8. Cartilage.-Snip off the thin edge of the omo- or xiphi-sternum and examine it as before in a drop of salt solution. Or, cut a thin section of the head of the humerus or femur with a razor.

Note the transparent, homogeneous matrix, containing numerous cellspaces or lacunce, in each of which is a nucleated cell : observe here and there the groups of cells formed by binary fission (Fig. 34). Stain as before. Sketch.

9. Bone.-For the examination of dried bone cut a very thin slice of one of the dried long bones with a fret-saw: fasten it to a slide with Canada balsam, and, when the balsam has dried quite hard, rub down the section on a hone until it is thin enough to be quite transparent. Or, a transverse section of a bone from a larger animal may be prepared in the same way or bought from a dealer in microscopic objects.

a. Examine first a transverse section of dry frog's bone (e.g., femur or humerus), and note the marroze-cavity, the lamelle, and the lacune and canaliculi; the two last will probably appear black, owing to their being filled either with air, or with bone-dust produced in grinding the section (Fig. 35). A section of human bone, such as is usually supplied ready prepared, or of the bone of some other larger animal, shows a more complicated structure : instead of a single system of lamelle, the bone consists of a number of such systems, each surrounding a central canal, in which blood vessels and nerves run, and which corresponds to the marrow-cavity in the simpler frog's bone described above. Sketch.

b. Compare with a section of decalcified frog's bone, ${ }^{1}$ and notice--the fibrons lamellee arranged in two layers, the outer of which is closely invested by the periosteum; the cell-spaces or lacuine, containing bonecells; and the outer and inner layers of osteoblasts (Fig. 36). Sketch.

(For the histology of nervous tissue see Chapter X.)

1 The method of preparing sections of this and other tissues will be described at the end of the next chapter. 


\section{CHAPTER VIIİ.}

THE FROG (continued): THE MICROSCOPIC EXAMINATION OF THE COMPOUND TISSUES-GLANDS-SECRETION AND ABSORPTION.

Wirt the exception of the tissues of the nervous system, which will be described later on (Chap. $\mathrm{X}$ ), we have now studied the principal simple tissues by the method of dissociation, i.e. by separating their constituent parts. We have now to consider the way in which these tissues are combined in the various organs, and for this purpose must adopt some method of examination by which they are seen in their natural relations.

The method adopted for this purpose is that of section cutting. You know how, by cutting sections, in various directions, of a bit of twig, the arrangement and natural relations of its various parts-wood, bark, and pith-can be ascertained. The same thing applies to the organs of the frog and other animals, but owing to their soft and nonresistant texture, it is impossible to cut them into sections thin enough for microscopic examination without special preparation. The methods employed are by no means easy for the beginner, especially without verbal instruction and the resources of a biological laboratory, but in the event of your wishing to make the preparations described and 
figured in this chapter for yourself, the directions given on pp. I 35-I 39 are simple enough to be carried out with very limited appliances.

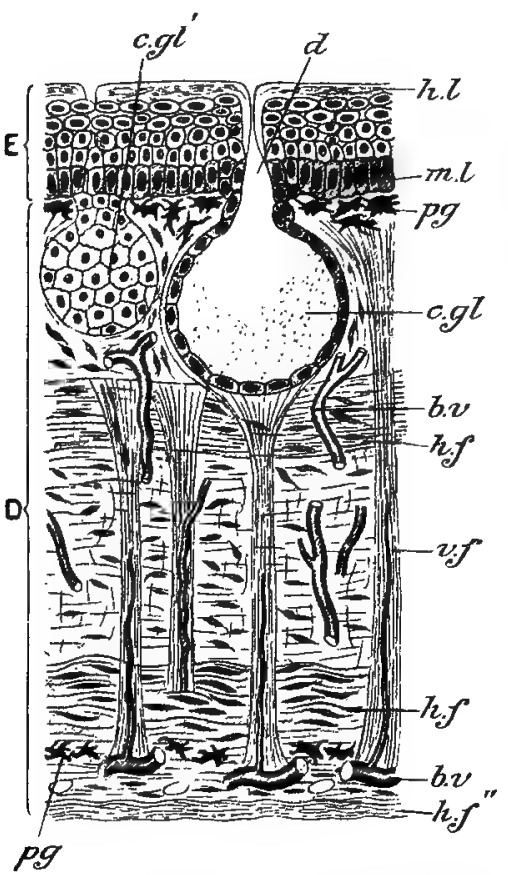

FIG. 38.-Vertical section of the frog's skin, highly magnified.

$D$. derm, formed of $h f . h f^{\prime} . h f^{\prime \prime}$. horizontal, and $v$. $f$. vertical fibres of connective issue, and containing $b . z^{\prime}$. blood-vessels, and $\not g$. pigment cells. $\mathbf{E}$, epiderm, consisting of $n$. $l$. active or Malpighian layer, and $k$. l. horny layer of epithelial cells; $c . g l$. cutaneous gland in section; $c . g l$. in surface view; $d$. duct. (After Howes.)

The Skin.-A vertical section of the skin, i.e. one taken at right angles to its surface, will be seen to have the following structure. 
The skin is clearly divisible into two layers, an outer, the epiderm (Fig. $38, \mathrm{E}$ ) and an inner, the derm (D). The epiderm is built up of several layers of epithelial cells. These differ greatly in form according to their position, those in the lower or internal layer $(m . l)$ being columnar, while those in the upper or external layer $(h . l)$ are squamous, and have their protoplasm converted into horny matter so as to furnish a comparatively hard and insensitive covering to the body.

The horny layer is cast off periodically in shreds, and to make up for this, the cells of the inner or deep layer multiply by binary fission, the increase in their number necessarily resulting in a pushing upwards of the superjacent layers. There is thus a constant travelling of cells from the inner to the outer surface of the epiderm: as they pass towards the outer surface they become more and more flattened, and at last squamous and horny. The whole process takes place in such a way that the multiplication of the columnar cells in the lower layer is just sufficient to make good the loss of the squamous cells in the superficial layer.

The derm (D) is formed of connective tissue, the fibres of which are mostly horizontal $\left(h . f, h . f^{\prime}, h . f^{\prime \prime}\right)$, or parallel to the surface of the skin, but at intervals are found bands of vertical fibres (v.f). The derm also differs from the epiderm in having an abundant blood-supply $(b . v)$, capillaries ramifying through it in all directions. It also contains nerves, the ultimate fibres of which have been traced into the deeper layers of the epiderm. Imbedded in the derm, especially in its external portion, are irregular cells $(p g)$, the protoplasm of which contains an intensely black pigment. It is to these pigment-cells, already referred to on p. 123, that the black patches in the frog's skin are due. 
In this, as well as in the other sections described in the present chapter, the structure of the nuclei of the various cells can be more easily made out than in the fresh preparations you have already examined. Each nucleus will be seen to be enclosed by a definite nuclear membrane, and to contain in its interior a number of minute bodies, which take up the stain more deeply than the rest of the nucleus. One or more of these bodies may correspond to the nucleoli already seen (p. Iog), but many of them are of a different nature and can often be seen to form a network : the material of which these are composed is known as chromatin, while the semi-fluid substance which surrounds them and forms the ground-work of the nucleus may be distinguished as the achromatin.

Cutaneous Glands-Secretion.-In the superficial part of the derm are seen numerous rounded spaces $\left(c . g l, c . g l^{\prime}\right)$, each of which can be proved, by taking sections in various directions, to be a nearly globular cavity, from which a narrow canal $(d)$, like the neck of a flask, passes through the epiderm to open on the external surface. Both the body and the neck of the flask are lined with epithelium, the cells lining the body being nearly cubical, those of the neck squamous.

These structures'are called cutaneous glands: they perform the function of manufacturing the slimy fluid which, as we have seen, is constantly exuding on the surface of the skin. The epithelial cells of the gland have the power of forming minute droplets of the fluid out of the materials supplied to them by the blood: the droplets escape from the cells and accumulate in the interior of the gland, whence the fluid is finally discharged by the duct.

The cells lining the duct are continuous on the one hand with those of the gland, and on the other with those of the 
epiderm. The whole gland with its duct is to be looked upon as a depression of the skin, lined by pushed-in epiderm cells.

Epithelial cells having the power of manufacturing and discharging a specific substance are called gland-cells, and the process of manufacture is known as secretion. We have already met with isolated gland-cells in the case of the
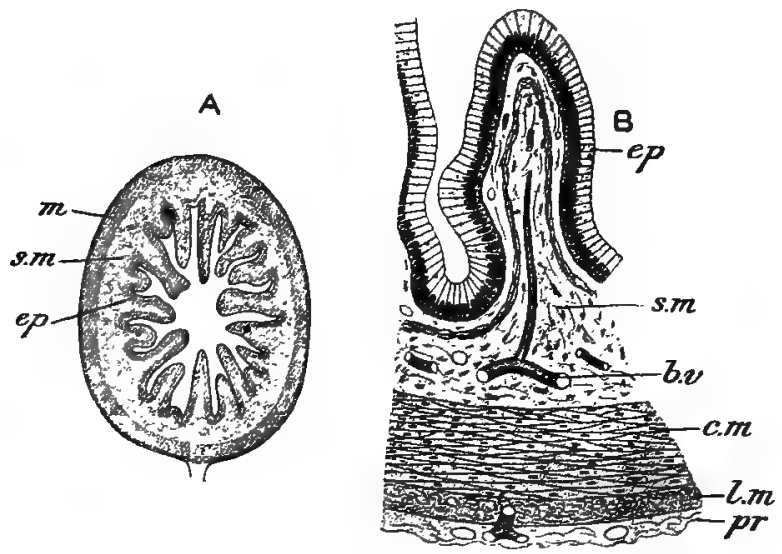

FIG. 39.-A, transverse section of frog's intestine; $\mathrm{B}$, small portion of the same highly magnified.

b. $v$. blood-vessel ; $c . m$. circular layer of muscle; ep. epithelium; 2 . $n$. longitudinal layer of muscle-fibres; pr. peritoneum; $m$. muscular layer; $s . m$. submucosa. (After Howes.)

goblet-cells of the intestine (p. Iog), which secrete mucus; but commonly, as in the present instance, gland-cells are aggregated into a definite organ called a gland.

The Intestine.-A transverse section of the intestine shows also a very definite and characteristic combination of simple tissues. The mucous membrane, like the skin, is composed of two layers, an epithelial layer (Fig. 39, epp), corresponding to the epiderm, and a connective tissue layer 
$(s . m)$, corresponding to the derm and called the submucosa. The epithelium consists of a single layer of cells only $(B, e p)$, all columnar, and with their long axes at right angles to the elevations into which, as we have seen (p. $7 \mathrm{I}$ ), the mucous membrane is thrown. Amongst the ordinary epithelial cells, numerous mucus-secreting goblet-cells will be recognised. The submucosa, like the derm, contains blood-vessels, lymphatics, and nerves.

The muscular layer $(\mathrm{A}, m)$ is also divisible into two: an outer layer of longitudinal fibres ( $\mathrm{B}, \mathrm{l} . \mathrm{m})$, running parallel with the long axis of the tube, and an inner, much thicker layer of circular fibres (c.m) which encircle it, and consequently lie at right angles to the longitudinal fibres. Thus in a transverse section, such as Fig. 39, the fibres of the circular layer are cut longitudinally, those of the longitudinal layer transversely, while the opposite would be the case in a longitudinal section.

The peritoneum ( $p r$ ) which, as we have seen (p. 27) forms an outer covering to the intestine, is formed of an inner layer of connective tissue and an outer of squamous epithelium.

The Stomach.-Transverse sections of the stomach (Fig. 40) show it to differ from the intestine not only in the much greater thickness of all its layers, but in the fact that the epithelium, instead of simply forming an even layer over the ridges of mucous membrane, is sunk into the submucosa in the form of simple or branched tubes, the gastric glands (g. $g l)$. These differ from the cutaneous glands in being not flask-shaped but test-tube shaped, each being a long, narrow tube, with an extremely small cavity ( $B$ and $c, c$ ). They are lined by a single layer of gland-cells, and open by minute apertures $(m)$ on the surface of the mucous membrane. 
The cells of the gastric glands have the power of forming, out of the materials supplied to them by the blood, the gastric juice, by which, as we have seen (p. 74), proteids are digested. Thus, while the raw material supplied to both cutaneous and gastric glands is the same, the manufactured article is entirely different in the two cases. Each kind of

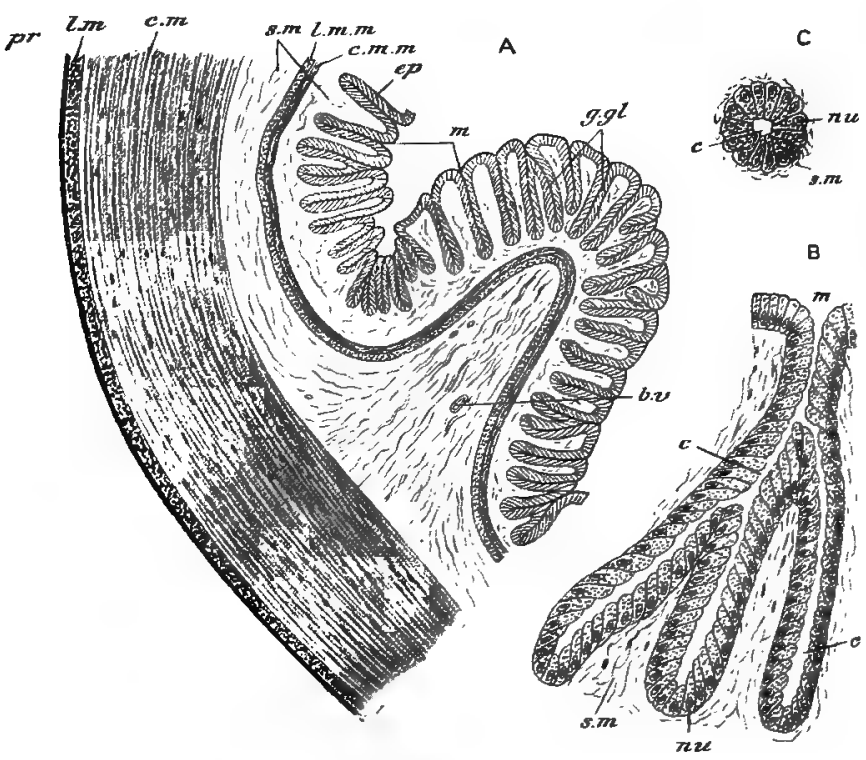

FIG. 40.-A, part of a transverse section of the frog's stomach; $B$, one of the gastric glands in longitudinal section, highly magnified; $C$, transverse section of a gastric gland.

b. v. blood-vessel; c. cavity of gastric gland; $c . m$. circular muscles; c. $m . m$. circular layer of muscularis mucosæ; ep. epithelium; $g . g l$. gastric glands; $l . m$. longitudinal muscles; $l, m . m$, longitudinal layer of muscularis mucos $m$. mouth of gastric gland; $z u$. nucleus; pr. peritoneum; s. m. submucosa.

gland-cell has the faculty of picking and choosing, the material supplied being worked up in the one case into the cutaneous secretion, in the other into gastric juice. 
The submucosa of the stomach is traversed by a narrow band of unstriped muscle, the muscularis mucose, formed, like the main muscular layer, of an outer layer of longitudinal $(l . m . m)$ and an inner of circular $(c . m . m)$ fibres.

A muscularis mucosa is also present in the intestine, but as it is very thin, it may easily be overlooked.

The Liver.-Sections of the liver show it to be made up of innumerable large, polyhedral cells (Fig. 4I, c), which are

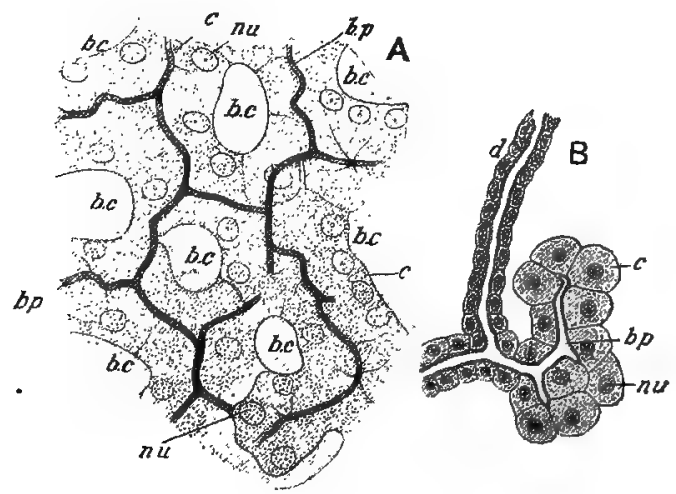

FIg $4 T_{*}-A$, portion of a section of the frog's liver; $B$, smal portion of the same, showing the origin of a bile-duct.

b. $c$. blood-capillaries, in section; $b$. $p$. bile-passages; $c$. liver cells; $d$, smallest bile-duct; -nz. nuclei. (After Hoffmann.)

so arranged as to bound extremely fine channels or bilepassages (b.p). These are found to open into one another, and finally to discharge into definite tubes $(B, d)$, lined with epithelium. These, in their turn, unite into larger and larger tubes, which form the hepatic ducts and ultimately open into the common bile-duct (p. 68).

The liver-cells are glandular and secrete the bile, which, as it is formed, drips into the bile passages and passes into 
the hepatic ducts, thence making its way either directly into the intestine or into the gall-bladder (p. 7o). The whole liver, which is the largest gland in the body, is traversed by a complex network of capillaries (b.c), supplied partly by the hepatic artery, partly by the portal vein; and, from the blood thus supplied, the liver-cells obtain the materials necessary to enable them to discharge their function of secreting the bile.

The liver-cells have, however, other functions, one of which is to manufacture, in addition to bile, a substance
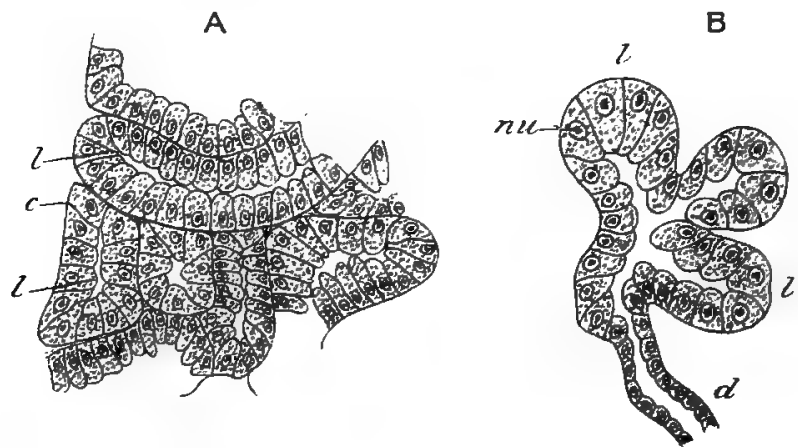

Frg. 42.-A, small portion of a section of the frog's pancreas; $\mathrm{B}$, diagram showing the connection between the lobules and ducts.

$c$. connective tissue covering of the gland; $d$. duct; $l$. lobules; $n u$. nuclei.

called glycogen or animal starch. This is stored up in the cells in the form of minute insoluble granules, which, being afterwards transformed into soluble sugar, pass into the blood and so to the tissues.

The Pancreas.-Sections of this gland (Fig. 42) show it to be made up of microscopic masses or lobules ( $l$, each of which consists of a cluster of gland-cells enclosing a very narrow central space. The cavities of adjacent lobules com- 
municate with one another and open into tubes or ducts $(d)$ lined with epithelium, which join with one another and finally discharge into the bile-duct as it traverses the pancreas (p. 70). The pancreas is distinguished as a racemose or grape-bunch gland : the duct is comparable to the branched stalk and the lobules to the grapes.

Connection of the foregoing facts with the physiology of nutrition.-You will now be able to understand more clearly the various processes connected with the nutrition of the frog, hitherto studied without the aid of histology.

When the food enters the enteric canal the various glandcells are stimulated into activity, and the gastric juice, bile, and pancreatic juice are poured out and mingle with the food, which is digested in the manner already described. The soluble products of digestion-peptones, sugar, salts, fatty acids, and glycerine, diffuse through the epithelium of the enteric canal into the blood-capillaries of the underlying submucosa, and the blood, now loaded with nutriment, is carried by the portal vein to the liver and thence by the hepatic and postcaval veins to the heart (see Fig. 23). At the same time the fats make their way into the lymphcapillaries and are finally pumped, by the lymph-hearts, into the veins. Thus the products of digestion all find their way ultimately into the blood, and are distributed, through the circulatory mechanism, to all parts of the body.

\section{PRACTICAL DIRECTIONS.}

Materials required for the Preparation and Sectioning of Animal Tissues.-In addition to the requisites mentioned in chapters I and VI, the following will be required :-

a. Corrosive sublimate: a saturated solution in water. Care shonld be taken in using this solution, as it is a very virulent poison. 
b. Absolute alcohol.

i. Turpentine.

d. Puraffin: sold in two grades, "hard" and "soft." It is best to get an ounce or two of each.

e. A solution of Canada balsam in chloroform or turpentine. This should be kept in a small glass bottle, with a ground glass cap-not a stopper or cork.

$f$. A solution of alcoholic borax-carmizze. This may be bought ready mixed, or made as follows :-Grind up in a mortar 2 grammes of carmine and 4 grammes of borax, and dissolve in Ioo c.c. of distilled water: to this solution add an equal volume of 70 per cent. alcohol: allow to stand for a day or two and filter.

g, A vater-bath, in which melted paraffin may be kept at a constant temperature. For a make-shift you can use a sancepan with a flat piece of tin over it; fill the saucepan about half full of water, and heat it over a spirit lamp or a small oil-lamp or gas-burner, regulating the distance of the flame so as to keep the temperature of the water at about $55^{\circ} \mathrm{C} .\left(13 \mathrm{I}^{\circ} \mathrm{F}.\right)$.

$h$. Two or three watch-glasses or other small shallow vessels for containing melted paraffin.

i. A sharp, flat-ground razor.

$j$. A section-lifter, made by beating out flat about half an inch of the end of a piece of stout copper wire, about 6 in. long, and bending the flattened portion at an obtuse angle with the rest.

\section{Preparation of Tissues for Section Cutting.}

a. Fixing, hardening, and decalcifying.

Sections may be cut from specimens which have been carefully preserved in alcohol, - first in 70 per cent, and after a day or two transferred to 90 per cent. But certain other reagents are more effective in effecting the $f$ ixing of the tissues-i.e., in quickly killing and coagulating the protoplasm of the cells with a minimum of shrinkage, and of these the one most generally useful is a solution of corrosive sublimate (see above), in which, from a freshly-killed frog, place small pieces of the various organs and tissues to be examined-e.g., skin, intestine, stomach, liver, pancreas, kidney, ovary, testis, and spinal cord, as well as the inner half of the eyeball. The intestine and stomach should be first washed out in salt solution, and then cut into pieces about 8 inch long; the liver should be 'cut into pieces not more than $\frac{1}{4}$ inch cube. After about half-an-hour to two hours, according to the size of the 
piece, place in water under a tap, and wash thoroughly for a quarter of an hour or more, until the corrosive sublimate is removed.

After washing, transfer to 50 per cent. alcohol for a few hours, and then to 70 per cent. for twenty-four hours, after which they may be stained at once (see below), or transferred to strong methylated spirit (90-93 per cent.), in which they may be kept until wanted. This completes the process of hardening : it is done gradually, by alcohols of increasing strength, in order to avoid shrinkage.

In order to decalcify such tissues as bone, from which the lime-salts must be extracted before cutting into sections, place a small piece for a few days in 70 per cent. alcohol, to which 2 per cent. of strong nitric acid has been added: then wash thoroughly, transfer to alcohol, and stain.

b. Staining.-Place the organs, cut into convenient sizes for imbedding-i.e., not more than $\frac{1}{4}$ inch long, and, in the case of such organs as the liver, $\frac{1}{8}$ inch in thickness-into borax-carmine for one or two days, or even more. They will become stained throughout, and the difficulty of staining the sections after cutting will thus be avoided. After staining, place them in weak alcohol (50-70 per cent.), slightly acidulated with hydrochloric acid : if a watch-glass or some such vessel is used, it is sufficient to dip the end of a glass rod into acid and stir it round in the alcohol. The effect of the acid is to remove much of the colour from the protoplasm of the cells, leaving the nuclei brightly tinted. After half-an-hour or less in the acid alcohol, place the tissues once more in strong methylated spirit.

c. Dehydrating.- Transfer from methylated spirit to absolute alcohol, which must be kept in a stoppered or tightly corked bottle, as it will otherwise deteriorate by absorption of water from the air. It has the effect of withdrawing the last traces of water from the tissues, an absolutely necessary step in order that they may be permeated with paraffin.

d. Imbedding. - Transfer the objects from absolute alcohol to turpentine. This fluid acts as an intermediary between alcohol and paraffin, being freely miscible with both : it gradually replaces the alcohol in the tissues, rendering then transparent. If they are not transparent in the course of an hour or so, the process of dehydration has not been complete, and they must be returned to absolute alcohol. In the meantime, melt some paraffin over the water-bath, using various mixtures of hard and soft according to the season : in a cold room in winter soft paraffin will be hard enough ; in the height of summer hard paraffin 
alone will be suitable. The temperature of the water-bath must never be allowed to rise more than a degree or two alonve the melting point of the paraffin. Transfer the objects from turpentine to melted paraffin and keep them in it for some hours-the time varies according to the size of the piece-until they are thoroughly permeated.

If you wish to cut sections by hand, get some ordinary medium-sized bottle-corks : around each wind a piece of paper, allowing it to project about $\frac{1}{2}$ an inch beyond one end of the cork, ancl fixing it with a pin, as in Fig. 43, A. Into the little cylindrical vessel or imbedding box thus formed pour some melted paraffin, and immediately transfer to it, by means of a warmed section-lifter or forceps, one of the prepared
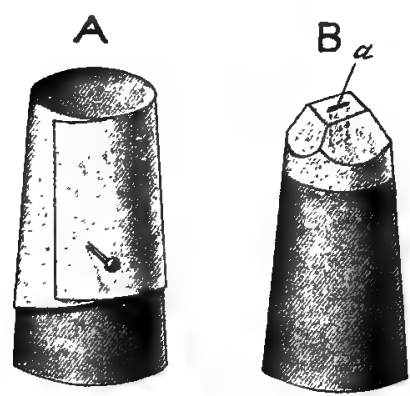

Fig. 43.-A, imbedding box made by wrapping paper round a cork; $\mathbf{B}$, cork after removal of the paper, showing the paraffin pared down to a convenient size for sectioning. $a$, object to be cut. pieces, adjusting its position by means of a heated needle. When the paraffin is quite cold remove the paper, and you will have fixed to the cork a solid block of paraffin containing the object to be cut.

e. Section-cutting.-Pare away the block of paraffin until the object comes into view: then trim the block, as in Fig. $43, \mathrm{~B}$, until its upper surface, with the object in the middle, is not more than $\frac{1}{4}$ inch square. Hold the cork firmly in the left hand, with the wrist resting on the table, and with a razor cut the thinnest possible slices of the paraffin block, including the imbedded object. The razor must be held firmly grasped at the junction of blade and handle, and kept with the surface of the blade parallel with that of the block : use almost the whole extent of the edge for each section. With a little practice you will be able to cut sections so thin as to be quite transparent under the high power. ${ }^{1}$

${ }^{1}$ If you are working in a properly furnished laboratory you will probably learn how to cut sections with a microtome, or section-cutting machine, which gives much better results and is absolutely necessary when a complete series of sections of the same object is required. 
f. Clearing and Mounting.-Place the section on a slide and warm it gently on the water-bath until the paraffin melts, and then add a large drop of turpentine in order to dissolve the paraffin. Then draw off the turpentine with blotting-paper and replace it by a fresh drop, repeating the process until all the paraffin is dissolved : put on a coverglass and examine.

If you wish to be sure that the parts of your sections are not displaced in mounting, or to mount several sections on your slide, the latter should first be smeared over with a very thin layer of a mixture of collodion and oil of cloves, in equal parts : then place the sections on the slide, warm, and immerse the whole slide in a small vessel of turpentine, leaving it until all the paraffin is dissolved.

In order to make a permanent preparation, remove the paraffin with turpentine, as above, draw off the turpentine, place a drop of Canada balsam on a cover-glass and very gently lower the cover-glass on the object, spreading out the balsam in a thin, even layer. Before long, the balsam will have set quite hard, and the sections may be preserved for an indefinite period; the halsam will set more quickly if you leave your preparations over the water-bath for a short time.

Remember that object, razor, slide, and cover must be kept free from water, the presence of which, from the stage of dehydration onwards, is fatal to success.

\section{Examination of Compound Tissues.}

Examine the following sections, prepared as clescribed above, first with the low, and then with the high power, noting the parts enumerated in each case, as well as the structure of the nuclei (nuclea) membrane, chromatin, and achromatin.)

I. Vertical section of skin (Fig. $3^{8}$ ).

a. Epiderm, stratified, divisible into outer (homy) and inner (Malpighian) layers.

b. Derm, connective-tissue fibres, blood-vessels and pigment-cells.

c. Cutaneous glands with their ducts. The apertures of the ducts on the surface you will probably have noticed already in your preparation of the epithelial cells of the skin (p. 124).

Sketch.

2. Transverse section of intestine (Fig. 39).

u. Mucous membrane : a superficial epithelial layer of columnar cells, 
with goblet cells amongst them ; and a deeper connective-tissue laver, the sabmucosa, enclosing blood-vessels, nerves, and lymphatics.

b. Muscular layer: an external longitudinal, and an internal circular layer of unstriped muscular fibres.

c. Peritoneal layer. This is very thin, and a careful examination of good preparations is required in order to make out its structure (p. I3I).

Sketch.

3. Transverse section of stomach (Fig. 40).-After recognising the layers as above, note :-

$a$. The gastric glands, and $b$. The muscularis mucosa.

Sketch.

4. Sections of liver (Fig. 4I).

a. Polyhedral gland-cells; b. Bile passages and ducts; c. Blood capillaries and vessels.

Sketch.

5. Sections of pancreas (Fig. 42).

a. Lobules, each consisting of gland-cells; b. Ducts.

Sketch. 


\section{CHAPTER IX.}

The Frog (continued): respiration and excretion.

IN the fifth chapter it was pointed out that a continual waste of substance goes on in the frog's body, the lost material taking the form of three chief waste products or products of excretion, water, carbon dioxide, and urea. It was further stated that these substances are got rid of by means of the lungs, the kidneys, and the skin.

The Organs of Respiration.-At their anterior ends the two lungs open into a small, thin-walled chamber (Fig. 44, l. $t r . c)$, which as it corresponds both with the larynx or organ of voice, and the trachea or windpipe in ourselves, is called the laryngo-tracheal chamber: it communicates with the pharynx through the glottis $(g l)$. The walls of the chamber and the edges of the glottis are supported by cartilages (ar).

The structure of the lung is best made out by distending it with air, and then placing it in spirit until thoroughly hardened: its walls contain so much elastic tissue that if cut when fresh, it contracts immensely, and its structure is then difficult to see. The inner surface of the lung is raised up into a complex network of ridges (A r. $\operatorname{lng}$ ), which project into the interior and produce the appearance of an 
irregular honeycomb. All these ridges are abundantly supplied with blood vessels fed by the pulmonary artery, the blood being carried away by the pulmonary vein.

The main substance of the lung is made of connective tissue containing elastic fibres and unstriped muscle, and traversed by a network of capillaries. Its cavity is lined by a layer of pavement epithelium, and its outer surface is covered with peritoneum.

Respiratory Movements.-In breathing, the frog depresses the floor of the buccal cavity (Fig. 45, A), and, the mouth

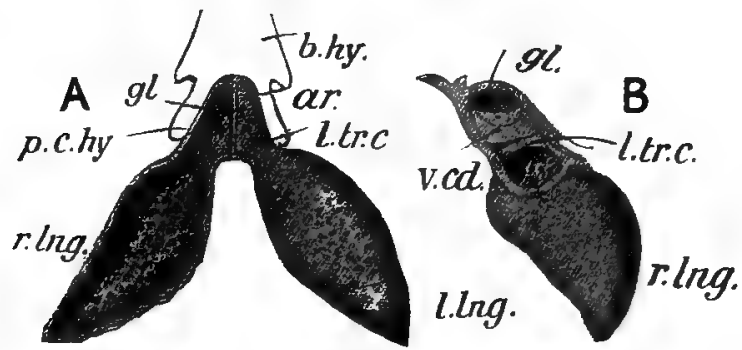

FIG. 44. -The respiratory organs of the frog from the ventral aspect; $B$, the laryngotracheal chamber in longitudinal section, with the right lung.

$a r$. the arytenoid, or principal cartilage of the larynx; $b . h y$. body of hyoid; $g l$. glot tis; l. lng. left lung; $l . t r . c$. laryngo-tracheal chamber; $p . c . h v$. posterior horn of hyoid ; r.lng. right lung, laid open in A to show its internal surface; v. cd. vocal cord. (After Howes).

being kept shut, air is drawn in through the nostrils. The floor of the mouth is then raised $(B)$, by muscles attached to the hyoid. At the same time the anterior end of the lower jaw presses upon the moveable premaxillæ $(p m x)$, the upward processes of which (p. 42, Figs. 8 and $9, P M X$ ) act upon certain cartilages in connection with the external nostrils in such a way as to produce closure of these apertures (Fig. 45, B). The gullet (gul) is so contracted, except during the act of swallowing, as to be practically 
closed. Thus when the floor of the mouth is raised the air contained in it can escape in one way only, viz., through the glottis into the lungs.

Thus inspiration, or breathing in, is produced by the buccal cavity acting as a force-pump: the lowering of its floor draws in air through the nostrils, the raising of its floor forces the imprisoned air into the lungs. Expiration, or
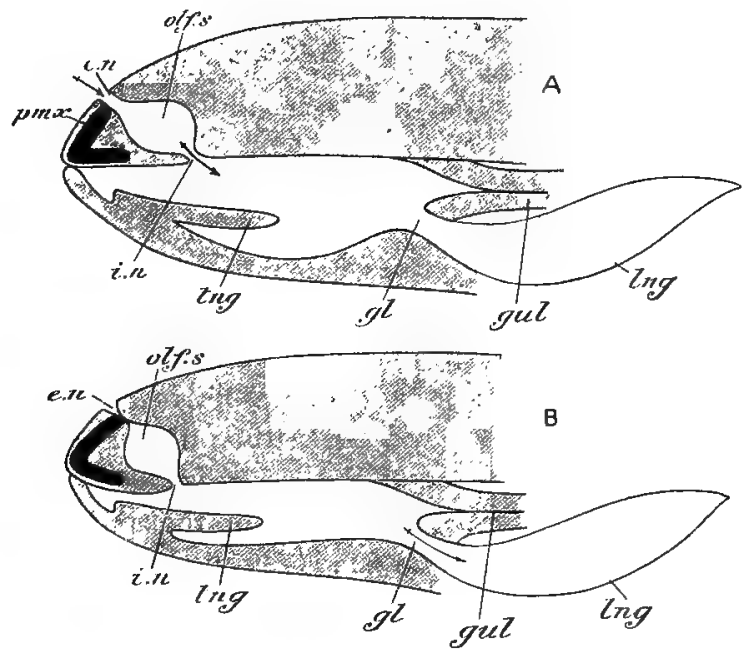

FIG. 45.-Diagrams illustrating the respiratory movements of the frog. In A the floor of the mouth is depressed and air is being drawn in through the nostrils; in $B$ the floor of the mouth is raised, the nostrils are closed, and air is being forced into the lungs.

e.n. external nostril; $g l$. glottis ; gul. gullet; $i . u$. internal nostril; lng. lung; olf. s. olfactory sac; phix. premaxilla; ing. tongue.

breathing-out, is due to a contraction of the elastic lungs, accompanied by a slight lowering of the tip of the lower jaw : the latter movement releases the premaxillæa and thus opens the external nostrils. 
Respiration.-By these alternate movements of inspiration and expiration fresh air passes into the lungs at regular intervals, while part of the air already contained in them is expelled. Now we saw, when studying the blood (p. 79), that dark purple blood drawn from a vein becomes bright scarlet when exposed to air, and we subsequently learnt (p. 107) that this change is due to the absorption of oxygen by the red corpuscles.

The blood brought to the lungs by the pulmonary artery is, as we have seen (p. 94), non-aërated, being the impure blood returned by the three caval veins to the right auricle. When this blood is pumped into the capillaries of the lungs it is separated from the air contained in those organs only by the extremely thin walls of the capillaries themselves and the equally delicate pavement epithelium lining the lungs (Fig. 23, p. I42, Ep. lng, Cp. lng). Under these circumstances an interchange of gases takes place between the air and the blood: the hæmoglobin of the red corpuscles absorbs oxygen, and the carbon dioxide in the blood, derived from the waste of the tissues, is given off into the cavities of the lungs. The blood in the pulmonary capillaries thus become aërated and is returned as red blood to the left auricle : at the same time it loses carbon dioxide, together with a certain amount of water, and these waste substances are expelled from the body with the expired air.

Voice.-It was mentioned above (p. r4I) that the glottis and laryngo-tracheal chamber are supported by cartilages. The largest of these are a pair of semilunar arytenoid cartilages (Fig. 44, ar), which bound the glottis to right and left. The mucous membrane on the inner or adjacent faces of the arytenoids is raised into a pair-right and left-of horizontal folds, the vocal cords $(v . c d)$. By means of muscles these folds can be stretched and relaxed, and can be brought 
into either a parallel or a divergent position. When they are parallel the air, passing to and from the lungs, sets their edges in vibration and gives rise to the characteristic croak, the pitch of which can be slightly altered by stretching or relaxing the cords.

Structure of the Kidneys. - The form and situation of the kidneys (Figs. 3,4, and $7, k d$ ) have already been referred to. Each is a flattened organ of a deep reddish-brown colour, its inner edge nearly straight but for one or two notches, its outer edge curved. Its ventral face is covered

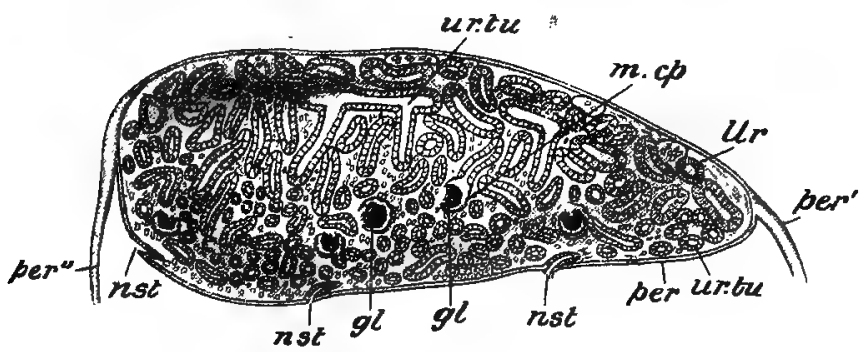

FIG. 46.-Transverse section of frog's kidney.

gl. glomerulus; $m . c p$. Malpighian capsule; nst. nephrostome; per. peritoneum covering ventral face of kidney; per'. fold of peritoneum supporting its outer border; $p z^{\prime \prime}$. fold supporting its inner border; $U R$. ureter; ur. tu. urinary tubules. (After Marshall and Bles.)

by peritoneum (Fig. 46 , per), continued on the one hand into the parietal layer (Fig. 5, p. per), of that membrane, on the other into the mesentery (mes.); its dorsal face is bathed by the lymph of the subvertebral sinus (sv. ly.s.) From the posterior end of its outer edge a delicate tube, the ureter (Figs. 3, 4, and 7, ur), passes backwards and opens into the dorsal wall of the cloaca. On its ventral face is a singular yellowish-white stripe, the adrenal or supra-renal body, an organ of unknown function (Fig. 7, between the lines from $k d$ and $t s$ ). 
A thin section shows the whole kidney to be made up of a tangled mass of microscopic tubes (Fig. 46, ur. $t u$ ), so

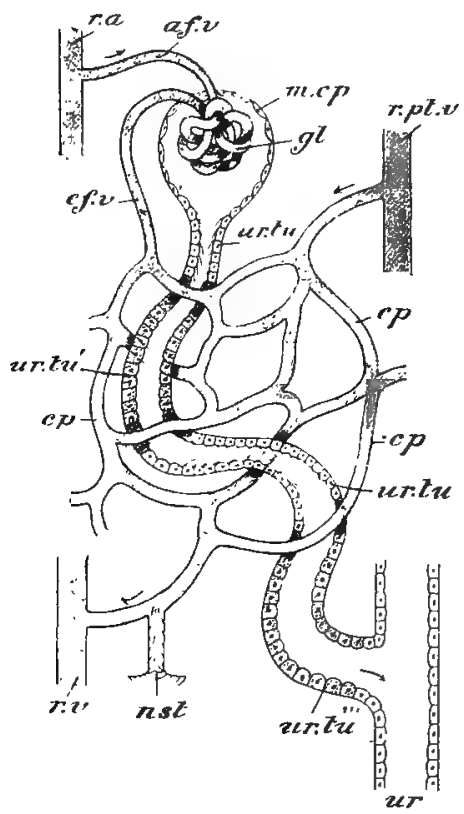

FIG. 47,-Diagram of a single urinary tubule with its blood-vessels, to illustrate the structure of the frog's kidney.

$a f . \nu$. afferent vessel of glomerulus; $c p$. capillary network of kidney; $e f . v$. efferent vessel of glomerulus; $g$ l. glomerulus; m.cp. Malpighian capsule, showing epithelium; nst. nephrostome showing cilia ; $r . a$. renal artery; $r . \not p t . v$. renal portal vein ; $r . v$. senal vein ; ur. ureter; ur.tu., ur. $t u^{\prime}$, , ur. $t w . ", u r . t u . "$ ", different portions of urinary tubule, showing epithelium and cilia. twisted together that any section cuts them in various planes, some transversely, when they appear as circles, others longitudinally or obliquely. Amongst these urinary tubules or nephridia, as they are called, are seen globular sacs, the Malpighian capsules (m. cp), each having in its interior a little irregular bunch, known as the glomerulus $(g l)$. Very accurate examination of numerous sections, as well as of teased-out specimens, shows that each Malpighian capsule (Fig. 47, $m .(p)$, is connected with a urinary tubule (ur.tu), to which it forms a blind, bulb-like end. The tubule itself winds through the substance of the kidney, is joined by other tubules, and finally discharges into the ureter (ur). The tubules are lined with somewhat cubical cells of glandular epithelium, which, in some parts (ur. tu, ur.tu') 
are ciliated. The Malpighian capsules are lined with flattened cells of pavement epithelium.

The arrangement of the bloodvessels is peculiar. Like other organs, the kidney is permeated by a network of capillaries $(\not p)$ which form a close mesh between the urinary tubules, so that the cavity of the tubule is separated from the blood only by the thickness of the gland-cells and of the capillary wall. The capillary network is supplied partly by the renal arteries $(r . a)$, partly by the renal portal vein $(r . p t . v)$, and is drained by the renal veins $(r . v)$. It is in the behaviour of the renal arteries that the chief peculiarity of the kidney-circulation lies. On entering the kidneys they break up into smaller and smaller arteries, but each of the ulffmate branches ( $a f . v$ ), instead of discharging into the general capillary network, passes to a Malpighian capsule, in the interior of which it breaks up into a little bunch of coiled capillaries $(g l)$, the glomerulus. From this the blood is carried off by a minute vessel (ef. $v$ ) by which it is poured into the general capillary network and finally discharged into the renal vein $(r . v)$.

Renal Excretion.-While circulating through the glomerulus, water and certain soluble matters are separated from the blood and make their way into the Malpighian capsule and thence into the urinary tubule. As the blood circulates through the general capillary network, the gland-cells of the tubules excrete, out of the materials brought to them by the blood, the nitrogenous waste matter urea, in the formation of which the liver plays an important part ; it is discharged from the cells into the cavity of the tubule where it is dissolved in the water separated in the glomerulus. In this way the urine is formed. Accumulating in the tubules it makes its way into the ureter and thence drop by drop into the cloaca, whence it is either expelled at once, or stored for a time in the bladder. 
Note that the formation of urine is a process of secretion of a similar nature to the secretion of gastric juice, bile, \&c. The fluid secreted, is, however, of no further use to the animal, and would, in fact, act as a virulent poison if retained in the system. It is therefore got rid of as soon as possible. Secretions of this kind, consisting not of useful but of harmful or waste matters, are distinguished as excretions.

Bile is also in part an excretion as it contains pigments due to the disintegration of hæmoglobin, and thus by its means the effete colouring matters of the blood are passed into the intestine and got rid of.

Pulmonary and cutaneous Excretion.--The lungs, besides being organs of respiration, take their share in excretion, since they get rid of the important waste product, carbon dioxide, together with a considerable quantity of water. Similar functions are discharged by the skin, which is also an organ both of respiration and of excretion. Interchange of gases take place between the outer air and the blood in the capillaries of the derm : the carbon dioxide of the non-aërated blood brought to the skin by the cutaneous artery (p. 93 and Fig. 23) is exchanged for oxygen, and the blood, in the the aerrated condition, is returned by the musculo-cutaneous vein to the heart. Moreover the cells of the cutaneous glands separate water and other constituents from the blood, and the fluid thus formed is poured out on the surface of the body. Here it serves to keep the skin moist, and is finally lost, either by evaporation or by mingling with the water in which the frog is immersed. The cutaneous secretion has also poisonous properties, and so probably serves as a defence against some of the animal's enemies.

Summary of the processes of Nutrition.-We are now in a position to understand the general features of the whole complicated series of processes which have to do with the nutrition of the frog, which are collectively spoken of 
as metabolism. These processes are illustrated in the diagram (Fig. 23, p. 91), which should be constantly consulted in connection with the following summary.

All parts of the body are placed in communication with one another by means of the bloodvessels, through which a constant stream of blood is flowing in a definite direction.

In all parts waste of substance (destructive metabolism) is continually going on, and the waste products, water, carbon dioxide, and some nitrogenous substance which ultimately takes the form of urea-are passed either directly into the blood, or first into the lymph and thence into the blood.

At the same time the tissues withdraw nutrient materials from the blood, whereby the waste of substance is made good, and the cells and other elements adequately nourished (constructive metabolism). Oxygen is also withdrawn from the blood ; like the air supplied to a fire it is essential to the oxidation or low temperature combustion with which the waste of the tissues is associated. By the withdrawal of its oxygen the hæmoglobin of the blood alters its colour from scarlet to purple.

Thus the blood as it passes through the body is constantly being impoverished by the withdrawal of nutrient matters and of oxygen and as constantly being fouled by the discharge into it of waste products. It reaches the capillaries of an organ as bright red, aërated blood, and leaves it as purple, non-aërated blood.

These changes, by which the blood loses nutrient matters and oxygen, and gains waste products, takes place all over the body. The converse processes by which nutrient matters and oxygen are absorbed and waste products got rid of are carried on in certain definite portions of the circulatory system.

In the walls of the enteric canal (Fig. 23, Ent. C), the 
products of digestion pass into the blood ( $C p . E n t . C)$, or in the case of fats, first into the lacteals and ultimately into the blood. In this way the due proportion of nitrogenous and other food materials is kept up.

In passing through the capillaries of the lungs (Cp. Lng) carbon dioxide is exchanged for oxygen and a certain amount of water is given off. In the capillaries of the skin $(C p . S k)$, a large quantity of water and smaller proportions of other waste matters are got rid of. In the kidneys $(C p . K d)$, a considerable quantity of water, together with the bulk of the urea, are removed from the blood and finally expelled from the body.

Note that all these changes are produced by the special activity of particular groups of epithelial cells, which, however alike they may be in general appearance and structure, have a marvellous selective faculty peculiar to themselves. Like all other parts of the body, they are constantly undergoing the usual processes of waste and repair, withdrawing nutrient matters and oxygen from the blood, and passing waste matters into it. But, in addition to the ordinary processes of nutrition, each particular group of cells has the power of withdrawing a specific substance from the blood or of passing substances into it. Thus the epithelial cells of the enteric canal $(E p . E n t)$ pass in digested food, those of the skin $\left(E_{p} . S k\right)$ and glomeruli $(\mathrm{g} l \mathrm{~m})$ withdraw water, those of the urinary tubules $(E p$. Ur. T), urea, and so on. Similarly, the various glandcells, such as those of the liver (Lvr. C), pancreas $(P n . C)$, gastric, and cutaneous ( $\mathrm{Cu}$. Gl) glands withdraw specific substances, or excretions, which are discharged on the free surface of the epithelium and serve various purposes.

We see that the blood loses-(I), nutrient matters and oxygen all over the body; (2), water in the skin, lungs, and 
kidneys ; (3), carbon dioxide in the lungs and skin ; (4) urea, principally in the kidneys ; and (5), various substances in the glands. It gains (I), waste products all over the body; (2), nutrient matters in the enteric canal; (3), liver-sugar in the liver ; (4), oxygen in the lungs and skin. It is therefore richest in oxygen and poorest in carbon dioxide as it leaves the lungs and skin, i.e., in the pulmonary aud musculocutaneous veins; richest in nutriment as it leaves the enteric canal, i.e., in the portal vein; poorest in urea as it leaves the kidneys, i.e., in the renal veins ; poorest in water as it leaves the skin and kidneys, i.e., in the cutaneous and renal veins.

In this way a single closed system of pipes not only supplies all parts of the body with everything necessary for their sustenance, but serves also as a drainage system to carry away their various waste products.

Notice that we must distinguish between the nutrition, respiration, and excretion of the frog as a whole, and of its various parts. Every one of the thousands of cells, fibres, \&c., in the entire body is nourished, breathes, and excretes, taking its nourishment and oxygen directly from the blood, and discharging its waste products into it. What are called the organs of nutrition and respiration are special portions of the body set apart for taking in fresh supplies of food or of oxygen for the organism as a whole, such supplies being finally distributed by the blood-system. Similarly, what are called the organs of excretion are special portions of the body by which the waste products, collected by the blood from all parts of the organism, are finally discharged.

Evolution of Heat.-The oxidation of the tissues, like that of coal or wood in a fire, is accompanied by a rise in temperature. But in the frog, as in other cold-blooded animals, the evolution of heat is never sufficient to raise the 
temperature of the body more than very slightly above that of the surrounding medium. In warm-blooded animals, such as ourselves, the temperature is regulated, according to the season, by a greater or less evaporation of water from the surface of the body. In the frog this is not the case. "The temperature of the animal is always nearly the same as that of the air or water in which it lives, and hence the frosts of winter would be fatal to it, but for the habit of hibernation (p. 8).

Death and Decomposition.-The decomposition undergone by a dead frog (p. I I), may be looked upon as an excessive process of waste unaccompanied by repair. Owing to the action of certain microscopic plants known as Bacteria, which will be referred to again in Part II, the proteids undergo oxidation, amongst the principal products of which are water, carbon dioxide, ammonia, and certain gases of evil odour, such as sulphuretted hydrogen and ammonium sulphide. The gases escape into the air, while the ammonia is finally converted into nitrous and nitric acids. These, combining with certain substances in the soil, give rise to salts called nitrates and nitrites, which furnish one of the chief sources of the food of plants.

\section{PRACTICAL DIRECTIONS.}

The Organs of Respiration and of Voice.-Pin out as frog in the ustual way (Pp. 31 and 32), remove the heart, and make out the precise relations of the lungs, first distending them with air through the glottis. The specimen already used for the dissection of the vascular system or alimentary canal will serve the purpose. IIarden thoroughly in spirit and note (Fig. 44) the laryngo-tracheal chamber, which communicates with the pharynx through the glottis on the one hand, and on the other with both lungs. Observe also the posterior horns of the hyoid which embrace the glottis, and then separate them from the laryngo- 
tracheal cliamber, so as to remove the latter, together with the lungs, from the body.

Then dissect off what remains of the mucous membrane of the pharynx around the glottis, and notice the small laryngeal musicles in connection with the laryngo-tracheal chamber: remove these, and pin the respiratory organs down under water, ventral surface uppermost, by means of a pin through each lung. Cut away the ventral wall of one lung, so as to expose the cavity and its connection with the laryngotracheal chamber. (You will very probably find some parasites in the lungs - small worms called Ascaris nigrovenosa, belonging to the group of Nematode worms.) Note :-

I. The two arytenoid cartilages, and a ring-shaped cartilage surrounding the base of the lungs.

2. The network of ridges on the inner surface of the lungs. Examine with a lens. Sketch.

3. The vocal cords. Observe these first in their natural position, and then with the scissors cut through the laryngo-tracheal chamber along the line of the glottis su as to divide it into right and left halves and thus expose the vocal cords from their surface. Sketch.

The Kidneys. - a. Examine these organs in silu (Figs. 3, 4, and 7) and note :-

I. Their form and position, and the relations of the peritoneum, which covers them on the ventral side only. (See Fig. 5).

2. The ureters (their openings into the cloaca may be scen at a later stage).

3. The yellowish adrenals.

Sketch.

$b$. Examine under the microscope a transverse section of the kidney, prepared as directed on p. 136 , and make out (Fig. 46) :-

I. The urinary tubules, cut through in various planes.

2. The Malpighian capsules and their glomeruli.

3. Blood-capillaries and vessels.

Sketch a portion under the high power. Compare with a section of kidney in which the blood-vessels have been injected with coloured gelatine (p. IOI). 


\section{CHAPTER X.}

THE FROG (continued): THE NERVOUS SYSTEM.

IN a machine of human construction, such as the engine of a steamer, the proper working of the whole depends, provided the parts of the machine itself are in good order, upon two things - the stoking or regulation of the fires, and the turning of certain cocks and levers by the engineer. In that very complex machine the frog, we have already studied what corresponds to stoking, viz., feeding and breathing. IVe must now direct our attention to what may be considered roughly to correspond with the work of the engineer-the means by which the whole complex machinery is kept under control, and its various parts made to work together to a common end.

How does it come about, for instance, that the various digestive glands begin to secrete actively as soon as food is taken into the enteric canal ? How is it that a touch on any part of the body, or even the sight of an enemy, is followed instantaneously by a series of vigorous muscular movements so ordered as to facilitate escape from the source of danger?

In the fourth chapter (p. 6z) we got so far as to learn that muscular contractions are induced by nervous impulses travelling from the brain or spinal cord, along the nerves, to to the muscles. It may therefore be inferred that the con- 
trolling apparatus, by which the functions of the body are regulated, is lodged in either the brain or the spinal cord, or both.

Divisions of the Nervous System.-The nervous system is divisible into ( $\mathrm{I}$ ) the central nervous system, consisting of the brain and spinal cord (Figs. 6 and 7), and (2) the peripheral nervous system, consisting of the nerves which pass from the central nervous system to the various parts of the body. The nerves are divisible into (I) cerebral nerves (Fig. 53), arising from the brain, and (2) spinal nerves (Fig. 5I) arising from the spinal cord.

The Spinal Cord.-In form the spinal cord (Figs. 6 and $7, s p . c d)$ is irregularly cylindrical. It is continuous in front with the brain, and tapers off posteriorily into a fine threadlike portion, the filum terminale $(f . t)$, while opposite the fore-limbs, and just anteriorly to the filum terminale, it presents a couple of enlargements, known respectively as the brachial and sciatic swellings. Along its dorsal surface runs a delicate longitudinal line, the dorsal fissure (Fig. 48, d.f), and a distinct groove, the ventral fissure $(v . f)$, extends along its lower surface.

The cord is covered with a delicate pigmented membrane known as the pia mater $(p . w)$ and the neural canal in which it lies is lined with a stout, tough membrane, the dura mater (Fig. 52, d.m). Between the two is a cavity filled with a lymphatic arachnoid fluid, which, like the pericardial fluid, preserves the contained organ from shocks.

Examination of a transverse section of the cord under a low power will show that the dorsal fissure is an extremely narrow vertical wall formed by an extension inwards of the pia mater. The ventral fissure is a distinct cleft. Thus the two fissures divide the cord into paired half cylinders, right and left, joined in the middle by a narrow bridge. This 
bridge is traversed from end to end by a very narrow longitudinal canal, the central canal $(c . c)$, so that the cord is not a solid cylinder, but a tube with an extremely narrow cavity and excessively thick walls.

The section also shows that the cord is not homogeneous, but is composed of two different substances. Its outer part is pure white and shining in the fresh cord, and is hence

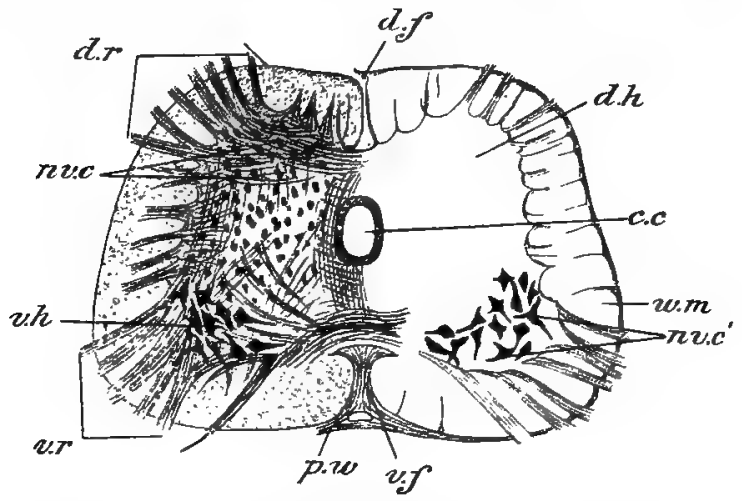

FIG. 48.-Transverse section of spinal cord of frog.

$c . c$. central canal ; $d . f$. dorsal fissure; $d . h$. dorsal horn of grey matter ' $d . r$. fibres of dorsal root of spinal nerve; $n v . c$. nerve cells of dorsal horn; $n v . c^{\prime}$. nerve cells of ventral horn; $p . w$. pia mater; $v$.f. ventral fissure; $v$. $h$. ventral horn of grey matter; $v . r$. fibres of ventral root of spinal nerve; $w$. $m$. white matter. (After Howes.)

called the white matter $(w . m)$. Its internal substance has a pinkish colour when fresh, and is called the grey matter $(d . h, v . h)$. The grey matter has a squarish outline in transverse section. It surrounds the central canal, and is continued upwards and downwards, forming what are called the dorsal (d.h) and ventral (v.h) horns of the grey matter.

The Brain.-In front the spinal cord passes insensibly into the brain (Fig. 49), which is of somewhat greater diameter than the cord, and is made up of several very dis- 
tinct parts or divisions. The hindermost division is called the bulb, or medulla oblongata (Med.obl); this appears to be simply a widening of the spinal cord $(S p . c d)$, except that on its dorsal surface is a triangular body $\left(\mathrm{D}, c h . p l x^{2}\right)$ of a reddish colour in the fresh condition, and called the posterior choroid plexus: it is simply a thickening of the pia mater containing abundant blood-vessels.

The choroid plexus forms a kind of lid to a triangular cavity (A and $\mathrm{D}, v^{4}$ ) excavated in the dorsal region of the medulla oblongata, and called the fourth ventricle. The apex of the cavity, which is directed backwards, opens into the central canal of the spinal cord (Fig. 50, $v^{4}, c . c$ ), and the the fourth ventricle is to be looked upon simply as the anterior part of the central canal which has become widened out and is covered only by a thickened portion of the pia mater.

The fourth ventricle is bounded in front by a narrow ledge of nervous matter (Fig. $49, C b$ ), which would be hardly worthy of being considered as a special division of the brain but for the fact that the corresponding part in many animals-e.g., dogfish, rabbit, man-is a large and important structure. It is called the cerebellum.

In front of the cerebellum comes a pair of rounded elevations, the optic lobes (Figs. 49 and 5o, Opt. l). Each contains a cavity, the optic ventricle (Fig. 50, Opt. v), communicating with a narrow median passage, the iter (Figs. $49 \mathrm{D}$ and $5 \circ, i$ ), which is continuous behind with the fourth ventricle. The bulb is continued forwards beneath the optic lobes as the crura cerebri $(\mathrm{Cr} . \mathrm{C})$.

In front of the optic lobes is an unpaired structure, the diencephalon or 'tween brain $(D i)$. On its upper surface is a small rounded vascular body, the anterior choroid plexus (Fig. $49 \mathrm{~A}, \mathrm{D}, \mathrm{ch} . p l x^{1}$ ), formed, like the posterior choroid 

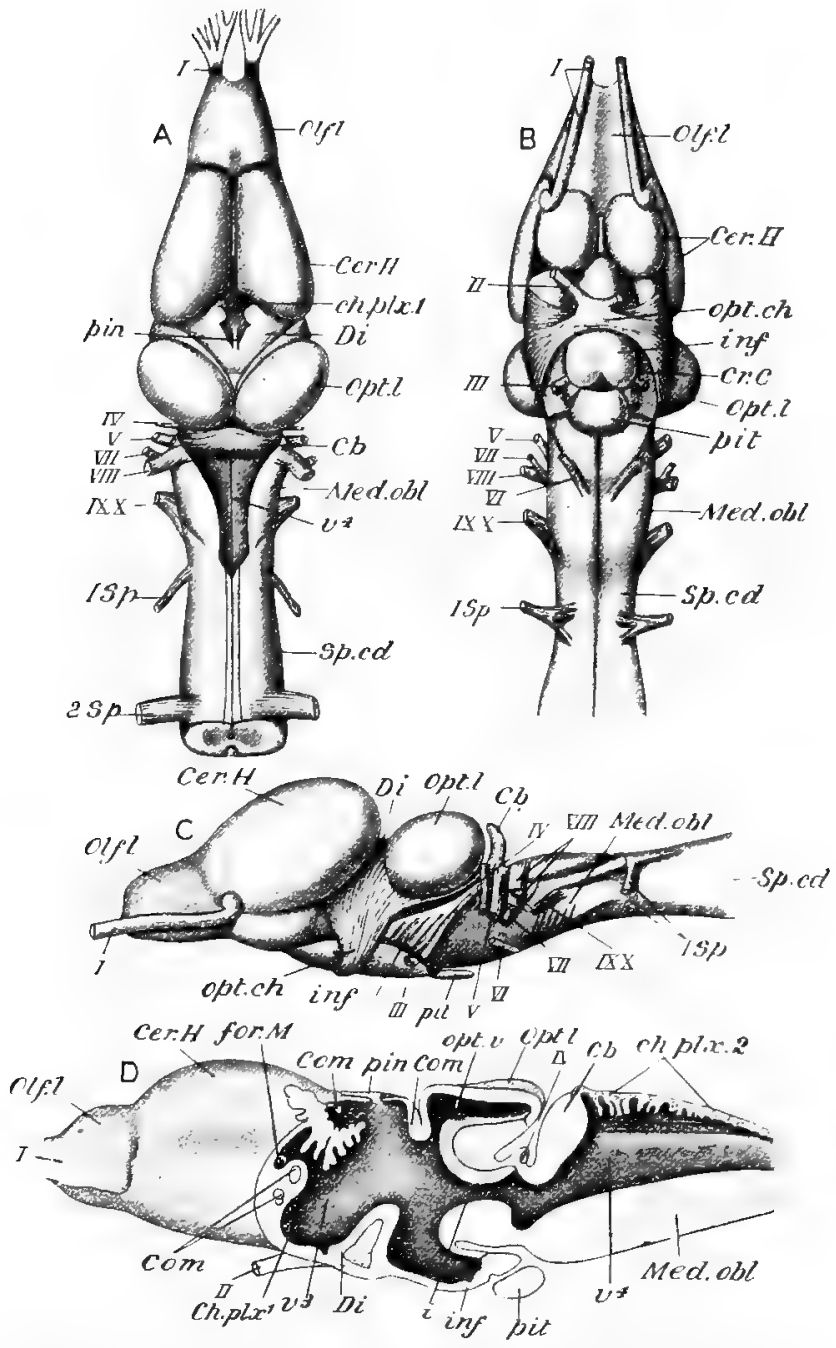

FiG. 49.-Brain of frog. A, from above; B, from below; C, from the side; $D$, in longitudinal vertical section.

Cb. cerebellum; Cer. $H$. cerebral hemispheres; $c h . p l x .1$ anterior and $c h . p l x .^{2}$ posterior choroid plexus; com. transverse bands of nerve fibres or commissures 
connecting the left and right halves of the brain; $C r C$ crura cerebri; $D i$. diencephalon; for. $M$. foramen of Monro; $i$. iter, or aqueduct or Sylvius ; i $\mathrm{N}$. infundibulum; Mlod.obl. medulla oblongata ; Olf.l. olfactory lobe; opt.ch. optic chiasma; Opt. $\%$ optic lobe; opt. $\tau^{\prime}$ optic ventricle; pin. stalk of pineal body, pit. pituitary body; $S f . c d$ spinal cord; $x^{*}$. third ventricle; $z^{4}$. fourth ventricle; I-X, cerebral nerves; $r S \% .2 S p$, first and second spinal nerves; (A-C, after Gaupp; D, from Wiedersheim, after Osilorn).

plexus, of a thickening of pia mater, containing numerous blood-vessels. It helps to roof over a narrow slit-like cavity, the third ventricle (Figs. $49 \mathrm{D}$ and $50, v^{3}$ ), the sides of which are formed by thickenings of nervous matter, the optic thalami $\left(D_{i}\right)$. On the ventral surface of the brain the diencephalon is continued into a funnel-like extension, the infundibulum (Fig. 49, inf), to which is attached a rounded structure, the pituitary body (pit). On the dorsal surface, just behind the choroid plexus, is the delicate stalk ( $p i n$ ) of the pineal body - the vestige of a sensory apparatus (Fig. ${ }_{4} 8$ C), part of which in some lizards, for example, has the structure of an eye, and which was probably functional in the ancestors of the frog. We shall meet with other examples of such vestigial organs in the

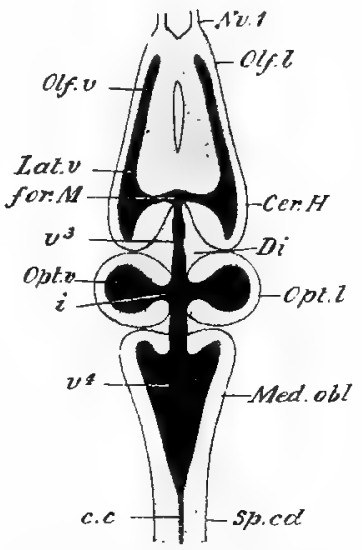

FIf;. 50.-Diagrammatic horizontal section of frog's brain.

c.c. central canal; $\mathrm{Cer}, \mathrm{H}$. cerebral hemisphere ; $D i$. diencephalon ; for: $M$. foramen of Monro ; $i$. iter ; Lat. ". lateral ventricle; $\mathrm{Med}$. ob2. medulla oblongata; $N^{\prime}, I$, olfactory nerve; Olf. $L$. olfactory lobe; $O l f . r$. olfactory ventricle; Opt.l. optic lobe; Opt. v. optic ventricle; $S p . c d$. spinal cord; $z, 3$, third ventricle; $v \cdot 4$, fourth ventricle. (After Ecker and Wiedersheim.) course of our studies.

In front of the 'tween-brain comes a pair of long, oval bodies, wider behind and narrower in front. These are the cerebral hemispheres (Cer. H). Each contains a cavity, the 
lateral ventricle (Fig. 50, Lat. v), which communicates with the third ventricle by a small aperture, the foramen of Monro (for. $M$ ).

Lastly, each cerebral hemisphere is continued forwards by a rounded olfactory lobe (Olf. $I$ ), which is fused with its fellow of the opposite side, the single mass lying in the posterior compartment of the girdle bone. The lateral ventricles are continued forwards into the olfactory lobes, forming the small olfactory ventricles (Fig. 50, Olf. v).

The brain, like the spinal cord, is formed of grey and white matter, but their relations are different. In the olfactory lobes, cerebral hemispheres, and 'tween-brain the white matter is internal, and the grey forms a thin outer layer or cortex. In the optic lobes and medulla the grey matter is mainly around the ventricles, and the white matter more external.

Like the spinal cord, the whole brain is covered with pia mater, densely pigmented in the region of the optic lobes, and the cranial cavity in which it is contained is lined with dura mater.

The Spinal Nerves. - The spinal nerves arise symmetrically from the spinal cord on the two sides of the body, and pass out at the neural canal through the intervertebral foramina (p. 38).

There are altogether ten pairs of spinal nerves in the adult frog (Fig. $5^{\mathrm{I}}, I-X$ ), each of which on leaving the neural canal divides into a smaller dorsal and a larger ventral branch (Fig. 53, I $s p-5 s p$ ). The first pair leave the cord through the intervertebral foramina between the first and second vertebra. They pass at first directly outwards, the large ventral branch of each, known as the hypoglossal, turning forwards, and going to the muscles of the tongue (Fig. 5 I, $I$, Fig. 53, I $S p$ ).

The second pair (Fig. $5 \mathrm{I}, I I$ ) are very large ; they emerge 
between the second and third vertebræ, and each is soon joined by the small third nerve (III) which emerges between the third and fourth vertebræ, as well as by a small branch or two from the first, thus forming a simple network or plexus -the brachial plexus (br. $p l$ ), from which pass off nerves to the fore-limb, supplying both skin and muscles.

The fourth, fifth, and sixth nerves take a very similar course. The fourth (IV) emerges between the fourth and fifth vertebra, the fifth ( $V$ ) between the fifth and sixth, and the sixth (VI) between the sixth and seventh. They all pass obliquely backwards, and supply the walls of the body, being distributed to both skin and muscles.

The seventh, eighth, and ninth nerves supply the muscles and skin of the hind limbs. The seventh (VII) leaves the neural canal between the seventh

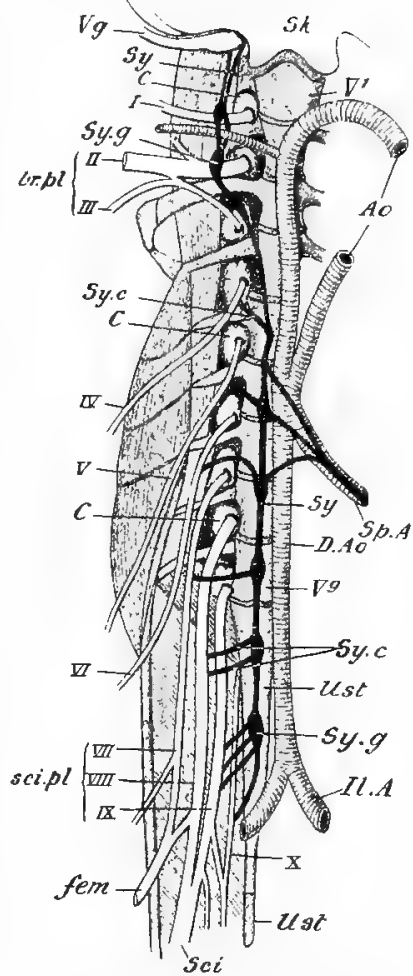

FIG. 5I,-The ventral branches of the spinal nerves and the sympathetic of the frog, from below: shown on the right side only,

$I-X$, spinal nerves; $A$ o. systemic arch; $b \%$. pl. brachial plexus; $C$. calcareous bodies which surround the spinal ganglia; D. Ao. dorsal aorta; fem. femoral nerve;,$I l$. $A$. iliac artery; sci. sciatic nerve; sci. pl. sciatic plexus; $S k$. skull; $S p$. A. splanchnic artery; $S y$. sympathetic cord; $S y$. c. commun.cating branches between the sympathetic and spinal nerves; $S_{y} . g$. sympathetic ganglia; Ust. urostyle ; $V .-V^{2}$. centra of vertebra; $\mathrm{Vg}$. vagus nerve, with its ganglion. (After Gaupp, slightly modified.) 
and eighth vertebra, the eighth (VIII) between the eighth and ninth, and the ninth $(I X)$ between the ninth vertebra and the urostyle. They all pass almost directly backwards, and are united with one another by oblique cross branches so as to form the sciatic plexus (sci.pl), from which are given off, amongst others, two nerves to the leg, the largest of which, the sciatic nerve ( $S c i$ ) being that already mentioned in the chapter on the muscular system.

The tenth $(X)$ is a very small nerve. It emerges through a small aperture in the side of the urostyle, and supplies the cloaca, urinary bladder, and adjacent parts. It is connected by cross branches with the ninth.

It will be noticed that while the large ventral part of the first spinal nerve-the hypoglossal-supplies muscles only, and is therefore a motor nerve, all the others go to both muscles and skin, and are therefore both motor and sensory, or mixed nerves. They all branch out in a complex manner, and are traceable to the remotest parts of the body.

The Sympathetic Nerves.-On either side of the dorsal aorta is a very delicate nerve, having at intervals little swelllings called ganglia, each of which is connected with a spinal nerve by a communicating branch (Figs. 5 I and $53 . S y, S y . g$, $S y . c)$. In front of the point where the dorsal aorta $(D . A o)$ is formed by the union of the two systemic trunks $(A o)$, these two sympathetic nerves, as they are called, are continued forward, one on either side of the vertebral column, towards the head, when they enter the skull and become connected with certain of the cerebral nerves.

Each sympathetic nerve has altogether nine or ten ganglia, each connected with one of the spinal nerves, and from the ganglia branches are given off which supply the heart and blood-vessels, the stomach, liver, kidneys, reproductive organs, rectum, and urinary bladder. 
Origin of the Spinal Nerves. - The mode of origin of the nerves from the spinal cord is peculiar and characteristic. Traced towards the cord, each nerve is found, on reaching the intervertebral foramen from which it emerges, to divide into two-a dorsal root which springs from the dorsal, and a ventral root which arises from the ventral region of the cord (Fig. 52, d.r, v.r). The dorsal root is distinguished from the ventral by being dilated into a ganglion $(g n)$. In Fig. 5 I these ganglia lie hidden within certain calcareous bodies $(C)$ in this region.

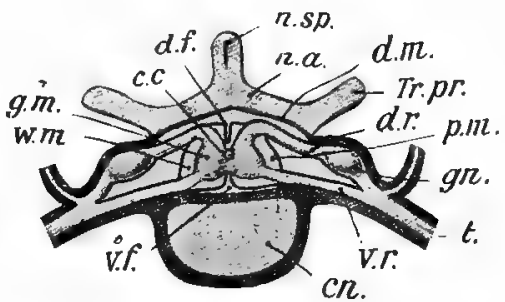

Fig, 52,-Transverse section through the vertebral column and spinal cord, to show the mode of origin of the spinal nerves.

$c . c$. central canal ; cth. centrum; $d$.f. dorsal fissure; $d . m$. dura mater ; $d . r$. dorsal root; $g . m$. grey matter; $g n$. ganglion of dorsal root; $n_{\text {. }} \alpha$. neural arch; $n . s p$. neural spine; $p .7 t$. pia mater (the reference line should stop at the margin of the cord); $t$. nerve trunk; Tr. pr. transverse process; $v_{0} f$. ventral fissure; v.r. ventral root; $w$. $m$. white matter. (After Howes.)

Cerebral Nerves. - There are ten pairs of cerebral nerves some of which are purely sensory, some purely motor, some mixed.

The first or olfactory nerves (Fig. $49, I$ ) arise from the olfactory lobes, and pass through the holes in the transverse partition of the girdle-bone. Each is distributed to the mucous membrane of the nasal sac or organ of smell of the same side, and is purely sensory.

The second or optic (Fig. $49 I I$ ) is a large nerve which springs from the ventral surface of the 'tween-brain. At 
their origin the right and left optic nerves have their fibres intermingled, forming a structure something like a St. Andrew's Cross and called the optic chiasma (opt. ch), the other limbs of the cross passing upwards and backwards to the optic lobes. The optic nerve makes its exit from the brain-case through the optic foramen, and is distributed to the retina, a delicate membrane which lines the eyeball, and is, as we shall see, the actual organ of sight. This nerve also is purely sensory.

The third or oculomotor (Fig. 49, III) is a small nerve arising from the crura cerebri beneath the optic lobes. It passes through a small hole in the cartilaginous side of the skull and supplies four out of the six muscles by which the eyeball is moved, and is purely motor.

The fourth or pathetic $(I V)$ is a very small nerve leaving the dorsal surface of the brain between the optic lobes and the cerebellum, and making its exit from the skull just above the optic nerve. It is also purely motor, supplying one of the muscles of the eye-the superior oblique.

The fifth or trigeminal (Figs. 49 and $53, V$ ) is a large nerve arising from the side of the medulla oblongata. Its root dilates to form a large ganglion, the Gasserian ganglion, and leaves the skull by the large aperture noticed in the pro-otic bone. It owes its name to the fact that it soon divides into three main branches, one, the ophthalmic (Fig. $\left.53, V^{1}\right)$, going to the skin of the snout, another, the maxillary $\left(V^{2}\right)$ to the upper lip and lower eyelid, and the third or mandibular $\left(V^{3}\right)$ to the muscles and skin of the lower jaw. The trigeminal is a mixed nerve.

The sixth or abducent (Fig. 49, VI) is a very small motor nerve arising from the ventral aspect of the bulb and supplying one of the muscles of the eyeball called the posterior rectus. 
The seventh or facial nerve (Figs. 49 and $53, V I I$ ) arises just behind the fifth and soon joins the Gasserian ganglion. Both it and the sixth leave the skull by the same aperture

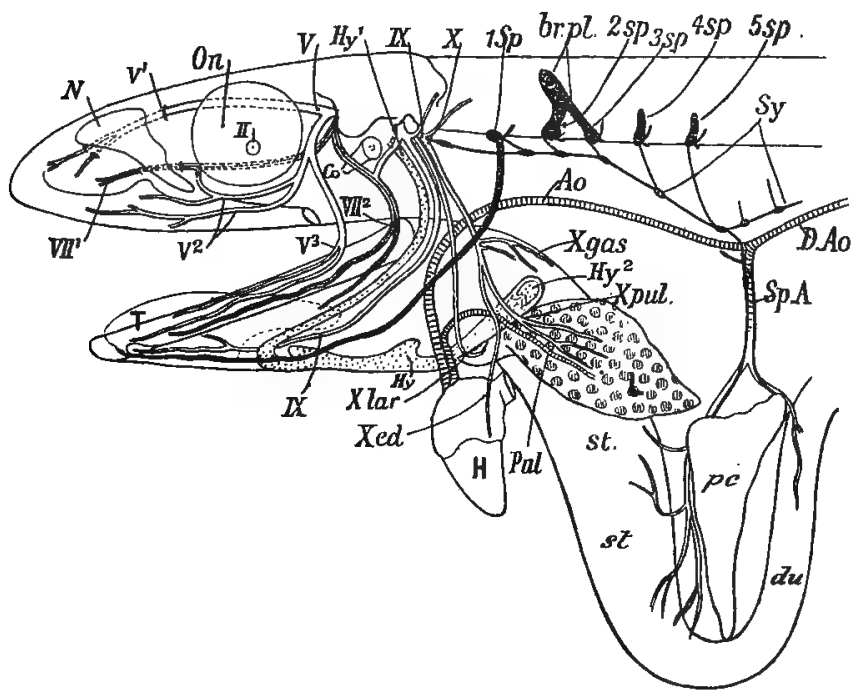

FIG. 53.-Dissection of the head and anterior part of the body of the frog from the left side, to show the distribution of the fifth, seventh, ninth and tenth cerebral nerves, as well as of the hypoglossal and part of the sympathetic.

Ao. systemic arch ; $b r$. pl. brachial plexus; $D . A o$. dorsal aorta ; clu. duodenum; $H$. heart ; $H y$. body of hyoid ; $H y y^{1}$. anterior, and $H y^{2}$. posterior horns of hyoid ; $L$. lung ; $N$. olfactory sac ; On. orbit ; $P u l$. pulmonary artery; $S p$. $A$. splanchnic artery ; $S t$. stomach; $S y$. sympathetic; $I T$. cut end of optic nerve; $V 1$. ophthalmic, $V^{2}$. maxillary, and $V^{3}$. mandibular branch of trigeminal ; $V I I^{1}$. palatine, and $V I I^{2}$. hyomandibular branch of facial ; $I X$. glossopharyngeal ; $X$. vagus ; $X a l$. cardiac, Xgas. gastric, Xlar. laryngeal, and $X p u l$. pulmonary branch of vagus ; $I s p$. first spinal nerve (hypoglossal); $2 s p .-5 s p$. second to fifth spinal nerves. (After Howes, slightly modified).

as the fifth. It divides into two branches, one of which, the palatine (Fig. 53,VII ${ }^{\mathrm{j}}$ ) supplies the mucous membrane of the roof of the mouth, and the other, or hyomandibular $\left(V I I^{2}\right)$ sends a branch to the skin and muscles of the 
lower jaw and to the muscles of the hyoid. It is a mixed nerve.

The eighth or auditory nerve (Figs. Io and 49, VIII) arises from the medulla just behind the seventh, passes through an aperture in the inner wall of the auditory capsule, and is distributed to the auditory organ or membranous labyrinth (see Chapter XI). It is the nerve of hearing, and is purely sensory.

The ninth or glossopharyngeal (Figs. 49 and $53, I X$ ) arises behind the auditory nerve. It sends a branch to join the facial and supplies the mucous membrane of the tongue and pharynx as well as certain small muscles connected with the hyoid. It is also a mixed nerve.

The tenth or vaigus (Figs. 49 and $53, X$ ) is a large nerve arising in common with the ninth, and dilating, shortly after leaving the skull, into a vagus ganglion. It supplies the larynx $(X l a r)$, the heart $(X c d)$, the lungs $(X p u l)$, and the stomach ( $X g a s)$, and is therefore often known as the pneumogastric. It has thus an extraordinarily wide distribution, being in fact the only cerebral nerve which supplies parts beyond the head. It is a mixed nerve, and contains many motor fibres, but its branches-some of which have to do with the regulation of the heart's contraction and with respirationare better described as efferent and afferent than as motor and sensory: the meaning of these terms will be explained later on. The ninth and tenth nerves leave the skull close together through the aperture noticed in the exoccipital bone.

The sympathetic nerve $(S y)$ extends forwards from its junction with the first spinal nerve, joins the vagus, and finally ends anteriorly in the Gasserian ganglion.

Microscopic Structure of Nervous Tissue.-Examination of a piece of nerve under the microscope shows it to be 
composed, like striped muscle, of cylindrical fibres, bound together by connective tissue. The latter is much more abundant than in muscle, and in particular forms a thick sheath round the nerve which must be torn off before the nerve-fibres are reached.

Each fibre (Fig. 54, A) is a cylindrical cord in which three parts can be distinguished. Running along the axis of the fibre is a delicate protoplasmic strand, the neuraxis or axis-fibre $(n x)$. Around this is a sheath formed of a fatty substance and known as the medullary sheath $(m . s) ;{ }^{1}$ and finally, investing the whole fibre is a delicate, structureless membrane, the neurilemma (ne). At intervals the medullary sheath is absent, and a node is produced, where the fibre consists simply of the neuraxis covered by the neurilemma. Directly beneath the neurilemma nuclei are found at intervals.

In the ganglia are found, not only nerve-fibres, but nerve-cells (Fig. 54): these are cells of a relatively large size, each with a large nucleus and nucleolus. In the spinal ganglia (B) the cell-body is produced into two processes, which may be united at their base. One of these processes is continuous with the neuraxis of a nerve-fibre; the other is also a protoplasmic process which passes into the spinal cord and sends off branches, each branch finally ending in a complicated branch-work or arborisation, which is interlaced, but not actually continuous with, a similar arborisation arising from a nerve-cell in the spinal cord or brain (Fig. 55).

The white matter of the brain and spinal cord consists of nerve-fibres, those in the cord having a longitudinal direction; the grey matter contains numerous much-branched (" multi-

${ }^{1}$ The medullary sheath may be absent in certain nerve-fibres (e.g., in the sympathetic and olfactory nerves.) 
polar ") nerve-cells (Figs. 48 and 54, A), each continuous with a neuraxis and enclosed in a tissue formed partly

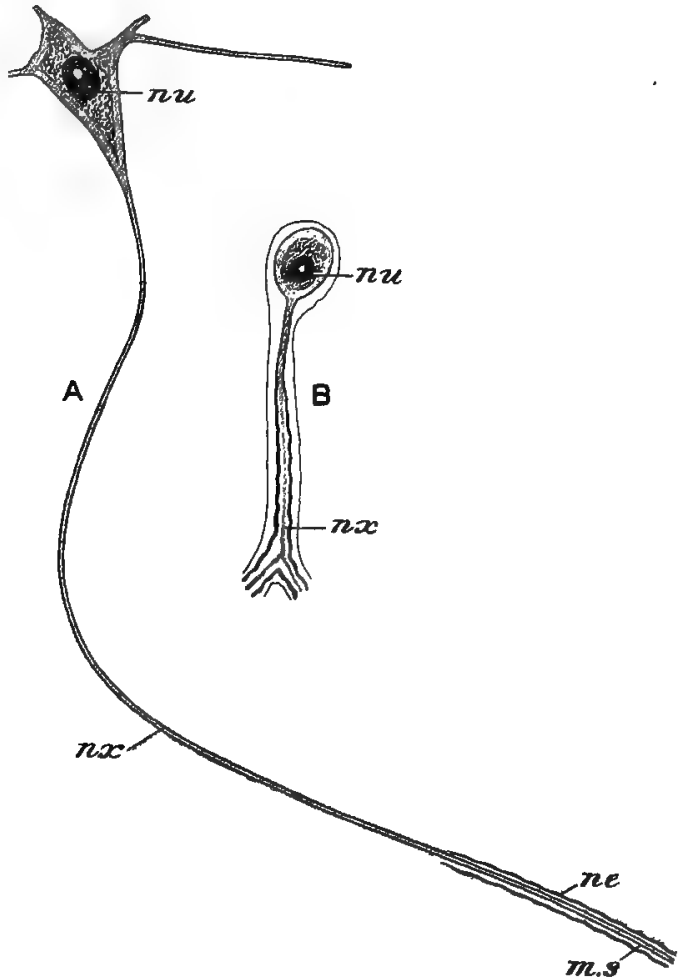

FIG. 54--A, nerve-cell from the grey matter of the spinal cord of a frog, and the nerve-fibre arising from it ; $\mathfrak{b}$, cell from the ganglion of a dorsal root. $n e$. neurilemma; $n u$. nucleus; $n x$. neuraxis; $m$. s. medullary sheath. (After Howes.)

of the axis-fibres of nerves which enter the grey from the white matter, losing their medullary sheath as they do so, 
and partly of a delicate fibro-cellular tissue called neuroglia, in which the other elements are imbedded.

Functions of the Nervous System: Reflex Action.-In the fourth chapter you learned that a muscle may be made to contract by a stimulus applied either to the muscle itself or to its nerve. You are now in a position to pursue the subject of the control of various parts of the body by the nervous system a little further.

A frog is first chloroformed, and then either decapitated or pithed, i.e., the medulla oblongata is severed and the brain destroyed (p. 103): there can thus be no question either of sensation or of voluntary action on the frog's part. It is then hung up by a hook or string, so that the legs are allowed to hang freely. If one of the toes is pinched with the forceps, the foot will be drawn up as if to avoid the pinch; or, if some very weak acid be applied to a toe, the foot will again be withdrawn, being raised every time it is touched with the acid with the regularity of a machine. Again, if acid be applied to varıous parts of the body, the foot of the same side will immediately try to rub off the irritating substance, or if that foot be held down, the other will come into play.

Movements of this kind are called refiex actions: the stimulus applied to the skin is transmitted by sensory nervefibres to the spinal cord, where it is, as it were, reflected in another form, and passed along motor fibres to one or more muscles, causing them to contract (p. 60).

As already stated, the spinal nerve-trunks are mixed, i.e., contain both sensory and motor fibres. It has been found by numerous experiments that as the nerve approaches the spinal cord these two sets of fibres separate from one another, the sensory passing into the cord by the dorsal root, the motor by the ventral root. As a consequence of 
this, if the dorsal root be cut and its proximal or central end-i.e., the end in connection with the cord--stimulated, muscular contraction will follow just as if the stimulus had been applied to the skin supplied by the nerve in question. If the other cut end-the distal or peripheral end-be stimulated, there is no result. On the other hand, if the ventral root be cut and its distal end stimulated, the

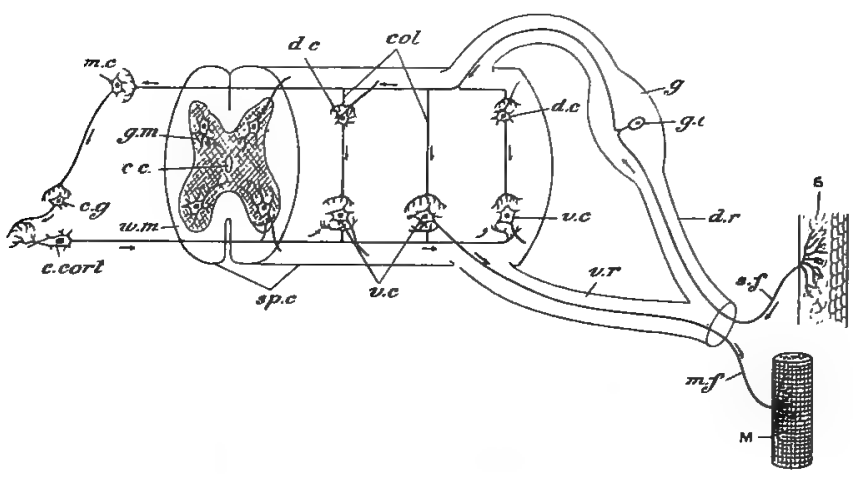

FIG. 55.-Diagram illustrating the paths taken by the nervous impulses.

c.c. central canal; col. collaterals; c. cort. cell in rind or cortex of the cerebral hemisphere; c. g. smaller cerebral cell; d.c. cells in dorsal horn of grey matter; $d .1$. dorsal root; $g$. ganglion of dorsal root; $g . c$. cell in ganglion of dorsal root; $g$. m. grey matter; M. muscle; $m$. $c$. cell in medulla oblongata; $m$. $f$. motor fibre; S. skin; s.f. sensory fibre; sp.c. spinal cord; $v$.c. cells in ventral horn of grey matter; $v$. $r$. ventral root; $w$. m. white matter. The arrows indicate the direction of the impulses.

muscles supplied by it will contract, while stimulation of the proximal end produces no result.

Very accurate observations have shown that the connection between the motor and sensory fibres is as follows (Fig. 55). A motor fibre $(m . f)$ is traceable from the nerve-trunk through the ventral root (v.r) into the white matter; and then, its medullary sheath being lost, passes into the ventral horn of the grey matter, its neuraxis being directly con- 
tinuous with the axis-fibre process of one of the large motor nerve cells $(v . c)$ : the remaining processes of these cells simply branch out in the neuroglia. The sensory fibres $(s . f)$ are traceable into the dorsal root $(d . r)$; in passing through the ganglion of the root $(g)$ they are found to be continuous with its simple ("bipolar") nerve-cells (g.c), and then pass into the cord. Instead, however, of entering the grey matter at once, they pass forwards as well as backwards for some distance, along the white matter of the cord, giving off numerous branches, or collaterals (col), which, losing their medullary sheaths, enter the dorsal horn of the grey matter and branch out into a complex series of fine fibres which interlace with the similar arborisations of the nerve-cells (p. I67).

The path of a nervous impulse will now be obvious. The stimulus applied to the skin (Fig. 55, S) is conducted by a sensory fibre to the nerve-trunk and by the dorsal root to the spinal cord; it then passes along the white matter of the cord, enters the grey matter, and is conducted by the collaterals to the nerve-cells of the ventral horn, either directly, or after passing through the cells of the dorsal horn: from one of the cells of the ventral horn it is conducted by an axis-fibre process continuous with the neuraxis of a nerve-fibre, which, leaving the cord by a ventral root, passes along the nerve-trunk and finally goes to a muscle (M) as a motor fibre.

It will be noticed that a single stimulus applied to the skin may result in the contraction of numerous muscles-as, e.g., when the application of a drop of acid to the toe causes the lifting of the leg, and that the movements are of such a nature as to withdraw the part stimulated from the irritating substance. Moreover, as shown by the experiment of applying acid to various parts of the body, the movements are 
varied according to circumstances; if one leg is prevented from rubbing off the irritating substance, the other immediately comes into play. Obviously, then, a simple stimulus reaching the spinal cord may be transmitted to numerous motor cells of the ventral horn, and through these to numerous motor nerves the particular nerve affected differing according to circumstances (compare Fig. 55). The spinal cord, therefore, is able, in response to a stimulus reaching it by a sensory nerve, to originate motor impulses causing complex muscular movements so adjusted as to serve definite purposes. Without such external stimulus, however, the spinal cord of a brainless frog is quite inactive, and the body of the animal will remain without movement until it dries up or decomposes.

In the uninjured frog, i.e., the frog with its brain intact, the case is very different. The animal no longer acts like an unintelligent machine, each stimulus producing certain inevitable movements and no others; but a single stimulus may produce varied movements, the nature and direction of which cannot be predicted; the animal will probably give a series of leaps, but the number and extent of these varies according to circumstances.

This is explained by the fact that certain nerve-fibres of the cord pass forwards to the brain, and that the nervecells in the grey matter of the cord are in communicationowing to the interlacing of their branching processes with those of the collaterals-with similar cells in the grey matter of the brain (Fig. 55, m.c, c.g, c.cort). In certain of these brain-cells (c.cort), voluntary impulses originate and exercise a controlling effect upon the cells of the spinal cord, so that these latter do not constitute, as in the brainless frog, a machine every movement of which can be accurately predicted. 
Moreover, it can be shown by experiment that the process of originating voluntary impulses is not performed by the whole brain, but is confined to the cerebral hemispheres. If the hemispheres and optic lobes are removed so as to leave nothing but the bulb and cerebellum, the frog no longer lies in any position in which it may be placed, exhibiting no movements beyond the beating of the heart, as is the case" when the whole brain is removed. It sits up in the ordinary attitude, breathes, swallows food placed in the mouth-while making no attempt to feed itself, turns over and sits up if placed on its back, and swims if placed in water. . If left alone, however, it remains in the sitting posture until it dies. Hence the bulb and cerebellum are evidently concerned with the co-ordination of muscular movements, but have no power of originating impulses. If the optic lobes as well as the medulla oblongata and cerebellum are left, the animal is affected by light, is able to perform complex balancing movements, and will even croak when stroked in a particular way. There is still, however, no voluntary action; without the application of stimuli, the animal sits motionless until it dies.

To sum up in the language of the illustration with which this chapter was begun, comparing the frog with an engine of human construction:-the grey matter of the brain may be compared with the engineer; much of the work of the engine may go on without him-certain levers, valves, \&c., acting automatically; but it is only by his controlling intelligence that the whole mechanism is adapted to the circumstances of the moment.

So far, we have considered the nervous system only in its relations to the skin or general surface of the body and to the muscles or organs of movement. The other parts of the body are, however, under nervous control. 
It has been mentioned that the heart continues to beat in a frog when the brain has been removed: not only so, but it pulsates with perfect regularity when removed from the body. This is due to the fact that the muscles of the heart, like the cilia of ciliated epithelium, have the power of contracting rhythmically quite independently of the nervous system, although the heart contains nerve-cells which were formerly supposed to serve as a special nervous system for this organ, originating all its motor impulses. It is, however, under the control of the central nervous system. We have seen that it is supplied by a branch of the vagus; when this is stimulated, the heart stops in the dilated state and begin to beat again only after a certain interval. A feebler stimulus to the vagus will not actually stop the heart, but will diminish the rate and the strength of its contractions and consequently the amount of blood propelled through the body. The vagus is accompanied by a branch of the sympathetic which has an exactly opposite effect; i.e., stimulation of it accelerates the heart's action. In this way, the general blood supply of the body is regulated by the central nervous system.

The blood supply of the various parts and organs is regulated by the vaso-motor nerves. These are traceable through the sympathetic into the spinal cord by the ventral roots : distally they send branches to the muscular coat of the arteries. Under ordinary circumstances, a constant succession of gentle stimuli pass along these from a group of nerve-cells in the medulla oblongata, and as a result, the arteries are ordinarily in a state of slight contraction. By various circumstances these stimuli may be diminished for any given artery and at the same time stimuli pass down another kind of vaso-motor fibres: the artery will then dilate and the blood supply of the organ to which it is distributed will 
be temporarily increased. For instance, the presence of food in the stomach acts, through the central nervous system, upon the coeliac branch of the splanchnic artery, causing a dilatation of all its capillaries and promoting an increased secretion of gastric juice. The secretion of other glands is regulated in the same way. In some cases, however, it has been proved that the nerves of a gland do not act simply by producing dilatation of the capillaries, but have a direct effect upon the gland-cells, causing an increased secretion.

You will thus note that there are nerve-fibres carrying impulses to the central nervous system which have nothing to do with sensation, and fibres carrying impulses from the central nervous system which have nothing to do with motion, but result in increased secretion or in stoppage of motion. It is therefore best to use the term afferent (which includes sensory) for a nerve carrying an impulse to the brain and spinal cord, and efferent (including motor) for one carrying an impulse in the other direction.

\section{PRACTICAL DIRECTIONS.}

I. The central nervous system (Fig. 6). Lay bare the brain and spinal cord as directed on p. 33, noting the dura mater and pia mater: the latter is densely pigmented over parts of the brain. The specimen in which this operation has already been performed will do, if the dissection has been done carefully.

Observe the origins of the cerebral and spinal nerves, noting the long dorsal and ventral roots of the latter (compare Fig. 52) which pass backwards for some distance before making their exit from the neural canal; and also the ganglia on the dorsal roots, lying just outside the canal and each hidden in a whitish calcareous body in this region (Fig. 5I, C). (The ganglia, however, can be more easily made out at a 
later stage). Then sever the nerves very carefully from the brain and spinal cord and remove the whole central nervous system from the neural canal : it is best examined after hardening in formaline or spirit. Lay it in a dissecting dish, under water, and make out its several parts as follows :-

a. The spinal cord.

I. Note its cylindrical form, the brachial and sciatic swellings, the flum terminale, and the dorsal and ventral fissures.

2. Examine a transverse section of the spinal cord, prepared as described on p. I36, under the low power of the microscope, and make out the dorsal and ventral fissures, the central canal, and the relations of the grey and white matter (Figs. 48 and 52). Sketch.

b. The brain (Fig. 49).

Beginning from the posterior end of the brain, where it passes into the spinal cord, make out its several divisions as follows :-

I. The bulb or medulla oblongata, with the posterior choroid plexus on its dorsal side : remove the latter, and notice that it roofs over the cavity of the fourth ventricle.

2. The small ledge-like cerebellum.

3. The two rounded optic lobes, and the crura cerebri beneath them.

4. The diencephalon, formed of a right and left optic thalamus. On its dorsal side is the anterior choroid plexus, roofing in the third ventricle; and on its ventral side the infundibulum, to which the bituitary body is attached; and, more anteriorly, the optic chiasma.

5. The cerebral hemispheres, continuous in front, with

6. The olfactory lobes, which are fused together in the middle line.

Sketch the whole central nervous system from above, and also the brain from below and from the side.

With the small scissors or a sharp scalpel, snip off a small piece of the wall of the hemisphere and optic lobe of one side-say the left, so as to expose the lateral ventricle and the optic ventricle (Fig. 50). Then with a sharp scalpel divide the whole brain into two by a longitudinal vertical cut very slightly to the left of the middle line, so as to reduce it to a longitudinal section (Fig. 49, D). Examine the cut surface of the right side under water, and make out as much as possible of the relations of the ventricles of the brain :-viz., the fourth ventricle, the iter and optic ventricle, and the third ventricle, which communicates with the lateral ventricle through the foramen of Monro. Sketch. 


\section{The Peripheral Nervous System.}

\section{a. The spinal nerves.}

Fasten out a frog with the ventral side uppermost, and remove the heart, enteric canal, reproductive organs, kidneys, and lungs with great care, leaving behind the systemic trunk and dorsal aorta (Fig. 5I). (One of the specimens already dissected will probably serve the purpose if the previous directions have been accurately followed.) Note the spinal nerves passing outwards from the vertebral column on either side, and the calcareous bodies close to their points of exit, covering up the ganglia of the dorsal roots (p. I63). If the centra of the vertebræ are removed, the nerve-roots and their origins from the spinal cord can be made out: the removal of the centra is rendered easier if the frog is first decalcified by being placed in to per cent. nitric acid for twenty-four hours and then thoroughly washed in running water.

Confine your attention to the large ventral branches of the ten pairs of spinal nerves, as described on p. 446.

b. The sympathetic nerves (Figs. 51 and 53.)

Examine the systemic trunk and dorsal aorta carefully with a lens. Closely connected with it will be seen on either side a sympathetic nervecord, covered by pigmented connective-tissue. Carefully dissect the cord away from the aorta, and note the ganglia and the branches (rani communicanies) connecting them with the spinal nerves. Sketch the spinal nerves and sympathetic.

c. The cerebral ner ves (Fig. 53.)

The dissection of these in the frog is not an easy task for a beginner, and directions for their examination in larger animals will be given in subsequent chapters. The origin of some of them from the brain, and the apertures through which certain of them pass out from the skull, have already been seen.

\section{The Microscopic Structure of Nervous Tissue.}

a. Examine your transverse section of the spinal cord (Fig. 48) under the high power of the microscope, and observe-

I. The nerve-cells, present in the grey matter only (compare Fig. 54, A). Note their branched form and their nuclei ; the larger motor cells are seen in the ventral horns of the grey matter. Sketch.

2. The nerve-fibres, in both grey and white matter, cut across transversely as well as in other directions, and each showing a deeply. stained central neuraxis. Sketch. 
b. Tease up a fresh spinal (Fig. 54, B) or sympathetic ganglion in saltsolution, and stain with methyl-green. Compare the form of the nervecells with those in the spinal cord. Sketch.

c. Cut off a very small piece of any fresh nerve (e.g., sciatic), and tease it out carefully, in a longitudinal direction, in salt solution. Note that it is made up of cylindrical, unbranched nerve-fibres, bound together by connective tissue.

Examine a single fibre under the high power (Fig. 54, A), and make out the neurilemma, the medullary sheath, and the nodes: at the nodes, the neuraxis can also be seen. Sketch.

Tease out another piece of fresh nerve in chloroform, so as to partially dissolve the medullary sheath, and note the central neuraxis. Sketch.

Tease out in glycerine a piece of nerve which has been treated with a I per cent. solution of osmic acid in water for an hour or two and then well washed in water. The medullary sheath will appcar nearly black, and the neurilemma, with its underlying nuclei, as well as the nodes, can be plainly seen. Sketch.

Reflex Action. The experiment described on p. 169 should be seen. 


\section{CHAPTER XI}

THE FROG (continued): THE ORGANS OF SPECIAL SENSE

IN the previous chapter you have learnt how the nervous system controls the various functions of the body and how voluntary action is absolutely dependent upon the connection of the brain, through the spinal cord, with the nerves. Obviously, in order that the power of voluntary action should be of full use to its possessor, some means of communication with the external world is not only desirable but necessary ; the frog, in order to adjust its actions to the circumstances in which it from time to time finds itself, must be able to distinguish friends from enemies, suitable from unsuitable food, darkness from light, heat from cold.

The avenues of communication between the animal and its surroundings are, as in ourselves, the senses of touch, taste, smell, sight, and hearing.

The sense of touch, including that of temperature, is lodged in the whole extent of the skin, which, as you have already learnt, is abundantly supplied with sensory nerves. Many of the nerves terminate in connection with what are known as tactile-cells--large flattened cells arranged in groups just below the epiderm and around which the ultimate fibres of the sensory nerves are distributed. Stimuli applied to the skin, either by direct touch or by the heat of the sun, 
are transmitted to the tactile-cells and thence through the sensory nerves to the brain. Notice that the stimulus is transmitted to the nerve-ends through the epithelial cells of the skin; if the skin be wounded and a stimulus applied directly to the tactile-cells or the nerves, the sensation is one, not of touch, but of pain.

The sense of taste is lodged in the mucous membrane of the mouth, especially in the tongue and in the neighbourhood of the vomerine teeth, but extending also as far back as the gullet. Certain of the epithelial cells have an elongated form and are arranged in groups known as taste-buds, to which the fibres of the ninth and palatine branch of the seventh cerebral nerves, or nerves of taste, are distributed; on the tongue, these taste-buds are situated on papille of the mucous membrane. In this case the stimulus is supplied, not by direct touch or by alteration of temperature, but by the contact of sapid or tasty substances. As before, the stimulus is applied to epithelial cells, and by them transmitted to the nerves and so to the brain, when the sensation of taste becomes manifest. Thus, just as common sensation may be abolished in any part of the body in three ways-by destruction of the skin, by cutting the sensory nerve, or by destroying the cerebral hemispheres - so the sense of taste is lost if either the mucous membrane of the mouth is injured, or if the glossopharyngeal and palatine nerves are cut, or if the cerebral hemispheres are destroyed.

The sense of smell is lodged in the nasal or olfactory sacs, which are enclosed in the olfactory capsules of the skull and separated from one another by a partition, the nasal septum. Each sac has two apertures, the external nostril, opening on the surface of the snout, and the internal nostril, opening into the mouth (p. I7). The sacs are lined by a 
delicate mucous membrane, some of the epithelial cells of which are of the ordinary columnar type, while others are extremely slender and produced into delicate processes at their free ends. With these latter the fibres of the olfactory nerve are connected, and they are distinguished as olfactory cells (Fig. 56). As the epithelial cells of the skin are affected by direct contact or by heat, so the olfactory cells are affected by the minute particles given off from odorous bodies: the contact of these particles acts as a stimulus, which, being transmitted by the olfactory nerve to the brain, gives rise to the sense of smell. This sense can be destroyed, as in the case of feeling and taste-either by destruction of the olfactory mucous membrane, or by cutting the olfactory nerves, or by destroying the brain.

The organ of sight or eye of the frog is so similar in structure to that of man, that the reader may be referred for details both of structure and of function to the text-books of Physiology, and it will only be necessary to give a brief outline here.

Each eye (compare Fig. 57) is a nearly globular organ, and when removed from the orbit and cleaned by dissecting away

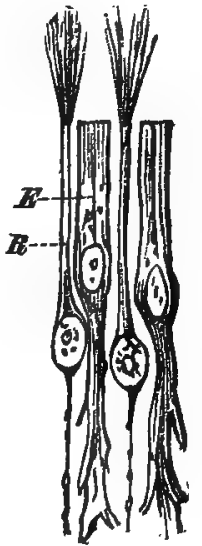

FIG. 56,-Epithelial cells of the olfac. tory nucous mem. brane of an Am. phibian.

$E$. interstitial cells ; $R$. olfactory cells. (From Wiedersheim's Vertebrata.) the attached muscles, \&c., two regions can be distinguished in it -an opaque portion of a dark bluish colour which forms some two-thirds of the entire globe and is hidden within the orbit in the entire animal ; and a clear, transparent, somewhat flattened portion which is directed outwards and freely exposed between the eyelids in the living frog. The outer 
coat of the concealed portion of the eyeball is the sclerotic $(S c t)$, and is formed of cartilage in the frog; its dark colour is due to the presence of a layer of black pigment which forms one of the internal coats; this will be referred to hereafter. Entering the sclerotic on its inner side, i.e., the side next the braincase, will be seen the cut end of the optic

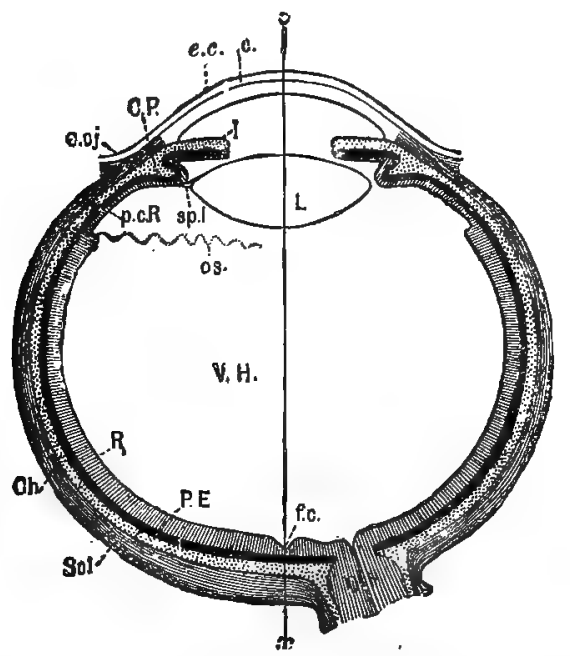

FIG. 57-Diagrammatic horizontal section of the human eye.

.. cornea ; $C h$. choroid (dotted); C.P. radiating folds of the choroid, known as the ciliary processes; $e . c, e . c j$. corjunctiva; $I$. .iris ; $L$. lens; $O . N$. optic nerve; os. ora serrata, a wavy line forming the boundary of the visual portion of the retina; $p . c . R$. anterior non-visual portion of retina; $P E$. pigmented epithelium (black); $R$. retina; $S c l$. sclerotic; $S p l$. suspensory ligament of lens; $V$. $H$. vitreous chamber. (From Foster and Shore's Physiology.)

nerve $(O . N)$. The transparent, exposed portion of the eyeball is the cornea $(c)$, a superficial thin layer of which, or conjunctiva (e.c., e.cj.), is continuous with the lining of the eyelids and thus with the skin covering the head; through it can be seen the coloured part of the eye, or iris $(I)$, with a black spot-really a hole -in its centre, the pupil. 
The interior of the globe $(V . H)$ is filled with a colourless, transparent jelly, the vitreous humour, surrounding which, everywhere but on its external face, is a thin semitransparent membrane, reddish when perfectly fresh, but becoming grey soon after death; this is the retina $(R)$. Between the retina and the sclerotic is a membrane called the choroid $(C h)$, the inner face of which, i.e., that in contact with the retina, is coloured black. It is this layer of black pigment which gives the dark tint to the semi-transparent sclerotic in the entire eye ; strictly speaking, it is the innermost layer of the retina, but actually it adheres to the choroid and appears like the innermost layer of that coat. The retina is readily detachable from the choroid, but at the place where the optic nerve enters (blind spot) it becomes continuous with the fibres of the latter, which pass through the sclerotic and choroid. The choroid is made up of connective tissue and contains numerous bloodvessels as well as pigment-cells.

Lying just internal to the pupil is a nearly globular body, perfectly transparent when fresh, the crystalline lens $(L)$; it is kept in place by a delicate membrane, the capsule of the lens. The iris, which covers the outer face of the lens except where it is perforated by the pupil, is covered on its inner surface with black pigment, and is continuous all round its outer margin with the choroid. Between the iris and the cornea is a space, the aqueous chamber of the eye, which contains a watery fluid, the aqueous humour. The main cavity of the eyeball, containing the vitreous humour, is called the vitreous chamber $(V . H)$.

The actual relations of these parts in the entire eye are best grasped in a vertical section, such as is represented in Fig. 57. The main part of the eyeball forms a chamber, enclosed by the sclerotic, darkened internally by the choroid, 
and lined by the retina. Into the outer side of this dark chamber is let a transparent window, the cornea; behind which, and separated from it by a space containing the aqueous humour, is a vertical curtain, the iris, perforated by an aperture, the pupil. Behind the iris and in close contact with it is the lens, and filling the whole of the dark chamber between the lens and iris in front, and the retina behind and at the sides, is the vitreous humour.

The whole eye thus has the structure of a camera obscura. The cornea, aqueous humour, lens, and vitreous humour are a series of lenses, so arranged that the rays of light from an external object are refracted and brought to a focus on the retina, where they form a greatly diminished and inverted image of the object. The iris is provided with muscles, by means of which the pupil can be enlarged or diminished; it therefore acts as a diaphragm and regulates the amount of light entering the eye. Attached to the capsule of the lens are delicate muscles, by means of which the lens can be made more or less convex; in this way the focus of the entire apparatus can be altered according to whether the object viewed is nearer or farther from the eye. This arrangement for accommodation is, however, much less highly developed in the frog than in man and the higher animals, in which the relatively smaller lens is flatter and distinctly biconvex in form (Fig. 57). Thus the various parts of this complicated organ are so adjusted as to bring the images of external objects to an accurate focus on the back part of the interior of the eyeball, i.e., on the retina.

A vertical section of the retina (Fig. $5^{8}$ ) reveals a very complex structure. On its inner face, i.e., the surface in contact with the vitreous humour, is a layer of nerve-fibres $(n . f$.$) , formed by the ramifications of the optic nerve, which,$ 
passing through the sclerotic and choroid, perforate the retina, and spreads out over its inner surface. Next comes a layer of nerve-cells $(g)$, and then several layers of fibres and nuclei $(g r, n c)$; and finally, forming the outer surface of the retina proper, is a layer of delicate, transparent bodies called, from their form, the rods (r) and cones $(c)$; these are known from their development to be modified epithelial cells. The whole of these structures are supported by a complex framework of connective tissue. In close contact with the outer or free ends of the rods and cones is a layer of cells the protoplasm of which is filled with a dense black pigment. It is this pigment-layer $(p . e p)$, which, as we have seen, is often counted as part of the choroid.|

In spite of its complex structure the retina is not much more than $\frac{1}{4}$ th $\mathrm{mm}$. ( $\frac{1}{100}$ th inch) thick, and is perfectly transparent. Hence, when an image is formed on it, the rays of light easily penetrate its whole thickness until they are stopped by the opaque layer of pigment. The rays are thus enabled to stimulate the rods and cones,

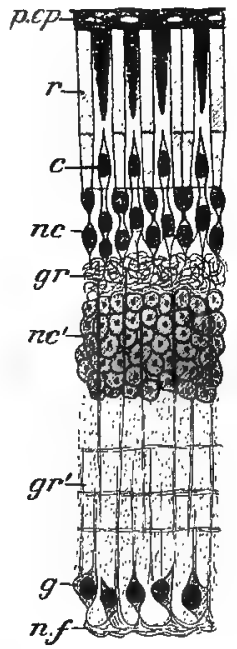

FIG. 58.-Vertical section of frog's retina.

c. cones; $g$. layer of nerve - cells ; $g r, g r^{\prime}$. outer and inner granular layers; $n c, n c$. outer and inner nuclear layers; $n$. $f$. nerve-fibre layer; $p$. ep. pigment epithelium ; $r$. rods. (After Howes.)

and the stimulus is transmitted through the layers of nuclei and nerve-cells to the fibres of the optic nerve, along which it is conveyed to the brain. Thus the actual organ of sight is not the eye as a whole, but the retina: all the rest is to be looked upon as an accessory apparatus, for focussing and for regulating the admission of light. 
As with the other sense-organs, sight may be destroyed by injury to the retina or actual organ of sight, by cutting the optic nerve, or by destroying the brain. But unlike the other sense-organs already considered, that of sight has a complex accessory or focussing apparatus in connection with it, and sight may also be rendered impossible by injury to the cornea or lens.

It is an obvious advantage to an organ of sight such as the frog's that it should be capable of movement in any direction, so as to allow the light from any object to enter the pupil. As a matter of fact, the animal can direct its gaze through a very wide range by means of eight muscles attached to the eyeball in the orbit. One of these, the levator bulbi, raises the whole eye, causing it to project further on the surface of the head. Another, the retractor bulbi, withdraws it, causing it to bulge into the mouth. Four others (compare p. 164 and Fig. I I7), the superior, inferior, anterior and posterior recti, rotate it respectively upwards, downwards, forwards, and backwards. And finally, two oblique muscles, the superior and inferior, produce a rotation along an axis joining the optic nerve with the middle of the cornea.

The conjunctiva, which covers the outer side of the eye and lines the eyelids, is kept moist by the secretion of a lachrymal gland, known as the Harderian gland, situated between the eyeball and the orbit in the antero-ventral aspect. The excess of this secretion is carried away into the olfactory chamber by means of a tube, the nasolachrymal duct.

Each organ of hearing, like that of sight, consists of an essential portion and an accessory apparatus. The essential organ of hearing is a structure called the membranous labyrinth, contained within the auditory capsule of the skull (Fig. Io), and consisting of a kind of bag of very peculiar 
and complicated form (Fig. 59). It is made up, in the first place, of two somewhat ovoid sacs separated by a constriction :" the dorsal one is called the utriculus (u), the ventral the sacculus $(s)$, and from the latter a small process, the cochlea ( $)$, projects backwards, and a narrow canal, the endolymphatic duct (d.c), upwards. With the utriculus are

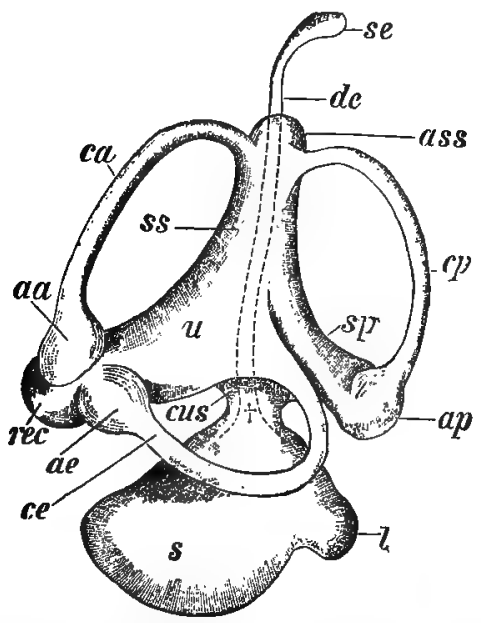

FIG. 59.-External view of left organ of hearing of a Vertebrate (semi-diagrammatic). $a \alpha$. ampulla of anterior canal, ae. of external canal, and $a p$. of posterior canal; ass. apex of utriculus; $c a$. anterior semicircular canal ; $c e$. horizontal canal ; $c p$. posterior canal ; cus. constriction between sacculus and utriculus; de, se. endolymphatic duct; 2 . cochlea; $r e c, s p, s s, u$. utriculus ; $s$. sacculus. (From Wiedersheim's Vertebrata.)

connected three tubes, called, from their form, the semicircular canals, each of which opens into the utriculus at either end. One of them, the anterior canal $(c a)$, is directed forwards; another, the posterior canal $(c p)$, backwards; both these are vertical in position and are united to one another at their adjacent ends. The third, the 
external canal $(c e)$, is directed outwards and has a horizontal position. Each canal has one end dilated into a bulb-like swelling or ampulla $(a a, a p, a e)$; those of the anterior and external canals are anterior in position, while that of the posterior canal is posterior.

The whole of this apparatus is filled with a fluid, the endolymph, in which are contained calcareous particles, the otoliths or ear-stones. It is made of connective tissue and

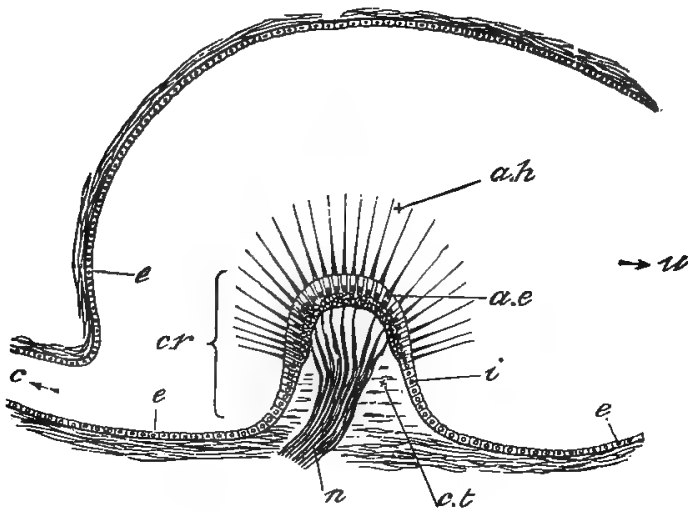

Fig. 6o.-Longitudinal section through an ampulla.

$a . e$. auditory epithelium; $a . h$. auditory hairs; $c$. part of semicircular canal; $c r$. acoustic spot and ridge; $c t$. connective tissue; $e$. epithelium; $n$. nerve; $u$. junction with utriculus. (From Foster and Shore's Physiology.)

lined with epithelium, the cells of which are cubical for the most part; but in certain places the wall is thickened, forming what are called acoustic spots, of which there is one to each ampulla, situated on a ridge (Fig. 60), while others occur in the utriculus and sacculus. On these acoustic spots the epithelial cells are greatly elongated and produced at the surface into delicate processes called auditory hairs: to these cells the fibres of the auditory nerve are distributed. 
The membranous labyrinth does not fit tightly into the cavity of the auditory capsule in which it is contained ; but a space is left between it and the surrounding bone and cartilage (Fig. Io). This space is filled by a fluid called perilymph, by which the membranous labyrinth is surrounded and protected from shocks. As you learnt in studying the skull, the outer wall of the auditory capsule is perforated by a small aperture, the fenestra ovalis (Fig. Io, fen. ov), in which is fixed the stapes (stp), a small nodule of cartilage connected with a bony rod or columella (Figs. 9 and ro, $c o l)$, the cartilaginous hammer-shaped outer end of which, or extra-columella, is fixed to the inner side of the tympanic membrane (Fig. 9, tymp. memb). The columella lies in the tympanic cavity (tymp. cav), which is bounded externally by the tympanic membrane, internally by the auditory capsule, and at the sides chiefly by muscles and connective tissue; while below it communicates with the mouth by the Eustachian tube (eus. $t$ ).

When sound-waves impinge on the tympanic membrane, the vibrations to which they give rise are transmitted by the columella to the stapes, and so to the perilymph. Thence they are communicated to the endolymph and act as stimuli to the auditory cells of the acoustic spots, and the impulses being carried to the brain by the auditory nerve, give rise to the sensation of sound. Whether or not all the acoustic spots are truly auditory in function is not known. It seems certain that the semicircular canals are really organs for the maintenance of equilibrium.

The sense of sound can be destroyed by injury to the membranous labyrinth, by cutting the auditory nerve, by destroying the brain, or-to a great extent at least-by injury to the tympanic membrane or columella.

Notice that the general plan of all the sensory organs, 
those of the skin, eye, and ear, is the same. They consist of certain peculiarly modified epithelial cells, specially sensitive to impulses of particular kinds, and in communication, by means of an afferent nerve, with nervecells of the brain. The three things-sensory cell, afferent nerve, and brain-form a chain, every link of which is necessary for the performance of the sensory function, so that the particular sense in question may be destroyed, not only by destruction of the sense-organs in the strict sense, but also by section of the afferent nerve or by destruction of the brain.

General Physiology-Summary.-Before going on to the next chapter it will be as well to take a final glance at the physiological processes of the frog as a whole (compare Fig. 23). The enteric canal is the manufactory in which the raw material of the food is worked up into a form in which it can be used by the various parts of the body. The circulatory organs are the communicating system by which the prepared food is taken to all parts ; and they also form a drainage system by which waste matters are collected from all parts and finally ejected by the three main sewers, the skin, lungs, and kidneys. The skin and lungs, besides getting rid of waste matters, serve for the supply of oxygen - a necessary form of gaseous food. The central nervous system forms a sort of headquarters staff by which the entire body is controlled, the means of communication being the nerves, and the muscles the executive by which the orders from headquarters are executed. And finally the sense-organs may be looked upon as the various branches of an intelligence department by which the headquarters are informed of what is going on outside. 


\section{PRACTICAL DIRECTIONS.}

\section{The Organs of Special Sense.}

I. Olfactory organ. Notice again the external and internal nostrils. Then remove the skin covering the snout, dissect off the nasal bones, and open up the olfactory sacs. Note the pigmented olfactory epithelium lining these, and make out the olfactory nerves and nasal septum. Sketch.

II. Eye.

u. Notice again the eyelids, iris and pupit. Then remove the skin covering the head so as to expose the nearly globular eyeballs, lying in the orbits.

In the antero-ventral angle of the orbit make out the Harderian gland, and the eye-muscles passing from the walls of the orbit to the eye-ball. The four recti and two oblique muscles can be more easily seen on a larger animal, and directions will be given for their examination in Part II.; but if you make a dissection of them in the frog, you should note at the same time the levator and rectractor bulbi, the latter underlying the eyeball, and the former situated internally to the recti muscles.

$\therefore b$. Remove the eyeball from a freshly-killed specimen, noticing as you do so the optic nerve, which is surrounded by the recti and retractor bulbi muscles: dissect away these "muscles and note the cartilaginous sclerotic, the cornea, iris, pupil, and the cut end of the optic nerve.

c. Divide the eyeball into an inner and an outer hemisphere by a rapid cut with scalpel or scissors taken vertically, midway between the cornea and the optic nerve, through the vitreous chamber. Place them both in a watch-glass or small dissecting dish, under water, and examine with a lens (compare Fig. 57). In the inner hemisphere note the vitreous humour, retina, pigmented choroid, and blind spot or entrance of the optic nerve; and in the outer hemisphere, the crystallize lens and the margin of the retina, or ora serrata. Sketch. Remove the lens, and notice the iris, continuous with the choroid, the pupil, and the aqueous chamber.

d. Examine sections through the wall of the inner hemisphere of the eyeball, prepared as directed on p. $\mathbf{3}^{6}$, first under the low, and then under the high-power of the microscope. Note:-

I. The cartilaginous sclerotic. 
2. The choroid, enclosing pigment-cells and blood-vessels.

3. The retina (Fig. 58) composed of a number of layers: notice the pigment epithelium, the rod-and cone-layer, and the various other layers of the retina, the innermost of which is composed of nerve-fibres continuous with the optic nerve.

Sketch.

The anatomy of the eye can be more easily made out by dissecting that of an ox or sheep, which is essentially similar to that of the frog, and directions for the examination of which will be given in Part II.

III. Auditory organ.

Notice again the tympanic membrane and tympanic ring, and then carefully cut away the former so as to expose the tympanic cavity. Observe the Eustachian tube, the fenestra ovalis, and the relations of the stapes, columella, and extra-columella (Fig. 10).

The essential part of the auditory organ (membranous labyrinth) is very small in the frog, and can be more satisfactorily studied in a goodsized fish (e.g. Dogfish or Cod). Directions for the preparation of the membranous labyrinth of the Dogfish will be given at a later stage, but if you have sufficient time and patience to dissect it out in the frog, proceed as follows :-

Place the head of a large frog in nitric acid (about Io per cent.) until the bone is dissolved. Wash well in water so as to remove the acid, and dissect away the muscle, etc., from the auditory capsule until the latter is thoroughly exposed. Then with a sharp scalpel slice away the roof of the capsule until the cavity it contains is exposed. Proceed now with great caution, removing the cartilage and decalcified bone, bit by bit, until the cavity is sufficiently enlarged to bring the membranous labyrinth into view (compare Figs. Io and 59.) Observe the utriculus, sacculus, ololiths, and the three semicircular canals with their ampullee. Sketch. 


\section{CHAPTER XII.}

THE FROG (continued): REPRODUCTION AND DEVELOPMENT.

So far we have considered those parts and organs of the frog which have to do with its welfare as an individual. We have now to consider the organs which are connected with the welfare of the frog as a race, that is, with the propagation of its kind.

The position of the reproductive organs has already been seen (pp. 23 and 25): they must now be examined in more detail. The essential part of these organs in each sex is a pair of bodies known as gonads, called in the male spermaries or testes, and in the female ovaries.

Reproductive Organs of the Male.-The spermaries (Fig. 3, r. spy, Fig. 5, spy, and Fig. $7, t s$ ) are a pair of ovoid bodies, each attached by a fold of the peritoneum to the corresponding kidney, and having connected with it a fat body $(c p, a d)$. From the inner margin of each spermary spring a number of delicate tubes, the efferent ducts (Fig. 6I, q) which run in the fold of the peritoneum to the kidney. Entering this organ near its inner edge, they open into a longitudinal tube $(L)$ from which transverse tubes pass horizontally across the kidney to open into the ureter $\left(U_{r}\right)$. The milt, or spermatic fluid (p. 9) is thus carried off by the same duct as the urine; the ureter is therefore often called the 
uringgenital duct. On the outer side of the ureter, and communicating with it by numerous short ducts, is a glandular body, the seminal vesicle (Figs. 3 and $7, v s .5 m$ ), which serves to store up the spermatic fluid.

The spermary itself contains a narrow, irregular, central cavity, from which the efferent ducts proceed and into which open a number of short tubes or crypts (Fig. 62, A).

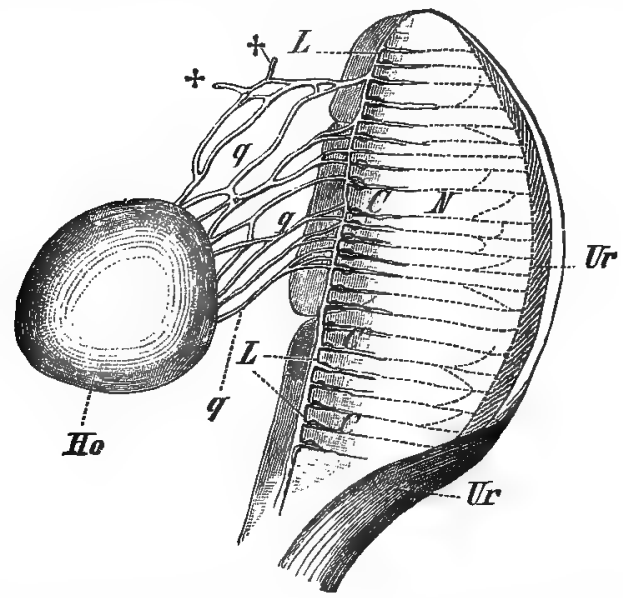

FIG. 6r.-Spermary and kidney of frcg showing the relations of the cfferent ducts (semidiagrammatic).

$C$. transverse tubes in kidney; Ho. spermary; $L$. longitudinal tube; $N$. kidney ; q. efferent ducts of spermary; Ur. ureter (urinogenital duct). (From Wiedersheim's Anatomy.)

These are lined with epithelium (t.e), the cells of which divide and subdivide, forming groups of smaller cells. Each of the latter undergoes a remarkable change, becoming converted into a rod-like body, produced into a long thread, which performs lashing movements, very much like those of the cilia in ciliated epithelium. These bodies are called sperms or spermatozoa (Fig. 62, 'A, sp, and B)r; in spite of 
their peculiar form, they are cells, the rod-like portion, or head, being the nucleus, and the delicate vibratile part, or tail, the protoplasm. In the breeding season the cavities of the testes are full of sperms floating in a fluid. Thus the spermatic fluid, like the blood, owes its distinctive character to the cells floating in it.
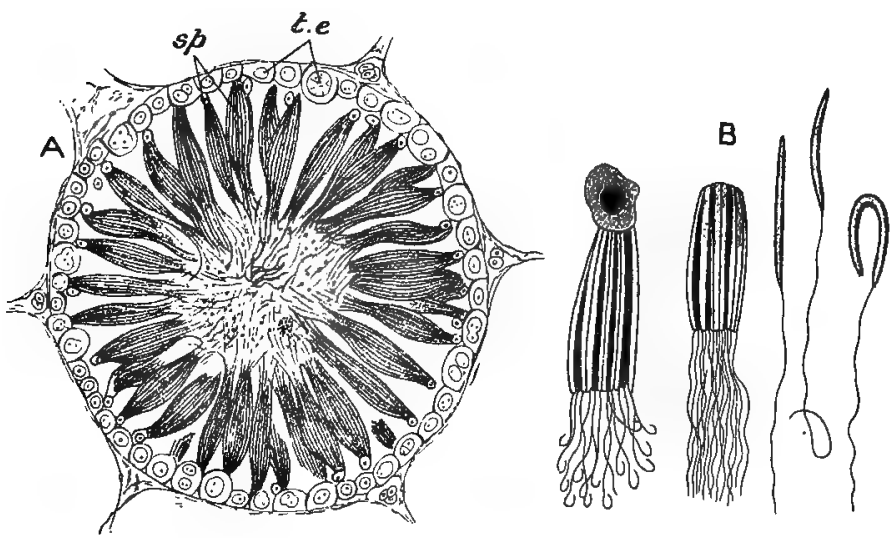

Fig. 6z.-A, transverse section of a crypt of the spermary. B, stages in the development of the sperms.

$S p$. bundles of sperms; t.e. germinal epithelium. (A, after Blomfield; B, after Howes).

Reproductive Organs of the Female-Each ovary (Fig. 4, l.ovy), is a greatly folded sac with thin walls and a large cavity, divided up by partitions. It is attached to the dorsal body-wall by a fold of peritoneum. As we have seen (p. 23), its surface is studded all over with little rounded projections, each of which is an ovisac, and contains an egg. The egg or ovum (Fig. 63) is a large globular cell with a clear nucleus $(n u)$ containing numerous nucleoli $\left(n u^{\prime}\right)$, and having its protoplasm ( $p r)$ full of yolk-granules-grains of proteid material which serve as nutriment for the growing 
embryo. It is covered with a delicate membrane, the vitelline membrane. By the time the egg is mature a superficial deposit of pigment takes place over one hemisphere.

In the young condition all the epithelial cells forming the walls of the ovary are alike, but as the organ reaches maturity, certain of them $(o)$ enlarge, and give rise to the

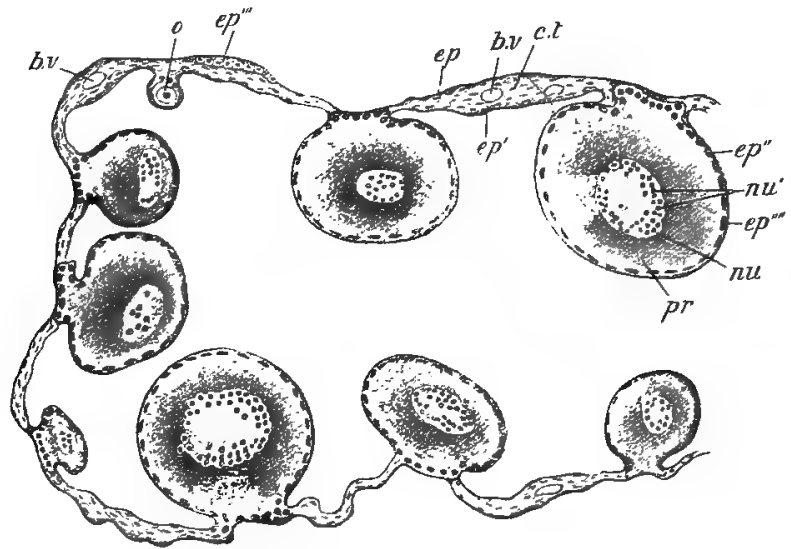

FrG. 63.-Transverse section of frog's ovary.

b. v. blood vessels; $c$. $t$. connective tissue; $e p$. outer layer, and $e \phi^{\prime}$, inner layer of epithelium; $e p^{\prime \prime}$. outer layer of ovisac, continuous with $e p^{\prime} . ; e p^{\prime \prime}$.' germinal epithelium, derived from $e p ; e p^{\prime \prime \prime}$. follicular epithelium, derived from $e p ; n u$. nucleus of ovum ; $n u^{\prime}$. nucleoli ; $o$. young ovum; pr. protoplasm of ovum containing yolk-granules. (After Marshall.)

ova, while others form an investment or follicle $\left(e p^{\prime \prime \prime}\right)$ for each ovum.

The oviduct (Fig. 4, r. ovd), as you have seen, is a long and greatly convoluted tube lying above or dorsal to the ovary. Its anterior end narrows considerably, runs parallel with the gullet, passes to the outside of the root of the lung, and then opens into the coelome by a small aperture $\left(r . o v d^{1}\right)$. The greater part of the oviduct is about as wide as the small intestine, and is thick-walled and lined 
with gland-cells, which secrete the jelly (p. 9) surrounding the eggs when laid. Posteriorly it suddenly dilates into a wide, thin-walled chamber ( $\left.r . o v d^{\prime \prime}\right)$ which opens into the dorsal wall of the cloaca. Notice that there is thus no connection between the generative organs and the kidneys in the female, such as occurs in the male, the ureters serving as renal ducts only.

In the breeding season the ovisacs burst and set free the eggs into the cœlome, whence by some means or other they find their way into the small openings of the oviducts, and so into these tubes, when each becomes surrounded by its little sphere of jelly secreted by the gland-cells. Passing down the oviducts the eggs accumulate in the dilated extremities, which they distend enormously, so that just before laying, the abdomen of a female frog is nearly filled with these two great egg-reservoirs; the ovaries, having lost so many of their eggs, are correspondingly reduced in size, and all the other organs are squeezed out of place.

Fertilization.--The eggs are now laid, and immediately the spawn is passed from the oviducts of the female into the water, the male sheds over them a quantity of spermatic fluid (p. 9). The sperms, swimming actively through the water, enter the spheres of jelly and come into contact with the eggs. A single sperm then penetrates the vitelline membrane of an ovum, loses its tail, and its head coming into contact with the nucleus of the egg, fuses or conjugates with it, so that a single nucleus is formed by the union of the egg-nucleus with the sperm-nucleus. We may speak of conjugating cells in general as gametes, the sperm in this case being the male gamete, and the ovum the female gamete, the body formed by the fusion of two gametes being known as a zygote.

This process is known as fertilization or impregnation. 
Without it, as we have seen, the egg is incapable of development; after it has taken place, the egg-or more strictly, the oosperm, since it is now an ovum plus a sperm-is potentially a young frog, since if left undisturbed in water, it will in course of time give rise to a tadpole, which in its turn will change into a frog.

It must be remembered in the first place that the fertilized egg is a single cell, comparable with a blood corpuscle or an epithelial cell. It is, however, peculiar in two respects : first in having its nucleus derived in part from a sperm, so as to contain matter from both the male and the female parent; and secondly in having its protoplasm distended with yolk-granules to such an extent that instead of being a minute body visible only under the microscope, it is easily visible by the naked eye. The yolk is not equally distributed : on one hemisphere it is less abundant than elsewhere, and it is this more protoplasmic hemisphere which is superficially blackened by a layer of pigment, and which always floats upwards in the water when the egg is laid.

Segmentation of the 0osperm.-Almost directly after being laid and fertilized, the egg undergoes a remarkable change. A furrow appears all around it, as if made with a blunt instrument, and deepening gradually, at last divides the oosperm into two hemispheres in contact with one another by their flat faces (Fig. 64, A). The examination of sections shows that this process is preceded by the division of the nucleus into two; its final result is the separation of the originally one-celled egg into two cells. Now if you refer to Chapter VIII, you will be reminded of the fact that the epithelial cells of the skin multiply by a similar process of simple fission, or division into two; the nucleus in each case dividing first and afterwards the protoplasm. 


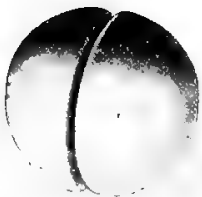

A

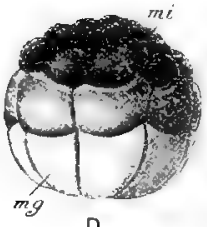

0
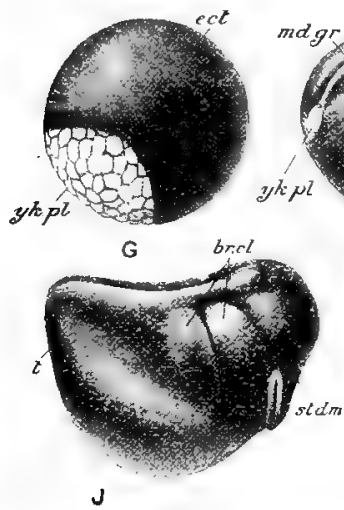

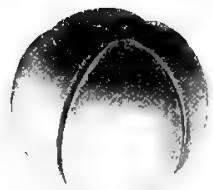

8

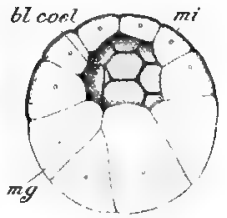

$E$

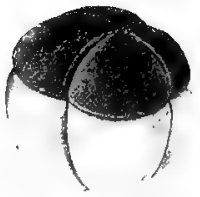

C

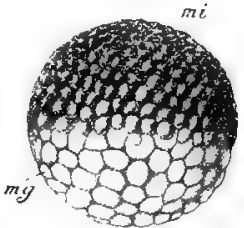

$F$
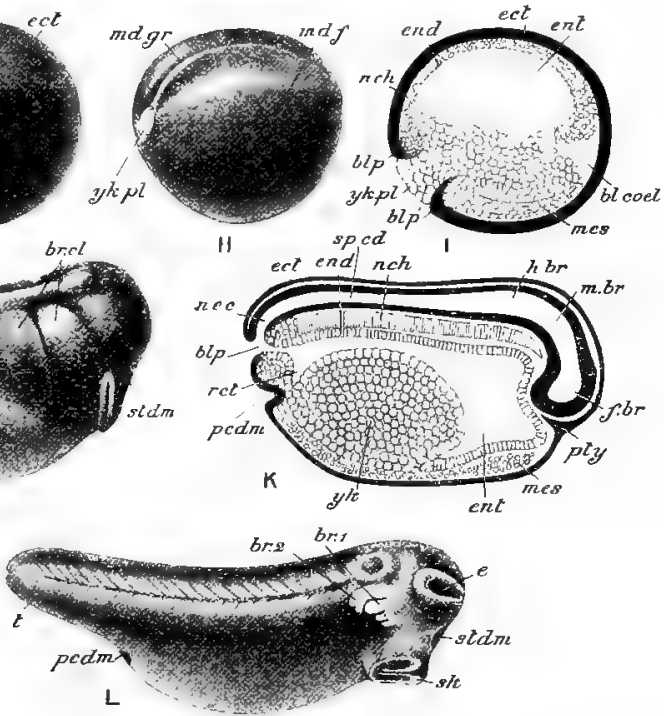

FiG. 64.-Development of the frog.

$\mathrm{A}-\mathrm{F}$, segmentation ; $\mathrm{G}$, overgrow th of ectoderm; $\mathrm{H}$, $\mathbf{I}$, establishment of germinal layers $; \mathrm{J}, \mathrm{K}$, assumption of tadpole-form and establishment of nervous system, notochord, and enteric canal ; L, newly-hatched tadpole.

bl. col. segmentation cavity; $b i p, b i p^{\prime}$. blastopore; $b r l, b r 2$. gills; $b r$. $c l$. branchial arches ; $e$. eye ; ect. ectoderm ; end. endoderm; ent. enteron; $f . b r$. fore-brain ; $h . b r$. hind-brain ; m. $b$. mid-brain; md. $f$. medullary fold; mnd.gr. medullary groove; mes. mesoderm; ng. large lower cells; mi. small upper cells; nch. notochord; $n_{\text {. }}$. c. neurenteric canal; pcdm. proctodaum; pty. invagination of ectoderm which will form the pituitary body; ret. commencement of rectum; sk. sucker; sp. cd. spinal cord; stdm. stomodzum;'t. tail; $y k_{\text {. }}$ yolk-cells; $y z-p h$. yolk-plug which fills the blastopore. $(A-D, F-H$, and $J$ rom Ziegler's models; $\mathbf{E}, \mathbf{I}, \mathrm{K}$, and $\mathrm{L}$ after Marshall.) 
The furrow which effects this division of the oosperm passes through both black and white poles, so that each of the two cells formed is half black and half white. Soon a second furrow is formed at right angles to the first, being like it, meridional, i.e., passing through the poles (Fig. 64, B). It divides what we must now call the embryo into four cells, each half black and half white. A third furrow is then formed, passing round the equator, but nearer the black than the white pole (C). It therefore divides the embryo into eight cells, four upper black and four lower white, the latter being obviously the larger.

This process of division or segmentation of the oosperm continues, the black cells dividing more rapidly than the white, so that before long the embryo consists of a mass of cells, the polyplast, somewhat resembling a mulberry, and therefore often called a morula, one hemisphere being composed of small cells containing much protoplasm and little yolk, and externally pigmented (Fig. 64, D-F, mi), and the other of larger cells containing little protoplasm and much yolk, and not pigmented $(m g)$. As segmentation goes on, a cavity appears in the interior of the embryo; it is called the segmentation cavity $(\mathrm{E}, b l$. cal), and is due to the fact that the work of segmentation produces a waste of substance, which there is at present no means of making good, so that for a time, while the size of the embryo remains the same, its bulk diminishes, some of its substance being used up; in other words, it feeds on itself.

Segmentation now proceeds to such an extent that the black cells become too small to be seen except with a lens of tolerably high magnifying power, so that with the amount of magnification used in Fig. 64, G, the black hemisphere shows no division into cells. At the same time the black hemisphere gradually encroaches on the white until only a 
small circular space-the blastopore, filled by a mass of yolkcells-the yolk-plug, is left uncovered (Fig. 64, G, H, yk. pl).

Development of the Chief Organs,-In the meantime a second cavity appears in the interior of the embryo, and as it increases in size, the segmentation cavity undergoes a proportional diminution and finally disappears. This new

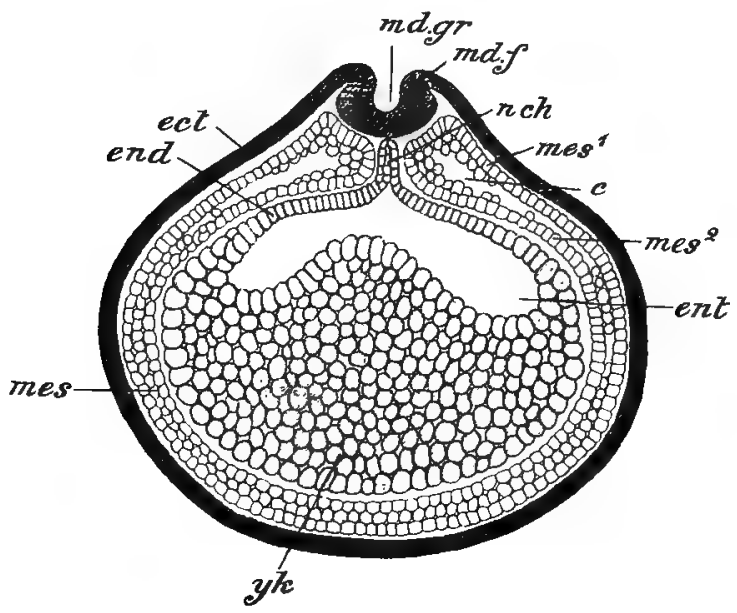

Fig. 65.-Transverse section through a frog-embryo during the formation or the medullary canal.

c. coelome ; ect. ectoderm ; cnd. endoderm ; ent. enteron; mes. mesoderm ; ntes $^{1}$. its outer (parietal) layer; mes $^{2}$. its inner (visceral) layer; md. f. medullary fold; md.gr. medullary groove; $n c h$. notochord; $y k$. yolk-cells. (After Marshall.)

cavity, or archenteron (Fig. $64, I$ and 65 , ent), is the rudiment of the enteric canal: it begins to be formed as a narrow slit at the edge of the blastopore through which it at first communicates with the exterior, but it soon becomes closed : at present there is neither mouth nor anus.

By this time the cells of which the embryo is composed have assumed different forms and become arranged in a 
very definite manner. First of all there are several layers of small pigmented cells, derived from the black cells of earlier stages, which cover the whole embryo with the exception of the yolk-plug: these constitute the ectoderm (Fig. 64, $\mathrm{G}, \mathrm{H}, \mathrm{I}, e c t)$. Forming the roof of the enteric cavity are other layers of cells derived from the yolk-cells of earlier stages and forming the endoderm $(\mathrm{I}, e n d)$ : the floor of the archenteron is at present formed of unaltered yolk-cells. Between the ectoderm and the yolk-cells on the ventral aspect are several layers of small cells which gradually spread until they form a complete layer between the ectoderm and the endoderm: these constitute the mesoderm (I, mes). The ectoderm, endoderm, and mesoderm are known as the three embryonic tissues or germinal layers.

While these processes have been proceeding, a longitudinal groove appears on the surface of the embryo: this is the medullary or neural groove, and is bounded by a pair of medullary folds (Figs. $64 \mathrm{H}$ and 65 , md.gr, md.f). The medullary groove marks the dorsal surface of the embryo and extends backwards to the blastopore, which now marks its posterior end. As development goes on, the folds approach one another and unite, so as to convert the groove into a canal: the cells lining this canal give rise to the central nervous system (Fig. $64 \mathrm{~K}, f . b r, m . b r$., h.br, $s p . c d)$, and its cavity becomes the central canal of the spinal cord and the ventricles of the brain. The anterior end of the hollow medullary cord thus developed enlarges to form three vesicles, one behind the other, and known respectively as the fore-brain, mid-brain, and hind-brain, each containing a ventricle. The fore-brain eventually gives rise to the cerebral hemispheres, produced in front into the olfactory lobes, and to the diencephalon; the mid-brain to the optic lobes and crura cerebri; and the hind-brain to the medulla 
oblongata and cerebellum. It will be seen (Fig. 65) that the medullary groove is lined by ectoderm and that therefore the whole central nervous system is ectodermic in origin; and this is also true of the nerves.

A longitudinal thickening of the endoderm on the dorsal side of the archenteron becomes constricted off as a solid rod of cells, lying between the medullary cord and the archenteron. This is the nolochord (Figs. $64 \mathrm{~K}$ and 65 , $n c h)$; it forms the primary axial skeleton around which the vertebral column is subsequently developed. The mesoderm is for some time a solid mass occupying all the space between the ectoderm and endoderm, so that there is no body-cavity. But at a later stage a cavity appears dividing the mesoderm into two layers, one in contact with the ectoderm (parietal layer, Fig. 65, mes $^{1}$ ), the other with the endoderm (visceral layer, mes ${ }^{2}$ ). 'The space between them is the coelome $(c)$. The dorsal part of the mesoderm on either side of the medullary cord and notochord becomes divided transversely, and gives rise to muscle-segments or myomeres (Fig. 64, L).

The embryo now begins to elongate in a definite direction (Fig. 64, J): its dorsal surface, still marked by the medullary grove, which now soon becomes closed, is slightly concave, its ventral surface very convex. The blastopore closes up, the yolk-plug becoming entirely covered by ectoderm; but for a short time the medullary canal and archenteron still communicate by the neurenteric canal $\mathrm{K}$, n.e.c). This soon disappears, and at the hinder end of the embryo a little conical outgrowth forms the rudiment of the tail $(t)$. The opposite end is rounded, and on its ventral surface appears a little half-moon shaped groove (which afterwards becomes subdivided into two, Fig. $66, \mathrm{C})$, the rudiment of the sucker by which the tad- 
pole attaches itself to weeds (J and $\mathrm{L}, s k)$. Just above the sucker a depression-the mouth-pit or stomodaum (L, stdm) - makes its appearance, and is the first indication of the mouth. A similar depression below the tail-the proctodoum $(p c d m)$-marks the anus : both at first are mere blind pouches and have no communication with the enteric canal. The head-region is further marked by two pairs of vertical ridges separated by depressions: the ridges are the branchial or gill arches $(\mathrm{J}, b r . c l)$, and the depressions the branchial clefts.

Further elongation takes place (Fig. 64, L), the head becomes distinctly marked off, the tail extends considerably beyond the anus, a thickening appears which marks the position of the eye (e), and a depression that of the ear (just above $b r^{2}$ in the figure); and from each branchial arch arises a little tuft, the rudiment of one of the external gills ( $b r{ }^{3}$, $\left.b r^{2}\right)$. In this condition the tadpole is hatched: it is still unable to feed, the stomodæum not yet being in communication with the enteric cavity; it is nourished therefore entirely by the yolk with which a large portion of the body is still filled.

Up to the stage shown in Fig. $64, \mathrm{I}$, the cells of which the tadpole is composed, although distinguishable into ectoderm, endoderm, and mesoderm, are all more or less similar : there are no muscles, no cartilage, no bone, no connective tissue. But shortly after the stage referred to, the permanent tissues begin to be formed: the outer ectoderm cells take on the form of epidermis, the endoderm cells become the epithelium of the enteric canal, and from offshoots of the enteric cavity-all of course lined by endoderm-are formed the lungs, the liver, the pancreas, and the urinary bladder.

The mesoderm undergoes much more extensive changes, giving rise to all the connective tissue, cartilage, bone, and muscle, as well as to other parts-i.e., to by far the greater 
part of the permanent tissues. In certain places the embryonic mesoderm cells, hitherto in close contact, separate from one another and become branched, while between them appear intercellular substance and fibres crossing one another in various directions: in this way the connective tissue which form so large a part of the adult body is produced.

The various parts of the skeleton first arise by the conversion of portions of the mesoderm into cartilage. The cells retreat from one another and between them a clear substance, the matrix, makes its appearance. For a considerable time almost the whole skeleton consists of cartilage, but subsequently much of this tissue is replaced by bone developed from a layer of cells on the inner surface of the perichondrium. The bone thus formed in connection with cartilage is cartilage-bone: membrane-bones arise in the connective tissue outside the cartilage (compare p. 43).

In the place where voluntary muscles are to appear the mesoderm cells elongate, their nuclei multiply by fission, and their protoplasm gradually becomes converted into the striated substance so characteristic of the adult muscular tissue.

These examples will suffice to illustrate the fact, which further study would show to be true, that all the permanent tissues are either-like epithelium-formed entirely of actual cells, or are-like connective tissue and striped muscle-derived from cells. The entire embryo in its earliest conditionthe oosperm stage-is a single cell, which multiplies repeatedly by simple fission, forming a group of cells ; and these, by assuming various forms and undergoing various changes, give rise to all the complex tissues-differing from one another both structurally and functionally-of the adult animal. So that every cell, fibre, or what not in the frog is 
a linear descendant, through repeated fission, of the oosperm ; and the oosperm itself is the product of the fusion of two cells, one-the ovum-derived from the female, the other - the sperm-from the male parent. Thus in passing from the oosperm to the adult animal there is a gradual structural or morphological differentiation accompanied by a differentiation of function or division of physiological labour.

The expression "division of physiological labour" was invented by the great French physiologist, Henri MilneEdwards, to express the fact that a sort of rough correspondence exists between lowly and highly organised animals and plants on the one hand, and lowly and highly organised human societies on the other. In primitive communities there is little or no division of labour : every man is his own butcher, baker, soldier, doctor, etc., there is no distinction between "classes" and "masses," and each individual is to a great extent independent of all the rest. Whereas in complex civilised communities society is differentiated into politicians, soldiers, professional men, mecharics, labourers, and so on, each class being to a great extent dependent on every other.

\section{Structure and Metamorphosis of the Tadpole-Develop-} ment of other Organs-Metamorphosis.-A sketch of the further development of the tadpole and of its metamorphosis into the frog has already been given (pp. 9-I I, Fig. I), and it is now necessary to add a few details.

A third pair of branchial arches appear behind the two already noticed, and on each a third external gill. The first two pairs increase greatly in size and all the gills become branched (Fig. 66, A). At the same time the branchial clefts come to open into the pharynx, and a current of water enters the mouth and passes out by the branchial clefts, thus providing the gills with a constant supply of aerated water for 
purposes of respiration. A thin median fold of the skin, the tail fin (Fig. r), arises all round the tail, both above and below, and the tail now forms a powerful swimming organ. The stomodæum (Fig. 66, B-E) and, at a still earlier period, the proctodæum (p. 204) open into the enteric cavity, so that there is now a complete enteric canal: it grows much faster than the body generally, and becomes coiled like a watch-spring $(\mathrm{D}, \mathrm{E})$. The mouth is very small, and is bounded by lips beset with little horny projections or papillæ and provided with a pair of horny jaws $(\mathrm{C}-\mathrm{E})$ with which the tadpole now browses upon the water-weeds which form its staple food. It is on account of this diet that the intestine is of such great proportionate length : vegetable diet contains less nourishment, bulk for bulk, than animal, and a longer intestine is required to extract all the nutriment from it.

Soon the external gills show signs of shrivelling, and on the inner portions of the branchial arches internal gills (Fig. 66, D ) are developed, like those of a fish. A fold of skin appears on either side in front of the gills and gradually grows backwards, covering the branchial clefts and the external gills, which latter soon disappear entirely: this is the operculum or gill-cover $(\mathrm{B}, \mathrm{C})$; it is quite like that of an ordinary fish, such as a cod or a perch, except that it is not supported by bone. The current of water from the pharynx now, of course, makes its final exit by a wide cleft between the edge of the operculum and the flank. The operculum gradually extends, and its free edge unites with the wall of the body posterior to the gills, so that the originally large aperture through which the water from the pharynx passes is much reduced. After some time, a complete union of the operculum with the flank takes place on the right side, the opercular aperture becoming closed: on the 
left side the spout-like aperture remains open for some time longer, and the water from both sides passes out through it (D, E). All this time the tadpole is to all intents and purposes a fish; apart from the possession of gills and a

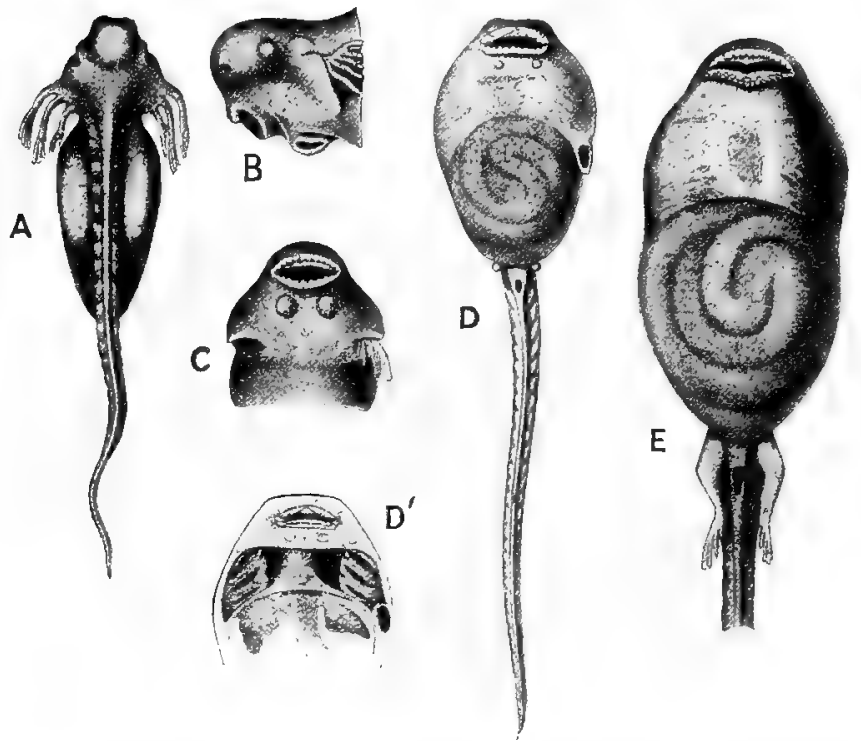

Fig. 66. - Stages in the later development of the tadpole.

A, stage with external gills; $\mathrm{B}$, stage showing the formation of the operculum; $\mathrm{C}$, later stage, in which the external gills are disappearing; $\mathrm{D}$, stage with internal gills and budding limbs; $D^{1}$, dissection of same stage to show the heart, gills and lungs; $\mathrm{E}$, later stage, in which the limbs are further differentiated. (After Howes.)

tail-fin, the structure of the circulatory organs and various other parts is more like that of a fish than of a frog.

The lungs $\left(D^{1}\right)$ now appear, and the tadpole is for a time truly amphibious, rising periodically to the surface of the water to breathe air. At a later stage, however, the single 
opercular aperture on the left side closes, and thenceforth respiration is purely aerial.

In the meantime the limbs are developed. The hindlimbs appear as little rounded buds, one on either side of the root of the tail (D): they gradually elongate and divide into thigh, shank and foot $(\mathrm{E})$. The fore-limbs appear beneath the operculum and are therefore hidden at first (E): at a later stage each divides into upper arm, fore-arm, and hand, and emerges from its concealment. As the limbs increase, the tail undergoes a progressive shrinking; its tissues become, as it were, digested, and are carried off by the blood; so that for a time the metamorphosing tadpole feeds largely on its own tail.

The mouth widens, the horny jaws and papillæ disappear and teeth are formed. The suckers vanish, and the intestine not only begins to grow less rapidly than the body, but even becomes reduced in length and loses its spiral arrangement, while vegetable is exchanged for animal diet. The dark colour of the tadpole gradually gives place to the bright hues of the frog. The little tailed frog can now leave the water and hop about on land: its tail is soon absorbed, and the metamorphosis is complete (Fig. I, 7,8 ).

It will be noticed that in the course of development of the frog a substitution or replacement of organs occurs. Thus, for instance, the notochord is replaced by a vertebral column, the gills by lungs, and the horny jaws by teeth.

Fate of the Germinal Layers.-In concluding this chapter we may enumerate in rather more detail the various parts and organs which are derived from each of the three embryonic tissues respectively. From the ectoderm are formed-the epidermis and the cutaneous glands; the whole of the nervous system, central and peripheral, and the essential parts of the sensory organs, as well as the crystalline Pract. Zool. 
lens of the eye; and also the epithelium lining the mouth (stomodæum) and outer part of the cloaca (proctodæum). The endoderm gives rise to the epithelium lining the enteric canal and its various offshoots, including the lungs, urinary bladder, gastric glands, bile and pancreatic ducts, as well as the glandular cells of the liver and pancreas; and to the notochord. From the mesoderm are developed the various parts which are situated between the ectoderm and endoderm with the exception of the notochord, viz., the connective tissue, cartilage, bone, striped and unstriped muscles, circulatory organs and peritoneum, as well as the urinary and reproductive organs and the accessory parts of the sensory organs.

\section{PRACTICAL DIRECTIONS.}

\section{Organs of Reproduction.}

Open a frog in the usual way: cut through the gullet, rectum, and mesentery, and remove all the digestive organs. In the female take especial care not to injure the roots of the lungs in severing the gullet. One of the specimens you have already dissected and kept in the preservative fuid should still contain the urinogenital organs intact in the case of the male: in the female, the ovaries and greater part of the oviducts will have been removed, but the relations of the two ends of each oviduct can still be made out.

Examine the reproductive organs, which will now be freely exposed, under water-or in the case of the female, in I per cent. salt solution.

I. Male Organs. (Figs. 3, 5, 7 and 6I.)

I. Notice again the spermaries or testes, each supported by a fold of peritoneum connecting it with the corresponding kidney; the efferent ducts; the branched fat-body; the ureter (urinogenital duct); and the seminal vesicle. Sketch after examining the cloaca (see below).

2. Tease up a bit of the spermary of a recently-killed frog in salt solution, and notice the form and movements of the sperms (Fig. 62, B). Sketch.

3. Examine under the microscope a transverse section of the spermary, prepared as directed on $p .136$, and note (Fig. 62, A) the numerous 
seminal tubes or crypts which open into it. Under the high power, observe the epithelial cells (germinal epithelizm), lining the tubes and their subdivision into smaller cells, which eventually give rise to the sperms, the tails of which project freely into the cavities of the tubes. Sketch.

II. Female Organs.

I. Notice again $(a)$ the ovaries, varying in size and appearance according to the time of year, and each suspended by a fold of peritoneum : they are studded all over with ovisacs, each of which contains an egg or ovum, pigmented when ripe ; and (b) the oviducts. (Fig. 4.) Trace the convoluted and glandular middle portion of the oviduct forwards, and make out the anterior thin-walled portion, running parallel to the gullet and opening into the coelome by a small aperture at the base of the lung; then trace the middle portion backwards, and notice the thinwalled, dilated, posterior portion, which opens into the cloaca. Sketch after examining the cloaca (see below).

If a female frog is examined in the spring, just before the eggs are laid, the ovaries will be seen to be reduced in size, and the posterior portions of the oviducts filled with eggs, each surrounded with a gelatinous coat secreted by the middle portion of the oviduct.

2. Examine under the microscope a section of the ovary, prepared as before (p. 136), first with the low, and then with the high power (Fig. 63). Note the epithelial cells (including the germinat epithelium) forming the walls of the ovary, and the ovisacs in different stages of development. Each ovisac contains an ovnum, surrounded by folliclecells. In the ova, note the protoplasm and its contained yolk-granules, the vitelline membrane, the nucleus, and the nucleoli. Sketch.

\section{Cloaca.}

Carefully cut through the pelvic symphysis in the middle line with a scalpel and press apart the two innominate bones, so as to expose the ventral surface of the cloaca. Inflate from the vent, and note that the large intestine is continuous with the cloaca and that the urinary bladder opens into it on the ventral side.

The entire urinogenital apparatus, together with the cloaca, may now be removed from the body, first cutting through the skin round the vent. Pin down under water, insert the small scissors into the vent, and slit open the cloaca very slightly to one side of the middle line, . so as not to injure the connection between the bladder and cloaca. 
Notice the openings into the bladder and large intestine respectively, and also :-

In the male, the apertures of the ureters (urinogenital ducts), situated on two small papillæe, lying close to one another on the dorsal side of the cloaca : insert a bristle into the ureter. Sketch.

In the female, the two small apertures of the ureters, and just in front of these, the two large apertures of the oviducts, all situated on the dorsal wall of the cloaca. Insert bristles into the ureter and oviduct. Sketch.

\section{Impregnation and Development.}

In the early spring (end of February or beginning of March), look about in ponds and ditches for frogs which have taken to the water for the purpose of laying eggs: This process can be watched more conveniently by catching a few frogs, male and female, and putting them in a large vessel of water or aquarium. If you have been unsuccessful in procuring frogs or frogs' spawn, toads will do equally well: their eggs are laid a few weeks later, and are arranged, each surrounded by its gelatinous envelope, not in clumps, like those of the frog, but in a string, like the beads on a necklace.

For purposes of observation, the spawn is best kept in a glass vessel, together with some water-weeds. Put only a small quantity of spawn in one vessel; if the water begins to get foul, change it at once. Examine the eggs every day with a magnifying-glass as development proceeds, and keep some of the tadpoles alive until metamorphosis takes place in June.

If you wish to see some of the stages in the development of the frog during other times of the year, you must obtain some preserved eggs and embryos, and, if possible, you should also examine the series of wax models, made on an enlarged scale, which are to be seen in most zoological museums.

The following are some of the more important things to be noticed. (Sketch a series of stages) :-

\section{Before hatching.}

I. The unsegmented oosperm, black above, and white below.

II. Farly stages in segmentation, during which the individual cells can be made out by means of a lens. (Fig. $64 \mathrm{~A}-\mathrm{F}$ ).

III. Later stages in segmentation, during which the individual cells cannot be distinguished without the aid of a microscope. Note the gradual enclosure of the white by the black hemisphere, until only a small 
rounded yolk-plug is left, filling in an aperture-the blastopore, in the black layer or ectoderm, as it is now called. The yolk-plug is continuous with a mass of yolk-cells, enclosed by the ectoderm, from which the endoderm and mesoderm are developed. (Fig, 64, G-I).

IV. The gradual flattening and elongation of the embryo, and the position of the blastopore at the posterior end of the dorsal side; the ormation of the medullary folds and groove along the dorsal side, and the closure of the blastopore. (Fig. 64, H-J).

$\mathrm{V}$. The closure of the medullary groove, the formation of the head with its suckers and of the tail with its tail-fin; as well as the appearance of the eyes, ears, branchial arches, external gills, and the involutions for the mouth and amus, which, however, do not open until after the tadpole is hatched. (Fig. 64, K-L).

\section{After hatching.}

VI. The appearance of the horny jares and papilla, and the further development of the head, external gills, and tail. (Fig. I, I-2 and Fig. $66 \mathrm{~A}-\mathrm{B}$ ).

VII. The formation of the operiulum, the closure of the right opercular aperture, and the disappearance of the external gills. The bud-like rudiments of the hind-limbs, and their further development. The fore-limbs remain for a long time hidden beneath the operculum. (Fig. 1, 3-4 and Fig. I, 66, C-E).

\section{Metamorphosis.}

VIII. The gradual change in the form and colour of the head and body; the widening of the month and loss of the horny jaws and suckers ; the appearance of the fore-limbs from beneath the operculum, and the shrinking of the tail (Fig. $r, 5-8$ ).

\section{Dissection of tadpole.}

Pin down a full-sized tadpole under water in a small dissecting dish, with the ventral side uppermost, inserting snall pins through the tail only. Carefully dissect off the ventral body walls, and note the coiled intestine, the heart, the internal gills, etc. (Fig. 66, D, D,$E$ ). Sketch.

It is rather a difficult task, and requires much time, to prepare sections of the early stages of the frog-embryo in order to make out the formation of the ectoderm, endoderm, and mesoderm, the relations of the segmentation cavity and archenteron, and the development of the central nervous system and notochord (Figs. 64, I, K, and 65); and directions for the study of the development of the chief organs of the chick will be 
given in a subsequent chapter. But if you wish to make the attempt, proceed as follows :-

Take out a few eggs every day from the time they are laid until the tadpoles are hatched : place these on a tile or piece of glass, and with a pair of needles dissect off the gelatinous covering. Then carefully transfer the segmenting eggs or embryos into corrosive sublimate for a quarter to half an hour, and after washing in running water, put them into weak and then strong alcohol; then stain, imbed, and cut sections as directed on p. I36. 


\section{CHAPTER XIII.}

THE FROG (continued): MEANING OF THE TERM SPECIESTHE PRINCIPLES OF CLASSIFICATION-EVOLUTIONONTOGENY AND PHYLOGENY-HEREDITY AND VARIATION-STRUGGLE FOR EXISTENCE-SELECTION-ORIGIN OF SPECIES.

THE frog which you have been studying is the only one commonly found in Great Britain. Another kind, very similar to it, is, like the common frog, abundant in Germany, and other parts of the European continent, but is rare in this country, only occurring in parts of the eastern counties ; and various other frogs, differing in certain minor respects from these are found in different parts of the world. This fact is expressed in the language of systematic zoology by saying that there are various species of frogs, belonging to the same genus, which are distinguished from one another by certain definite characteristics as regards form, structure, and colour.

According to the system of binomial nomenclature introduced by Linnæus, each kind of animal receives two namesone, the generic name, common to all the species of the genus; the other the specific name, peculiar to the species in question. Both generic and specific names are Latin in form, and are commonly Latin or Greek in origin, although 
frequently modern names of persons or places with latinized terminations are employed. In giving the name of an animal, the generic name is always placed first, the specific name following it, and being written as a rule with a small letter. Thus the common English frog is called Rana temporaria, and the continental form referred to above, often spoken of as the edible frog, Rana esculenta.

You will probably have noticed certain differences in colour and markings in the different individual frogs you have examined, and it is matter of common observation that no two individuals of a species are exactly alike. In the case of human beings and many of the more familiar animals this is very apparent to every one : in other cases a more careful examination of the individuals is necessary in order to tell them apart ; thus, for instance, the individuals in a flock of sheep appear all alike to the casual observer, but the shepherd can easily distinguish them from one another. These differences we designate individual variations, and it is often difficult to decide whether two kinds of animals should be considered as distinct species, or as varieties of a single species, and no universal rule can be given for determining this point. Among the higher animals, mutual fertility is a fair practical test, the varieties of a species (e.g., common pigeon, fowl, dog, horse) usually breeding freely with one another and producing fertile offspring, while distinct species usually do not breed together, or else produce infertile hybrids or mules. Compare, for instance, the fertile mongrels produced by the union of the various breeds of domestic dog with the infertile mule produced by the union of the horse and ass. But this rule is not without exception, and in the case of wild animals is, more often than not, impossible of application; failing it, the only criterion of a "good species" is usually the presence of constant differences from 
allied species, whereas if there is a complete series of gradations between two forms, they will be considered to form a single variable species.

In the previous chapters it will have been evident that an animal may be studied from two chief points of view, firstly, from the point of view of its structure, and secondly from that of the functions performed by its various parts and the way in which these work together for the welfare of the whole. The branch of Zoology dealing with the former is known as Morphology, and with the latter Physiology.

It is evident that a knowledge of Morphology is necessary as a preliminary to the study of Physiology, and also that, as animals have to be distinguished from one another largely by structural characters, the foundations of a scientific Zoology must be laid in Morphology, which, as we have seen, deals not only with the external characters and the anatomy and histology of the adult animal, but also with the changes undergone during the development of the egg into the adult form, i.e., with embryology. Given a sound knowledge of the anatomy, histology, and embryology of animals, their classification may be attempted : that is, we may proceed to arrange them in groups and sub-groups, each capable of accurate definition. In doing so we must be careful to distinguish between homologous parts, or those which correspond structurally (compare p. 39), and analogous parts, which have merely a similar function, and are of no value for purposes of classification. Thus the fore-limbs of a frog are homologous with-i.e., are formed on a similar plan to-the wings of a bird, although differing from them in function; they are only analogous to those of an insect, for though the function is similar in both, the structure is widely different. In the same way the wing of the bird is only analogous to that of an insect. 
The general method of classification employed by zoologists may be illustrated by reference to the different kinds of frogs already referred to in explanation of the terms genus and species.

The common frog (Rana temporaria) is distinguished from the "edible frog" ( $R$. esculenta $)$ by its smaller size and brown colour, by the large black patch in the tympanic region, and by the rudimentary character of a pair of inflatable vocal sacs at the sides of the head in the male, which are very large and highly distensible in $R$. esculenta. On the other hand, these two frogs agree with one another and with all the other species of the genus Rana, in having teeth on the upper jaw, and in not having the transverse processes of the sacral vertebra dilated. Comparing all these with our English toads, of which there are two species, the common toad and the rarer "natterjack," we find that in them the skin is comparatively dry and covered with glandular warts, the hind-limbs are proportionally shorter, there are no teeth, and the transverse processes of the sacral vertebra are more or less dilated. These differences are so great as not only to necessitate placing the toads in another genus, the genus Bufo, but also to relegate the frogs just mentioned to one family - the Ranida, and the toads to another-the Bufonida. All frogs and toads, however, agree with one another in having no tail in the adult, while the trunk is relatively short and broad, and the hind-limbs are longer than the fore-limbs. They therefore differ fundamentally from such animals as the common English newts and the salamanders, which retain the tail throughout life, and in which the fore- and hind-limbs are of approximately equal size. The differences here are obviously far greater than those between the families mentioned above, and are emphasised by placing the frogs and toads in the crder 
Anura, the newts and salamanders in the order Urodela.

The Urodela and Anura, although differing from one another in many important respects, agree, e.g., in possessing gills during part or the whole of their existence, and in nearly always possessing lungs. They usually pass through a metamorphosis, the young being hatched in the form of gilled larvæ; their skin is soft and glandular, and the toes are in nearly all cases without claws. These and numerous other structural characters separate them from reptiles, in which gills are never developed, and the young do not pass through a metamorphosis, while the skin is provided with scales and the toes have claws. The differences here are considerably more important than those between the orders referred to above, and are expressed by placing the latter in the class Amphibia, while reptiles consticute the class Reptilia. In the same way the finned, water-breathing fishes form the class Pisces, the feathered birds the class Aves, and the hairy animals which suckle their young the class Mammalia.

Mammals, Birds, Reptiles, Amphibians, and Fishes all agree with one another in the possession of red blood and an internal skeleton, an important part of which in the embryo is the notochord (p. 203), which is nearly always replaced in the adult by a backbone or vertebral column; and in never having more than two pairs of limbs. They thus differ in some of the most fundamental features of their organisation from such animals as Crayfishes, Insects, Scorpions, and Centipedes, which have colourless blood, a jointed external skeleton, and numerous limbs. These differences-far greater than those between classes - are expressed by placing the back-boned animals in the phylum or sub-kingdom Vertebrata, the many-legged armoured forms 
in the phylum Arthropoda. Similarly, soft-bodied animals with shells, such as mussels and snails, form the phylum Mollusca; various worms, such as the earthworm, the phylum Annulata; polypes and jelly-fishes the phylum Calenterata; the simplest animals, mostly minute, such as Amarba, the phylum Protozoa. Finally, the various phyla recognised by zoologists together constitute the kingdom Animalia.

Thus the animal kingdom is divided into phyla, the phyla into classes, the classes into orders, the orders into families, the families into genera, and the genera into species; while the species themselves are assemblages of individual animals agreeing with one another in certain definite characteristics. It will be seen that the individual is the only term in the series which has a real existence; all the others are mere groups, formed, more or less arbitrarily, by man.

Thus the zoological position of the common frog is expressed as follows:-

Kingdom-ANimalia.

Phylum-Vertebrata.

Class-Amphibia.

Order-Anura.

Family-Ranida.

Genus-Rana.

Species-temporaria.

Let us now briefly consider some of the reasons which have led zoologists to adopt the system of classification now in general use.

It is obvious that there are various ways in which animals may be classified, and the question arises-which of these, if any, is the right one? Is there any standard by which we can judge of the accuracy of a given classification, or 
does the whole thing depend upon the fancy of the classifiers, like the arrangement of books in a library? In other words, are all possible classifications of living things more or less artificial, or is there such a thing as a natural classification?

Suppose we were to try and classify all the members of a given family-parents and grandparents, uncles and aunts, cousins, second cousins, and so on. Obviously there are a hundred ways in which it would be possible to arrange them-into dark and fair, tall and short, curly-haired and straight-haired, and so on. But it is equally obvious that all these methods would be purely artificial, and that the only natural way, i.e., the only way to show the real connection of the various members of the family with one another would be to classify them according to bloodrelationship; in other words, to let our classification take the form of a genealogical tree.

There are two theories which attempt to account for the existence of the innumerable species of living things which inhabit our earth: the theory of special creation and the theory of evolution.

According to the theory of creation, all the individuals of every species existing at the present day-the tens of thousands of dogs, frogs, oak-trees, and what not, are derived by a natural process of descent from a single individual, or from a pair of individuals_-in each case precisely resembling, in all essential respects, their existing descendants-which came into existence by a process outside the ordinary course of nature and known as creation. On this hypothesis each species of frog is derived from a common ancestral pair which came into existence, independently of the progenitors of all the other species, at some previous period of the earth's history. 
Notice that on this theory the various species of frogs are no more actually related to one another than is either of them to a Newt, or for the matter of that, to Homo. The individuals of any one species are truly related since they all share a common descent, but there is no more relationship between the individuals of any two independently created species than between any two independently manufactured chairs or tables. The words affinity, relationship, etc., as applied to different species are, on the theory of creation, purely metaphorical, and mean nothing more than that a certain likeness or community of structure exists; just as we might say that an easy chair was more nearly related to a kitchen chair than either of them to a threelegged stool.

We see, therefore, that on the hypothesis of creation, the varying degrees of likeness and unlikeness between the species receive no explanation, and that we get no absolute criterion of classification: we may arrange our organisms, as nearly as our knowledge allows, according to their resemblances and differences, but the relative importance of the characters relied on becomes a purely subjective matter.

According to the rival theory - that of Descent or Organic Evolution, with which the name of Darwin is inseparably connected-every species existing at the present day is derived by a natural process of descent from some other species which lived at a former period of the world's history. If we could trace back from generation to generation the individuals of any existing species, we should, on this hypothesis, find their characters gradually change, until finally a period was reached at which the differences were so considerable as to necessitate the placing of the ancestral forms in a different species from their descendants at the 
present day. And in the same way, if we could trace back the species of any one genus, we should find them gradually approach one another in structure until they finally converged in a single species, differing from those now existing, but standing to all in a true parental relation.

It will be seen that, on this hypothesis, the relative likeness and unlikeness of the various species of frogs are explained as the result of their descent with greater or less modification or divergence of character from the ancestral form: and that we get an arrangement or classification in the form of a genealogical tree, which, on this hypothesis, is a strictly natural one, since it shows accurately the relationship of the various species to one another and to the parent stock. So that on the theory of evolution, a natural classification of any given group of allied organisms is simply a genealogical tree, or, as it is usually called, a phylogeny.

Now it is evident that the only way in which we could be perfectly sure of an absolutely natural classification of the species of any kind of animal - the frog, for examplewould be by obtaining specimens as far back as the distant period when the genus first came into existence.

Forming part of the solid crust of the earth are a series of sedimentary or stratified rocks, and the researches of geologists have shown that these present a general order of succession, the lowest, when undisturbed, being in every case older than the more superficial layers. Imbedded in these rocks are found the remains of various extinct animals in the form of what are called fossils; and it might perhaps, on first considering the subject, be supposed that, had a process of evolution taken place, we ought to be able to find in the rocks belonging to the various geological ormations a complete series of animal remains, represent- 
ing all the stages in the evolution of the highest from the lowest forms. But owing to various causes which we cannot further consider here, the record of the succession of life on the globe is very imperfect and incomplete in the case of any individual species: but the evidence furnished by Palæontology - as the study of fossils is called-is very important in supplying proofs that there has been a gradual evolution of the higher from the lower forms.

Further evidence in the same direction is furnished by Morphology and Embryology, as will be more apparent to you when you have studied a number of other kinds of animals. You have, however, seen that the frog begins life as a single cell, and that it is possible to trace a series of modifications which gradually convert the unicellular oosperm into a tadpole-which is to all intents and purposes a fish, and that the tadpole subsequently undergoes metamorphosis into the more highly organised frog. According to the theory of recapitulation, these facts indicate that the frog repeats, during its single life, the series of changes passed through by its ancestors in the course of ages. In other words, ontogeny, or the evolution of the individual, is, in its main features, a recapitulation of phylogeny, or the evolution of the race. At the same time you must bear in mind that it is not always an easy matter to determine which characters are of phylogenetic significance, and which have been secondarily acquired owing to various causes. To take an example:-the horny jaws of the tadpole might be taken to indicate that frogs were descended from ancestors with horny jaws and without teeth, though in all probability these organs have been secondarily acquired as adaptations in connection with the habits of the tadpole, and have therefore, unlike the gills, for instance, no ancestral significance. 
It is obvious that the evolution of one species from another presupposes the occurrence of variations in the ancestral form. As we have seen, such individual variation is of universal occurrence. This may be expressed by saying that heredity, according to which the offspring tends to resemble the parent in essentials, is modified by variability, according to which the offspring tends to differ from the parent in detail. If, from any cause, any well-marked individual variation is perpetuated, there is produced what is known as a variety of the species, and according to the theory of the origin of species by evolution, such a variety may, in course of time, become a new species. Thus a variety is an incipient species, and a species is a (relatively) permanent variety.

One other important factor in evolution must be briefly referred to before concluding this chapter. In order that every species of animal and plant may flourish, certain conditions are necessary. Thus the frog requires, for example, a moist place to live in, and water in which to lay its eggs. For spots presenting the necessary favourable conditions, there is constantly going on a competition between individuals of one species and between the members of different species. The nature of this struggle is well seen when a piece of garden-ground is allowed to run to waste. Its surface is soon overgrown by weeds of many kinds, which kill out nearly all the original gardenplants by depriving them of light and food. By and by the more hardy weeds exterminate and replace such weaker forms as may first have obtained a footing, till an entirely new set of weeds may take the place of those that first appeared.

A struggle for existence goes on on all sides among animals. To begin with, before there is any struggle for 
existence in the strict sense, there is-particularly in animals which, like the frog, produce eggs in great numbers annually -a very great indiscriminate destruction of ova and young embryos. Only a few of them reach maturity; a large proportion are destroyed at one stage or other, some failing to reach a spot favourable for their further development, others becoming the food of other animals. But such of the young as are less adapted to escape the various dangers to be encountered, and less fitted to procure the necessary food, are more likely to be destroyed. This is one phase-and the most important, perhaps, of all -of the struggle for existence amongst animals. But there is also a struggle for existence, not only between individual animals of the same kind, but between animals of different kinds. This struggle, in so far as it relates to the competition for food and shelter, is more severe between nearly related species; for in such a case the food and the favourable conditions required are the same, or nearly so, in the two competitors. Again, a struggle for existence of a constant and severe kind also goes on between carnivorous animals and the animals on which they prey-e.g., between the frog and the insects and worms on which it feeds, and between the snake and the frog: a struggle in which the defensive qualities of the prey-such as swiftness, power of eluding observation, or of resisting attack-are opposed to the predatory powers of the attacker.

There can be little doubt that, in the long run, such individuals will survive as are best fitted to cope with the conditions to which they are subject. According to Darwin's theory of Natural Selection, it is assumed that such surviving individuals would transmit their special properties to their progeny, and there would thus be a gradual approximation towards a perfect adaptation of the 
species to its surrounding conditions by virtue of this "survival of the fittest."

Let us suppose the conditions to change. Gradual alterations in climate and other conditions are known to take place, owing to subsidence or elevation of the land. But conditions might be changed in many other ways : some animal or plant previously used as food might become exterminated, or a new enemy might find its way into the district inhabited by the species. Then such individuals as presented variations which enabled them better to cope with the new surroundings, would have the advantage over the others, and would have a much better chance of surviving and leaving progeny. The useful variations thus produced and transmitted to the progeny would tend to increase, generation after generation, until a form sufficiently distinct to be regarded as a new species had become developed from the original one.

That very different varieties of animals and plants can, and have been produced, in a comparatively short time, by man selecting those forms which tend to vary in a desired direction, is a well-known fact. All the breeds and varieties of our domestic animals have been produced by this process of artificial selection; and "if man can by patience select variations useful to him, why, under changing and complex conditions of life, should not variations useful to Nature's living products often arise, and be preserved or selected?"

It does not come within the scope of the present work to discuss either the causes of variability or those which determine the elevation of a variety to the rank of a species : both questions are far too complex to be adequately treated except at considerable length, and anything of the nature of a brief abstract would only be misleading. As a pre- 
liminary to the study of Darwin's Origin of Species, the student is recommended to read Romanes's Evidences of Organic Evolution, in which the doctrine of Descent is expounded as briefly as is consistent with clearness and accuracy. 


\section{P A R T I I}

\section{CHAPTER I}

AMEBA-UNICELLULAR AND MULTICELLULAR ANIMALS.

From your study of the frog you will have learnt some of the more important facts with regard to the Morphology and Physiology of a comparatively highly-organised animal, and will have overcome a number of preliminary difficulties in acquiring a knowledge of zoological terminology and technique. You will now, therefore, be in a better position to undertake a systematic and comparative examination of a number of other animals-some much less complicated, some more complicated, than the frog-working upwards from the simple to the complex forms and beginning with a very instructive animalcule belonging to the genus Amaba.

$\Lambda$ mobæ are often found in the slime at the bottom of pools of stagnant water, adhering to weeds and other submerged objects. They are mostly invisible to the naked eye, rarely exceeding $\frac{1}{4}$ th of a millimetre $\left({ }_{1} \frac{1}{0} 0_{0}\right.$ th inch) in diameter, so that it is necessary to examine them entirely by the aid of the microscope. Though they can be seen and recognised with the low power, the high power is necessary for the accurate examination of their structure. 


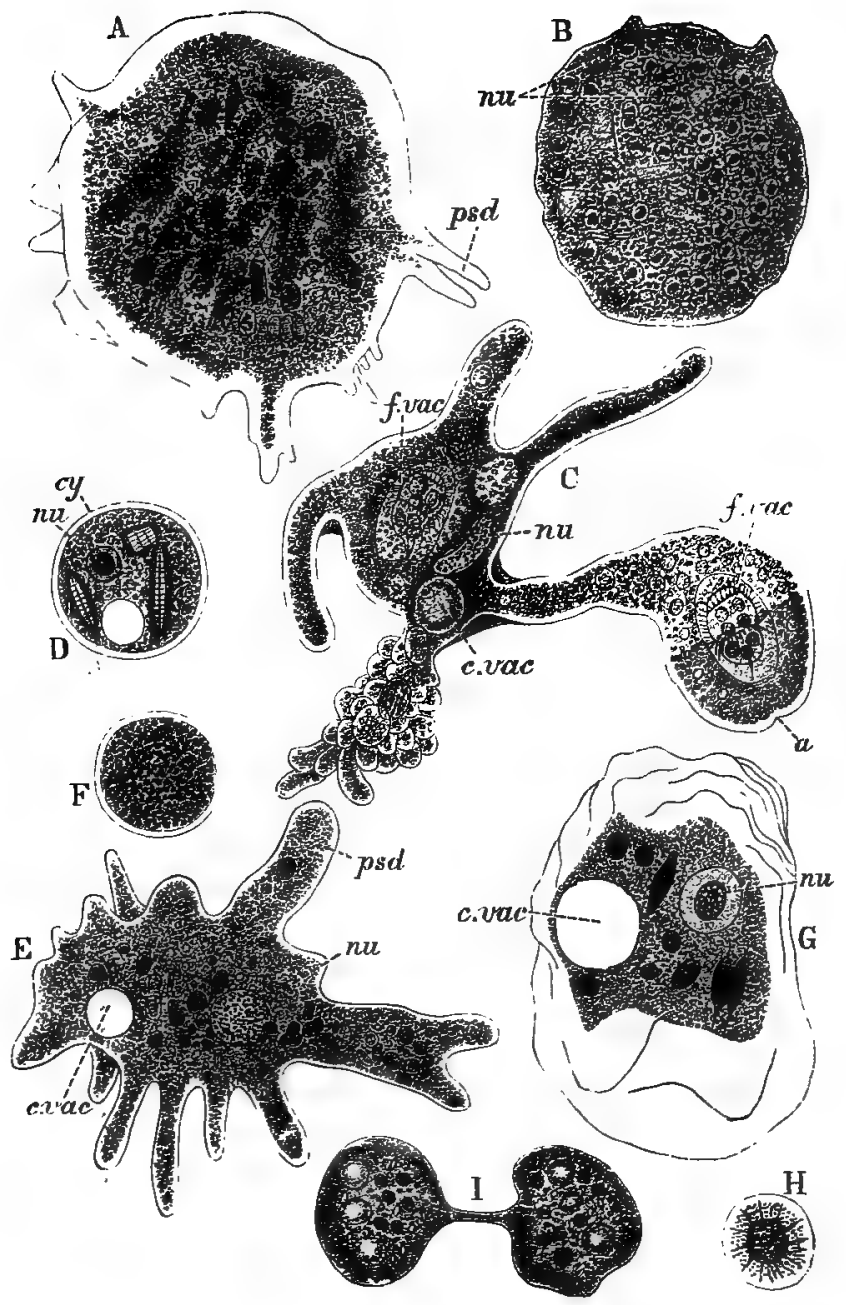

FIG. 67.-A, Amaba quarla, a living specimen, showing granular endoplasm surrounded by clear ectoplasm, and several pseudopods ( $p s t$ ), some formed of ectoplasm only, others containing a core of endoplasm. The larger bodies in the endoplasm are mostly food-particles (300 diameters).

$\mathbf{B}$, the same species, killed and stained with carmine to show the numerous uuclei (nic) present in this species (compare p. 269$)\left(\times 3^{\circ 0}\right)$. 
C, Ameda proteus, a living specimen, showing large irregular pseudopods, nucleus (nu), contractile vacuole (c. vac), and two food vacuoles ( $f . v a c)$, each containing a small infusor (see p. 26I) which has been ingested is food. The letter $a$ to the right of the figure indicates the place where two pseudopods have united to enclose the food vacuole. The contractile vacuole in this figure is supposed to be seen through a layer of granular protoplasm, whereas in the succeeding figures (D, E, and $G$ ) it is seen in optical section, and therefore appears clear.

$D$, an encysted Amoba, showing cell-wall or cyst (cy), nucleus (nu), clear contractile vacuole, and three microscopic plants (diatoms) ingested as food.

E, Amaba protons, a living specimen, showing several large pseudopods ( $p s d$ ), single nucleus ( $n u)$ and contractile vacuole (c. vac), and numerous food-particles embedded in the granular endoplasm $\left(x, 33^{\circ}\right)$.

F, nucleus of the same after staining, showing a ground substance of achromatin, containing deeply-stained granules of chromatin, and surrounded by a distinct membrane ( $x$ Ioro).

$\mathrm{G}$, Amceba verrucosa, living specimen, showing wrinkled surface, nucleus $(n u)$, large contractile vacuole (c. vac), and several ingested organisms $(\times 330)$.

$\mathrm{H}$, nucleus of the same, stained, showing the chromatin aggregated in the centre (X roro).

1, Amaba proteus, in the act of multiplying by binary fission ( $\times 500$ ). (From Parker's Biology: A, B, E, F, G, and H after Gruber; C and I after Leidy; D after Howes.)

Examined under the high power (Fig. 67), the Amœba appears like a little shapeless blob of jelly, nearly or quite colourless, and closely resembling a colourless blood corpuscle or leucocyte of one of the higher animals (p. 105). The central part of it, or endoplasm, is granular and semitransparent-something like ground-glass-while surrounding this inner mass is a border of perfectly transparent and colourless substance-the ectoplasm.

One very noticeable thing about the Amœba is that, like the leucocyte, it is never of quite the same form for long together, owing to the protrusion of pseudopods ( $p s d)$, by means of which it creeps along slowly. The occurrence of amoboid movements is alone sufficient to show that it is an organism, or'living thing, and no mere mass of dead matter. Moreover, it consists of protoplasm and contains a nucleus $(\mathrm{C}-\mathrm{H}, m u)$, which is rendered more apparent by staining and consists of chromatin and achromatin. The Amœba is therefore a cell (compare p. I06).

A very important difference is thus at once seen between the Amceba and the frog: the Amoba is unicellular, i.e. it consists of a single cell, while the frog is, as we have seen 
a multicellular animal, built up of innumerable cells which are incapable of an independent existence for any length of time.

Besides the nucleus, there is another structure frequently visible in the living Amoba and not present in the leucocyte. This is a clear, rounded space in the ectoplasm (c. vac), which periodically disappears with a sudden contraction and then slowly reappears, its movements reminding one of the beating of a minute colourless heart. It is called the contractile vacuole, and consists of a cavity in the ectoplasm containing a watery fluid.

We must now study the Physiology of our animalcule. First of all, as we have already seen, it is contractile: although it has no muscles, it can move about from place to place. Its movements, like the voluntary movements of the frog (pp. 7, 173), may occur without the application of any external stimulus, i.e., they are spontaneous or automatic; or they may be induced by external stimuli-by a sudden shock or by coming in contact with an object suitable for food. Movements of this latter kind, like those resulting from the stimulation of the nerves in a brainless frog, are the result of the irritability of the protoplasm; the animalcule is therefore both automatic and irritable, although it possesses neither nerves nor sense-organs.

Under certain circumstances an Amceba temporarily loses its power of movement, draws in its pseudopods, and becomes a globular mass around which is formed a thick, shell-like coat, called the cyst or cell-wall (Fig. 67, D, cy). This is formed by the protoplasm by a process of secretion (p. I 30) : its composition is not known; it is certainly not protoplasmic, and very probably consists of some nitrogenous substance allied in composition to horn and to the chitin (see Chapters V \& VI) which forms the external shell of crustaceans, insects, \&c. 
The formation of the cyst is probably of great importance in preserving the animalcule from destruction by drought, so that should the pool in which it is living dry up, it may still remain alive, protected by its shell-like case, until the conditions for its active life are once more restored, when it escapes from this encysted condition by the rupture of the cell-wall.

Very often an Amcba in the course of its wanderings comes in contact with a still smaller organism of some kind or other. When this happens the Amcba may be seen to extend itself round the lesser organism until the latter becomes sunk in its protoplasm in much the same way as a marble might be pressed into a lump of clay (Fig. $67, \mathrm{C}, a$ ). The diatom or other organism becomes in this way completely enclosed in a cavity or food-vacuole ( $f$. vac), which also contains a small quantity of water necessarily included with the prey. The latter is taken in by the Amoba as food: so that the Amoeba, like the frog, feeds. It is to be noted that the reception of food takes place in a particular way, viz. by ingestion-i.e. it is enclosed entire by the organism.

When the prey is thus ingested, its protoplasm becomes digested, any insoluble portions being passed out or egested, as fæces (p. 75), from the surface of the Amœba as it creeps slowly on. Note that all this is done without either ingestive aperture (mouth), digestive cavity (stomach), or egestive aperture (anus): the food is simply taken in by the flowing round it of protoplasm, digested as it lies enclosed in the protoplasm, and those portions for which it has no further use are got rid of by the Amœba flowing away from them.

We have seen that the frog possesses certain digestive glands, the function of which is to secrete digestive fluids which have an important chemical action on the food 
swallowed, rendering it soluble and diffusible before it passes through the epithelial cells of the intestine into the blood: the gastric juice, for example, has the power of converting proteids into peptones by means of the ferment pepsin (p. 74); the digestion here takes place outside the cells, i.e. is extracellular. There can be little doubt that the protoplasm of Amoeba is able to render that of its prey soluble and diffusible by the agency of some substance analogous to pepsin, and that the dissolved matters diffuse through the body of the Amœba until the latter is, as it were, soaked through and through with them. The process of digestion in Amœba thus takes place within a single cell, i.e. it is intracellular.

It has been proved by experiment that proteids are the only class of food which Amœba can make use of : it is unable to digest either starch or fat (p. 72). Mineral matters must, however, be taken with the food in the form of a weak watery solution, since the water in which the animalcule lives is never absolutely pure.

The Amoeba being thus permeated, as it were, with a nutrient solution, the elements of the solution, hitherto arranged in the form of peptones, mineral salts, and water, become rearranged in such a way as to form new particles of living protoplasm, which are deposited among the preexisting particles. In a word, the food is assimilated, or converted into the actual living substance of the Amœba, which must therefore grow, if nothing happens to counteract this formation of new protoplasm.

We have seen, however, that work results in a proportional amount of waste (p. 66), and just as in the frog or in ourselves, every movement of the Amceba, however slight, is accompanied by a proportional oxidation or low temperature combustion of the protoplasm, i.e. the constituents of the 
protoplasm combine with oxygen, forming waste or excretory matters-carbon dioxide, water, and certain nitrogenous substances of simpler constitution than proteids, such as urea. These products of excretion, formed in the case of Amœba without the agency of any special excretory organs (e.g. kidneys), are given off partly from its general surface, but partly, it would seem, by the agency of the contractile vacuole, by means of which the water taken in with the food is also got rid of.

With this breaking down of proteids the vital activity of all organisms are invariably connected. Just as useful mechanical work may be done by the fall of a weight from a given height to the level of the ground, so the work done by the organism is a result of its complex proteids falling, so to speak, to the level of simpler substances. In both instances potential energy or energy of position is converted into kinetic or actual energy.

The statement just made that the protoplasm of Amœba constantly undergoes oxidation presupposes a constant supply of oxygen. The water in which the animalcule lives invariably contains that gas in solution, and diffusion takes place, oxygen passing into the interior of the Amoba while carbon dioxide passes out into the water. This is the process of breathing or respiration (p. I44), and it occurs in Amœba without the agency of lungs or other respiratory organs. Thus the carbon dioxide is got rid of, and at the same time a supply of oxygen is obtained for further combustion. The oxidation of the protoplasm of the Amoba is doubtless accompanied by an evolution of heat, as in higher animals (p. I 5 I), although this has never been proved.

IVe thus see that a very elaborate series of chemical processes is constantly going on in the interior of Amœba, as in the frog, the whole series of which is spoken of collectively 
as metabolism-constructive and destructive (p. 149). Living protoplasm is thus the most unstable of substances; it is never precisely the same thing for two consecutive seconds ; its existence, like that of a waterfall or a fountain, depends upon the constant flow of matter into it and away from it.

It follows from what has been said that if the income of an Amœba, i.e., the total weight of substances taken in (food plus oxygen plus water) is greater than its expenditure or the total weight of substances given out (fæces plus excreta proper plus carbon dioxide) the animalcule will grow : if less it will dwindle away: if the two are equal it will remain of the same weight or in a state of physiological equilibrium.

It is evident that Amoeba must also be able to perform the function of reproduction. You have learnt that the cells of the frog multiply by simple or binary fission (p. 106): the nucleus first divides into two, and then the surrounding, protoplasm; and precisely the same thing occurs in Amœba, the reproduction of which therefore takes place by the simplest method known, without any special reproductive organs. The animalcule simply divides into two Amcebæ, each exactly like itself; and in doing so ceases to exist as a distinct individual. Instead of the successive production of offspring from an ultimately dying parent, we have the simultaneous production of offspring by the division of the parent, which does not die, but becomes simply merged in its progeny. There can be no better instance of the fact that reproduction is discontinuous growth.

From this it seems that an Amceba, unless suffering a violent death, is practically immortal, since it divides into two completely organised individuals, each of which begins life with half of the entire body of its parent, there being therefore nothing left of the latter to die: it therefore 
appears certain that "death has no place as a natural recurrent phenomenon" in that organism.

It is said that occasionally two Amœba come into contact and undergo complete fusion, just as the gametes of the frog (sperm and ovum) unite in the processes of fertilization (p. 197). This process of conjugation has been more accurately observed in other unicellular organisms (pp. 268 and 278 ), and it is important to bear in mind that reproduction can take place in all these quite independently of such a process.

Amoba may also be propagated artificially. If a specimen is cut into pieces, each fragment is capable of developing into a complete animalcule provided it contains a portion of nuclear matter, but not otherwise. From this it is obvious that the nucleus exerts an influence of the utmost importance over the vital processes of the organism.

If an Amoba does happen to be killed and to escape being eaten it will, like a dead frog, undergo gradual decomposition, becoming converted into various simple substanstances of which carbon dioxide, water and ammonia are the chief (p. I 52).

Death results if the temperature to which an Amœba is exposed reach about $40^{\circ} \mathrm{C}$, and at freezing point its movements cease entirely and it becomes inert.

We thus see that complex organs, composed of various tissues, each consisting of cells of characteristic form, are not necessary in order that the vital functions may be performed : the only essential is nucleated protoplasm. As we pass from the unicellular Amœba to the higher multicellular animals we shall find-just as we found in tracing the development of the frog from the unicellular oosperm (p. 206), - that a differentation of structure accompanied by a division of physiological labour becomes more and more 
marked, some cells giving rise to organs of locomotion, others to organs of reproduction, and so on. But every function necessary for the life of an animal-or a plant-is due in the first instance to protoplasm, and a simple cell, like the Amoba, can perform them all.

In the next two chapters we shall study certain other unicellular organisms which show an advance on Amceba in possessing a certain amount of morphological and physiological differentiation. But the structural differentiations, as they are merely parts of one cell, cannot be spoken of as "organs" in the sense in which we have used the word hitherto, as they are not composed of numerous cells. They are, however, organs in the physiological sense, as they perform different functions.

\section{PRACTICAL DIRECTIONS. ${ }^{1}$}

\section{Amcba.}

Examine a drop of water containing Amobre, from the bottom of a pond, with the low power, first putting on a cover-glass: if the water does not contain small particles of sand or mud, place a small piece of paper under the edge of the cover so as to avoid crushing the organisms. When you have found a specimen, put on the high power, and note- I. The irregular and changing form of the animal, the protoplasm running out into blunt pseredopods.

2. The granular character of the protoplasm, the granules usually

${ }^{1}$ You should, if possible, try and obtain specimens of Amœbæ and the other fresh-water organisms described in this and the two following chapters for yourself, by collecting stagnant pond-water, together with a little of the mud at the bottom and sone water-weeds, and letting it stand for a few days in a glass jar or bottle. If you are unable to find the organisms you require, they, as well as most of the other animals described in this book, may be obtained from dealers in Natural History objects (see e.g. the advertisements in Nature). 
not extending to the periphery, so that a clear ectoplasm can be distinguished from a granular endoplasm. The granules rencler the flowing movements of the protoplasm visible.

3. The food-vacuoles in the protoplasm, containing fluid, and often also food particles.

4. The contractile vacuole, containing fluid, and its rhythmical contractions.

5. The protrusion and retraction of the psendopods. Sketch a specimen several times at short intervals, noting the direction in which the granules flow.

6. Look out for specimens undergoing mueltiplication by binary fission, and also for encysted individuals.

7. Run a little dry carmine or indigo under the cover-glass, and note that the particles can be taken in at all parts of the surface.

8. Stain with methyl-green (see p. I2I). This will kill the animal, and render the mucleus distinct.

9. Permanent preparations, showing the nucleus, may be made on the slide as follows :-

Place a drop of water containing Amœbæ on a slide, and soak up with blotting-paper as much of the water as is possible without carrying the Amobre along with it. Fix (see p. 136) with a drop of absolute alcohol, stain (a staining-fluid called picrocarmine is better than borax-carmine for this purpose), wash carefully with weak and then with absolute alcohol, and add a drop of turpentine-or better, oil of cloves. Soak off the excess of oil of cloves with blotting-paper, and mount in Canada balsam. 


\section{CHAPTER II}

HÆMATOCOCCUS AND EUGLENA-MONADS AND BACTERIADIFFERENCES BETWEEN ANIMALS AND PLANTS-SAPROPHYTES.

THE rain-water which collects in puddles, open gutters, \&c., is frequently found to have a green or red colour. The colour is due to the presence of various organismsplants or animals-one of the commonest of which is Hamatococcus (or, as it is sometimes called, Protoccocus or Spharella) pluvialis.

Like Amœba, Hæmatococcus is so small as to require a high power for its examination. Magnified three or four hundred diameters it has the appearance (Fig. 68, A) of an ovoidal body, somewhat pointed at one end, and of a bright green colour, more or less flecked with equally bright red.

Like Amcba, moreover, it is in constant movement, but the character of the movement is very different in the two cases. An active Hæmatococcus is seen to swim about the field of the microscope in all directions and with considerable apparent rapidity. We say apparent rapidity because the rate of progression is magnified to the same extent as the organism itself, and what appears a racing speed under the microscope is actually a very slow crawl 
when divided by 300. It has been found that such organisms as Hæmatococcus travel at the rate of one foot in from a quarter of an hour to an hour; or, to express

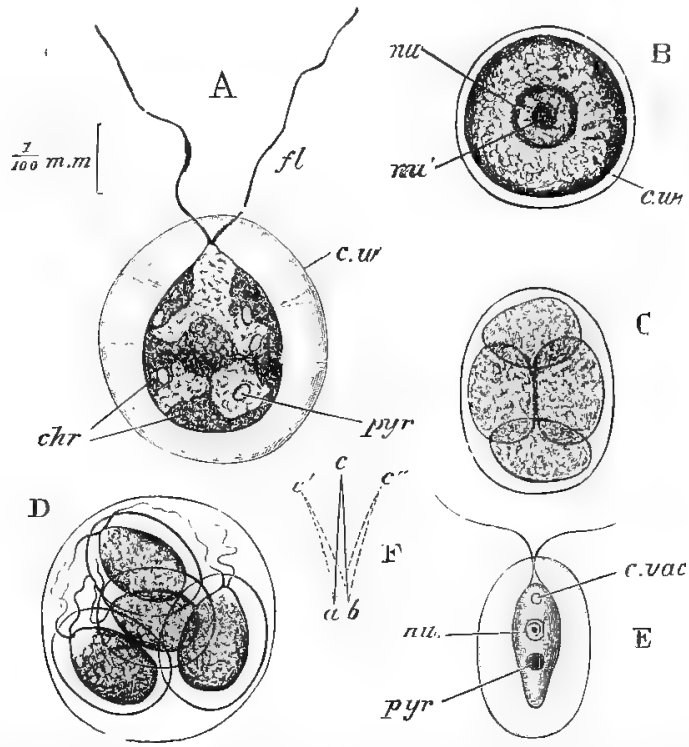

Fic. 68.-A, Hlematococons plaviaiis, motile phase. Living specimen, showins protoplasm with chromatophores $(c k r)$ and pyrenoids ( $\left.\beta y^{\prime} r\right)$, cell-wall $(c$. w) connected to cell-body by protoplasmic filaments, and flagella $(A)$. The scale to the left applies to Figs. A-D.

$\mathrm{B}$, resting stage of the same, showing nucleus ( $(u n)$ with nucleolus (mu'), and thich cell-wall $(c . v o)$ in contact with the protoplasm.

C, the same, showing division of the cell-body in the resting stage into four daughter-iells.

$\mathrm{D}$, the same, showing the development of flagella and detached cell-wall by the daughter cells before their liberation from the enclosing mother-cell-wall.

E, Ifematococcus lacustris, showing nucleus $(n u)$, single large pyrenoid $(\not p y)$, and contractile vacuole $(c, v a c)$,

$F$, diagram illustrating the movement of a flagellum; $a b$. its base; $c, c^{\prime}, c^{\prime \prime}$. different positions assumed by its apex. (From Parker's Biology: It, after Buitschli.)

the fact in another and fairer way, that they travel a distance equal to two and a half times their own diameter in one second. In swimning the pointed end is always directed 
forwards, and the forward movement is accompanied by a rotation of the organism upon its longer axis.

Careful watching shows that the outline of a swimming Hæmatococcus does not change, so that there is evidently no protrusion of pseudopods, and at first the cause of the movement appears rather mysterious. Sooner or later, however, the little creature is sure to come to rest, and there can then be seen projecting from the pointed end two excessively delicate colourless threads (Fig. 68, A, $f$ ), each about half as long again as the organism itself; these resemble the cilia on the epithelial cells lining the frog's mouth (p. 1 og), except that they are few in number, and do not vibrate rhythmically; they are therefore usually distinguished as flagella. In a Hæmatococcus which has come to rest these can often be seen gently waving from side to side: when this slow movement is exchanged for a rapid one the whole organism is propelled through the water, the flagella acting like a pair of extremely fine and flexible fins or paddles. Thus the movement of Hæmatococcus is not amoboid, i.e., produced by the protrusion and withdrawal of pseudopods, but is ciliary, i.e., due to the rapid vibration of cilia or flagella.

By staining and other tests it is shown that Hæmatococcus, like Amokba, consists of protoplasm, and that the flagella are simply filamentous processes of the protoplasm.

The green colour of the body is due to the presence of a special pigment called chlorophyll, the substance to which the colour of leaves is due. That this is something quite distinct from the protoplasm may be seen by treatment with alcohol, which simply kills and coagulates the protoplasm, but completely dissolves out the chlorophyll, producing a clear green solution. The solution, although green by transmitted light, is red under a strong reflected light, 
and is hence fluorescent: when examined through the spectroscope it has the effect of absorbing the whole of the blue and violet end of the spectrum as well as a part of the red. The red colour which occurs in so many individuals, sometimes entirely replacing the green, is due to a colouring matter closely allied in its properties to chlorophyll, and called hamatochrome.

At first sight the chlorophyll appears to be evenly distributed over the whole body, but accurate examination under a high power shows it to be lodged in a variable number of irregular structures called chromatophores (Fig. 68, A, chr), which together form a layer immediately beneath the surface. Each chromatophore consists of a protoplasmuc substance impregnated with chlorophyll.

After solution of the chlorophyll with alcohol a nucleus $(\mathrm{B}, n u)$ can be made out; like the nucleus of Amœba, it is rendered more distinct by staining. Other bodies which might easily be mistaken for nuclei are also visible in the living organism. These are small ovoidal structures (A, pyr) with clearly defined outlines occurring in varying numbers in the chromatophores. When treated with iodine they assume a deep, apparently black, but really dark blue colour. The assumption of a blue colour with iodine is the characteristic test of the carbohydrate, $\operatorname{starch}(p .72)$, as can be seen by letting a few drops of a weak solution of iodine fall upon some ordinary washing starch. The bodies in question have been found to consist of a proteid substance covered with a layer of starch, and are called pyrenoids.

In Hæmatococcus pluvialis there is no contractile vacuole, but in another species, $H$. lacustris, this structure is present as a minute space near the anterior or pointed end (Fig. 68, $\mathrm{E}, c . v a c)$.

There is still another characteristic structure to which no 
reference has yet been made. This appears at the first view something like a delicate haze round the red or green body, but by careful focussing is seen to be really an extremely thin globular shell $(\Lambda, c . z v)$, composed of some colourless transparent material, and separated, by a space containing water, from the body, to which it is connected by very delicate radiating strands of protoplasm. It is perforated by two extremely minute apertures for the passage of the flagella. Obviously we may consider this shell as a cyst or cellwall, differing from that of an encysted Amœba (Fig. 67, D) in not being in close contact with the protoplasm.

A more important difference, however, lies in its chemical composition. The cyst or cell-wall of Amœba, as stated in the preceding chapter (p. 232), is very probably nitrogenous; that of Hæmatococcus, on the other hand, is formed of a carbohydrate called cellullose, allied in composition to starch, sugar, and gum, and, like starch, having the formula $\mathrm{C}_{6} \mathrm{H}_{10} \mathrm{O}_{5}$. Many vegetable substances, such as cotton, consist of cellulose, and wood is a modification of the same compound. Cellulose is stained yellow by iodine, but iodine and sulphuric acid together turn it blue, and a similar colour is produced by a solution of iodine and potassium iodide in zinc chloride known as Schulze's solution. These tests are quite easily applied to Hæmatococcus: the protoplasm stains a deep yellowish brown, around which is seen a sort of blue cloud, due to the stained and partly-dissolved cell-wall.

It has been stated that in stagnant water in which it has been cultivated for a length of time Hæmatococcus sometimes assume an amoboid form. In any case, after leading an active existence for a longer or shorter time, it comes to rest, loses its flagella, and forms a thick cellwall of cellulose (Fig. 68, B), thus becoming encysted. So 
that, as in Amoba, there is an alternation of an active or motile with a stationary or resting condition.

In the matter of nutrition, the differences between Hrematococcus and Amoba are very marked, and indeed fundamental. As we have seen, Hæmatococcus has no pseudopods, and therefore cannot take in solid food after the manner of Amœba; moreover, even in its active condition, it is usually surrounded by a cell-wall, which of course quite precludes the possibility of ingestion. As a matter of observation, also, however long it is watched it is never seen to feed in the ordinary sense of the word. Nevertheless it must take in food in some way or other, or the decomposition of its protoplasm would soon bring it to an end.

Hæmatococcus lives in rain-wațer. This is never pure water, but always contains certain mineral salts in solution, especially nitrates, ammonia salts, and often sodium chloride or common table-salt. These salts can and do diffuse into the water which is a constituent part of the protoplasm of the organism, so that we may consider its protoplasm to be constantly permeated by a very weak saline solution, the most important elements contained in which are oxygen, hydrogen, nitrogen, potassium, sodium, calcium, sulphur, and phosphorus. It must be remarked, however, that the diffusion of these salts does not take place in the same uniform manner as it would through parchment or other dead membrane. The living protoplasm has the power of determining the extent to which each constituent of the solution shall be absorbed.

If water containing a large quantity of Hæmatococcus is exposed to sunlight, minute bubbles are found to appear in it, and these bubbles, if collected and properly tested, are found to consist largely of oxygen. Accurate chemical 
analysis has shown that this oxygen is produced by the decomposition of the carbon dioxide contained in solution in rain-water, and incleed in all water exposed to the air ; the gas, which is always present in small, quantities in the atmosphere, being very soluble in water.

As the carbon dioxide is decomposed in this way, its oxygen being given off, it is evident that its carbon must be retained. As a matter of fact it is retained by the organism, but not in the form of carbon; in all probability a double decomposition takes place between the carbon dioxide absorbed and the water contained in its protoplasm, the result being the liberation of oxygen in the form of gas and the simultaneous production of some extremely simple form of carbohydrate, i.e., some compound of carbon, hydrogen and oxygen with a comparatively small number of atoms to the molecule.

The next step seens to be that the carbohydrate thus formed unites with the ammonia salts or the nitrates absorbed from the surrounding water, the result being the formation of some comparatively simple nitrogenous compound. Then further combinations take place, substances of greater and greater complexity are produced, sulphur from the absorbed sulphates enters into combination, and proteids are formed. From these, finally, fresh living protoplasm arises.

From the foregoing account, which only aims at giving the very briefest outline of a subject as yet imperfectly understood, it will be seen that, as in Amœba, the final result of the nutritive process is the manufacture of protoplasm, and that this result is attained by the formation of various substances of increasing complexity. But it must be noted that the steps in this process of constructive metabolism are widely different in the two cases. In Amœba we start 
with living protoplasm-that of the prey--which is killed and broken up into diffusible proteids, these being afterwards re-combined to form now molecules of the living protoplasm of Amœba. So that the food of $\Lambda$ moeba is, to begin with, as complex as itself, and is first broken down by digestion into simpler compounds, these being afterwards re-combined into more complex ones. In Hæmatococcus, on the other hand, we start with extremely simple compounds, such as carbon dioxide, water, nitrates, sulphates, \&c. Nothing which can be properly called digestion, i.e., a breaking up and dissolving of the food, takes place, but its various constituents are combined into substances of gradually increasing complexity, protoplasm, as before, being the final result.

To express the matter in another way: Amœba can only make protoplasm out of proteids already formed by some other organism: Hæmatococcus can form it out of simple liquid and gaseous inorganic materials.

Speaking generally, it may be said that these two methods of nutrition are respectively characteristic of the two great groups of living things. Animals require solid food con. taining ready-made proteids, and cannot build up their protoplasm out of simpler compounds. Green plants, i.e., all the ordinary trees, shrubs, weeds, \&c., take only liquid and gaseous food, and build up their protoplasm out of carbon dioxide, water, and mineral salts. The first of these methods of nutrition is conveniently distinguished as holozoic, or wholly-animal, the second as holophytic, or wholly-vegetal.

It is important to note that only those plants or parts of plants in which chlorophyll is present are capable of holophytic nutrition. Whatever may be the precise way in which the process is effected, it is certain that the decomposition of carbon dioxide which characterises this form of nutrition 
is a function of chlorophyll, or to speak more accurately, of chromatophores, since there is reason for thinking that it is the protoplasm of these bodies and not the actual green pigment which is the active agent in the process.

Moreover, it must not be forgotten that the decomposition of carbon dioxide is carried on only during daylight, so that organisms in which holophytic nutrition obtains are dependent upon the sun for their very existence. While Amoba derives its energy from the breaking down of the proteids in its food (see p. 235), the food of Hæmatococcus is too simple to serve as a source of energy, and it is only by the help of sunlight that the work of constructive metabolism can be carried on. This may be expressed by saying that Hæmatococcus, in common with other organisms containing chlorophyll, is supplied with kinetic energy (in the form of light or radiant energy) directly by the sun.

As in Amoba, destructive metabolism is constantly going on side by side with constructive. The protoplasm becomes oxidised, water, carbon dioxide, and nitrogenous waste matters being formed and finally got rid of. Obviously then, absorption of oxygen must take place, or in other words, respiration must be one of the functions of the protoplasm of Hæmatococcus as of that of Amœba. In many green, i.e., chlorophyll-containing, plants, this has been proved to be the case : respiration, i.e., the taking in of oxygen and giving out of carbon dioxide, is constantly going on, but during daylight is obscured by the converse process-the taking in of carbon dioxide for nutritive purposes and the giving out of the oxygen liberated by its decomposition. In darkness, when this latter process is in abeyance, the occurrence of respiration is more readily ascertained.

Owing to the constant decomposition, during sunlight, of carbon dioxide, a larger volume of oxygen than of carbon 
dioxide is evolved; and if an analysis were made of all the ingesta of the organism (carbon dioxide plus mineral salts flus respiratory oxygen) they would be found to contain less oxygen than the egesta (oxygen from decomposition of carbon dioxide plus water, excreted carbon dioxide, and nitrogenous waste) ; so that the nutritive process in Hæmatococcus is, as a whole, a process of deoxidation. In Amœba, on the other hand, the ingesta (food plus respiratory oxygen) contain more oxygen than the egesta (fæces plus carbon dioxide, water, and nitrogenous excreta), the nutritive process being therefore on the whole one of oxidation. This difference is, speaking broadly, characteristic of plants and animals generally; animals, as a rule, take in more free oxygen than they give out, while green plants always give out more than they take in.

But destructive metabolism is manifested not only in the formation of waste products, but in that of substances simpler than protoplasm which remain an integral part of the organism, viz., cellulose and starch. The cell-wall is probably formed by the conversion of a thin superficial layer of protoplasm into cellulose, the cyst attaining its final thickness by frequent repetition of the process. The starch of the pyrenoids is apparently formed by a similar process of decomposition or destructive metabolism of protoplasm.

We see then that destructive metabolism may result in the formation of (a) waste products and (b) plastic products, the former being got rid of as of no further use, while the latter remain an integral part of the organism.

Let us now turn once more to the movements of Hæmatococcus, and consider in some detail the manner of their performance. 
Each flagellum (Fig. 68, A, $f$ ) is a thread of protoplasm of uniform diameter except at its distal or free end, where it tapers to a point. The lashing movements are brought about by the flagellum bending successively in different directions ( $\mathrm{F}$ ). Thus the ciliary movement of Hæmatococcus, like the amœboid movement of Amcba, is a phenomenon of contractility. Imagine an Amœba to draw in all its pseudopods but two, and to protrude these two until they became mere threads; imagine further these threads to contract rapidly and more or less regularly instead of slowly and irregularly; the result would be the substitution of pseudopods by flagella, i.e., of temporary slow-moving processes of protoplasm by permanent rapidly-moving ones.

To put the matter in another way: in Amœba the function of contractility is performed by the whole organism; in Hæmatococcus it is discharged by a small part only, viz., the flagella, the rest of the protoplasm being incapable of movement.

Hæmatococcus multiplies after becoming quiescent or in the encysted condition (Fig. 68, C, D) ; as in Amoba its protoplasm undergoes simple or binary fission, but with the peculiarity that the process is immediately repeated, so that four daughter-cells are produced within the single mothercell-wall. By the rupture of the latter the daughter-cells are set free as the ordinary motile form, acquiring their flagella and-detached cell-wall before making their escape (D).

Under certain circumstances the resting form divides into eight or more instead of four daughter-cells, and these when liberated are found to be smaller than the ordinary motile form, and to have no cell-wall. Hæmatococcus therefore occurs, in the motile condition, under two distinct forms, i.e., is dimorphic: the larger or ordinary form with detached 
cell-wall is called a megazooid, the smaller form without a cell-wall a microzooid.

We will now examine another small organism which is often found in puddles and pools, frequently in such vast numbers as to give the water a green colour. This organism is known as Euglena viridis.

Euglena is also microscopic, its length varying from $\frac{1}{9}-\mathrm{mm}$. to $\frac{1}{6} \mathrm{~mm}$. The body is spindle-shaped, wide in the middle and narrow at both ends (Fig. 69, $A-\mathrm{E}$ ) : one extremity is blunter than the other, and from it proceeds a single long flagellum $(f)$ by the action of which the organism swims with great rapidity, the flagellum being, as in Hæmatococcus, directed forwards. Besides its rapid swimming movements, Euglena frequently performs slow movements of contraction and expansion, something like those of a short worm, the body becoming broadened out first at the anterior end, then in the middle, then at the posterior end, twisting to the right and left, and so on $(\Lambda-D)$. These movements are so characteristic of the genus that the name euglenoid is applied to them.

The body consists of protoplasm covered with a very delicate membrane or cuticle which is often finely striated, and is to be looked upon as a superficial hardening of the protoplasm. The green colour is due to the presence of chlorophyll, which tinges all the central part of the body, the two ends being colourless. It is difficult to make out whether the chlorophyll is lodged in one chromatophore or in several.

In Hæmatococcus we saw that chlorophyll was associated with starch $(p, 243)$. In Euglena there are, near the middle of the body, a number of grains of paramylum $(\mathrm{H}, p)$, a carbohydrate of the same composition as starch 
$\left(\mathrm{C}_{6} \mathrm{H}_{10} \mathrm{O}_{5}\right)$, but differing from it in remaining uncoloured by iodine.
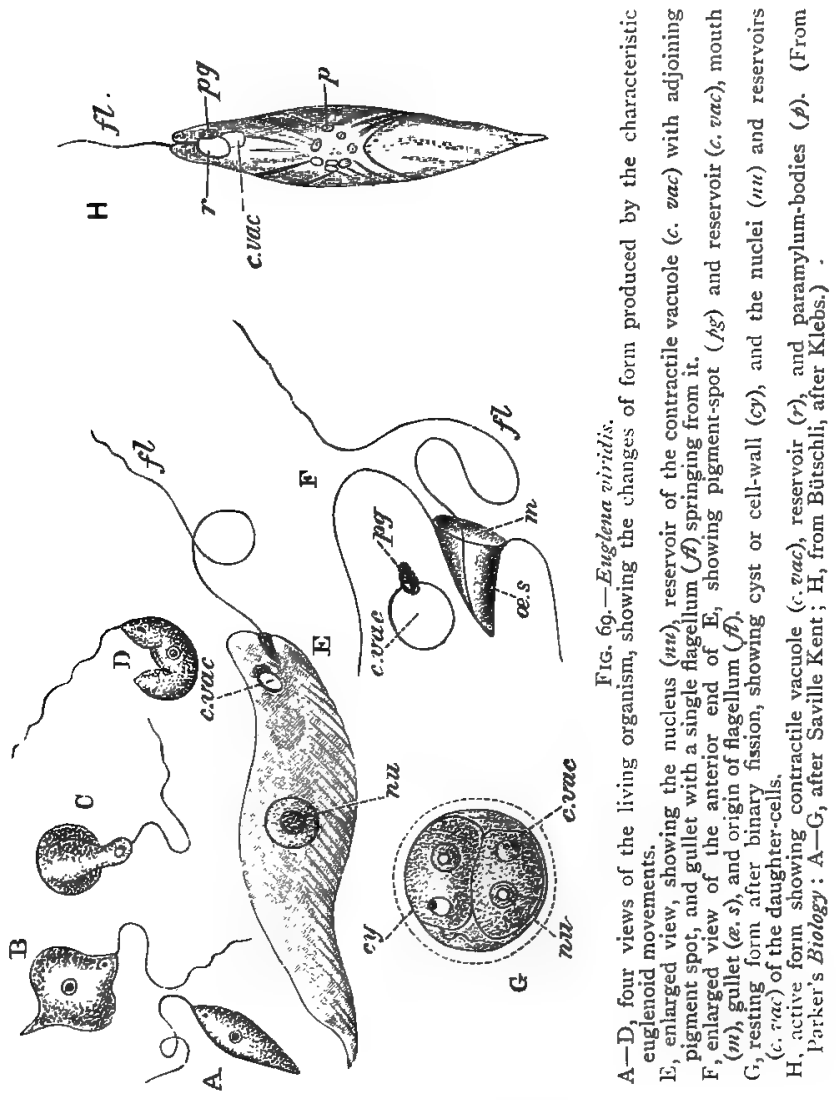

Water containing Euglena gives off bubbles of oxygen in sunlight : as in Hæmatococcus the carbon dioxide in solution in the water is decomposed in the presence of chlorophyll, 
its oxygen evolved, and its carbon combined with the elements of water and used in nutrition. For a long time Euglena was thought to be nourished entirely in this way, but there is a good deal of reason for thinking that this is not the case.

When the anterior end of a Euglena is very highly magnified it is found to have the form shown in Fig. 69, $\mathrm{r}$. It is produced into a blunt snout-like extremity, at the base of which is a conical depression ( $a . s$ ) leading into the soft internal protoplasm :-just the sort of depression one could make in a clay model of Euglena by thrusting one's finger or the end of a pencil into the clay. From the bottom of this tube the flagellum arises, and by its continual movement gives rise to a sort of whirlpool in the neighbourhood. By the current thus produced, minute solid food-particles are swept down the tube and forced into the soft internal protoplasm, where they doubtless become digested in the same way as the substances ingested by an Amceba. That solid particles are so ingested by Euglena has been proved by diffusing finely powdered carmine in the water, when the coloured particles were seen to be swallowed in the way described.

The depression in question serves therefore as a gullet, and its external aperture or margin $(m)$ as a mouth. Euglena, like Amoba, takes in solid food, but instead of ingesting it at almost any"part of the body, it can do so only at one particular point where there is a special ingestive aperture or mouth. This is clearly a case of specialisation or differentiation of structure : in virtue of the possession of a mouth and gullet, Euglena is more highly organised than Amceba.

It thus appears that in Euglena nutrition is both holozoic and holophytic (p. 247): very probably it is mainly holophytic during daylight and holozoic in darkness. 
Near the centre of the body or somewhat towards the posterior end is a nucleus (E, $n u$ ) with a well-marked nucleolus, and at the anterior end is a clear space $(c, v a c)$, looking very like a contractile vacuole. It has been shown, however, that this space is in reality a non-contractile cavity or reservoir $(\mathrm{H}, r)$ into which the true contractile vacuole (c. vac) opens, and which itself discharges into the gullet.

In close relation with the reservoir is found a little bright red speck $(p g)$, called the pigment spot or stigma. It consists of hæmatochrome (p. 243), and is curiously like an eye in appearance, so much so that it is often known as the eye-spot. Recent experiments seem to show that it is specially sensitive to light, and is therefore a true eye in the sense of a light-perceiving organ, although having no actual visual function.

As in Hæmatococcus a resting condition alternates with the motile phase: the organism loses its flagellum and surrounds itself with a cyst of cellulose $(G, c y)$, from which, aftêr a period of rest, it emerges to resume active life.

Reproduction takes place by simple binary fission of the resting form, the plane of division being always longitudinal (G). Sometimes each product of division or daughter-cell divides again: finally the two, or four, or sometimes even eight daughter-cells emerge from the cyst as active Euglenæ. A process of multiple fission has also been described, numerous, simple, minute, active bodies or spores being produced, which gradually assume the ordinary form and size.

We have seen that typical animal-cells, such as those of the frog (Part I, Chap. VII) are not provided with a cellulose cell-wall and do not contain chlorophyll. It is 
characteristic, on the other hand, of most plant-cells-which also consist of nucleated protoplasm-that they are surrounded with a cellulose cell-wall, and that, in the case of green plants, they contain chlorophyll. Speaking generally, the nutrition of animals is holozoic, and that of green plants holophytic; and in correspondence with this difference in the character of the food, most animals have an ingestive aperture or mouth for taking in the solid food, and some kind of digestive cavity, either permanent (stomach), or temporary (food-vacuole); they also have, as a rule, some kind of excretory apparatus. Moreover, animals are usually capable of automatic movement, while in most plants the organism, as a whole, exhibits no automatism, but only the slow movements of growth.

Let us now apply these definitions to the simple forms described above and see how far they will help us in placing those organisms in one or other of the two "kingdoms" (p. 220) into which living things are divided.

Amceba has a cell-wall, probably nitrogenous, in the resting condition: it ingests solid proteids, its nutrition being therefore holozoic: it has a contractile vacuole : and it performs amoboid movements. It may therefore be safely considered as an animal.

Hæmatococcus has a cellulose wall : it contains chlorophyll and its nutrition is purely holophytic: a contractile vacuole is present in $\mathrm{H}$. lacustris but absent in $\mathrm{H}$. pluvialis : and its movements are ciliary.

Euglena has a cellulose wall in the encysted state : in virtue of its chlorophyll it is nourished by the absorption of carbon dioxide and mineral salts, but it can also ingest solid food through a special mouth and gullet: it has a contractile vacuole, and performs both euglenoid and ciliary movements. 
In both these organisms we evidently have conflicting characters: the cellulose wall and holophytic nutrition would place them both among plants, while from the contractile vacuole and active movements of both genera, and from the holozoic nutrition of Euglena, we should group them with animals. That the difficulty is by no means easily overcome may be seen from the fact that both genera are claimed at the present day both by zoologists and by botanists.

Another mode of nutrition occurs in certain organisms which must now be referred to very briefly.

When animal or vegetable matter is placed in water and allowed to stand at the ordinary temperature, the well-known process of decomposition or putrefaction (p. II and I52) sooner or later sets in, the water becoming turbid and acquiring a bad smell. A drop of it examined under the nicroscope is then found to teem with very minute unicellular organisms, some of which are known as Monads, much smaller than Euglena. Like Hæmatococcus, the Monad swims about by means of two flagella, but it contains no chlorophyll. The putrefying infusion in which it lives contains proteids in solution, in part split up by the process of decomposition into simpler compounds, some of which are diffusible. As the Monad contains no chlorophyll, its nutrition is evidently not holophytic, and, apart from the fact that it possesses neither mouth nor pseudopods, observation seems to show pretty conclusively that it is not holozoic.

There remains only one way in which nutrition can take place, namely, by absorption of the proteids and other nutrient substances in the solution: the Monad may be said to live immersed in an immense cauldron of broth which it is for ever imbibing, not by its mouth, for it has none, but by the whole surface of its body. This is the saprophytic 
mode of nutrition, and resembles that which occurs in certain plants which contain no chlorophyll-the fungi. It will be seen that the main difference between saprophytic and holozoic nutrition is that in the former digestion, i.e., the process of rendering food-stuffs soluble and diffusible, takes place outside the body, so that constructive metabolism can begin at once.

In the Monad, the characters are again conflicting: the probable absence of cellulose, the presence of a contractile vacuole, and the flagella all have an "animal" look, but the mode of nutrition is that of a fungus.

Decomposition, as already stated (p. 1 $5^{2}$ ), is due in the first instance to the action of certain other minute organisms, known as Bacteria, which appear in the earlier stages of the putrefaction of an organic infusion. The nutrition of these organisms, like that of Monads, is usually saprophytic and the novements are generally ciliary; but as they have a definite cell-wall and no contractile vacuole, they are almost universally classed amongst plants, while Monads are as constantly included in the animal kingdom.

We see then that while it is quite easy to divide the higher organisms into the two distinct groups of plants and animals, any such separation is by no means easy in the case of the lowest forms of life. It was in recognition of this fact that Haeckel proposed, many years ago, to institute a third " kingdom," called Protista, to include all unicellular organisms. Although open to many objections in practice, there is a great deal to be said for the proposal. From the strictly scientific point of view it is quite as justifiable to make three subdivisions of living things as two: the line between animals and plants is quite as arbitrary as that between protists and plants or between protists and animals, and no more so: the chief objection to the change is that it 
doubles the difficulties by making two artificial boundaries instead of one.

The important point for the student to recognise is that these boundaries are artificial, and that there are no scientific frontiers in Nature. As in the liquefaction of gases there is a "critical point" at which the substance under experiment is neither gaseous nor liquid: as in a mountainous country it is impossible to say where mountain ends and valley begins: as in the development of an animal it is futile to argue about the exact period when, for instance, the egg becomes a tadpole or the tadpole a frog: so in the case under discussion. The distinction between the higher plants and animals is perfectly sharp and obvious, but when the two groups are traced downwards they are found gradually to merge, as it were, into an assemblage of organisms which partake of the characters of both kingdoms, and cannot without a certain violence be either included in or excluded from either. When any given "protist" has to be classified the case must be decided on its individual merits : the organism must be compared in detail with all those which resemble it closely in structure, physiology, and life-history: and then a balance must be struck and the doubtful form placed in the kingdom with which it has, on the whole, most points in common.

It will no doubt occur to the reader that, on the theory of evolution (p. 222), we may account for the fact of the animal and vegetable kingdoms being related to one another like two trees united at the root, by the hypothesis that the earliest organisms were protists, and that from them animals and plants were evolved along divergent lines of descent. 


\section{PRACTICAL DIRECTIONS.}

Hæmatococcus. Examine a drop of water containing Hæmatococcus, first with the low power, and then, after putting on a coverglass, with high power. Note-

I. Their rounded form and green (or red) colour ; the thick cellulose cell-wall; the protoplasm enclosing (a) chromatophores, containing chlorophyll (the red colour is due to another colouring matter, hamatochrome), and (b) a central nucleus, seen better later; and in the active forms, the two flagella. Sketch.

2. Dissolve out the chlorophyll by adding alcohol ; the nucleus will then be visible, and may be made more distinct by staining with methyl-green, magenta, or iodine. After treatment with iodine, a bluish colouration will be seen around the small starch-containing pyrenoids.

3. Treat some specimens either with strong iodine solution and then with sulphuric acid ( 75 per cent.), or with Schulze's solution, which is prepared as follows :-Make about $25 \mathrm{cc}$. of a syrupy solution of zinc chloride, and let it stand for a short time ; then pour off the clear fluid, saturate it with potassium iodide and add iodine until the solution has about the colour of sherry. Note the colouration of the cell-wall.

4. In the living specimens note also the mode of division into 4 megazooids or into 16 or 32 microzooids, and observe their movements when set free. Sketch.

Euglena. Examine a drop of water containing Euglena with the low power, then cover, and put on the high power. Note-

I. The spindle-shaped form of the body, and its changes of form in contraction and expansion.

2. The long flagellum.

3. The superficial cuticle, and the mouth and conical depression (gullet) at the anterior end.

4. The central part of the body, which contains chlorophyll, except at the two ends. Near the middle is a nucleus enclosing a nucleolus, and near the anterior end a non-contractile space into which a contractile vacuole opens, close to which a red pigment-spot is situated; the colour of this is due to hæmatochrome. Grains of paramylum may be recognised near the centre of the. body. Sketch before and after staining as above. 
5. Look for specimens in the resting condition, and observe if any of them are undergoing division. Sketch.

Racteria and Monads. Examine a drop of water containing some putrefying animal or vegetable substance. An infusion of hay is convenient for this purpose :-pour boiling water on a handful of hay and strain the fluid through blotting-paper; shortly afterwards decomposition will set in.

The first organisms to appear in such a putrefying infusion are Bacteria, which are so minute as to appear as mere specks under the high power -some of them being only $\frac{1}{50} \mathrm{~mm}$. ( $\frac{1}{2} \frac{1}{500}$ inch) in length, or even less. Careful examination will show globular, rod-like, and spiral forms, all capable of movement at one phase of their existence. Monads will appear somewhat later, and may be recognised by their ovoidal form and their two flagella, which, however, like those of bacteria, can only be seen under a very high power. 


\section{- CHAPTER III}

PARAMCECIUM, OPALINA, VORTICELLA AND ITS ALLIESPARASITIC AND COLONIAL ORGANISMS-BIOGENESIS AND ABIOGENESIS-CLASSIFICATION OF THE UNICELLULAR ORGANISMS EXAMINED.

WE have now to consider certain organisms in which differentiation has gone much further than in the unicellular forms already considered: which have, in fact, acquired many of the characteristics of the higher animals and plants while remaining unicellular (compare p. $23^{8}$ ). The study of several of these more or less highly differentiated though unicellular forms will occupy the present chapter.

It was mentioned above that, in the earlier stages of the putrefaction of an organic infusion, bacteria only were found, and that later, monads made their appearance (p. 257). Still later organisms much larger than monads are seen, generally of an ovoidal form, moving about very quickly, and seen by the use of a high power to be covered with innumerable fine cilia. These are called ciliate Infusoria, in contradistinction to monads which are often known as flagellate Infusoria: many kinds are common in putrefying infusions, some occur in the intestines of the higher animals 
while others are anong the commonest inhabitants of both fresh and salt water.

A very common ciliate infusor is the beautiful "slipper animalcule," Paramocium, which from its comparatively large size and from the ease with which all essential points of its organization can be made out is a very convenient and interesting object of study.

Compared with the majority of the organisms which have come under our notice it may fairly be considered as gigantic, being no less than $\frac{1}{5}-\frac{1}{4} \mathrm{~mm}$. $(200-260 \mu)$ in length: in fact it is just visible to the naked eye as a minute whitish speck.

Its form (Fig. 70, A) can be fairly well imitated by making out of clay or stiff dough an elongated cylinder rounded at one end and bluntly pointed at the other; then giving the broader end a slight twist; and finally making on the side rendered somewhat concave by the twist a wide shallow groove beginning at the broad end and gradually narrowing to about the middle of the body, where it ends in a tolerably deep depression.

The groove is called the buccal groove (A \& B, buc. gr): at the narrow end is a small aperture the mouth $(n t h)$, which, like the mouth of Euglena (Fig. 69), leads into the soft internal protoplasm of the body. The surface of the creature on which the groove is placed is distinguished as the ventral surface, the opposite surface being upper or dorsal; the broad end is anterior, the narrow end posterior, the former being directed forwards as the animalcule swims. These descriptive terms being decided upon, it will be seen that the buccal groove begins on the left side of the body, and gradually curves over to the middle of the ventral surface. 

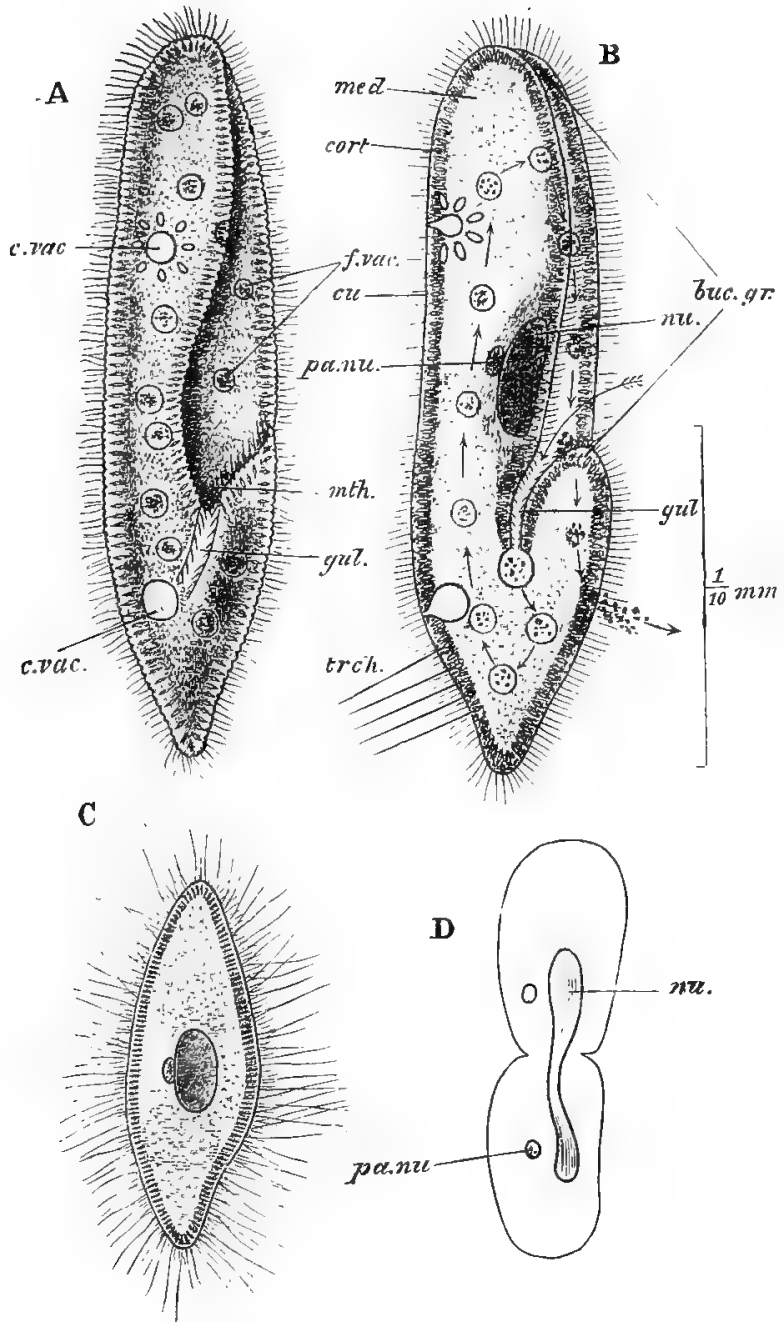

FIG. 7o.-Paramacium caudutum.

A, the living animal from the ventral aspect, showing the covering of cilia, the buccal groove (to the right) ending posteriorly in the mouth (mth) and gullet $(g u l)$; several food vacuoles $(f \cdot v a c)$, and the two contractile vacuoles (c. vac). 
$\mathrm{B}$, the same in optical sections showing cuticle (cus), cortex (cort), and medulla (mted); buccal groove (buc. $g r$ ), mouth, and gullet ( $g u l)$; numerous food vacuoles ( $f$. vac) circulating in the direction indicated by the arrows, and containing particles of indigo, which are finally ejected at an anal spot; meganucleus (nu), micronucleus (pa. $n u$ ), and trichocysts, some of which (trch) are shown with their threads ejected. The scale to the right of this figure applies to A and B.

$C$, a specimen killed with osmic acid, showing the ejection of trichocyst-threads, which project considerably beyond the cilia.

D. diagram of binary fission; the micronucleus ( $p a$. $u u$ ), has already divided, the meganucleus (nu) is in the act of dividing. (From Parker's_Biology: D, after Lankester.)

As the animal swims its form is seen to be permanent, exhibiting no contractions of either an amœboid or a euglenoid nature. It is however distinctly flexible, often being bent in one or other direction when passing between obstacles such as entangled masses of weed. This permanence of contour is due to the presence of a tolerably firm though delicate cuticle $(B, c u)$ which invests the whole surface.

The protoplasm thus enclosed by the cuticle is distinctly divisible into two portions-an external somewhat dense layer, the cortical layer or cortex (cort), and an internal more fluid material, the medullary substance or medulla (med). It will be remembered that a somewhat similar distinction of the protoplasm into two layers is exhibited by Amcba (p. $23 \mathrm{I}$ ), the ectoplasm being distinguished from the endoplasm simply by the absence of granules. In Paramœcium the distinction is a far more fundamental one: the cortex is radially striated and is comparatively firm and dense, while the medulla is granular and semi-fluid, as may be seen from the fact that food-particles ( $f$. vai, see p. 266) move freely in it, whereas they never pass into the cortex. The medulla has a reticular structure similar to that of the protoplasm of the ordinary animal-cell, consisting of a delicate granular network the meshes of which are filled with a transparent material. In the cortex the meshes of the network are closer, and so form a comparatively dense substance. The cortex also exhibits a superficial oblique striation, forming what is called the myophan layer. 
'The mouth (mth) leads into a short funnel-like tube; the gullet (gul), which is lined by cuticle and passes through the cortex to end in the soft medulla, thus making a free communication between the latter and the external water.

The cilia with which the body is covered are of approximately equal size, quite short in relation to the entire animal, and arranged in longitudinal rows over the whole outer surface. They consist of prolongations of the cortex, and each passes through a minute perforation in the cuticle. They are in constant rhythmical movement, like the cilia on the epithelial cells of the frog's mouth (p. I09), and are thereby distinguished from the flagella of Hæmatococcus, Euglena, \&c., which exhibit more or less intermittent lashing movements.

Near the middle of the body, in the cortex, is a large oval nucleus $(\mathrm{B}, . n u)$ which is peculiar in taking on a uniform tint when stained, showing none of the distinction into chromatin and achromatin which is so marked a feature in many of the nuclei we have studied. It has also a further peculiarity : against one side of it in $P$. caudatum is a small oval structure $(p a . n u)$ which is also deeply stained by, e.g., magenta or carmine. This is the micronucleus : it is to be considered as a second, smaller nucleus, the larger body being distinguished as the meganucleus. In the closely allied $P$. aurelia, there are two micronuclei.

There are two contractile vacuoles $(c . v a c)$, one situated at about a third of the entire length from the anterior end of the body, the other at about the same distance from the posterior end: they occur in the cortex.

The action of the contractile vacuoles is very beautifully seen in a Paramœecium at rest : it is particularly striking in a specimen subjected to slight pressure under a cover glass, but is perfectly visible in one which has merely temporarily 
suspended its active, swimming movements. It is then seen that during the diastole, or phase of expansion of each vacuole, a number-about six to ten-of delicate, radiating, spindle-shaped spaces filled with fluid appear round it, like the rays of a star (upper vacuole in A \& B): the vacuole itself contracts or performs its systole, completely disappearing from view, and immediately afterwards the radiating canals flow together and refill it, becoming themselves emptied and therefore invisible for an instant (lower vacuole in A \& B) but rapidly appearing once more. There seems to be no doubt that the water taken in with the food is collected into these canals, emptied into the vacuole, and finally discharged into the surrounding medium.

The process of feeding can be very conveniently studied in Paramœcium by placing in the water some finely-divided carmine or indigo. When the creature comes into the neighbourhood of the coloured particles, the latter are swept about in various directions by the action of the cilia: some of them however are certain to be swept into the neighbourhood of the buccal groove and gullet, the cilia of which all work downwards, i.e., towards the inner end of the gullet. The grains of carmine are thus carried into the gullet, where for an instant they lie surrounded by the water of which it is full : then, instantaneously, probably by the contraction of the tube itself, the animalcule performs a sort of gulp, and the grains with an enveloping globule of water or food-vacuole are forced into the medullary protoplasm. This process is repeated again and again, so that in any well-nourished Paramoecium there are to be seen numerous globular spaces filled with water and containing particles of food-or in the present instance of carmine or indigo. At every gulp the newly-formed food-vacuole pushes, as it were, its predecessor before it : contraction of the medullary pro- 
toplasm also takes place in a definite direction, and thus a circulation of food-vacuoles is produced, as indjcated in Fig. 70 в by arrows.

After circulating in this way for some time the water of the food-vacuoles is gradually absorbed, being ultimately excreted by the contractile vacuoles, so that the contained particles come to lie in the medulla itself (refer to figure). The circulation still continues, until finally the particles are brought to a spot situated about half way between the mouth and the posterior end of the body : here if carefully watched they are seen to approach the surface and then to be suddenly ejected. The spot in question is therefore to be looked upon as a potential anus, or aperture for the egestion of fres or undigested food-matters. It is a potential and not an actual anus, because it is not a true aperture but only a soft place in the cortex through which, by the contractions of the medulla, solid particles are easily forced.

Of course when Paramœcium ingests, as it usually does, not carmine but minute living organisms, the latter are digested as they circulate through the medullary protoplasm, and only the non-nutritious parts cast out at the anal spot. It has been found by experiment that this infusor can digest not only proteids but also starch and perhaps fats. The nutrition of Paramocium is therefore characteristically holozoic.

It was mentioned above (p. 264) that the cortex is radially striated in optical section. Careful examination with a very high power shows that this appearance is due to the presence in the cortex of minute spindle-shaped bodies (A and $\mathrm{B}, \operatorname{trch}$ ) closely arranged in a single layer and perpendicular to the surface. These are called trichocysts.

When a Paramœcium is killed, either by the addition of some poisonous reagent or by simple pressure of the cover 
glass, it frequently assumes a remarkable appearance. Long delicate threads suddenly appear, projecting from its surface in all directions (c) and looking very much as if the cilia had suddenly protruded to many times their original length. But these filaments have really, nothing to do with the cilia ; they are contained under ordinary circumstances in the trichocysts, probably coiled up; and by the contraction of the cortex consequent upon any sudden irritation they are projected in the way indicated. In Fig. 70 B, a few trichocysts (trch) are shown in the exploded condition, i.e., with the threads protruded. Most likely these bodies are weapons of offence like the very similar structures (nematocysts) found in polypes (see p. 295, Fig. 75).

Paramœcium multiplies by simple fission, the division of the body being always preceded by the elongation and subsequent division of the mega- and micronucleus (Fig. 70, D).

Conjugation (p. 237) also occurs, usually after multiplication by fission has gone on for some time. Two Paramocia come into contact by their ventral faces, and in each of these conjugating individuals or gametes the meganucleus and micronucleus undergo a somewhat complicated series of changes, the essential part of the process being the fusion of two products of the division of the micronuclei, one from each gamete, each of which then contains a single nuclear body, the conjugation-nucleus, formed by the union of nuclear matter derived from two distinct individuals, and therefore comparable to the nucleus of the oosperm in the higher animals (p. 197). In this case, however, the two entire gametes do not unite into one, but separate after the process is complete and begin once more to lead an independent existence, when once more ordinary transverse fission takes place. 
It will be noticed that, in the present instance, conjugation is not a process of multiplication: it has been ascertained that during the time two infusors are conjugating each might have produced several thousand offspring by continuing to undergo fission at the usual rate. The importance of the process lies in the exchange of nuclear material between the two conjugating individuals: without such exchange these organisms are said to undergo a gradual process of senile decay characterized by diminution in size and degeneration in structure.

Some ciliate Infusoria are parasites. Parasites are organisms which live in association with other organisms, the readydigested food of which they utilize or even nourish themselves from the tissues of the forms they infest. It will be interesting to compare Paramœcium with an Infusor which lives in the intestine of the common frog, and is known as Opalina ranarum.

Opalina has a flattened body with an oval outline (Fig. 7 r, $\mathrm{A}, \mathrm{B})$, and full-sized specimens may be as much as one millimetre in length. The protoplasm is divided into cortex and medulla, and is covered with a cuticle; the cilia are equal-sized and uniformly arranged in longitudinal rows over the whole surface.

On a first examination no nucleus is apparent, but after staining, a large number of globular nucleican be $\operatorname{seen}(\mathrm{B}, n u)$ : these nuclei multiply within the body of the infusor.

The presence of numerous nuclei in Opalina is a fact worthy of special notice. The unicellular organisms we have studied are uninucleate as well as unicellular (compare, however, Fig. $67, \mathrm{~B}$ ) : the higher animals consist of numerous cells each with a nucleus: Opalina, on the other hand, is multinucleate but its protoplasm is undivided, so that it 
presents a condition of things intermediate between the unicellular and the multicellular types of structure.
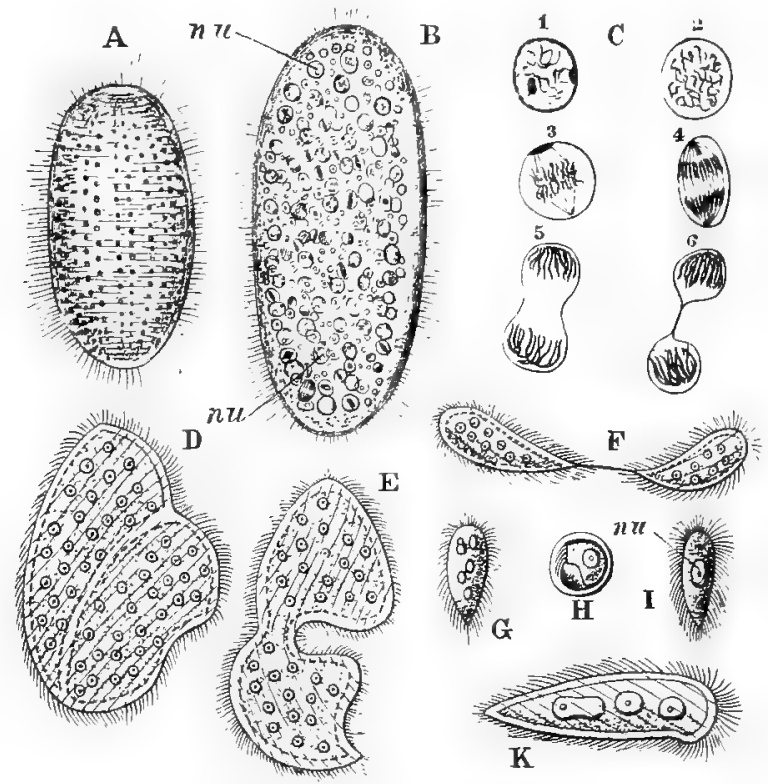

FiG. 71.-Opalina ranarum.

A, living specimen, surface view, showing longitudinal rows of cilia.

$B$, the same, stained, showing numerous nuclei $(n u)$ in various stages of division.

$C, 1-6$, stages in the division of a nucleus.

$\mathrm{D}$, longitudinal fission.

E, transverse fission.

$\mathbf{F}$, the same in a specimen reduced in size by repeated division.

$G$, final product of successive divisions.

$H$, encysted form.

I, uninucleate form produced from cyst.

$\mathbf{K}$, the same after multiplication of the nucleus bas begun. (From Parker's Biology: $A-C$, after Pfitzner ; D-K, from Saville Kent, after Zeller.)

There is no contractile vacuole, and no trace of either mouth or guilet, so that the ingestion of solid food is impossible. The creature lives, as already stated, in the intestine 
of the frog: it is therefore, like the worms you have probably noticed in the frog's urinary bladder and lungs (pp. 33 and 153), an internal parasite, or endoparasite, having the frog as its host. The intestine contains the partially-digested food of the frog, and it is by the absorption of this that the Opalina is nourished. Having no mouth, it feeds solely by imbibition: whether it performs any kind of digestive process itself is not certainly known, but the analogy of other mouthless parasites leads us to expect that it simply absorbs food ready digested by its host, upon which it is dependent for a constant supply of soluble and diffusible nutriment.

Thus Opalina, in virtue of its parasitic mode of life, is saved the performance of certain work-the work of digestion, that work being done for it by its host. This is the essence of internal parasitism: an organism exchanges a free life, burdened with the necessity of finding food for itself, for existence in the interior of another organism, on which, in one way or another, it levies blackmail.

Note the close analogy between the nutrition of an internal parasite like Opalina and the saprophytic nutrition of a monad (p. 256). In both, the organism absorbs proteids rendered soluble and diffusible, in the one case by the digestive juices of the host, in the other by the action of putrefactive bacteria.

The reproduction of Opalina presents certain points of interest largely connected with its peculiar mode of life. It is obvious that if the Opalinæ simply went on multiplying, by fission or otherwise, in the frog's intestine, the population would soon outgrow the means of subsistence: moreover. when the frog died there would be an end of the parasites, What is wanted in this, as in other internal parasites, is some 
mode of multiplication which shall serve as a means of dispersal, or in other words, enable the progeny of the parasite to find their way into the bodies of other hosts, and so start new colonies:instead of remaining to impoverish the mother country.

Opalina multiplies by a somewhat peculiar process of binary fission : an animalcule divides in an oblique direction (Fig. 7 I D), and then each half, instead of growing to the size of the parent cell, divides again transversely $(\mathrm{E})$. The process is repeated again and again $(F)$, the plane of division being alternately oblique and transverse, until finally small bodies are produced $(\mathrm{G})$, about $\frac{1}{20}-\frac{1}{0} \mathrm{~mm}$. in length, and containing from two to four nuclei.

If the parent cell had divided simultaneously into a number of these little bodies the process would have been one of multiple fission (p. 254): as it is, it forms an interesting link between simple and multiple fission.

Opalina ranarum multiplies in this way in the spring-i.e. during the frog's breeding season. Each of the small products of division $(\mathrm{G})$ becomes encysted $(\mathrm{H})$, and in this passive condition is passed out with the frog's excrement, probably falling on to a water-weed or other aquatic object. Nothing further takes place unless the cyst is swallowed by a tadpole, as must frequently happen when these creatures, produced in immense numbers from the frogs' eggs, browse upon the water-weeds which form their chief food.

Taken into the tadpole's intestine, the cyst is burst or dissolved, and its contents emerge as a lanceolate mass of protoplasm ( $\mathrm{r}$ ), containing a single nucleus and covered with cilia. This, as it absorbs the digested food in the intestine of its host, grows, and at the same time its nucleus divides repeatedly $(\mathrm{K})$ in the way already described, until by the time the animalcule has attained the maximum size it has also 
acquired the large number of nuclei characteristic of the genus.

Here, then, we have an interesting case of progressive differentiation or development (p. 9): the organism begins life as a very small uninucleate mass of protoplasm, and as it increases in size increases also in complexity by the repeated binary fission of its nucleus.

The next organism we have to consider is a ciliated infusor even commoner than those just described. It is hardly possible to examine the water of a pond with any care without finding in it, sometimes attached to weeds, sometimes to the legs of water-fleas, sometimes to the sticks and stones of the bottom, numbers of exquisitely beautiful little creatures, each like an inverted bell with a very long handle, or a wineglass with a very long stem. These are the well-known "bell-animalcules;" the commonest among them belong to various species of the genus Vorticella.

The first thing that strikes one about Vorticella (Fig. 72, A) is the fact that it is permanently fixed, like a plant, the proximal or near end of the stalk being always firmly fixed to some aquatic object, while to the distal. or far end the body proper of the animalcule is attached.

But in spite of its peculiar form it presents certain very obvious points of resemblance to Paramœcium and Opalina. The protoplasm is divided into cortex (c, cort) and medulla (med), and is invested with a delicate cuticle $(c u)$. There is a single contractile vacuole $(c . v a c)$ the movements of which are very readily made out owing to the ease with which the attached organism is kept under observation. There is a meganucleus $(n u)$ remarkable for its elongated band-like form, and having in its neighbourhood a small rounded micronucleus. Cilia are also present, but the way in which

PrACT. ZOOz. 


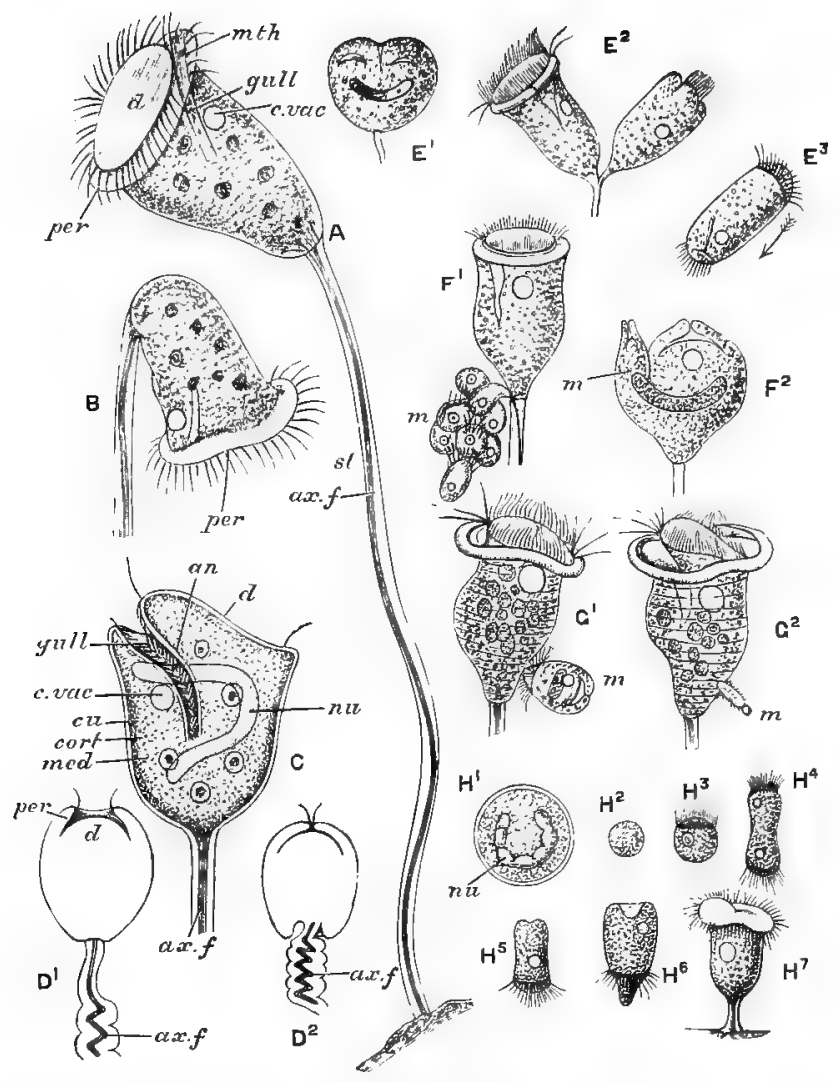

FIG. 72 - Lorticilla.

1, living specimen fully expanded, showing stalk (st) with axial fibre (ax. $f$ ), peristome $(p e r)$, disc $(d)$, mouth ( $n t h)$, gullet $(g u l l)$, and contractile vacuole. 1), the same, bent on its stalk and with the disc turned away from the observer.

$C$, optical section of the same, showing cuticle (cw), cortex (cort), medulla (mcd), nucleas ( $m$ m), gullet ( $\mathrm{s} u l l)$, several food-vacuoles, and anus $(a n)$, as well as the structures shown in A.

$\mathrm{U}^{1}$, a half-retracted and $\mathrm{D}^{2}$ a fully-retracted specimen, showing the coiling of the stalk and overlapping of the disc by the peristome.

$\mathbf{E}^{1}$, commencenent of binary fission; $\mathbf{E}^{2}$, completion of the process; $\mathbf{E}^{3}$, the barrel-shaped product of division swimming freely in the direction indicated by the arrow.

Fl, a specimen dividing into a megazooid and several microzooids (m); $\mathrm{F}^{2}$, rivision into one mega- and one microzooid.

$\mathrm{G}^{1}, \mathrm{G}^{2}$, two stages in conjugation showing the gradual absorption of the microgamete $(m)$ into the megagamete.

$\mathrm{H}^{\mathrm{i}}$. multiple fission of encysted form, the nucleus dividing into numerous masses: $\mathrm{H}^{3}$, spore formed by multiple fission; $\mathrm{H}^{3}-\mathrm{H}^{7}$, development of the spore; $\mathrm{H}^{4}$ is andergoing binary tission. (From Parker's Biology : E-H after Saville Kent. 
they are disposed is very peculiar and characteristic. To understand it we must study the form of the body a little. more closely.

The conical body is attached by its apex or proximal end to the stalk: its base or distal end is expanded so as to form a thickened rim, the peristome (per), within which is a plate-like body elevated on one side, called the disc (d), and looking like the partly raised lid of a chalice. Between the raised side of the disc and the peristome is a depression, the mouth (mth), leading into a conical gullet ( $\mathrm{gull}$ ).

There is reason for thinking that the whole proximal region of Vorticella answers to the ventral surface of Paramoecium, and its distal surface with the peristome and disc to the dorsal surface of the free-swimming genus : the mouth is to the left in both.

A single row of cilia is disposed round the inner border of the peristome and continued on the one hand down the gullet, and on the other round the elevated portion of the disc; the whole row of cilia thus takes a spiral direction The rest of the body is completely bare of cilia.

The movements of the cilia produce a very curious optical illusion: as one watches a fully-expanded specimen it is hardly possible to believe that the peristome and disc are not actually revolving - a state of things which would imply that they were discontinuous from the rest of the body. As a matter of fact the appearance is due to the successive contraction of all the cilia in the same direction, and is analogous to that produced by a strong wind on a field of corn or long grass. The bending down of successive blades of grass produces a series of waves travelling across the field in the direction of the wind. If instead of a field we had a large circle of grass, and if this were acted upon 
by a cyclone, the wave would travel round the circle, which would then appear to revolve.

Naturally the movement of the circlet of cilia produces a small whirlpool in the neighbourhood of the Vorticella, as can be seen by introducing finely-powdered carmine into the water. It is through the agency of this whirlpool that food particles are swept into the mouth, surrounded, as in Paramœcium, by a globule of water: the food-vacuoles (c) thus constituted circulate in the medullary protoplasm, and the non-nutritive parts are finally egested at an anal spot (an) situated near the base of the gullet.

The stalk $(\mathrm{A}, s t)$ consists of a very delicate, transparent, outer substance, which is continuous with the cuticle of the body and contains a delicate axial fibre $(a x . f)$ running along it from end to end in a somewhat spiral direction. This fibre is a prolongation of the cortex of the body (c): under a very high power it appears granular or delicately striated, the strix being continued into the cortex of the proximal part of the body.

A striking characteristic of Vorticella is its extreme irritability, i.e., the readiness with which it responds to any external stimulus. The slightest jar of the microscope, the contact of some other organism, or even a current of water produced by some free-swimming form like Paramœcium, is felt directly by the bell-aninalcule, and is followed by an instantaneous change in the relative position of its parts. The stalk becomes coiled into a close spiral so as to have a mere fraction of its original length, and the body from being bell-shaped becomes globular, the disc being withdrawn and the peristome closed over it $\left(\mathrm{D}^{\mathrm{I}}, \mathrm{D}^{2}\right)$.

The coiling of the stalk leads us to the consideration of the particular form of contractility called muscular, which is met with in multicellular animals, e.g. the frog (p. 60). It 
was mentioned above that while the stalk in its fully expanded condition is straight, the axial fibre is not straight, but forms a very open spiral, i.e., it does not lie in the centre of the stalk, but at any transverse section is nearer the surface at one spot than elsewhere, and this point as we ascend the stalk is directed successively to all points of the compass.

Now suppose that the axial fibre undergoes a sudden contraction, that is to say, a decrease in length accompanied by an increase in diameter, since as we have already seen there is no decrease in volume in protoplasmic contraction. There will naturally follow a corresponding shortening of the elastic cuticular substance which forms the outer layer of the stalk. If the axial fibre were entirely towards one side of the stalk, the result of the contraction would be a flexure of the stalk towards that side, but, as its direction is spiral, the stalk is bent successively in every direction, that is, is thrown into a close, spiral coil.

The axial fibre is therefore a portion of the protoplasm which possesses the property of contractility in a special degree; in which moreover contraction takes place in a definite direction - the direction of the length of the fibreso that its inevitable result is to shorten the fibre and consequently to bring its two ends nearer together. This is the essential characteristic of a muscular contraction, and the axial fibre in the stalk of Vorticella is therefore to be looked upon as the first instance of a clearly differentiated muscle which has come under our notice amongst unicellular animals.

There are some interesting features in the reproduction of Vorticella. It multiplies by binary fission, dividing through the long axis of the body (Fig. $72, E^{1}, E^{Z}$ ). Hence it is generally said that fission is longitudinal, not transverse, as 
in Paramœcium. But on the theory (p. 275) that the peris tome and disc are dorsal and the attached end ventral, fission is really transverse in this case also.

It will be seen from the figures that the process takes place by a cleft appearing at the distal end $\left(\mathbf{E}^{1}\right)$ and gradually deepening until there are produced two complete and fullsized individuals upon a single stalk $\left(\mathrm{E}^{2}\right)$. This state of things does not last long: one of the two daughter-cells takes on a nearly cylindrical form, keeps its disc and peristome retracted, and acquires a new circlet of cilia near its proximal end $\left(\mathrm{E}^{3}\right)$ : it then detaches itself from the stalk, which it leaves in the sole possession of its sister-cell, and swims about freely for a time in the direction indicated by the arrow. Sooner or later it settles down, becomes attached by its proximal end, loses its basal circlet of cilia, and develops a stalk, which ultimately attains the normal length.

The object of this arrangement is obvious. If, when a Vorticella divided, the plane of fission extended down the stalk until two ordinary fixed forms were produced side by side, the constant repetition of the process would so increase the numbers of the species in a given spot that the foodsupply would inevitably run short. This is prevented by one of the two sister-cells produced by fission leading a free existence long enough to enable it to emigrate and settle in a new locality, where the competition with its fellows will be less keen. The production of these free-swimming zooids is therefore a means of dispersal (p. 272): contrivances having this object in view are a very general characteristic of fixed as of parasitic organisms.

Conjugation occasionally takes place, and presents certain peculiarities. A Vorticella divides either into two unequal halves $\left(\mathrm{F}^{2}\right)$ or into two equal halves, one of which divides again into from two to eight daughter-cells $\left(\mathrm{F}^{1}\right)$. There are 
thus produced from one to eight microzooids which resemble the barrel-shaped form $\left(E^{3}\right)$ in all but size, and like it become detached and swim freely by means of a basal circlet of cilia. After swimming about for a time, one of these microzooids comes in contact with an ordinary form or megazooid, when it attaches itself to it near the proximal end $\left(\mathrm{G}^{1}\right)$, and undergoes gradual absorption $\left(\mathrm{G}^{2}\right)$, the mega- and microzooids becoming completely and permanently fused to form a zygote ( $\mathrm{p}$ 197). As in Paramœcium, conjugation is followed by increased activity in feeding and dividing.

Notice that in this case the conjugating bodies or gametes are not of equal size and similar characters ; but one, which is conveniently distinguished as the microgamete (= microzooid) is relatively small and active, while the other or megagamete (= megazooid, or ordinary individual) is relatively large and passive. As we have seen in the case of the frog (pp. I95 and I96), this differentiation of the gametes is precisely what we get in the higher animals, and, in fact, in almost all organisms with two sexes: the microgamete being the male, the megagamete the female conjugating body (see p. 197).

The result of conjugation is somewhat different in the two cases already studied : in Paramœecium no zygote is formed, conjugation being a mere temporary union (p. 268): in Vorticella the zygote is an actively moving and feeding body, indistinguishable from an ordinary individual of the species.

Vorticella sometimes encysts itself (Fig. $72, \mathrm{H}^{1}$ ), and the nucleus of the encysted cell has been observed to break up into a number of separate masses, each doubtless surrounded by a layer of protoplasm. After a time the cyst bursts, and a number of small bodies or spores $\left(\mathrm{H}^{2}\right)$ emerge from it, each containing one of the products of division of the nucleus. 
These acquire a circlet of cilia $\left(\mathrm{H}^{3}\right)$, by means of which they swim freely, and they are sometimes found to multiply by simple fission $\left(\mathrm{H}^{4}\right)$. Finally, they settle down $\left(\mathrm{H}^{5}\right)$ by the end at which the cilia are situated, the attached end begins to elongate into a stalk $\left(\mathrm{H}^{6}\right)$, this increases in length, the basal circlet of cilia is lost, and a ciliated peristome and disc are formed at the free end $\left(\mathrm{H}^{7}\right)$. In this way the ordinary form is assumed by a process of development (p. 273), and, moreover, the free-swimming young $\left(\mathrm{H}^{3}\right)$, to which the spores formed by division of the encysted protoplasm give rise, differ strikingly in form and habits from the adult. This is expressed by saying that development is in this case accompanied by a metamorphosis, this word literally meaning simply a change, being always used in zoology to express a striking and fundamental difference in form and habit between the young and the adult; as, for instance, between the tadpole and the frog (p. 9) or between the caterpillar and the butterfly. It is obvious that in the present instance metamorphosis is another means of ensuring dispersal.

In Vorticella, as we have seen, fission results not in the production of equal and similar daughter-cells, but of one stalked and one free-swimming form. It is however quite possible to conceive of a Vorticella-like organism in which the parent cell divides into two equal and similar products, each retaining its connection with the stalk. If this process were repeated again and again, and if, further, the plane of fission were extended downwards so as to include the distal end of the stalk, the result would be a branched, tree-like stem with a Vorticella-like body at the end of every branch.

As a matter of fact, this process takes place not in Vorticella itself, but in some nearly allied infusors, such as 
Carchesium and Epistylis. Each of these forms consists of a main stem attached by its proximal end and giving off at its distal end several branches, on each of which numerous bell-animalcules with short stalks are borne, like foxgloves or Canterbury-bells on their stem.

We see, then, that Carchesium and Epistylis differ from all our previous types in being compound organisms. The entire "tree" is called a colony or stock, and each separate bellanimalcule borne thereon is an individual or zooid, morphologically equivalent to a single Vorticella or Paramœcium. The colony is therefore an individual of a higher grade than the zooid, and such a multicellular animal as a frog is an individual of a higher grade still.

As in Vorticella, the stem of Carchesium consists of a cuticular sheath with an axial muscle-fibre which, at the distal end of the main stem, branches like the stem itself, a prolongation of it being traceable to each zooid; so that the muscular system is common to the whole colony, and any shock causes a general contraction of all the zooids. The stalk of Epistylis, on the other hand, is noncontractile.

The study of the foregoing living things and especially of Bacteria (p. 152), the smallest and probably the simplest of all known organisms, naturally leads us to the consideration of one of the most important problems of biology-the problem of the origin of life.

In all the higher organisms we know that each individual arises in some way or other from a pre-existing individual : no one doubts that every bird now living arose by a process of development from an egg formed in the body of a parent bird, and that every tree now growing took its origin either from a seed or from a bud produced by a parent plant. 
But there have always-until comparatively recently, at any rate-been upholders of the view that the lower forms of life, bacteria, monads, and the like, may under certain circumstances originate independently of pre-existing organisms: that, for instance, in a flask of some organic infusion boiled so as to kill any living things present in it, fresh forms of life may arise de novo-may in fact be created then and there.

We have therefore two theories of the lower organisms, the theory of Biogenesis, according to which each living thing, however simple, arises by a natural process of budding, fission, spore-formation, or what not, from a parent organism: and the theory of Abiogenesis, or as it is sometimes called Spontaneous or Equivocal Generation, according to which fully formed living organisms sometimes arise from not-living matter.

In former times the occurrence of abiogenesis was universally believed in. The expression that a piece of meat has "bred maggots"; the opinion that parasites such as the gall-insects of plants or the tape-worms in the intestines of animals originate where they are found; the belief still held in some rural districts in the occurrence of showers of frogs, or in the transformation of horse-hairs kept in water into eels; all indicate a survival of this belief.

As accurate enquiries into these matters were made, the number of cases in which equivocal generation was supposed to occur was rapidly diminished. It was not surprising however, considering the rapidity with which Bacteria and Monads were found to make their appearance in organic substances and infusions, that many men of science imagined them to be produced abiogenetically. The rapid multiplica tion of these forms means, of course, that a certain amount of fresh living protoplasm has been formed out of the 
constituents of the hay-infusion, through the agency in the first instance of a single living Bacterium. The question naturally arises, Why may not the formation of protoplasm take place independently of this insignificant speck of living matter?

It must not be thought that this question is in any way a vain or absurd one. That living protoplasm has at some period of the world's history originated from not-living matter seems a necessary corollary of the doctrine of evolution, and is obviously the very essence of the doctrine of special creation (p. 22r); and there is no $\dot{a}$ priori reason why it should be impossible to imitate the unknown conditions under which the process took place. But at present we have absolutely no data towards the solution of this fundamental problem.

Experiments conducted with proper precautions, however, all tell the same tale: they prove conclusively that in putrescible infusions that have been properly sterilized,-i.e. thoroughly boiled so as to kill any organisms they may contain-and adequately protected from the entrance of atmospheric germs, no micro-organisms ever make their appearance. So that the last argument for abiogenesis has been proved to be fallacious, and the doctrine of biogenesis shown, as conclusively as observation and experiment can show it, to be of universal application as far as existing conditions known to us are concerned. It is also necessary to add that the presence of microbes in considerable quantities in our atmosphere has been proved experimentally.

There is another question intimately connected with that of Biogenesis, although strictly speaking quite independent of it. It is a matter of common observation that, in both animals and plants, like produces like: that a cutting from 
a willow will never give rise to an oak, nor a snake emerge from a hen's egg. In other words, ordinary observation teaches the general truth of the doctrine of Homogenesis.

But there has always been a residuum of belief. in the opposite doctrine of Heterogenesis, according to which the offspring of a given animal or plant may be something utterly different from itself, a plant giving rise to an animal or vice versî, a lowly to a highly organised plant or animal and so on. Perhaps the most extreme case in which heterogenesis was once seriously believed to occur is that of the "barnacle-geese." Buds of a particular tree growing near the sea were said to produce barnacles, and these falling into the water to develop into geese. This sounds absurd enough, but within the last twenty years two or three men of science have described, as the result of repeated observations, the occurrence of quite similar cases among microscopic organisms. For instance, the blood corpuscles of the silkworm have been said to give rise to fungi, Euglenæ to thread-worms, and so on.

It is proverbially difficult to prove a negative, and it might not be easy to demonstrate, what all competent naturalists must be firmly convinced of, that every one of these subposed cases of heterogenesis is founded either upon errors of observation or upon faulty inductions from correct observations.

It is obvious that the only way in which a case of heterogenesis could be proved would be by actually watching the transformation, and this no heterogenist has ever done; at the most, certain supposed intermediate stages between the extreme forms have been observed-say, between a Euglena and a thread-worm--and the rest of the process inferred. On the other hand, innumerable observations have been made on these and other organisms, the result being that 
each species investigated has been found to go through a definite series of changes in the course of its development, the ultimate result being invariably an organism resembling in all essential respects that which formed the starting point of the observations: Euglenæ always giving rise to Euglenæ and nothing else, Bacteria to Bacteria and nothing else, and so on.

'There are many cases which imperfect knowledge might class under heterogenesis, such as the origin of frogs from tadpoles or of jelly-fishes from polypes (see Chapter IV.), but in these and many other cases the apparently anomalous transformations have been found to be part of the normal and invariable cycle of changes undergone by the organism in the course of its development: the frog always gives rise ultimately to a frog, the jelly-fish to a jelly-fish. If a frog at one time produced a tadpole, at another a trout, at another a worm: if jelly-fishes gave rise sometimes to polypes, sometimes to infusoria, sometimes to cuttle-fishes, and all without any regular sequence-that would be heterogenesis.

It is perhaps hardly necessary to caution the reader against the error that there is any connection between the theory of heterogenesis and that of organic evolution. It might be said-if, as naturalists tell us, dogs are descended from wolves and jackals, and birds from reptiles, why should not, for instance, thread-worms spring from Euglenæ or Infusoria from Bacteria? To this it is sufficient to answer that the evolution of one form from another takes place by a series of slow, orderly, progressive changes going on through a long series of generations (p. 222); whereas heterogenesis presupposes the casual occurrence of sudden transformations in any direction-i.e., leading to either a less or a more highly organised form-and in the course of a single generation. 
Each of the organisms which we have studied in this and the two previous chapters consists of a single cell-or in the case of Carchesium and Epistylis of a colony of cells to a large extent independent of one another. They are therefore placed in the lowest primary division of the animal kingdom-the phylum Protozoa (p. 220). This phylum is subdivided into a number of classes, examples of certain of which we have examined. Those in which, like the Amœba, the amoboid form is predominant constitute the class Rhizopoda: those in which, like the Monads and Euglenæ (Flagellata), the flagellate form is predominant are often included with the ciliated forms (Ciliata) - such as Paramocium, Opalina and Vorticella-in a single class, the Infusoria.

The animals above the Protozoa are classed, as we have seen, in a number of different phyla, but as they are all multicellular they are often spoken of collectively as the Metazoa, one of the simplest of which we must next examine.

\section{PRACTICAL DIRECTIONS.}

Paramœcium.--Spread a little cotton-wool on a slide over a drop of water containing Paramœcia, in order to entangle them in its meshes, and put on a cover-glass. Examine first with the low power and then with the high power. Note-

I. The elongated form of the animal; its anterior (more rounded) and posterior (more pointed) end; its flattened dorsal and ventral surfaces; and its buccal groove and mouth, on the ventral surface.

2. The active movements, due to the cilia covering the body.

3. The marked distinction between cortex and medulla.

4. The characters of the elastic cortex:-(a) the superficial cuticle, and deeper striated layer; $(b)$ the cilia arising from the deeper layer, and projecting through the cuticle ; $(c)$ the trichocysts-small oval sacs, imbedded in the deeper layer; $(d)$ the two spherical contractile vacuoles, situated in the deeper layer on the dorsal side: note that canals radiate 
from them when they contract; $(e)$ the cilia lining the buccal groove. (The potential anus behind the mouth can only be seen at the moment of defacation.) Compare the mode of feeding with that of Amoba.

5. The characters of the meduila:-(a) The food vacuoles and their circulation; (b) the meganucleus and micronucleus, which can be better seen when stained. Sketch.

6. Add methyl green. Then note again the structure of cortex and medulla, as well as-(a) the oval meganucleus, near the middle of the body; $(b)$ the micronucleus, a smaller body, close to the meganucleus; (c) the extruded trichocysts. Sketch.

7. Look out for specimens undergoing transverse fission, and also for others in process of conjugation. Sketch.

Permanent preparations may be made as directed in the case of Amcha (p. 239).

Opalina.-Make an incision in the rectum or small intestine of a freshly-killed frog, and mount a little of its contents in water on a slide. Note the oval and flattened form of the Opalina and their uniform covering of cilia; the cuticle, cortex and medulla; and the absence of a mouth and contractile vacuole. Stain as before, and make out the numerous nuclei. Look out for the products of division free and encysted.

Vorticella. Mount some specimens in a drop of water, and examine with the low power. The Vorticella will be seen to have the form of a wine-glass or bell with a long stem. The stem frequently contracts spirally, the edge of the bell being at the same time turned in, so as to give the animal a rounded form. Put on the high power and note-

I. The form of the bell, its thickened rim or peristome, and the disc, which forms a cover to the bell.

2. The mouth and grullet, opening on one side between the peristome and disc, which is here slightly raised. (The anus opens into the oral depression, but can only be seen at the moment of defrcation.)

3. The single row of cilia round the peristome and extending down the gullet on the one hand, and on to the raised portion of the disc on the other. Run in a little finely powdered indigo or carmine under the cover-glass, and note the currents produced by the cilia: the granules of pigment will be carried down the gullet.

4. The contractions of the bell and stalk.

5. The structure of the cortex, which is similar to that of Paramocium, except that the cilia have a restricted distribution, and that 
there are no trichocysts. Moreover, in the stalk (into which the medulla does not extend) the deeper layer of the cortex gives rise to a central contractile axial fibre, by means of which the stalk can contract spirally.

6. The medulla, which circulates and contains food-vacuoles, as in Paramœcium. The single spherical contractile vacuole is also situated in it, as well as an elongated and curved meganucleus and a small micronuclezts (not easy to distinguish). Sketch.

7. Make preparations as directed under Paramœcium. Sketch.

8. Look out for specimens undergoing fission, noting the different stages, and the second, aboral ring of cilia on one of the daughter individuals, which eventually becomes detached. Note these free swimming forms, and also search for conjugating individuals-a small free Vorticella uniting with a large stationary one. Sketch.

Carchesium or Epistylis.-You will very likely find specimens of one of these, or of an allied genus, amongst the Vorticellæ. Note that several individual zooids are borne upon a branched stalk, together forming a colony. 


\section{CHAPTER IV}

IITDRA : BOUGAINVILLEA-ALTERNATION OF GENERATIONSCHARACTERS OF THE PHYLUM COEIFNTERATA.

A CAREFUL search in ponds will often result in the capture of some small organisms known as "fresh-water polypes," belonging to the genus Hydra.

Although far from uncommon in pond-water, Hydra is not always easy to find, being rarely abundant and by no means conspicuous. In looking for it the best plan is to fill either a clear glass bottle or beaker, or a white saucer, with weeds and water from a pond and to let it remain undisturbed for a short time. If the gathering is successful there will be seen adhering to the sides of the glass, the bottom of the saucer, or the weeds, little white, tawny, or green bodies, about as thick as fine sewing cotton, and $2-6 \mathrm{~mm}$. in length. They adhere pretty firmly by one end, and examination with a pocket lens shows that from the free extremity a number of very delicate filaments, barely visible to the naked eye, are given off.

Under the low power of a compound microscope a Hydra (Fig. 73) is seen to have a cylindrical oody attached by a flattened base to a weed or other aquatic object, and bearing at its opposite or distal end a conical structure, the 


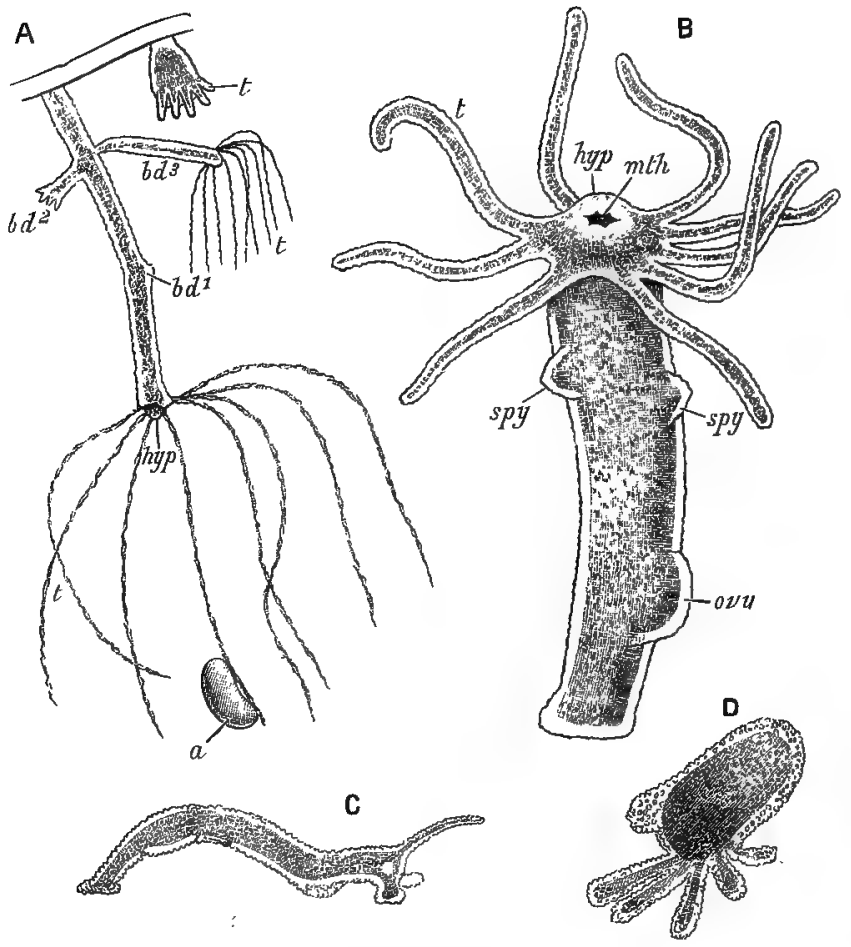

FIG. 73.-Hydra.

c. A, two living specimens of H.viridis attached to a bit of weed. The larger specimen is fully expanded, and shows the elongated body ending distally in the hypostome $(h y t)$, surrounded by tentaeles $(t)$, and three buds $(b d .1, b d .2, b d .3)$ in different stages of development; a small water-flea $(a)$ has been captured by one tentacle. The smaller specimen (to the right and above) is in a state of complete retraction, the tentacles $(t)$ appearing like papilla.

E B, $H$. fisca, showing the mouth (tnth) at the end of the hypostome (hyp), the circlet of tentacles $(t)$, two spermaries $(s p y)$, and an ovary (ovy)

$C$, a Hydra creeping on a flat surface by looping movements.

$\mathrm{D}$, a specimen crawling on its tentacles. (From Parker's Biology: C and D after W. Marshall.)

hypostome (hyp), at the apex of which is a circular aperture, the mouth (mth). At the junction of the hypostome with the body proper are given off from six to eight long delicate 
tentacles $(t)$ arranged in a circlet or whorl. A longitudinal section shows that the body is hollow, containing a spacious cavity, the enteron (Fig. 74, A, ent. cav), which communicates with the surrounding water by the mouth. The tentacles are also hollow, their cavities communicating with the enteron.

Thus it will be seen that the Hydra is not bilaterally symmetrical, like the frog-i.e., equally divisible into two lateral halves by a median vertical plane passing through the axis of the body,-but is radially symmetrical, i.e., the body is divisible into similar parts radiating from a common central axis.

There are three kinds of Hydra commonly found: one, $H$. vulgaris, is colourless or nearly so ; another, $H$. fusca, is of a pinkish-yellow or brown colour; the third, $H$. viridis, is bright green. In the two latter it is quite evident, even under a low power, that the colour is in the inner parts of the body-wall, the outside of which is formed by a transparent colourless layer (Fig. 73).

An examination of the living animal shows, in the first place, that its form is continually changing. At one time (Fig. 73 , A, left-hand figure) it extends itself until its length is fully fifteen times its diameter and the tentacles appear like long delicate filaments: at another time (right-hand figure) it contracts itself into an almost globular mass, the tentacles then appearing like little blunt knobs.

Besides these movements of contraction and expansion, Hydra is able to move slowly from place to place. This it usually does after the manner of a looping caterpillar (Fig. $73, \mathrm{c})$ : the body is bent round until the distal end touches the surface: then the base is detached and moved nearer the distal end, which is again moved forward, and so on. It has also been observed to crawl like a cuttle-fish (D) 
by means of its tentacles, the body being kept nearly vertical.

It is also possible to watch a Hydra feed. It is a very voracious creature, and to see it catch and devour its prey is a curious and interesting sight. In the water in which it lives are always to be found numbers of "water-fleas," minute animals of about a millimetre or less in length, belonging to the class Crustacea.

Water-fleas swim very rapidly, and occasionally one may be seen to come in contact with a Hydra's tentacle. Instantly its hitherto active movements stop dead, and it remains adhering in an apparently mysterious manner to the tentacle. If the Hydra is not hungry it usually liberates its prey after a time, and the water-flea may then be seen to drop through the water like a stone for a short distance, but finally to expand its limbs and swim off. If however the Hydra has not eaten recently it gradually contracts the tentacles until the prey is brought near the mouth, the other tentacles being also used to aid in the process. The waterflea is thus forced against the apex of the hypostome, the mouth expands widely and seizes it, and it is finally passed down into the digestive cavity. Hydræ can often be seen with their bodies bulged out in one or more places by recently swallowed water-fleas.

The precise structure of Hydra is best made out by cutting it into a series of extremely thin sections and examining them under a high power of the microscope. The appearance presented by a vertical section through the long axis of the body is shown in Fig. 74, A.

The whole animal is seen to be built up of cells, each consisting of protoplasm with a large nucleus $(\mathrm{B}-\mathrm{D}, n u)$, and with or without vacuoles. As in the case of most animal cells, there is no cell-wall. 
The essential feature in the arrangement of the cells is that they are disposed in two layers round the central digestive cavity or enteron (A, ent. cav) and the cavities of tentacles (ent. cav'). So that the wall of the body is formed throughout of an outer layer of cells, the ectoderm (ect), and of an inner layer, the endoderm (end), which bounds the enteric cavity (compare p. 202). Between the two layers is a delicate transparent membrane, the mesoglaa, or supporting lamella (msgl). A transverse section (B) shows that the cells in both layers are arranged radially.

Thus Hydra is a two-layered or diploblastic animal, and may be compared to a chimney built of two layers of radially arranged bricks with a space between the layers filled with mortar or concrete.

Accurate examination of thin sections, and of specimens teased out or torn into minute fragments with needles, shows that the structure is really much more complicated than the foregoing brief descripton would indicate.

The ectoderm cells are of two kinds. The first and most obvious ( $\mathrm{B}, e c t$, and $\mathrm{C}$ ) are large cells of a conical form, the bases of the cones being external, their apices internal. Spaces are necessarily left between their inner or narrow ends, and these are filled up with the second kind of cells (int. c), small rounded bodies which lie closely packed between their larger companions and are distinguished as interstitial cells.

The inner ends of the large ectoderm cells are continued into narrow, pointed prolongations $(c, m$. pr), placed at right angles to the cells themselves and parallel to the long axis of the body. There is thus a layer of these longitudinallyarranged muscle-processes lying immediately external to the mesogloa (B, m. pr). 'They appear to possess, like the axial 


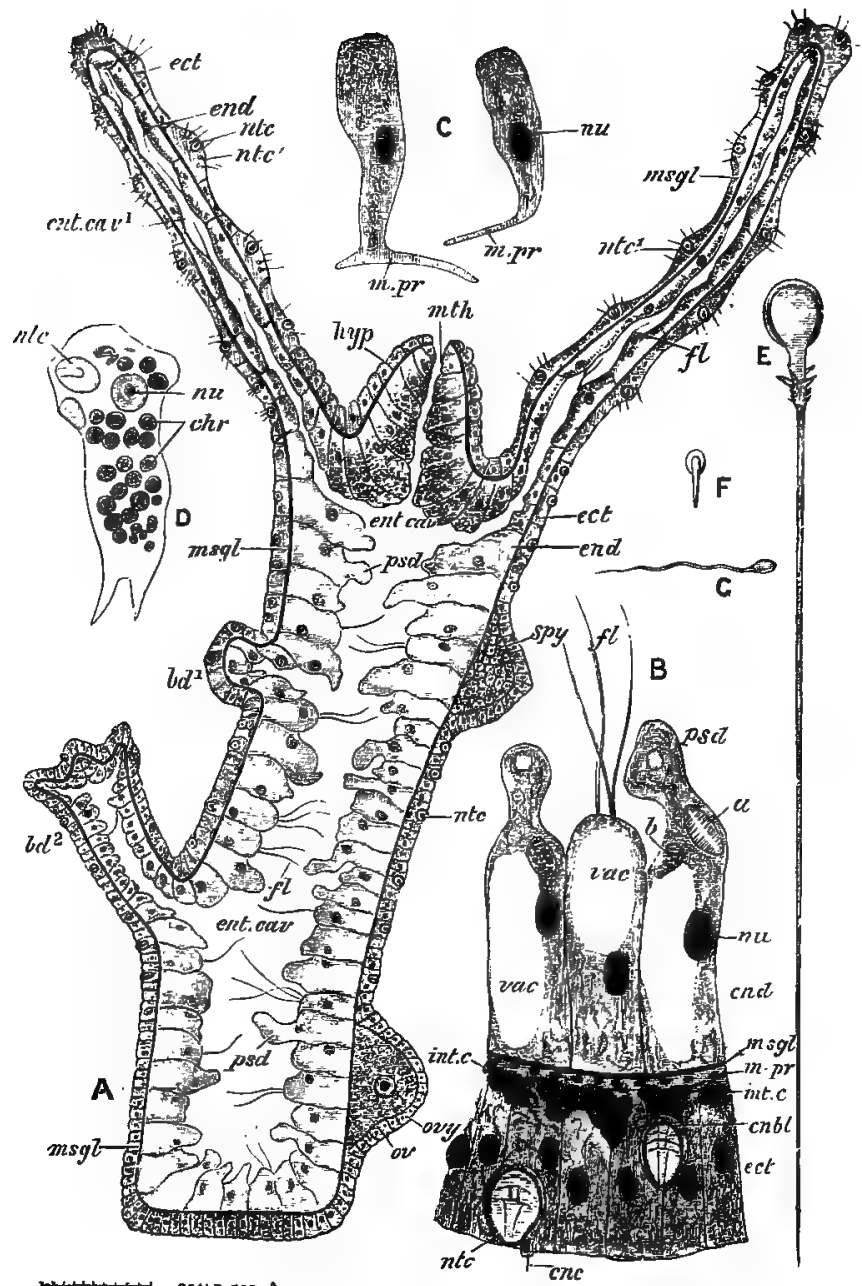

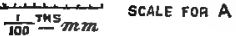

FIG. 74, - Hydra.

$\Lambda$, vertical section of the entire animal, showing the ${ }^{-}$pody-wall composed of ectoderm $(e c t)$ and endoderm (end), enclosing an enteric cavity (cut.cat), which, as well as the two layers, is continued (ent. cav') into the tentacles, and opens 
externally by the mouth ( $m$ th) at the apex of the hypostome ( $h y p)$. Between the ectoderm and endoderm is the mesogloea ( $m s g l)$, represented by a black line. In the ectoderm are seen large (ntc) and small $\left(n t c^{\prime}\right)$ nematocysts; some of the endoderm cells are putting out pseudopods ( $p s d)$, others flagella $(A)$. Two buds $\left(b d .^{1} b d^{2}\right.$.) in different stages of developmept are shown on the left side, and on the right a spermary (spy) and an ovary (ovy) containing a single ovum (ov).

$B$, portion of a transverse section more highly magnified, showing the large ectoderm cells $(e c t)$ and interstitial cells (int. $c$ ); two cnidoblasts (cnbl) enclosing nematocysts ( $n t c)$, and one of them produced into a cnidocil (cnc); the layer of muscle-processes ( $m$. pr) cut across just external to the mesogloea ( $m$ sgl); endoderm cells (end) with large vacuoles and nuclei (uz), pseudopods ( $\left.p s a d^{\prime}\right)$ and flagella $(\mathscr{A})$. The endoderm cell to the right has ingested a diatom $(a)$, and all enclose minute black granules.

$\mathrm{C}$ two of the large ectoderm cells, showing nucleus $(n w)$ and muscle-process $(n, p r)$. $I$, an endoderm cell of $H$.viridis, showing nucleus (nk), numerous chomato* phores $(c h r)$, and an ingested nematocyst $(n t c)$.

$\mathrm{E}$, one of the larger nematocysts with extruded thread barbed at the base.

$F$, one of the smaller nematocysts.

$\mathrm{G}$, a single sperm. (From Parker's Biology : D after Lankester; $\mathrm{F}$ and $\mathrm{G}$ after Howes.)

fibre of Vorticella (p. 276), a high degree of contractility, the almost instantaneous shortening of the body being due, in great measure at least, to their rapid and simultaneous contraction. It is probably correct to say that, while the ectoderm cells are both contractile and irritable, a special degree of contractility is assigned to the muscle-processes, the cells themselves being eminently irritable, the slightest stimulus applied to them usually being followed by an immediate contraction of the whole body.

Imbedded in and between some of the large ectoderm cells are found clear, oval sacs $(n t c)$, with very well-defined walls, and called thread-cells or nematocysts. Both in the living specimen and in sections they ordinarily present the appearance shown in Figs. 74, B, and 75, A, but are frequently met with in the condition shown in Figs. 74 , E, and 75, B, that is, with a short conical tube protruding from the mouth of the sac, armed near its distal end with three recurved barbs besides several similar processes of smaller size, and giving rise distally to a long, delicate, flexible filament.

Accurate examination of the nematocysts shows that the structure of these curious bodies is as follows. Each con. sists of a tough sac (Fig. 75, A), one end of which is turned 

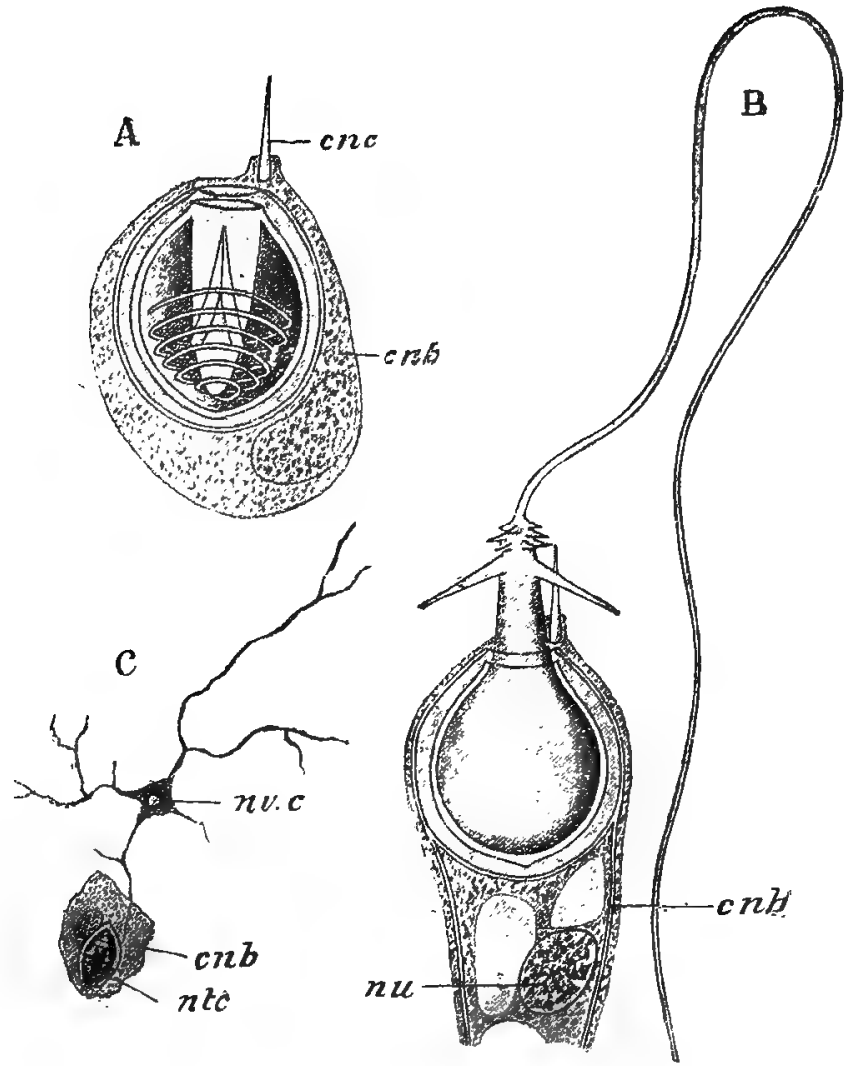

FIG. 75.-Hydra.

$A$ a nematocyst contained in its cnidoblast ( $c n b)$, showing its coiled filament and the cnidocil (cre).

$B$, the same after extrusion of the thread, showing the larger and smaller barbs at the base of the thread; $n u$. the nucleus of the cnidoblast.

$\mathrm{C}$, a cnidoblast, with its contained nematocyst, connected with one of the processes of. a nerve-cell $(n v, c)$. (From Parker's Biology ; after Schneider.)

in as a hollow pouch: the free end of the latter is continued into a hollow coiled filament, and from its inner surface 
project the barbs. The whole space between the wall of the sac and the contained pouch and thread is tensely filled with fluid. When pressure is brought to bear on the outside of the sac the whole apparatus goes off like a harpoon-gun (B), the compression of the fluid forcing out first the barbed pouch and then the filament, until finally both are turned inside out.

It is by means of the nematocysts - the resemblance of which to the trichocysts of Paramœcium (p. 267) should be noted-that the Hydra is enabled to paralyse its prey. Probably some specific poison is formed and ejected into the wound with the thread : in the larger members of the group to which Hydra belongs, such as jelly fishes, the nematocysts produce an effect on the human skin quite like the sting of a nettle.

The nematocysts are formed in special interstitial cells called cnidoblasts (Figs. 74, B, and 75, cnbt), and are thus in the first instance at a distance from the surface. But the cnidoblasts migrate outwards, and so come to lie quite superficially either in or between the large ectoderm cells. On its free surface the cnidoblast is produced into a delicate pointed process, the cnidocil or "trigger-hair" (cnc). In all probability the slightest touch of the cnidocil causes contraction of the cnidoblast, and the nematocyst, thus compressed, instantly explodes.

Nematocysts are found in the distal part of the body, but are absent from the foot or proximal end, where also there are no interstitial cells. They are especially abundant in the tentacles, on the knob-like elevations of which-due to little heaps of interstitial cells--they are found in great numbers. Amongst these occur small nematocysts with short threads and devoid of barbs (Fig. 74, A, ntt' and F).

In connection with the cnidoblasts small irregular cells 
with large nuclei occur (Fig. $75, \mathrm{C}, n v . c$ ); they are supposed to be nerve-cells, and to constitute a rudimentary nervous system (compare p. I67).

The ectoderm cells of the foot differ from those of the rest of the body in being very granular (Fig. 74, A). The granules are probably the material of the adhesive secretion by which the Hydra fixes itself, and these cells are therefore glandular (p. I3o).

The endoderm consists for the most part of large cells which exceed in size those of the ectoderm, and are remarkable for containing one or more vacuoles, sometimes so large as to reduce the protoplasm to a thin superficial layer containing the nucleus (Fig. 74, A and $\mathrm{B}$, end). Then again, their form is extremely variable, their free or inner ends undergoing continual changes of form. This can be easily made out by cutting transverse sections of a living Hydra, when the endoderm cells are seen to send out long blunt pseudopods $\left(\not s d^{2}\right)$ into the digestive cavity, and now and then to withdraw the pseudopods and send out from one to three long, delicate flagella $(f)$. Thus the endoderm cells of Hydra illustrate in a very instructive manner the essential similarity of flagella and pseudopods already referred to (p. 250). In the hypostome the endoderm is thrown into longitudinal folds, so as to allow of the dilatation of the mouth in swallowing.

Amongst the ordinary endoderm cells are found long narrow cells of an extremely granular character. They are specially abundant in the distal part of the body, beneath the origins of the tentacles, and in the hypostome, but are absent in the tentacles and in the foot. There is no doubt that they are gland-cells, their secretion being a fluid used to aid in the digestion of the food. 
In Hydra viridis the endoderm-cells (Fig. 74, D) contain chromatophores ${ }^{1}(c h r)$ coloured green by chlorophyll, which performs the same function as in plants (p. 247), so that in this species holozoic is supplemented by holophytic nutrition. In $\mathrm{H}$. fusca bodies resembling these chromatophores are present, but are of an orange or brown colour, and devoid of chlorophyll.

Muscle-processes exist in connection with the endoderm cells, and they are said to take a transverse or circular direction, i.e., at right angles to the similar processes of the ectoderm cells.

When a water-flea or other minute organism is swallowed by a Hydra, it undergoes a gradual process of disintegration. The process is begun by a solution of the soft parts due to the action of a digestive fluid secreted by the gland-cells of the endoderm ; it is apparently completed by the endoderm

1 In the substance of certain animals-e.g., the orcler Radiolaria of the class Rhizopoda (p. 286), small Hæmatococcus-like cells occur, which have been proved to be independent organisms, called Zooxanthella. Such a living together of two organisms is known as symbiosis. It differs essentially from parasitism (see p. 27 I), in which one organism preys upon another, the host deriving no benefit but only harm from the presence of the parasite. In symbiosis, on the contrary, the two organisms are in a condition of mutually beneficial partnership. The carbon dioxide and nitrogenous waste given off by the Radiolarian serve as a constant food-supply to the Zooxanthella: at the same time the latter by decomposing the carbon dioxide provides the Radiolarian with a constant supply of oxygen, and also with two important food stuffs-starch and proteids, which, after sointion, diffuse from the protoplasm of the Zooxanthella into that of the Radiolarian. The Radiolarian may therefore be sail to keep the Zooxanthellix constantly manured, while the Zooxanthellix in return supply the Radiolarian with abundance of oxygen and of ready-digested food. There is some reason for believing that the chromatophores of Hydra viridis are also to be regarded as symbiotic organisms. 
cells seizing minute particles with their pseudopods and engulfing them quite after the manner of Amœbæ. It is often found that the protrusion of pseudopods during digestion results in the almost complete obliteration of the enteric cavity.

It would seem therefore that in Hydra the process of digestion or solution of the food is to some extent intracelinular, i.e., takes place in the interior of the cells themselves, as e.g., in Amœba or Paramœcium : it is however mainly extra-cellular or enteric, i.e., is performed in a special digestive cavity lined by cells (p. I 3 I).

The ectoderm cells do not take in food directly, but are nourished entirely by diffusion from the endoderm. Thus the two layers have different functions : the ectoderm is protective and sensory; it forms the external covering of the animal, and receives impressions from without; the endoderm, removed from direct communication with the outer world, performs a nutrient function, its cells alone having the power of digesting food.

The essential difference between digestion and assimilation is here plainly seen : all the cells of Hydra assimilate, all are constantly undergoing waste, and all must therefore form new protoplasm to make good the loss. But it is the endoderm cells alone which can make use of raw or undigested food : the ectoderm has to depend upon various products of digestion received by diffusion or osmosis from the endoderm.

It will be eviclent from the preceding description that Hydra is comparable to a colony of Amobæ in which particular functions are made over to particular individuals-just as in a civilised community the functions of baking and butchering are assigned to certain members of the community, and not performed by all. Hydra is therefore an 
example of individuation: morphologically it is equivalent to an indefinite number of unicellular organisms: but, these acting in concert, some taking one duty and some another, form, physiologically speaking, not a colony of largely independent units (compare p. 281), but a single multicellular individual.

Hydra has two distinct methods of reproduction, asexual and sexual.

Asexual multiplication takes place by a process of budding. $A$ little knob appears on the body (Fig. $73 \mathrm{~A}, b d^{1}$ ), and is found by sections to arise from a group of ectoderm cells; soon however it takes on the character of a hollow outpushing of the wall containing a prolongation of the enteron, and made up of ectoderm, mesoglœa, and endoderm. (Fig. $\left.74, A, b d^{1}\right)$. In the course of a few hours this prominence enlarges greatly, and near its distal end six or eight hollow buds appear arranged in a whorl (Figs. 73, A, and 74, $\Lambda$, $\left(d d^{2}\right)$. These enlarge and take on the characters of tentacles, and a mouth is formed at the distal end of the bud, which thus acquires the character of a small Hydra (Fig. 73, $\left.\Lambda, b d^{3}\right)$. Finally the bud becomes constricted at its base, separates from the parent, and begins an independent existence. Sometimes, however, several buds are produced at one time, and each of these buds again before becoming detached: in this way temporary colonies are formed. But the buds always separate sooner or later, although they frequently begin to feed while still attached.

It is a curious circumstance that Hydra can also be multiplied by artificial division: the experiment has been tried of cutting the living animal into pieces, each of which was found to grow into a perfect individual

The sexual organs or gonads (p. 193) are of two kinds, 
spermaries and ovaries. Both are found in the same individual, Hydra being hermaphrodite or monocious.

The spermaries (Figs. 73, B, and 74, A, spy) are white conical elevations situated near the distal end of the body: as a rule not more than one or two are present at the same time, but there may be as many as twenty. They are perfectly colourless, even in the green and brown species, being obviously formed of ectoderm alone.

In the immature condition the spermary consists of a little heap of interstitial cells covered by an investment of somewhat flattened cells formed by a modification of the ordinary large cells of the ectoderm. When mature each of the small internal cells becomes converted into a sperm (p. 194), consisting of a small ovoid head formed from the nucleus of the cell, and of a long vibratile tail formed from its protoplasm (Fig. 74, G). By the rupture of the investing cells or wall of the spermary the sperms are liberated and swim freely in the water.

The ovaries (Figs. 73, B, and 74, A, ovy) are found nearer the proximal end of the body, and vary in number from one to eight. When ripe an ovary is larger than a spermary, and of a hemispherical form. It begins, like the spermary, as an aggregation of interstitial cells, so that in their earlier stages the sex of the gonads is indeterminate. But while in the spermary each cell is converted into a sperm, in the ovary one cell (Fig. 74, A, ov), soon begins to grow faster than the rest, and becomes amœboid in form, sending out pseudopods amongst its companions and ingesting the fragments into which they become broken up, thus continually increasing in size at their expense. Ultimately the ovary comes to consist of this single amœboid ovum and of a layer of superficial cells forming a capsule for it (compare p. I95). As the ovum grows, yolk-granules 
are formed in it, and in Hydra viridis it also acquires green chromatophores.

When the ovary is ripe the ovum draws in its pseudopods and takes on a spherical form: the investing layer then bursts so as to lay bare the ovum and allow of the free access to it of the sperms. One of the latter conjugates with the ovum producing an oosperm (p. 198) or unicellular embryo.

The oosperm undergoes segmentation, dividing into a number of cells which constitute a morula or polyplast (p. 200), the outermost cells of which become changed into a hard shell or capsule. The embryo, thus protected, falls to the bottom of the water, and after a period of rest develops into a Hydra, its cells becoming differentiated into ectoderm and endoderm, the enteron and mouth being formed, and the tentacles budding out round the latter.

It was stated on p. 301 that in a budding Hydra the buds do not always become detached at once, but may themselves bud while still in connection with the parent, temporary colonies being thus produced.

Suppose the state of things to continue indefinitely: the result would be a tree-like colony or compound organism consisting of a stem with numerous branchlets each ending in a Hydra-like zooid. Such a colony would bear much the same relation to Hydra as Carchesium or Epistylis bears to Vorticella (p. 28I).

As a matter of fact this is precisely what happens in a great number of animals allied to Hydra and known by the name of Zoophytes or Hydroid polypes.

Every one is familiar with the common Sertularians of the sea-coast, often mistaken for sea-weeds : they are delicate, much-branched, semi-transparent structures of a horny 
consistency, the branches beset with little cups, from each of which, during life, a Hydra-like body is protruded.

A very convenient genus for our purpose is Bougainvillea, a hydroid polyp found in the form of little tufts a few centimetres long, attached to rocks and other submarine objects. Fig. 76 , A, shows a colony of the natural size, B, a part of it magnified : it consists of a much-branched stem of a yellowish colour attached by root-like fibres to the support. The branches terminate in little Hydra-like bodies called hydranths ( $\mathrm{B}$, hyd), each with a hypostome (hyp) and circlet of tentacles $(t)$. Lateral branchlets bear bell-shaped structures or meduse (med) : these will be considered presently.

Sections show that the hydranths have essentially the structure of a Hydra, consisting of a double layer of cells -ectoderm and endoderm-separated by a supporting lamella or mesogloea and enclosing a digestive cavity (ent. cav) which opens externally by a mouth placed at the summit of the hypostome.

The tentacles, however, differ from those of Hydra in two important respects. In the first place they are solid: the endoderm, instead of forming a lining to a prolongation of the enteron, consists of a single axial row of large cells with thick cell-walls and vacuolated protoplasm. Then in the position of the muscle-processes of Hydra there is a layer of spindle-shaped fibres, many times longer than broad, and provided each with a nucleus. Such muscle-fibres are obviously cells greatly extended in length ( $p$. I I r), so that the ectoderm cell of Hydra with its continuous muscle-process is here represented by an ectoderm cell with an adjacent musclecell. We thus get a partial intermediate layer of cells between the ectoderm and endoderm, in addition to the gelatinous mesogloa ; and so, while a hydroid polyp is, like Hydra, diploblastic (p. 293), it shows a tendency towards the 


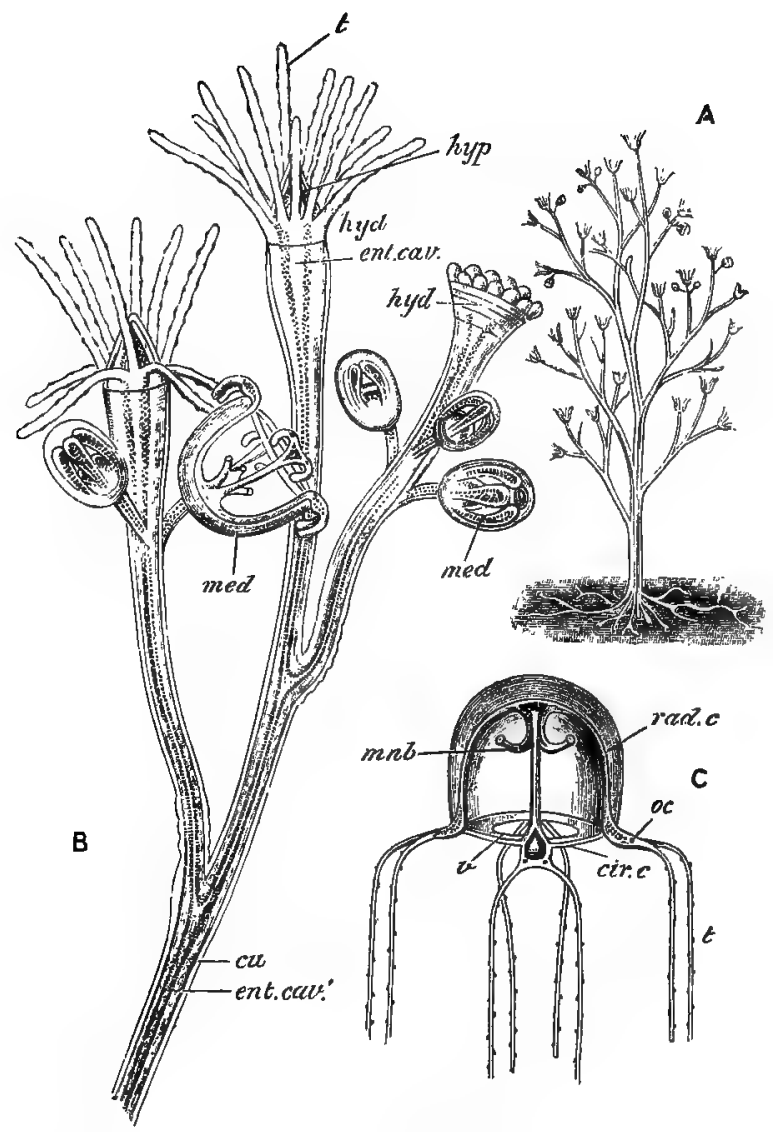

FIG. 76.-Bougainvillea ramosa.

A, a complete living colony of the natural size, showing the branched stem and root-like organ of attachment.

$B$, a portion of the same magnified, showing the branched stem bearing hydranths (hyd) and medusx (med), one of the latter nearly mature, the others immature; each hydranth has a circlet of tentacles $(t)$ surrounding a hypostome (hyp), and contains an enteric cavity (ent.cav) continuous with a narrow canal (ent. cav') in the stem. The stem is covered by a cuticle (cat).

$C$, a medusa after liberation from the colony, showing the bell with tentacles $(t)$, velum (z), manubrium (mnb), radial (rad.c) and circular (cir.c) canals, and eye-spots (oc). (From Parker's Biologv, after Allman.)

Pract, Zool 
assumption of a three-layered or triploblastic condition (compare p. 202).

The stem is formed of the same layers and contains a cavity (ent. $\left.c a v^{\prime}\right)$ continuous with those of the hydranths, and thus the structure of a hydroid "polyp is, so far, simply that of a Hydra in which the process of budding has gone on to an indefinite extent and without separation of the buds.

There is however an additional layer added in the stem for protective and strengthening purposes. It is evident that a colony of the size shown in Fig. 76, A, would, if formed only of soft ectodermal and endodermal cells, be so weak as to be hardly able to bear its own weight even in water. To remedy this a layer of transparent, yellowish substance of horny consistency, called the cuticle, is developed outside the ectoderm of the stem, extending on to the branches and only stopping at the bases of the hydranths and medusa. It is this layer which, when the organism dies and decays, is left as a semi-transparent branched structure resembling the living colony in form except that hydranths and medusæ are wanting. The cuticle is therefore a supporting organ or skeleton, not, like our own bones, formed in the interior of the body (endoskeleton, p. I6), but like the shell of a crayfish or lobster, lying altogether outside the soft parts (exoskeleton).

As to the mode of formation of the cuticle:- we saw that many organisms, such as Hæmatococcus and Amoba, are able to form a cyst or cell-wall, by secreting or separating from the surface of the protoplasm a succession of layers either of cellulose or of a transparent horn-like substance (pp. 232 and 244). But Amoba and Hæmatococcus are unicellular, and are therefore free to form this protective layer at all parts of their surface. The ectoderm cells of 
Bougainvillea, on the other hand, are in close contact with their neighbours on all sides and with the mesogloea at their inner ends, so that it is not surprising to find the secretion of skeletal substance taking place only at their outer ends. As the process takes place simultaneously in adjacent cells, the result is a continuous layer common to the whole ectoderm instead of a capsule to each individual cell. It is to an exoskeletal structure formed in this way, i.e. by the secretion of successive layers from the free faces of adjacent cells, that the name cuticle is in strictness applied in multicellular organisms.

The medusæ ( $\mathrm{B}$, med, and $\mathrm{c}$ ), mentioned above as occurring on lateral branches of the colony, are found in various stages of development, the younger ones having a nearly globular shape, while when fully formed each resembles a bell attached by its handle to one of the branches of the colony and having a clapper in its interior. When quite mature the medusæ become detached and swim off as little jelly-fishes (c).

The structure of a medusa must now be described in some detail. The bell or umbrella (c) is formed of a gelatinous substance (Fig. 77, D, $7 / \mathrm{hgl}_{\mathrm{g}}$ ) covered on both its inner surface or sub-umbrella and on its outer surface or ex-umbrella by a thin layer of delicate cells (ect). The clapper-like organ or manubrium (Fig. 76, $\mathrm{C}$ and Fig. $77 \mathrm{D}$ and $\mathrm{D}^{\prime}$, mnb) is formed of two layers of cells, precisely resembling the ectoderm and endoderm of Hydra, and separated by a thin mesogloea ; it is hollow, its cavity (Fig. 77, ent. cav) opening below, i.e. at its distal or free end, by a rounded aperture, the mouth $(m t h)$, used by the medusa for the ingestion of food. At its upper (attached or proximal) end the cavity of the manubrium is continued into four narrow, radial canals (Fig, $76, \mathrm{c}, \mathrm{rad} . c$, and Fig. $77, \mathrm{D}$ and $\mathrm{D}^{\prime} \mathrm{rad}$ ) which extend 


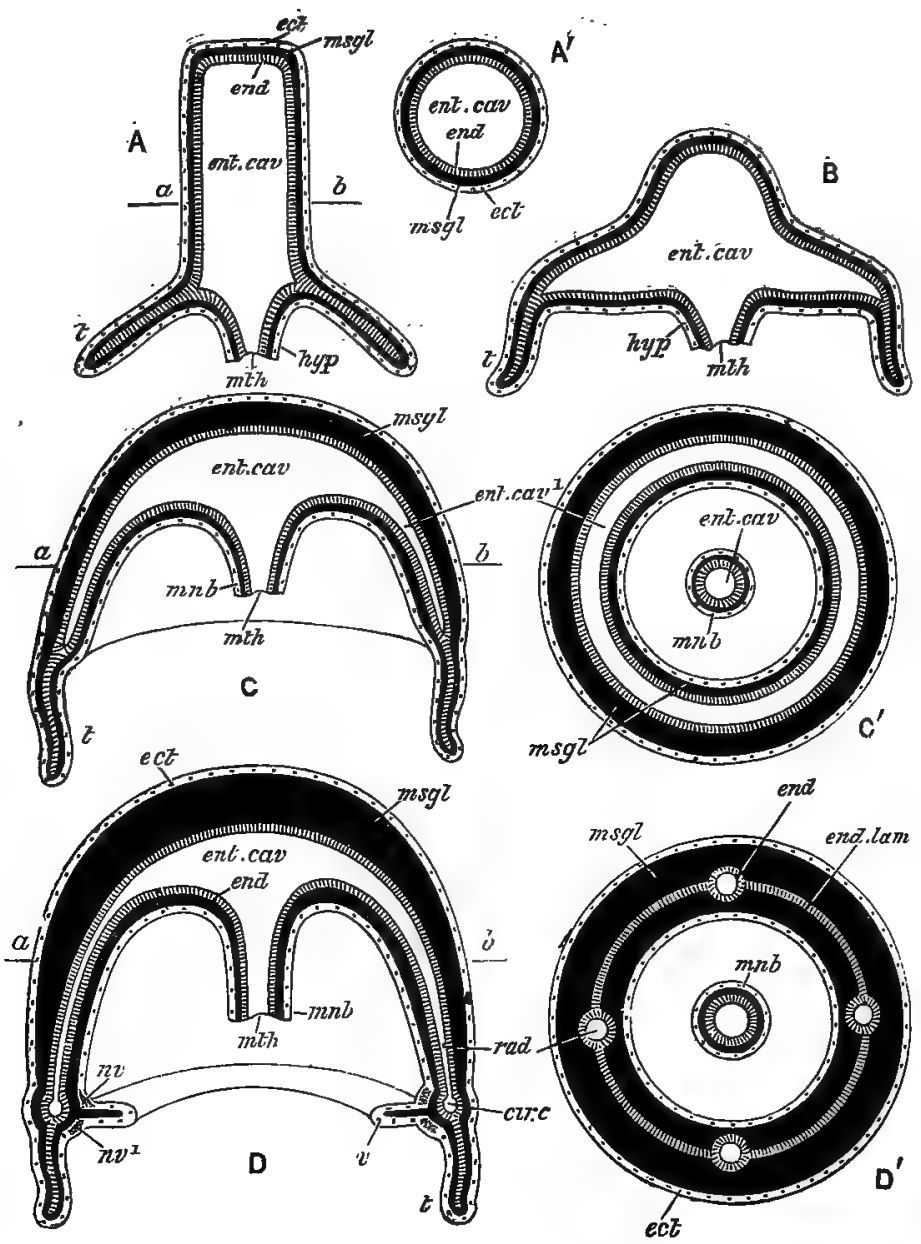

FIG. 77.-Diagrams illustrating the derivation of the medusa from the hydranth. In the whole series of figures the ectoderm $(e c t)$ is dotted, the endoderm (end) striated, and the mesogloa (msgl) black.

A, longitudinal section of a simple polyp, showing the tubular body with enteric cavity (ent.cav), hypostome (hvp), mouth (mth.), and tentacles $(t)$.

$A^{\prime}$, transverse section of the same through the plane $a b$.

$B$, the tentacular region is extended into a hollow disc.

C, the tentacular region has been further extended and bent into a lell-like form 
the enteric cavity being continued into the urabrella (ent. cav'); the hypostume now forms a manubrium $(m n b)$.

$C^{\prime}$, transverse section of the same through the plane $a b$, showing the continuous cavity (ent. cav') in the umbrella.

$D$, fulfy formed medusa; the cavity in the umbrella is reduced to the radial ( $r a d)$ and circular (cir.c) canals, the velum (v) is formed, and a double nervering $\left(n v, n v^{\prime}\right)$ is produced from the ectoderm.

$\mathrm{D}^{\prime}$, transverse section of the same through the plane $a b$, showing the four radial canals ( $\mathrm{rad}$ ) united by the endoderm-lamella (end. lam), produced by partial obliteration of the continuous cavity (est.cav') in C. (From Parker's Biology.)

through the gelatinous substance of the umbrella at equal distances from one another, like four meridians, and finally open into a circular canal (cir. c) which runs round the edge of the umbrella. The whole system of canals is lined by a layer of cells (Fig. 77, D and $\mathrm{D}^{\prime}$, end) continuous with the inner layer or endoderm of the manubrium; and extending from one canal to another in the gelatinous substance of the umbrella, is a delicate sheet of cells, the endoderm-lamella ( $\mathrm{D}^{\prime}$, end. lam).

From the edge of the umbrella four pairs of tentacles (Figs. $76, \mathrm{c}$ and $77, t$ ) are given off, one pair corresponding to each radial canal, and close to the base of each tentacle is a little speck of pigment (Fig. 76, or), the ocellus or eyespot. Lastly, the margin of the umbrella is continued inwards into a narrow circular shelf, the velum (v).

At first sight there appears to be very little resemblance between a medusa and a hydranth, but it is really quite easy to derive the one form from the other.

Suppose a simple polyp or Hydra-like body with four tentacles (Fig. 77, A, $A^{\prime}$ ) to have the region from which the tentacles spring puiled out so as to form a hollow, transversely extended $\operatorname{disc}(\mathrm{B})$. Next, suppose this disc to become bent into the form of a cup with its concavity towards the hypostome, and to undergo a great thickening of its mesoglcea. A form would be produced like c, i.e. a medusa-like body with umbrella and manubrium, but with a continuous cavity ( $c^{\prime}$, ent. cav') in the thickness of the umbrella instead 
of four radial canals. Finally, suppose the inner and outer walls of this cavity to grow towards one another and meet, thus obliterating the cavity, except along four narrow radial areas ( $\mathrm{D}, \mathrm{rad}$ ) and a circular area near the edge of the umbrella (cir. c). This would result in the substitution for the continuous cavity of four radial canals opening on the one hand into a circular canal and on the other into the cavity of the manubrium (ent. cav), and connected with one another by a membrane-the endoderm-lamella ( $D^{\prime}$ end. $(a m)$-indicating the former extension of the cavity.

It follows from this that the inner and outer layers of the manubrium are respectively endoderm and ectoderm: that the gelatinous tissue of the umbrella is an immensely thickened mesogloea : that the layer of cells covering both inner and outer surfaces of the umbrella is ectodermal : and that the layer of cells lining the system of canals, together with the endoderm-lamella, is endodermal.

Thus the medusa and the hydranth are similarly constructed or homologous structures (p. 217), and the hydroid colony is dimorphic (p. 250), bearing zooids of two kinds. In some allied forms, this individuation may go still further, the zooids being of very various forms and performing diverse functions: such a colony is said to be polymorphic.

Sooner or later the medusæ separate from the hydroid colony and begin a free existence. Under these circumstances the rhythmical contraction-i.e. contraction taking place at regular intervals - of the muscles of the umbrella causes an alternate contraction and expansion of the whole organ, so that water is alternately pumped out of and drawn into it. The obvious result of this is that the medusa is propelled through the water by a series of jerks. The movement is performed by means of the muscle-processes 
and muscle-fibres of the sub-umbrella and velum, some of which differ from the similar structures in the hydranth in exhibiting a delicate transverse striation.

There is still another important matter in the structure of the medusa which has not been referred to. At the junction of the velum with the edge of the umbrella there lies, immediately beneath the ectoderm, a layer of peculiar, branched cells, containing large nuclei and produced into long fibre. like processes. These nerve-cells (p. r67) are so disposed as to form a double ring round the margin of the bell, one ring (Fig. 77, I, 'nv) being immediately above, the other $\left(n v^{\prime}\right)$ immediately below the insertion of the velum. An irregular network of similar cells and fibres occurs on the inner or concave face of the bell, between the ectoderm and the layer of muscle-fibres. The whole constitutes the nervous system of the medusa; the double nerve-ring is the central, the network the peripheral nervous system (p. 155).

Some of the processes of the nerve-cells are connected with ordinary ectoderm-cells, which thus as it were connect the nervous system with the external world: others, in some instances at least, are probably directly connected with muscle-fibres.

We thus see that while the manubrium of a medusa has the same simple structure as a hydranth, or what comes to the same thing, as a Hydra, the umbrella has undergone a. very remarkable differentiation of its tissues. Its ordinary ectoderm cells, instead of being large and eminently contractile, form little more than a thin cellular skin or epithelium (p. I 09 ) over the gelatinous mesogloea: they have largely given up the function of contractility to the muscleprocesses or fibres, and have taken on the functions of a protective and sensitive layer.

Similarly the function of automatism, possessed by the 
whole body of Hydra, is made over to the group of specially modified ectodermal cells which constitute the central nervous system. If a Hydra is cut into any number of pieces each of them is able to perform the ordinary movements of expansion and contraction, but if the nerve-ring of a medusa is removed by cutting away the edge of the umbrella, the rhythmical swimming movements stop dead: the umbrella is in fact permanently paralysed.

It is not, however, rendered incapable of movement, for a sharp pinch, i.e. an external stimulus, causes a single contraction, showing that the muscles still retain their irritability. But no movement takes place without such external stimulus, each stimulus giving rise infallibly to one single contraction: the power possessed by the entire animal of independently originating movement, i.e. of supplying its own stimuli, is lost with the central nervous system (compare p. I72).

Another instance of morphological and physiological differentiation is furnished by the pigment spots or ocelli (Fig. 76, c, oc) situated at the bases of the tentacles. They consist of groups of ectoderm cells in which are deposited granules of deep red pigment, and which serve as organs of sight.

The two forms of zooid - hydranth and medusa (p. $3^{\circ}$ ) are respectively nutritive and reproductive in function, the reproductive zooids becoming detached and swimming off to found a new colony elsewhere : the hydranths are purely nutritive zooids; the medusæ, although capable of feeding, are specially distinguished as reproductive zooids. The gonads are found in the walls of the manubrium, between the ectoderm and endoderm, some medusæ producing ovaries, others spermaries only. Thus while Hydra is monoecious (p. 302), Bougainvillea is, like the frog, dioecious, 
certain individuals producing only male, others only female products.

The medusæ, when mature, become detached and swim away from the hydroid colony. The sperms of the males are shed into the water and carried to the ovaries of the females, where they fertilize the ova, converting them, as usual, into oosperms.

The oosperm undergoes segmentation, forming a polyplast or morula (p. 200): ectoderm and endoderm become differentiated, and the ectoderm cells acquire cilia, by means of which the embryo now swims freely in the water. An enteron appears in the endoderm, and in this stage the embryo, which has an elongated form, is known as a planula. It then loses its cilia and settles down on a rock, shell, sea-weed, or other submarine object, assuming a vertical position with its broader end fixed to the support.

The attached or proximal end widens into a disc of attachment, a dilatation is formed a short distance from the free or distal end, and a thin cuticle is secreted from the whole surface of the ectoderm. From the dilated portion short buds arise in a circle: these are the rudiments of the tentacles: the narrow portion beyond their origin becomes the hypostome. Soon the cuticle covering the distal end is ruptured so as to set free the growing tentacles: an aperture, the mouth, is formed at the end of the hypostome, and the young hydroid has very much the appearance of a Hydra with a broad disc of attachment, and with a cuticle covering the greater part of the body. Extcnsive budding next takes place, the result being the formation of the ordinary hydroid colony.

Thus from the oosperm or impregnated egg-cell of the medusa the hydroid colony arises, while the medusa is produced by budding from the hydroid colony. We have 
what is called an altemation of generations, the asexualgeneration or agamolium (hydroid colony) giving rise by budding to the sexual reneration or gamobium (medusa), which in its turn produces the agamobium by a sexual process, i.e. by the conjugation of ovum and sperm.

Hydra and Bougainvillea both belong to the simplest class-the Hydrozoa-of the phylum Colenterata: this phylum includes all the polypes or zoophytes, the jelly-fishes, and the anemones and corals. In all there is an ectoderm and an endoderm, separated by a mesogløea, which may consist, as in Hydra, of a structureless membrane containing no cells, or may be gelatinous as in the medusa, and may even contain cells, thus assuming more the character of an intermediate cell-layer or mesoderm. There is no bodycavity or colome (p. 2o) surrounding the digestive cavity or enteron, and tentacles are present round the mouth. Organs of offence occur in the form of thread-cells or nematocysts.

In all the higher phyla a definite mesoderm is developed in the embryo in addition to the ectoderm and endoderm (triploblastic condition), and in nearly all cases there is a definite cavity or coelome present in the mesoderm: hence all these animals are often included together as the Cœlomata.

\section{Hydra.}

\section{PRACTIC.IL DIRECTIONS.}

Examine some living Hydre in a vessel of water, with the naked eye or with a pocket lens, and note the differences in form according to the clegree of contraction. The animal is usually attached to foreign bodies (weeds, \&c.), at one end, and at the other end a number of tentacles (usually six to eight) are given off. In the expanded state the body and tentacles are greatly elongated and thread-like, while when contracted the body is more globular, and the tentacles appear like smal! knobs. 
Note the brown colour in $H$. fusca, and the green colour in $X I$. viritis. Observe the method of seizing foorl. Place a specimen on a slide in a drop of water, together with a small piece of water-weed or paper to prevent crushing, and then put on a cover-glass. Wait till the animal is fully expanded, and then examine with the low power. Note:-

I. The body, enclosing the digestize cavity or enteron, which opens by the mouth on the free or distal end of the animal, at the summit of a conical hypostome. At the proximal end is the flattened foot or disc of attachment.

2. The tentackes, arranged in a single circlet or zehor around the base of the hypostome. They are hollow, and their cavities communicate proximally with the general digestive carity of the body. On their surface are a number of small knobs.

3. The great contractility of the animal, ancl especially of the tentacles.

4. The structure of the body-zeall, which is made up of $(a)$ an outer layer of colourless cells (ectoderm); and $(b)$ an inner layer (brown in $H$. fusca and green in $H$. viridis) of cells (endoderm) lining the digestive cavity. Between these two layers is a thin gelatinous non-cellular supporting lamella or mesoglua, not easily seen with the low power. (The tentacles have a similar structurc, the details of which cannot be made out with the low power.) Sketch.

Put on the high power and examine a tentacle, focussing on to the surface as well as deeper, so as to get an optical section. Note:-

5. The relations of the ectoderm, endoderm, and supporting lamella, and the nuclei of the ectoderm and endoderm cells.

6. The structure of the ectodern:-(a) las"ge conical cells, with their broader ends outwards, arranged in a single row, and differing in form according to the state of contraction. The spaces between the inner narrower ends of these are filled up with (b) smaller rounded interstitial cells (absent on the foot); (c) thread-cells or nematocysts (Fig. 75)-oval capsules containing a spirally-wound thread, leveloped within certain of the interstitial cells called cnidoblasts, and when fully formed, found imbedded in or between the large ectoderm-cells. They are much more numerous on the tentacles than on the body, causing the knobs referred to above. Each cnicloblast gives rise to a small process-the triggerhair or cnidocil, which projects from the surface. Notice the discharged thread-cells, and observe that each consists of a flask-like base (to which part of the protoplasm and the nucleus of the burst cnicloblast usually 
remains attached) and a long filament, with three large and several smaller spines or barbs at its proximal end. (Smaller thread-cells, with thicker threads and no spines, are also present; some of these have long, spirally coiled threads, others shorter, straight threads. These can be seen better later on.)

7. The endoderm, consisting of a single layer of large amoboid cells, which in $H$. viridis contain 'green chromatophores. Note the currents in the tentacles, which are produced by long vibratile fagella present on many of the endoderm cells.

8. The thin transparent supporting lamella. Sketch.

9. Treat a specimen with methyl-green. A slight pressure on the cover-glass will crush the animal, and render the interstitial cells and thread-cells especially distinct. Note also other isolated cells of the ectoderm and endoderm. Sketch.

IQ. Examine a specimen with buds in different stages of development, and note as much as possible of the mode of asexual reproduction by gemmation. Sketch.

II. If none of your specimens bear sexual organs, try and procure a mounted preparation which shows them, and examine first with the low, and then the high power. Note-(a) The spermaries-several conical swellings, usually not far below the tentacles. They are covererl with large ectoderm cells, and contain numerous interstitial cells, each of which eventually gives rise to a sperm with a "head" and vibratile "tail," These are discharged at the apex of the cone, which when ripe may be ruptured by a slight pressure on the cover-glass. (b) The ovaries (sometimes only one), generally situated near the proximal end of the body. They are larger than the spermaries and more spherical, but at first have a similar structure. When ripe a single orum is found in each. Sketch.

I r Place some Hydræ in a watch-glass with a very small amount o water, and when they have expanded, pour quickly over them a warm saturated solution of corrosive sublimate in alcohol. Wash several times with weak alcohol, stain for a few minutes with borax-carmine, and wash with weak and then with stronger alcohol. Place in absolute alcohol for a few minutes, and afterwards in turpentine or oil of cloves; mount in balsam. Work through $\$ \S 5$ again, noting especially the characters of the various cells and their nuclei, as well as-

12. The contractile processes coming off from the inner ends of the 
large ectoderm cells. These extend longitudinally, and lie against the onter surface of the supporting lamella. Sketch.

Examine transverse sections through the body or tentacles, prepared as directed on p. 136, after killing and fixing the specimens as above. Work through $6-8$ again, noting the various cells and their nuclei, \&c. Observe especially-

13. (a) The contractile processes of the ectoderm cells, which will be cut across transversely, so as to appear as dots just outside the supporting lamella ; $(b)$ the amoboid and vacuolated character of the endoderm cells. (Special methods of preparation are necessary in order to show the flagella.) Sketch.

\section{Bougainvillea. ${ }^{1}$}

If possible, examine first alive, and then kill and stain as directed int the case of Hydra. Examine under the microscope and note:--

a. Colonial stage. I. The hydranths, attached to a common branching stem : compare their structure with that of Hydra. 2. The medusa= buds. 3. The cuticular exoskcleton supporting the colony.

b. Meducsa stage. I. The temlirella, tentacles, velum, manubrium, mouth, radial and circular canals, ocelli, and gonads.

${ }^{1}$ Specimens living or preserved, both of the colonial and medusa stage of Bougainvillea or some allied form (e.g. Obelia), can be obtained from any Marine Biological Laboratory; or the fresh-water Cordylophord will answer the purpose as far as the colony is concerned, but it has nu medusa stage. 


\section{CHAPTER V}

THE LARTHWORM-CHARACTERS OF THE PHYLUM ANNULATA

THE getleral form and appearance of an earthworm are familiar to every one. In this country there are a number of different species of earthworms belonging to several genera, the commonest of which are Lumbricus and Allolobophora; but the differences between these are of minor importance to the beginner, and any one of the common forms will serve our purpose,

Earthworms burrow into the soil and live on decaying leaves and other organic matter, which they swallow together with a considerable quantity of earth. This earth, mingled with the undigested portions of the food, is passed from the body on to the surface of the ground in the form of the well-known little heaps or "castings " which you must have noticed in gardens and fields, especially after rain, when the worms come more frequently to the surface. In this way, a quantity of finely divided earth, mixed with the fæces of the worms, is constantly being spread out on the surface of the soil, and Darwin calculated that on an average a layer of earth about onefifth inch in thickness or about ten tons an acre is thus brought to the surface in the course of a year. Earthworms are therefore good friends to the gardener and 
agriculturist, as they are continually ploughing and manuring the soil, and in doing so, they gradually cover up stones and other objects lying on the surface.

The body of the earthworm is long and narrow, approximately cylindrical in shape, and bilaterally symmetrical (p. 291): in the common forms it reaches a length of about six inches. Anteriorly it is bluntly pointed, while more posteriorly it is somewhat flattened, its greatest diameter being reached at about a third of the entire length from the anterior end. In the ordinary creeping movements of the animal, which are effected by the alternate contraction and extension of its body, the anterior end is directed forwards. The colour is pinkish in most species, and is paler on the lower or ventral than on the upper or dorsal side.

The surface of the body is distinctly marked by transverse annular grooves into body-segments or metameres (Fig. 79), the number of which is about 150 , more or less : the segments are rather longer towards the anterior end than they are further back. At the extreme anterior end is a small finger-shaped head-lobe or prostomium, which overhangs the mouth, situated on the antero-ventral surface of the next segment, which is therefore called the peristomium, and is counted as the first metamere. The anus is a slit-like aperture on the hinder surface of the last or anal segment. The earthworm is thus a metamerically seg. mented animal, and the segments are serially homologous with one another (p. 39).

In adult worms a prominent glandular swelling is noticeable on the dorsal and lateral surfaces of the body, extending through about six segments beginning at about the thirtieth; this is known as the clitellum, and, as we shall see, is important in the process of im- 
pregnation and in forming a case or coccoon for the eggs. On the ventral part of this region are some small glandular swellings, which are more conspicuous in young worms before the clitellum is developed.

The whole of the body is invested with a delicate, iridescent membrane or cuticle (p. 306) formed as a secretion of the epiderm or outer epithelial layer of the body (compare p. 128). Every segment, except the first and the last, is provided with eight small cuticular spines or seta (Fig. 78, set) —slightly curved bodies with tapering ends composed of a horn-like substance called chitin-each of which is developed in a small sac formed as an involution of the epiderm, and is provided with muscles by means of which it can be protruded and retracted. Thəse setæ are arranged in couples, forming two double rows along each latero-ventral region of the body, and their points can be distinctly felt on drawing the worm through the fingers : they serve to prevent the animal from slipping backwards as it moves along on the surface of the ground or in its burrows.

We have seen that the earthworm takes in its food, together with quantities of earth, by the mouth, and after retaining it for a longer or shorter time in the body, expels it by the anus. It is obvious, therefore, that there must be some kind of digestive cavity into which the food passes by the mouth, and from which effete matters are expelled through the anus. Sections (Figs. 78 ) show that this cavity is not a mere space excavated in the interior of the body, but a definite tube, the enteric or alimentary canal (p. 23), which passes in a straight line from mouth to anus, and is separated in its whole extent from the walls of the body by a wide space, the body-cavity or calome (cal), as in the frog (, 20$)$. So that the general structure of the earthworm might be imitated by taking a wide tube, 
stopping the ends of it with corks, boring a hole in each cork, and then inserting through the holes a narrow tube of the same length as the wide one. The outer tube would represent the body-wall, the inner the enteric canal, and the cylindrical space between the two the cœlome. The inner tube would communicate with the exterior by each of its ends, representing respectively mouth and anus; the space between the two tubes, on the other hand, would have no communication with the outside. A transverse section of the body has, therefore, the general character of two concentric circles.

It will be remembered that a transverse section of Hydra has the character of two concentric circles, formed respectively of ectoderm and endoderm (Fig. 74, p. 293), the two layers being, however, only separated by the thin mesogloea. At first sight then, it seems as if we might compare the earthworm to a Hydra in which the ectoderm and endoderm, instead of being in contact, were separated by a wide interval; we should then compare the body-wall of the earthworm with the ectoderm of Hydra, and its enteric canal with the endoderm. But this comparison would only express part of the truth.

A thin transverse section (Fig. 78) shows the body-wall of the earthworm to consist of four distinct layers. Outside is a thin transparent cuticle (cut) showing no structure beyond a series of intersecting oblique lines. Next comes a layer of epithelium, the epiderm, or deric epithelium (epid). Within this is a very thin connective tissue layer representing the derm (p. I28), and a double layer of muscle-fibres by means of which the movements of the body are produced-an outer, in which the fibres extend transversely round the body (circ. mus), and a much thicker inner layer consisting of longitudinal fibres, in section arranged like the barbs of a 
feather on a central axis (long. mus). Finally, within the muscular layer and lining the cœlome is a thin peritoneal membrane (parietal layer, compare p. 26), on the inner

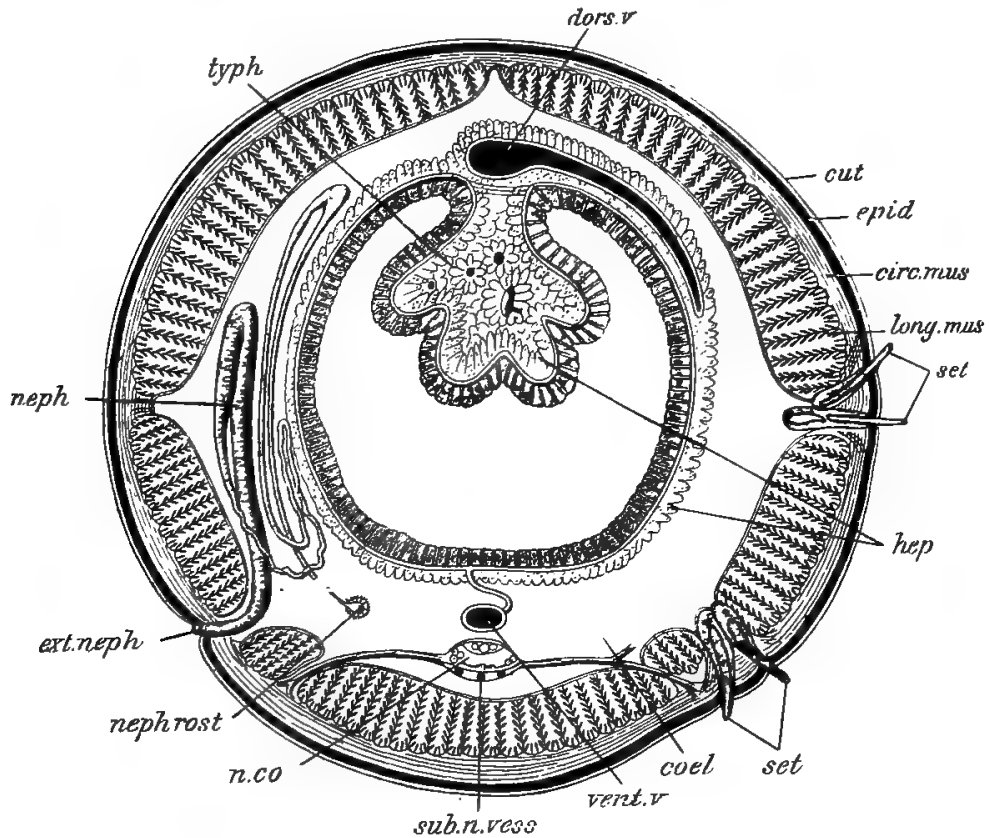

FIG. 78.-Lumbricus, transverse section iof the middlefregion of the body. circ. mus. layer of circular muscular fibres?; cal. colome; cut. cuticle ; dors. $v$. dorsal vessel; epid. epiderm; ext. neph. nephridropore; hep. layer of yellow cells; long. mus. longitudinal muscles; neph. nephridium; nephrost. nephrostome; nerv.co. nerve cord; set. seta; sub. n. vess. sub-neural vessel; typh. typhlosole; vent.v. ventral vessel. (From Parker and Haswell's Zoology, after Marshall and Hurst.)

surface of which is a very thin layer of cells the calomic epithelium.

A transverse section of the intestine shows an inner layer of ciliated, columnar enteric epithelium (compare $\mathrm{p}$. rog), a thin middle layer composed of muscle-fibres and 
connective tissue, and an outer layer of large yellow cells, the function of which is not thoroughly understood, and which correspond to a special development of the cœlomic epithelium covering the visceraly layer of the peritoneal membrane which invests the intestine.

We are now in a better position to compare the transverse section of Hydra and of the earthworm. The epiderm of the earthworm being the outermost cell-layer is to be compared with the ectoderm of Hydra, and its cuticle with the layer of the same-name which, though absent in Hydra, is present in the stem of hydroid polypes, such as Bougainvillea (p. 306). The enteric epithelium of the earthworm, bounding as it does the digestive cavity, is clearly comparable with the endoderm of Hydra. So that we have the double layer of musclefibres and the two layers of peritoneum not represented in Hydra, in which their position is occupied merely by the mesogloea. The muscle-fibres are not of the striped kind, like those in the corresponding position in the frog (p. II 2).

But it will be remembered that in Medusæ there is sometimes found a layer of separate muscle-fibres between the ectoderm and the mesoglœea, and it was pointed out (p. 304) that such fibres represented a rudimentary intermediate cell-layer or mesoderm. We may therefore consider the muscular layer and the peritoneum of the earthworm as mesoderm, and we may say that in this animal, as in the frog (p. 203 and Fig. 65), the mesoderm is divisible into an outer or parietal layer, an inner or visceral layer.

The parietal layer is in contact with the ectoderm or deric epithelium, and with it forms the body-wall; the visceral layer is in contact with the endoderm or enteric epithelium, 
and with it forms the enteric canal. The cœlome separates the parietal and visceral layers from one another, and is lined throughout by coelomic epithelium.

The relation between the diploblastic polype and the triploblastic worm may therefore be expressed in a tabular form as follows-

\section{Hydroid \\ Earthworm. ${ }^{1}$}

Cuticle . . . . . Cuticle.

Ectoderm . . . . . Deric epithelium or epiderm.

Mesoderm . .
(rudimentary) $\left\{\begin{array}{c}\text { Parietal } \\ \text { layer }\end{array}\left\{\begin{array}{l}\text { Connective tissue and muscle- } \\ \text { fibres. } \\ \text { Peritoneum with its colomic } \\ \text { epithelium (parietal layer). }\end{array}\right.\right.$
Visceral
layer $\left\{\begin{array}{c}\text { Peritoneum with its coelomic } \\ \text { epithelium (visceral layer). } \\ \text { Connective tissue and muscle- } \\ \text { fibres. }\end{array}\right.$

Endoderm . . . . Enteric epithelium.

Strictly speaking this comparison does not hold good of the anterior and posterior ends of the worm : at both mouth and anus the deric passes insensibly into the enteric epithelium, and the study of development shows that the cells lining both the anterior and posterior ends of the canal are ectodermal (compare pp. 204 and 207). For this reason the terms deric and enteric epithelium are not mere synonyms of ectoderm and endoderm respectively.

It is important that you should, before reading further, understand clearly the general composition of a tripoblastic

1 It will be seen that the relations of these layers in the earthworm and frog are similar, except that in the latter the cuticle is wanting (compare Figs. 5, 38, and 39). 
animal as typified by the earthworm, which may be summarised as follows. It consists of two tubes formed of epithelial cells, one within and parallel to the other, the two being continuous at either end of the body, where the inner tube (enteric epithelium) is in free communication with the exterior; the outer tube (deric epithelium) is lined by a layer of connective tissue and muscle-fibres, within which is a thin peritoneum lined by colomic epithelium, the three together forming the body-wall; the inner tube (enteric epithelium) is covered externally by a layer of muscle-fibres and connective tissue and a thin peritoneum covered by colomic epithelium, which form with it the enteric canal; lastly, the body-wall and enteric canal are separated by a considerable space, the colome.

The enteric canal is not, as might be supposed from the foregoing description, connected with the body-wall only at the mouth and anus, but is supported in a peculiar way. There is no dorsal mesentery as in the frog (p. 27), but a series of transverse vertical partitions or septa (Fig. 79) extend right across the body-cavity, each being perforated by the canal. The septa are regularly arranged and correspond in position with the external grooves by which the body is divided into metameres. Thus the transverse or metameric segmentation affects the cœlome as well as the body-wall, the former being divided up into a series of chambers, which, however, communicate with one another ventrally, where the septa are incomplete. Each septum is composed of a sheet of connective tissue and muscle-fibres, and is covered on both sides by colomic epithelium. The cœlome communicates with the exterior by a series of dorsal pores situated in the grooves between all the segments except about the first ten. 


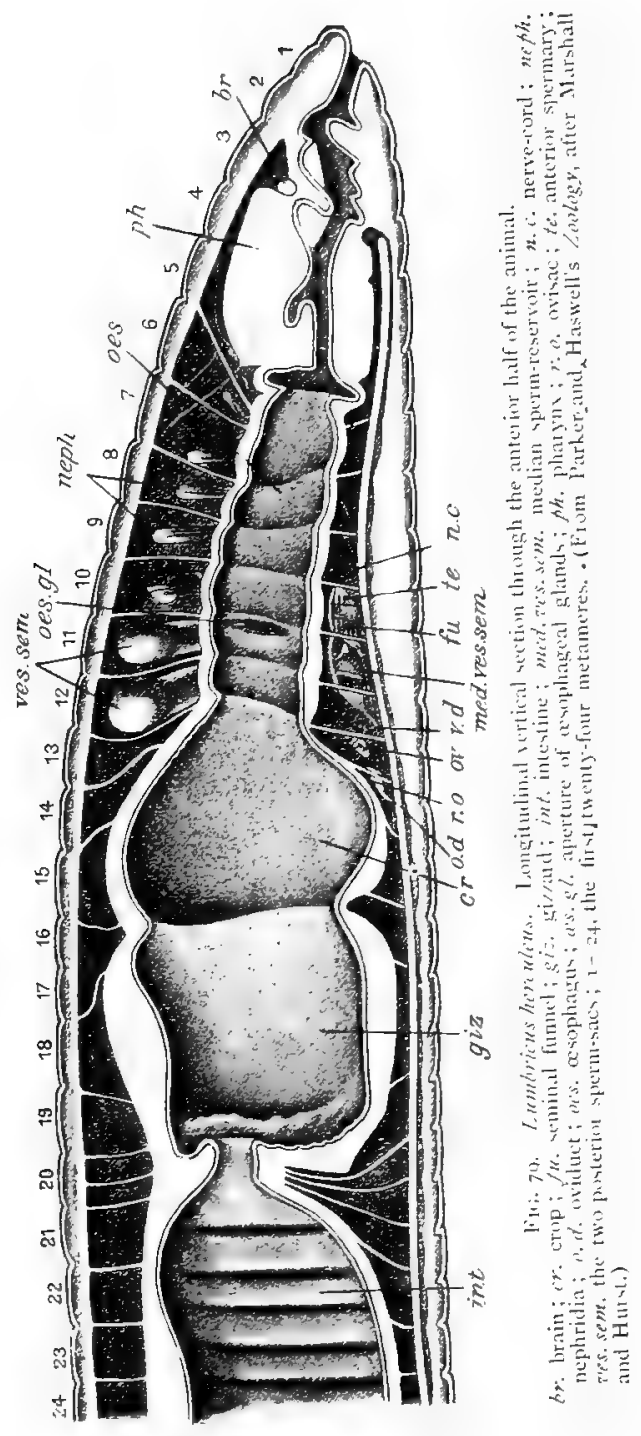


The digestive canal is not a simple tube of even calibre throughout, but is divisible into several portions. The mouth is bounded by a soft lip and leads into a small buccal cavity, which communicates with a thick-walled pharynx (Fig. 79, ph), extending through about five segments and connected with the body-wall by a number of radially arranged muscle-fibres, the septa being absent in this region. When the worm feeds, the buccal cavity is everted, and the muscles serve to draw it and the pharynx back again, as well as to dilate the pharynx. The latter is followed by a narrow gullet or asophagus (oes.) extending through about eight segments, which is provided towards its hinder end with three lateral pairs of glandular swellings of a yellowish colour-the cesophageal glands: these contain a calcareous substance which serves to neutralise the organic acids present in the food swallowed. The first two pairs communicate with the third pair, which open into the œsophagus (oes, $g l$ ). The gullet opens into a dilated, thin-walled receptacle, the crop ( $c r)$, and this, again, communicates posteriorly with a large gizzard (giz) with thick and muscular walls, which in about the 2 oth segment communicates with the intestine (int). The intestine has a similar character throughout, and extends from the gizzard to the anus: its dorsal wall is folded inwards so as to produce a longitudinal ridge or typhlosole (Fig. 78, typh), which serves to increase the absorptive surface and in the interior of which the yellow cells are very numerous.

Certain of the cells lining the enteric canal, and especially those along the typhlosole, are very granular, and like the endoderm cells of the hypostome of Hydra (p. 298) are to be considered as unicellular glands. They secrete a digestive juice which-mixing with the various substances containing organic acids taken in by the mouth, and 
neutralised by the calcareous secretion of the csophageal glands-dissolves the proteids and other digestible parts so as to allow of their absorption. It is very probable that the process is purely extra-cellular or enteric, the food being dissolved and rendered diffusible entirely in the cavity of the canal (p. 300). By the movements of the canal-caused partly by the general movements of the body and partly by the contraction of the muscles of the canal and septa, aided by the action of the cilia, the contents are gradually forced backwards and the earth and other indigestible matters are expelled at the anus.

The colome is filled with a colourless transparent colomic fluid in which are suspended amœboid corpuscles or leucocytes like those of the frog's blood and lymph (p. 105). The function of this cœlomic fluid is probably to distribute the digested food in the enteric canal to all parts of the body. In Hydra, where the lining wall of the digestive cavity is in direct contact with the simple wall of the body, the products of digestion can pass at once by diffusion from endoderm to ectoderm; but in the present case a means of communication is wanted between the enteric epithelium and the comparatively complex and distant body-wall. The peptones and other products of digestion diffuse through the enteric epithelium into the cœlomic fluid, and by the continual movement of the latter-due to the contractions of the body-wall-are distributed to all parts. Thus the external epithelium and the muscles, as well as the nervous system and reproductive organs not yet described, are wholly dependent upon the enteric epithelium for their supply of nutriment.

The earthworm, like the frog, possesses a series of blood- 
vessels, containing red blood, the whole of which form a single closed ivascular system, there being no communication between them and any of the other cavities of the body. The main trunks have a longitudinal direction, the chief ones being a large dorsal vessel, running along the dorsal surface of the enteric canal, and a ventral or subintestinal vessel, below the canal (Fig. 78. dors. v, vent. v). In addition to these there are three smaller longitudinal trunks in relation to the nerve-cord, which, as we shall see, extends along the ventral side of the cœlome: these are a median subneural (sub. n. vess) and two lateral neural vessels. All these longitudinal trunks give off branches to the various parts of the body, and certain of them are connected with one another by a pair of lateral commissural vessels : in the region of the gullet there are about five or six pairs of large vessels connecting the main dorsal and ventral trunks; and the dorsal and subneural trunks are also connected in each segment all along the body by a pair of smaller commissural vessels, running in the inner surface of the body-wall.

Notice that there is here no distinction into arteries and veins, as in the frog (p. 27), and also that there is no heart. The vessels gradually divide up into smaller and smaller branches in the various parts of the body, and then again unite to form larger and larger vessels which eventually open into one or other of the main trunks.

The circulation of the blood is effected by the rhythmical, peristaltic contraction ( $p .75$ ) of certain of the larger vessels: thus the dorsal trunk. contracts from behind forwards, and the large commissural vessels-often spoken of as "hearts" - which connect it anteriorly with the ventral trunk, from above downwards, so that the blood passes forwards in the dorsal, backwards in the ventral vessel. The 
blood from the enteric canal is returned by a series of vessels into the dorsal trunk. The vessels of the excretory organs, to be described presently, arise from the ventral trunk, and the blood from these organs is returned into the commissural vessels in the body-wall which connect the dorsal and subneural trunks. By means of branches of these parietal vessels the body-wall is plentifully supplied with blood.

The red colour of the blood is due to hamoglobin ( $\mathrm{p}$. 107), which is not, as in the 'frog, contained in red bloodcorpuscles, but is dissolved in the plasma, in which, however, minute colourless corpuscles can be recognised. The function of hæmoglobin in the process of respiration has already been described (p. I44); but in the earthworm, as in many other lower animals, there are no specialised respiratory organs (lungs or gills), the necessary exchange of gases being performed by the entire surface of the body, the minute branches of the blood-vessels in the body-wall being only separated from the air by the single layer of epidermic cells, and even penetrating amongst the latter in the region of the clitellum: this is an exceptional occurrence, for as we have seen, capillaries do not, as a general rule, extend amongst epithelial cells (compare, e.g., Figs. $38-40$ ).

In discussing in a previous chapter the differences between plants and animals, we found (p. 255) that in the unicellular organisms previously studied, the presence of an excretory organ in the form of a contractile vacuole was a characteristic feature of such undoubted animals as the ciliate Infusoria. But the reader will have noticed that Hydra and its allies have no specialised excretory organ, waste products being apparently discharged from any part of the surface. In the earthworm we meet once more with an animal in which excretory organs are present, although, 
in correspondence with the complexity of the animal itself, they are very different from the simple contractile vacuoles of Paramœcium or Vorticella, and are more nearly comparable with those of the frog ( $p$. I46).

The excretory organs of the earthworm consist of little tubes called nephridia, of which each metamere-. except the first three and the last-possesses a pair, one on either side (Figs. 78 , 79 , and 80 neph). You will remember that in the frog all the nephridia are connected together to form a pair of kidneys, each with a single duct communicating with the cloaca ( $p$. 145). In the earthworm each nephridium is a lon and extremely delicate tubc, arranged in three main loops (Fig. 80), opening at one end into the cœlome by a nephrostome and at the other communicating with the exterior directly

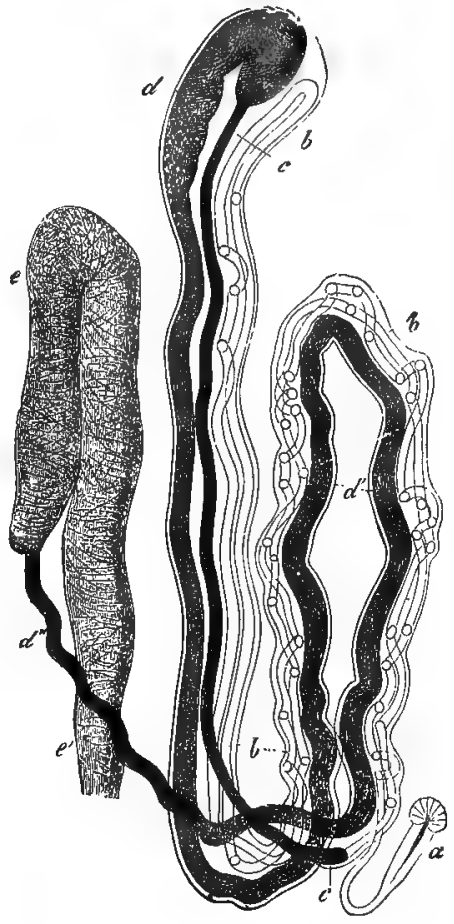

Eig. 80.-A nephridium of Limbricus, showing the three main loops into which the different parts of the tubule are ar. ranged, as well as the different portions of the tubule.

a. nephrostome; b. b.b. slender portion of the tubule into which the nephrostome opens; $c . c$. second ciliated portion; $d$. glandular portion; e. muscular portion; $\ell^{\prime}$. end of $e$ at which the nephridiopore opens. (From Gegenbaur.)

(Fig. 78). ${ }^{1}$ The tubes are

1 In the frog the nephrostomes lose their connection with the nephridia, and open in the adult into the renal veins (Fig. 47, p. I46). 
attached to the posterior faces of the septa. Each nephrostome $(a)$ is ciliated, and projects through the corresponding septum so as to communicate with the segment of the body-cavity next in front of that in which the main part of the tubule is situated. The nephrostome opens into a long and slender transparent part of the tube lined with ciliated cells in part of its course and extending along the first and second loops $(b)$; this part is succeeded by a wider, ciliated portion in the second loop $(c)$, which communicates with a still wider portion (d) lined by granular, non-ciliated, glandular cells, also lying in the second loop; the glandular portion opens to a much wider muscular part of the tube $(e)$ which constitutes the third loop and opens into the exterior by a small pore-the nephridiopore-near the outer seta of the inner couple (Fig. 78).

Thus the nephridia, which are abundantly supplied with blood-vessels, are lined in part by gland-cells and in part by cilia which work towards the exterior. Water and nitrogenous waste from all parts of the body pass by diffusion into the blood and are conveyed to the nephridia, the gland-cells of which withdraw the waste-products and pass them into the cavities of the tubes, whence they are finally discharged from the body. The granular yellow cells on the walls of the intestine also appear to contain excretory products, which become set free in the body cavity and are thence got rid of through the nephridia. It will be noticed that a certain amount of loss of the colomic fluid must take place through the dorsal pores as well as through the nephridia.

In discussing the hydroid polypes we found that one of the most important points of difference between the locomotive medusa and the fixed hydranth was the presence in 
the former of a well-developed nervous system (p. 3 I r) consisting of an arrangement of peculiarly modified cells, to which automatic action was seen to be due. It is natural to expect in such an active and otherwise highly-organised animal as the earthworm a nervous system of a considerably higher degree of complexity than that of a medusa.

The central nervous system consists of two parts, the brain and the ventral nervecord. The brain (Fig. 79 br, and Fig. 8I, cer. gang) consists of a pair of white pearshaped swellings or ganglia situated on the dorsal side of the buccal sac where it is continued into the pharynx. The ventral nerve-cord (n. $c$ and ne.co) is a

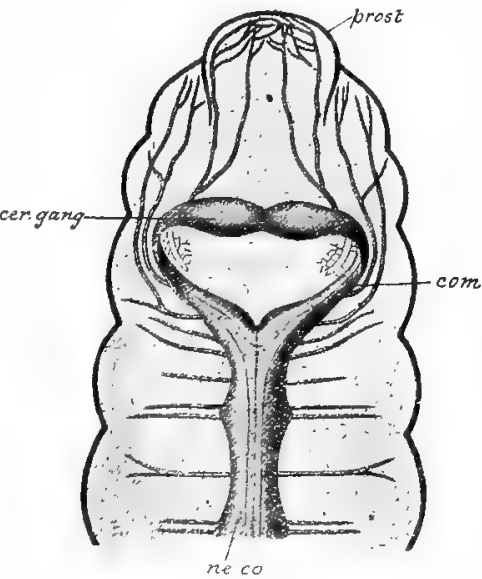

FIG. 8I.-Anterior portion of nervous system of Lumbricus.

cer.gang. cerebral ganglia or brain; com. cesophageal connectives; ne.co. ventral nerve-cord ; prost. prostomium. (From Parker and Haswell's Zoology, after Leuckart.)

longitudinal band extending along the whole middle ventral line of the body, internally to the longitudinal muscular layer, from the third to the anal segment, and slightly swollen in each segment. The brain is connected with the anterior end of the ventral nerve-cord by a pair of nervous bands, the asophageal connectives (com) which pass respectively right and left of the buccal sac, and thus form a nerve-collar. 
It is to be noted that one division of the central nervous system - the brain-lies altogether above and in front of the enteric canal, the other division - the ventral nerve-cordaltogether beneath it, and that, in virtue of the union of the two divisions by the cesophageal connectives, the enteric canal perforates the nervous system. Both brain and cord are composed of delicate nerve-fibres and iof nerve-cells, the latter being situated in the ventral and lateral regions of the cord along its whole length, so that there is here hardly any distinction into ganglia and connectives, although the swellings are often spoken of as ganglia. Along the dorsal side of the cord are three transparent tube-like structures, known as giant-fibres, the function of which is not known (Fig. 78). The whole cord is enclosed in a sheath consisting of connective tissue and muscular fibres.

The peripheral nervous system consists of a number of nerves, both sensory and motor (p. r62), which arise from the central nervous system and supply the various parts of the body. From the brain a number of nerves are given off to the prostomium, and from each ganglionic enlargement two pairs of nerves can be traced into the bodywall, while between these enlargements one pair is given off which supply mainly the septa.

Comparing the nervous system of the earthworm with that of a medusa it is important to notice the concentration of the central nervous system in the higher type, and the special concentration at the anterior end of the body to form a brain. When, again, we compare the central nervous system of the earthworm with that of the frog (pp. 28 and $\mathrm{r}_{55}$ ) several important points of difference are noticeable. In the former it lies freely in the colome, and with the exception of the brain, is situated on the ventral side of the 
body; while in the frog it is enclosed in a neural canal and is dorsal in position. The brain of the frog is a complicated structure, and the whole nervous system is hollow, there being ventricles in the brain and a central canal surrounded by nerve-cells in the spinal cord; while in the earthworm the brain consists merely of a pair of cerebral ganglia, and it and the ventral cord are solid.

The whole nervous system is capable of originating automatic action. It is a well-known fact that if the body of an earthworm is cut into several pieces each performs independent movements ; in other words, the whole body is not, as in the higher animals, paralysed by removal of the brain (p. 172). There can, however, be little doubt that complete co-ordination, i.e., the regulation of the various movements to a common end, is lost when the brain is removed.

The earthworm is devoid of organs of sight or hearing. It exhibits sensitiveness to bright light, which may be due to direct action on the central parts of the nervous system. The sense of hearing appears to be absent; but a faculty analogous to taste or smell, enabling the animal to distinguish between different kinds of food, is well developed. Groups of narrow sensory cells in the epiderm, which are most abundant on the prostomium and peristomium, have probably to do with this faculty.

There are two matters of general importance in connection with the structure of the earthworm to which special attention must be drawn.

Notice in the first place how in this type, far more than in Hydra, we have, as in the frog, certain definite parts of the body set apart as organs (p. $3^{\circ}$ ) for the performance of particular functions: it is clear that differentiation of 
structure and division of physiological labour play a far more obvious and important part than in any of the lower organisms described in the four previous chapters.

Notice in the second place the vastly greater complexity of microscopic structure, the body being divisible into tissues (p. I I 8) each clearly distinguishable from the rest. We have epithelial tissue with its cuticle, muscular tissue, and nervous tissue, as well as blood and colomic fluid. One result of this is, that, to a far greater extent than in Hydra, we can study the morphology of the earthworm, as we have done that of the frog, under two distinct heads: anatomy and histology (p. 104).

Asexual reproduction does not take place normally in the earthworm, but it frequently happens by accident that a worm is cut into two or more parts. When this occurs, each end is able to reproduce the missing portion: this process is known as regeneration.

The earthworm, like Hydra, is monœcious or hermaphrodite (p. 302), and besides the essential organs of sexual reproduction-ovaries and spermaries - which are, as in the frog, developed from certain parts of the cœlomic epithelium, it possesses various accessory organs. The whole reproductive apparatus is situated in segments 9-15.

The ovaries (Figs. 7900 , and 820 ) are a pair of minute bodies about I mm. in length, attached by a short stalk, one on either side, to the posterior face of the septum separating segments twelve and thirteen, not far from the nerve-cord. The proximal end of each ovary, nearest the stalk, is composed of a mass of undifferentiated cells of germinal epithelium (compare Figs. 62 and 63): nearer its middle, certain of these are seen to increase in size so as to be recognisable as young ova: while the distal end 
contains the ripe ova, arranged in a single row, each enclosed in a vitelline membrane and containing a large nucleus and nucleolus and a number of granules of food-yolk (p. 195). The eggs are discharged into the colome and are received into the female gonoducts or oviducts (Fig. 79 o.d., and Fig. 82) - two short tubes, each with a wide ciliated mouth placed opposite the corresponding ovary. The oviduct perforates the next following septum (i.e. that between segments thirteen and fourteen) to open by a minute aperture on the fourteenth segment, near the inner double row of setæ. Connected with the mouth of each oviduct is a small egg-sac (Fig. 79, r. o, Fig. 82 , e. s), developed as an out-

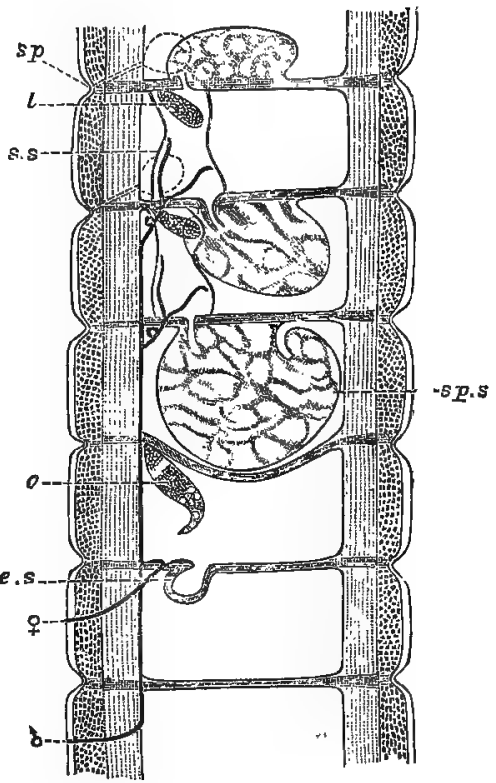

FIG. 82. Diagrammatic longitudinal section o part of a Lumbricus, showing segments 9-15 and the contained generative organs of one side : $x_{3}$. In the body-wall the cuticle is indicated by a clear space, the circular muscles by irregular dots, the longitudinal muscles by dotted longitudinal lines, and the peritoneal membrane by a thin line.

e. $s$. egg-sac ; 0. rovary; $s p$. aperture of anterier spermotheca - both spermotheca are indicated by dotted lines; $s p$. s. posterior sperm-sac, the anterior and middle sacs are not lettered; ss. sperm-sac; $t$. anterior spermary-the posterior is not lettered; 9 . aperture of oviduct; $\delta$. aperture of spermiduct. The oviduct, spermiduct, and seminal funnels are indicated by thick lines. (From the Cambridge Natural History, after Hesse.)

growth from the same septum and extending back into the cavity of segment fourteen.

PrAct. Zool. 
Certain globular sacs called spermothecee (Fig. 82) also belong to the female part of the reproductive apparatus. Of these there are usually two pairs (sometimes more than two in Allolobophora) situated in the ninth and tenth segments, and opening to the exterior between the ninth and tenth, and tenth and eleventh segments respectively; their function will be mentioned presently.

The earthworm possesses two pairs of very minute spermaries attached to the posterior face of the septum between the ninth and tenth, and tenth and eleventh segments respectively (Figs. $79 t e$, and $82, t$ ). They have a flattened form and their free or distal ends are produced into finger-shaped processes. Behind each spermary, and in the same segment, is a ciliated seminal funnel opening into the colome and produced backwards through the septum next behind into an efferent duct, the two ducts of either side communicating with a main spermiduct or vas deferens (Figs. 79 and 82). Each of these extends backwards in the ventral body* wall to open by a tumid lip on the fifteenth segment, near the inner couple of setæ.

The most prominent portions of the reproductive apparatus are certain large whitish bodies-the sperm-sacs or seminal vesicles (Figs. 79, ves. sem, and $82, s p . s$ ) which are very apparent in the adult worm as soon as the colome is cut open in this region. Of these there are three (Lumbricus) or four (Allolobophora) pairs, situated in segments 9-r 2. They arise as outgrowths of the septa, and communicate with the cœlome; but in Lumbricus, the anterior pairand the two posterior pairs respectively become joined across the middle line so as to form two median sperm-reservoirs (Figs. 79; med. ves. sem, and $82, s .5)$, each of which encloses a cœlomic cavity in which one pair of spermaries and seminal funnels becomes enclosed. 
The cells of which the spermaries are composed do not develop into sperms in the testes themselves, but pass into the sperm-sacs, where they undergo division into rounded masses of cells looking very much like a segmenting oosperm in the polyplast stage. Each of these products of division of the testicular cells becomes elongated, and gradually takes on the form of a sperm with a rod-like head and a vibratile tail (compare pp. 194 and 302). When set free, the sperms pass into the spermiducts through the ciliated funnels.

It is well known that many flowers (the reproductive organs of higher plants) contain the generative cells of both sexes, enclosed within the ovules and anthers respectively; yet in very many cases self-fertilization does not occur owing to contrivances of various kinds for its prevention. It has been proved in numerous instances that cross-fertilizationi.e., the impregnation of the ovum in one individual by the male cell of another-is of great importance in keeping up the strength and vigour of the plant from generation to generation. The same is true amongst animals; and though in some monœcious forms, such as the Hydra, there is no special arrangement for the prevention of self-impregnation if the male and female gametes of the same individual ripen at the same time, in others, such as the earthworm, the ova are always fertilized by the sperms from another individual.

This is effected in the earthworm in the following way. Two individuals, their anterior ends pointing in opposite directions, become applied together by their ventral surfaces and attached to one another in this position by a viscid secretion from the clitellum. The sperms are then passed from the male apertures of one into the spermothecæ of the other individual, and the two worms afterwards separate. The clitellum then secretes a tough chitinous tube or cocoon 
which forms a broad ring round the body in this region, and which is gradually siipped forwards. As it passes over the apertures of the oviducts and spermothecx, ova and sperms (the latter derived from the other individual) are passed into it, as well as albumen secreted by certain glands present in this region. When the worm has entirely withdrawn itself from the cocoon, the latter closes up at the ends in virtue of its elasticity, and the eggs, after fertilization, undergo segmentation.

The cells of the polyplast soon become differentiated into an outer ectoderm and an inner endoderm enclosing the archenteron, which communicates with the exterior by the blastopore (compare p. 201). A mesoderm (p. 202) is then developed, and each layer gradually gives rise to the corresponding parts in the adult animal, much as in the frog (compare p. 209), except that the greater part of each nephridium is apparently derived from the ectoderm, only the inner end of the tube, not the whole of it, arising from the mesoderm: the mesoderm undergoes segmentation; the colome appearing in it as a cavity (p. 203)-or rather as a series of cavities, one in each segment. The young worm is then hatched, and it is to be noticed that it passes through no metamorphosis (p. I I).

In the marine worms belonging to the same class as the earthworm, on the other hand, the young is hatched in the form of a larva known as the trochosphere, which swims by means of cilia arranged in circles round the body, and gradually undergoes metamorphosis into the adult form.

There are a number of different kinds of animals commonly known as "worms," but many of these (e.g. the parasitic worms in the lungs and bladder of the frog, the tapeworms, \&c.), are very different from the earthworm in structure, and are placed in several different 
phyla. The earthworm is a member of the phylum Annulata, which also includes a number of other worms living in the sea and in fresh-water, as well as the leeches, \&c. In all these the body is elongated and divided into metameres; there is usually an extensive cœlome; and the nervous system and nephridia are similar to those of the earthworm. The class Chatopoda, in which the earthworms and their fresh-water and marine allies are included, receives its name from the fact that all its members are provided with cuticular setæ, which in the order to which the marine forms belong (Polychata) are usually long, and of varied forms, and are much more numerous than in the earthworms and fresh-water worms, which constitute the order Oligochata. This order includes several families, both Lumbricus and Allolobophora belonging to the family Lumbricide.

\section{PRACTICAL DIRECTIONS.}

\section{EARTHWORM.}

Select a large earthworm, and after noting its movements and mode of progression, kill by immersion in spirit for a few minutes and then place in a dish and let the tap run on it for a short time.

A. External Characters :-

I. Note:-a. The form and colour of the body and its division into metameres; 6 . the anterior end terminating in the prostomium and followed by the peristomium; $c$. the clitellum; and $d$. the last or anal segment.

2. If the worm be drawn through the fingers backwards, the seta will be felt : examine with a lens and observe their position and the number in each segment.

3. Make out the following apertures: $a$. the mouth; $b$. the anus; c. the dorsal pores (p. 325); $d$. the two apertures of the spermiducts, with thickened lips, on the fifteenth segment.

(It requires careful examination to see the other apertures, viz:those of the oviducts, spermotheca, and nephridia).

Sketch from below' or from the side, 


\section{B. Dissection :-}

I. Take a freshly-killed worm in the left hand, and carefully insert the point of the fine scissors into the integument about one-third of the way down the body, close to the middle dorsal line. Place a drop of the calomic fluid which exudes on a slide, add a drop of salt solution, and cover. Examine with the low and high powers, and note the structure and movements of the amaboid corpuscles. Sketch at intervals.

II. Continue the cut forwards to the prostomium, keeping very slightly to one side of the median dorsal line, and taking care that the point of the scissors does not penetrate deeper than the integument : note the iridescent cuticle. Place the animal in a dish with just enough water to cover it, and carefully insert a pin between the integument and the yellow intestine on either side, near the posterior end of the incision, so as to expose the calome: note the septa connecting the body-wall with the intestine. Then insert more pins, obliquely, so as to expose the cœlome and enteric canal up to the anterior end, taking especial care not to tear the ventral parts of the septa and to stretch the animal longitudinally as much as possible. Then note :-

I. The sperm-sacs-three or four pairs of large white bodies in segments IX-XII, and varying greatly in size and form according to the size of the animal. If your specimen is a Lumbricus, you will notice that the anterior and the two posterior pairs are respectively united across the middle line, beneath the enteric canal, to form the two sperm-reservoirs.

2. The enteric canal and its subdivisions:-a. buccal sac; $b$. pharynx; c. gullet (largely hidden by the sperm-sacs); d. crop; gizzard; and e. intestine, covered with a layer of yellow cells.

3. The dorsal blood-vessel, containing red blood, and giving off branches to the enteric canal; the large rhythmically contractile commissural vessels connecting the dorsal with the ventral vessel : the latter will be seen later.

4. A pair of small whitish coiled bodies, the nephridia, attached to the posterior face of each septum exposed (except the first three), on either side of the alimentary canal. Carefully remove one of these in the region of the intestine-(take hold of the septum with the fine forceps, and cut around the nephridium with the small scissors)-mount in salt solution or water, and examine first with the low power, and then with the high power. Note that the nephridium consists of a long 
coiled tube, plentifully supplied with blood-vessels, and that long vibratile cilia can be seen in parts of it. (For details see $\$ V I$ ).

Add a little methylated spirit to the water in your clissecting dish and sketch your dissection.

5. The ovaries-Examine segment XIII closely, being very careful not to injure its contents, and the ovaries may then be seen projecting backwards into this segment, one on either sicle, just in front of the crop. They can easily be recognised by their shape, and by the fact that they hang freely into the colome, as can be seen by touching them with a seeker. Carefully seize the septum between segments XII and XIII with the small forceps, and cut around the attachment of an ovary so as to remove it. Stain with methyl-green and mount in glycerine (or else fix, stain, and mount in balsam, as directed on p. 136). Note the mass of undifferentiated cells at the proximal, attached end of the ovary, and the gradual development of the ova towards the distal, narrower end. Examine an ovum, and observe the nucleus, nucleolus, and granules of food-yolk. Sketch the ovary.

:. (The oviducts and ovisacs are not easy to make out in dissec. tions).

6. The globular spermothece (usually two pairs) in segments IX and $\mathrm{X}$.

III. Tease out a small portion of a sperm-sac, stain with magenta, and mount in glycerine. The following stages in the development of the sperms can then be made out:- $a$. The sperm-mother-cells (developed in the spermary) in different stages of division: the products of division, each with a nucleus, become arranged in a single peripheral row, the central mass of protoplasm remaining undivided. $b$. The gradual elongation of these small cells; and $c$. the conversion of each into a sperm, the nucleus forming the rod-like "head," and the protoplasm giving rise to the delicate "tail." $d$. Free sperms (also to be found in the spermothecæ). Slsetch a series of stages.

It is difficult to make out the two pairs of spermaries and the spermiducts by dissection, and they can be more easily studied by examining transverse sections, prepared as directed below. (In Lumbricus the spermaries and seminal funnels are enclosed within the median sperm-reservoirs.) The spermiducts are partly embedded in the body-wall.

IV. Remove the sperm-sacs carefully, and make out further details as regards the enteric canal (see \$ II, 2). Note the asophageal glands. 
Cut open part of the intestine along one side, and observe the thick dorsal fold or typhlosole projecting into it. Sketch.

V. I. Note the small cerebral ganglia or brain on the dorsal side of the buccal sac, and then cut through the anterior part of the pharynx just behind the brain. Carefully remove the alimentary canal, noting as you do so the ventral or sub-intestinal blood-vessel. The nervous-system will now be exposed. Observe again the paired cerebral ganglia, from which arise a pair of connectives, forning a small nerve-ring or collar around the buccal sac, and continuous ventrally with the ventral nerve-cord, consisting of lateral halves fused together and extending along the whole length of the ventral body-wall, passing through spaces in the septa, and expanding slightly in each segment, so as to form ganglionic swellings. Three pairs of nerves are given off in each segment. Sketch.

2. Remove the nerve-ring and a small portion of the ventral cord, and examine with the low power. Sketch.

3. A lateral neural vessel can be seen close to the ventral cord on either side. Remove a portion of the cord, and note the sub-neural vessel.

VI. Further details as regards the structure of the nephridia are best made out on a worm which has been preserved in spirit. Very carefully remove the enteric canal as directed above, so as not to injure the septa more than necessary: the nephrostomes can then be seen with a lens, looking like small whitish dots. Remove an entire nephridium carefully as before ( $\$$ II. 4), stain, and mount in glycerine or balsam. Note the three loops, and- $a$. the ciliated nephrostome; $b$. the first, slender part of the tube with its cilia; c. the second wider, ciliated part; $d$. the third still wider, glandular part; and $e$. the fourth much wider, muscular part, which opens on to the exterior by the nephridiopore. Sketch.

VII. Remove a small piece of the integument containing setæ, and separate the latter out with needles. Mount in water, and examine. Sketch.

C. Transverse Sections.-For the preparation of these, it is best to keep a worm in coffee-grounds or small pieces of blotting-paper moistened with water, in order that the gritty contents of its intestine may be replaced by a soft substance which will not blunt the razor. Kill the worm, cut a small piece about $\frac{1}{2}$ inch in length from the region of the intestine, $i$ and fix, stain, and cut into transverse sections as 
directed on p. I36. Examine a section with the low power, and note :-

I. $a$. The thin cuticle; $b$. the epiderm, enclosing goblet-cells (unicellular glands); $c$. the very thin derm; $d$. the sete, with their sacs and muscles, if your section passes through one or more of them.

2. The muscles of the body-zuall. a. The external circulay layer; and $b$. the thicker longitudinal layer, appearing feather-like in transverse section, and broken up into bands at the lines of the dorsal pores and setæ. Note that the muscles are unstriped.

3. The calome and peritoneal membrane.

4. The intestine, with its thick dorsal typhlosote. It is lined by a single layer of columnar cells (enteric epithelium), outside which is a thin muscular and connective tissue layer. Externally to this, again, are the elongated and granular yellow cells, which are especially abundant in the typhlosole.

5. The dorsal, ventral, and intestinal blood-vessels.

6. The ventral nerve-cord, just internal to the longitudinal muscular layer. It is enclosed in a muscular and connective-tissue sheath, imbedded in which the sub-neural and lateral neural vessels can be seen. Along the dorsal side are three clear.looking "giant fibres." Observe the nerve-cells along the cord ventrally and laterally, the nerves coming off from the cord, and the symmetrical halves of which the cord is composed.

7. The nephridia:-these will be seen cut through in various planes.

The thin septa will be cut through in different directions, and their relations are therefore not easily seen in sections; note the circular and radial muscular fibres in the septa. Sketch the lateral half of your section, and then put on the high power and work through $\$ \S I-7$ again. Sketch as many details as possible.

(If time permits, prepare and examine a series of transverse sections through the genital region, and observe any important points not already made out in your dissection. Note especially the spermaries and spermiducts). 


\section{CHAPTER VI}

THE CRAYFISH.-CHARACTERS OF THE PHYLUM ARTHROPODA.

WE have now to study an animal formed on a very similar plan of structure to the Earthworm as regards segmentation and arrangement of many of the organs, but which reaches in every respect a far higher grade of organisation.

The common British Fresh-water Crayfish is usually known as Astacus fluviatilis, and is found in many of the streams and rivers of England and Ireland, hiding under stones, or in holes, into which it darts very suddenly on the approach of danger. Its ordinary creeping movements are slow, and are effected by means of a number of jointed limbs, for which reason it is included, together with insects, spiders, scorpions, \&x, in the phylum Arthropoda.

In colour, the Crayfish is greenish grey, and in form it is very similar to the marine Lobster (Homarus oulgaris), to which the following description will apply almost equally well.

In addition to the presence of paired limbs or appendages, one of the most striking points of difference between an Earthworm and a Crayfish is the smaller and constant number of segments or metameres in the latter, as well as the fact that certain of these are more or less 
firmly united with one another-i.e., have undergone concrescence. The result of this fusion of the segments is that two distinct regions can be distinguished in the body-an anterior cephalothorax and a posterior abdomen (Fig. 86, tth, $a b$ ).

The cephalothorax is unjointed, and is covered by a cuirass-like structure, the carapace; and the abdomen is divided into distinct segments, movable upon one another in a vertical plane. The cephalothorax is divided into two regions, an anterior-the head, and a posterior-the thorax, by a transverse depression, the cervical groove. The carapace is developed from the dorsal and lateral regions of both head and thorax; it is free at the sides of the thorax, where it forms a flap or gill-cover (Fig $87, k d$ ) on each side, separated from the actual body-wall by a narrow space in which the gills are contained.

The limbs spring from the ventral surface. Both trunk and appendages are covered with a sort of shell, formed of chitin (p. 320), strongly impregnated with carbonate of lime in most parts, so as to be hard and but slightly elastic.

The abdomen is made up of seven segments: the first six of these are to be considered as metameres in the sense in which the word is used in the case of the earthworm. Each has a ring-like form, presenting a broad dorsal region or tergum (Fig. 83, T), a narrow ventral region or sternum (S), and downwardly directed lateral processes, the pleura (PL). The seventh division of the abdomen is the telson (Fig. 86, $t$ ): it is flattened horizontally and divided by a transverse groove into anterior and posterior portions. All seven segments are calcified, and are united to one another by uncalcified articular membranes: the first segment is similarly joined to the thorax. Thus the exoskeleton of Astacus is a continuous cuticular structure, discontinuously calcified so as 
to have the character of a hard, jointed armour. Tufts of minute feather like cuticular structures, or sete, are present on various parts of the exoskeleton both of the body and appendages.

It has been stated that the abdominal segments are movable upon one another in a vertical plane: i.e., the whole

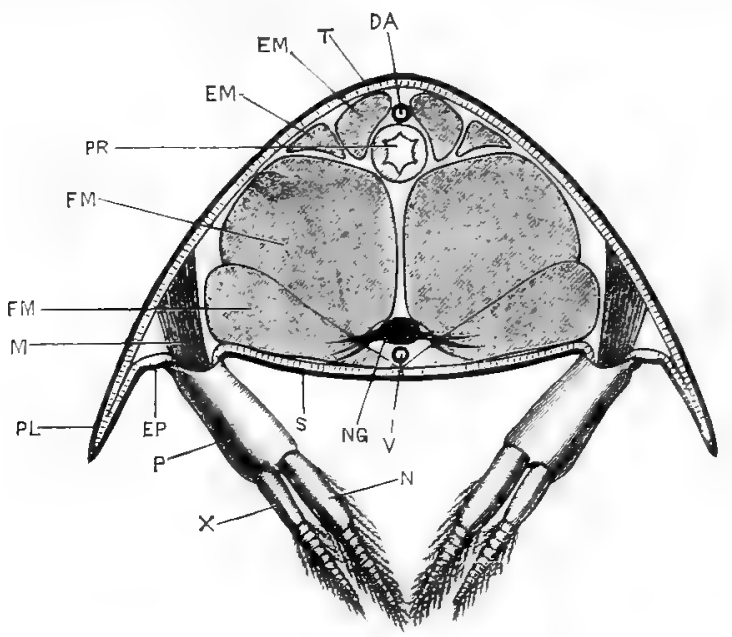

FIG. 83,-Transverse section of abdomen of Crayfish.

DA. dorsal abdominal artery; EMI. dorsal muscles of the abdomen; EP. space between the pleuron and the appendage; FMI. ventral muscles of the abdomen; M. muscles of the appendage; $N$. endopodite; NG. nerve-ganglion; P. protopodite ; $\mathrm{PL}$. pleuron; $\mathrm{PR}$. hind-gut ; $\mathrm{S}$. sternum ; $T$. tergum; $\mathrm{V}$. ventral abdominal artery ; $\mathrm{X}$. exopodite. (From Marshall and Hursi's Zoology).

abdomen can be extended or straightened, and flexed or bent under the cephalothorax; the segments are incapable of movement from side to side. This is due to the fact that, while adjacent segments are connected dorsally and ventrally by flexible articular nembranes, they present at each side a joint, placed at the junction of the tergum and 
pleuron, and formed by a little peg-like process of one segment fitting into a depression or socket in the other. A line drawn between the right and left joints constitutes the axis of articulation, and the only possible movement is in a plane at right angles to this axis.

Owing to the presence of the carapace the thoracic region is immovable, and shows no distinction into segments either on its dorsal (tergal) or lateral (pleural) aspect. But on the ventral surface the sterna of the thoracic segments are clearly marked off by transverse grooves, and the hindmost of them is slightly movable. Altogether eight thoracic segments can be counted.

The ventral and lateral regions of the thoracic exoskeleton are produced into the interior of the body in the form of a segmental series of calcified plates, so arranged as to form a row of lateral chambers in which the muscles of the limbs lie and a median tunnel-like passage or sternal canal, containing the thoracic portion of the nervous system (Fig. 87). The entire endophragmal system, as this series of plates is called, constitutes a kind of internal skeleton.

The head exhibits no segmentation: its sternal region is formed largely by a shield-shaped plate, the epistoma, nearly vertical in position. The ventral surface of the head is, in fact, bent so as to face forwards instead of downwards. The cephalic region of the carapace is produced in front into a large median spine, the rostrum: immediately below it is a plate from which spring two movably articulated cylindrical bodies, the eye-stalks, bearing the eyes at their ends.

The appendages have very various forms, and are all, like the abdomen, jointed or segmented, being divisible into freely articulated limb-segments or podomeres. You will at once notice the long feelers attached to the head, the 
five pairs of legs springing from the thorax, and the little fin-like bodies arising from the sterna of the abdomen. It will be convenient to begin with the last-named region.

The third, fourth, and fifth segments of the abdomen bear each a pair of small appendages, the swimming feet or pleopods (Fig. 83, P, N, X). A pleopod (Fig. 84, тo) consists of an axis or protopodite having a very short proximal (Fig. 84 $p r$. I) and a long distal ( $p r .2)$ podomere, and bearing at its free end two jointed plates, fringed with setæ, the endopodite (en) and exopodite (ex). These appendages act as fins, moving backwards and forwards with a regular swing, and probably aiding in the animal's forward movements.

In the female a similar appendage is borne on the second abdominal segment, while that of the first is more or less vestigial (p. 159). In the male the first and second pleopods (9) are modified to form incomplete tubes which serve to transfer the spermatophores (p. 368 ) to the body of the female. The sixth pair of pleopods (I I) are alike in the two sexes: they are very large, both endopodite and exopodite having the form of broad, flat plates: in the natural position of the parts they lie one on each side of the telson, forming with it a large five-lobed tail fin: they are therefore conveniently called uropods or tail-feet. The telson itself bears no appendages.

The thoracic appendages are very different. The four posterior segments bear long, slender, jointed legs (8), with which the animal walks : in front of these is a pair of very large legs terminating in huge claws or chelee, and hence called chelipeds (Fig. 86, bf. 4). The three anterior thoracic segments bear much smaller appendages, more or less leg-like in form, but having their bases toothed to serve as jaws : they are distinguished as maxillipeds or footjaws. 

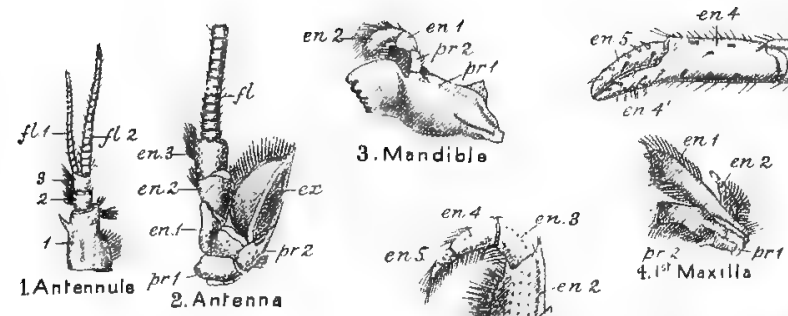

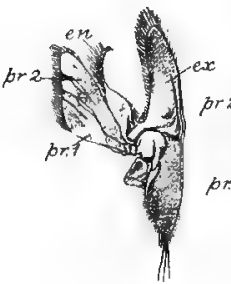

5. 2nd Moxilla

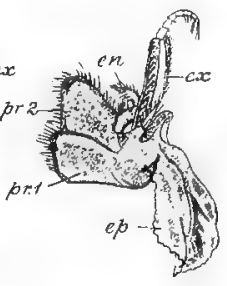

6. 1? Maxilliped
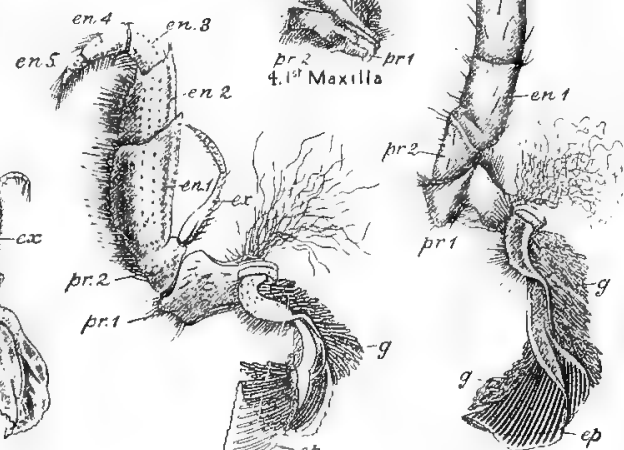

8. 3 r.d $^{\mathrm{d}} \cdot \theta 8$

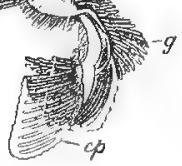

7. 3.d Maxillipad

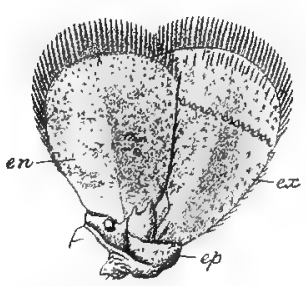

11. Uropod

Fig. 84.-The principal appendages of the Fresh-water Crayfish, placed in the same position, with the protopodite ( $p r)$ and epipodite (ep) downwards, the endopodite $(e n)$ to the left, and the exopodite $(e x)$ to the right.

The protopodite is typically formed of two podomeres ( $p r .1, p r .2)$, the endopodite of five (en. I-en, 5); a gill $(g)$ may be attached to the epipodite.

The three proximal segments of the antennule are marked $\mathrm{I}-3$, its flagella $A$. and $f .2$; the distal end of the endopodite of the antenna is a flagellum $(f)$. (The tufts of threads in 7 and 8 are very long seta which extend between the gills). - (From Parker and Haswell's Zoology, after Huxley.)

$\therefore$ - The structure of these appendages is best understood by a consideration of the third maxilliped (Fig 84, 7). The 
main portion of the limb is formed of seven podomeres arranged in a single series, strongly calcified, and-with the exception of the second and third, which are fused-moveably articulated with one another. The second podomere, counting from the proximal end, bears a many-jointed feeler-like organ $(e x)$, and from the first springs a thin, folded plate $(e p)$ having a plume-like gill $(g)$ attached to it. The first two segments of the axis form the protopodite $(p r . \mathrm{I}, 2)$, its remaining five segments the endopodite (en. I, 5), and the feeler, which is directed outwards, or away from the median plane, the exopodite $(e x)$. The folded plate is called the epipodite : in the natural position of the parts it is directed upwards, and lies in the gill-cavity between the proper wall of the thorax and the gill-cover.

The five legs (8) differ from the third maxilliped in their greater size, and in having no exopodite: in the fifth or last the epipodite also is absent. The first three of them have undergone a curious modification, by which their ends are converted into pincers or chele: the fourth segment of the endopodite (sixth of the entire limb, en. 4) is produced distally so as to form a claw-like projection (en. $\left.4^{\prime}\right)$, against which the terminal segments (en. 5) bites. The first leg is much stouter than any of the others, and its chela is of immense size and forms an important weapon of offence and defence. The second maxilliped resembles the third, but is considerably smaller : the first (6) has its endopodite greatly reduced, the two segments of its protopodite large and Ieaf-like, and no gill is connected with the epipodite.

The head bears a pair of mandibles and two pairs of maxilla in relation with the mouth, and in front of that aperture a pair of antennules and.one of antenna. The hindmost appendage of the head is the second maxilla (5), a leaflike Iappendage, its protopodite being cut up into 
lobes, while the exopodite is modified into a boomerangshaped plate, which, as we shall see, is an important accessory organ of respiration. The first maxilla (4) is a very small organ, having neither exopodite nor epipodite. The mandible (3) is a large strongly calcified body, toothed along its inner edge, and bearing on its anterior border a little three-jointed, feeler-like body, the palp, the two distal seg. ments of which represent the endopodite, its proximal segment, together with the mandible proper, the protopodite.

The antenna (2) is of great size, being nearly as long as the whole body. It consists of an axis of five podomeres, the fifth or last of which bears a long, flexible, many.jointed structure, or flagellum ( $f$ ), while from the second segment springs a scale-like body or squame (ex). It is fairly obvious that the two proximal segments represent the protopodite, the remaining three, with the flagellum, the endopodite, and the squame the exopodite.

The antennule ( $\mathrm{I}$ ) has an axis of three podomeres ending in two many-jointed flagella $(f, 1, f .2)$, which are sometimes considered as corresponding to the endopodite and exopodite. But in all the other limbs, as we have seen, the exopodite springs from the second segment of the axis, and the probabilities are that there is no exact correspondence between the parts of the antennule and those of the remaining appendages.

The eye-stalks, already noticed, arise just above the antennules, and are formed each of a small proximal and a large distal segment. They are sometimes counted as appendages serially homologous with the antennæ and legs, but are more properly to be looked upon as articulated processes of the prostomium. It is probable that the antennuies are also prostomial and not metameric structures: assuming this to be the case, it will be seen that the PRACT. Zoor, A A 
body of the crayfish consists of a prostomium, eighteen metameres, and a telson, which is probably composed of an anal segment plus a post-anal extension. The prostomium bears eye-stalks and antennules: the first four metameres are fused with the prostomium to form the head, and bear the antennæ, mandibles, first maxillæ, and second maxillæ: the next eight metameres (5th-I 2 th) constitute the thorax, and bear the three pairs of maxillipeds and the five pairs of legs: the remaining six metameres ( $^{\text {th }}$ I8th), together with the anal segment, constitute the abdomen, and bear five pairs of pleopods and one of uropods.

The articulation of the various podomeres of the appendages is on the same plan as that of the abdominal segments (p. 348). The podomeres are, it must be remembered, rigid tubes: they are connected with one another by flexible articular membranes (Fig. 85, art. $m$ ), but at two points the adjacent ends of the tubes come into contact with one another and are articulated by peg-and-socket joints $(h)$, the two joints being at opposite ends of a diameter which forms the axis of articulation. The two podomeres can therefore be moved upon one another in a plane at right angles to the axis of articulation and in no other direction, the joints being pure hinge-joints. As a rule the range of movement is from the perpendicular to a tolerably extensive flexion on one side-the articulations are single-jointed, like our own elbows and knees. The whole limb is, however, capable of universal movement, owing to the fact that the axes of articulation vary in direction in successive joints: the first joint of a limb bending, for instance, up and down, the next obliquely, the next backwards and forwards, and so on. In some cases-e.g., the pleopods-peg-and-socket joints are absent, the articulation being formed merely by an annular 
articular membrane, movement being therefore possible in any plane.

Sections show the body-wall to consist of an integument composed of a layer of deric epithelium (epiderm) secreting a thick cuticle, and a layer of connective tissue forming the derm, beneath which is a very thick layer of large and complicated muscles which fill up a great part of the interior of the body. Neither on the epiderm nor elsewhere are there any cilia, the absence of these structures being generally characteristic of Arthropods.

The cuticle is of great thickness, and except at the joints between the various segments of the body and limbs, is impregnated with lime salts so as to form a hard, jointed armour. It thus constitutes a cuticular exoskeleton, forming a continuous investment over the whole body but discontinuously calcified. It is shed entire and renewed periodically-once a year during adult life--the process being known as ecdysis, growth taking place during the period between ecdysis and renewal while the animal is soft.

The muscular system shows a great advance in complexity over that of the earthworm: and consists entirely of transversely striated fibres (compare p. I I 2). In the abdomen the muscles are of great size, and are divisible into a smaller dorsal and a larger ventral set. The dorsal muscles (Figs. 83 and $86, e m$ ) are paired longitudinal bands, divided into segments called myomeres (p. 203), and inserted by connective tissue into the anterior border of each segment: anteriorly they are traceable into the thorax, where they arise from the side-walls of that region. When these muscles contract they draw the anterior edge of each tergum under the posterior edge of its predecessor, and thus extend or straighten the abdomen. 
The ventral muscles (Figs. 83 and $86, f . m$ ) are extraordinarily complex and cannot be described in detail here. They partly aid the dorsal muscles in extending the abdomen, but are chiefly important in producing an approximation of the sterna, and thus in flexing the abdomen. The ventral muscles are, like the dorsal, traceable into the thorax, where they arise from the endophragmal system (p. 349). The flexor muscles are immensely powerful, and produce, when acting together, a sudden and violent bending of the abdomen upon the cephalothorax causing the crayfish to dart backwards with great rapidity.

It will thus be seen that the body-muscles of the crayfish cannot be said to form a layer of the body-wall, as in the earthworm (Fig. 78), but constitute an immense fleshy mass, filling up the greater part of the body-cavity, and leaving a very small space around the enteric canal.

In the limbs the essential arrangement of the muscles in relation with the joints in Arthropods is more easily seen (Fig. 85): each podomere is acted upon by two muscles situated in the next proximal podomere. These muscles are inserted, by chitinous and often calcified tendons, into the proximal edge of the segment to be moved, the smaller (ext) on the extensor, the larger $(f)$ on the flexor side, in each case half-way between the two hinges, so that a line joining the two muscular insertions is at right angles to the axis of articulation.

The digestive organs are constructed on the same general plan as those of the earthworm, but present many striking differences. The mouth lies in the middle ventral line of the head and is bounded in front by a shield-shaped process, the labrum, at the sides by the mandibles, and behind by a pair of delicate lobes, the paragnatha. It leads by a short wide gullet (Fig. $86, a$ ) into a capacious gizzard, usually 
spoken of as the stomach, which occupies a great part of the interior of the head, and is divided into a large ante. rior division (cs), and a smaller posterior division $(p s)$ : the latter passes into the intestine, which consists of a narrow and very short mid-gut ( $m d$ ) from which a somewhat wider hind-gut ( $h d)$ extends to the anus (an), situated on the ventral surface of the telson.

The outer layer of the enteric canal consists of connective tissue containing striped muscular fibres: within this is a single layer of columnar epithelial cells, none of them glandular. In the gullet and gizzard and in the hind-gut the epithelium secretes a layer of chitin, which thus constitutes the innermost layer of those cavities. It is proved by development that the midgut, which has no chitinous lining, is the only part of the enteric canal developed from

FIG. 85.-A leg of the Fresh-water Crayfish with part of the exoskeleton removed to show the muscles.

$e n .2-e n .5$, segments of endopodite; $t h$. hinges ; art. $m$. articular membrane; $e x t$. extensor muscle; $A$. flexor muscle. (From Parker and_Haswell's Zoology.)

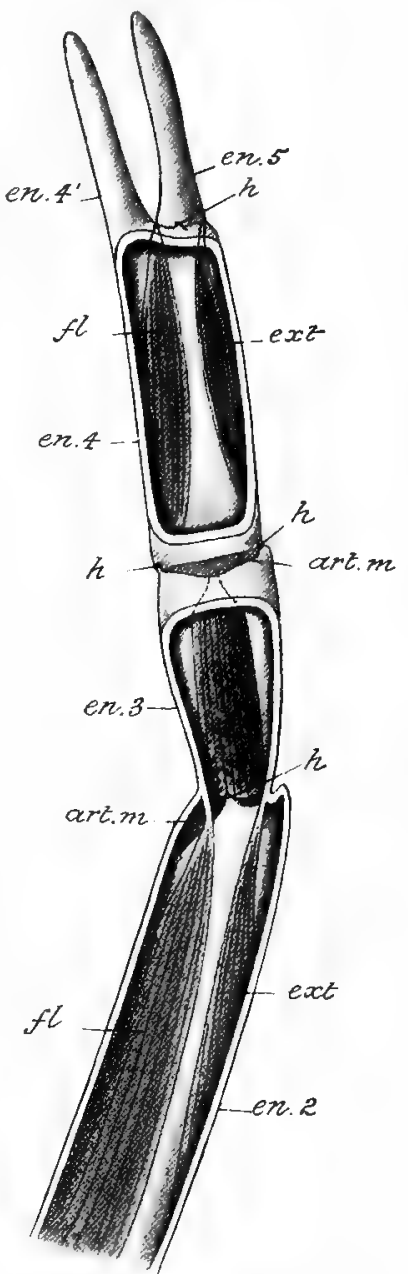
A A $2^{*}$ 


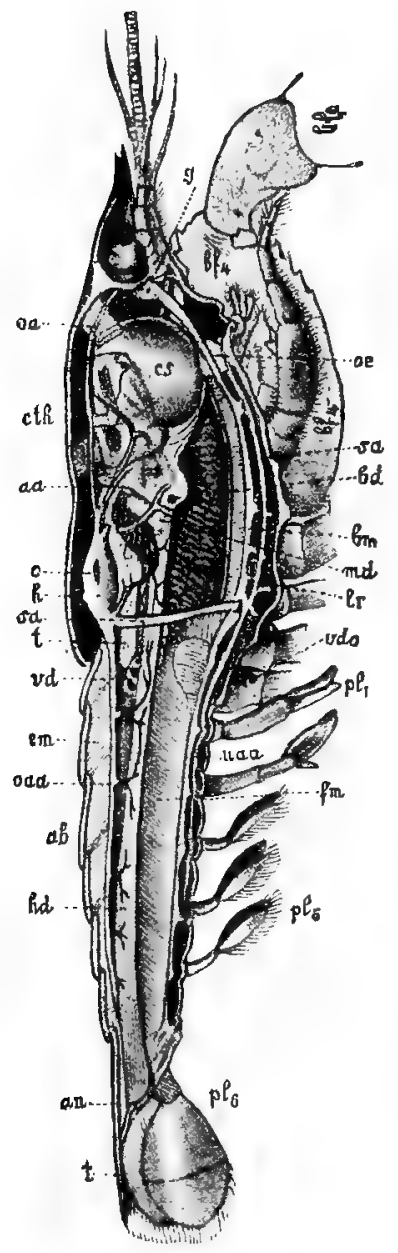

the enteron of the embryo : the gullet and gizzard (fore-gut) arise from the stomodæum, the hind-gut from the proctodæum (p. 204). Thus only a very small portion of the enteric epithelium is endodermal.

In the anterior division of the gizzard the chitinous lining is thickened and calcified in certain parts, so as to form a complex articulated framework, the "gastric mill," on which are borne a median and two lateral teeth, strongly calcified and projecting into the cavity of the gizzard. Two pairs of strong muscles arise from the carapace, and are inserted into the gizzard (Fig. 86) : when they contract

FIG. 86.-Dissection of Fresh-water Crayfish made by removing the exoskeleton with the appendages, and the muscles, digestive gland and excretory organ, of the right side.

$a a$. antennary artery; $a b$. abdomen; $a n$. anus; $b . d$. aperture of right digestive duct exposed by removal of gland; $b f$. 4, cheliped; bn. ventral nerve cord. cs. anterior division of gizzard; $c t h$. cephalothorax; $e m$. dorsal muscles; fiz. ventral muscles; $g$. brain; $h$. heart; $h d$. hind-gut; $l r$. left digestive gland; wd. mid-gut; 0 . right lateral ostium of heart; oa, ophthalmic artery

oax. dorsal abdominal artery; $\propto$. gullet; $\not h .1-5$, pleopods; $\not p l .6$, uropod; $p s$. posterior division of gizzard; s. a. sternal artery; $t$. (near heart), spermary; $t$. (below anus), telson; waa. ventral abdominal artery; $v . d$. spermiduct; vdo. male genital aperture. (From Lang, after Huxley.) 
they move the mill in such a way that the three teeth meet in the middle line and complete the comminution of the food begun by the jaws. The separation of the teeth is effected partly by the elasticity of the mill, partly by delicate muscles in the walls of the gizzard. The posterior division of the gizzard forms a strainer: its walls are thickened and produced into numerous setæ, which extend quite across the narrow lumen and prevent the passage of any but finely divided particles into the intestine. Thus the gizzard has no digestive function, but is merely a masticating and straining apparatus-in fact it is not a stomach at all in either the embryological or in the physiological sense. On each side of the anterior division is found, at certain seasons of the year, a plano-convex mass of calcareous matter, the gastrolith or "crab's-eye," which apparently merely serves to store up reserve calcareous material for use after the next ecdysis.

The digestion of the food, and to some extent the absorption of the digested products, are performed by a pair of large glands (Fig. 86, lr), lying one on each side of the gizzard and anterior end of the intestine. They are formed of finger-like sacs or caca, which discharge into wide ducts opening into the mid-gut, and are lined with glandular epithelium derived from the endoderm of the embryo. The glands are often spoken of as the liver, but as the yellow fluid they secrete digests proteids as well as fat, the name hepato-pancreas is often applied to them, or they may be called simply digestive glands. The crayfish is carnivorous, its food consisting largely of decaying animal matter.

The digestive organs and other viscera are surrounded by an irregular cavity, which is in free communication with the blood-vessels and itself contains blood. This cavity is not 
lined by epithelium, and is to be looked upon as a large blood-sinus, and not as a true ccelome.

There are well-developed respiratory organs in the form

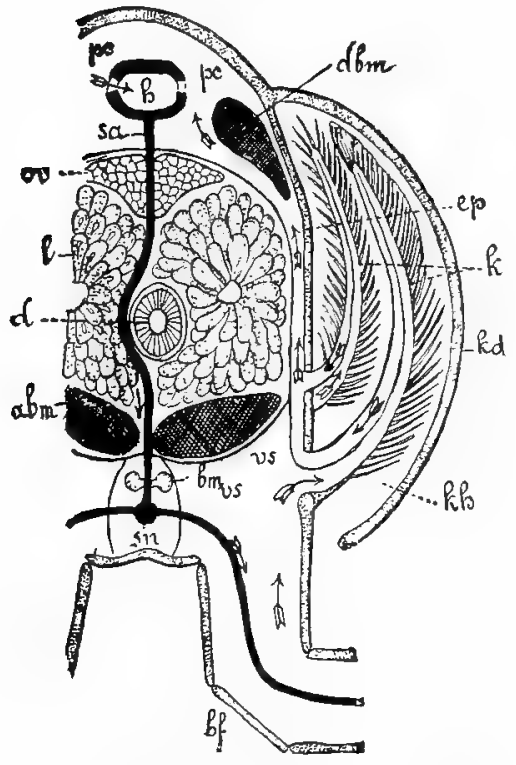

FIC. 87.-Transverse section of thorax of Crayfish, diagrammatic.

$a b m$. ventral abdominal muscles; bf. leg; bm. ventral nerve cord; $d$. intestine; $d b m$. dorsal abdominal muscles; $e p$. wall of thorax; $h$. heart; $k$. gills; $k d$. gill-cover; $l$, digestive glands; $o v$. ovary; $p c$. pericardial sinus ; $s \alpha$, sn. sternal artery; ws. ventral blood-sinus. The arrow shows the direction of the bloodcurrent. (From Lang's Comparative Ana. tonty.) of gills (Figs. 84, g, and $87, k)$, contained in a narrow branchial chamber, bounded internally by the proper wall of the thorax, externally by the gill-cover or pleural region of the carapace. Each gill consists of a stem giving off numerous branchial filaments, so that the whole organ is plume-like. The filaments are hollow and communicate with two parallel canals in the stem - an external, the afferent branchial vein, and an internal, the efferent branchial vein (Fig. 89). The gill is to beconsidered as an out-pushing of the body-wall specially modified for respiration (compare p. 204), and it contains the same layers-a thin layer of chitin externally, then a single layer of epithelial cells, and bereath

- this connective tissue, hollowed out for the blood channels. 
According to their point of origin the gills (Figs. 84 and 87 ), are divisible into three sets-first, podobranchs, or foot-gills springing from the epipodites of the thoracic appendages, from which they are only partially separable; secondly, arthrobranchs, or joint-gills, springing from the articular membranes connecting the thoracic appendages with the trunk ; and thirdly, pleurobranchs, or wall-gills, springing from the lateral walls of the thorax, above the attachment of the appendages. The total number of gills is eighteen, besides two filaments representing vestigial (p. I59) or vanishing gills, which are represented by functional organs in some allied forms.

The water in the branchial chamber is constantly renewed in the living Crayfish by the action of the plate attached to the second maxilla (p. 352), the movement to and fro of which bales out the water in front, and consequently causes fresh water to flow in behind. Thus a fresh supply of water, containing air in solution, is continually being passed over the gills. The fact that the podobranchs are attached to the bases of the limbs must also result in bringing their surfaces more easily in contact with the water when the animal uses its legs in walking.

The excretory organs differ both in position and in form from those of the earthworm. At the base of each antenna is an organ of a greenish colour, the antennary or green gland (Fig. 88), by which the function of renal excretion is performed. The gland is cushion-shaped, and contains canals and irregular spaces lined by glandular epithelium: it discharges its secretion into a thin-walled sac or urinary bladder (bl), which opens by a duct on the proximal segment of the antenna. The green glands are to be looked upon as corresponding to peculiarly modified nephridia.

The circulatory organs are in a high state of development 
The heart (Fig. 86, $h$ ) is situated in the dorsal region of the thorax, and is a roughly polygonal, muscular organ pierced by three pairs of apertures or ostia (o), guarded by valves which open inwards. It is enclosed in a spacious pericardial sinus (Fig. 87, pc) which contains blood. From the heart-spring a number of narrow arteries (compare p. 27), which serve to convey the blood to various parts of the body. At the origin of each artery from the heart are

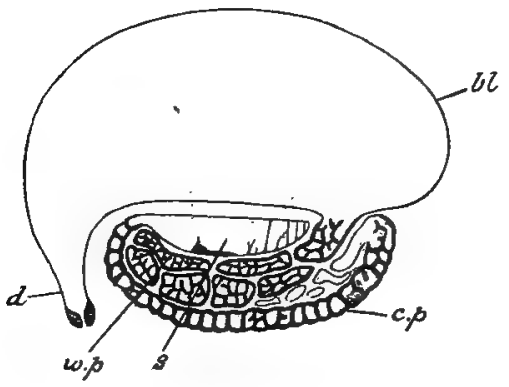

Fig. 88.-Diagram of excretory organ of Crayfish.

bl. bladder; c.p. outer or cortical green portion; $d$. duct; s. yellowish sac-like portion; $20 . p$. white tubular portion. (From Parker and Haswell, after Marchal.) valves which allow of the flow of blood in one direction only, viz., from the heart to the artery. From the anterior end of the heart arise five vessels - the median opthalmic artery (Fig. 86, oa), which passes forwards to the eyes ; paired antennary ar: teries (ac), going to the antennules, antennæ, green glands, \&c., and sending off branches to the gizzard; and paired hepatic arteries, going to the digestive glands. The posterior end of the heart gives off two unpaired arteries practically united at their origin-the dorsal abdominal artery ( 0 a $a$ ), which passes backwards above the intestine, sending branches to it and to the dorsal muscles; and the large sternal artery (Figs. 86 and $87, s(x)$, which extends directly downwards, indifferently to right or left of the intestine, passing between the connectives uniting the third and fourth thoracic ganglia, and then turns forwards and runs in the sternal canal, immediately beneath the nerve-cord, sending 
off branches to the legs, jaws, \&c. At the point where the sternal artery turns forward it gives off the median ventral abdominal artery (Fig. 86, uaa), which passes backwards beneath the nerve-cord, and supplies the ventral muscles, pleopods, \&c.

All these arteries branch extensively in the various organs they supply, becoming divided into smaller and smaller offshoots, which finally end in microscopic capillaries (p. 95). These latter end by open mouths, which communicate with the blood-sinuses-spacious cavities lying among the muscles and viscera, and all communicating, sooner or later, with the sternal sinus, a great median canal running longitudinally along the thorax and abdomen, and containing the ventral nerve-cord and the sternal and ventral abdominal arteries. In the thorax the sternal sinus (Fig. 87, vs, and Fig. 89, st.s), sends an offshoot to each gill in the form of a well-defined vessel, which passes up the outer side of the gill, and is called the afferent branchial vein (af. br. v). Spaces in the gillfilaments place the afferent in communication with the efferent branchial vein (ef. br. $v)$, which occupies the inner side of the gill-stem. The eighteen efferent branchial veins open into six branchiocardiac veins (br. c. v) which pass dorsally in close contact with the lateral wall of the thorax and open into the pericardial sinus.

The whole of this system of cavities is full of blood, and the heart is rhythmically contractile. When it contracts the blood contained in it is prevented from entering the pericardial sinus by the closure of the valves of the ostia, and therefore takes the only other course open to it, viz., into the arteries. When the heart relaxes the blood in the arteries is prevented from regurgitating by the valves at their origins, and the pressure of blood in the pericardial sinus forces open the valves of the ostia and so fills the 
heart. Thus in virtue of the successive contractions of the heart and of the disposition of the valves, the blood is kept constantly moving in one direction-from the heart by the arteries to the various organs of the body, where it receives carbon dioxide and other waste matters; thence by sinuses into the great sternal sinus; from the sternal sinus by afferent branchial veins to the gills, where it exchanges

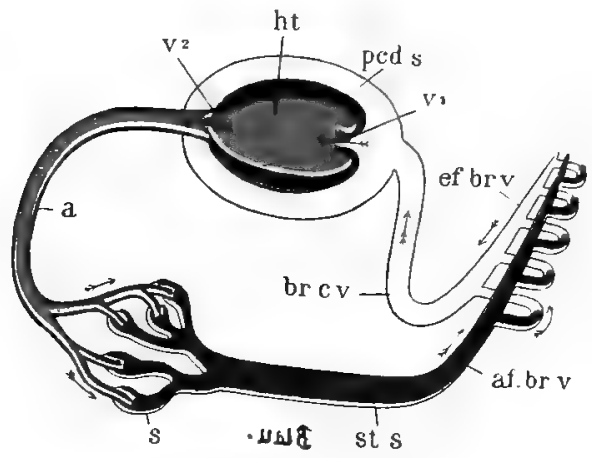

FIG. 89.-Diagram illustrating the course of the circlulation of the blood in the Crayfish. Heart and arteries red; veins and sinuses containing non-aërated blood blue; veins and sinuses containing aërated blood pink. The arrows show the direction of the flow.

The blood from the pericardial sinus ( $p c d$. $s$ ) enters the heart ( $h t$ ) by a valvular aperture $\left(\tau l_{0}\right)$ and is propelled into arteries $(a)$, the orifices of which are guarded by valves $\left(v^{2}.\right)$; the ultimate branches of the arteries discharge the blood into sinuses $(s)$, and the sinuses in various parts of the body debouch into the sternal sinus (st. s); thence the blood is taken by the afferent branchial veins $(a f . b r, v)$ into the gills, where it is purified and is returned by effertnt branchial veins $(e f . b r, v)$ into the branchiocardiac veins $(b r, c, v)$ which open into the pericardial sinus. (From Parker and Haswell's Zoology.)

carbon dioxide for oxygen; from the gills by efferent branchial veins to the branchiocardiac veins, thence into the pericardial sinus, and so to the heart once more.

It will be seen that the circulatory system of the crayfish, like that of the frog, consists of three sections-(I) the heart, or organ of propulsion; (2) a system of out-going channels, the arteries, which carry the blood from the heart to the 
body generally ; and (3) a system of returning channelssome of them, the sinuses, mere irregular cavities, others, the zeins, with definite walls-these return it from the various organs back to the heart. The respiratory organs, it should be observed, are interposed in the returning current, so that blood is taken both to and from the gills by veins.

Comparing the blood-vessels of the Crayfish with those of the earthworm, it would seem that the ophthalmic artery, heart, and dorsal abdominal artery together answer to the dorsal vessel, part of which has become enlarged and muscular, and discharges the whole function of propelling the blood. The horizontal portion of the sternal artery, together with the ventral abdominal, represent the ventral vessel, while the vertical portion of the sternal artery is a commissure, developed sometimes on the right, sometimes on the left side, its fellow being suppressed.

The blood when first drawn is colourless, but after exposure to the air takes on a bluish-grey tint. This is owing to the presence of a colouring matter called hamocyanin, which becomes blue when combined with oxygen; it is a respiratory pigment, and serves, like hæmoglobin (pp. $1 \circ 7$ and 330 ), as a carrier of oxygen from the external medium to the tissues. The hæmocyanin is contained in the plasma of the blood : the corpuscles are all leucocytes (pp. 105 and 328).

The nervous system consists, like that of the earthworm, of a brain (Fig: 86, $g$ ) and a ventral nerve-cord $(b n)$, united by cesophageal connectives. But the ganglia of the ventral nerve-cord are more distinct, and to them the nerve-cells are confined, the longitudinal connectives between them consisting of nerve-fibres only. The brain supplies not only the eyes and antennules, but the antennæ as well, and it is found by development that the two pairs of ganglia belonging to the antennulary and antennary segments have fused with the 
brain proper. Hence we have to distinguish between a primary brain or archi-cerebrum-the ganglion of the prostomium, and a secondary brain or syn-cerebrum formed by the union of one or more pairs of ganglia of the ventral cord with the archi-cerebrum. A further case of concrescence of ganglia is seen in the ventral nerve-cord, where the ganglia of the last three cephalic and first three thoracic segments have united to form a large compound sub-esophageal ganglion. All the remaining segments have their own ganglia, with the exception of the telson, which is supplied from the ganglion of the preceding segment. There is a visceral system of nerves supplying the gizzard, originating in part from the brain and in part from the oesophageal connectives.

The eyes have a very complex structure. The chitinous cuticle covering the distal end of the eye-stalk is transparent, is divided by delicate lines into areas or facets which are mostly square, and constitutes the cornea. Beneath each facet of the cornea is an apparatus called an ommatidium, consisting of an outer segment or vitreous body having a refractive function, and an inner segment or retinula, enclosing a striated body, the rhabdome, and forming the actual visual portion of the apparatus. The ommatidea are optically separated from one another by black pigment, so that each is a distinct organ of sight, with a very limited visual area, and the entire eye is called a compound eye. The optic nerve $(o p . n)$ dilates to form an optic ganglion $(o p . g n)$ in close connection with the inner ends of the ommatidea; the latter are thus turned towards the light, and are not, like the rods and cones of the vertebrate eye (p. I85), covered by a layer of nerve-fibres, \&c., through which the light must first penetrate.

Each antennule bears two sensory organs, to which are 
usually assigned the functions of smell and hearing respectively. The olfactory organ is constituted by a number of extremely delicate alfactory setre, borne on the external flagellum, and supplied by branches of the antennulary nerve. The auditory organ is a sac formed by invagination of the dorsal surface of the proximal segment, and is in

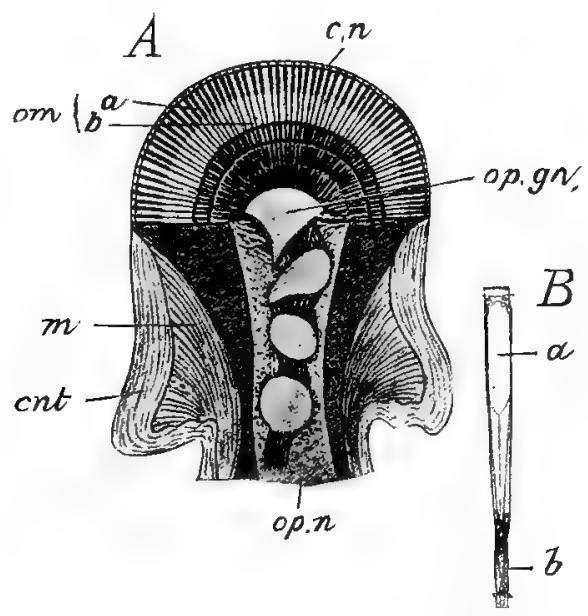

FIG, go.-A, Longitudinal section of eyestalk; B, a single ommatideum ; $a$, vitreous body; $b$, retinula; $c n$, cornea, continuous with cnt, cuticle of eyestalk; $m$, muscles; om, ommatidea; op.gn, optic ganglion; $\phi p, n$, optic nerve. (After Howes.)

free communication with the surrounding water by a small aperture. The chitinous lining of the sac is produced into delicate feathered auditory setce, supplied by branches of the antennulary nerve, and in the water which fills the sac are minute sand grains, which take the place of the otoliths (p. 188) found in most auditory organs, but which, instead of being formed by the animal itself, are taken in after each ecdysis when the lining of the sac is shed. Many of the 
setæ on the body generally have a definite ncrve-supply, and are probably tactile organs.

The crayfish is diœcious (p. 3 r 2 ), and presents a very obvious sexual dimorphism or structural difference between male and female, apart from the actual organs of reproduction. The abdomen of the female is much broader than that of the male : the first and second pleopods of the male are modified into tubular or rather spout-like organs (p. 350); and the reproductive aperture is situated in the male on the proximal podomere of the fifth leg, in the female on that of the third.

The spermary (Fig. 86,t) lies in the thorax, just beneath the floor of the pericardial sinus, and consists of paired anterior lobes and an unpaired posterior lobe. From each side goes off a convoluted spermiduct or vas deferens (vd), which opens on the proximal segment of the last leg $(v d o)$. The sperms are curious, rounded, non-motile bodies produced into a number of stiff processes : they are aggregated into vermicelli-like spermatophores by a secretion of the vas deferens.

The ovary is also a three-lobed body, and is similarly situated to the spermary : from each side proceeds a thin-walled oviduct, which passes downwards, without convolutions, to open on the proximal segment of the third or antepenultimate leg. The eggs are of considerable size and contain a large amount of yolk.

Both ovary and spermary are hollow organs, discharging their products internally. Their cavities, lined by germinal epithelium (p. 336), represent the colome, and their ducts are organs of the same general nature as nephridia (p. 33I). The ova, when laid, are fastened to the setæ on the pleopods of the female by the sticky secretion of glands occurring both on those appendages and on the segments 
themselves : they are fertilized immediately after being laid, the male depositing spermatophores on the ventral surface of the female's body just before oviposition.

The process of segmentation of the gosperm presents certain striking peculiarities. The nucleus divides repeatedly (Fig. $91.1, n u$ ), but no corresponding division of the protoplasm takes place, with the result that the polyplast-stage (p. 200), instead of being a heap of cells, is simply a multinucleate body (compare p. 269). Soon the nuclei thus formed retreat from the centre of the enbryo, and arrange themselves in a single layer close to the surface (1): around
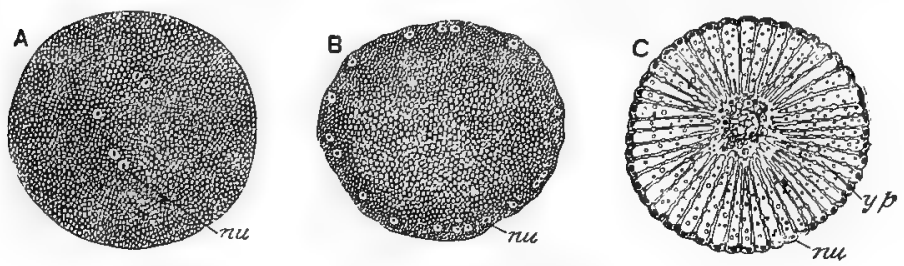

FIG. 9r. - Two stages in the early development of the Crayfish.

In A the products of division of the nucleus ( $n z$ ) are seen in the centre of the yolk; in 13 the nuclei have become arranged in a peripheral layer, each surrounded by protoplasm, so as to form the blastodern. (From Parker and Huswell's Zoology, after Morin.)

each of these protoplasm accumulates, the central part of the cmbryo consisting entirely of yolk-material. We thus get a superficial segmentation, characterised by a central mass of yolk and a superficial layer of cells collectively known as the blastoderm. From this the ectoderm and endoderm are derived, the latter enclosing a relatively small enteron communicating with the exterior through a blastopore (p. $20 \mathrm{I}$ ).

Very soon the embryo becomes triploblastic; or threelayered, by the budding off of cells from the endodern in the neighbourhood of the blastopore: these accumulate between the ectoderm and endoderm, and constitute the mesoderm. 
Before long the blastopore closes, and a stomodæum and proctodæum (p. 204) are formed as invaginations of the 'ectoderm, which eventually communicate with the enteron, forming a complete enteric canal (p. 357). On each side of the mouth or aperture of the stomodæal depression (Fig. 92) three elevations appear, the rudiments of the antennules $\left(a^{1}\right)$, antennæ $\left(a^{2}\right)$, and mandibles $(m):$ in front of them is another pair of elevations on which the eyes $(A\rangle$ subsequently appear. An unpaired elevation $(T A)$ behind the mouth, having the anus or aperture of the proctodæal depression at its summit $(A)$, is the rudiment of the thorax and abdomen. The embryo is now in the nauplius stage.

Many allied forms are hatched in the form of a free-swimming larva (compare pp. II and 340), to which the name nauplizes is applied, characterised by the presence of three pairs of appendages used for swimming, and becoming the antennules, antennæe, and mandibles of the adult. In the crayfish there is no free larva, and the nauplius stage is passed through before hatching.

The embryo is gradually transformed into the crayfish by the appearance of fresh appendages, in regular order, behind the first three; by the elongation of the rudiment of thorax and abdomen; and by the gradual differentiation of the appendages. When hatched the young animal agrees in all essential respects with the adult, but its proportions are very different, the cephalothorax being nearly globular and the abdomen small. For some time after hatching the young crayfishes cling in great numbers to the pleopods of the nother by means of the peculiarly hooked chela of the first pair of legs.

All the members of the phylum Arthropoda (p. 346) are characterised by each typical segment of the body bearing a 
pair of appendages divisible into podomeres; in addition to this, there is an almost universal absence of cilia in the phylum, the sperms are usually non-motile, the muscles arc nearly always of the striped kind, and the body-cavity, which

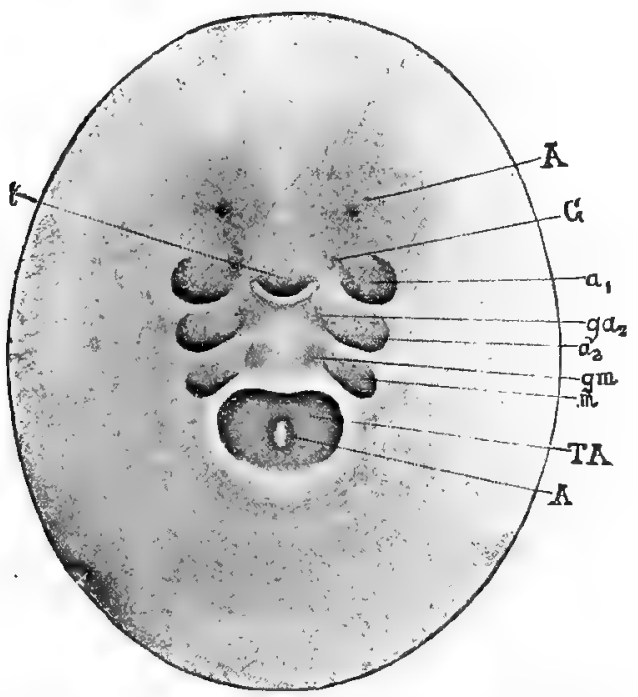

FiG. 92.-Early embryo of the Crayfish in the nauplius stage.

$A$ in the upper part of the figure is the eye; $l$. the labrum overhanging the mouth, on each side of which are the rudiments of the antennules $\left(a^{1}.\right)$, antennas $\left(a^{2}.\right)$, and mandibles $(m$.$) ; behind them is the rudiment of the thorax and abdomen$ ( $T A$ ) with the anus $(A)$. The rudiments of the first three pairs of ganglia $\left(G . g \alpha^{2}, g m,\right)$ are seen through the transparent ectoderm. (From Lang, after Reichenbach.)

does not represent a true colome, is largely represented by blood-spaces in free communication with the circulatory system.

The phylum is divided into several classes, which are all air-breathing except the Crustacea, the class to which the Crayfish belongs and which also includes the Lobsters, Crabs, Shrinups, Wood-lice, Barnacles, Water-fleas, \&c. 


\section{PRACTICAL DIRECTIONS.}

\section{CRAYFISH}

In a living specimen note the cephalcthorax, abdonten, jointed appendages, and exoskeleton, as well as the mode of walking and swimming. Holding the animal out of water between the thumb and finger, observe that bubbles are continually being formed on either side of the lower part of the head (respiratory movements, see $\S B, I$ ). Kill with chloroform (p. 3I), and at the end of the day's work, preserve your dissection in 3 per cent. formaline or 70 per cent. spirit.

\section{A. External Characters.}

I. Note again the cephalothorax and abdomen, and that the abdomen consists of seven movable segments or metameres. Examine the ventral side of the cephalothorax, and note that it also is composed of a number of segments all fused together, except the ventral part of the last.

2. Examine the third or fourth abdominal segment closely, and note that it is connected with the segments in front and behind by a sort of peg- and socket-joint on either side, and that the chitinous exoskeleton at the joints is soft and pliable, forming an articular membrane, while elsewhere it is calcified. Distinguish between the dorsal convex tergum, the ventral sternum, and the pleuron projecting downwards on either side from the tergum.

3. Examine the appendages of the same segment: they are attached to the sterna, near the pleura, by articular membranes. Each consists of a basal or proximal portion-the protopodite, to which two distal, many. jointed parts are attached-an inner endopodite, and an outer exopodite. The cuticle covering the segments of the limb, or podoneres, is more or less calcified, and the distal segments are covered with feathery setce.

4. The second to the sixth abdominal segments are essentially similar to one another, except as regards the appendages of the second in the male and of the sixth in both sexes (see p. 350). The first abdominal segment is smaller than the others, and its pleura are reduced. The sixth abdominal appendages are very large, and, together with the anal segment or telson, form the tail-fin.

5. The cephalothorax is reckoned as consisting of a prostomizum and I 2 metameres, which are completely fused together dorsally and laterally, forming a large calcified shield-the carapace. Thus the entire number of segments is 20 (prostomium +18 metameres + telson). The sternal region of the head is bent upwards.

6. Note:- a, the transverse cervical groove on the dorsal surface of the 
carapace, extending forwards laterally and forming the boundary between the head (prostomium +4 metameres) and the thorax (8 metameres); $b$, the two longitudinal branchiocardiac grooves on the tergal region of the thoracic portion of the carapace, about tinch apart: the part of the exoskeleton between these covers the heart, and the part below each groove forms a large plate, the gill-cover, at the sicle of the thorax; c, the rostrum, movable eye-stalks, and epistoma (p. 349).

7. Note the following apertures: Median-a, the mouth, on the ventral surface of the head, between the jaws; $b$, the anus, on the ventral side of the telson: Paired-c, the auditory aperture, on the dorsal side of the basal joint of the smaller feeler or antennule (this will be seen better later on); $d$, the renal aperture, on a conical ventral elevation of the basal joint of the larger feeler (antenna); $e$, the genital aperture, in the male on the basal joint of the last thoracic leg, and in the female on the last thoracic leg but two.

\section{B. Respiratory Organs.}

I. Carefully cut away the left gill-cover with scissors, and fix the animal under water on its right side, so as to expose the left gillchamber containing the feathery-looking gills. The inner wall of the chamber is formed by the proper wall of the thorax, and the chamber is open behind and below. In front of the gills is a groove, in which a flattened plate (see p. 353) works backwards and forwards during life, driving the water out in front, and causing the bubbles already noticed,

2. The gills are 18 in number, and each has the form of a bottlebrush. The six podobranchs are external to the arthrobranchs and pleurobranchs (p. 36I), and each is attached to a large folded and corrugated epipodite (p. 352). The gills are related to definite metameres, as will be seen from the following table, in which ep stands for epipodite, and $v$ for the vestige of a gill. Note that the first pair of thoracic limbs bears a simple large epipodite only.

\begin{tabular}{|c|c|c|c|c|c|c|c|c|c|}
\hline $\begin{array}{l}\text { Thoracic } \\
\text { SEgMENTS. }\end{array}$ & I. & II. & III. & IV. & V. . & VI. & VII. & VIII. & Total. \\
\hline Podobranchs... & $o+e p$ & $I+e p$ & $\mathrm{I}+c p$ & $I+e p$ & $I+e p$ & $\mathrm{I}+e p$ & $\mathrm{I}+e p$ & $\circ$ & $6+7 e p$ \\
\hline Arthrobranchs & 0 & I & 2 & 2 & 2 & 2 & 2 & o & IT \\
\hline Pleurobranchs & o & $\circ$ & o & 0 & - & $\nu$ & $v$ & I & $x+2 \pi$ \\
\hline TOTAL.... & $0+e p$ & $2+e p$ & $3+e p$ & $3+e p$ & $3+e p$ & $3+v+e p$ & $3+v+e p$ & $I:$ & $18+2 v+7 e p$ \\
\hline
\end{tabular}


3. Turn down the podobranchs and make out the relations of the arthrobranchs from the above table. Then turn these down, or cut them off, and note the single complete pleurobranch and the two vestigial ones. Cut off an arthrobranch and examine its structure, noting the afferent and efferent blood-vessels in its stem. Sketch.

4. Note the branchiocaratiac veins on the inner side of the thoracio wall. Blow air or injeot French blue into the cut bases of the gills removed, and note that the branchiocardiac trụnks extend upwards to the pericardial-sinus (see below) from the gills.

\section{General Dissection.}

Holding the animal in your left hand, insert a scalpel carefully beneath the hinder edge of the carapace on the dorsal side, so as ta separate the exoskeleton from the soft integument, and then with the large scissors cut along the outer side of each branchiocardiac groove, and remove the median portion of the carapace. Note the pigmented integument and then remove it, when some of the nearly colourless blood will ooze out.

I. I. Examine a drop of blood ander the microscope, adding salt solution. Note the amoboid nucleated corpuscles. Sketch.

2. The pericardial sinus will now be exposed, containing the hoart with three pairs of valvular ostia (only the dorsal ostia can be seen at present), through which the blood enters the heart from the pericardial sinus.

Inject some French blue (see p. 99) into the heart through one of the ostia, so as to fill the arteries (tying is unnecessary). Then remove the clorsal part of the exoskeleton and integument bit by bit, all along the thorax and abdomen, as well as the pair of longitudinal extensor muscles lying just beneath the dorsal integument of the abdomen. Pin down under water, dorsal surface uppermost, and note :-

3. The absence of a continuous muscular layer in the body-wall and of a true colome, and the presence of irregular spaces (blood sinuses) between the viscera and muscles.

4. The delicate arteries, arising from the anterior and posterior ends of the heart :- $a$, the anterior median ophthalmic artery, running forwards to the eye-stalks ; $b$, the paired antennary artery, on either side of $a$, and passing forwards̀ and downwards to supply the gizzard, renal organ, feelers, \&c. ; $c$, the hepatic artery (also paired), rather further back and more ventral, extending into and supplying the digestive gland ; $d$, the median dorsal abdominal artery, arising from the 
posterior end of the heart, and running along the dorsal side of the intestine, giving off branches in each metamere; $e$, the sternal artery, arising just beneath $a_{3}$ and passing directly ventralwards to one side of the intestine (this will be seen better later on: compare Figs, 86 and $S_{7}$ ) it perforates the ventral nerve-chain, supplies the segmenis and appendages of the thorax, and gives off i ventral abdominal artery, supplying the segments and appendages $o_{4}$ the abclomen (this artery can be seen in injected specimens through the transparent cuticle).

5. Note the position of the following parts before dissecting further:$a$, the gizzard, a large sac in the head; with two pairs of muscles passing to the integument (now cut through); $b$, the adductor musiles of the mandible, just external to $a$; $c$, the paired, brownish or greenish digestive gland on either side of, and extending further back than the gizzard; above it are $d$, the gonuds, on either side of and behind the pericardial sinus. In the male, the spemmary is small and whitish, and each spermiduct is a coiled, clensely white tube; in the female, the ovary $\therefore$ a larger, brownish organ, containing prominent ova. (In both sexes, the paired character of the gonads is partly lost by fusion : a pair of anterior lobes and a single posterior lobe can be seen in each.) Sketch.

By slightly raising the surrounding parts the gonaducts can be seen to pass ventralwards to their external apertures (p. 373), the oviducts being thin-walled and straight. White masses, the spermatophores (p. 368); will very likely be found stuck on to the sternal region of the body.

6. Tease up a small portion of the spermary or of a spermatophore; stain, and mount in glycerine. Examine under the microscope and note the rounded and flattened sperms each with in number of stiff, curved processes coming off from the periphery. The sperms are non-notile. Sketch.

Remove the heart and reproductive organs carefully, noting the sternal artery (see above) as you do so, and taking especial care not to injure the surrounding parts. Examine the heart under water, and note the six ostia.

II. The enteric canal. Note-I. The oval mouth, bounded by the labrum in front, leading into a short and wide gullet (this will be seen later on), which dilates to form the large gizzard (Fig. 86), filling up a considerable portion of the head and extending into the thorax : a transverse constriction divides it into an anterior and a posterior portion; both gullet and gizzard are lined by chitin. The chitinous 
cuticle of the gizzard is calcified in places to form the sclerites or so-called "ossicles" of the gastric mill. Note the two median sclerites bounding the transverse constriction in front and: behind respectively. To then the anterior and posterior pairs of muscles (p. 375, \& 5) are attached.

n 2. Following on the gizzard is the short, thin-walled mid-gut, on the florsal side of which is a small cacum. It has no chitinous lining, and the large duct of the digestive gland opens into it on either side. The digestive gland is made up of three main lobes on either side and consists of a number of small blind tubes.

3. The hind-gut, which runs straight to the anus. Its inner surface is raised into longitudinal ridges which take a slightly spiral course, and it is lined by a thin chitinous cuticle.

4. Carefully press the gizzard backwards and note $a$, the brain, just behind the bases of the small feelers; $b$, the gullet; and $c$, a pair of white nerve cords (connectives) coming off from the brain and embracing the gullet. Taking care not to injure these parts of the nervous system, cut through the gullet, just above the connectives, and then gently remove the whole enterie canal together with the digestive gland from the body, cutting through the intestine just in front of the nus. Examine the whole digestive sy'stem under water.

5. Note again the mid-gut and the digestive glands and ducts; then remove the digestive gland of one side, and sketch the enteric canal from the same side. Slit up the hind-gut so as to see the ridges and cuticle.

6. Clean the walls of the gizzard and note the other sclerites of the gastric mill.

- Articulated to each end respectively of the two median sclerites already referred to, in each lateral wall of the gizzard, is a lateral sclerite, the two articulating with one another at their other ends, so that these six sclerites together form a sort of hexagonal frame. Two other median sclerites, arising from the median ones above-mentioned respectively, extend downwards into the constriction between the two portions of the gizzard, and these join below at an angle, where they bear a median tooth. Each of the posterior lateral sclerites bears a lateral tooth.

Cut open the anterior end of the gizzard, and note the strongly calcified, brownish, median tooth, and the two large lateral teeth. Seize hold of the two median assicles with two pairs of forceps, one in each 
hand, and pull gently backwards and forwards (in the direction in which the muscles pull). It will then be seen that the median and lateral teeth come together in the micldle line so as to act as a gastric mill. Note the slit-like lumen of the part of the gizzard behind this and the arrangement of the setc which act as strainers. Make sketches as you proceed.

III. I. The chief muscles of the body are:- $a$, the paired and segmented dorsal extensor, arising from the side walls of the thorax, and extending into the abdomen above the intestine, giving off slips to each segment of the abdomen (this has already been removed); and $b$, the large and complex ventral muscles, the lateral halves of which are not separate from one another, the fibres being interwoven, somewhat like those of a rope; slips are given off to the abdominal sterna. These act mainly as a flexor of the abdomen.

2. Muscles pass from the body to the proximal joints of the limb, and between successive podomeres :-these latter will be examined at a later stage $(\S \mathrm{D})$.

3. Note again the paired adductor of the mandible (p. 375), and trace its calcified tendon downwards to its insertion on to the mandible.

4. Tease out a small piece of muscle so as to separate its fibres from one another. Stain, and mount in glycerine. Note the transverse striations, sarcolemma, and nuclei (compare Fig. 32). Sketch.

Remove the muscles of the body described above, noting the sternal artery ( $p .375)$, and taking especial care to leave the abdominal nerve. cord in situ when removing the large ventral muscles. Note that in the thorax, the nerve-cord passes into a sternal canal, formed by a series of ingrowths of the exoskeleton-the endophragmal system-from which the muscles passing to the thoracic limbs arise. Insert the scissors into the sternal canal, and cut away and remove its roof, bit by bit. The whole of the central nervous system will then be exposed.

IV. Observe that a more marked distinction into ganglia and connectives is seen than in the case of the Earthworm, and that the fusion of the two lateral halves of the cord or chain has only affected the ganglia, the connectives being double all the way along.

I. Note:- $a$. The brain, or f'used, compound supra-cesophageal ganglia; $b$, the cesophageal connectives, and $c$, the postoral ventral nerve-cord, consisting of a large compound sub-assophageal ganglion and of I2 segmental ganglia, united by paired connectives. Beneath the cord, the 
sternal and ventral abdominal arteries (1. 375) will be seen, the sternal artery passing letween the connectives joining the fifth and sixth postoral ganglia.

2. The brain gives off nerves to the eyes and the two pairs of feelers: the suboosophageal ganglion supplies the mandibles and four following pairs of appendages with their segments. Each of the other ganglia supplies one segment (with its appendages) only, except in the case of the last or 6th abdominal ganglion, which gives off nerves to the telson. (A small anterior visceral nerve, arising from the brain and nesophageal connectives and supplying the fore-gut, will have been removed, and a small posterior visceral nerve, supplying the hind-gut, arises from the last abdominal ganglion.) Sketch.

$\checkmark$. The antennary or green-gland's are situated just behind the bases of the large feelers. Blow through the renal aperture of one side (p. 373) and note the duct and urinary bladder on the dorsal side of the gland. Then cut through the duct, remove the whole gland, and examine under water. Sketch.

D. The Appendages.-Remore the appendages of one side, beginning with the last, one by one, cutting through the articular membrane with a scalpel, and then taking hold of the basal joint with the forceps and pulling the appendage away. Work through the de. scription on pp. 350-353 and sketch typical appendages from each region.

Note the delicate paragnatha behind the month and the labrum in front of it (p. 356).

The arrangement of the joints and muscles of the limbs can be well seen by examining the large first leg or cheliped. Note that each joint works in a different plane, and then cut away the exoskeleton from one side of the two or three distal podomeres, so as to expose the muscles (compare Fig. 85). Then remove these, and note their chitinous tendons. Observe that the flexor muscle and its tendon, which moves the pincers, is much larger than the extensor muscle.

\section{E. Sensory Organs.}

1. Tactile organs. Snip off some setæe from the body or appendages. Examine under the microscope. Sketch.

2. "Olfactory" organs. Examine the outer flagellum of the antennule under the low power, and note the tufts of spatula-like olfactory selie on the ventral surface. Sketch.

3. Auditory organ. Carefully cut away the convex ventral side of 
the basal segment of the antennule with scissors, so as to expose the auditory sac. Cut this out and place it on a slide, carefully removing the muscles surrounding it, as well as the sete around its aperture. Note the contained grains of sand (otoliths), and then wash them away. Stain with magenta and mount in glycerine, flattening the sac out with a cover-glass. Note that the sac is an involution of the integument lined by cuticle, and that it contains simple, jointed auditory seta of various sizes, arranged in rows, and that branches of the antennulary nerve run up the stem of each seta. Sketch.

4. The Eyos. Remove one of the eye-stalks, and note the apparently black, uncalcified, oval portion of the cuticle (cornea) at its distal end. Strip this off, and note that it is transparent. Then wash off any pigment which may have come away with it and mount in water. Observe the comeal facets. Then eut the eye-stalk into two longitudinal halves with a knife, and examine with a lens under water. Note the optic nerve entering the stalk, and enlarging to form the optic ganglion, from which a number of bodies (ommatidea) radiate outwards to the corresponding facets of the cornea. The ommaticlea are separated from one another by pigment. Sketch.

5. Examine longitudinal sections of the eye-stalks, decalcified and prepared as directed on p. I36, and note in detail the above parts. Each -ommatideum lies beneath the corresponding corneal facet, and is made up of an onter vitreous body or crystalline cone, and an inner retinula formed of sensory cells and enclosing a transversely. striated, spindlesshaped, refractive body or rhabdome, and closely connected with the optic ganglion. Note also the pigment between the ommatidea. Sketch.

\section{F. Structure of the Exoskeleton.}

In order to follow this ont in greater detail, proceed as follows :-

I. Cut through the thorax and abdomen of a crayfish transversely, and note the relations of the hard and soft parts. (Compare Figs. 83 and 87).

2. Dip a crayfish into hot water, so that the soft parts come away easily. Open up the cephalothorax from the dorsal side, separate some of the abdominal segments, and clean thoroughly. Examine the joints and make out the relations of the endophragnal system (p. 377), looking something like a lattice-gircler.

3. Examine youx preparation of the eye once more under the microscope and notice the part where the section passes through the outer 
wall of the eye-stalk, so as to make out the microscopic structure of the integument and exoskeleton, which apart from the calcification, is similar in all parts. Notice the epiderm, and the thick, laminated, chitinous cuticle, the superficial layer of which.is uncalcified throughout.

\section{G. Dissection from the Side.}

Cut through the carapace on the dorsal side as before, but be careful to keep your cut very slightly to one sicle-say the left-of the middle line, and when the pericardium is exposed, inject the arteries through the heart as before ( $p .374$ ). Then remove the gill-cover of the same side, and examine the gills once more.

Remove and examine again all the appendages of the same side, and then carefully continue cutting longitudinally through the exoskeleton, both dorsally and ventrally, close to the middle line, taking care you do not injure any median organs. Remove the entire exoskeleton of this side, as well as the dorsal and ventral muscles, eutting through the latter in the median line very carefully.

After your dissection has been pinned down under water, the greengland, digestive gland, and gonad of the same side should also be removed, and the gizzard cut open. Then tidy up the dissection, which will now be reduced to a longitudinal section like that represented in Fig. 86.

Once more carefully follow out the structure and relations of all the organs exposed, and sketch your dissection. 


\section{CHAPTER VII}

THE FRESH-WATER MUSSEI.-CHARACTFRS OF THE PHYLUM MOLLUSCA-ENUMERATION OF THE CHIEF PHYLA OF THE ANIMAL KINGDOM.

IN the mussel we meet with an entirely new type of structure: the animal, like the worm and crayfish, is bilaterally symmetrical, but there is no trace of metameric segmentation; the power of locomotion is greatly restricted; and food is obtained passively by ciliary action, as in Infusoria, not by the active movements of definite seizing organs -tentacles, limbs, or protrusible mouth-as in most of the Metazoa (p. 286).

Fresh-water mussels are found in rivers and lakes in most parts of the world. Anodonta cygnea, the swan-mussel, is the commonest species in England; but the pearl-mussel, Unio margaritifera, is found in mountain streams, and other species of the same genus are universally distri'tuted.

The mussel is enclosed in a brown shell formed of two separate halves or valves hinged together along one edge. It lies on the bottom, partly buried in the mud or sand, with the valves slightly gaping, and in the narrow cleft thus formed a delicate, semi-transparent substance is seen, the edge of the mantle or pallium. The mantle really conisists of separate halves or lobes corresponding with the valves of 
the shell (compare Fig. 95), but in the position of rest the two lobes are so closely approximated as to appear simply like a membrane uniting the valves. At one end, however, the mantle projects between the valves in the form of two short tubes, one (Fig. 93, ex. sph) smooth-walled, the other (in. sph) beset with delicate processes or tentacles. By diffusing particles of carmine or indigo in the water it can be seen that a current is always passing in at the fringed tube -hence called the inhalant siphon, and out at the smooth or exhalant siphon. Frequently a semi transparent, tongue-like body $(f t)$ is protruded between the valves at the opposite side from the hinge and at the end furthest from the siphons: this is the foot, by means of which the animal is able slowly to plough its way through the sand or mud. When the mussel is irritated the foot and siphons are withdrawn and the valves tightly closed. In a dead animal, on the other hand, the shell always gapes, and it can then be seen that each valve is lined by the corresponding lobe of the mantle, and that the exhalant siphon is formed by the union of the lobes above and below it and is thus an actual tube; but that the boundary of the inhalant siphon facing the gape of the shell is simply formed by the approximation of the mantle-lobes, so that this tube is a temporary one.

The hinge of the shell is dorsal, the gape ventral, the end bearing the siphors posterior, the end from which the foot is protuded anterior: hence the valves and mantle-lobes are respectively right and left.

In a dead and gaping mussel the general disposition of the parts of the animal is readily seen. The main part of the body lies between the dorsal ends of the valves: it is produced in the middle ventral line into the keel-like foot: and on each side between the foot and the corresponding mantle-lobe are two delicate, striated plates, the gills or 
ctenidia, as they are often called. Thus the whole animal has been compared to a book, the back being represented by the hinge, the covers by the valves, the fly-leaves by the mantle-lobes, the two first and the two last pages by the gills, and the remainder of the leaves by the foot. (Fig. 95).

When the body of the mussel is removed from the shell the two valves are seen to be united, along a straight hingeline, by a tough, elastic substance, the hinge-ligament (Fig. $95, l g$ ) passing transversely from valve to valve. It is by the elasticity of this ligament that the shell is opened : it is closed, as we shall see, by muscular action : hence the mere relaxation of the muscles results in opening the shell. In Anodonta the only junction between the two valves is afforded by the ligament, but in Unio each is produced into strong projections and ridges, the hinge-teeth, separated by grooves or sockets, and so arranged that the teeth of one valve fit into the sockets of the other.

The valves are marked externally by a series of concentric lines parallel with the free edge or gape, and starting from a swollen knob or elevation, the umbo, situated towards the anterior edge of the hinge-line. These lines are lines of growith. The shell is thickest at the umbo, which represents the part first formed, and new layers are deposited under and concentrically to this original portion as secretions from the mantle, the shell being, like the armour of the crayfish, a cuticular exoskeleton. As the animal grows each layer projects beyond its predecessor, and in this way successive outcrops are produced, giving rise to the markings in question. In the region of the umbo the shell is usually more or less eroded by the action of the carbonic acid in the water.

The inner surface of the shell also presents characteristic markings. Parallel with the gape, and at a short distance from it, is a delicate streak caused by the insertion into the 
shell of muscular fibres from the edge of the minaitle: the streak is hence called the pallial line. Beneath the anterior end of the hinge the pallial line ends in an oval mark, theanterior adductor impression, into which is inserted one of the muscles which close the shell. A similar, but larger, posiefior adductor impression lies beneath the posterior end of the hinge (compare Fig 93, $a . a d, p . a d$ ). Two smaller markings close behind the anterior adductor impression, dorsal and ventral respectively, mark the origin of the anterior retractor and of the protractor muscle of the foot: one just anterior to the posterior adductor impression, that of the posterior retractor of the foot. From all these impressions faint converging lines can be traced to the umbo: they mark the gradual shifting of the muscles during the growth of the animal.

The shell consists of three layers, the outer layer, as in the crayftsh, being uncalcified. Outside is a brown hornlike layer, the periostracum, composed of conchiolin, a substance allied in composition to chitin. Beneath this is a prismatic layer formed of minute prisms of calcium carbonate, separated by thin layers of conchiolin; and, lastly, forming the internal part of the shell is the nacre, or "mother-of-pearl," formed of alternate layers of carbonate of lime and conchiolin arranged parallel to the surface. The periostracum and the prismatic layer are secreted from the edge of the mantle only, the pearly layer from the whole of its outer surface. The hinge-ligament is continuous with the periostracum, and is to be looked upon simply as a median uncalcified portion of the shell, which is therefore, in strictness, a single continuous structure.

By the removal of the shell the body of the animal is seen to be elongated from before backwards, narrow from side to side, produced on each side into a mantle-lobe, and con- 
tinued ventrally into a keel-like visceral mass, which passes below and in front into the foot (Fig. 93, ft). Thus each valve of the shell is in contact with the dorso-lateral region of the body of its own side together with the corresponding mantle-lobe, and it is from the epithelium covering these parts that the shell is formed as a cuticular secretion. The whole space between the two mantle-lobes, containing the gills, visceral mass, and foot is called the mantlecavity.

A single layer of epithelial cells, the deric epithelium or epiderm, covers the whole external surface-i.e. the body proper, both surfaces of the mantle, the gills, and foot; that of the gills and the inner surface of the mantle is ciliated. Beneath the epiderm come connective and muscular tissue, which occupy nearly the whole of the interior of the body not taken up by the viscera, the colome being, as we shall see, much reduced. The muscles are all unstriped, and are arranged in distinct bands or sheets, many of them being very large and conspicuous. The largest are the anterior and posterior adductors (Figs. 93 and 95,a.ad, p. ad), great cylindrical muscles which pass transversely across the body and are inserted at either end into the valves of the shell, which are approximated by their contraction. Two muscles of much smaller size pass from the shell to the foot, which they serve to draw back : they are the anterior and posterior retractors of the foot. A third foot-muscle arises from the shell close to the anterior adductor, and has its fibres spread fan-wise over the visceral mass which it serves to compress, thus forcing out the foot and acting as a protractor of that organ. The substance of the foot itself consists of a complex mass of fibres, the intrinsic muscles of the foot, many of which also act as protractors. Lastly, all along the border of the mantle is a row of delicate pallial muscles?

Pract. Zool. 
which, by their insertion into the shell, give rise to the line already seen.

The colome is reduced to a single ovoidal chamber, the pericardium (Figs. 93 and 95, pc), lying in the dorsal region of the body and containing the heart and part of the intestine : it is lined by cœlomic epithelium, and does not correspond with the pericardial sinus of the crayfish, which is a blood-space (p. 362). In the remainder of the body the space between the epiderm and the viscera is filled by the muscles and connective tissue.

The mouth (Fig. 93, mth) lies in the middle line just below the anterior adductor. On each side of it are two triangular flaps, the internal and external labial palps; the external palps unite with one another in front of the mouth, forming an upper lip; the internal are similarly united behind the mouth, forming a lower lip : both are ciliated externally. The mouth leads by a short gullet (gul) into a large stomach (st), which receives the ducts of a pair of irregular, dark-brown, digestive glands $(d . g l)$. The intestine (int), which is lined by a ciliated epithelium, goes off from the posterior end of the stomach, descends into the visceral mass, where it is coiled upon itself, then ascends parallel to its first portion, turns sharply backwards, and proceeds, as the rectum ( $r c t)$, through the pericardium where it traverses the ventricle of the heart, and above the posterior adductor, finally discharging by the anus (a) into the exhalant siphon, or cloaca. The wall of the rectum is produced into a longitudinal ridge, or typhlosole (Figs. 93 and 95, ty), and two similar ridges begin in the stomach and are continued into the first portion of the intestine. The stomach contains at certain seasons of the year a gelatinous rod, the crystalline style.

The gills consist, as we have seen, of two plate-like 
bodies on each side between the visceral mass and the mantle: we have thus a right and a left outer, and a right and a left inner gill (Fig. 95, ext. gl, int. gl.). Seen from
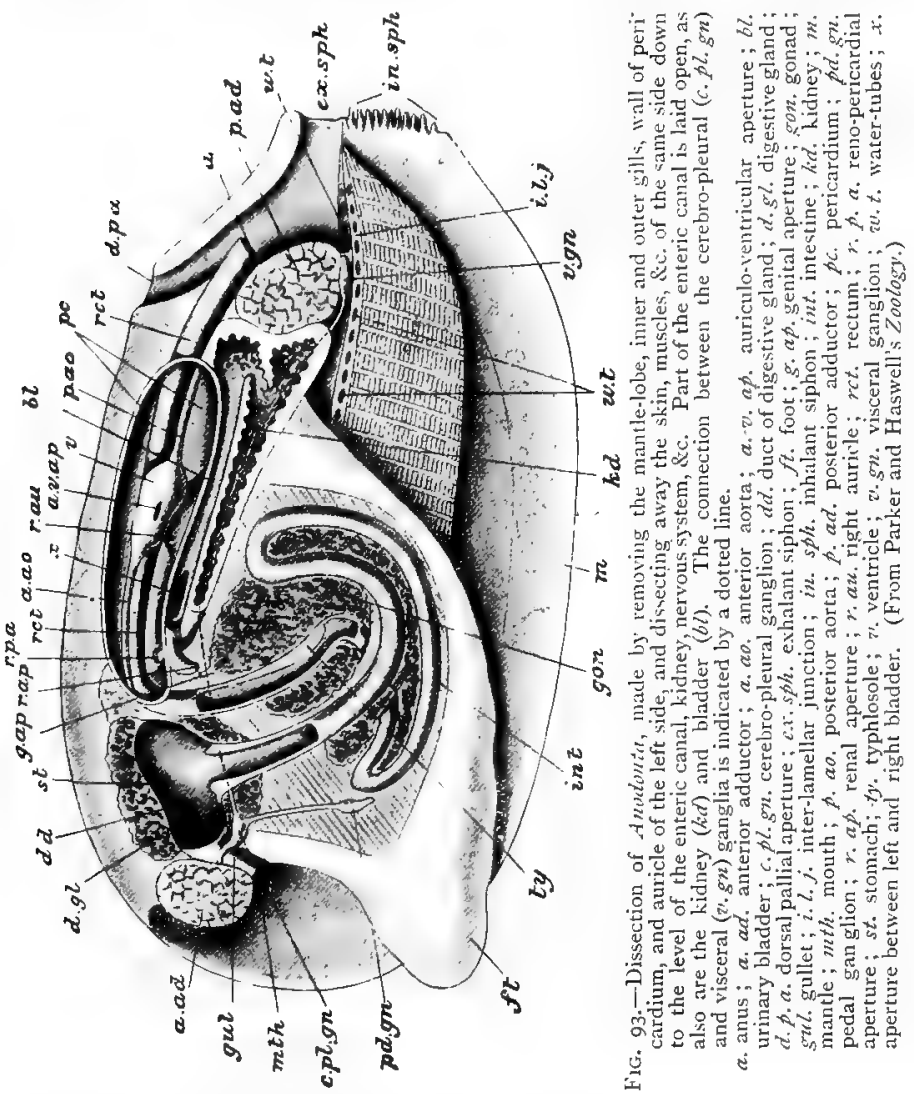

the surface (Fig. 93), each gill presents a delicate double striation, being marked by faint lines running parallel with, and by more pronounced lines running at right angles to, 
the long axis of the organ. Moreover, each gill is double, being formed of two similar plates, the inner and outer lamelle, united with one another along the anterior, ventral, and posterior edges of the gill, but frec dorsally. The gill has thus the form of a long and extremely shallow bag open above (Figs. 94 and 95) : its cavity is subdivided by vertical plates of tissue, the inter-lamellar junctions (i.l.j), which

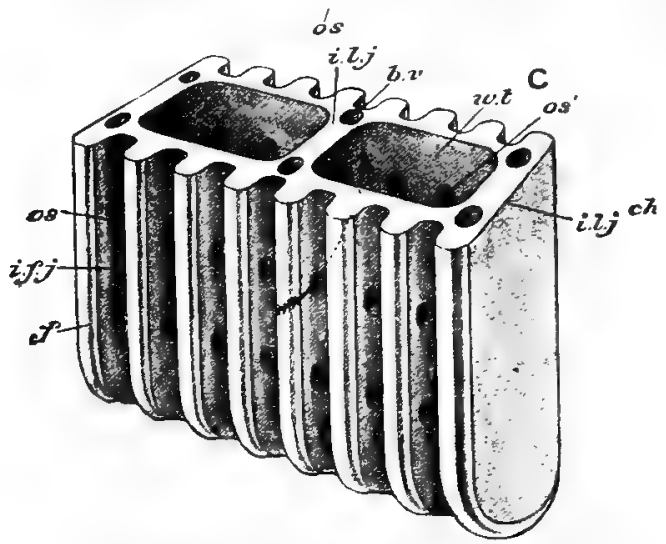

FIG. 94--Diagram of the structure of the gill of Anodonto.

The gill is made up of V-shaped gill-filaments $(f)$ arranged in longitudinal series and bound together by horizontal inter-filamentar junctions $(i, f \cdot j)$ which cross them at right angles, forming a kind of basket-work with apertures, the ostia $(o s)$, leading from the outside and opening (os) into the cavity of the gill. The latter is divided by vertical partitions, the inter-lamellar junctions $(i, l, j)$ into compartments or water-tubes $(z, t)$ which open also into the supra-branchial chamber; $b, v$, blood-vessels. (From Parker and Haswell's Zoology.)

extend between the two lamellie and divide the intervening space into distinct compartments or zeater tubes (Figs. 93 and $94, w . t)$, closed ventrally, but freely open along the dorsal edge of the gill. The vertical striation of the gill is due to the fact that each lamella is made up of a number of close-set gill-filaments (Fig. 94, $f$ ) : the longitudinal striation to the circumstance that these filaments are connected by horizontal bars, the inter-filamentar junctions $(i . f . j)$. At the thin free 
or ventral edge of the gill the filaments of the two lamellæ are continuous with one another, so that each gill has actually a single set of $\mathrm{V}$-shaped filaments, the outer limbs of which go to form the outer lamella, their inner limbs the inner lamella. Between the filaments, and bounded above and below by the inter-filamentar junctions are minute apertures or ostia (os), which lead from the mantle-cavity through a more or less irregular series of cavities into the interior of the water-tubes. The filaments themselves are supported by chitinous rods, and covered with ciliated epithelium, the large cilia of which produce a current running from the exterior through the ostia into the water-tubes, and finally escaping by the wide dorsal apertures of the latter. The whole organ is traversed by blood-vessels $(b . v)$.

The mode of attachment of the gills presents certain features of importance (compare Fig. 95, A, B, C). The outer lamella of the outer gill is attached along its whole length to the mantle: the inner lamella of the outer, and the outer lamella of the inner gill are attached together to the sides of the visceral mass a little below the origin of the mantle : the inner lamella of the inner gill is also attached to the visceral mass in front, but is free further back. The gills are longer than the visceral mass, and project behind it, below the posterior adductor (Figs. 93 and $95 \mathrm{C}$ ), as far as the posterior edge of the mantle : in this region the inner lamellæ of the inner gills are united with one another, and the dorsal edges of all four gills constitute a horizontal partition between the pallial cavity below and the exhalant chamber or cloaca above. Owing to this arrangement it will be seen that the water-tubes all open dorsally into a supra-branchial chamber (Fig. 95, s.br.c), continuous posteriorly with the cloaca and thus opening on the exterior by the exhalant siphon.

The physiological importance of the gills will now be 
obvious. By the action of their cilia a current is produced which sets in through the inhalant siphon into the pallial cavity, through the ostia into the water-tubes, thence into the supra-branchial chamber, and out at the exhalant siphon. The in-going current carries with it not only oxygen for the aeration of the blood, but also diatoms, infusoria, and other microscopic organisms, which are swept into the mouth by the cilia covering the labial palps. The out-going current carries with it the various products of excretion and the freces passed into the cloaca. The action of the gills in producing the food current is of more importance than their respiratory function, which they share with the mantle.

The excretory organs are a single pair of curiously-modified nephridia, situated one on each side of the body just below the pericardium. Each nephridium consists of two parts, a brown, spongy, glandular portion or kidney (Figs. 93 and 95, $k d)$, and a thin-walled, non-glandular part or bladder $(b l)$. The two parts lie parallel to one another, the bladder being placed dorsally and immediately below the floor of the pericardium : they communicate with one another posteriorly, while in front the kidney opens into the pericardium $(r . p . a$.$) ,$ and the bladder on the exterior by a minute aperture $(r, a p)$, situated between the inner gill and the visceral mass. Thus the whole organ, often called after its discoverer, the organ of Bojanus, is simply a tube bent upon itself, opening at one end into the ccelome, and at the other on the external surface of the body: it has therefore the normal relations of a nephridium (p. 33I). Two bladders communicate anteriorly, and their epithelium is ciliated, producing an outward current.

It seems probable that an excretory function is also discharged by a large glandular mass of reddish-brown colour, called the pericardial sland or Kebes's organ (Fig. 95, k.o). It lies in the anterior region of the body just in front of the pericardium, into which it discharges. 
The circulatory system is well developed. The heart lies in the pericardium, and consists of a single ventricle (Figs. 93 and $95 \mathrm{~B}, v)$ and of right and left auricles (au). The ventricle is a muscular chamber which has the peculiarity of surrounding the rectum $(r c t)$ : the auricles are thin-walled chambers communicating with the ventricle by valvular apertures opening towards the latter. From each end of the ventricle an artery is given off, the anterior aorta (Fig 93, $a$. ao) passing above, the posterior aorta ( $p . a 0)$ below the rectum. From the aortæ the blood passes into arteries (Fig. 96, art., ${ }^{1}$ art ${ }^{2}$ ) which ramify all over the body, finally forming an extensive network of vessels, many of which are devoid of proper walls and have therefore the nature of sinuses. The returning blood passes into a large longitudinal vein, the vena cava (Figs. 95 and 96, v. c), placed between the nephridia, whence it is taken to the kidneys themselves (Fig. $96, n p h . v$ ), thence by afferent branchial veins (af. br. v) to the gills, and is finally returned by efferent branchial veins (ef. $b r . v)$ to the auricles. The mantle has a very extensive blood supply, and probably acts as the chief respiratory organ (p. 390): its blood (art $t^{1}$ ) is returned directly to the auricles without passing through either the kidneys or the gills. The blood is colourless and contains leucocytes. There is no communication between the blood-system and the pericardium.

The nervous system is formed on a type quite different from anything we have yet met with. On each side of the gullet is a small cerebro-pleural ganglion (Fig. 93, c.pl. gn), united with its fellow of the opposite side by a nerve-cord, the cerebral commissure, passing above the gullet. Each cerebro-pleural ganglion also gives off a cord, the cerebropedal connective, which passes downwards and backwards to a pedal ganglion ( $p d . g n$ ) situated at the junction of the 
visceral mass with the foot: the two pedal ganglia are so closely united as to form a single bilobed mass. From each cerebro-pleural ganglion there further proceeds a long cerebrovisceral connective, which passes directly backwards through the kidney and ends in a visceral ganglion (Figs. 93 and 95,

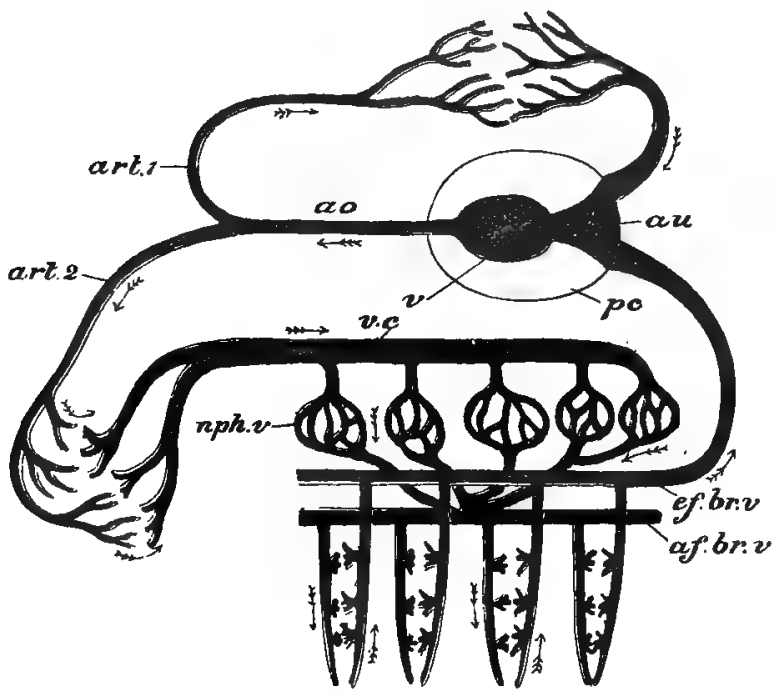

F IG. 96.-Diagram of the circulatory system of Anodonta.

The blood received from the auricles $(a u)$ is pumped by the ventricle $(v)$ into the aorta $(a 0)$ and thence passes to the mantle $(a r t 1$.$) and to the body generally$ $\left(a r t^{2}\right)$. The blood which has circulated through the mantle is returned directly to the auricle : that from the body generally is collected into the vena cava $(v . c)$, passes by nephridial veins $(n p h . v)$ to the kidneys, thence by afferent branchial veins $(a f, b r . v)$ to the gills, and is returned by efferent branchial veins (ef.br. $v)$ to the auricles; $p c$. pericardium. (From Parker and Haswell's Zoology.)

C, v. $g n$ ) placed on the ventral side of the posterior adductor muscle. The visceral, like the pedal ganglia, are fused together. The cerebro-pleural ganglia supply the labial palps and the anterior part of the mantle; the pedal, the foot and its muscles; the visceral, the enteric canal, heart, 
gills, and posterior portion of the mantle. The nerve-cells are confined to the ganglia.

It will be seen that the cerebral commissures and cerebropedal connectives, together with the cerebro-pleural and pedal ganglia, form a nerve-ring which surrounds the gullet: the cerebro-pleural ganglia may be looked upon as a supracesophageal nerve mass corresponding with the brain of the Earthworm and Crayfish, and the pedal ganglia as an infraœsophageal mass representing the ventral nerve-cord.

Sensory organs are poorly developed, as might be expected in an animal of such sedentary habits. In connection with each visceral ganglion is a patch of sensory epithelium forming the so called olfactory organ or, better, osphradium, the function of which is apparently to test the purity of the water entering by the respiratory current. Close to the pedal ganglion a minute " auditory sac" or otocyst is found, the nerve of which is said to spring from the cerebropedal connective, being probably derived from the cerebral ganglion. Sensory cells, probably tactile, also occur round the edge of the mantle, and especially on the tentacles of the inhalant siphon.

The sexes are separate. The gonads (Fig. 93 gon) are large, paired, racemose (p. 135) bodies, occupying a considerable portion of the visceral mass amongst the coils of the intestine : the spermary is white, the ovary reddish. The gonad of each side has a short duct which opens $(g \cdot a p)$ on the surface of the visceral mass, just in front of the renal aperture.

In the breeding season the eggs, extruded from the genital aperture, pass into the supra-branchial chamber, and so to the cloaca. There, in all probability, they are impregnated by sperms introduced with the respiratory current. The oosperms are then passed into the cavities of the outer gills, 
which they distend enormously. Thus the outer gills act as brood-pouches, and in them the embryo develops into the peculiar larval form presently to be described.

As in the frog and earthworm the cells formed by the segmentation of the oosperm are of two sizes, small cells composed entirely of protoplasm, and large cells loaded with yolk-granules. The large become enclosed by the small cells, but the enteron thus formed is very small and quite unimportant during early larval life, the young mussels being
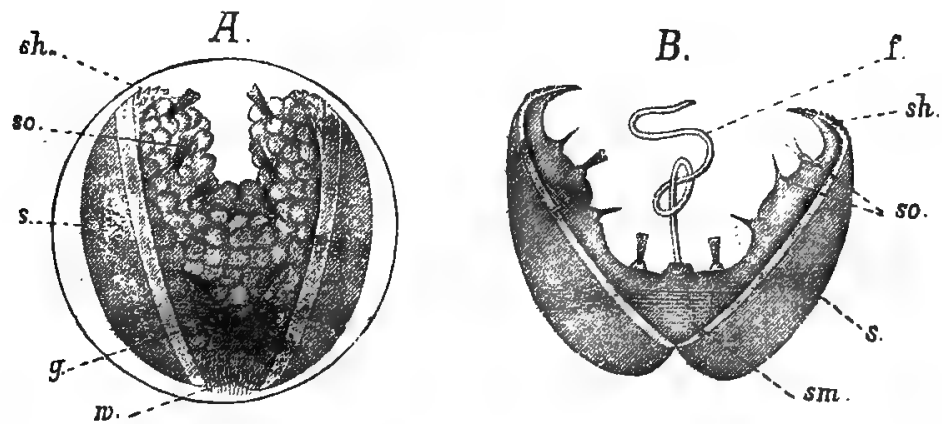

F'G. 97.-A, advanced embryo of Anodonta enclosed in the egg-membrane. $B$, free larva or glochidium.

$f$. byssus ; $g$. lateral pits; $s$. shell ; sh. hooks; sm. adductor muscle; so. sensory hairs; w. ciliated area. (From Korschelt and Heider.) $\cdot j$

nourished, after the manner of parasites (p. $27 \mathrm{I}$ ), by a secretion from the gills of the parent.

The dorsal surface of the embryo is soon marked out by the appearance of a deep depression, the shell-gland, which secretes, in the first place, a single median shell. This is, however, soon replaced by a bivalved larval shell (Fig. 97, $s)$ of triangular form, the ventral angles being produced into hooks $(s h)$. The body at the same time becomes cleft from below upwards (A), forming the right and left mantle-lobes. On the ventral surface, between the lobes of the mantle, is 
formed a glandular pouch, which secretes a bunch of silky threads, the byssus $(f)$. The larva is now called a glochidium, the subsequent history of which furnishes another example of a means of ensuring dispersal in a sedentary animal (compare pp. 272 and 278 ).

The glochidia, entangled together by means of their byssal threads, escape from the gills of the parent by the exhalant siphon, and eventually attach themselves by their hooked valves to the body of a passing fish, such as a stickleback. Here they live for a time as external parasites, gradually undergoing metamorphosis ; and finally drop from the host and assume the sedentary habits of the adult.

This mode of development is exceptional amongst bivalves, in most of which (e.g. oyster) the egg gives rise to a larva resembling the trochosphere of many annelids (p. 340), the prostomial' region then growing out into a thickened rim which bears the circlet of cilia in front of the mouth, the larva at this stage being distinguished as a veliger.

The Mussel belongs to the phylum Mollusca, which includes, in addition to the bivalved "shell-fish" - such as mussels, oysters, cockles, - the snails, slugs, whelks, periwinkles, \&c.-most of which possess a univalved shell, as well as the cuttle-fishes and their allies. These are all sharply distinguished from the Arthropods by the absence of segmentation, and by having, as a rule, an exoskeleton in the form of a shell. The bivalves are included in the class Pelecypoda or Lamellibranchiata, the essential structure of which you will have learnt from your study of the Mussel.

You have now examined examples of the following chief divisions or phyla of the animal kingdom (compare p. 219):

Protozoa.

Annulata.

Mollusca.

Colenterata. Arthropoda. Vertebrata.

In addition to these, you will probably have seen, when dissecting the frog, certain parasiles. One of these, Polystorram (p. 33), belongs 
to a lowly group of worms of a flattened form, constituting the phylum Platyhelminthes, which includes the parasitic liver-flukes and tapeworms as well as certain free-living forms; another, Ascaris (p. 153), belongs to the phylum Nemathelminthes in which all the parasitic thread-worms are placed. A part from certain other smaller groups, which include such animals as " wheel-animalcules," "sea-mats," "lamp-shells," \&c., there only remains one other of the larger phyla of which you will not have studied an example, viz., the phylum Echinodermata, which is constituted by the star-fishes, sea-urchins, sea-cucumbers, \&c. : these are all inhabitants of the sea, and are peculiar in exhibiting a more or less pronounced radial arrangement of their parts, and in possessing a curious calcareous exoskeleton developed within their integument, consisting of small particles or of definitelyshaped plates. All the phyla with the exclusion of the Vertebrates are spoken of collectively as the Invertebrata.

\section{PRACTICAL DIRECTIONS.}

FRESH-WATER MUSSEI.

Examine a living specinen in a vessel of water with some sand at the bottom, and note the greenish-brown bivalve shell, the foot, and the manner in which the animal buries itself, anterior end downwards, with the pallial openings projecting posteriorly. Observe the currents of water passing in at the fringed inhalant aperture, and out at the exhalant aperture: these can be more easily observed if a little finelypowdered indigo or carmine is placed in the water.

In order to kill the animal in as fully-extended a condition as possible, place it in a saucepan or beaker of water, and heat over a gas-burner or spirit-lamp until the water is warmed up to a temperature of about $40^{\circ} \mathrm{C}$, when the foot will be protruded. Keep at this temperature until the mussel is dead. The animal is best preserved from day to day in 3 per cent. formaline, but 70 per cent. spirit will answer the purpose.

\section{A. External characters.}

Compare the shell with the carapace of the crayfish, and observe that it covers the whole body and is densely calcified except along the dorsal hinge-line, where it forms an elastic ligament connecting the two lateral values of the shell. Note the lines of growth, and the umbo near the hinge-line. The anterior end is rounded, the posterior end more pointed. In dead specimens the valves gabe, owing to the elasticity of 
the ligament and to the relaxation of the adductor muscles (see below), and they can than be seen to be lined by a fold of the integument, the mantle or pallizim. By wedging the valves open still further, the anterior and posterior adductor muscles are seen connecting the two valves; also the foot, visceral mass and gills, between the two mantle folds.

\section{B. Dissection.}

I. Carefully insert a scalpel between the left valve and mantle-edge, and separate them all round the margin of the valve. Then cut through the anterior and posterior adductor muscles close to the shell, and remove the left valve, cutting through the ligament.

I. Compare the positions of the cut muscles on the body with the muscular impressions on the shell:-a. the large anterior adductor near the dorsal and anterior end ; and close behind it $-b$. the protractor and $c$. the anterior retractor muscles of the foot, the latter dorsal to the former ; $d$. the large posterior adductor near the dorsal and posterior end, on the upper and anterior margin of which is $e$. the posterior retractor. Note also the thickened ventral edge of the mantle, the corresponding pallial line on the valve, as well as the lines from the nuscular impressions converging towards the umbo, the smooth longitudinal hinge (with hinge-teeth in Unio), the liganent, and the iridescent inner layer of the shell. Sketch the inner surface of the valve.

2. Break the shell across, and examine the edge with a lens, noting the three layers of which it is composed :- $a$. the thin outer uncalcified periostracum; $b$. the thicker middle prismatic layer; and $c$. the inner pearly or nacreans layer.

Pin down the animal firmly under water, leaving it in the right valve : insert the pins through the ligament and muscular part of the foot, and obliquely against the edges of the shell. Note :-

3. The left mantle-lobe uniting with the right above the middle of the anterior adductor muscle anteriorly, and behind the posterior adductor muscle posteriorly. Just behind the posterior adductor muscle the margins of the mantle are much thickened; and in life, the two approximated nantle-edges here separate to form the exhalant and inhalant apertures, the latter of which is provided with short tentacles.

4. Turn back the left mantle-lobe and note the mantle-cavity and its contents:- $a$. the foot and visceral mass; $b$. the left pair. of gills at the sides of the visceral mass; and anteriorly $\iota$. the left pair of small, triangular, labial palps. (Note that there is no distinct head). These parts are situated between the two mantle-folds in the larg 
ventral mantle-cavity. Note also the position of the pericardium on the clorsal side of the gills, the pericardial gland, and the left excretory organ (nephridium), between the pericardium and gills, and extending back to the posterior adductor muscle.

5. Pass a seeker into the exhalant aperture and note that it enters a short exhalunt siphon or cloaca, separated from the mantle-cavity by the bases of the gills. Slit this chamber open, and note that it extends above the posterior adductor muscle, as well as above the gills. The hinder end of the rectum will he seen just above the posterior adductor muscle, opening by the anus into the cloaca. Insert a seeker into the mouth, between the anterior adductor muscle and the anterior edge of the foot.

II. I. Remove the left mantle-lobe, cutting very carefully along the bases of the palps and gills. Then slit open the pericardium longituclinally, a short distance clorsally to the gills, and note the rectum, rentricle, and clelicate left auricle. Cut away sufficient of the wall of the pericarrlium to expose these parts, taking care not to injure the auricle. Note-

2. The rectum, which runs straight through the pericardium, and in its middle is surrounded by the elongated muscular ventricle, which is bilolsed posteriorly. The thin-walled, triangular left autricle can be seen opening into the ventricle by a valvular aperture : the base of the auricle is attached along the dorsal border of the gills. (Note the right auricle on the other side). Inject French-blue (p. 99) into the left auriculoventricular aperture, and note $u$. the anterior aorta above the rectum, and $b$. the posterior aorta below the rectum. In the micldle line of the floor of the pericardium the venza cava can be seen.

3. Examine the gills. Note the left outer and inner gill, and that in the female the former is often distended with eggs or larve. Each gill consists of an outor and an inner lamella, forming a kind of trelliswork, with small meshes or ostia, separated by horizontal and vertical bars. Cut away a piece of the outer lamella, noting that the two lamella of each gill are united at intervals by interlamellar junctions.

4. Make out the mode of attachment of the gills (see p. 389, and Fig. 95). Sketch your dissection with the gills in their natural position.

5. Pass a seeker forwards from the exhalant siphon along the suprabranchial passages-two longitudinal canals at the bases of the gills communicating with the interlamellar spaces or water zabes (the inner 
passage unites with its fellow behind the visceral mass, see Fig. 95c). The inner canal also communicates with the branchial chamber through the slit between the visceral mass and the middle part of the lamella of the inner gill. Cut open the canals carefully, and examine their relations.

6. Turn up the gills, and insert the scissors in the slit just mentioned; cut forwards through the inner lamella of the inner gill for a short distance. The renal aperture or nephridiopore can then be seen opening into the suprabranchial passage, and just below and in front of it is the genital aperture. Then note the paired reno-pericardial aperture (nephrostome) in the anterior end of the pericardium, just below the point at which the rectum enters it : this can be more easily seen if the rectum is cut through and raised up.

7. Blow through the nephridiopore, and note the thin-walled sac-like bladder into which it opens : this lies just below the pericardium, and communicates with its fellow anteriorly. Just beneath and internal to the bladder is the glandular portion of the kidney, which is dark in colour and extends further back than the bladder, beyond which it forms a large mass just in front of the posterior adductor muscle : it communicates with the hinder end of the bladder. Sketch.

III. I. Remove both gills carefully, cutting along their bases; stain and mount a small piece of one lamella. Note that the lamella is made up of close-set vertical bars or gill-filaments, connected by numerous tranverse inter-filamentar junctions; the bars are covered with ciliated epithelial cells, and each is strengthened by pairs of small chitinous rods. The whole gill is traversed by blood vessels. Sketch.

2. A small portion of a gill should be preserved, stained, inbedded, and cut into sections (p. 136) passing transversely through the gillfilaments. Compare with Fig. 94, and sketch.

3. Mount in salt-solution a small piece of fresh gill, and also of the mantle, and observe the movements of the cilia.

IV. If your specimen is a female, and contains ova or larvæ in the outer gill, examine some under the microscope. The ova are provided with a canal or micropyle perforating the vitelline membrane, for the entrance of the sperms. Note the form of the larvæ or glochidia (Fig. 97).

$\mathrm{V}$. The nervous system consist of three pairs of small orange-coloured ganglia, with connectives between them.

I. Cut away the left labial palps very carefully and look for the left 
cerebro-pleural gangtion, situated just below the attachment of the mantle lobe close to the mouth and directly beneath the epithelium :it may already have been exposed by removing the mantle-lobe. The two cerebro-pleural ganglia are connected by a commissure above the gullet.

2. On the under surface of the posterior adductor muscle are the two closely applied visceral ganglia, which will be at once seen when the membranous covering of this muscle is dissected off.

3. The pedal ganglia are more difficult to find, as they are more deeply situated, at the junction of the foot and visceral mass. Slit up the foot by a median longitudinal incision, and carefully dissect the two halves apart until the two closely apposed ganglia are seen; then remove the left half of the foot.

4. Carefully dissect out the cerebro-pedal connective, which runs straight from the cerebro-pleural to the pedal ganglion. Then follow out the cerebro-visceral connective from behind forwards :- each extends forwards from the corresponding visceral ganglion along the inner surface of the renal organ, and then passes slightly downwards across the visceral mass to the cerebro-pleural ganglia. Sketch.

VI. The sensory organzs are nol easy to make out. In order to see the osphradizm and tactile-cells, it is necessary to cut microscopic sections. The otocyst may be found by examining under the microscope a portion of the tissue just behind the pedal ganglion; but is is much more easily observed in the small fresh-water bivalve Cyclas, often to be found in ponds : it is only necessary to examine the entire animal in water under a low power after removing the shell in order to see the two globular otocysts, each lined by epithelial cells and containing an otolith, which is in constant, trembling motion.

VII. The alimentary organs consist of a gullet, stomach, paired digestive-gland, and coiled intestine, imbedded in solid tissue in the visceral mass, the hinder part of the intestine or rectum alone being surrounded by a coelomic cavity (pericardium). The anus has already been seen.

I. Dissect away the epiderm to the left side of and above the stomach (if this has not already been done), and note the brown digestive gland in the anterior dorsal part of the visceral mass. Then insert a seeker into the stomach through the mouth, to act as a guide, and slit up the short gullet and stomach. Note the ducts of the digestive gland opening into the stomach.

Pract, Zoot.

D.D 
2. In the same way slit up the whole intestine, either from the stomach backwards or from the rectum forwards, using the seeker as a guide the whole way, and first examining Fig. 93 to see the direction which the coils take. Note the ventral typhlosole in the rectum, beginning at the last coil. Sketch.

VIII. I. The gonads appear very similar in both sexes, and fill ip a large part of the visceral mass between the coils of the intestine : their ducts open at the genital apertures (\$ II., 6).

2. Tease up a bit of the ovary or spermary in salt solution, stain with magenta, and examine.

C. Transverse sections (Fig. 95).

With a sharp scalpel or razor cut a specimen hardened in formaline or spirit into transverse sections about $\frac{1}{4}$ inch thick, passing obliquely through-

I. The anterior part of the pericardial cavity, visceral mass, and foot. Note the relations of the mantle-folds, the gonads, the enteric canal (inclucling the rectum with its typhlosole), the gills and supprabranchial canals, the anterior corta and vena cava, and the kidneys and bladders.

2. The ventricle, auricles, and hinder part of the visceral mass. Note the relations of the mantle-folds, enteric canal, gills, suprabranchial canals, vena cava, and kidney's and bladders.

3. The posterior adductor muscle, hinder part of gills, and rectun. Note the relations of the various parts. Sketch the three sections. 


\section{CHAPTER VIII}

CHARACTERS OF THE PHYLUM VERTEBRATA - AMPHIOXUS:

FROM your study of the frog, you have learnt something about a vertebrate animal, and we will now examine a few more examples of the phylum Vertebrata, which, as we have seen (p. 219), includes several classes, the chief of which are-the Pisces, Amphibia, Reptilia, Aves, and Mammalia. Leaving aside the lampreys and their allies, which present certain peculiarities and are therefore placed in a class by themselves, these all agree with-one another and resemble the frog in the following essential characters.

They all possess :- a vertebral column-or at any rate a notochord (p. 203), which is nearly always replaced by a vertebral column in the adult_-and a skull with upper and lower jaze's; a hollow, dorsal, nervous system, consisting of brain and spinal cord; paired olfactory organs, eyes, and auditory organs, which take on a close connection with the skull; a pharynx, which, at an early stage at any rate, is perforated by a small number (never more than seven) of paired gill-clefts (p. 204); a mouth which is ventral and anterior, and an anus which is ventral and posterior; kidneys which are composed of numerous urinary tubules or nephridia; a chambered heart and red blood-corpuscles; a liver, 
and a hepatic portal system; usually two pairs of limbs, and never more than two pairs; and a series of body-muscles

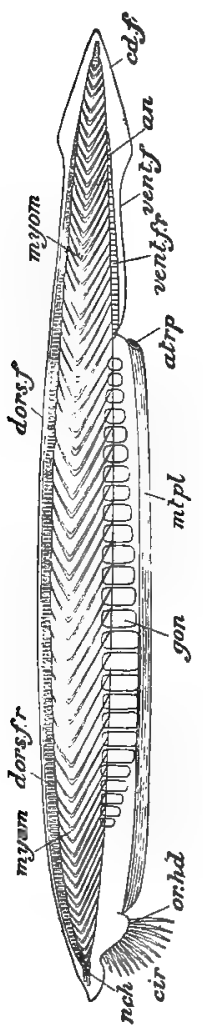
which are divided into segments or myomeres (p. 203), at any rate in early stages.

Now in various parts of the world, occurring only rarely off the English coasts, certain small fishlike animals called lancelets are found, the commonest species of which is known as Amphioxus lanceolatus (Fig. 98). This animal, which possesses a median fin like that of the tadpole (p. 207), is not more than a couple of inches in length, and lives in the sea near the shore, burrowing in the sand; it is especially interesting owing to the fact that it presents certain characteristics indicating a near relationship to the primitive ancestors of Vertebrates. It possesses a notochord, a dorsal hollow nervous system, a pharynx perforated by gill-slits, a hollow outgrowth of the intestine representing a simple liver, with a hepatic portal system, and a series of nephridia. But it differs from all the higher Vertebrates in the following important points.

- The epiderm consists of a single layer of cells. There is no distinct head and no skull; the persistent noto 
chord (Fig. 99, nch) extends to the anterior end of the animal, and there are no paired limbs. A brain (br) can hardly be said to be present, and there are no paired olfactory, optic, or auditory organs. The pharynx is relatively very large, and is perforated by very numerous oblique gill-slits (br. cl), which do not open on the exterior directly, but are surrounded by a chamber, the atrium (Figs. 99, atr, and Ioo A, b) ; this opens externally by a pore (Figs. 98 and 99, atrp), and though differing in its mode of deve. lopment from the branchial chamber of the tadpole ( $p$. 207), has somewhat similar relations. There is no heart

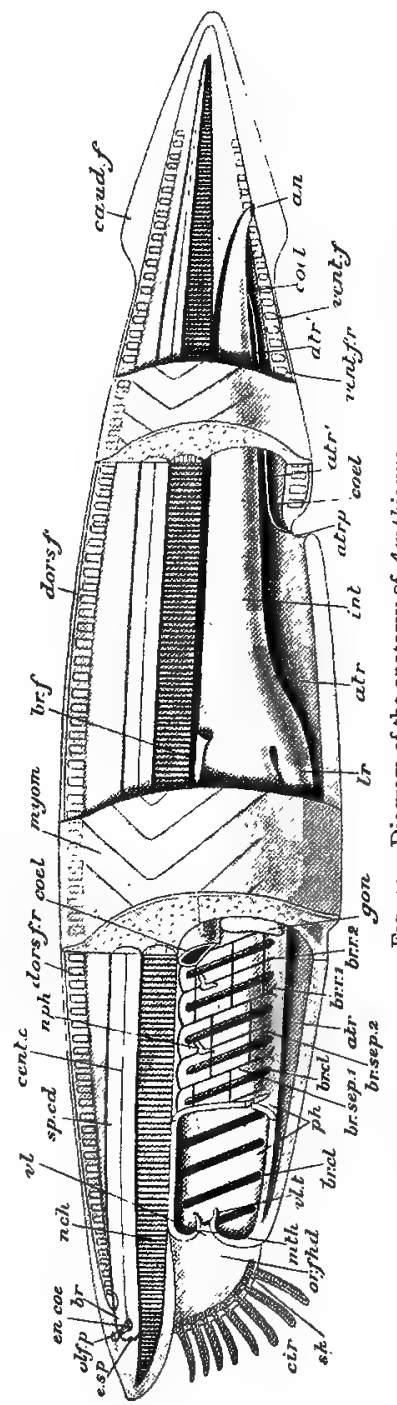

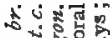
. ‥ 过颌 矛趿

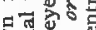
考的的

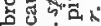
4. रิษ 일 भing o

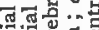
돈 要 证路 . Tno sิ

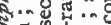

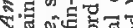

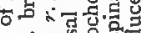
के ..... द वुं

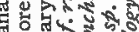
응.

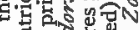

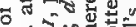

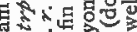

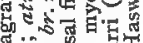

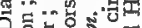

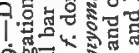

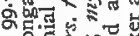
응현뎐 出

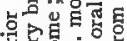
政专和出 ơ 없 ๓ . 航要 हैं.

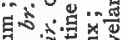
至.. 式苟

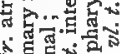
है in - त्व 试密守

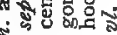


(Fig. IOr), and the colourless blood contains no red corpuscles. The nephridia remain distinct, not being united into a single kidney on either side: they are situated anteriorly, in the neighbourhood of the pharynx (Fig. 99, $n p h$ ); the gonads (Figs. 98 and 99, gon, and Fig. I00 A, g) are metamerically arranged, and have no ducts.

In certain of its characters Amphioxus resembles the members of a group of animals-the Tunicata, commonly known as "sea-squirts," in which the body is enclosed in a "test" or mantle, consisting largely of cellulose (p. 244). These, like Vertebrates, possess a notochord and a dorsal, hollow nervous system in young stages, and in the adult retain numerous pharyngeal gill-slits : they are almost certainly degenerate descendants of primitive animals from which the Vertebrata also arose.

These numerous and marked differences between the lancelet and the higher Vertebrates make it necessary to place Amphioxus in a separate division of the Vertebrata, called-from one important negative character-the Acrania, while all the other Vertebrates, which possess skulls, are included in the division Craniata.

The external appearance of Amphioxus is represented in Fig. 98. In addition to the points already referred to, it will be seen that the mouth is surrounded by a fold, the oral hood $(o r . h d)$, from which a number of tentacles or cirri (cir) are given off ; and that there is a lateral or metapleural fold (mtpl) along either side of the body extending backwards as far as the atrial pore, in addition to the median fin-fold (dors. $f$, $c d . f$, vent. $f$ ) extending round the tail as a caudal fin. In the young animal the gill slits open directly to the exterior, but a median canal is subsequently formed along the ventral side of the body, and as this extends inwards to form the atrium, it gradually surrounds the pharynx at the sides, pushing the coelome before it, so that the latter becomes greatly reduced in this region (Fig. 100 $\mathrm{A}, c 0$ ).

The skeleton is very simple; besides the notochord there 
are rod-like bars of a substance somewhat resembling cartilage supporting the oral hood and cirri (Fig. 99, sk), chitinoid rods (br. r) supporting the gill-bars between the

$A$

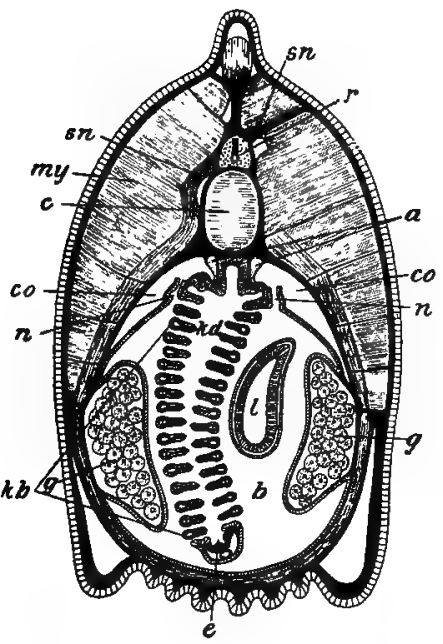

B

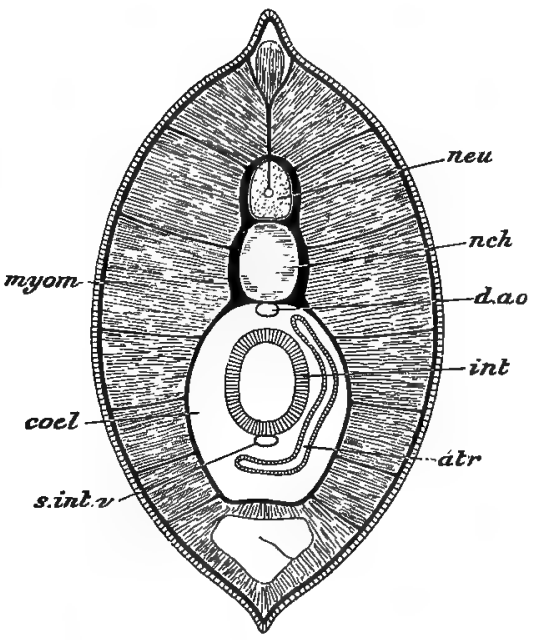

FIG. roo.-Amphioxus lanceolatus. A, transverse section of the pharyngeal region. $a$. dorsal aorta; $b$. atrium; $c$. notochord; $c o$. coelome; $e$. endostyle; $g$. gonad (ovary); kb. branchial septa; $k d$. pharynx; l. liver; $m y$. myomere; $n$. nephridium; $r$. spinal cord : $s \pi$, $s \pi$, dorsal and ventral spinal nerves.

$\mathrm{B}$, transverse section of the intestinal region. atr. atrium; coel. colome; $d, a o$. dorsal aorta ; int. intestine ; myom. myomere; nch. notochord ; neu. spinal cord ; s. int. $v$. sub-intestinal vein. (From Parker and Haswell's Zoology : A, from Hertwig, after Lankester and Boveri; B, partly after Rolph.)

clefts, and short rods of connective-tissue, the fin-rays, in the dorsal and ventral fins (Figs. 98 and 99, dors. f.r, vent. $f . r)$.

In the course of development, each primary gill-slit becomes divided into two by the growth, from above downwards, of a tongue-like process, the secondary gill-bar or septum (Fig. 99, br. r. 2), so that in the adult the slits and 
intervening bars are seen to be arranged in couples, the supporting rods (br, $r, I$ ) of the primary bars (br. sep. I) being forked below. A further complication is produced by the formation of transverse connections, supported by skeletal rods, between the gill-bars. The gill-slits are more numerous than the muscle-segments or myomeres (myom), and owing to their obliquity, a large number of them always appear in a transverse section (Fig. Ioo, A).

On the ventral wall of the pharynx is a longitudinal groove, the endostyle (Fig. Ioo A, e), lined by ciliated and glandular cells; and a somewhat similar groove, the epipharyngeal groove, extends along the dorsal aspect of the pharynx: these serve as channels along which the particles of food pass into the intestine. The minute mouth (Fig. 99, $m t h$ ) leads from the cavity of the oral hood into the pharynx, and is surrounded by a membrane, the velum $(v l)$, produced at its edge into a number of tentacles $(v \%$. $t)$.

The intestine is straight (Fig. 99, int) and the hepatic cacum, or rudimentary lizer, arises from its anterior end on the ventral side, and extends forwards to the right of the pharynx (Fig. 99, lr and Fig. 100, l).

Although no heart is present, the blood-vessels present some similarities to those of the Craniata. Their arrangement may be seen from the accompanying diagram (Fig. Ior), which should be compared later on with Fig. I Io. The nephridial tubes (Fig. $99 n p h$ ) are situated above the pharynx on either side and open on the one hand into the reduced colome in this region, and on the other into the atrium (Fig. I00, $n$ ).

The central canal of the spinal cord widens out in front (Fig. 99, br) to form a small cerebral zentricle (en. coe) in the front wall of which is an unpaired pigment spot, representing a simple kind_of eye $(e . s p)$, and at its anterior 
end is a ciliated depression usually known as the olfactory pit (olf. $p$ ). There is no auditory organ. The dorsal and ventral nerves (Fig. Ioo $A, s n$ ) remain separate, and do not unite to form a nerve-trunk (compare p. $\mathrm{I}_{3}$ ).

The sexes are distinct, and the metamerically arranged gonads (Fig. 98-roo, gon. g) are situated in pouches, the cavities of which represent part of the coelome and which project into the atrium on either side of the pharynx. When

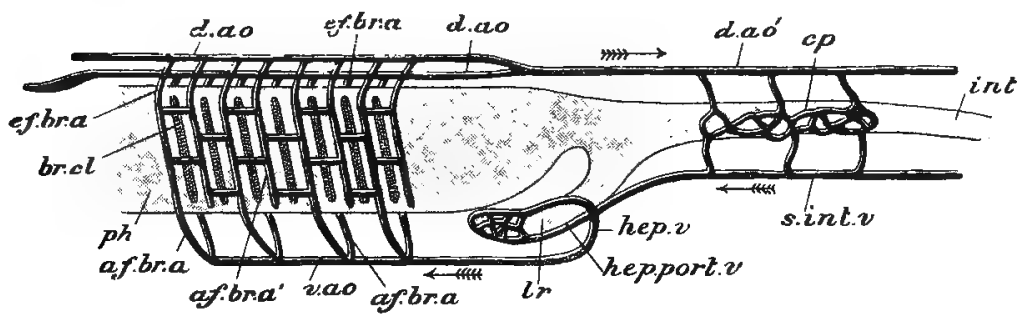

FIG. ror.-Diagram of the vascular system of Amphioxus.

af. br. a. afferent branchial artcries; $b r . c l$. gill-slits; $c p$. interstinal capillaries; d. ao. paired dorsal aorta; $d$. a $o^{\prime}$. median dorsal aorta; ef. br. a. efferent branchial arteries; hep. port. $z$. hepatic portal vein; hep.v. hepatic vein; int. intestine; lr. liver; ph. pharynx: s. int. $v$. sub-intestinal vein; $v$. ao. ventra aorta. (From Parker and Haswell's Zoology.)

ripe, the eggs and sperms are shed into the atrium and find their way out through the atriopore.

The oosperm undergoes segmentation, and gives rise to a simple larva, which gradually develops into the adult form. The embryology of Amphioxus, which is very instructive, will be referred to in greater detail in Chapter XI. 


\section{PRACTICAL DIRECTIONS.}

AMPHIOXUS.

\section{A. External Characters.}

Examine an entire specimen and note:-I, the form of the body; $z$, the continuous median fins-dorsal, caudal, and ventral; 3 , the paired lateral fin or metapleure, extending along the body anterior to the ventral fin; 4, the oral hood, anterior and ventral, with its cirri; 5, the anus, a short distance from the posterior end, just on the left side of the caudal fin; 6, the median and ventral atriopore, at the junction of the lateral and ventral fins; 7 , the myomeres.

\section{B. Anatomy.}

I. Place a young specimen for a short time in absolute alcohol, and then transfer to oil of cloves. If the specimen is a very small one, it may with advantage be slightly stained first. Then transfer to a hollow slide or, if you use an ordinary slide, support the cover-glass on two small pieces of wood of the thickness of the specimen, and mount. The preparation may be examined in oil of cloves, but it is better to make a permanent preparation and replace the cloves by thick balsam. Examine, and note in addition to the above points :-

I. The notochord, extending from the anterior to the posterior end or the body, rather nearer the dorsal than the ventral side.

2. The neural canal enclosing the spinal cord, lying just above the notochord, and having pigment in its walls.

3. The oral skeleton, consisting of a segmented basal bar along the sides of the oral hood, with a rod passing down each cirrus.

4. The longitudinal series of connective tissue compartments, which are filled with gelatinous substance, forming the fin-rays.

5. The elastic, horny rods supporting the gill-bars, and the arrangement of the gill-slits in pairs:-the primary gill-bars separating the successive pairs, the rods supporting them being forked ventrally; and the secondary gill-bars between the members of a pair, the rods of these being unsplit ventrally. Note also the horizontal bars connecting the primary and secondary gill-bars and making the walls of the pharynx appear like a meshwork.

6. The cavity of the oral hood, bounded by lateral folds, the muscular velum between it and the pharynx, and the minute mouth.

7. The intestine, running straight from the hinder end of the pharynx 
to the anus, and giving off near its anterior end the hepatic cocum or liver, extending forward on the right side of the pharynx.

8. The myomeres and the internituscular sepia between them, arranged like a series of $\mathrm{V}^{\prime} \mathrm{s}$, the apices of which point forwards. The muscular fibres run longitudinally from septum to septum.

9. The gonads (ovaries or spermaries), arranged in a single row on either side of and rather further back than the pharynx, extending as far as the atriopore. Note in the female the large ova, which, when ripe, cause a great distension of the body; and in the male the minute sperm-cells, the structure of which cannot be made out in entire specimens. Sketch.

II. Cut a specimen into short pieces, about an eighth of an inch in length, and select portions from- $a$, between the hinder end of the atrium and the anis (Fig. IOo, B) or through the latter; $b$, just in front of or through the atriopore; $c$, through the anterior part of the pharynx; and $d$, through the posterior part of the pharynx (Fig. 100, A.). Stain and imbed these, and prepare a few sections from each (p. 136). Examine in the order given above, first with the low, and then with the high power. Sketch a section from each region.

u. I. The oval form of the section, the median dorsal and ventral fins, and the integument (columnar epiderm and thin derm).

2. The myonzeres cut across in various planes, and appearing as squarish masses separated by the septa. The muscle-fibres are mostly striated.

3. The central notochord, oval in transverse section, with transverse wavy lines indicating the boundaries of the vacuolated notochordal cells. It is surrounded by a connective tissue sheath, continuous with the connective tissue investment of the neural canal above, and with the intermuscular septa at the sides: the latter pass into the derm peripherally.

4. The spinal cord, lying in the neural canal. It is ovoid in transverse section, and has a dorsal fissure (see p. I 55) extending downwards nearly to the central canal, which is nearer the ventral than the dorsal surface. See if your section happens to pass through a dorsal or a ventral nerve. The dorsal nerves arise by large single roots from about the middle of the sides of the cord, while the ventral nerves have numerous fine roots, and arise from the ventro-lateral angles of the cord. The dorsal and ventral nerves are not in the same transverse lane (compare p. I63), but have ạn alternatịng arrangement. 
5. The gelatinous connective tissue forming the fin-rays.

6. The intestine, with its single layer of long epithelial cells. If the section passes through the anus, the latter will be seen opening on the left side of the ventral fin.

7. The calome, surrounding the intestine, and also enclosing the subintestinal or portal veins (Fig. IOI).

8. The dorsal aorta, just below the notochord.

b. Go over $2,3,4,5,6$, and 8 again-all much as in $\alpha$. Note-

I. The dorsal and lateral fins (metapleural folds), ventral body-wall, small metapleural lymph-canals, and atriopore, if present in your section.

2. The calome, a narrow space round the intestine, in which three or four subintestinal (portal) weins ean be seen.

3. The atrial cavity just outside the coelome, and separated from it by the atrial membrane. If the section passes through the atriopore, it will be seen in the mid-rentral line, putting the atrial cavity in communication with the exterior.

،. Note-I. The triangular form of the body in transverse section; the dorsal fin, the ventral surface, with its folded integument and thin transverse ventral muscles; the metapleural folds and their contained large canals. Then go over a. 2, 3, 4, and 5 again, which are all much as in $a$ and $b$.

2. The pharynx, lying just below the notochord, and extending nearly to the ventral body walls. Note- $(a)$ the deep, dorsal epibranchial groove; (b) the ventral endostyle projecting into the pharyngeal cavity, and enclosing a colomic canal; (c) the gill-bars, a great number of which will be cut through obliquely, longer primary alternating with smaller secondary ones. Note the skeletal rods near their outer surfaces, and, in the primary bars, the small calomic spaces on the outer sides of the rods; $(d)$ the dorsal suspensory folds of the pharynx, which enclose the two dorsal calonic canals.

3. The atrial cavity, surrounding the pharynx except at the middorsal line where the pharynx is attached to the sheath of the notochord, and communicating with the cavity of the pharynx through the gill-slits. It is enclosed by the two atrial folds, which are united in the mid-ventral line; the atrial epithelium is often much folded ventrally.

4. The single ventral aorta, in the endostylar colomic canal, and the paired dorsal aorta, on either side of the epibranchial groove.

d. Note again- a. $2,3,4,5 ; c, \mathrm{r}, 3,4$, and also:- 
1. The pharynx, much as in $6 \mathbf{2}$, but laterally compressed. The ondostyle is now converted into a groove.

2. The hepatic coeum, on the right side of the pharynx, and covered by the atrial epithelium. Note the colomic space around it, in which are the portal veins, and the hepatic veins (dorsal side).

3. The gonads (ovaries or spermaries)-large masses on either side 0 . the pharynx, projecting into the atrial cavity and covered by the atrial folds. The spaces around them represent portions of the colome. Note the small sperm-cells in the male, and in the female, the large ova. (The nephridia can only be made out satisfactorily in good sections of very well preserved specimens.) 


\section{CHAPTÉR IX}

CHARACTERS OF THE CLASS PISCES-THE DOGFISH

The class Pisces (see p. 403) includes a number of aquatic Vertebrates which present a considerable amount of differ. ence in form and structure. Excluding, however, one small group, the "Mud-fishes" or Dipnoi-which differ so much from other fishes that they are sometimes placed in a separate class, intermediate between Fishes and Amphibians -the Pisces are distinguished from the Amphibia, as a whole, by certain constant characteristics, of which the following are the chief.

The organs of respiration and locomotion are adapted for life in the water. The former consist, as in the tadpole (p. 207), of a series of vascular processes, the gills, attached to the arches separating the gill-clefts and persisting throughout life; and lungs are never developed. The pectoral and pelvic limbs have the form of paddle-like fins, which, like the median fins (p. 406), are supported by skeletal fin-rays; the median fin is usually subdivided into separate dorsal, ventral, and caudal portions. In addition to the endoskeleton, there is usually an exoskeleton, developed in the derm and consisting of scales; and peculiar integumentary sense-organs, supplied by special nerves not represented in terrestrial Vertebrates, are always present. 
The cerebellum is relatively large; internal nostrils and a tympanic cavity and membrane are not present. There is only one auricle in the heart and no post-caval vein. A urinary bladder developed as an outgrowth of the enteric canal (p. 210), is wanting. There is never such a marked metamorphosis as in the case of the frog.

The two most important sub-classes of the Pisces are the Elasmobranchii and the Teleostomi. The Elasmobranchs are all marine forms, and include the dogfishes, sharks, rays, and skates : their endoskeleton is composed almost entirely of cartilage, like that of the tadpole. The Teleostomi, in which the skeleton is mainly or to a large extent bony, include by far the greater number of fishes-both marine and fresh water forms-such as the Salmon, Cod, Herring, Perch, as well as the Sturgeon and its allies.

- The Dogfishes are small Sharks, and include a number of genera and species. They are all powerful swimmers, and feed voraciously on other fishes, crustaceans, etc.

The commonest British forms are the Rough Hound (Scyllium canicula), the Lesser Spotted Dogfish (S. catulus), the Piked Dogfish (Acanthias vulgaris), and the Smooth Hound (Mustelus vulgaris). The following description, though referring mainly to Scyllium, will apply, in essential respects, to any of these.

External Characters and General Structure. - The dogfish has a spindle-shaped body, ending in front in a bluntly-pointed snout and behind tapering off into an upturned tail. On the ventral surface of the head is the large, transversely elongated mouth (Fig. 108), supported by a pair of jaws which work in a vertical, and not, like those of the crayfish, in a transverse plane : they are, in fact, like those of the frog, portions of the skull, having nothing to 
do with limbs. They are covered with teeth which vary in form in the different species. In front of the mouth, on the ventral surface of the snout, are the paired nostrils, each leading into a cup-like nasal or olfactory sac, and (in Scyllium) connected with the mouth by a groove. The eyes are placed one on each side of the head, above the mouth: they are protected by folds of the skin forming upper and lower eyelids, the latter of which can be closed over the eye. Behind the mouth are five pairs of slit-like apertures arranged in a longitudinal series: these are the gill-clefts or external branchial apertures (p. 403). Just behind each eye is a small aperture, the spiracle: like the gill-clefts, it communicates with the pharynx, and it is found by development to be actually the functionless first gill-cleft.

On the ventral surface of the body, about half-way between its two ends, is the vent or anus, leading into the cloaca (p. 23), and on either side of it a small pouch into which opens a minute hole, the abdominal pore, communicating with the cœlome, which is therefore not a completely closed cavity, as in the frog. From the end of the snout to the last gill-cleft is considered as the head of the fish; from the last gill-cleft to the anus as the trunk; and the rest as the tail.

A number of symmetrically arranged, minute apertures on the skin of the head, particularly numerous on the snout, lead into a series of tubes known as sensory canals, which are situated beneath the skin in this region; and a single tube, known as the lateral line canal, the position of which is indicated by a very faint longitudinal line, extends along either side of the body and tail. The whole apparatus constitutes an important, but imperfectly understood, in- 
tegumentary sense-organ : it is represented in the tadpole, but disappears at metamorphosis.

Springing from the body are a number of flattened folds, called the fins, divisible into median and pxired. The median folds are not continuous, as in the tadpole and Amphioxus, but are subdivided into several distinct parts, viz., two dorsal fins along, the middle line of the back, a caudal fin lying along the ventral edge of the upturned tail, and a ventral fin behind the anus. The paired folds are the pectoral fins, situated one on each side of the trunk just behind the last gill-cleft, and the pelvic fins, one on each side of the vent: these correspond to the pectoral and pelvic limbs of the frog. In the male there is connected with the inner border of each pelvic fin a grooved, rod-like structure known as the clasper, which serves as a copulatory organ.

It is very possible that the paired fins, like the median fins, are specialised portions of a primarily continuous fin-fold-extending along either side of the body like the lateral or metapleural folds of Amphioxus.

The fish swims by vigorous strokes of the tail: the pectoral fins are used chiefly for steering, and the dorsal and ventral fins serve, like the keel of a boat, to maintain equilibrium.

The skin or external part of the body-wall consists, as usual, of two layers, an outer layer of deric epithelium or epiderm (Fig. 102, Der. Epthm), formed, like that of the frog, of several layers of cells; and an inner layer of connective tissue, the derm. In the derm are innumerable close-set, calcified bodies, each consisting of a little irregular plate of bone produced into a short spine-composed, like the teeth present in the frog and most Vertebrates, of a calcified tissue harder than bone known as dentine capped with Pract. Zool. 
a still harder tissue called enamel-which projects through the epiderm and gives a rough, sandpaper-like character to the skin. These placoid scales or dermal teeth together constitute the exoskeleton of the dogfish: it is a discontinuous, mainly dermal, exoskeleton (p. 429), and not a continuous cuticular one like that of the crayfish.

Beneath the derm is the muscular layer, which, as in Amphioxus and in the tail of the tadpole, is metamerically segmented. The muscles are divided into myomeres, following one another from before backwards, and having a zigzag disposition. The fibres composing them are longitudinal, and are inserted at either end into fibrous partitions or myocommas which separate the myomeres from one another. ${ }^{\mathrm{I}}$ The muscular layer is of great thickness, especially its dorsal portion. The fibres of all the body muscles are, as in the frog and Vertebrates generally, of the striped kind.

There is a large colome (Figs. I02 and ro8), which, as in other Vertebrates, is confined to the trunk, both head and tail being, in the adult, acœlomate. 'The cavity is divisible into two parts : a large abdominal cavity, containing most of the viscera, and a small anterior and ventral compartment, the pericardial cavity (Fig. I08, pcd. cav), containing the heart and communicating with the abdominal cavity by a canal which opens on the ventral surface of the gullet. Both are lined by colomic epithelium (Fig. 102, Col. Epthm) underlain by a layer of connective tissue, a strong lining membrane being thus produced, called, as in the frog, peritoneum in the abdominal, pericardium in the pericardial cavity.

Another very characteristic Vertebrate feature is that the

I In the adult frog a segmentation can still be seen in the rectus $\mathrm{m}$ uscle of the abdomen (Fig. I6). 
dorsal body-wall is tunnelled, from end to end, by a median longitudinal neural cavity, in which the central nervous system is contained. The greater part of the cavity is narrow and cylindrical, and contains the spinal cord (Figs. 102 and $108, s p, c d)$ : its anterior or cerebral portion is dilated, and contains the brain.

Skeleton. - Imbedded in the body-wall and extending into the fins are the various parts of the endoskeleton. This characteristic supporting framework is mainly composed, as in the tadpole and in embryos of Vertebrates generally, of cartilage, which may be more or less impregnated with lime salts, so as to have, in part, the appearance of bone, but in most parts differing in structure from true bone and consisting merely of calcified cartilage (p. 46).

The entire skeleton consists of separate pieces of cartilage, calcified or not, and connected with one

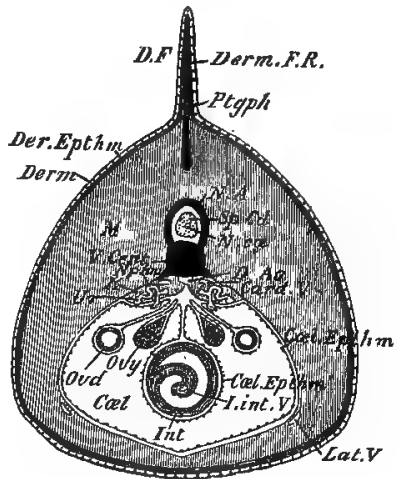

F1G. 102. - Diagrammatic transverse section through the trunk of a female dogfish. The ectoderm is dotted, the endoderm radially striated, the mesoderm evenly shaded, and the colomic epithelium represented by a beaded line.

Card. $V$. cardinal vein ; Col. coelome; Coel. Epthm. parietal, and $\mathrm{Col}$. Epthm'. visceral layer of cœlomic (peritoneal) epithelium; $D$. Ao. dorsal aorta; Derm. derm; Dern. $F$. $R$. dermal fin-ray; $D$. F , dorsal fin $;.$, int. $V$. ventral intra-intestinal vein; $I n t$. intestine; $K$. kidney; Lat. $V$. lateral vein; $M$. myomeres; $N . A$. neural arch; $N$ cos. central canal of spinal cord ; Nph. nephridium; Ot'd. oviduct ; Ovy. ovary; $S p . C d$. spinal cord; $U_{r}$, ureter ; V. Cent, vertebral centrum. (From Parker's Elementary Biology.) another by ligaments (p. 57): as in the frog, it is divisible into skull, vertebral column, and skeleton of the paired fins, with their arches or girdles; in connection with the skull are certain cartilaginous visceral arches, forming the upper 
and lower jaws and supporting the gills; and there are also skeletal parts in the median fins.

The cranium or brain-case (Fig. $103, \mathrm{Cr}$ ) is an irregular, cartilaginous box containing a spacious cavity for the brain, very similar to the chondrocranium of the frog (p. 43). It is produced into two pairs of outstanding projections: a posterior pair, called the auditory capsules (aud. $c p$ ), for the lodgment of the organs of hearing, ridge-like projections on which indicate the position of the semicircular canals (p. I87); and, in front of the brain-cavity, an anterior pair, the olfactory capsules (olf. cp), for the organs of smell, open below, and separated from one another by a septum. Between the olfactory and auditory capsules, on either side, the cranium is hollowed out into an orbit (or), bounded by a supra-orbital and a sub-orbital ridge, for the reception of the eye. In front the brain-case is produced into three cartilaginous rods forming the rostrum ( $r$ ) and supporting the snout. On its posterior face is the foramen magnum (p. 40), on each side of which is an oval condyle for articulation with the first vertebra. On the roof of the skull, between and behind the olfactory capsules, there is a fontaneile ( $\mathrm{p} .43$ ), closed over by connective tissue only; and between the two auditory capsules is a depression into which open the two endolymphatic ducts of the ears (p. 187). The nerve apertures will be referred to at a later stage (p. 445).

In the human and other higher vertebrate skulls the upper jaw, as we have seen to be the case in the frog (Fig. 9 ), is firmly united to the cranium, and the lower alone is free. But in the dogfish both jaws $(u p . j, l . j)$ are connected with the cranium by ligaments $\left(l g, l g^{\prime}\right)$ only, and each consists of strong, paired (right and left) moieties, united with one another by fibrous tissue. The upper jaw, which corre- 


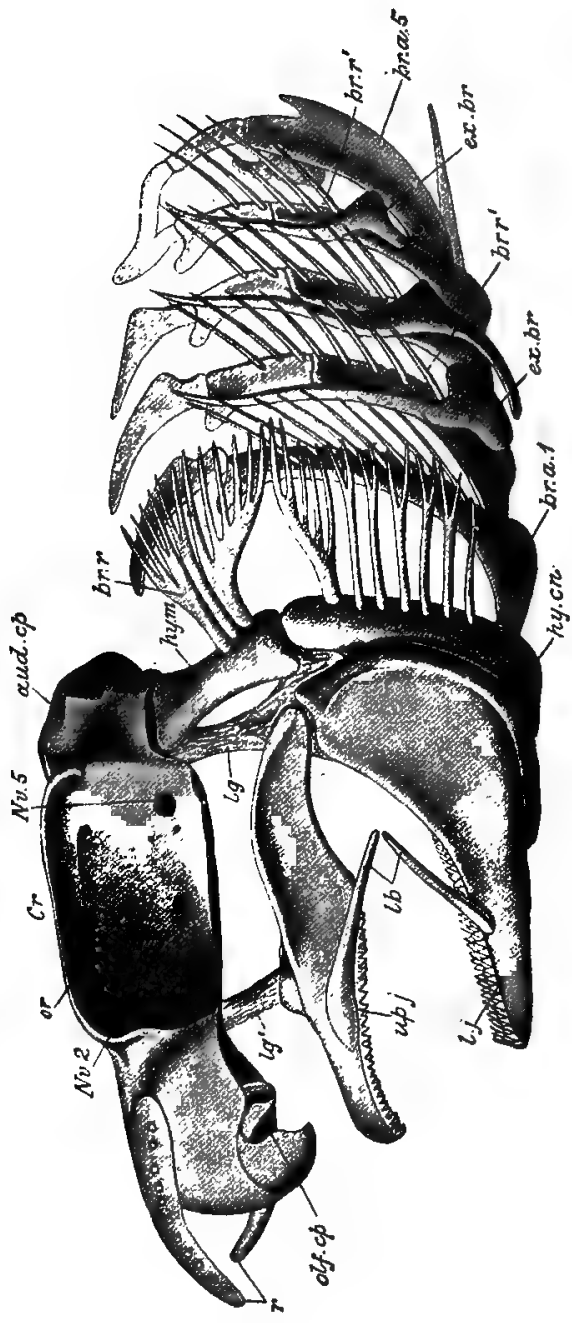

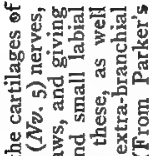

可高

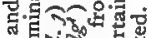

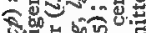

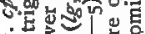

की

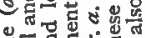

공

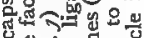

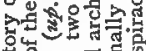

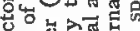

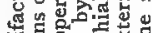

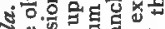

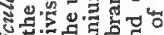

证娄

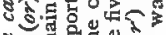

늘

空它它。品

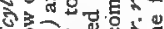

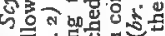

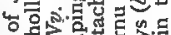

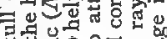

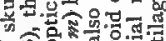

फ 8

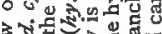
영 है.

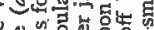

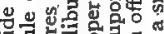

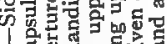

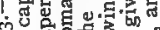
ติ

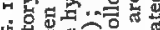

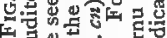

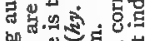
bo

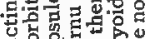

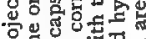

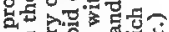

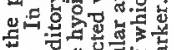

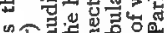
在

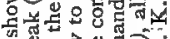

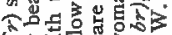

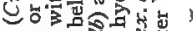

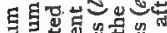

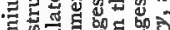

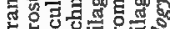
0

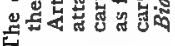


sponds to the palatoquadrate cartilage of the frog (p. 44), presents at its posterior end a rounded surface against which fits a corresponding concavity on the lower jaw, so that a free articulation is produced, the lower jaw, or Meckel's cartilage (p. 44) working up and down in the vertical plane.

The upper and lower jaws of either side correspond to the first of a series of seven pairs of visceral arches, and are therefore often spoken of as the mandibular arch. The remaining six pairs have the form of cartilaginous half-hoops, lying in the walls of the pharynx, and united with one another below so as to form a basket-like apparatus supporting the gills. The second of these arches is distinguished as the hyoid, and is situated immediately behind the jaws. It consists of two parts: a strong, rod-like hyomandibular (Fig. I03, hy. m), which articulates above with the auditory capsule and is connected below by fibrous tissue with the jaws, thus helping to suspend them to the cranium and serving as a suspensorium (p. 4o); and a hyoid cornu or horn, which curves forwards inside the lower jaw, and is connected with its fellow of the opposite side by a median basi-hyal plate which supports the tongue.

The remaining five arches (br. $a . \mathrm{I}-b r . a .5)$ are called the branchial arches. Each is formed of several separate pieces, united by fibrous tissue so as to render possible the distension of the throat during swallowing: the fifth is connected below with its fellow by a large median basibranchial plate, which supports the roof of the pericardial cavity. Both they and the hyoid give attachment to delicate cartilaginous branchial rays $(b r, r, b r . r$, Fig. $109, r)$ which support the gills.

In the frog-tadpole the gills are similarly supported by cartilaginous arches, which become greally reduced and modified at meta- 
morphosis: the tongue skeleton of the adult (p. 44) represents the hyoid and first branchial arch.

In addition to the parts of the skull clescribed above there are certain small cartilages of minor importance in relation with the nostrils, spiracle, mouth, and outer sides of the branchial arches $(e . g ., 16$ and ex. br in Fig. I03).

The vertebral column has the general character of a jointed tube surrounding the spinal portion of the neural
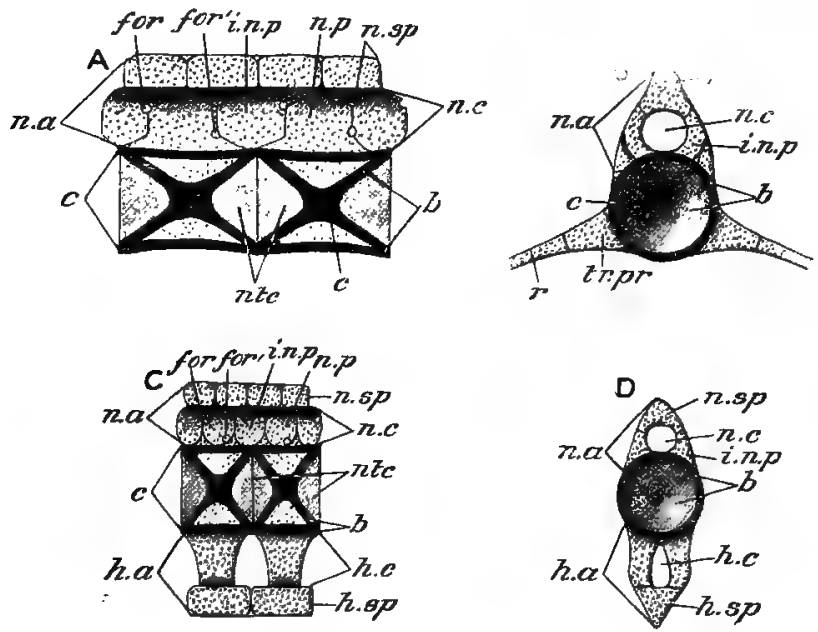

FIG. I04,-Vertebræ of Scyllinum canicula.

$\mathbf{A}$ and $\mathbf{B}$ from the trunk, $\mathbf{C}$ and $\mathbf{D}$ from the middle of the tril; $\mathbf{A}$ and $\mathbf{C}$ two vertebras in longitudinal section; $B$ and $D$ single vertebra viewed from one end. b. bony portion of centrum; $c$. centrum; for. foramen for dorsal, and for'. for ventral root of spinal nerve; $h . a$. hæmal arch; $h . c$. hæmal canal; $h$. sp. hæemal spine; $i . n$. $p$. interneural plate; $n$. $a$. neural arch; $n . c$. neural canal; $n$. $p$. neural plate; $n . s p$. neural spine; $n t c$. iutervertebral substance (remains of notochord) $t r$. $p r$. transverse process; $r$. proximal portion of rib.

canal. I.ying beneath this cavity, i.e., between it and the colome, is a longitudinal row of biconcave or amphicolous discs, the vertebral centra (Fig. I04, $c$; Fig. I08, $c n$ ) : they are formed of cartilage, but have their anterior and posterior 
faces converted into bone. The biconcave intervals between them are filled with a soft intervertebral substance (Fig. I04 ntc), which is also present between the first vertebra and the skull and which represents part of the embryonic notochord (see pp. 203 and 404). The centra are united by ligament, so that the whole vertebral chain is very flexible.

In the frog it will be remembered that the completely ossified centra are procalous, and are articulated with one another (pp. $3^{6}$ and 57).

Connected with the dorsal aspect of the series of centra is a cartilaginous tunnel, consisting of the neural arches (Figs. 104 and 108, n. a) enclosing the spinal cord: it is divided into segments, corresponding with, but usually twice as numerous as the centra owing to the presence of intercalary pieces. Arising from each centrum on either side is a neural process, which in the adult fuses with a neural plate (Fig. 104,n.p) and is perforated posteriorly for the exit of the ventral root of a spinal nerve ( $f \circ r^{\prime}$ ) ; and fitting between two consecutive neural plates is an intercalary piece, the interneural plate (i.n.p), perforated by an aperture for the dorsal root of a spinal nerve (for). The arch is completed above by the neural spines ( $n . s p$ ), which fit. in between the neural and interneural plates respectively, and are thus, like the lateral elements of the arch, twice as numerous as the centra. The first vertebra has facets for articulation with the condyles of the skull.

In the anterior part of the vertebral column the centra give off paired outstanding transverse processes, (Fig. 104 B, tr. $p r)$, to the end of each of which is articulated a short, cartilaginous rod, the rib ( $r)$. Further back the transverse processes are directed downwards, instead of outwards, and in the whole caudal region they unite 
below, forming hamal arches and spines (Fig. то4 D, $h$. $a$, $h$. sp., and Fig. Io8, $h . a$ ), which together constitute a kind of inverted tunnel in which lie the artery and vein of the tail. In the region of the caudal fin the hæmal spines are elongated and act as supports for the fin. A centrum, together with the corresponding neural arch and transverse processes, or hæmal arch, form a vertebra or single segment of the vertebral column.

In the frog we have seen that there are no independent ribs, and that the caudal vertebra are represented by a single bone, the urostyle (p. 39).

It should be noticed that in the vertebral column we have another instance of the metameric segmentation of the vertebrate body. The vertebræ do not, however, correspond with the myomeres, but alternate with them. The myocommas are attached to the middle of the vertebræ, so that each myomere acts upon two vertebræ and thus produces the lateral flexion of the body.

In the embryo dogfish, as in the tadpole, before the development of the vertebral column, an unsegmented, cellular rod with an elastic sheath, the notochord, resembling that of Amphioxus (p. 404), lies beneath the neural cavity in the position occupied in the adult by the line of centra, by the development of which it is largely replaced. Cartilages appear round the notochord, which on the one hand give rise to the arches, and on the other constrict the notochord at regular intervals, so as to replace it completely in those regions which will form the middle parts of the vertebral bodies, leaving the notochordal cells in the biconvex spaces between the centra (Fig. I04, ntc). Thus much of the notochord persists as the soft intervertebral substance.

The skeleton of the median fins consists of a series of parallel cartilaginous rods, the fin-rays or pterygiophores, the proximal ends of which are more or less fused together to form basal cartilages or busalia. The free edges of the fins 
are supported by a double series of delicate horn-like fibres, the dermal fin-rays.

The paired fins are also supported proximally by cartilaginous pterygiophores, fused together at their bases to form basal cartilages which articulate with the corresponding. arch or girdle, and distally by horny, dermal fin-rays. The pelvic arch (Fig. I05, BP) is a transverse bar of cartilage situated just in front of the vent, and representing the pubic and ischiatic portions of the girdle in the frog, an iliac region, extending dorsally, and coming into connection

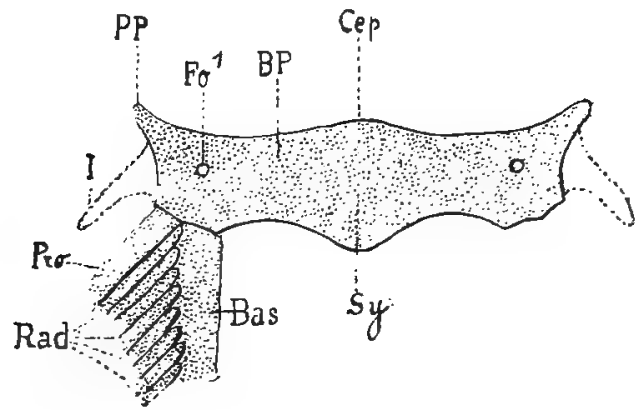

FIG. 105.-Diagram of the Elasmobranch pelvic arch $(B P, C e p, P P, S y)$ and fin.

Bas. basal cartilage; $F o^{\prime}$. nerve foramen; $I$. iliac process; $P$ ro. anterior ray articulating directly with the arch; $R$ rd. the remaining radial cartilages. (From Wiedersheim.)

with the vertebral column, being hardly represented. On its posterior edge are articular facets for the pelvic fins, each of which has a single very large basal cartilage (Bas), but one or two of the anterior rays $\left(P_{70}\right)$ may articulate separately with the arch. In the male, the skeleton of the clasper (p. 4I7) is comnected with the distal end of the basal cartilage.

The shoulder-girdle (Fig. 106) is a strong inverted arch of cartilage situated just behind the last branchial 
arch. On each side of its outer surface it presents three articular facets for the pectoral fin; the presence of these allows of the division of each side of the arch into a narrow, pointed, dorsal portion corresponding to the scapular region of the frog (pct. $g$ ), and a broader

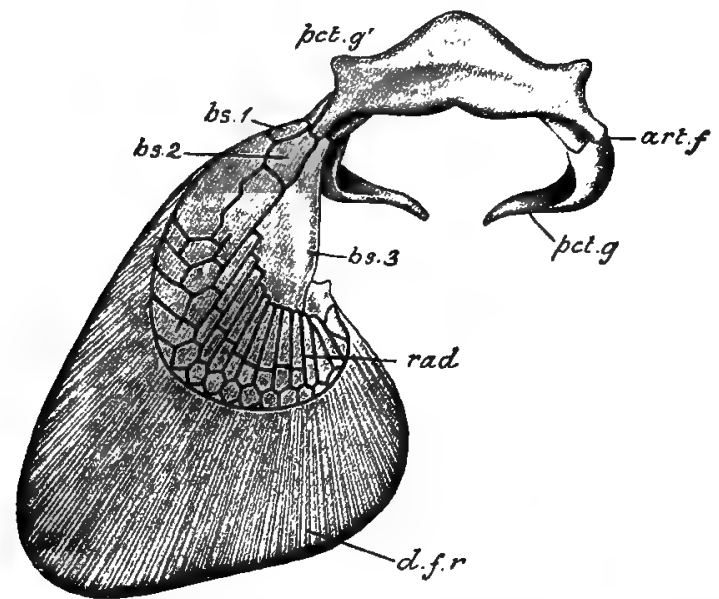

FIG ro6.-Ventral view of pectoral arch os Scyllium with right pectoral fin. The pectoral arch is divisible into dorsal or scapular $(p c t . g)$ and ventral or coracoid $\left(p c t . g^{\prime}\right)$ portions separated by the articular facets $(a r t . f)$ for the fin. The pectoral fin is formed of three basal cartilages $(b s .1-3)$ and numerous radials (rad); its free edge is supported by dermal rays $(d . f . r)$. (Modified from Marshall and Hurst.)

ventral portion, answering to the coracoid ( $\left.p c t . g^{\prime}\right)$ united in the middle line with its fellow of the opposite side : there is no sternum. The pectoral fin is formed of pterygiophores ( $\mathrm{rad})$, fused proximally to form basals which are three in number (bs. r. - -3), the third, like the main basal of the pelvic fin, being the largest and supporting the greater number of the cartilaginous rays, which give rise distally to a series of polygonal plates. 
It will be noticed that while the skeleton of the crayfish is a series of articulated tubes, with the muscles inside them, that of the dogfish and frog is a series of articulated rods with the muscles outside. The joints, formed by two rods applied at their ends and bound together by ligament, are not confined to movement in one plane, like the hingejoints of the crayfish, but are capable of more or less rotalory movement.

Digestive organs. - The mouth, as we have seen, is a transverse aperture bounded by the upper and lower jaws.

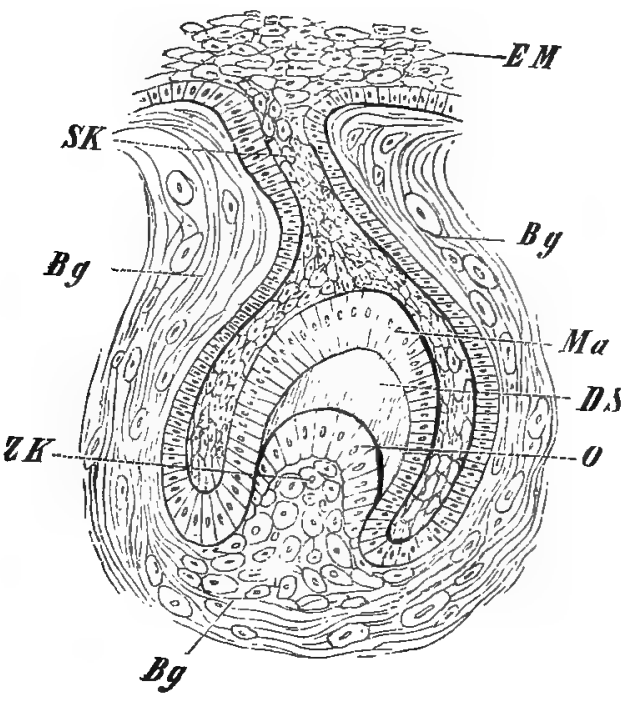

FIG. xo7,-Diagram of the development of a tooth.

$B g, B g$. mesoderm; $D S$. dentine ; $E M$. epithelium of mouth ; $M \pi$. epithelium of enamel organ ; $O$. odontoblasts ; $S K$. dental lamina; $Z K$. dental papilla. (From Wiedersheim's Comparative Anatony.)

In the mucous membrane covering the jaws are imbedded large numbers of teeth-conical, calcified bodies, with enamelled tips, arranged in transverse rows. They are to be looked upon as special developments of the 
placoid scales or dermal teeth enlarged for the purpose of seizing prey, and are continually renewed on the inner sides of the jaws as they are worn away on the outer sides.

The teeth, in Vertebrates generally, are developed in the following manner. The ectodermal epithelium of the mouth (stomodicum, p. 204) -or in the case of the dermal teeth of the dogfish that covering the body generally-grows inwards to form a ridge or dental lamina (Fig. 107, $S K$ ) which projects into the underlying mesodermal connective tissue and becomes enlarged distally to form a bell-shaped cnamel-organ, into the base of which a mesodermal dental papilla $(Z K)$ extends : the superficial part of this papilla forms a layer of cells known as odontoblasts $(O)$. The dentine $(D S)$ is formed, in successive layers, from the odontoblasts, and gradually accumulates between them and the epithelium lining the interior of the enamel organ $(M a)$, which gives rise, also in successive layers, to the enamel, or hardest part of the tooth. Around the base of the papilla more or less bony matter-the cement-is formed. It will thus be seen that while the teeth are mainly mesodermal structures, a part of them-the enamel-is ectodermal in origin.

The mouth leads into an oral cavity, on the floor of which is a rudimentary tongue (Fig. 108, $t n g$ ) capable of very little movement, and which passes insensibly into the throat or pharynx $(p h)$, distinguished by having its walls perforated by five pairs of slits, the internal branchial apertures (i. br. a) as well as by the inner opening of the spiracle $(s p)$. The pharynx is continued by a short gullet (gul) into a capacious, U-shaped stomach, consisting of a wide cardiac division ( $c d . s t$ ) and a narrow pyloric division $(p y l . s t)$. The pyloric division communicates by a narrow valvular aperture with the intestine (int), a wide, nearly straight tube having its lining membrane produced into a spiral fold, the spiral valve $(s p . v l)$, which practically converts the intestine into a very long, closely-coiled tube, and greatly increases the absorbent surface. Finally the intestine opens into a large chamber, the cloaca $(c l)$, which communicates with the exterior by the vent. 


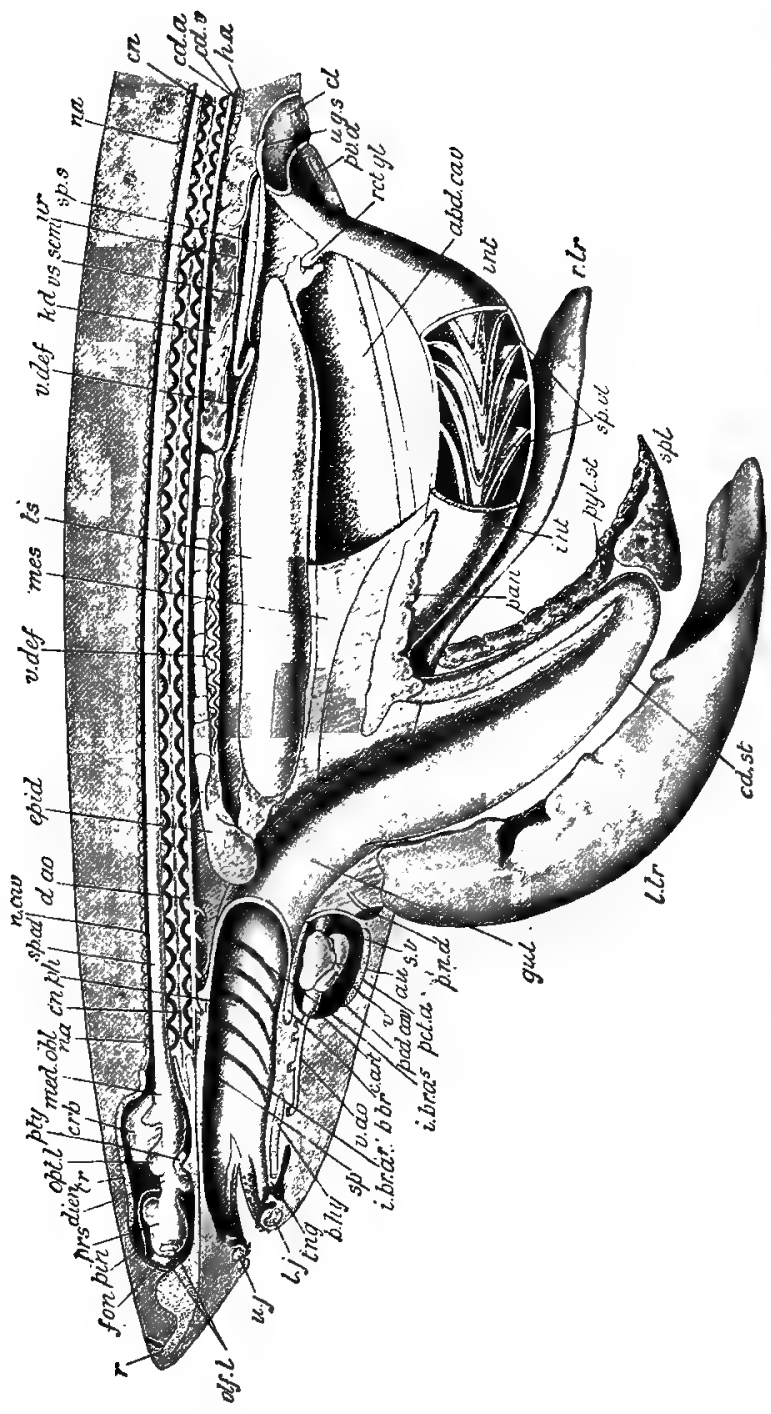

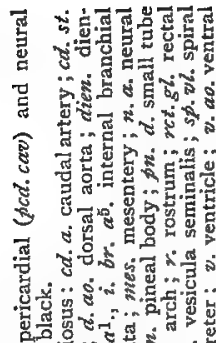
(1.)

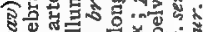
U u

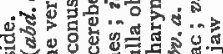
फ

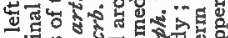
" 可 5 तो

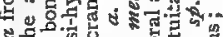
s 5 w w in

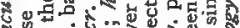

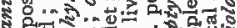

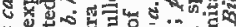
son 0 on $x$. \%

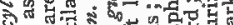
$\checkmark$ 은 o 0 . .

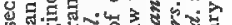
品

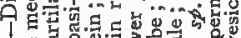

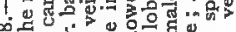

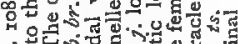

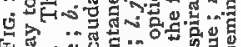

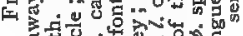
of

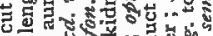

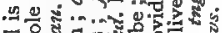

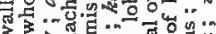

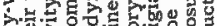

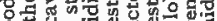

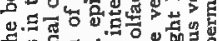
5. 4.50 \&

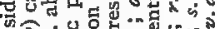
Q

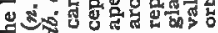


From the gullet backwards the enteric canal is contained in the abdominal division of the colome, to the dorsal wall of which it is suspended by a median mesentery (Figs. IO2, and 108, mes). The greater part of the canal is developed from the enteron of the embryo, and is consequently lined by endoderm ; the oral cavity is formed from the stomodæum, and the cloaca from the proctodæum (p. 204). Outside the enteric epithelium are connective tissue and muscular layers, the latter formed of unstriped fibres : it is generally characteristic of vertebrates that the voluntary muscles are striped, the involuntary unstriped (compare pp. II I and I 2 ).

The immense liver, divided into two lobes $(l . l r, r . l r)$, is situated below the stomach along the whole length of the abdomen, to the wall of which it is attached by a fold of peritoneum. It discharges its secretion, the bile, into the anterior end of the intestine by a tube, the bile-duct, which gives off a blind offshoot terminating in a gall-bladder (p. 69).

-The pancreas (pan) lies against the anterior end of the intestine, with which it communicates independently by the pancreatic duct. Opening into the hinder part of the intestine or rectum, is a small finger-like rectal gland $(r c t . g l)$, the function of which is uncertain. In addition to these there are, as in all Vertebrates, minute tubular gastric glands sunk in the mucous membrane of the stomach (p. I 3 I).

The spleen $(s p l)$ is an irregular, dark-red, gland-like body, of considerable size, attached by peritoneum to the stomach (compare pp. 23 and 98).

Other so-called "ductless glands" - which are also represented in the frog, and the functions of which are not thoroughly understoodare the thyroid in the throat and the thymus in connection with the dorsal ends of the branchial arches; there are also suprarenal and interrenal bodies in relation with the kidneys (compare p. 145). 
Respiratory Organs. The gills consist of five pairs of pouches, 'each opening by one of the internal branchial apertures (Fig. I08, i.br.a) into the pharynx, and by one of the external branchial apertures on the exterior. The walls of the pouches, or inter-branchial septa, are supported

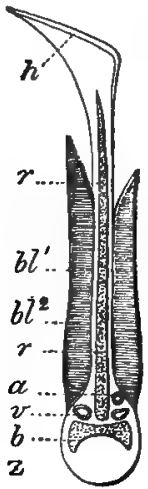

Fig. rog.-Transverse section through a gill of an Elasmobranch.

a. afferent branchial artery; $b$. branchial arch; $b l$. anterior, and $b z^{2}$. posterior hemibranch; $h$. septum ; $r$. branchial ray; $z$. efferent branchial artery. (From R. Hertwig's Zoology.) by the visceral arches and branchial rays (Fig. I03, br. $r$ and Iog, $r$ ), and are lined with mucous membrane raised into horizontal ridges, the branchial filaments, which are abundantly supplied with blood-vessels and are the actual organs of respiration. As the fish swims, water enters the mouth and passes by the internal clefts into the branchial pouches, and thence outwards by the external clefts, a constant supply of oxygen being thus ensured. The gill-pouches are developed as offshoots of the pharynx, and the respiratory epithelium is therefore endodermal, not ectodermal, as in the crayfish and mussel (compare also pp. 204 and 207).

As already mentioned, the walls of the pharynx are supported by the cartilaginous visceral arches, which surround it like a series of incomplete hoops, each half-arch being embedded in the inner or pharyngeal side of an interbranchial septum. Thus the visceral arches alternate with the gill-pouches, each being related to the posterior set of filaments of one pouch and theanterior set of the next. An entire gill or holobranch therefore consists of two half-gills or hemibranchs-the sets of branchial filaments belonging to the adjacent sides of two consecutive gill-pouches (Fig. IO9). On the other hand, a gill-pouch is equivalent to the posterior hemibranch of one gill and the anterior hemibranch of its immediate successor. 
The first pouch is situated between the hyoid and the first branchial arch, and the hyoid thus bears a hemibranch only. The first four branchial arches bear each a holobranch, and the fifth is without gillflaments. There is a vestigial hemibranch, or pseudobranch, on the anterior wall of the spiracle.

Now it is known that parts which have become useless tend to disappear more or less completely (e.g. pineal body of the frog-p. I59, and certain of the gills in the crayfish-p. 36r). In some cases, however, such vanishing parts take on new relations with other organs and thus once more become useful in other ways, undergoing a change of function. Thus in higher Vertebrates the spiracle is utilised in connection with the auditory organ, and instead of disappearing entirely, as do the other gill-clefts, it gives rise to the tympanic cavity and Eustachian tube.

Circulatory organs. The heart is situated in the pericardial cavity or anterior compartment of the cœlome, and is a large muscular organ composed of four chambers. Posteriorly and dorsally is a small, thin-walled sinus venosus (Fig. 108 and $110, s$. v), opening in front into a single, capacious, thin-walled auricle $(a u)$; two auricles are present only in those Vertebrates which possess lungs. The auricle communicates with a very thick-walled ventricle (v), from which is given off in front a tubular chamber, also with thick muscular walls, the conus arteriosus (c. art). There are valves between the sinus and the auricle, and between the auricle and ventricle, and the conus contains three longitudinal rows of valves : all the valves are arranged so as to allow of free passage of blood from sinus to auricle, auricle to ventricle, and ventricle to conus, but to prevent any flow in the opposite direction.

The conus gives off in front a single blood-vessel (v. ao), having thick elastic walls composed of connective and elastic tissue and unstriped muscle. This vessel, the ventral aorta, passes forwards beneath the gills, and gives off on each side paired lateral branches, the afferent branchial arteries Pract, Zool. 


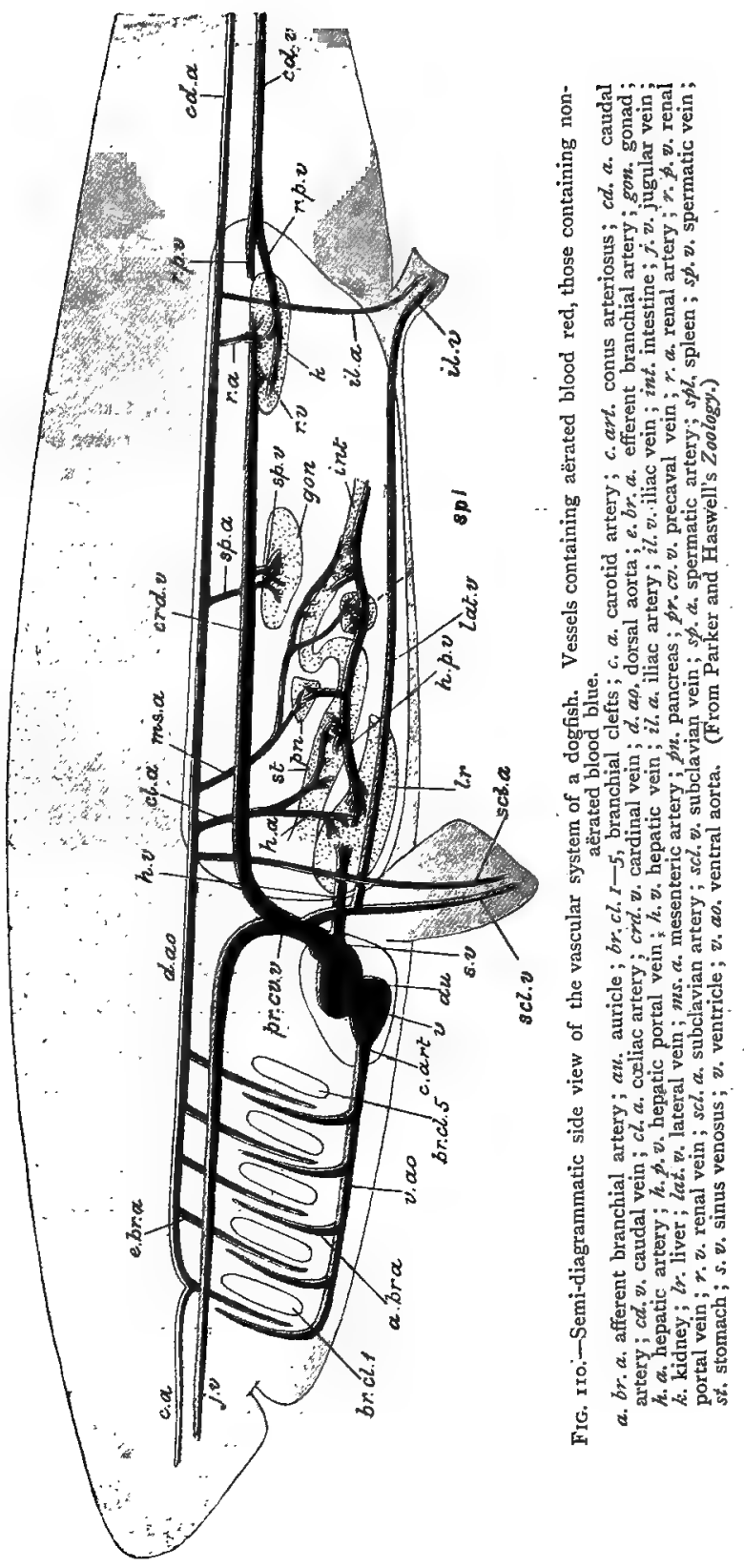


(Fig. I I0,"a.br. a). Each afferent artery passes to the corresponding gill, and there branches out into smaller and smaller arteries, which finally open into a network of delicate capillaries (p. 95), with which the connective tissue of the branchial filaments is permeated. The blood in these respiratory capillaries is therefore brought into close relation with the surrounding water, and as the blood flows through them it exchanges its carbon dioxide for oxygen, obtained from the air dissolved in the water.

From the respiratory capillaries the blood is collected into minute arteries which join into larger and larger trunks, and finally unite into efferent branchial arteries. $(e$. br. a) by which the purified blood is carried from the gills. The efferent arteries of the right and left sides unite in a median longitudinal artery, the dorsal aorta (d.ao), which passes backwards, immediately beneath the vertebral column, to the end of the tail.

From the efferent branchial arteries and the dorsal aorta are given off numerous arteries supplying the whole of the body with blood. The most important of these are paired carotid arteries (c. a) to the head, and subclavians (scl. a) to the pectoral fins ; unpaired splanchnic arteries (cl.a, ms.a), to the enteric canal, liver, pancreas, and spleen; numerous paired renals $(r, a)$ to the kidneys, spermatic $(s p . a)$ or ovarian arteries to the gonads, and a pair of iliacs (il. a) to the pelvic fins. The posterior part of the dorsal aorta, supplying the tail, is contained in the hæmal canal of the caudal vertebræ, and is often spoken of as the caudal artery $(c d . a)$.

The arrangement of the arteries in the tadpole (p. 206) is very similar to that described above, and the diagram (Fig. I Io) would serve almost equally well for a tadpole as for a fish. In the former there are four pairs of afferent and efferent branchial arteries (corresponding to the 
second to the fifth in Fig. IIO) in relation with the corresponding branchial arches and their gills. The afferent and efferent vessels at first communicate with one another through the respiratory capillaries, but later on cach afferent becomes directly connected with the corresponding efferent artery; and at metamorphosis, when the gills gradually

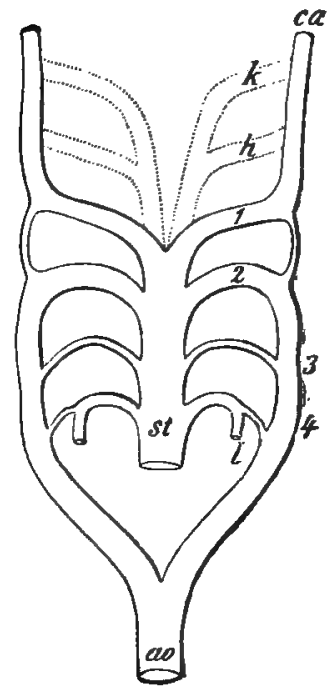

FIG. 11x.-Diagram of the arterial arches of an Amphibian.

$\mathrm{I}-4$, the four arterial arches which pass up the corresponding branchial arches; ao, dorsal aorta ; $c$. a. carotid artery ; $k$. embryonic arterial arch of the mandibular arch, and $h$. of the hyoid; $l$. pulmonary artery; st. ventral aorta. (From Wiedersheim's Anatomy, after Boas.) disappear, all the blood thus passes directly from the ventral to the dorsal aorta, through the four arterial arches (Fig. III). The first arch (I) gives rise in the adult frog to the carotid trunk ( $c a$ ), and loses its connection with the second at the dorsal end; the second (2) forms the systemic trunk; the third (3) disappears; and the fourth (4), losing its connection with the dorsal aorta, forms the pulmo-cutaneous trunk (l).

Having now traced the main course and arrangement of the chief arteries, there are a few minor points of detail to be noticed in the dogfish (Fig. I12). The five afferent branchial arteries (af. br $1--5$ ) of either side do not arise regularly and symmetrically fron: the ventral aorta, as represented in the diagrammatic Fig. I 10. The anterior end of the ventral aorta divides into right and left branches, each of which again subdivides to form the first two afferent branchial arteries, which supply respectively the hemibranch on the hyoid arch and the holobranch of the first branchial arch. The third afferent

branchial artery arises from about the middle of the ventral aorta, and supplies the holobranch of the second branchial arch; a short distance behind it, the fourth and fifth come off close together, and supply the gills on the third and fourth branchial arches respectively : it will be remembered that the fifth branchial arch bears no gill-filaments. After 
aeration, the blood from each hemibranch passes into an efferent branchial artery (ef. br), which, except in the case of the last (ef. $\left.b r^{9}\right)$, joins with is fellow of the same cleft and thus forms a loop surrounding the cleft, the two halves of adjacent loops being connected in the middle by a commissural vessel. From the upper ends of each of the four loops arises an epibranchial artery $(e p . b r)$ which is connected with

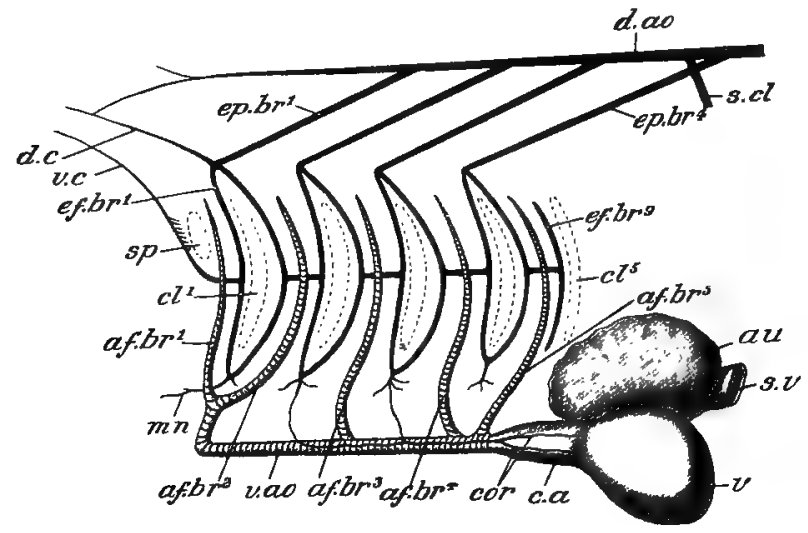

FIG. 112. -The heart and branchial arteries of Scylliwm, from the side.

af. br. ${ }^{1-5}$, afferent branchial arteries; au. auricle ; $c . a$. conus arteriosus ; $c l .1-5$, branchial clefts; cor. coronary artery; $d . a o$. dorsal aorta; $d . c$. dorsal carutid artery ; $e f^{\prime} b r^{1}{ }^{1}-9$, efferent branchial arteries; $e p . b r .1-4$, epibranchial arteries : $n n$. mandibular artery : sp. spiracle; $s . c l$. subclavian artery ; s. $v$. sinus venosus : v. ventricle; $v . a \sigma$. ventral aorta; $v$. c. ventral carotid artery.

the dorsal aorta ( $d$. ao), the blood from the last hemibranch passing into the fourth loop.

From the dorsal end of the first efferent branchial, a dorsal carotid artery $(d, c)$ is given off : this passes forwards and inwards, gives off a cranch to the upper jaw and snout, and then runs inwards in a groove on the skull-flour, which it penetrates in the middle line so as to reach the tranial cavity. A vessel arises from the middle of the first efferent branchial, and supplies the pseudobranch, from which the blood is collected by a ventral carotid artery (v.c) which passes through the orbit into the cranium, giving off branches to the brain and anastomosing with the dorsal carotid. From the ventral end of the first efferent branchial a small mandibular artery (mn) passes to the lower jaw. The F $\mathrm{F}^{*} *$ 
dorsal aorta is continued forwards, anteriorly to the first epibranchial artery, as a slender vessel which soon bifurcates and anastomoses with the dorsal carotid. From the ventral ends of the efferent branchial loops small arteries are given off which supply the lower parts of the head, the branchial region, and the heart (cor). The subclavian arteries $(s . c l)$ arise from the aorta just before it is joined by the last epibranchials.

In the short-bodied frog we have seen (p. 8o) that there is only a single splanchnic artery, which soon divides into a coliac and a mesenteric. In the dogfish there are four splanchnic arteries, arising separately from the dorsal aorta, viz., a caliac, supplying the proximal ends of the stomach and intestine, the liver, and pancreas ; an anterior mesen. teric, arising a short distance further back and supplying the intestine, \&c. ; a lieno-gastric, coming off from the aorta close behind the anterior mesenteric and guing to the spleen and part of the stomach and pan. creas; and a small posterior mesenteric supplying the rectal gland.

As in all Vertebrates, the arteries branch and branch again in the various parts to which they are distributed, their ultimate ramifications opening into a capillary network with which all the tissues except the cartilages and epithelia are permeated. From these systemic capillaries the blood is collected into larger and larger efferent, thin-walled, trunks or veins, parts of many of which are greatly dilated to form sinuses.

The blood from the head is brought back by a pair of jugular veins (Figs. I I,$j . v$. and $\mathrm{I}$ I 3 jug. $v$ ) : each of these enters a large precaval sinus (pr.cv. $v, d c t . c)$ which passes vertically downwards and enters the sinus venosus. The blood from the tail is returned by a caudal vein (cd. $v$, caud. v) lying immediately beneath the caudal artery in the hæmal canal ; this vessel enters the cclome and then divides into right and left branches, the renal portal veins ( $r . p . v$, $r$ port. $v$ ), which pass to the kidneys and join with the capillaries of these organs, the impure blood brought from the tail mingling with the pure blood of the renal arteries (Fig. I Io, r, a). From the kidneys the blood is returned 


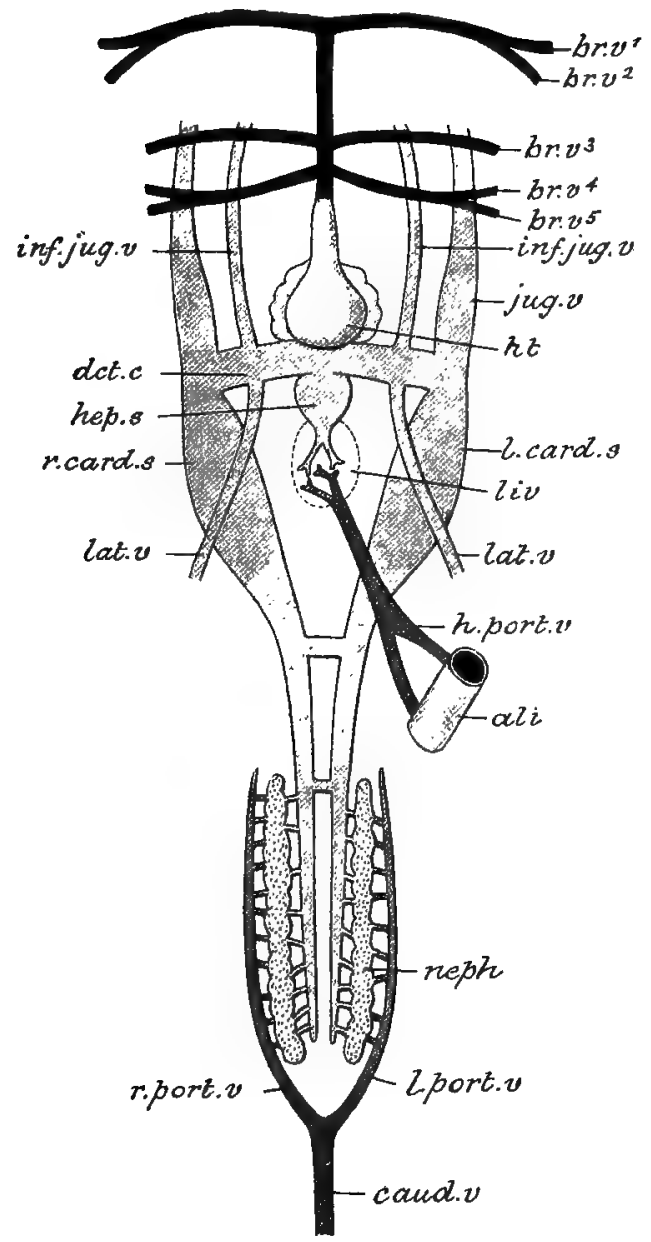

FIG. Ir3.-Diagram of the chief veins together with the ventral aorta and afferent branchial arteries of a dogfish (Cheiloscyllium).

ali. enteric canal; $b r . v . l-b r . v .^{5}$ afferent branchial arteries; caud. $v$. caudal vein; dct. c. precaval sinus; $h t$. heart; $h$. port. $v$. hepatic portal vein; hep.s. hepatic sinus; inf.jug. $v$. inferior jugular vein or sinus; jug. jugular vein or

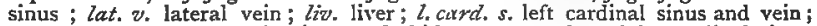
$l$. port. $v$. left renal portal vein; meph. kidney; $r$. card. s. right cardinal sinus and vein; $r$. port. $v$. right renal portal vein. (From Parker and Haswell's Zoology.) 
into a pair of cardinal veins (crd. $v$ ) which pass forwards, become swollen to form sinuses (Fig. I I 3, l.card.s, r.card.s), receive veins from the reproductive organs, muscles, \&c., and finally enter the precaval sinus.

From the stomach, intestine, spleen, and pancreas the blood is collected by numerous veins, which all join to form a large hepatic portal vein ((Fig. Iro, h.p.v, Fig. II 3 , $h$. port. v). This behaves in the same way as the renal portal: instead of joining a larger vein on its way to the heart, it passes to the liver and breaks up to connect with the capillaries of that organ; its blood, deprived of oxygen but loaded with nutrient matters from the enteric canal, mingling with the oxygenated blood brought to the liver by a branch of the coliac artery. After circulating through the capillaries of the liver the blood is taken by a pair of hepatic sinuses (h. $v$, hep.s) to the sinus venosus.

The course and arrangement of the veins, like that of the arteries, is very similar to that existing in the tadpole, in which several important changes occur at metamorphosis. With the disappearance of the tail and caudal vein, the renal portal veins receive their blond from the hind-linbs only. The hinder parts of the two cardinal veins, situated between the kidneys, fuse into one, and their anterior parts disappear, a new vessel being developed which conducts the blood from the fused cardinals to the sinus venosus: the whole of the great vein thus formed is the postcaval (p. 82, fig. 21), which is present in all Vertebrates above the fishes.

The iliac veins of the dogfish (Fig. $110, i l . v$ ) pour the blood from the pelvic fins into the lateral veins (Figs. I Io and II 3 , lat. $v$ )-paired trunks running forwards in the side walls of the body to the sinus venosus, and receiving at their anterior ends the subclavian veins ( $s c l . v)$ from the pectoral fins. In addition to the dorsally situated jugular veins, there are paired inferior jugulars (Fig. I I 3, inf. jug. v), 
bringing back the blood from the ventral parts of the head, and each opening into the corresponding precaval.

As we have seen, several of the veins, e.g., the precavals, jugulars, cardinals, and the genital veins, are dilated into spacious cavities called sinuses (Fig. II3). These are, however, of a totally different nature from the sinuses of the crayfish, which are mere spaces among the tissues devoid of proper walls. In the dogfish, as in the frog and Vertebrates generally, the blood is confined throughout its course to definite vessels; the heart, arteries, capillaries, and veins invariably forming a closed system of communicating tubes.

The general course of the circulation will be seen to agree with that already described in the frog, as well as in the crayfish and mussel, i.e., the blood is driven by the contractions of the heart through the arteries to the various tissues of the body, whence it is returned the heart by the veins or sinuses (Fig. I14). But "whereas in both crayfish and mussel the respiratory organs are interposed in the returning current-both their afferent and efferent vessels being veiris, in the dogfish they are interposed in athe outgoing current-their afferent and efferent vessels being arteries. An artery, it must be remembered, is a vessel taking blood from the heart to the tissues of the body and having thick walls; a vein is a thin-walled vessel bringing back the blood from the tissues to the heart.

Moreover, the circulation in the dogfish is, as in the frog, complicated by the presence of the two portal systems, renal and hepatic. In both of these we have a vein, renal portal or hepatic portal, which, instead of joining with larger and larger veins and so returning its blood directly to the heart, breaks up, after the manner of an artery, in the kidney or liver, the blood finding its way into the ordinary venous channels after having traversed the capillaries of the gland in question.

Thus an ordinary artery arises from the heart or from an artery of higher order and ends in capillaries; an ordinary vein arises from a capillary network, and ends in a vein of higher order or in the heart. But the hepatic and renal portal veins end in capillaries after the manner of arteries, and the efferent branchial arteries begin in capillaries after the manner of veins. 
With regard to the general morphology of the blood-system, the dorsal aorta with the caudal artery may be considered as a dorsal vessel (compare Earthworm, p. 329, Crayfish, p. 365, and Amphioxus, Fig. IOI); the caudal vein, hepatic portal vein, and ventral aorta as together representing a ventral vessel; the afferent and efferent branchial arteries as commissural vessels; and the lateral veins as lateral vessels. It will be seen that the heart of Vertebrates is a muscular dilatation of the ventral vessel, as is also shown by a study of its development.

The blood, like that of the frog (pp. I04-106), consists of a colourless plasma containing red corpuscles-the colour

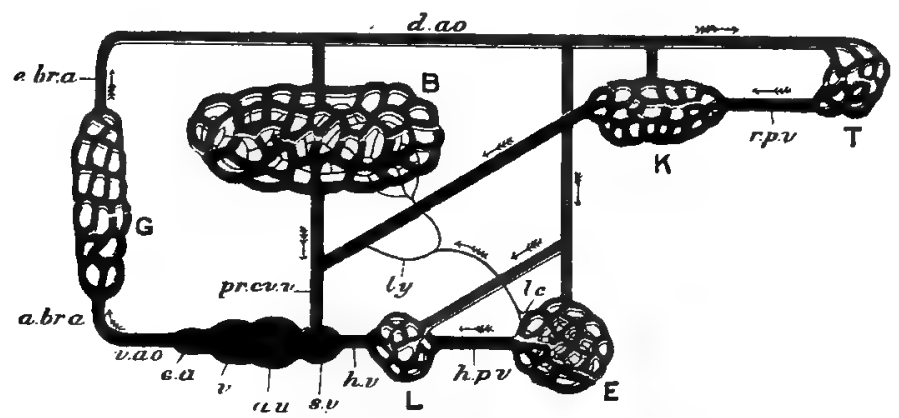

FIG. I14.-Diagram illustrating the course of the circulation in the dogfish. Vessels containing oxygenated blood red; non-oxygenated blood blue.

B. capillaries of the body generally; $\mathbf{E}$. of the enteric canal ; G. of the gills; $K$. of the kidneys; $L$. of the liver; $T$. of the tail. a.br.a. afferent branchial arteries; $a u$. auricle; $c . a$. conus arteriosus; $d$. ao. dorsal aorta ; $e$. br. a. afferent branchial arteries; $h . p . v$. hepatic portal vein; $h . v$. hepatic vein; $l c$. lacteals; $l y$. lymph-

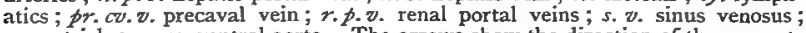
$v$. ventricle; $v$. al. ventral aorta. The arrows show the direction of the current. (From Parker and Haswell's Zoology.)

of which is due to hæmoglobin-and leucocytes. It must be remembered that the ventral aorta and the afferent branchial arteries (Fig. I14), like the pulmonary artery of the frog (p. I44), contain venous blood. As in the frog, there are in addition to the blood-vessels, a set of lymphatic vessels.

Nervous System. - The nervous system is constructed on a similar plan to that of the frog (compare Part I, Chapter X) 
and of Vertebrates in general. The central nervous system is dorsal in position and consists of a brain contained within the cranial cavity, and continuous posteriorly with a spinal cord contained in the neural canal of the vertebral column: it consists of grey and white matter, and its cavity or neurocole, lined with epithelium, gives rise to the ventricles of the brain and to the central canal of the spinal cord.

In correspondence with the form of the body, the spinal cord is relatively much longer than in the frog, and it is not swollen opposite the paired appendages.

In the brain (Fig. II5) the bulb or medulla oblongata $(N H)$ broadens out anteriorly to form lateral swellings, and its contained fourth ventricle $(F . r h o)$ is roofed over by the pia mater. The cerebellum $(H H)$, which is very small in the frog, is here relatively enormous, and its surface is marked by slight grooves : it overlaps the bulb behind and the optic lobes in front, and contains a ventricle communicating with the fourth ventricle. The oval optic lobes $(\dot{M} H)$ are hollow, their cavities communicating with the median ventricle or iter (p. I57); and ventrally to them are the crura cerebri. The diencephalon $(Z H)$ is relatively narrower than in the frog. From its thin roof, which covers over the third ventricle, is a delicate tube-like structure $(G p)$, which extends upwards and forwards and ends in a small knob attached to the roof of the skull:- this is the pineal body (p. I59). From the ventral surface of the diencephalon arises the infundibulum, with an oval swelling on either side, to which is attached the pituitary body with a vascular sac on each side of it and a median tubular body on its ventral surface. In front of the infundibulum is the optic chiasma.

Apart from the large size of the cerebellum, the most 
marked difference between the brain of the dogfish and that of the frog is seen in its anterior portion. In the frog, the

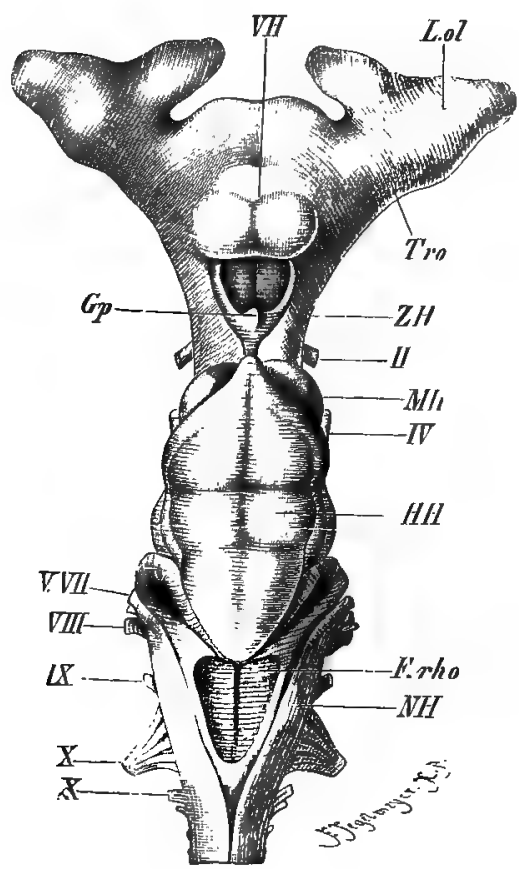

FiG. II5.-Dorsal view of the brain of Scyllizm canicula.

The posterior division of the brain is the medulla oblongata $(N H)$, enclosing the fourth ventricle $(F, r h o)$. The large cerebellum $(H H)$ nearly covers the optic lobes $(M H)$ The diencephalon $(Z H)$ shows in the middle the third ventricle, and the place of attachment of the pineal stalk $(G p)$. The prosencephalon $(V H)$ gives off the olfactory lobes (Tro. L.ol). The origins of the following nerves are shown:-optic $(I I)$, trochlear $\left(I^{*}\right)$, trigeminal $(V)$, facial $(V I I)$, auditory $(V I I I)$, glossopharyngeal $(I X)$, and vagus $(X)$. (From Wiedersheim's Anatomy.)

diencephalon is continuous anteriorly with the paired cerebral hemispheres (159, Fig. 49) : in the dogfish there is in this region a relatively smaller, unpaired portion of the brain, marked in front by a slight groove, and known as the prosencephalon $(V H)$, which represents the cerebral hemispheres of the higher Vertebrates but which does not become subdivided externally into paired lobes. Anteriorly it gives off, right and left, a large, oval olfactory lobe ( $L$. ol) each connected with the prosencephalon by a short, stout stalk (Tro) and applied distally to the corresponding olfactory capsule. The prosencephalon contains paired lateral ventricles, which 
communicate posteriorly with the third ventricle and anteriorly are continued into the olfactory lobes.

Each spinal nerve arises, as in the frog (p. 163), by two roots, which, however, are not in the same transverse plane, the dorsal root being slightly anterior to the corresponding ventral root, so that successive dorsal and ventral roots of either side alternate with one another: they also alternate on the right and left sides. As already mentioned (p. 424), the two roots of each nerve passes out from the neural canal independently, uniting on the outside of the canal to form the spinal nerve. A sympathetic is represented.

The origin and distribution of the cerebral nerves is in the main similar to that already described in the case of the frog (p. I63), the chief differences, characteristic respectively of air-breathing and of branchiate Vertebrates in general, being as-follows.

In fishes, there are certain nerves, usually considered as belonging to the facial and vagus, which supply the sensory canals of the integument (Fig. II $6, V I I o p, b, e . m$, and $X l$ ): these organs are not present in terrestrial forms, and their nerves are consequently also wanting. The vagus, moreover, gives off a series of branchial branches $\left(b r^{1}{ }^{1}-^{4}\right)$ to the gills instead of a pulmonary branch, and the glossopharyngeal $(I X)$ is also a branchial nerve.

The olfactory nerves (Fig. II6, I) arise from the olfactory lobe on each side, which is situated in a large aperture in the skull communicating between the cranial and olfactory cavities. The optic nerve (II) is continued outwards from the optic chiasma, and passes through a foramen in about the middle of the orbit, towards the ventral side. The oculomotor (III), arising from the crura cerebri, makes its exit from the skull a short distance behind and slightly above the optic nerve. The tathetic (IV), coming off from the dorsal side of the front end of the bulb and supplying the superior oblique muscle, pierces the 

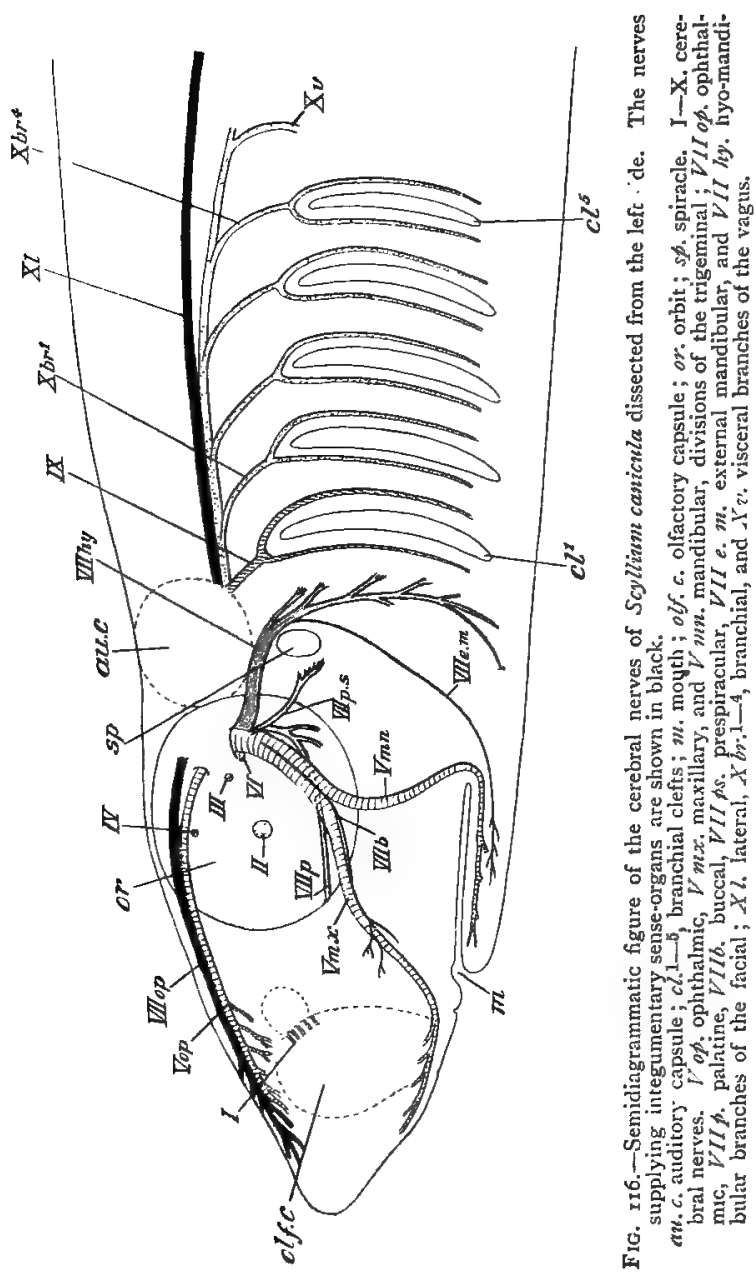
cranial wall almost directly above the optic foramen (compare also Fig. 117). All the other nerves arise from the ventro-lateral regions of the medulla oblongata, the abducent, supplying the external rectus muscle, coming off nearer the middle line than, and anterior to, the others. The abducent (VI) and the main parts of the trigeminal (V) and facial (VII) nerves pass out through a single foramen in the skull in the posterior and ventral part of the orbit, just anterior to the auditory capsule. A short distance above this foramen are two others, the ventral slightly anterior to the dorsal: these transmit the ophthalmic branches (see below) of the trigeminal and facial (V op, VII op) respectively, and from them grooves pass along the dorsal side of the orbit to an aperture just behind the olfactory capsule, the nerves emerging again on the dorsal side of the skull. The auditory nerve passes through $=$ large foramen on the inner side of the auditory capsule to supply the membranous labyrinth. The glossopharyngeal (IX) emerges behind the auditory capsule at the posterior end of a horizontal groove in this region, and the vagus $(\mathrm{X})$ passes out through a foramen between the glossopharyngeal and the foramen magnum.

The nerves supplying the integumentary sensory organs are as follows: (I) The ophthalmic branch of the facial (VII op) runs, as we have seen, dorsally to the similarly named branch of the trigeminal, close under the skin, and supplies the sensory tubes and ampullæ (see p. 448) of the upper part of the snout; those of the lower part of the snout are innervated by (2) a buccal branch (VII b), which extends along the floor of the orbit just above the maxillo-mandibular division of the trigeminal ; and those in the region of the hyomandibular by a small (3) external mandibular branch (VII $e, m$ ), arising from the large hyomandibular nerve (see below). The lateral line canal, extending along the body and tail, is supplied by (4) the lateral branch of the vagus (X $l$, which runs backwards to the inner side of the rest of the nerve and dorsally to the spinal nerves, along the inner side of the body-wall, giving off branches which extend outwards between the great lateral muscles to the lateral canal.

The other branches of the facial are :-a small palatine (VII $p$ ), which extends along the floor of the orbit, just behind the trigeminal, and supplies the roof of the mouth; and a large hyomuzndibular (VII $h y$ ) which passes behind the spiracle-first giving off small prespiracular branches (VII $p . s$ ) to its anterior wall, extends along the anterior border of the auditory capsule and the posterior wall of the orbit, just beneath the 
skin, to the anterior side of the hyoid arch: it thus forks over the spiracular or mandibulo-hyoid cleft.

The glossopharyngeal (IX) forks above the first gill-cleft, thus giving rise to two branches, one passing down the posterior side of the hyoid, and the other down the anterior side of the first branchial arch. The main part of the vagus extends backwards to the outer side of the lateral nerve and gives off four branchial nerves (X br. ${ }^{1-4}$ ) forking over the second to the fifth gill-clefts respectively, and is then continued into the visceral nerves (X v), which supply the stomach and heart.

Sensory Organs.-The dogfish possesses, as we have seen, a series of peculiar integumentary sense-organs supplied by the nerves just described, the function of which is not known with certainty. They are situated within a number of epithelial canals, developed from the epiderm, the openings of which on the head have already been noticed (p. 4 Io).

The tubes are of two kinds, known respectively as sensory and ambullary canals: the former, which are present in all Vertebrates with gills ( $\mathrm{p} .4 \mathrm{I} 4$ ), are all continuous with one another and are situated along certain definite regions in the head and jaws, a canal extending along the body and tail as the lateral line canal. The ampullary canals, which are peculiar to Elasmobranch fishes, and which contain a gelatinous material, are not continuous with one another, but run side by side, converging to form large masses in the snout and at the sides of the head; at their blind ends they are swollen to form ampulla, to which the nerves are distributed. The sensory cells are arranged in little conical masses in the lining epithelium of the canals or of the ampullæ, a section of one of which nearly resembles that of an ampulla of a semicircular canal of the ear (Fig. 6o).

The olfactory organs are a pair of cup-like sacs in the snout, enclosed by the olfactory capsules and opening externally on the ventral side by the nostrils. Notice that there are no internal nostrils, as in the frog: these are only present in Vertebrates which possess lungs. The sacs are lined by the olfactory epithelium, which is supplied by the 
olfactory nerves and is raised up into ridges so as to increase the surface.

The structure of the eye, as well as of the accessory apparatus in connection with it, is in all essential respects the same as in the frog (p. I8I), except for the differences in the eyelids (pp.5 and 41o), the absence of a lachrymal apparatus (p. 186), and for the fact that the four recti muscles

(Fig. I I 7) do not ensheath the optic nerve, which emerges into the orbit a short distance in front of their point of origin.

The membranous labyrinth of the ear (compare Fig. 59, p. 187 ) is also very similar to that of the frog, but being larger, and the auditory capsules being composed entirely of cartilage, it can be dissected out

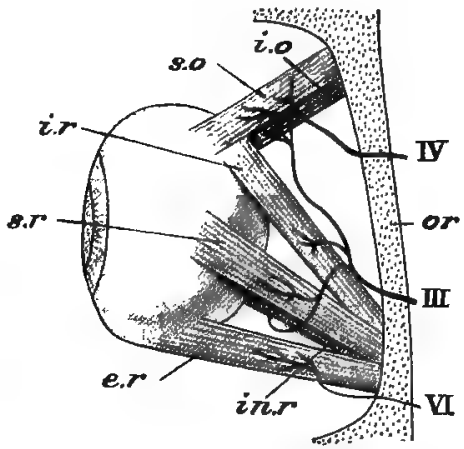

FIG. II7.-Semidiagrammatic figure of the eyemuscles and their nerves of an Ellasmobranch. $I I I$. oculomotor, $Y V$. pathetic, and $V I$. abducent nerve; e.r. posterior rectus muscle; i. o. inferior oblique; $i r . r$. inferior rectus; $i, r$. anterior rectus; or.wall of orbit; s.o. superior oblique; s. $r$. superior rectus. (From Parker and Haswell's Zoology.)

with comparative ease by slicing away the capsule with a knife.

A tube given off from the sacculus, called the endolymphatic duct (Fig. 59), which in the frog communicates with the lymphatic system, opens to the exterior on the top of the head in the dogfish, and thus the endolymph is in free communication with the surrounding sea-water.

As we have seen, the membranous labyrinth is the essential part of the ear, and it, together with its enclosing capsule, is often spoken PRACT. Zool. 
of as the internalear. In the frog there is also an accessory apparatusthe tympanic cavity and membrane, together with the columella-which is called the middle ear (compare p. 49 and 189).

Urinogenital organs.-In order to understand the morphology of the kidneys, and the close relations existing in most Vertebrates between them and the generative organs, it is necessary to know something of the development of these parts. In the embryo, the kidneys appear in the form of separate, segmentally arranged tubes having the general character of nephridia, opening on the one hand by nephrostomes into the colome, and on the other into a longitudinal duct which discharges into the cloaca. Thus the primitive structure of the vertebrate kidney furnishes another example of metamerism, which can no longer be distinctly recognised in the adult kidney. (Figs. 46 and 47.)

At a later stage of development in most vertebrate orders two longitudinal ducts can be recognised on either side, which in some cases (e.g. Dogfish) are formed by the subdivision of the single primary duct. These are known respectively as the Wolfian and the Mïllerian ducts: the former takes on the function of a spermiduct in the male, although it may (e.g., in the frog, p. I93) retain also its function as a ureter; the latter gives rise to the oviduct in the female.

In the dogfish the kidneys (Fig. I $8, e p, k$ ) are long, narrow, lobulated organs, lying close to the vertebral column on either side, covered ventrally by the thick peritoneum, and extending primarily along almost the whole length of the colome. But in the course of development, certain important modifications take place in them and in their ducts (Wolffian ducts). In the male, about the anterior half of the kidney takes on a 
close relation with the generative organs, and gives rise to a glandular body - the epididymis $(A, e p)$-with which the long, convoluted Wolffian duct $(s p d)$, serving exclusively as a spermiduct, is closely connected ventrally : in the female, this part of the kidney and its duct becomes vestigial $\left(B, k^{\prime}\right)$. The hinder half of the embryonic kidney in each sex is retained in the adult as the renal organ $(k)$, which is somewhat swollen posteriorly. The ureters $(u r)$ are independently developed tubes, about five in number on each side. In the female they open separately into the swollen persistent posterior ends of the Wolffian ducts, which unite together to form a median urinary sinus $(B, u . s)$, opening by a single aperture into the cloaca: while in the male $(A, u r)$ most of them unite to form a wide main ureter before communicating with a similar median sinus, which, as it receives the products both of the spermaries and kidneys, is called the urinogenital sinus (u.g.s).

The spermaries are a pair of large, elongated, soft organs united with one another posteriorly, and suspended to the dorsal body-wall by a fold of peritoneum. From the anterior end of each $(A, t s)$ arise delicate efferent ducts (ef. $d$ ), which pass to the epididymis to become connected with the convoluted spermiduct. The latter dilates posteriorly, where it underlies the functional kidney, forming an elongated, spindle-shaped seminal vesicle (s.v) which opens $\left(s . v^{\prime}\right)$ into the base of a thin-walled blind reservoir of about the same length, the sperm-sac (sp.s); and just to the inner side of its aperture are the openings of the ureters $\left(u r^{\prime}\right)$. The sperm-sac is continuous posteriorly with the urinogenital sinus, the opening of which into the cloaca is situated on a papilla.

The female Scyllium has a single ovary. $(B, o v)$, suspended by a fold of peritoneum. In the adult it is studded all over with rounded ova in different stages of development, 


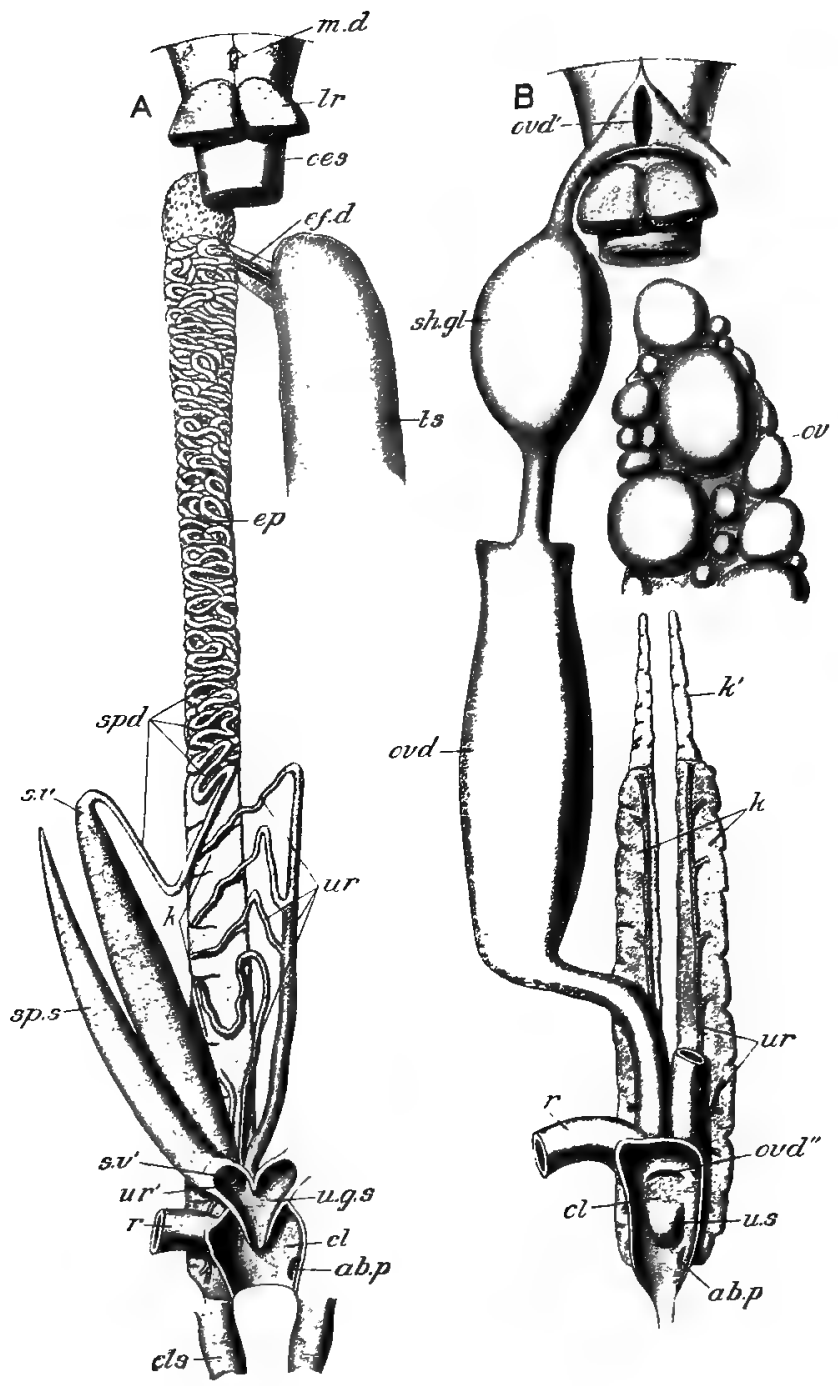

Fig. rr8.-The urinogenital organs of Scyilinem canicula from the ventral side. $A$, male, and B, female. Only the anterior end of the gonad is represented in each figure, and except that in $B$ both kidneys are shown, the organs of the right 
size only are drawn. In $\mathbf{A}$ the seminal vesicle and sperm-sac are dissected away from the kidneys and displaced outwards, and the ureters inwards.

$\alpha b . \not$. depression into which the abdominal pore opens ; $c l_{\text {. cloaca ; }} c l s$. clasper;

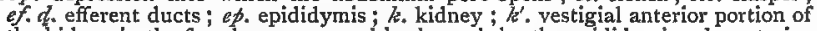
the kidney in the female, represented in the male by the epididymis; $l r$. anterior portion of liver; $m . d$. vestigial Müllerian duct in the male; ces. gullet; ov. ovary ; ovd $z^{\prime}$. its coelomic aperture; $o v d^{\prime \prime \prime}$. the common aperture of the oviducts into the cloaca; $r$. rectum ; sh.gl. shell-gland; $s p d$. spermiduct; $s p$. s. spermsac; $s, v$. seminal vesicle; $s . v^{\prime}$ its aperture into the urinogenital sinus; $t s$. spermary ; $u . g_{.} s_{0}$ urinogenital sinus ; $u r$. ureters ; $u r^{\prime}$. their apertures into the urinogenital sinus ; $k$. s. urinary sinus.

varying in diameter from I2-1.4 $\mathrm{mm}$. downwards : in other Vertebrates which produce large eggs, a similar reduction of one ovary may take place (e.g., Birds). The oviducts (ovd) are paired, and extend along the whole length of the dorsal wall of the coelome, below the kidneys: anteriorly they unite with one another below the gullet and just in front of the liver, where they communicate with the coelome by a common aperture $\left(o v d^{\prime}\right)$; posteriorly they open together by a single aperture ( $o v d^{\prime \prime}$ ) into the cloaca, behind the rectum (r). About the anterior third of each oviduct is narrow and thin-walled; the posterior two-thirds is wide and distensible, and at the junction of the two parts is a yellowish, glandular mass, the shell-gland (sh.gl).

Development.--Impregnation is internal, and is effected through the agency of the claspers of the male. The eggs, when ripe, break loose from the surface of the ovary into the colome, and thence pass, through the common oviducal aperture, into one or other of the oviducts, where fertilization occurs. As it passes into the dilated portion of the oviduct, the oosperm of Scyllium becomes surrounded first by a gelatinous substance, and then by a horny egg-shell or "Mermaid's purse" 1 secreted by the shell-gland, and having the form of a pillow-case produced at each of its four angles into a long, tendril-like process. The eggs are laid among sea-weed, to which they become attached by their tendrils.

1 An egg is contained in the oviduct figured (Fig. I I8 B). 
In Acanthias and Mustelus (p. 4I5) a mere vestige of the egg-shell is formed, and the eggs undergo the whole of their development in the oviducts, the young being eventually born alive with the form and proportions of the adult.

The great size of the egg is due to the immense quantity of yolk it contains: its protoplasm is almost entirely aggregated at one pole in the form of a small disc. When segmentation of the oosperm takes place it affects the protoplasm alone, the inactive yolk taking no part in the process (compare Crayfish, p. 369). The polyplast stage consequently consists of a little mass of cells, the blastoderm

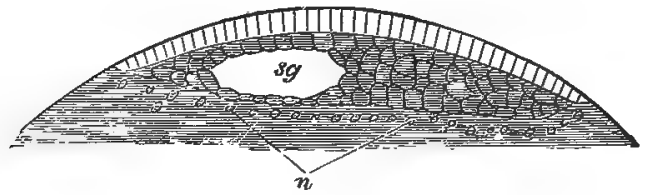

Fir. Irg.--Section of the upper part of the oosperm of a Dogfish which has undergone segmentation to form the blastoderm. The blastoderm is formed of a single layer of ectoderm cells (white), and of several rows of cells (shaded) which subsequently give rise to endoderm and mesoderm.

sg. segmentation cavity; below the blastoderm is the unsegmented yolk containing scattered nuclei $(n)$. (From Balfour.)

(Fig. I I9), at one pole of an undivided sphere of yolk. The cells of the blastoderm become differentiated into the three embryonic layers-ectoderm, mesoderm, and endoderm. At the same time the blastoderm extends in a peripheral direction so as to gradually cover the yolk, and its middle part becomes raised up into a ridge-like thickening, which is moulded, step by step, into the form of the embryo fish. The head, trunk, and tail acquire distinctness, and become more and more completely separated off from the bulk of the egg, the latter taking the form of a yolk-sac (Fig. I20, $A, y k . s$ ) attached by a narrow stalk to the ventral surface of the embryo. 
- In this condition the various parts of the adult fish can be recognised, but the proportions are different and the head presents several peculiarities. The gill-filaments $\left(b r . f^{\prime}\right)$ are so long as to project through the external branchial apertures and the spiracle in the form of long threads, abundantly supplied with blood-vessels, and apparently serving for the absorption of nutriment-the albumen in the egg-shell in the case of Scyllium, secretions of the oviduct

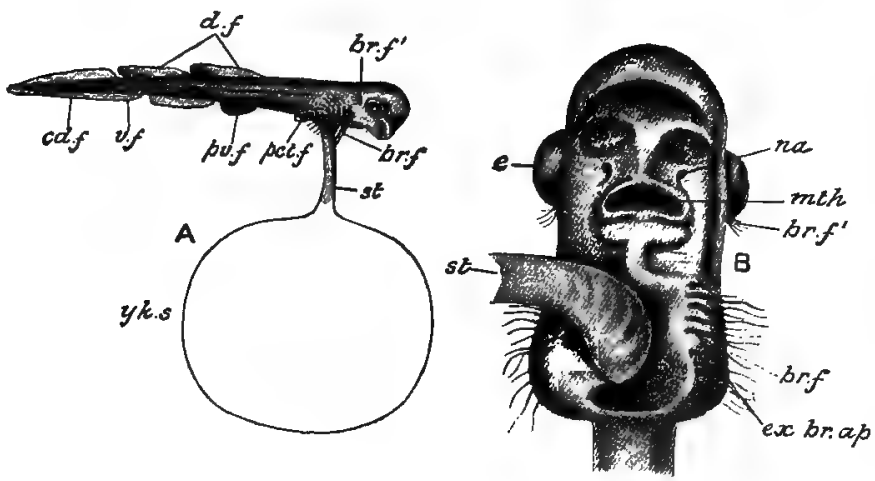

FIG. I2o.-A, embryo of Scyllizm with yolk-sac ' $\left(X I \frac{1}{2}\right) ; B$, under-side of head, enlarged, $b r . f$. branchial filaments protruding through gill-clefts; $b r$. $f^{\prime}$. branchial filaments protruding through spiracle; $c d . f$. caudal fin; $d$. $f$. dorsal fins ; e. eye ; ex. br. ap. external branchial apertures; nth. mouth ; na. nostrils ; pct. $f$. pectoral fin; $b v . f$. pelvic fin; st. yolk-stalk; $v . f$. ventral fin; $y / k . s$. volk-sac. (From Parker's Biology, after Balfour, slightly altered.)

in the viviparous forms referred to above. Besides this mode of nutrition, the yolk-sac communicates with the intestine by a narrow duct $(s t)$, through which absorption of its contents is constantly going on. By the time the young fish is ready to be hatched or born, the greater part of the yolk-sac has been drawn into the colome, a mere remnant of it still dangling from the ventral surface of the body. 


\section{PRACTICAL DIRECTIONS.}

Dogfishes are best preserved in 4 per cent. formaline, which has the additional merit over spirit of not coagulating the blood, so that the vessels can be injected in preserved specimens. They can be obtained, fresh or ready preserved, from any Marine Biological Station.

A. External characters : see pp. 4I5-4I8. Sketch from the side. Isolate some of the dermal teeth by boiling a small piece of skin in caustic potash, and make out the bony basal plate, and the spine com. posed of dentine tipped with enamel. Sketch.

B. Skeleton. (If you are working on a fresh fish, and wish to dissect the soft parts before preservation, the examination of the skeleton may be postponed until later.)

It is advisable to have one skeleton prepared entire, and one in which the parts have been disarticulated. Obtain a common butcher's or cook's pointed knife (a strong pocket-knife will do) for cutting through the rough skin and for the coarser work of preparation. Prepare as directed on p. 53, and when the greater part of the muscles has been removed, disarticulate the skull from the vertebral column, leaving the branchial apparatus attached to it, and also remove the paired fins and their arches. Disarticulate the hyomandibular cartilage from the cranium so as to separate the visceral arches, including the jaws (compare Fig. I03): these should then be thoroughly cleaned without further immersion in hot water, as the cartilages of which they are composed come apart very easily. The other parts may be dipped into hot water for a few seconds from time to time, but care should be taken that the more delicate elements do not thereby become separated. It is useful to prepare a second cranium as well as a few trunk- and caudal vertebræ, which should be bisected vertically into right and left halves. When prepared, the skeleton should be kept in weak spirit or formaline, and not allowed to dry, or the cartilages will of course shrink, unless the following method is resorted to:-

Thoroughly clean a skeleton, or typical parts of it (e.g. skull, limb. skeleton, and a few trunk- and caudal vertebre), and then transfer from weak into strong methylated spirit for a day or so, and afterwards into absolute alcohol for a few hours. Place in a vessel filled with turpentine for another day, and then transfer into melted paraffin in the waterbath until the parts are thoroughly permeated, after which they should 
be suspended in the water-bath in order to drain off the superfluous paraffin, and then allowed to cool. Any superfluous paraffin still remaining may then be removed with a hot wire.

With the specimens before you, work through pp. 419-427, noting first of all the relations of the parts in the entire skeleton (viz., cranial and visceral portions of the skull, trunk- and caudal vertebræ, and the skeleton of the median and paired fins). When examining the skull, note the nerve foramina ( $\mathrm{pp} .445-447$ ).

Sketch-(a) the skull (including visceral arches) from the side, and the cranium in longitudinal section; $(b)$ trunk- and caudal vertebræe from the side or in longitudinal section and from the anterior or posterior face; (c) the pectoral arch, from the sicle, with the pectoral fin attached; and $(d)$ the pelvic arch and fin.

\section{General dissection : Enteric Canal; \&c.}

I. - Fix the animal down on the dissecting board with the ventral surface uppermost by means of strong pins inserted through the paired fins, and make a median longitudinal cut with a common knife (see p. 456) through the skin and underlying muscular layer which is closely connected with the skin, from the pectoral to the pelvic arch. At each end of this incision cut through the body-walls transversely, and reflect and pin down the two flaps. Cut through the pelvic arch slightly to one side of the median line, so as not to injure the cloaca. The abdoninal cavity, lined by the peritoneum, will then be exposed. (In the course of your dissection you will probably find many parasitic thread-worms belonging to the phylum Nenathelminthes (see p. 397). Make out :-

I. The liver, with the gall-bladder:partly embedded in it close to the junction of its two lobes; the gullet, U-shaped stomach, and the branches of the vagus nerve on its walls; the wide intestine, narrowing into a short rectum posteriorly; the cloaca; the pancreas, spleen, and rectal gland; and the incomplete mesentery. Pass a seeker backwards, on one side of the cloaca, through an abdominal pore.

2. In the male, the spermaries, fused together posteriorly; and in the female, the single ovary and the oviducts and shell-glands. The peritoneum covering the kidneys is so thick that at present they can only be recognised as slightly convex ridges.

II. - Remove the skin from the dorsal surface of the head between and slightly in front of and behind the eyes, and then slice away part of the 
roof of the skull with a knife until the brain is exposed, being careful not to injure some nerves which you will see close beneath the skin on either side of the brain-case. Then cut off the tail transversely, a short distance behind the pelvic fins, and on the cut surface note-

I. The integument, in which runs the sensory canal of the latera. line.

2. The centrum and neural and homal arches of the vertebra, and the soft intervertebral substance (remains of the notochord); the spinal cord; and the caudal artery and vein.

3. The myomeres and myocommas; and if your section passes through a dorsal fin, the cartilaginous pierygiophores and the horny fin-rays. Sketch.

III. - The dorsal aorta and its branches may now be injected (see p. 99) through the cut end of the caudal artery, into which a cannula should be inserted for some distance (tying is unnecessary). Now return to the examination of the abdominal viscera, and make out:-

I. The bile-dzcct, opening into the intestine just behind the pylorus. The pancreatic duct runs in the wall of the intestine, and careful dissection is required to make out its course (see $\$ I V, I$ ).

2. The hepatic porlal vein and its factors, entering the liver near the median plane. If the blood has escaped from it, try to blow it up with a blowpipe.

3. The position of the dorsal aorta, which "will be seen better at a later stage, but the chief branches of which should now be traced to their distribution, as follows: $a$, the caliac artery, extending downwards and backwards along the stomach from above the posterior end of the gullet; $b$, the anterior mesenteric artery, arising about $1 \frac{1}{2}$ inch behind the coeliac; $c$, the lieno-gastric artery, arising close behind the anterior mesenteric; and $d$, the small posterior mesenteric artery, passing downwards to the rectal gland.

4. The large hepatic sinus, immediately in front of the liver, below the gullet : slit it open, and note the veins entering it from the liver. On either side of the gullet in this region, along the dorsal surface of the cœlome, a capacious cardinal sinus will be seen : make an aperture in this, and pass a seeker backwards, noting that the sinus narrows into the cardinal vein, which passes along the inner side of the corresponding kidney and parallel to the aorta. The genital (spermatic or ovarian) sinus communicates with the cardinal.

5. The lateral veins (Figs. 1 ro and II3), running on either side of 
the body, just beneath the peritoneum. Cut through the body-wall on one side, a short distance behind the pectoral fin; insert a cannula, directed forwards, into the cut end of the lateral vein, and inject. The vein will then be seen running forwards as far as the pectoral arch, when it turns towards the dorsal side.

6. In the female, the united anterior ends of the ovidacts and their colomic aperture, ventral to the gullet and just in front of the liver.

IV.-Taking care not to injure the anterior ends of the oviducts and to leave part of the hepatic sinus in situ, remove the liver, together with the stomach and intestine, without injuring the bile-duct, cutting through the stomach at its junction with the gullet and the intestine iust in front of the rectal gland. Wash out the portion of the enteric canal thus removed under the tap, fill it with water, and place the whole under water in a dissecting-dish. Cut away portions of the wall of both stomach and intestine, and make out-

I. The course of the bile ducts and pancreatic ducts, and their apertures into the intestine.

2. The pyloric valve, and the spiral valve of the intestine, which makes about seven or eight close turns, appearing like a series of cones one within the other.

3. The characters of the mucous membrane of the stomach and intestine.

Sketch your dissection.

\section{Urinogenitál organs.}

I. - After noting again the gonads, and in the male the delicate efferent ducts of the spermaries (Fig. II8, A), remove in the male all but the anterior ends of the latter, and in the female the entire ovary; then carefully dissect away the thick peritoneum covering the kidneys, noting as you do so the dorsal aorta and its various branches, and once again the cardinal veins ( $\mathrm{C}, \S$ III, 4), which may be-inflated with air. The renal portal veins are not easily traced without injection, which may be done from the cut end of the caudal vein.

Note-

I. The brownish kidneys, and in the male their anterior, whitish sexual part-the epididymes.

2. In the male : $a$, the convoluted spermiduct, indistinguishable from the epididymis anteriorly and enlarging posteriorly to form the elongater seminal vesicle; and $b$; the grooved, eversible claspers, which are 
supported by cartilages and have each a gland at the base of the groove.

II.-Cut through the skin round the vent, and dissect the entire cloaca and the kidneys (together with the epididymes in the male) away from the body, and pin them down, ventral side uppermost, under water. Clear away with great care the connective tissue which binds the ureters and generative ducts to the kidneys posteriorly, slit open the cloaca, and make out-

I. In the male (Fig. $118, A$ ) the aperture of the rectum and the urinogenital papilla. Insert the small scissors into the aperture at the apex of the latter, and slit open the urinogenital simus, continuing the cut into the two spernt-sacs; make out the apertures of the seminal vesicles and ureters. Pass a seeker or probe into each of these apertures (the main ureter may be injected), and then dissect out, on one side- $a$, the elongated and pointed, thin-walled sperm-sac; and $b$, the delicate ureters, three or four of which unite to form a widish common tube, situated towards the inner border of the kidney, before opening into the urinogenital sinus. Sketch.

2. In the female (Fig. II8, B), the thin-walled, anterior united ends of the oviducts, their thick-walled posterior portion, the shell-glands, the apertures of the rectum and oviducts into the cloaca, and the urinary papilla. Insert the point of the scissors into the aperture on the apex of the latter, and slit open the urinary sinus, in which several openings of the ureters will be seen on either side. Cut open the oviducts, and note, if present, the eggs enclosed in horny egg-cases. Sketch.

\section{E. Circulatory ${ }^{1}$ and Respiratory Organs, \&c.}

I. -After noting the spinal nerves, exposed by the removal of the kidneys, the body may be cut through first behind the pectoral arch, and the posterior portion thrown away. Pin down the head and anterior portion of the body, ventral side uppermost, make a median longitudinal incision through the skin from the lower jaw to the pectoral arch, and dissect it away on either side as far as the gill clefts. Then, without injuring the lateral vein (p. 458), remove the middle portion of the pectoral arch and expose the pericardial cavity and heart. Insert a seeker, pointing backwards, along the dorsal side of the heart, through 
the canal which communicates between the pericardial and abdominal cavities : it opens on the ventral side of the gullet by two apertures. Make out-

I. The form and relations of the chambers of the heart (sinus venosus, auricle, ventricle and conus arteriosus).

2. The ventral aorta, to expose which the muscles in front of the pericardium must be carefully removed; but before doing so, it is better to inject the ventral aorta, cutting a small hole in the ventricle, and inserting and tying the cannula into the conus arteriosus: use a blue injection if you have already used red for the dorsal aorta.

3. The five afferent branchial arteries (compare Fig. II2): trace these outwards, and note their distribution. Sketch your dissection.

II. - Cut through the ventral aorta at its junction with the conus arteriosus and through both ends of the sinus venosus, carefully separating the latter from the walls of the pericardium and noting the entrance of the hepatic sinus (p. 458). Remove the entire heart, pin it down under water, ventral side uppermost, and cut open the ventricle and conus arteriosus. Note-

I. Their cavities and walls; the auriculo-ventricular aperture and valves; the valves in the conus arteriosus, of which there are two sets, consisting of three in each set. Sketch.

2. The cavity and walls of the auricle and the simu-auricular aperture, which are best made out by turning the heart over, with the dorsal side uppermost, before cutting open the auricle. Sketch.

3. Insert a seeker into one of the cut distal ends (still left in situ) or the sinus venosus, and slit it up so as to expose the precaval sinus of the same side; by means of a seeker find the apertures into it of the following veins or sinuses (compare Fig. II3) $-a$, the jugular; $b$, the injerior jugular; $c$, the cardinal; and $d$, the lateral vein.

III. -Insert the scissors into each external gill-cleft of one side, one by one, and extend them by cutting dorsally and ventrally, so as to expose the gill-pouches, communicating with the pharynx by the inzternal gill-clefts. Make out-

1. The branchial filaments, and observe that there are four complete gills on the first four branchial arches, and a half-gill or hemibranch on the posterior face of the hyoid arch. Note also the psezdobranch on the anterior side of the spiracle.

2. The structure of the gills. Remove two entire gills; dissect one, and cut the other across transversely (compare Fig. 109), noting the 
relations of the septum, cartilaginous branchial arch and rays, branchial filaments, and single afferent and paired efferent branchial artery. Sketch.

IV.-Cut through the floor of the pharynx and mouth close to the middle line, just on one side of the ventral aorta, and extend the cut through the lower jaw. On one side, turn the floor outwards, and pin it back in this position, so as to expose the roof of the mouth and pharynx and the internal gill-clefts; dissect away the mucous membrane lining the roof, and trace out on one side (Fig. 112)-

I. The epibranchial and efferent branchial arteries, and the dorsal aosta.

2. The carotid and subclavian arteries, \&c.

\section{F. Nervous System and Sense-Organs.}

I. - Remove with the knife the rest of the skull-roof and a few of the anterior neural arches, so as to expose the entire brain and the anterior part of the spinal cord. In doing so, be careful not to injure the contents of the orbit, the nerves referred to on p. $45^{8}$, or the auditory capsule of one side. After noting the dura mater and pia mater, make out-

I. The subdivisions of the brain (olfactory labes, prosencephaton, diencephalon, optic lobes, cerebellum, and medulla oblongata). Sketch.

2. The origins of the cerebral nerves from the brain and the points at which they penetrate the walls of the skull (pp. 445-447).

3. The spinal cord, and the alternating dorsal and ventral roots of the spinal nerves. Then cut through the spinal cord just behind the medulla oblongata, and through the origins of the cerebral nerves. Remove the brain and place it in formaline or spirit.

II.-Carefully dissect away the skin covering the head and pharyn. geal region on the undissected side, expose the orbit, and remove the delicate connective tissue surrounding its contents. Pin down firmly, and dissect out the following from the side (compare Fig. II6 and pp. 445-449).

I. The ophthalmic division of the facial nerve, and immediately below it that of the trigenina: trace them backwards to their foramina in the skull-wall and forwards through a canal between the olfactory capsule and the cranium, to their distribution.

2. The large mass of sensory (ampullary) canals on the dorsal side of the snout. 
3. The four recti and the two oblique eye-muscles (Fig. II7), and the nerves (III, IV, VI) supplying them.

4. The eye, and the optic nerve-anterior to the recti muscles. The eye may now be removed by cutting through the muscles and optic nerve, and dissected as directed on p. I9I, noting its structure as before.

5. The large, flat, maxillo-mandibular division of the trigeminal, running forwards and outwards along the floor of the orbit, and there dividing into maxillary and mandibular branches.

6. The facial nerve, entering the orbit close behind the maxillo-mandibular nerve, and giving off: behind the spiracle-a large hyomandilular branch, passing along the anterior border of the auditory capsule and posterior wall of the orbit, and down the anterior side of the hyoid arch just beneath the skin: and in front of the spiracle-a palatine and prespiracular branches. Of the branches to the sensory canals, the ophthalmic has already been seen; the buccal and external mandibular require very careful dissection in order to make them oút satisfactorily.

7. The glossopharyngealand vagus nerves. To expose these, slice away sufficient of the auditory capsule (noting as you do so the semicircular canals and the endolymphatic duct) to expose the foramina by which they emerge from the skull, behind the auditory capsule, and separate the mass of muscles lying alongside the vertebral column from the branchial apparatus, by dissecting away the connective tissue. Trace the glosso. pharyngeal to its bifurcation over the first gill-cleft, and in the vagus follow out $-a$, the four branchial branches, forking over the remaining gill-clefts; $b$, the visceral branch; and $c$, the lateral line branch, above and to the inner side of the branchial branches.

8. Separate some of the ampullary sensory tubes rrom one another, and note the ampulle and the nerves supplying them.

9. Carefully slice away the cartilage of the auditory capsule of the side you have not already dissected so as to expose the membranous labyrinth. Examine under water, and make out the vestibule with its contained otolithic mass, the three semicircular canals with their ampulle, and the branches of the auditory nerve (compare Fig. 59).

III. Now examine the preserved brain from above, from below, and from the side, making out, in addition to the parts already noticed (\$ F, I, I)-

I. The optic chiasma, infundibulum- with an oval swelling and a 
vascular sac on either side, pituitary body, crura cerebri, and, as far as possible, the origins of the nerves. Sketch from below.

2. On one side of the brain, cut into the olfactory lobe, prosencephalon, optic lobe, and cerebellum from above, so as to expose the olfactory ventricle, lateral ventricle, optic ventricle, and cerebellar ventricle. Then bisect the entire brain into right and left halves with a sharp scalpel, and examine the uninjured half in longitudinal section, noting in addition to the parts mentioned above, the third ventricle, foramen of Monro, iter, and fourth ventricle. Sketch.

G. Transverse Sections.-Cut thick transverse sections of an entire dogfish with a knife through-a. the anterior, and $b$. the posterior part of the head (pharyngeal region); c. about the middle of the body; and $d$. the tail. Make out the relations of the various parts and organs, and sketch the lateral half of each section.

A more satisfactory method than this is to obtain a very young dogfish, not more than $\frac{1}{2}$ inch in diameter, and after cutting it transversely into pieces about $\frac{1}{4}$ inch in thickness in the regions named above, stain, imbed, and mount a few sections from each piece (see p. 136). These can first be examined with a lens or with the low power of the microscope, and then, by putting on the high power, important points in the histology can be made out. In addition to the minute structure of the tissues and organs described in Part I. of this book, the structure of the notochord (p. 425), integumentary sense-organs (pp. 414, 418), dermal teeth (p. 4I7), \&c., should be studied.

H. Side Dissection.-It is very instructive to supplement and recapitulate your work on the anatomy of the dogfish by dissecting another specimen from the side (compare Fig. 108), as in the case of the crayfish. Open up the abdominal cavity as before, very slightly to the left side of the middle line. Continue the cut forwards through the pectoral arch and backwards through the pelvic arch : the arteries may be injected at this stage. Then dissect off the left half of each arch with the corresponding fin, and cut away the left body-wall. Cut through the skin in the mid-dorsal line, and then, working from the left side, remove the left half of the skull and visceral arches, as well as the left side of the vertebral column, so as to expose the brain and spinal cord. Remove the left kidney (and left oviduct if your specimen is a female). Pin down under water, and tidy up your dissection so as to reduce it to a neat longitudinal section, in 
which all the unpaired soft organs are left intact. Without tearing the mesentery, pin out the liver, stomach, and intestine beyond the ventral limits of the body-wall, so that the other abdominal organs are not hidden. Follow out the relations of the various organs as before, and sketch your dissection.

It is important at this stage to refresh your memory of the anatomy of the Frog, and to compare it with the Dogfish, by making a dissection from the side as directed above (compare Fig. 7). Sketch. 


\section{CHAPTER X}

CHARACTERS OF THE CLASS MAMMALIA-THE RABBIT

BEFORE examining a Rabbit, as an example of the highest class of Vertebrates-the Mammalia, it will be well to recapitulate some of the characters of the frog, the organisation of which is higher than that of a fish.

The frog, taken as an example of the class Amphibia, differs from a fish in the following points, amongst others. Its paired limbs have not the form of paddle-like fins, the forelimb consisting of upper arm, fore-arm, wrist and hand, and the hind-limb of thigh, shank, ankle, and foot, each with its characteristic skeletal parts; it has no median fin in the adult, and that of the tadpole is not supported by finrays ; there is no hard, dermal exoskeleton; respiration in the adult is pulmonary, and internal nostrils are present; there are two auricles in the heart, and the cardinal veins are replaced by a postcaval; there is a urinary bladder formed as an outgrowth of the cloaca. Moreover the skeleton, unlike that of the dogfish, is in the adult composed mainly of bone.

In all these characters the frog resembles the rabbit. But the Mammal differs from the Amphibian in many important respects, some of the chief of which are:-the presence o an epidermic exoskeleton consisting of hairs; the high 
temperature of the blood, which remains almost uniformly within a few degrees of $100^{\circ} \mathrm{Fahr}$., and does not vary to any appreciable extent with the temperature of the air; the absence of nuclei in the red corpuscles of the blood; the presence of mammary glands beneath the skin in the female, which secrete milk for nourishing the young; the subdivision of the body-cavity into two portions-thorax and abdomenby a transverse partition, the diaphragm; the presence of two ventricles as well as of two auricles in the heart, and of a single systematic left aortic arch; the higher differentation of the brain, and also of the skeleton; and the mode of articulation of the lower jaw. The teeth, again, of the rabbit, like those of the large majority of Mammals, are differentiated into front-teeth for biting or seizing the food and into cheek-teeth or grinders, and their succession is limited to two sets; an external ear or pinna is present; there is no cloaca, the anus and urinogenital apertures opening separately on the exterior, while the ureters open directly into the bladder; the ova are minute, and the young undergo their early development in the oviduct, where they are nourished by diffusion from the blood-system of the parent by means of an organ known as the placenta, and after birth, they are suckled by the mother.

Bearing in mind these essential characters of the higher Mammalia as compared with the Vertebrates previously studied, we can now proceed to examine the structure of the rabbit in greater detail.

External characters. The Rabbit (Lepus cuniculus) is a very abundant and widely distributed animal which in the wild state makes burrows in the earth in which the young are born; there are a number of varieties, the habits and general appearance of which have been modified by domestication (compare p. 227). 
In addition to head, trunk, and short tail, the rabbit possesses a distinct neck, and the whole animal, including the limbs and even the soles of the feet, is covered with a soft fur consisting of hairs (Fig. I2 I). In the wild rabbit, the fur is of a brownish colour, lighter below, and white under the tail : in the many domesticated varieties the colour is very varied.

The hairs correspond to modified epidermic cells, which become converted into a horny material; they are developed in tube-like involutions of the epiderm called hair-sacs, into the swollen base of each of which a mesodermal hair-papilla projects, the substance of the hair being formed from the epidermic cells covering the papilla. Into the hair-sacs open the ducts of sebaceous glands, the secretion of which serves to lubricate the hair.

There are five digits in the hand or manus, and four in the foot or pes, each terminated by a pointed and curved horny clarw, developed, like the hairs, from the epiderm. Along the ventral surface of the body in the female are four or five pairs of papillæ-the teats, on which open the ducts of the milk-glands, which correspond to modified sebaceous glands. The various parts of the skeleton (Fig. I I I) can be felt through the skin, and in addition to those already enumerated in the Vertebrates previously studied, it will be noticed that the anterior part of the trunk, or thorax, is surrounded by ribs, many of which meet below with a breast-bone or sternum, and which are absent in the posterior part of the trunk, or abdomen.

Beneath the anterior end of the snout is the transverse mouth which has a narrow gape and is bounded by upper and lower lips: the upper lip is divided by a longitudinal cleft which is continuous with the oblique, slit-like external nostrils. Just inside the lips are the upper and lower front teeth or incisors, which are chisel-shaped, and behind them the 
hairy integument is continued on either side into the cavity of the mouth. The eyes are protected by movable upper and lozeer hairy eyelids, as well as by a hairless third eyelid or nictitating membrane (compare p. 5), supported by cartilage and situated in the anterior corner of the eye, over which it can be partly drawn : it corresponds to the little red lump in the inner corner of the human eye. On the upper lip and above and below the eye are certain very long and stiff hairs-the "whiskers" or vibrissa, and behind the

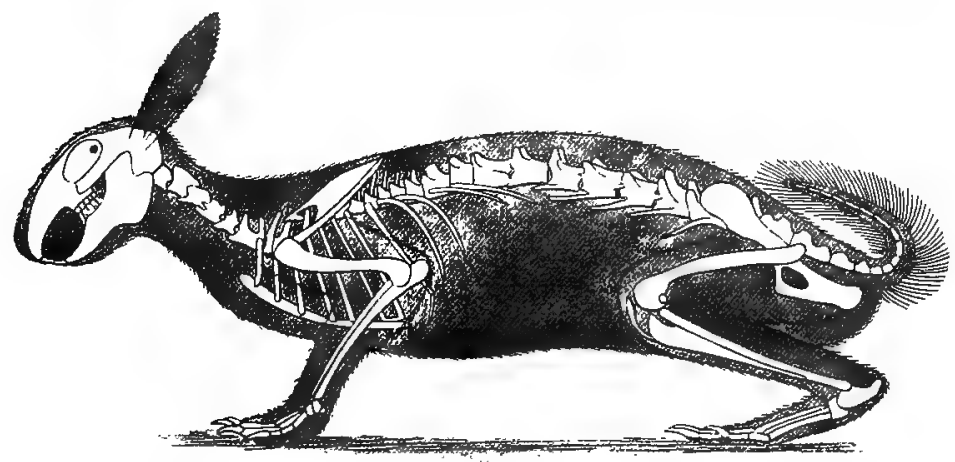

Fig. I2I.-Rabbit.

Lateral view of skeleton with outline of body. (From Parker and Haswell's Zoology.)

eyes are a pair of long and movable external ears or pinne: these are supported by cartilage and are somewhat spoutshaped, leading to the external auditory openings.

Below the root of the tail is the anus, and in front of and below this, the urinogenital aperture, the space between them being known as the perincum. On either side of these apertures is a hairless depression of the skin on which open the ducts of the perinceal glands, the secretion of which has a strong and characteristic odour. In the female the slit- 
like urinogenital aperture is called the vulva; in the male the aperture is smaller and situated on the conical apex of a cylindrical organ, the penis, which can be retracted within a fold of skin, the foreskin or prepuce. On either side of the penis is an oval pouch of the skin, the scrotal sac, not very apparent in young animals, in each of which a spermary or testis is contained.

Skeleton. The skeleton of the rabbit consists almost entirely of bone, but it must be remembered that in addition to certain cartilages described below, all articular surfaces are covered or lined by a thin layer of cartilage, and that the various parts of the skeleton are connected together by ligaments.

In the skull, both cartilage and membrane bones (p. 43) are much more numerous than in the frog, and the structure of the entire skull is far more complicated and highly differentiated. A posterior, relatively large, cranial region, in the side walls of which auditory capsules are embedded, can be distinguished from an anterior, somewhat conical, facial region, constituting the skeleton of the snout (Fig. I22). Just behind the junction of these two regions on either side is a large orbit, separated from its fellow by a thin interorbital septum, perforated by a foramen for the optic nerve (opt. fo), At the sides of the foramen magnum are the two rounded occipital condyles; the auditory apertures (aud. me) are situated at the sides of the posterior part of the cranium, and the external nostrils open at the anterior end of the snout. Most of the bones remain more or less distinct throughout life, and are in contact along lines or sutures, many of which are wavy or zig-zagged: others, again, become completely fused together, in the adult so that their limits are no longer distinguishable. 


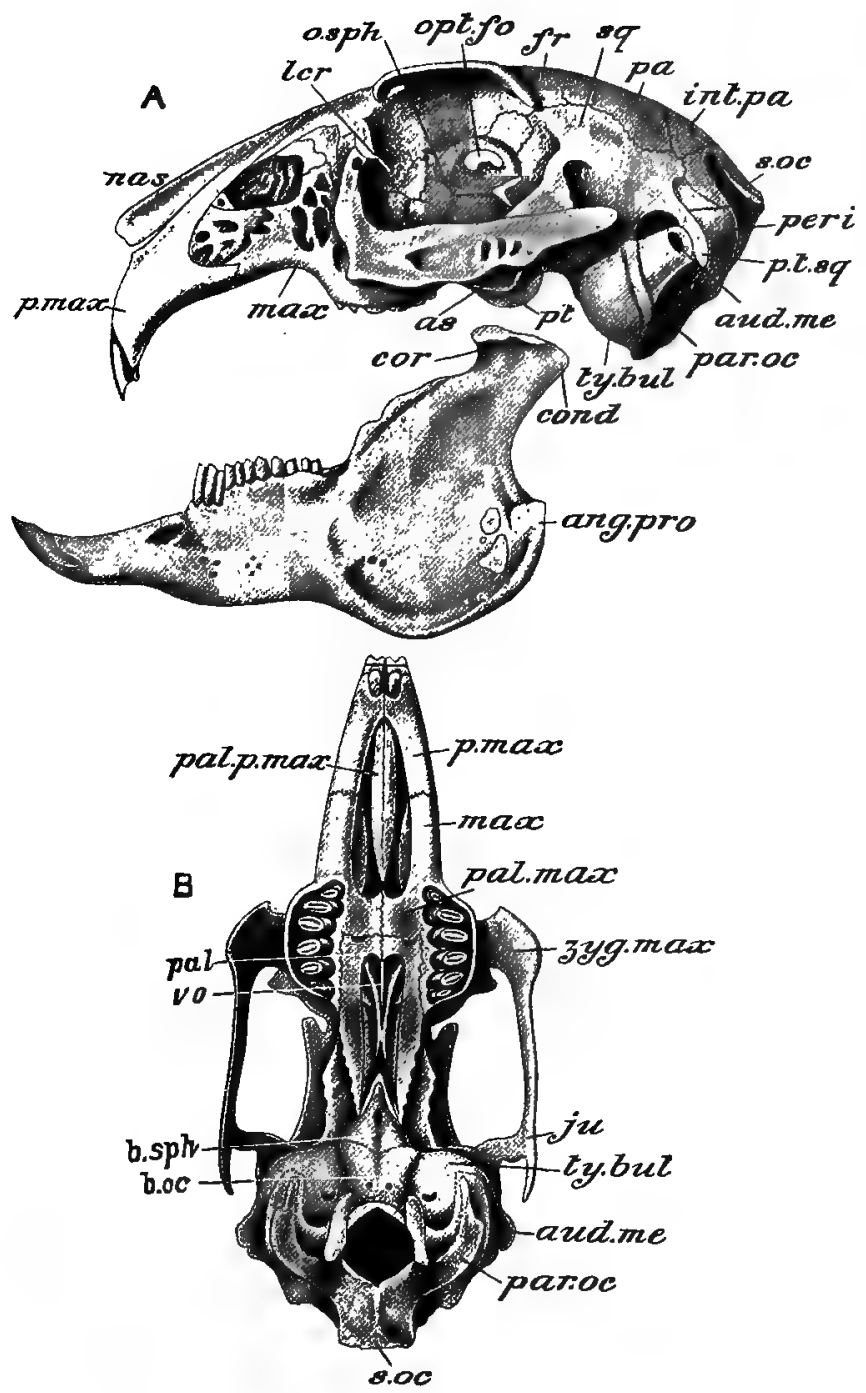

FIG. 122.-Skull of Rabbit. A, lateral view with lower jaw; B, ventral view. ang. proc. angular process of mandible; as, alisphenoid (external pterygoid process); aud. whe. external auditory aperture in tympanic bolne; $6.0 c$. basioccipital; 
b. sph. basisphenoid ; cond. is wrongly lettered-cor points just below the condyle; fr. frontal ; int. pa. interparietal; ju. jugal ; lcr. lachrymal ; max. maxilla; nas. nasal ; opt. fo. optic foramen; o. sph. orbitosphenoid ; pa. parietal ; pal. palatine; pal. max. palatine process of maxilla ; par. oc. paroccipital process of exoccipital ; pal.p. max. palatine process of premaxilla; p.max. premaxilla; peri. periotic; $p t$. pterygoid ; $p$. t.sq. post-tympanic process of squamosal ; s. oc. supraoccipital . sq. squamosal ; ty.bul. tympanic bulla ; vo. vomer ; zyg. max. zygomatic process of maxilla. (From Parker and Haswell's Zoology.)

The upper jaw forms part of the facial region, which encloses the olfactory chambers, and the lower jaw, consisting of a single bone on either side, articulates directly with the sides of the cranium without the intervention of a hyomandibular, as in the dogfish (p. 422) or of a quadrate cartilage, as in the frog (p. 44). The rest of the visceral portion of the skull, representing the hyoid and first branchial arch, forms the so-called hyoid bone, which is embedded in the base of the tongue (Fig. $x 25, h y$ ).

The bones ${ }^{1}$ which form the walls of the brain-case are arranged in three rings or segments, the middle and posterior of which are separated by the auditory bones (Figs. I 23 and I22, A, peri).

The posterior, or occipital segment, consists of three bones, which in the adult become completely united with one another. The lower of these is the basioccipital (b.oc), a flattened bone bounding the foramen magnum below, and forming the hinder part of the base of the skull and the lower part of each occipital condyle (oc.c). The two exoccipitals (e.oc) bound the foramen magnum at the sides, and form the upper part of the occipital condyles: each is produced downwards into a paroccipital process (par.oc) which fits closely against the posterior surface of a swollen bone ( $t y . b u t)$ to be described presently, which is produced into a tube surrounding the auditory aperture (aud. me). The occipital segment is completed above by the supraocipital (s.oc),

${ }^{I}$ In the following description, the membrane bones are distinguished by an asterisk from the cartilage bones. 
bounding the foramen magnum above ; it has a pitted surface and is marked externally by a shield-shaped prominence.

The middle, or parietal segment, consists of five bones, - - a basisphenoid (b.sph) below, an alisphenoid (a.sph.a.s) on either side, and two parietals* $(p a)$ above. The broad posterior end of the basisphenoid is connected with the basiocci-

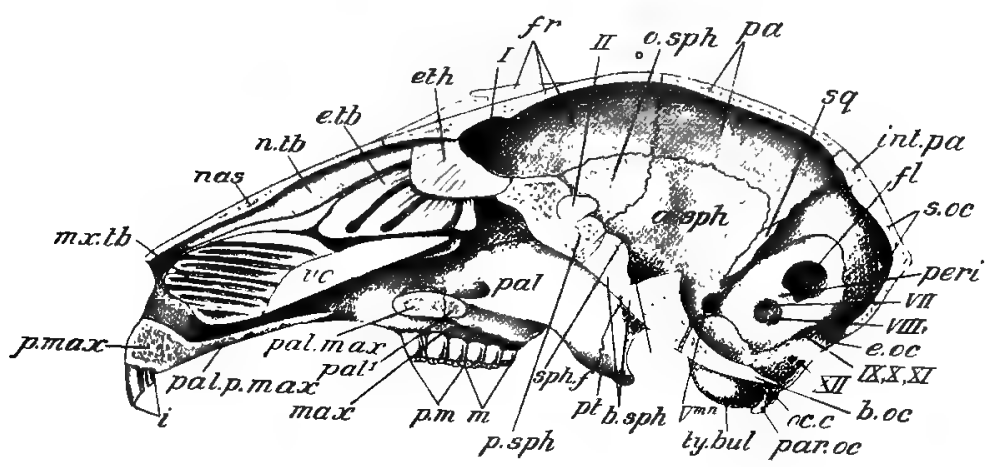

FIG. 123.-Skull of Rabbit in longitudinal vertical section. The cartilaginous nasal septum is removed.

a. sph. alisphenoid ; e. oc. exoccipital ; e. $t b$. ethmo-turbinal ; $e t h$. ethmoid ; $f$. fossa for flocculus of brain; $i$. incisors; $m$. molars ; $m x$. $t b$. maxillary turbinals ; $n . t b$. naso-turbinals; oc. $c$. occipital condyle; pal. palatine; $p a l^{\prime}$. palatine portion of the bony palate; $p . m$. premolars; $\not$. $s p h$. presphenoid; I. point at which the olfactory nerves leave the skull; iI. optic foramen; sph. $f$. sphenoidal fissure (compare Fig. I22 A, just below opt. fo.); $\mathrm{V} n n$. foramen for mandibular division of trigeminal; VII. for facial nerve; VIII. for auditory nerve; IX, X, XI, for glossopharyngeal, vagus, and spinal accessory ; XII. for hypoglossal. Other letters as in Fig. I22 : the unlettered line above $b$. sph points to the sella turcica, or depression in which the pituitary body lies.

pital by a thin plate of cartilage, and tapers in front to a blunt point: it is perforated at about its middle by an oval pituitary foramen, and on its upper surface is hollowed out to form a depression in which the pituitary body lies. The alisphenoids are wing-like bones, directed upwards and outwards, and firmly united with the basisphenoid : each is produced ventrally into a pterygoid process (as), consist- 
ing of two laminæ which converge and unite with one another anteriorly. The parietals are a pair of thin, slightly arched bones, forming a considerable part of the roof of the brain-case, and united with one another by suture along the middle line; the outer edge of each gives off a thin, ventral process which is covered by the squamosal $(s q)$, a bone which will be referred to presently and which separates the parietal from the alisphenoid. Interposed between the parietals and the supraoccipital is a small median interparietal* (int.pa).

The frontal segment also consists of five bones-a presphenoid, ( $p . s p h)$ two orbitosphenoids (o. sph) and two frontals* $(f r)$. The small presphenoid is laterally compressed and is connected with the basisphenoid by cartilage, so that in the dry skull there is a considerable interval between the two bones; it forms the inferior and anterior boundary of the optic foramen (opt. fo, $I I$ ), which puts the two orbits in communication with one another and both in communication with the cranial cavity. The orbitosphenoids are two wing-like laminæ directed outwards and slightly backwards, and completely fused with the presphenoid; they surround the rest of the optic foramen. The frontals form the, roof and side-walls of the anterior part of the brain-case and are united by suture with one another in the middle line and with the parietals behind; below they meet with one another anteriorly on the floor of the braincase and unite with the presphenoid by suture; the outer part of each forms a prominent crescentic ridge, the supra-orbital process.

The brain-case is closed in anteriorly by a bone riddled with numerous small holes for the passage of the olfactory nerves: this is the cribriform plate of the ethmoid (eth). 
It will be remembered that in the frog the occipital region is ossified by exoccipitals only, the parietals and frontals of either side are fused together, there are no ali- or orbito-sphenoids, the cartilaginous walls of the anterior part of the cranium are ossified as a sphenethmoid, and that the floor of the skull is supported by membrane bone, the parasphenoid.

The auditory capsules are comparatively loosely wedged in laterally between the parietal and occipital segments; in the embryo each is ossified from three centres, instead of one (the pro-otic) as in the frog, but these early unite to form the periotic bone (peri), as the ossified auditory capsule is called. The internal or petrous portion of this bone (Fig. I23) encloses the membranous labyrinth of the ear and is very dense and hard; posteriorly it is produced outwards as the porous mastoid portion, which is visible on the outer side of the skull (Fig. I24 A). Closely applied to the outer surface of each periotic is a bone called the tympanic,* consisting of a tubular portion above-the edge of which surrounds the auditory opening (aud. me) to which the cartilage of the pinna is attached, and of a swollen portion, or tympanic bulla (ty. bul) below: this encloses the tympanic cavity, and in it, at the base of the tubular portion, is an incomplete bony ring to which the tympanic membrane is attached (Fig. 133). The tympanic is incomplete on its inner side, where its cavity is closed by the outer wall of the periotic, and between the two, at the antero-inferior angle of the former, is the aperture by which the Eustachian tube leaves the tympanic cavity (compare p. 45). When the tympanic is removed, two small holes are seen on the outer wall of the periotic: the anterior of them is the fenestra ovalis and is plugged by the stapes-which together with two small bones, the malleus and incus (Fig. r33), forms the chain of auditory ossicles to be described later in connection with 
the organ of hearing; the posterior aperture is called the fenestra rotunda. On the internal or cranial surface of the periotic is a large depression $(f l)$ which lodges the flocculus of the cerebellum (Fig. $\mathrm{r}_{3} \mathrm{r}$ ).

The olfactory capsules are roofed in by two long and narrow nasal bones* (nas), which meet together in the middle line and unite by suture with the frontals posteriorly. Their side walls are formed by the bones which bear the teeth of the upper jaw-the premaxillæ $(\not$. max) and maxilla (max), and in the median line below is a single long and slender bone, deeply grooved on its upper surface, and formed by the fusion of the two vomers* $(v 0)$. The two nasal chambers are separated from one another in the middle line by a median vertical plate of cartilage, the nasal septum (Fig. I 25 n.s) embraced below by the vomer. This cartilage together with the cribriform plate and a median vertical plate of bone (eth) extending forwards from the latter into the septum, constitutes the mesethmoid. Within the nasal chambers certain scroll-like folds of the mucous membrane (Fig. 125) are present in order to increase the surface, and in these, cartilages are developed. The cartilages become ossified, and the resulting turbinal bones unite with certain of the bones enclosing the olfactory organs, and are named accordingly. The ethmoid turbinals (Fig. I 23 , e.tb) or true olfactory scrolls are two complicated, folded bones united to the cribriform plate of the ethmoid, and are covered in the fresh condition by the olfactory epithelium; the maxillo-turbinals ( $m x . t b$ ) are similar but more complex bones in the antero-ventral part of the nasal cavities; and the naso-turbinals (n.tb) are thin, folded bones, much less complex, and fused with the inferior surface of the nasals.

In the front wall of each orbit, fitting comparatively loosely 
between the frontal and maxilla, is a small bone, the lachrymal,* (Fig. I $22 \mathrm{~A}, \mathrm{lcr}$ ) with a notch near its outer border through which the naso-lachrymal duct passes (compare p. 186.)

As in the frog, the chief bones of the upper jaw on either side are the premaxilla * (p. max) and the maxilla* (max), and nearer the middle line are the palatine ${ }^{*}(p a l)$ and pterygoid* $(p t)$ : in the embryo the position of the two last mentioned bones is taken by cartilage representing the upper jaw of the dogfish (compare Figs. ro3 and 9). The premaxillæ, in which the sockets for the front or incisor teeth are situated, form the anterior boundary of the snout, and articulate with one another in the median line and with the maxilla behind: each gives off a nasal process passing backwards between the nasal and maxilla to the frontal, and a palatine process (pal. p. max) extending backwards along the palate in contact with its fellow of the opposite side. The maxillæ are large and irregular bones, parts of the sides of which are fenestrated, and in which the cheek-teeth are situated. From the inner and inferior edge of each, opposite the first two cheek-teeth, a horizontal palatine process (pal. max) is given off, which, articulating with its fellow of the opposite side, forms the anterior part of the bony support of the hard palate-this is of much less extent in the rabbit than in most mammals : from its outer side arises a zygomatic process (zyg. max) which forms the anterior part of the strong zygomatic arch extending below and externally to the orbit.

The palatines are thin, nearly vertical, bony laminæ, internal to the maxillæ, to which they are attached in front, while above they join the presphenoid and the pterygoid process of the alisphenoid. They bound the passage of the internal nostrils, and from the 
inner and anterior region of each is given off, opposite the third cheek-tooth, a horizontal, inwardly directed process $\left(p a l^{\prime}\right)$, which, articulating in the middle line with its fellow of the opposite side and in front with the palatine process of the maxilla, forms the posterior part of the bony support of the hard palate. The pterygoids are small irregular plates of bones attached to the posterior edge of the corresponding palatine and the pterygoid process of the alisphenoid; each ends ventrally in a backwardly curved process.

The squamosals* $(s q)$ are a pair of plates which overlap and complete the side-walls of the brain-case (p. 474) in front of the periotics: they articulate with the frontals, parietals, orbitosphenoids, and alisphenoids. From the outer face of each is given off a strong zygomatic process, which bears on its under surface the articular facet for the lower jaw, and further back a slender process (p.t.sq.) arises which is applied to the outer surface of the periotic.

The zygomatic processes of the squamosal and maxilla respectively are united by a flat bar of bone, the jugal* $(j u)$, which in the adult is fused with the last-named bone. All these three bones therefore take part in forming the zygomatic arch.

Most of the apertures for the transmission of the cerebral nerves have so far not been mentioned : the branches of the olfactory nerve, as we have seen, pass out through the numerous apertures in the cribriform plate (Fig. I23, eth), and the optic foramen (II) is situated between the orbitosphenoid and presphenoid. Behind and below the optic foramen is a vertical aperture-the sphenoidal fissure (sph. $f$ ) - between the basisphenoid and alisphenoid, which transmits the third, fourth, and sixth nerves, as well as the opththalmic and maxillary divisions of the fifth. Between the periotic and alisphenoid is a large space (Vmn), through the anterior part of which the mandibular division of the trigeminal 
leaves the skull. ${ }^{1}$ Between the mastoid portion of the periotic and the posterior border of the tympanic, at the juncture of the tubular and bulbous portions of the latter bone, is a small aperture-the stylomastoid foramen-which transmits the seventh nerve : it and the eighth (VII, VIII) enter the periotic just below the depression for the flocculus of the cerebellum $(f)$. A space (IX, X, XI) between the occipital condyle and tympanic bulla gives exit to the ninth and tenth, as well as to the eleventh nerve-which is not represented in the dogfish and frog; and the hypoglossal, which in mammals is counted as the twelfth cerebral nerve, passes out through two small apertures (XII) in the exoccipital, just anterior to the condyle. Various other apertures will be noticed in the skull and jaws: through some of these branches of certain of the above-mentioned nerves pass, while others transmit blood-vessels.

The lower jaw or mandible (Fig. I 22 A) consists of two halves or rami, each corresponding essentially to the dentary of the frog, which unite with one another in front, at the symphysis, by a rough surface, while behind they diverge like the limbs of the letter $V$. Each ramus is a vertical plate of bone, broad behind and tapering towards the front, where it bears the incisor teeth : further back, on its upper margin, are the sockets for the cheek-teeth, and behind them is an ascending portion which bears the condyle (cor) for articulation with the facet on the squamosal : in front of the condyle is a curved coronoid process. The postero-inferior border, which is rounded and inflected, is known as the angular process (ang. pro).

The hyoid is a small bone situated at the root of the tongue, anterior to the larynx (Fig. I $25 h y$ ). It consists of a stout body or basi-hyal, a pair of small anterior horns, representing the ventral ends of the hyoid arch of lower Vertebrates, and a pair of longer, backwardly-projecting pos-

1 In most mammals (e.g. dog, cat), the maxillary division of the trigeminal passes out through a separate foramen, behind the sphenoidal fissure; and the anterior part of the space referred to above is separated off as is a distinct foramen for the mandibular division, 
terior horns or thyro-hyals, attached to the larynx and representing the lower ends of the first branchial arch.

The vertebral column includes about forty-five bony vertebræ, each consisting of a centrum, a neural arch, ard various processes (compare pp. $36-38$ ), but becoming simplified towards the end of the tail. The centra have flat anterior and posterior surfaces, and are not connected by synovial articulations, as in the frog, but interposed between them are elastic intervertebral discs of fibrocartilage. In addition to the ossification which gives rise to the main part of the centrum, a separate flat disc of bone (Fig. I $24 e p$ ) is formed on the anterior and posterior surface of each. These epiphyses are characteristic of the vertebræ of all or nearly all mammals : they unite comparatively late with the centrum proper, and so in disarticulated skeletons of young animals they often come away from the main mass of the centrum and remain attached to the intervertebral discs.

In correspondence with the differentiation of the parts of the body, the vertebral column is divisible into five regions (Fig. I 2I) : the cervical in the neck, including seven vertebræ, the first two of which-called respectively the atlas and axis-are peculiarly modified in order to allow the skull free movement; the thoracic in the thorax, twelve or thirteen in number, and bearing ribs; six or seven lumbar in the abdominal region: three or four sacral in the sacral region: and about fifteen or sixteen caudal in the tail.

Examining one of the anterior thoracic vertebræ first (Fig. I24), we see that the centrum $(c)$ is continuous above with the neural arch (n. $a)$, the lower part of which, on either side, presents an anterior and a posterior notch $(i, v . w)$, so that when the vertebræ are in their 
natural position, an inlervertebral foramen is formed for the passage of a spinal nerve. The roof of the arch is continued into a long neural spine ( $n . s p$ ) projecting upwards and backwards, and just above the intervertebral notches are a pair of anterior and posterior articular processes or zygapophyses (pr. z, pt. z), which articulate synovially with the vertebre next in front and behind respectively. The articular surface of each pre-zygapophysis looks upwards and oulwards, that of the post-zygapophysis downwards and inwards. Arising laterally from each side of the arch is an outstanding transverse process $(t . p r)$, on the under surface of which is an articular tubercular facet, $(t . f)$ with which the upper fork of the rib (p. 483) articulates. The lower fork or head of the rib articulates with a facet $(c, f)$ formed partly by the anterior edge of the corresponding centrum just at the base of the neural arch, and partly by the posterior edge of the centrum next in front, so that each centrum bears half a capilatlar facel, as it is called, on cither side, both anteriorly and posteriorly $\left(c . f^{\prime}, \imath . f^{\prime \prime}\right)$.

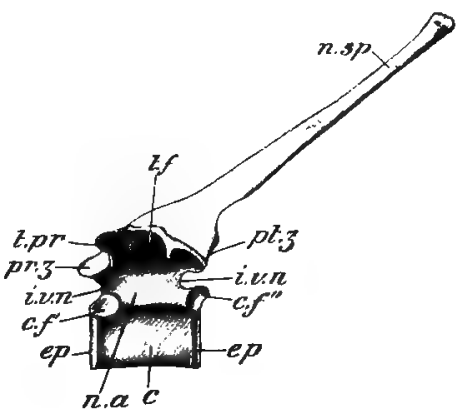

FIG. 124--Fifth thoracic vertebra of the rabbit, from the left side $\left(x+\frac{1}{2}\right)$.

c. centrum ; c. $f^{\prime}$. capitular half-facet for fifth, and $c . f^{\prime \prime}$. for sixth rib; $c p$. epiphysis; $i . v . n$. intervertebral notch; $n$. a. neural arch; $n . s p$. neural spine; pr. $z$. pre-zygapophysis ; $p t . z$. post-zygapophysis; t.f. tubercular facet for fifth rib; t. pr. transverse process.

There are no free ribs in the vertebræe of other regions, in which, however, they are represented in the embryo, but early fuse with the corresponding transverse processes.

The first cervical vertebra, or atlas, is ring-shaped, and its lower portion is narrow and unlike the other centra. The neural spine is small, and the transverse processes are broad horizontal plates, each perforated at its base by a vertebrarterial canal, through which the vertebral artery runs. On the anterior face of the lateral parts of the atlas are two concave articular facets for articulation wilh the occipital condyles of the skull, and on its posterior face are two smaller acets for articulation with the second vertebra. The second cervical vertebra, or Pract, Zool. 
axis, has its centrum produced anteriorly into a conical odontoid process, which fits into the lower part of the ring of the atlas and is held in its place by a ligament extending transversely across the latter : it is ossified from a distinct centre, and probably represents the true centrum of the atlas. The neural spine of the axis is elongated and compressed, and its transverse processes small and perforated each by a vertebrarterial canal. Zygapophyses are present only on the posterior face of the arch. In all the other cervical vertebræ, the transverse processes are also perforated by the vertebrarterial canal, and except in the seventh or last, are divided into dorsal and ventral lamellix. The zygapophyses resemble those of the thoracic vertebra described above. The seventh cervical vertebra has a longer spine than the others, and bears a pair of half facets on the posterior surface of its centrum with which the first pair of ribs in part articulate.

A typical thoracic vertebra has already been described. In the tenth, the neural spine is vertical, and in the remaining two or three, which are larger than the others, it slopes forwards. In the posterior three or four there are no tubercular facets, the ribs in this region not being forked; and the capitular facets are entire, and are situated on the corresponding centrum only. Additional processes are present above the pre-zygapophyses from the ninth thoracic vertebra onwards.

The lumbar vertebra are relatively large, increasing in size from before backwards, and their various processes are greatly developed. The neural spines are directed upwards and forwards, the transverse processes are large and project outwards, downwards, and forwards. As in the posterior thoracic vertebræ, there are stout processes above the pre-zygapophyses, and there is also a pair of more slender processes below the post-zygapophyses and a median ventral process projecting downwards from the centrum in the first two.

The sacral vertebræare fused together to form the sacrum, which supports the pelvic arch. The first-and to a less extent the second also-has large, expanded, transverse processes which articulate with the ilia: these are the sacral vertebrae proper, the others, which decrease in size from before backwards, are really the anterior caudal vertebræ which fuse with the true sacral vertebræ to form a compound sacrum.

The more anterior caudal vertebræ resemble those of the sacral region, but on passing backwards all the processes are seen to diminish in size, until nothing but the centra are left at the end of the tail. 
There are twelve or occasionally thirteen pairs of ribs, which have the form of curved rods, situated in the walls of the thorax, and articulating with the thoracic vertebra above and-in the case of the first seven-with the breastbone or sternum below: the remaining ribs do not reach the sternum (Fig. 12 I).

Each rib consists of a bony, dorsal, vertebral portion, and of a ventral, sternal portion consisting of cartilage which is calcified or only incompletely ossified. The dorsal end-the head or capitulum of the rib-articulates with the capitular facet on the centra, and the first nine have also a tubercle, a short distance from the capitulum, which articulates with the tubercular facet; just externally to the tubercle is a short, vertical process.

The sternum, which is developed in the embryo by the fusion of the ventral ends of the ribs, consists of six segments or sternebre, the first of which, or manubrium, is larger than the rest, and has a ventral keel. With the last is connected a rounded, horizontal, cartilaginous plate, the xiphisternum. The ribs articulate between the successive sternebræ except in the case of the first pair, the articulations of which are on the manubrium.

The chief bone of the pectoral arch is the flat, triangular scapula, the coracoid portion (compare p. 47) becoming early fused with it and forming a small, inwardly curved, coracoid process, situated anteriorly to the glenoid cavity at the lower end or apex of the scapula: the apex lies over against the first rib, and the bone inclines upwards and backwards to its dorsal base, which in the fresh condition, consists of a strip of cartilage, the supra-scapula. On its outer surface is a prominent ridge or spine, the free ventral edge of which is called the acromion, from which a process, the metacromion, projects backwards. The collar-bone or clavicle is never strongly developed in Mammals in which the fore-limb only 
moves in one plane-forwards and backwards: in the rabbit it is a small, curved, rod-like bone, attached by fibrous tissue at one end to the sternum and at the other to the coracoid process of the scapula, there being small cartilages at either end of it.

The relative positions of the bones of the fore-limb are at first sight somewhat difficult to understand owing to their having become altered in the course of development. In your own fore-arm the bones can be rotated on one another, so that the thumb can be made to point outwards or inwards; while in the rabbit the first digit has permanently the same position, pointing inwards. To understand this, extend your arm outwards with the thumb pointing away from the ground. The back of the hand and arm, continuous with the dorsal surface of the body, or back, is its dorsal surface; the palm of the hand, and the surface of the arm continuous with the chest, is its ventral szerface: the border of the arm and hand continuous with the thumb is the preaxial border; and that continuous with the little finger the postaxial border. This position is called the position of supination: if the fore-arm and hand be now rotated, so that the thumb points inwards, the position is that of pronation. While in this position, bend the elbow at right angles and bring it inwards close to the body; the preaxial border of the hand will now be on the inner side, and an examination of the bones of the fore-arm shows that they cross on another. It is in this position that the bones of the rabbit's forelimb are permanently fixed (Fig. Ior, and compare Fig. 8).

The proximal extremity of the humerus bears a rounded head for articulation with the glenoid cavity, in front of which is a groove for the tendon of the biceps muscle (p. 6I) ; and certain tuberosities for the attachment of muscles will also be observed. Its distal extremity presents a large, pulley-like surface or trochlea for the articulation of the bones of the fore-arm, and a deep depression or fossa, perforated by a foramen, on its posterior side, for the reception of the end of the ulna. The radius is the shorter, inner (preaxial) bone of the fore-arm, and is slightly curved. 
Its head presents a large clouble surface for articulation with the trochlea of the humerus, and its distal extremity a pair of slight concavities for the bones of the carpus: the shaft is flattened where it abuts against the corresponding flattened surface of the ulna. Near the proximal end of the last-mentioned bone is a cavity for the articulation of the humerus, and proximally to this, at the elbow, the ulna is produced to form a large olecranon process, which is received into the fossa on the humerus when the limb is extencled: its small distal end articulates with the carpus.

The carpus consists of a proximal and a distal row of small, nodular bones, which articulate with one another where they are in contact. The bones of the proximal row, beginning at the inner (preaxial) side, are the radiale and intermedium, articulating with the radius, and the ulnare, articulating with the ulna. In the distal row are five bones, the middle one of which is distinctly proximal to the other four, so as really to lie in the middle of the carpus : this is the centrale, the others constituting a row of distal carpals. Of these the first three articulate with the corresponding digits, the fourth, on the outer (postaxial) side, supporting the fourth and fifth digits, and really consisting of two carpals fused together. A small bone, the pisiform, articulating with the ulna and ulnare on the ventral side, is usually looked upon as a sesamoid bone, i.e., an ossification in the tendon of a muscle; but it may represent the vestige of a sixth digit.

The hand or manus consists of five digits, each made up of a metacarpal and phalanges, articulating with one another. The innermost (preaxial) digit-the thumb or pollex-is the shortest, and the third the longest: the former has two phalanges, the others three each, the distal or ungual phalanx of all the digits having a conical form, its dorsal 
surface being grooved for the firmer attachment of the horny claw. Small sesamoid bones are situated on the under or palmar side of the joints of the digits.

The ends of the long bones in both limbs are separately ossified as exiphyses (compare p. 480), which eventually unite with the shaft of the bone in question.

The pelvic arch consists of two lateral halves or innominate bones, the long axis of which is almost parallel with that of the vertebral column (Fig. I 2 I), and which are firmly united anteriorly and internally with the transverse processes of the sacral vertebræ by a rough surface, while ventrally they are connected together by cartilage at the pelvic symphysis. On the outer surface of each innominate bone, at about the middle of its length, is a deeply concave cup, the acetabulum, for articulation with the head of the femur : in it, in young rabbits, a triradiate suture can be seen, marking the boundaries of the three bones of which the innominate is composed (p. 50). Of these, the antero-dorsal is the ilium, which is connected with the sacrum. The postero-ventral portion of the innominate is perforated by a large aperturethe obturator foramen, through which a nerve of that name passes, the bone above and behind it being the ischimm, and that below it the pubis. Behind the obturator foramen the ischium has a thickened posterior edge or tuberosity, and then curves round and becomes continuous with the pubis, both bones taking part in the symphysis.

In young rabbits it will be noticed that the part of the pulbis which enters the acetabulum consists of a small, distinct epiphysis.

The hind-limb has undergone rotation forwards (Fig. 12I), so as to be brought, like the fore-limb, into a plane parallel with the median vertical plane of the body; but the rotation being forwards, and the bones of the shank not being crossed, the preaxial border is internal in the whole liml), and the original dorsal surface looks, on the whole, forwards. 
Close to the proximal end of the femur, on its inner (preaxial) border, is a rounded, projecting head for articulation with the acetabulum: the actual end of the bone is formed by a strong process, the great trochanter, while just distal to the head is a lesser trochanter, and opposite this, on the outer (postaxial) side, a third trochanter. The distal end of the bone bears two large condyles, separated from one another by a notch, for articulation with the tibia: this notch is continuous with a groove extending for a short distance along the anterior (dorsal) surface of the femur in which a large sesamoid bone, the knee-cap or patella, slides : the patella lies in the tendon of the extensor muscles of the leg, and is connected by ligament with the tibia. Two other sesamoid bones, the fabella, occur on the opposite side of the knee-joint.

The tilia, or inner (preaxial) bone of the shank, is much larger than the fibula, the distal half of which in the adult becomes completely fused with it. The proximal end of the tibia bears two slightly concave articular surfaces for the condyles of the femur, and distally it articulates with the tarsus : a prominent ridge-the cnemial crest, extends along the proximal end of its anterior (dorsal) surface. The distinct part of the slender fibula is attached proximally to the tibia.

The tarsus consists of six bones arranged in three rows. In the proximal row are two tarsals, of which the inner (preaxial) or astragalus-probably corresponding to two bones fused together, the tibiale and intermedium-has a large pulley-like surface for articulation with the tibia: while the outer (postaxial) - the fibulare or calcaneum-articulates with the fused end of the fibula, and is produced into a strong heel or calcaneal process. In the middle row is a single bone, the centrale of the tarsus, and the distal 
row is made up of three bones, the true first, together with the corresponding digit (hallux), being absent as a distinct bone. The second (apparent first) distal tarsal articulates proximally with the centrale, and distally with the innermost (preaxial) metatarsal: the third (apparent second) with the astragalus and the corresponding metatarsal: the fourth (apparent third), which corresponds to the two outer (postaxial) tarsals fused together, with the centrale and calcaneum and the remaining two digits.

The foot or pes consists of tour metatarsals with their phalanges, of which there are three to each digit. The metatarsal of the hallux, together with the corresponding distal tarsal, is probably represented by a distinct ossification which in the adult becomes fused with the second (apparent first) metatarsal, and forms a process on that bone which articulates with the centrale. The phalanges are similar to those of the manus, and sesamoid bones are also present on the under surface of the foot.

Muscles and Body-wall. - It will be remembered that in Amphioxus and the dogfish the muscles of the trunk arc divided up into myomeres (pp. 404 and 418), while in the adult frog the only indication of such a segmentation of the muscles is seen in the recti of the abdomen (Fig. I6, rct. $a b d$ ). In the rabbit nearly all trace of a segmentation of the muscles has also disappeared, and the muscular system, although similar in its general arrangement to that of the frog (compare Fig. 16), is more complicated and highly differentiated. $\mathrm{We}$ shall have occasion to notice certain of the muscles in the course of our examination of other organs.

Immediately beneath the skin, which consists of epiderm and derm (p. 1 28), the whole ventral region of the trunk 
and neck is covered by a thin cutaneous muscle, by means of which the rabbit is able to twitch its skin. Internally to this muscle in the female are the mammary glands (p. 467), which, when secreting, appear as long, whitish, branched masses, the ducts of which can be traced to the teats, on the apices of which they open by numerous small apertures.

A whitish band of connective tissue passes along the mid-yentral line of the abdomen from the xiphisternum to the pubis : this separates two longitudinal bands of muscle, the recti abdominis, from one another; and laterally to them, the aldominal wall consists of three thin layers of muscle with their fibres running in different directions-the externai oblique, the internal oblique, and the transversalis, the latter being lined on its inner surface by the peritoneum. A fibrous cord, known as Poupart's ligament, beneath which the blood-vessels and nerves pass outwards to the leg, extends upwards and forwards from each pubis to the anterior part of the corresponding ilium. In the thorax the nuscles of the body-wall are broken up into separate portions by the ribs, and thus form a series of ,intercostal muscles, which are, like the oblique muscles of the abdomen, arrangerl in two layers, external and internal, and are important in respiration.

Extending from the thorax to the fore-limb of either side are the large pectoral muscles; and a number of other muscles can be seen in the neck, in the ventral middle line of which, covered by the cutaneous muscle, the windpipe or trachea is visible. The trachea is strengthened by a series of cartilaginous rings, and ends in front in the larynx, situated between the two rami of the mandible; and just in front of the larynx is the hyoid bone (p. 479), embedded in a mass of muscle.

The Colome and its contents.-On cutting open the body-cavity, it will be seen to be divided into two main chambers - the thoracic and abdominal cavities-by means of the diaphragm (Fig. I25, d). The relatively small thoraxwhich is lined by a serous membrane corresponding to the 
peritoneum of the abdomen and known as the pleura-contains the lungs, as well as the heart enclosed in a pericardium, on the ventral surface of which is an organ known as the thymus (see p. 43I): the gullet and main blood-vessels also pass through the thorax. The abdomen encloses the greater part of the enteric canal, together with the liver, and pancreas, the spleen, and the urinogenital organs. The diaphragm is convex on its anterior side, towards the thorax: it consists of a central, thin, tendinous portion into which radial muscles are inserted. 'These arise from the vertebral column and posterior ribs, and are especially strong on the dorsal side, where they form two bands known as the pillars of the diaphragm. When the muscles contract, the diaphragm is made flatter, and thus the thoracic cavity is enlarged.

Digestive organs. - The mouth-cavity (Fig. I25) is large, and the small gape is bounded by upper and lower lips, behind which are the incisor teeth $(i)$. On either side of the cavity are the borders of the upper and lower jaws from which the cheek-teeth project: these are separated from the incisors by a considerable interval or diastema. Close behind the upper incisors are a pair of small openings leading into the naso-palatine canals (n.p.c), which communicate with the nasal cavities but must not be confounded with the internal nostrils. The roof of the oral cavity is formed by the palate, the anterior part of which, or hard palate $(h . p)$, is transversely ridged and partly supported by bone $\left(h . p^{\prime}\right.$, p. 477$)$; while the posterior part, or soft palate $(s . p)$ is smooth, its hinder, free edge forming a pendulous flap, the velum palati, on each side of which is a tonsil consisting of connective and lymphatic tissue and having the form of a small pit with a broad papilla on its 


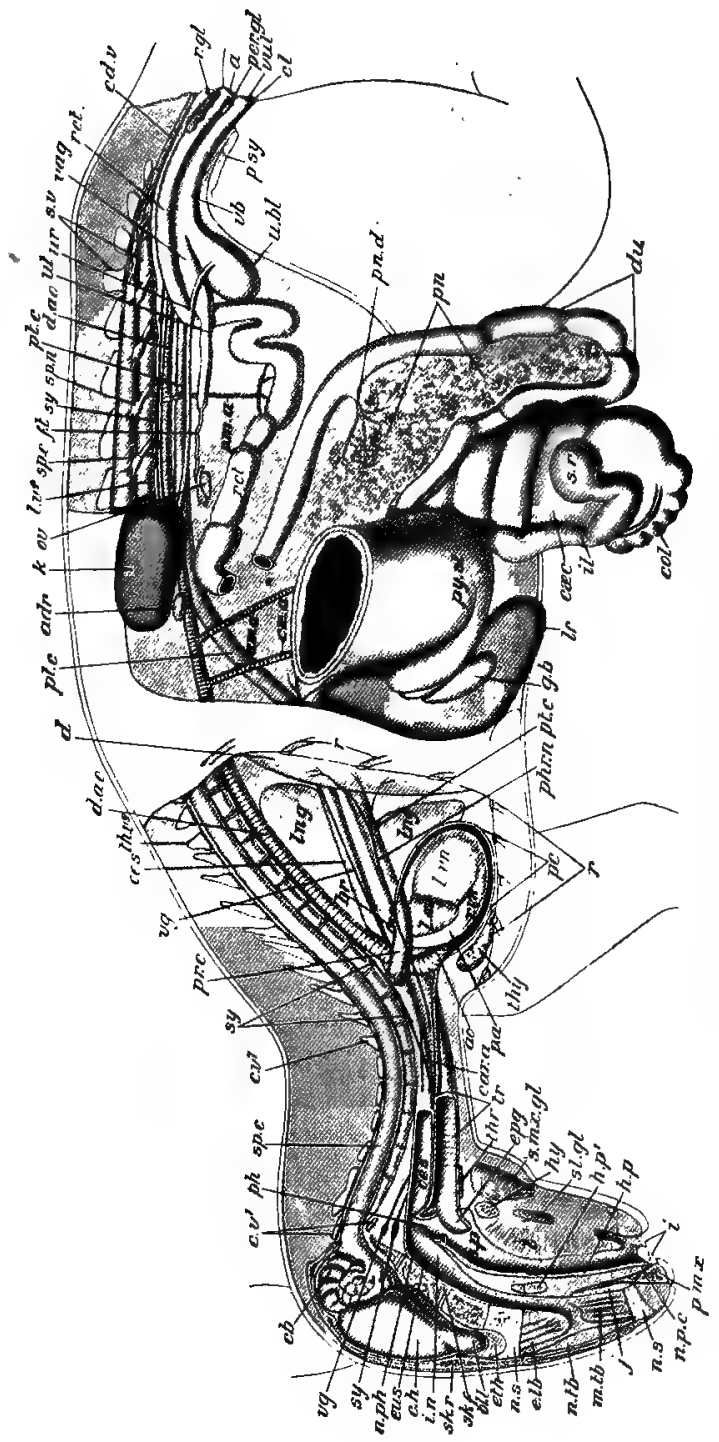

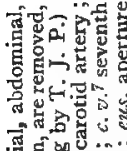

है हो

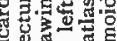

否

过

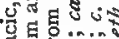

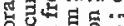

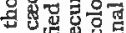

晏

पू 8 है

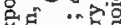

ง

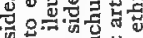

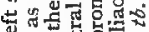

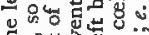

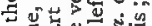

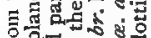

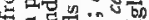

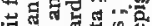

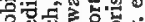

전

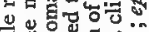

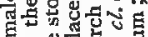

造 8 夏的

त

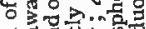

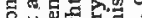

.

罂安

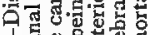

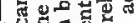

in

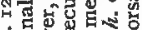

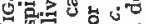

I

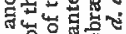
$=0.0$ \& 공 = तु 저

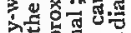
ํ.. की

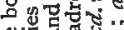

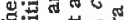

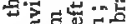

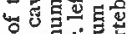

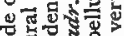
는

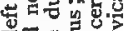

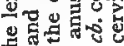
तै 
of Fustachian tube ; f.t. Fallopian tube ; $g . b$. gall-bladder; $h . p$. hard palate; $h$. $p^{\prime}$. bones of hard palate; $h y$. hyoid ; $i$. incisors ; $i l$. ileum ; $i . n$. passage of internal nostrils; $j$. Jacobson's organ; $k$. left kidney; $l$. au. left curicle; lng. left lung, seen through pleura; $l r$. liver; $l v_{0} 4$. fourth lumbar vertebra; $l . v n$. left ventricle; $m$. th. maxillary turbinal; $n ., p . c$. naso-palatine canal; $n . \not h h$. naso-pharynx; $n . t b$. naso-turbinal ; $n$.s. nasal septum (middle part cut away); as. gullet; $o l . l$. olfactory lobe; $o v$. left ovary; $p . a$. pulmonary artery; $p c$. pericardium; per. gl. perineal gland; ph. pharynx; phr. n. phrenic nerve (origin not shown); $p . m$. $a$. posterior mesenteric artery; $p$. mx. premaxilla; $\not n$. pancreas; $p n . d$. pancreatic duct; $p r . c$. left precaval; $p . s y$. pelvic symphysis ; pt. $c$. postcaval; $\not y$. st. pyloric region of stomach; r. ribs; rct. rectum; $r . g l$. rectal gland; $r$. vnt. right ventricle; sk. f. floor, and $s k$. $r$. roof of skull; sl. gl. sublingual gland; $s . m x . g l$. submaxillary gland; s. p. soft palate, ending in the velum palati, on the lower sicle of which a tonsil is seen; sp. $c$. spinal cord; $s p . \eta$. spinal (lumbar) nerves; $s . r$ sacculus rotundus of ileum; $s . v$. first sacral vertebra; sy. sympathetic (the interior end shown on the right side, the rest on the left); $t$. tongue; thr. thyroid; th. $x^{2} \cdot 9$ ninth thoracic vertebra; thy. thymus; $t \nu$ trachea; $u . b l$. urinary bladder; $w$. ureter; ut. utenss; zag. vagina; $v b$. vestibule; $v g$. vagus (the anterior end shown on the right side, the rest on the left); zul. vulva.

outer margin. The tongue $(t)$ lies on the floor of the mouth to which it is attached below, its anterior, rounded end being free : the surface of its posterior part is elevated, and clsewhere-but more particularly on the tip-its covering of mucous membrane is produced into minute, fingershaped papilla, on which some of the microscopic organs of taste are situated (compare p. I 80). Taste-organs are also present on a pair of circumvallate papilla on the dorsal side of the tongue towards its posterior end, and on a pair of transversely ridged areas-the foliate papilla, situated laterally, slightly anterior to the-former. The main substance of the tongue is composed of muscles, some extrinsic-i.e. arising from other parts, and others intrinsic-i.e. entirely confined to the organ in question.

The teeth (Figs. 122 and 123 ), as we have seen, are not all alike, as in the dogfish and frog : there are incisors and cheekteeth or grinders, the latter being divisible into two seriesthe premolars and the molars. In most Mammals there is also a pair of canine teeth, situated between the incisors and premolars, and these are especially long and pointed in such carnivorous animals as the dog and cat. In the dogfish and frog, again, the teeth are continually renewed as they 
become worn out, but in Mammals there are never more than two functional sets, which are known respectively as the deciduous or "milk"-teeth, and the successional or permanent teeth: certain of the former may even be absorbed before birth, as is the case with the incisors of the rabbit. The incisors and premolars (and in Mammals in which they are present the canines also) have deciduous predecessors, the molars developing behind the premolars and having no predecessors.

All the teeth are embedded in sockets or alveoli of the jaw-bones, and each contains a pulp-cavity (Fig. I26, $P H$ ) extending into it from the base and containing bloodvessels and nerves. In the case of the rabbit, the aperture of the pulpcavity $\left(P H^{\prime}\right)$ remains wide open in each tooth, and the substance of the tooth is continually added to at its base as it wears away at the other end: in many Mammals, however (e.g., dog, cat, man), the aperture becomes narrowed and

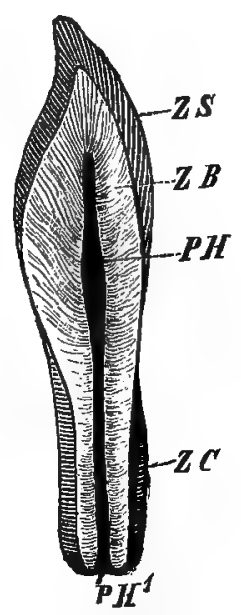

FIG. I26. - Longitudinal section of a mammalian tooth, semidiagrammatic.

$P H$, pulp-cavity; $P H^{\prime}$. opening of same; $Z B$. dentine; $Z C$. cement; $Z S$. enamel. (From Wiedersheim's $A$ na. tomy.) growth ceases after a time, the base of the tooth forming one or more roots or fangs. The main substance of each tooth is formed of dentine $(Z B)$, into which the pulp-cavity extends for a considerable distance and round which the enamel $(Z S)$ forms an external layer, which may become more or less folded inwards (as in the cheek-teeth and front upper incisors of the rabbit), the cement $(Z C)$ extending into the folds (compare p. 429). 
The number of the various teeth in the jaws is conveniently expressed by a dental formula, in which the kind of tooth (incisor, canine, premolar, or molar) is indicated by the initial letter $i, c, p m$, or $m$; and the whole formula has the arrangement of four vulgar fractions, in each of which the numerator indicates the number of teeth in the upper, the denominator that of those in the lower jaw, only those of one side being indicated, since the teeth of the right and left sides are always the same. Thus the dental formula of the rabbit is $i \frac{2}{1}, c \frac{0}{0}, p m \frac{3}{2}, m \frac{\overrightarrow{3}}{3}=28$.

The anterior incisors in the upper jaw of the rabbit are long and greatly curved. They are surrounded by enamel, which is much thicker on the anterior surface, where it presents a median groove; the posterior upper incisors are much smaller and are situated behind the anterior ones. In the lower jaw the single pair of long and curved incisors have no median groove, and they bite between the anterior and posterior upper incisors : owing to the thick layer of enamel anteriorly, they, like the large upper incisors, wear away less quickly in this region, and thus remain sharp, like a chisel, at their biting edges. The premolars and molars, on the other hand, are modified for grinding the food, to do which satisfactorily it is necessary that they should have broad crowns with a surface which remains uneven. This is effected in most of the cheek-teeth by the enamel becoming involuted along the outer side in a longitudinal direction, so as to form a groove extending into the dentine almost to the other side, the groove becoming filled up with cement. As the enamel is harder than the dentine and cement, it thus gives rise to ridges as the crown wears. The first upper premolar and the last upper and lower molars are simpler than the others, and the first lower premolar presents two grooves.

Connected with the mouth-cavity are several pairs of salivary glands, not present in the other Vertebrates we have examined, the secretion of which-saliva-contains a ferment called ptyalin, which is capable of converting starch into sugar. The food taken into the mouth is ground up 
or masticated and mixed with the saliva before passing down the gullet, and thus digestion begins in the mouth.

The infra-orbital salivary gland is a large, lolulated, pinkish mass situated in the antero-ventral region of 'the orbit, below and in front of the eyeball : its duct passes downwards to open into the mouth, nearly opposite and externally to the second premolar. The parotid gland is a soft, irregular, flattened organ, lying close beneath the skin, just below and in front of the base of the external ear; its duct passes forward and opens close to the duct of the infra-orbital gland. The sub. maxillary gland (Fig. 125, s.mx. $g l$ ), is a reddish, ovoidal, compact body situated inside the angle of the lower jaw and near the middle line, somewhat anterior to the larynx : its duct runs forward to open into the mouth a short distance behind the lower incisors. The sublingual gland (sl. gl) is an elongated structure situated on the inner side of the mandible, and having several ducts opening independently into the mouth.

The oral cavity is continued backwards as the phorynx $(p h)$ : this begins at the velum palati, above which it extends forwards as the naso-pharynx (n. ph); the latter is continuous with the passage of the internal nostrils, and into it open the Eustachian tubes (eus, compare pp. I 7 and 45). On the floor of the pharynx, behind the base of the tongue, is the glottis, which leads into the larynx and is guarded. in front by an elastic, leaf like, cartilaginous flap, the epiglottis $(e p g)$ : this projects upwards towards the velum palati and is capable of being pressed backwards over the glottis during the passage of food from the mouth to the gullet.

The gullet (Figs. 125 and 127 , es) is a narrow but dilatable tube which passes backwards along the neck and through the thorax, entering the abdomen through an aperture in the diaphragm and then opening into the stomach ( $p y . s t, c a . s t)$, a wide, curved sac, elongated transversely and greatly dilated at the cardiac end, which lies towards the 


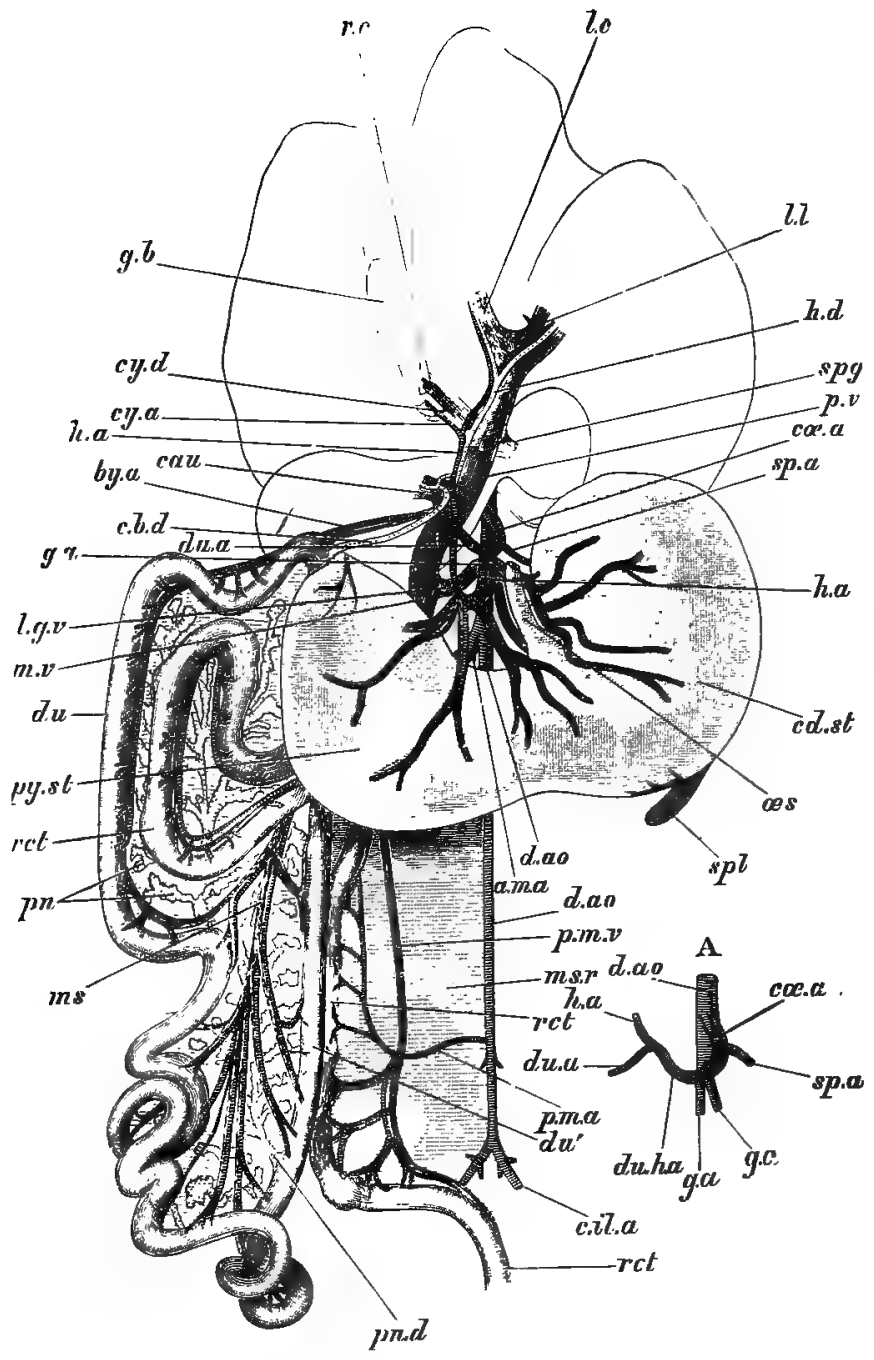

Fig. 127.-The stomach, duodenum, posterior portion of rectum, and liver (in outline) of the Rabbit, with their arteries, veins and ducts. $A$, the colliac artery of another specimen (luoth $\times \frac{2}{3}$ ). The gullet is cut through and the stomach some- 
what displaced backwards to show the ramifications of the coeliac artery $\left(c a^{3}, a\right)$; the duodenum is spread out to the right of the subject to show the pancreas $(m)$; the branches of the bile-duct $(c, b . d)$, portal vein $(\not . v)$ and hepatic artery $(h, a)$ are supposed to be traced some distance into the various lobes. of the liver.

a. $m$. $a$. anterior mesenteric artery ; can. caudate lobe of liver with its artery, vein and bile-duct; $c . b . d$. common bile-duct; $c d$. st. cardiac portion of stomach; $c . i l . a$. common iliac artery; $c e . a$. coeliac artery; $c y . a$. cystic artery; $c y . d$. cystic duct ; $d$. ao. dorsal aorta ; $d t$. proximal, and $d u^{\prime}$. distal limbs of duodenum ; du. $a$. and $b y . a$. duodenal artery; $d u . h . a$. (in $A$ ), duodeno-hepatic artery; $g . a$. gastric artery and vein; $g . b$. gall bladder; $h . a$. hepatic artery; $h . d$. left hepatic duct; l.c. left central lobe of liver, with its artery, vein, and bile-duct; $l . g . v$. lieno-gastric vein ; $l . l$ left lateral lobe of liver, with its artery, vein, and bile-duct; $m s$. branch of mesenteric artery and vein to duodemum; ms. $r$. mesentery of the rectum; $m . v$. chief mesenteric vein ; as. gullet; $p . m . a$. posterior mesenteric artery; $\not, m, v$. posterior mesenteric vein; $p, n$. pancreas; $p n, d$. pancreatic duct; $\not . v$. portal vein; $\not y$. st. pyloric portion of stomach; $r c t$. rectum; $r$. c. right central lobe of liver, with artery, vein, and bile-duct; spg. Spigelian lobe of liver with its artery, vein, and bile-duct; $s p l$. spleen; $s p$. $a$. splenic artery, (From Parker's 7ootomy.)

left side of the body: the pyloric end, from which the duodenum arises, towards the animal's right, is less dilated and has much thicker muscular walls. The mucous membrane of the stomach, in which the microscopic gastric glands (p. I $3 \mathrm{I}$ ) are contained, is raised into ridges or rugæ, and there is a circular pyloric valve at the entrance to the intestine.

The duodenum ( $d u$ ) extends backwards along almost the whole length of the abdomen and then turns forwards again, forming a slightly coiled, U-shaped loop, and becoming continuous with the very long and coiled, second portion of the small intestine or ileum (Fig. $125, i$ ), which finally dilates to form a rounded sac $(s . r)$ opening into the proximal end of the dark-coloured colon ( $c o l)$ or first portion of the large intestine. The colon has a much greater diameter than the small intestine, and presents a series of sacculations arranged in three rows, separated by flat regions of its wall : it passes insensibly into the second portion of the large intestine or rectum ( $r c t)$, which is of about the same diameter as the small intestine, and is recognisable by its rounded swellings containing the pill-like fæces: it passes into the pelvic cavity to open by the anus $(a)$. At the junction of the ileum and colon is a relatively enormous blind-gut or

Praçt. Zool, K K 
crecum (cac) - a structure not met with in either the dogfish or the frog, and only reaching such a relatively large size as in the rabbit in certain other herbivorous Mammals in which the stomach has a simple form: in those which possess a complicated stomach (viz., Ruminants) the cæcum is comparatively small. It is continuous with the proximal end of the colon, which contains an intra-colic valve and into which the round sac at the distal end of the ileum opens by a circular aperture provided with an ileo-colie valve. From this point arises the thin-walled cæcum, which lies coiled on itself amongst the folds of the rest of the intestine: it is about an inch in diameter, and a spiral constriction is seen on the outside marking the attachment, on the inside, of a spiral valve-like that of the dogfish's intestine but narrower-which makes about twenty-four turns and ends at the base of a blind, finger-shaped process, the vermiform appendix, which forms the apex of the cæcum. The whole canal is supported by a mesentery (p. 27) which has a very complicated arrangement in correspondence with the numerous folds of the intestine.

It will be noticed that the intestine is much more differentiated as regards its subdivisions than in the Vertebrates previously examined, and also that it is relatively much longer, being fifteen or sixteen times as long as the body.

On cutting open the small intestine, its mucous membrane is seen to be raised into minute, finger-shaped elevations or villi, and here and there certain patches present a honeycombed appearance: these portions are known as Peyer's patches, and, like the tonsils, thymus, and spleen, consist of masses of lymphoid follicles composed of a connective-tissue framework in which numerous leucocytes are imbedded. Other so-called "lymphatic glands" or adenoids are present in the mesentery and elsewhere. Peyer's patches also occur in the proxinal end of the colon, close to the ileo-colic aperture; and the round sac with which the colon communicates, as well as the vermiform appendix, are lined with 
similar lymphoid tissue. The mucous membrane of the colon has no villi, but, like that of the spiral valve, is raised into small papillæ; while that of the rectum is smooth.

The liver (Figs. 1 25, lr, and I 27 ) is a large organ, consisting of five lobes. Its anterior surface is convex and is applied to the diaphragm, its posterior concave surface fitting against the stomach. A median vertical fold of the peritoneum attaches it to the diaphragm, and marks the boundary between the right and left central lobes (Fig. I 27 r.c, l.c). Externally to the left central lobe, between it and the cardiac end of the stomach, is the left lateral lobe (l. l), and externally to the right central lobe the caudate lobe (cau), applied to the pyloric end of the stomach and hollowed posteriorly, when it fits over the right kidney: a small Spigelian lobe (spg) fits closely against the concave anterior surface of the stomach. The bile-duct is made up of several hepatic ducts ( $h . d)$ from the various lobes of the liver, as well as of a cystic duct (cy. d) from the pear-shaped gallbladder $(g . b)$, which is imbedded in the right central lobe of the liver: the common bile-duct $(c . b . d)$ thus formed opens into the dorsal side of the duodenum by a prominent aperture a short distance beyond the pylorus.

The pancreas (Figs. 125 and $127 p n$ ) is a diffuse gland, consisting of a number of small lobules looking not unlike masses of fat, spread all over the mesentery which connects the two limbs of the duodenum. The small ducts from the various lobules run together to form the main pancreatic duct $(p n . d)$ which opens into the distal limb of the duodenal loop a couple of inches or so beyond the bend.

The spleen (Fig. I $27, s p l$ ) is a long, flat body of a dark red colour, attached to the cardiac end of the stomach by a sheet of peritoneum. 
Organs of respiration and voice. - Owing to the presence of a neck, the lungs are situated some distance from the glottis (Fig. 125), and, instead of a short laryngo-tracheal chamber, as in the frog (p. I4I), there is a windpipe or trachea (tr) extending along the neck (p. 489), just ventrally to the gullet, its anterior end forming the larynx or organ of voice, and communicating with the pharynx through the glottis. The cartilaginous rings of the trachea are incomplete dorsally, and the cartilages of the larynx are more highly differentiated than in the frog, apart from the presence of an epiglottis (epg, p. 495). The largest and most anterior laryngeal cartilage is the thyroid, which, like the epiglottis, is peculiar to Mammals : it has the form of a broad ring, incomplete dorsally, and is the part of the larynx which can be felt externally. The second cartilage is the cricoid, represented in the frog by a ring-shaped cartilage at the base of the lungs (p. I 53): its form is somewhat like that of a signet ring, being broad dorsally-where it lies mainly between the edges of the thyroid-and narrow ventrally. A pair of arytenoid cartilages are articulated to the dorsal and inner surface of the cricoid, and each is produced into a projecting process situated between the two edges of the thyroid cartilage. The vocal cords (p. 144) are a pair of elastic folds extending across the cavity of the larynx from the thyroid below to the arytenoids above, each bounded in front by a depression.

In the position of rest, the vocal cords lie at an acute angle to one another, as in the frog; they can be brought into parallelism and regulated by the action of a number of intrinsic and extrinsic muscles, and are set in vibration by the respiratory current of air.

After entering the thorax, the trachea divides into two bronchi (Fig. I 25, br), one entering each lung and giving off branches to its different lobes : the bronchi, like the trachea, 
are supported by incomplete cartilaginous rings at their anterior ends, but these gradually disappear after they have entered the lungs.

The elastic lungs (Figs. I 25 and I 28, lng) are not hollow sacs, like those of the frog, but are spongy bodies, of a light pink colour, situated on either side of and above the heart, and filling the greater part of the thoracic cavity but collapsing as soon as the wall of the thorax is perforated. Each is subdivided into two main lobes, and the right lung has in addition two small accessory lobes, an anterior and a posterior, the latter lying in the median line, behind the heart, and being closely applied to the gullet.

Each pulmonary artery (Fig. I25, p,a,) crosses the main bronchus anteriorly to the point at which it branches into the various lobes, except in the case of the anterior accessory lobe, the bronchus to which comes off in front of the artery and may even arise from the trachea before its bifurcation. Microscopic examination shows that the bronchi divide and subdivide to form a ramifying system of tubes, each ultimate branch of which opens into a minute chamber or infundibulum, which in structure closely resembles a frog's lung in miniature.

The parietal layer of the pleura (p. 490) lines the cavity of the thorax, and is reflected over each lung at the entrance of the bronchus to form the visceral layer : in the median line it forms a vertical partition, the mediastinum, with which it is continuous ventrally to the vertebral column above and beneath the pericardium below (Fig. 128). Thus each lung (l. lng, r.lng) has its own separate pleural cavity (l. pl,r.pl), separated from its fellow by the right and the left mediastinum, the space between which is called the mediastinal space. The anterior and dorsal parts of this space are narrow and enclose the posterior part of the trachea and the bronchi, as well as the gullet (oes) and main blood-vessels (aort, $a z . v$, pt.cav) ; its middle part is wide, and encloses the heart 
(l. vent, $r$. vent $)$, the mediastinum here fusing with the visceral layer of the pericardium (vis. per) and thus obliterating the space; below this it again narrows to form the ventral mediastinal space ( $v$. med), in which the thymus (p. 490) is situated.

In the entire animal, the air-tight pleural cavities are

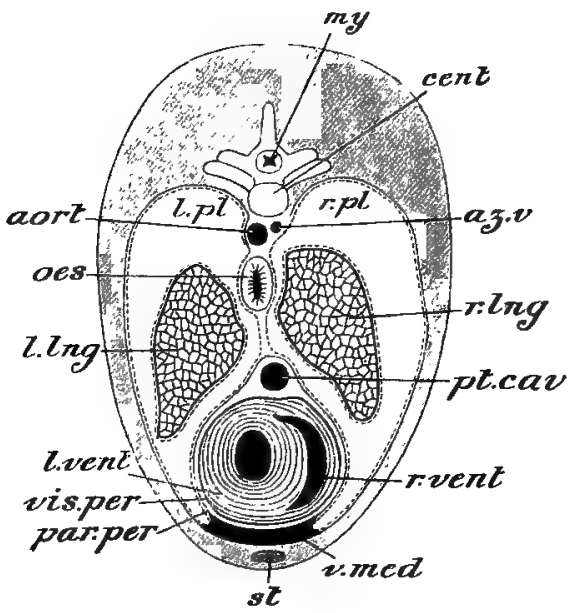

FIG. 128.-Diagrammatic transverse section of the rabbit's thorax in the region of the ventricles, to show the relations of the pleurse and mediastinum (dotted line), etc. The lungs are contracted.

aort. dorsal aorta; az. $v$. azygos vein ; cent. centrum of thoracic vertebra; l. lug. left lung; $l . p l$. left pleural cavity; $l$. veut. left ventricle; $m t y$, spinal cord; oes. gullet; par. per. parietal layer of pericardium; $p t$. cav. postcaval, close to its entrance into right auricle; $\%$. log. right lung; $\% \phi l$. right pleural cavity ; $r$. vent. right ventricle; $s t$. sternum; vis. per. visceral layer of pericardium; v. med. ventral mediastinal space. (From Parker and Haswell's Zoology.)

completely filled by the lungs, so that the parietal and visceral layers of the pleuræ are practically in contact, there being only a lubricating serous fluid lymph between them. The pressure of the air in the bronchial cavities of the lungs is therefore sufficient to keep them distended; but as 
soon as the pleural cavities are perforated, the pressure is equalised, and the elasticity of the lungs comes into play, causing them to collapse. When the muscles of the diaphragm contract (p. 490) air is drawn into the lungs, and this process is aided by the external intercostal muscles (p. 489) and, in forced respiration, by other muscles of the body-wall also. The mechanism of respiration may therefore be compared with a suction-pump, while that in the frog resembles a force-pump (p. 142).

On either side of the larynx is a soft, vascular, gland-like thyroid body, consisting of two lateral portions connected ventrally by a median bridge. Its function is not thoroughly understood : morphologically it represents a gland developed from the pharynx, but it loses its connection with the latter and thus has no duct. The glandular vesicles of which it is composed give rise to an albuminous substance containing iodine, which is passed into the blood and lymph; if extirpated in the living animal, various functional disturbances result. We are also ignorant of the function of the thymus, which is largest in young animals, becoming reduced in size in adults.

Organs of circulation.-The heart, as in all Vertebrates, is enclosed in a pericardium consisting of parietal and visceral layers (Fig. I28), between which is a serous pericardial fluid. There is a complete separation between the arterial and venous blood in the heart, for in addition to an auricular septum, as in the frog (p. 88), the ventricular portion is divided into right and left chambers by a partition (Fig. 128), the arterial blood from the lungs entering the left auricle and thence passing into the left ventricle to be pumped into the aorta, and the venous blood entering the right auricle and thence into the right ventricle to pass to the lungs through the pulmonary artery. A distinct conus arteriosus and sinus venosus (pp. 79 and 433) can no longer be recognised, the former having become practically absorbed 
into the ventricular portion of the heart, and the latter into the right auricle ;: so that the aorta-together with the carotids-and the pulmonary artery now arise directly from the left and right ventricles respectively, and the precavals and postcaval enter the right auricle independently (Figs. 125 and $\mathrm{I} 30$ ).

The line of separation between the two ventricles can be seen externally as an oblique depression extending from the base of the heart backwards and to the right, but not reaching the apex, which is formed by the left ventricle only. The small, irregular cavity of the latter is enclosed by very thick muscular walls, and is partly surrounded by the right ventricle, the cavity of which is crescentic in transverse section (Fig. I 28), while its walls are much thinner than those of the left ventricle, as it has only to pump the blood to the lungs. The auricles have thin walls : each is produced into a little flap or appendix which envelops the base of the corresponding ventricle, and the walls of which are strengthened by a network of muscular bands.

In the auricular septum is a thin, oval area, the fossa ovalis (Hig. 129, $f .0 z$ ), which in the cmbryo is perforated, and so allows the blood from the body to pass directly into the right auricle without going to the lungs, which are not, of course, functional until the animal is born.

The two auriculo-ventricular apertures are guarded by valves - that of the left side, or mitral valve, consisting of two membranous flaps, that of the right, or tricuspid valve (Fig. I29, tri. ) of three flaps: the valves are attached by their bases to the margins of the apertures, their apices extending into the corresponding ventricles. Attached to their edges are tendinous cords arising from conical elevations of the ventricular walls known as papillary muscles, which are nuch larger in the left ventricle than in the right 
(m. pap): these serve to prevent the valves from being pushed into the auricles when the ventricles contract.

The right ventricle narrows towards its base, on the ventral side of the heart, to form a conical prolongation from which arises the pulmonary artery (Figs. I 25 and $\mathrm{i} 30$, $p . a)$, its aperture being guarded by three pocket-like, semilunar valves (Fig. 129, sem. v) : the aperture of the aorta from

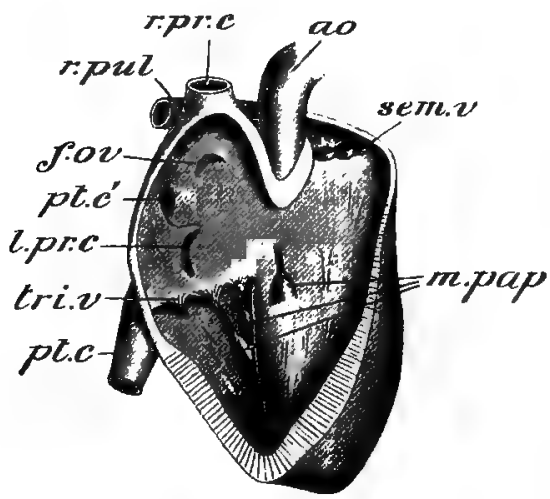

HII. I2g. - Heart of the rablsit, seen from the right side, the walls of the right auricle and right ventricle partly removed so as to expose the cavities.

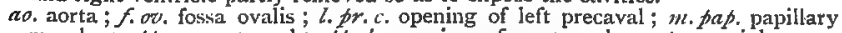
muscles; $p t . c_{0}$ postcaval ; $p t . c^{\prime}$. opening of postcaval; $r p r_{.} c_{0}$ right precaval; $r$. pul. right pulmonary artery; sem. $v$. semilunar valves; tri. $z$. tricuspid valve. (From Parker and Haswell's Zoology.)

the left ventricle is similarly provided with three semilunar valves. The two precavals (l. pr.c,r.pr.c) and the postcaval ( $p t . c)$ communicate, as we have seen, directly with the right auricle, the right precaval opening into it anteriorly, the left precaval posteriorly, the aperture of the postcaval being just anterior to that of the left precaval. The pulmonary veins from each lung unite and open together into the left auricle. 
Membranous folds, the Eustachian and Thebesian valves, extend into the right auricle between the apertures of the postcaval and left precaval : in the embryo these help to conduct the blood through the aperture in the auricular septum (p.504): they afford another example of vestigial structures, representing the remains of the sinu-auricular valve.

You will remember that in the frog (p. 80) there are two systemic trunks, representing the second arterial arch of the tadpole and fish (p. 435, and Fig. II I), and uniting above to form the dorsal aorta. In the Mammal, one of these-the right-disappears in the course of development and all the blood from the left ventricle passes into the single left aortic arch (Figs. 125 and 130 ) from the base of which both carotid arteries arise, the aortic arch then curving over the left bronchus to pass into the dorsal aorta (d. ao).

Close to the origin of the aortic arch, just beyond its semilunar valves, two small coronary arteries are given off to the walls of the heart; and more anteriorly, at the curve of the arch, arise the vessels which supply the head and forelimb. There is a certain amount of variation as to the origin of these, which is asymmetrical, and is usually as follows. Springing from the arch of the aorta towards the right side is an innominate artery (Fig. $\mathrm{r} 30$, in), which gives off close to its origin the left common carotid (l.c.c), and then, passing forwards, divides into the right common carotid (r.c.c) and the right subclavian (s. cl.a), the left subclavian (br) taking its origin independently from the left side of the arch. Each common carotid passes forwards along the neck, close to the trachea, and at about the level of the larynx divides into an internal carotid (i.c), which supplies the brain, and an external carotid (e.c), which goes to the head and face. Each subclavian forms several branches, the most important of which are a brachial (br) to the fore-limb, a vertebral 
(vr) which passes through the vertebrarterial canal of the cervical vertebræ (p. 48I) and supplies the spinal cord and brain, and an anterioriepigastric or internal mammary (a.epg) running along the inner side of the ventral wall of the thorax. The aorta gives off, in the thorax, a series of small paired intercostal arteries (i.cs) to the body-walls, and then passes into the abdomen, between the pillars of the diaphragm.

A short distance behind the diaphragm the coliac artery (Figs. I 27 and I30, $c \infty$ ) arises, and supplies the liver; stomach, and spleen; and about half or three quarters of an inch further back is the anterior mesenteric artery $(a . m . a)$, the branches of which pass to the small intestine, pancreas, cæcum, and colon. Close behind the anterior mesenteric is the right-and rather further back the left-renal artery (Fig. I $30, r)$, and still more posteriorly, a posterior mesenteric $(p . m)$ to the rectum, and a pair of spermatic or ovarian arteries $(s p m)$ to the spermaries or ovaries, as the case may be. A small caudal artery (m. sc), corresponding to the caudal continuation of the aorta, arises from the dorsal surface of the posterior part of the latter just in front of a pair of large common iliac arteries (c.il.a), which appear like a bifurcation of the aorta. These are continued outwards and backwards towards the hind-limbs, each giving off an itio-lumbar artery (i.l) to the dorsal body-wall and then dividing into an internal iliac (i.il.a) passing along the dorsal side of the pelvic cavity, and an external iliac (e.il.a) which gives off an artery to the bladder, (s.vs) and in the female one to the part of the oviduct known as the uterus $(u t)$; and then, passing beneath Poupart's ligament (p. 489) to the hind-limb, becomes the femoral artery ( $f m, a)$, from the proximal end of which a posterior epigastric ( $p . e p g$ ) runs forwards in the ventral abdominal wall. Small lumbar 


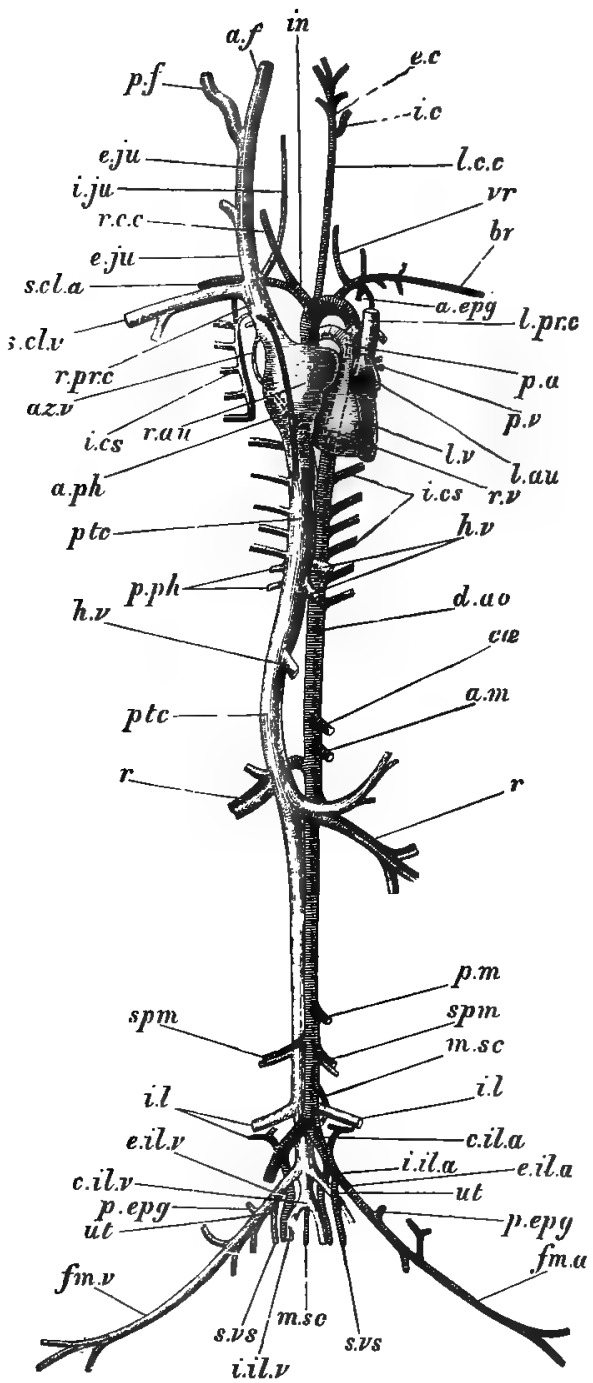

Fic. I30.-The vascular system of the Rabbit from the ventral side. The heart is somewhat displaced towards the left of the subject; the arteries of the right and the veins of the left side are in great measure removed. 
$a$. epg. anterior epigastric artery; $a . f$. anterior facial vein; $a . m$, anterior mesenteric artery; $a . p h$. anterior phrenic vein; $a z . z \nu_{0}$ azygos vein; br. right brachial artery; c.il.a. common iliac artery; c.il.v. hinder end of postcaval; co. coeliac artery; d. no. dorsal aorta; $e . c$. external carotid artery; e.jl. a. external iliac artery; $e$. il. $v$. external iliac vein ; $e . j u$. external jugular vein ; $m$. $a$. femoral artery; $f m$, $v$. femoral vein; $h$. $v$. hepatic veins ; $i, c$. internal carotid artery; $i$.cs. intercostal vessels; $i . j u$. internal jugular vein ; $i . l$. iliolumbar artery and vein; in. innominate artery; l. au. left auricle; l.c.c. left common carotid artery; $l$. pr. c. left precaval vein; $l . v$. left ventricle; $m$, sc. caudal artery; $p . a$. pulmonary artery; $p . e p g$. posterion epigastric artery and vein; $p . f$. posterior facial vein; $p . m$. posterior mesenteric artery; $p . p h$. posterior phrenic veins; $p t c$. postcaval vein; $\not$. $v$. pulmonary vein ; $r$. renal

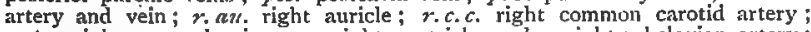
$r$, prc. right precaval vein; $r$. $\imath^{\prime}$, right ventricle; scl. $a$. right subclavian artery; scl. 7 . right subclavian vein; $s p m$. spermatic artery and vein; $s$. $v s$, vesical artery and vein; $u t$. uterine artery and vein; $z^{\prime} r$ verteloral artery. (From Parker's Zootomy.)

arteries are also given off from the aorta to the walls of the abdomen.

The pulmonary artery $(p . a)$ divides soon after its origin from the right ventricle into two branches, one supplying each lung. Just before its bifurcation it is connected by a short cord, known as the ductus arteriosus, with the aorta : this is the solid vestige of the embryonic connection between the fourth arterial arch and the aorta (compare $\mathrm{p}$. 436, and Fig. I I I).

Each precaval (l. pr. c, r. pr. c) receives-a subclavian (s. cl.v) from the fore-limb; an external jugular (e. $j u$ ) from the head, running along the neck just beneath the skin; a small anterior epigastric from the ventral thoracic wall, as well as small vessels from some of the anterior intercostal spaces $(i . c s)$ and the anterior surface of the diaphragm $(a . p h)$; and a small internal jugular $(i . j u)$ from the brain, opening into the corresponding external jugular nearly opposite the subclavian. An azygos vein (az.v), representing part of the right cardinal of the embryo (compare p. 440) and receiving blood from the posterior intercostal spaces, also opens out the base of the right precaval.

There is no renal portal system, as in the dogfish and frog 
(pp. $43^{8}$ and 85 ). A pair of internal iliac veins (i.il.v) in the pelvic cavity unite to join a median vessel $(c . i l . v)$, the hinder end of the postcaval, which receives on either side an external iliac (e. il. v), constituted by-a femoral vein ( $f m . v)$ from the hind-limb; a posterior epigastric (p.epg), from the ventral walls of the abdomen, entering the femoral just external to Poupart's ligament ; and by small veins from the bladder as well as from the uterus in the female. Slightly in front of the external iliacs the postcaval receives a pair of large ilio-lumbar veins $(i . l)$ from the body-walls : the left ilio-lumbar sometimes runs forwards to open into the corresponding renal vein. Rather more anteriorly still are a pair of spermatic $(\mathrm{spm})$ or ovarian veins, and a large renal vein ( $r$ ) enters the postcaval from each kidney. As the postcaval passes through the dorsal border of the liver, it receives several large hepatic veins (Figs. 127 and $\mathbf{1} 30, h . v^{\prime}$ ) from the lobes of that organ. Other small veins from the body-walls and from the posterior surface of the diaphragm also open into the postcaval, which then passes through the central tendon of the diaphragm and runs forward in the mediastinal space (Fig. 128, pt. cav) to open into the right auricle.

The hepatic portal vein (Fig. $127, p . v$ ) is a large vessel situated in the mesentery, ventral to the postcaval. Anteriorly it passes into and divides up in the liver, sending a branch to each lobe : posteriorly it is constituted by a large anterior mesenteric vein $(m . v)$ returning the blood from the small intestine, colon, and cæcum, and by smaller veins from the stomach, spleen, and duodenum, as well as by a posterior mesenteric vein $(p, m . v)$ from the rectum.

The pulmonary zeins have already been described (p. 505). In the freshly-killed animal a number of the delicate, transparent, lymphatic vessels (p. 97) can be made out, those from the intestine. (lacteals) 
running in the mesentery. They come into connection with numerous adenoids (p. 498) in the mesentery and elsewhere, and most of them communicate with a main trunk-the thoracic duct-which extends from the abdomen through the thorax on the left and upper side of the aorta. The thoracic duct also receives the lymphatics from the right side of the head and neck and the fore-limb, and opens into the veins at the junction of the left external jugular and subclavian : the lymphatics of the right side of the head and neck and right fore-limb communicate with the corresponding veins of the right side.

Nervous System.-The brain (Figs. I 25, r 3 I, and $\mathrm{I}_{32}$ ) reaches a much higher development than in the other Vertebrates we have already studied. The prosencephalon is subdivided into two cerebral hemispheres (ch), of much larger relative size than those of the frog (Fig. 49) and forming about two-thirds of the whole brain. They are closely applied to one another along theirflat internal surfaces, and are roughly conical in form, narrower in front (frontal lobes), broadening out posteriorly (parietal lobes) where they overlap the diencephalon and optic lobes and abut against the cerebellum, and produced downwards into the prominent temporal lobes which partly overlap the crura cerebri below. Their external layer or cortex is formed of grey matter, and their surfaces are smooth, except for the presence of slight fissures between the lobes : in many Mammals the surface of the hemispheres is highly convoluted, i.e. raised into numerous winding elevations or gyri, separated by narrow grooves or sulci. A broad transverse band of nerve-fibres forms a commissure connecting the two hemispheres known as the corpus callosum (Fig. I3I cp. $c$, and Fig. I32,cp.cl): this structure is confined to the Mammalia, and is even wanting in certain of the lower members of the class. The olfactory lobes (olf) are club-shaped, and extend backwards along the ventral surface of the hemispheres in the form of narrow bands as far as the temporal lobes. 
The diencephalon consists of a right and left optic thalamus (o. th.) between which is the slit-like third ventricle $\left(v^{3}\right)$

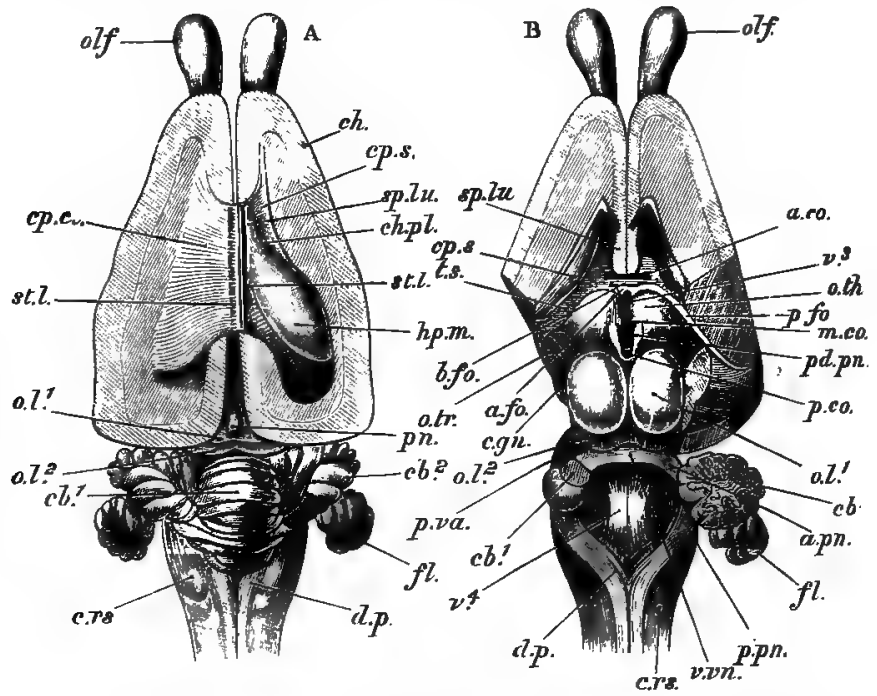

Fig. r3r. Two dissections of the Rabbit's brain, from above (nat. size). In A, the left hemisphere is dissected down to the level of the corpus callosum; on the right side the lateral ventricle is exposed. In $B$, the hemispheres are dissected down to a little below the level of the anterior end of the corpus callosum; only the frontal lobe of the left hemisphere is retained, of the right a portion of the temporal lobe atso is left ; the choroid plexus and pineal body are removed, as well as the greater part of the body of the fornix and the whole of its left posterior pillar; the cerebellum is removed with the exception of a part of its right lateral lobe with the flocculus.

u. co. anterior commissure; $a$. fo. anterior pillar of fornix ; $a$. ph. anterior peduncles of cerebellum; $b$. fo. body of fornix; $c b^{1}$. central lobe of cerebellum; $c b^{2}$. its lateral lobe; c.gn. elevation on the optic thalamus; $c h$. cerebral hemisphere; $c h$. pl. part of choroid plexus; $c p . c l$. corpus callosum ; $c p$. s. corpus striatum; $c$. $r s$. and $d$. $p$. elevations on the bulb; $f$. flocculus; $h f . m$. hippocampus; $m$.co. middle commissure; $0.2^{11} .0 . l^{2}$. optic lobes ; olf. olfactory lobe ; 0. th. optic thalamus; $o$. $t r$. optic tract (continuation of chiasma); $p . c o$. posterior commissure; $p . f o$. posterior pillar of fornix; $p n$. pineal body; $p d . p n$. its peduncle; $p . p n$. posterior peduncles of cerebellum; $p$. va . fibres of pons Varolii forming middle peduncles of cerebellum ; sp. lu. septum lucidum; st.l. line on corpus callosum; $t$. s. band of white matter lying beneath choroid plexus; 7 . $z \mathrm{~m}$. valve of Vieussens; $v^{3}$. third ventricle; $v^{4}$. fourth ventricle. (From Parker's Zootomy'.)

roofed over by a thin membrane, on the uppersurface of which is a vascular choroid plexus (Fig. $\mathrm{r}_{32}, v l$. ip), and from the 
hinder part of which arises a stalk bearing at its end a small, rounded pineal body $(p n)$. The floor of the diencephalon is produced downwards to form the infundibulum (inf), to which the pituitary body (pty) is attached. In front of the infundibulum is the optic chiasma $(o . c h)$ and behind it a small, rounded lobe (c. ma).

Each optic lobe is divided into two by a transverse furrow, so that there are four rounded elevations in this region-an anterior, larger pair $\left(o . l^{1}\right)$, and a posterior, smaller pair $\left(o . l^{2}\right)$. Below the optic lobes are the crura cerebri (c. c)-two strong, diverging bands passing forwards and outwards from the bulb to the hemispheres.

The bulb or medulla oblongata. (m.o) is slightly flattened dorso-ventrally, and passes behind into the spinal cord, the dorsal and ventral fissures of which are continued into it: the fourth ventricte $\left(v^{4}\right)$ which it contains is roofed over by the thin pia mater only (p. 155). Ventrally its anterior border is marked by a stout band of nerve-fibres running transversely, and known as the pons $\operatorname{Varalii}(p . v a)$. The cerebellum is connected with the dorsal surface of the brain by three pairs of peduncles (Fig. I $3 \mathrm{I}, a . p n, p . v a$, $p \cdot p n)$, and consists of a median central lobe $\left(c b^{1}\right)$ and of two lateral lobes $\left(c b .{ }^{2}\right)$, on the outer side of each of which is a smaller floccular lobe $(f l)$. The grey matter is superficial, and the surface is marked by numerous folds which in section present a tree-like pattern (arbor vita), brought about by the arrangement of the grey and white matter (Fig. I 32).

The fourth ventricle is not prolonged into the cerebellum to any extent: it is continued forwards as the iter, from which no optic ventricles are given off (compare pp. I 5 I and 443) and which passes into the narrow but deep third ventricle in front (Fig. 132): this is bounded anteriorly by PRACT._Zool. 
a thin wall, the lamina terminalis (l.t), and extends into the infundibulum below. At its anterior end are the foramina of Munro $(f . m)$, leading into the middle of the lateral ventricles in the hemispheres (Fig. I3I). In this region each lateral ventricle is broad from side to side, but narrow from above downwards; it extends forwards into the frontal

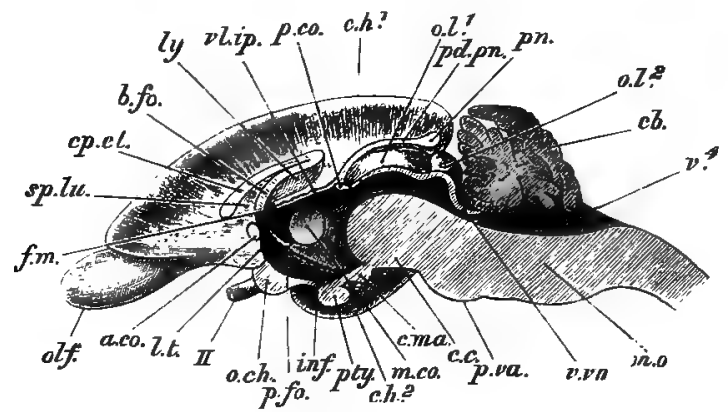

FIG. 132.--Rabbit. Longitudinal vertical section of the Rabbit's brain (nat. size).

Letters as in preceding figure; in addition $c b$. central lobe of cerebellum, showing arbor vita; c.c. crus cerebri; $c_{*} h_{*} 1$ parietal, and $c_{*} h_{.} 2$ temporal lobe of cerebral hemisphere; $c$. $m a$. elevation behind the infundibulum; $f, m$, foramen of Monro; inf. infundibulum; l.t. lamina terminalis; $l y$. part of hippocampus; m. o. medulla oblongata; $0 . c h$. optic chiasma; ply. pituitary body; $v l . i f$. choroid plexus; $I I$. optic nerve. (From Parker's Zootony.)

lobe, backwards into the parietal lobe, and downwards into the temporal lobe. The olfactory lobes are solid.

A prominent, convex ridge of white matter-the hippocampus (Fig. I $3 \mathrm{I}, h p . m_{0}$ ) projects into the inner side and floor of each lateral ventricle where it descends into the temporal lobe, and closely applied to it is a continuation of the choroid plexus $(c h . p l)$, which passes from the the roof of the third ventricle into the lateral ventricle through the foramen of Monro. In front of the hippocampus the outer side and floor of the anterior part of the lateral ventricle is thickened to form an eminence of grey matter, the corpus striatum (cp.s) Just beneath the corpus callosum the internal wall of each lateral ventricle is thin, and is known as the septum lucidum (sp. lu ; and below it and above the foramina of Monro is another commissure known as the body of the fornix (Figs. I $13 \mathrm{I}$ and $\mathrm{I} \mathfrak{Z}^{2}, b$. fo) which is continuouns on each side with 
two bands-one (posterior pillar) lying along the anterior edye of the hippocampus, and the other (anterior pillar) passing backwards in the side walls of the third ventricle. Connecting the two optic thalami are three tranverse bands of nerve fibres, known respectively as the anterior (a.co), midale ( $m, c o$ ) and posterior ( $\beta, c o$ ) commissures: the middle commissure, which is much the largest, is not represented in the lower Vertebrata.

The spinal cord (Fig. I 25, sp.c) is similar in structure to that already described in other Vertebrates (pp. I 55 and 443). It extends through the entire neural canal, ends in a flum terminale, and is swollen opposite the fore- and hindlimbs, where the nerves arise which form the limb-plexuses (pp. I6r and 162).

The dorsal and ventral roots of the spinal nerves lic in the same transverse plane, as in the frog (p. 163), but are relatively shorter than in that animal ; and after uniting to form the nerve-trunks, pass directly outwards through the intervertebral foramina. The brachial plexnes is formed from the four posterior cervical and the first thoracic nerve, and gives off a number of nerves to the shoulder and fore-limb. The sciatic or lumbo-sacral plexus is constituted by the two or three hindermosi lumbar and the first two or three sacral nerves, and gives off branches to the pelvic region and hind-limb, the chief of which are a femoral and peroneal going to the dorsal extensor muscles, and a large sciatic and an obturator (which passes through the obturator foramen, p. 486) supplying the ventral flexor muscles. Arising from the fourth cervical spinal nerve of either side is a phrenic nerve (Fig. 125, phr. n), which passes backwards, between the heart and lungs, to supply the muscles of the diaphragm ; and a large auricular nerve, arising from the third cervical nerve, supplies the external ear.

In addition to the ten cerebral nerves enumerated in the frog (p. 163 ) and dogfish (p. 445), two others-the spinal accessory and the hypoglossal (represented by the first spinal nerve in the frog, p. I60) emerge from the skull and are counted respectively as the eleventh and twelfth cerebral nerves. The former arises from the side of the spinal cord 
and bulb by numerous fibres, the posterior of which are opposite the fifth spinal nerve, from which point it runs forwards between the dorsal and ventral roots and leaves the skull together with the glossopharyngeal and vagus (p. 479), supplying certain muscles of the neck and shoulder. The hypoglossal arises by a number of fibres from the ventral surface of the bulb, passes out through the condylar foramen and supplies the muscles of the tongue as well as certain muscles of the neck.

The origin and distribution of the first ten pairs of cerebral nerves correspond in their main features with those seen in the frog. The facial is almost entirely a motor nerve and is chiefly important in supplying the facial muscles, which are very highly developed in Mammals.

The relations of the sympathetic nerves (Fig. I 25, sy) are also essentially similar to those occurring in the frog (p. 162). Each passes backwards along the neck close to the vagus (vg) and alongside the carotid artery, enlarging at each end of this region to form an anterior and a posterior cervical ganglion. In the thorax it runs just beneath the heads of the ribs, having a ganglion in each intercostal space: it then passes into the abdomen, lying close to the centra of the vertebræ and having ganglia at intervals. From all the sympathetic ganglia branches are given off connecting them with the spinal nerves, others going to the bloodvessels: others again, in the thorax and abdomen, are connected with plexuses from which nerves pass to the heart and abdominal viscera. In the abdomen these plexuses can be seen in the mesentery, a large celiac plexus being present close to the origin of the coliac and mesenteric arteries.

Sensory Organs. - The sense of touch is situated in microscopic tactile organs in the skin, and groups of cells, called 
taste-buds, are present on the papillae of the tongue (p. 492) and on the soft palate (compare Pp. I 79 and i80).

The organs of smell are situated in the olfactory capsules, the form of which has already been described (p. 476). They open externally by the external nostrils, and are produced backwards above the palate into the passage of the internal nostrils, which communicate with the naso-pharynx (Fig. I $25 i . n, n . p h$, p. 495). The olfactory epithelium, supplied by the olfactory nerves, is situated on the ethmoturbinal $(e . t b)$ : the mucous membrane of the maxillo-turbinal $(m, t b)$ probably serves merely to warm the inspired air.

On the ventral side of the nasal septum is a pair on small, tubular structures lnown as Jacobson's organs (Fig. $125, j$ ), lined by epithelium and enclosed in cartilages situated just to the imer side of the palatine processes of the premaxillæ (p. 477). Each of them opens anteriorly into the corresponding naso-palatine canal (p. 490), and receives a special branch of the olfactory nezve. The function of these organs is not understood.

The structure of the eye (Fig. 57) is similar to that already described in other Vertebrates (pp. I8I and 449), except that the sclerotic is not cartilaginous, but is composed of dense fibrous tissue, and the lens is relatively smaller than in the dogfish and frog and is markedly biconvex in form, the outer surface being rather flatter than the inner: it is capable of adjustment by means of the ciliary muscles contained in the radiating ciliary processes (C.P) into which the choroid is thrown just externally to the iris (compare p. I 84 ).

The eyelids have already been clescribed on p. 469. The four recti muscles ensheath the optic nerve, as in the frog (p. 186, compare Fig. I17), but the superior oblique, instead of arising-ilike the inferior oblique -in the anterior part of the orbit, takes its origin further back, near the recti, passes forwards through a fibro-cartilaginons pulley at the anterior angle of the orbit, and then backwards and ontwards to its insertion on the eyeball. 
Between the wall of the orbit and the eyeball are two glands, the secretion of which, passing through ducts perforating the conjunctiva lining the eyelids, serves to keep the outer surface of the eye moist, and is then conducted into the nasal chambers by means of the naso-lachrymal duct (pp. I86 and 477). These two glands correspond to special differentiations of a primarily continuous structure : one, the Harderian gland-already met with in the frog-is situated in the antero-ventral region of the orbit: the other, or lachrymal gland proper, in its postero-dorsal region. Besides these, a series of small Meibomian glands are present on the inner side of the edges of the eyelids, and produce a fatty secretion.

The essential part of the auditory organ consists, as in other Vertebrates, of the membranous labyrinth with its three semicircular canals (pp. 186 and 449) enclosed in the auditory capsule (periotic bone, p. 475), and constituting the internal ear. The small outgrowth of the sacculus seen in the dogfish and frog, and known as the cochlea (Fig. 59, l), is represented by a relatively larger structure, coiled on itself in a spiral manner. 'The part of the periotic bone which directly surrounds the cavity in which the membranous labyrinth lies is especially hard, and when the outer portion of the bone is cut away, is seen to form a sort of cast of the enclosed organ, the form of which it repeats: this is known as the bony labyrinth (Fig. 133). Internally it is separated from the membranous labyrinth by a narrow space all round, containing the perilymph (p. 189) and only shut off from the tympanic cavity at the fenestra ovalis and fenestra rotunda (p. 475) by a membrane which closes them.

The membranous cochlea does not run up the middle of the spiral or the bony cocblea, but is attached between its onter wall and a spiral shelf arising from its inner wall. Thus the entire cochlea show's 
three cavities in transverse section-a middle, the membranous cochlea or scala media, and a scala vestibuli and a scala tympani on either side of it respectively, which communicate with one another at the apex of the cochlea and with the perilymphatic cavity surrounding the rest of the membranous labyrinth at its base, where the scala tympani abuts against the membrane of the fenestra rotunda, and the scala vestibuli against that of the fenestra ovalis. On the wall of the scala media which separates it from the scala tympani is a specially modified series of auditory cells forming what is known as the organ of Corti, which receives nerve-fibres from a branch of the auditory nerve extending along the spiral shelf of the cochlea.

The midale ear (p. 450) is constituted by the tympanic cavity in the tympanic bulla (p. 475), and communicates with the pharynx by the Eustachian tube (Fig. I33, E). The tympanic membrane ( $\mathrm{M})$, situated obliquely at the boundary of the bulbous and the tubular portions of the tympanic bone, separates the middle ear from the external ear, consisting of the auditory passage $(\mathrm{Ex})$ and the pinna (p. 469).

The fenestra ovalis is plugged by a small stirrup-shaped bone, the stapes (Fig. I33, $\mathrm{O}^{\mathrm{l}}$ ), one of the three auditory ossicles ( $p$. 475) connecting the internal ear with the tympanic membrane, and probably corresponding morphologically to the cartilaginous stapes of the frog (Fig. Io, stp): with it is connected a small stapedius muscle, serving to keep the membrane of the fenestra ovalis on the stretch. 'The middle bone of the chain is the incus (Fig. I33, $\mathrm{O}^{2}$ ), a short process of which is articulated to the stapes by the intermediation of a small bony nodule, while its body articulates with the outer bone of the series, the malleus $\left(O^{3}\right)$. Arising from the body of the malleus is a handle-like process or manubrium, which is attached to the tympanic membrane $(M)$ : this has the form of the roof of a tent, and is kept on the stretch by a small muscle, the tensor tympani, 
arising from the wall of the tympanic cavity and inserted on to the manubrium of the malleus.

The study of development indicates that the malleus corresponds to the articular part of Meckel's cartilage of lower Vertebrates, and the incus to the quadrate (p. 44): the articulation of the bony mandible

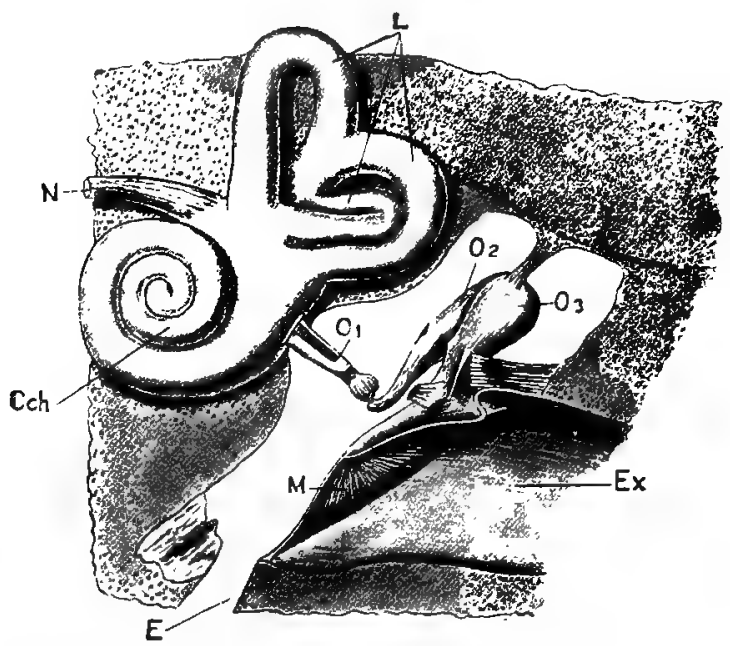

IIG. 133.-Diagram of the mammalian bony labyrinth, tympanic cavity, and external auditory passage.

$C i h$. bony cochlea; $E$. Eustachian tube ; $E x$, external auditory passage ; $L$. bony semicircular canals; $M$. tympanic membrane; $N$. auditory nerve; $O$. stapes; $\mathrm{O}^{2}$. incus; $\mathrm{O}^{3}$. malleus. (After Headley.)

with the squamosal in Mammals has rendered these parts unnecessary for their original purpose, and they have undergone a change of function, forming an accessory part of the auditory apparatus.

Urinogenital Organs.-CThe kidney's (Fig. I 25, $k$ ) are of a somewhat compressed, oval shape, with a notch or hilus on the inner side. They are in close contact with the dorsal wall of the abdominal cavity, the right being somewhat in advance of the left. Towards the hilus, the tubules of 
which the kidney is composed (p. I46) converge to open into a wide chamber or pelvis, which forms the dilated commencement of the ureter. When the kidney is cut across, its substance is seen to be divided into a central mass or medulla and a peripheral portion or cortex. The former appears radially striated, owing to the tubules in this region being straight and converging to open on the surface of a conical process or pyramid, which projects into the pelvis: the cortex contains the coiled portions of the tubules and the Malpighian bodies, and thus has a dotted appearance. The ureter (Figs. I25 and $134, u r$ ) runs backwards along the dorsal wall of the abdomen to open into the urinary bladder $(u . b l, b l)$, a pyriform sac with elastic walls which vary in thickness according as the organ is dilated or contracted: in the male the openings of the ureters are situated nearer the posterior, narrower end or neck than in the female. Near the front end of each kidney, towards its inner side, is a small yellowish adrenal or supra-renal body (Fig. $125, a d r$ ).

In the male Rabbit the spermaries are oval bodies which in the young animal are situated close to the kidneys, on the dorsal wall of the abdomen, but which pass backwards and downwards as the animal approaches maturity until they come to lie each in a scrotal sac (p. 47o), situated at the side of the urinogenital opening. The cavity of each scrotal sac is in free communication with the cavity of the abdomen by an opening-the inguinal canal. A convoluted epididymis (p. 45 I), closely adherent to the spermary and connected with the distal end of the scrotal sac, forms the proximal part of the spermiduct or vas deferens (Fig. $\mathrm{r} 34 \Lambda, v . d$ ), which, together with the blood vessels and nerves of the spermary, passes through the inguinal canal: it then loops round the corresponding ureter, and extends back between 
the neck of the bladder and a median sac on the dorsal side of the latter, known as the uterus masculinus $(u, m)$. The neck of the bladder is continued backwards, through the

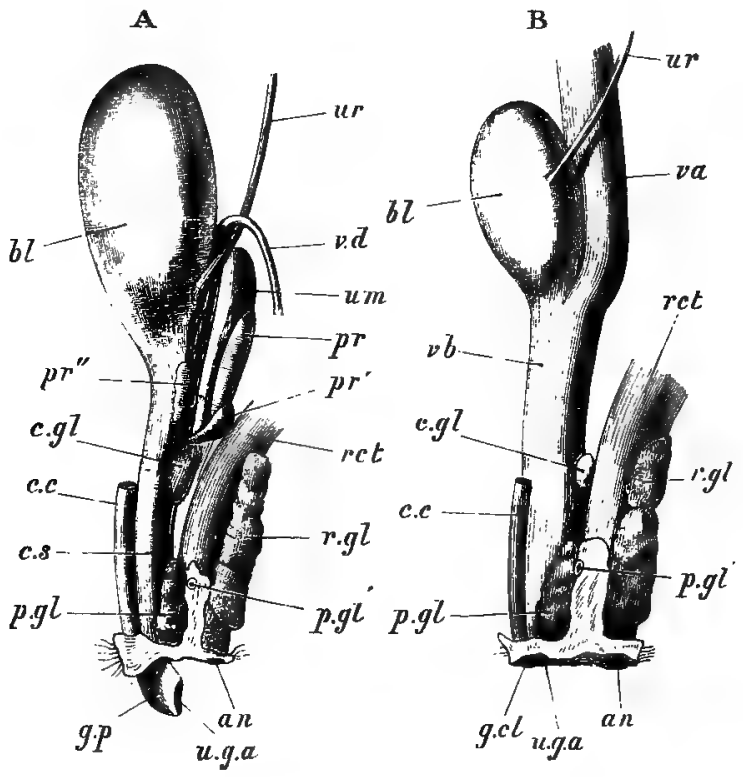

FIG. T34--The urinogenital organs of the Rabbit. A, of male; $\mathbf{B}$, of female : from the left side (half nat. size). The kidneys and proximal ends of the ureters, in A the spermaries, and in $B$ the ovaries, Fallopian tubes, and uteri are not shown. $a n$. anus; $b l$. urinary bladder ; $c$. $c$. corpus cavernosum; $c$. s. corpus spongiosum; $c . \xi l$. Cowper's gland; $g . c l$. glans clitoriclis ; $g . p$. apex of penis; $p . g l$. perineal gland; p. $g l^{\prime}$. aperture of its duct on the perineal space ; $p r$. anterior, $p r^{\prime}$. posterior, and $p^{\prime H}$. lateral lobes of prostate; rct. rectum; $r . g l$. rectal gland; $7 t . g . a$. urino-

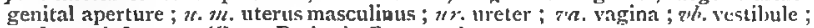
7.d. vas deferens. (From Parker's Znotomy'.)

pelvic cavity, as the urinugenital canal or urethra, on the dorsal side of which, just in front of a rounded elevation, is an aperture by means of which the uterus masculinus and vasa deferentia open into it. 
A prostate gland (pr), consisting of several lobes, is imbedded in the walls of the uterus masculinus and opens by small ducts on either side of the elevation just referred to ; and a pair of smaller, ovoid Cozeper's glands communicate with the urinogenital canal further back.

The terminal part of the urethra traverses the copulatory organ or penis (p. 470), the posterior or dorsal wall of which is constituted by a soft vascular portion, the corpus spongi$\operatorname{osum}(c, s)$, while the opposite surface is strengthened by two harder bodies, the corpora cavernosa $(c . c)$, which are closely applied together through the greater portion of their length, but diverge proximally and are attached to the ischia:

In both sexes a pair of perineal glands ( $p . g l$ ) open on the perineal spaces (p. 469) at the sides of the penis, and two larger rectal glands $(r . g l)$ lie at the side of the rectum.

In the female the ovaries (Figs. I 25 and $135,0 v$ ) are small, ovoid bodies attached by peritoneum to the dorsal wall of the abdomen behind the kidneys, the ovarian follicles or ovisacs (p. 195) forming very small, rounded projections on their outer surface.

The oviducts, instead of remaining separate along their whole length, are fused proximally to form a wide, median portion, the ragina (Figs. I $34 \mathrm{~B}$ and $\mathrm{x} 35, v a$ ), opening into the urinogenital canal or vestibule $(v b)$, with which the bladder communicates and which opens externally at the vulva (Fig. I $34 \mathrm{~B}, u \cdot g: a$ ). Into the other or distal end of the vagina, the paired, thick-walled uteri (Fig. I $35, r . u t, l . u t$ ), or middle portions of the oviducts, open by separate thick-walled apertures. The eggs undergo development in the uteri, which vary in size according to whether or not they contain embryos, and according to the stage of development of these. Each uterus is continued forwards as a narrow, slightly coiled tube-the anterior section of the oviduct, or Fallopian tube ( $f t, t)$ which communicates with. 
the cœlome by a small aperture $\left(f t, t^{\prime}\right)$ surrounded by a wide, membranous funnel with thin walls and folded margins, which is applied to the outer surface of the corresponding ovary. On the ventral or anterior wall of the hinder or proximal end of the urinogenital canal is a small, hard, rodlike body, the clitoris (Fig. $125, c l$ ), corresponding to the

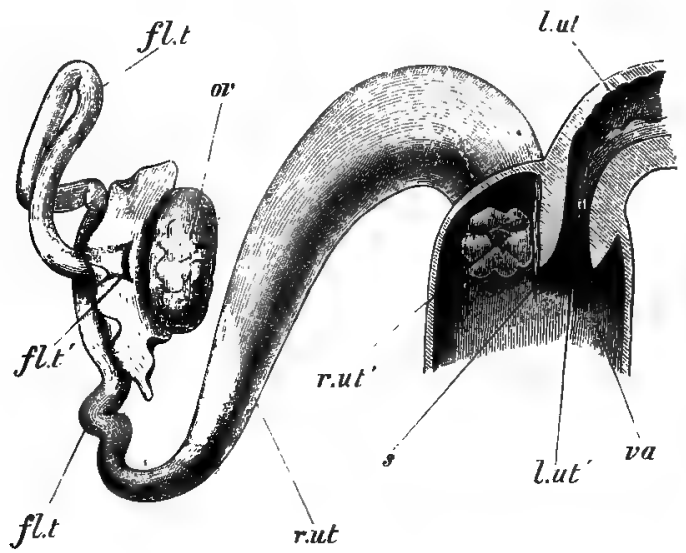

FIG. 135.-The anterior end of the vagina, with the right uterus, Fallopian tule and ovary of the Rabbit (nat. size). Part of the ventral wall of the vagina is removed, and the proximal end of the left uterus is shown in longitudinal section. $f . t$. Fallopian tube ; $f_{.} t^{\prime}$. its colomic aperture; $l . u t$. left uterus; $l . u t^{\prime}$. aperture of same (os uteri) into vagina; ov. right ovary; $r$. zt. right uterus; $r, u t$. right os uteri ; s. vaginal septum; za. vagina. (From Parker's Zootomy.)

penis of the male, and composed of two small corpora cavernosa (Fig. I $34, \mathrm{~B}$, c.c) attached at their proximal ends to the ischia.

The Rabbit is viviparous. The minute ova undergo development in the uterus, in which each develops into a fotus, as the intra-uterine embryo is termed, and is nourished by means of an organ known as the placenta, which will be described in the next chapter. The young animal escapes 
from the uterus in a condition in which all the parts have vecome fully formed except that it is practically hairless; the eyelids are at first coherent. As many as eight or ten young are produced at a birth, and the period of gestation, i.e., the time elapsing between the fertilization of the ovum and the birth of the young animal is thirty days. Fresh broods may be born once a month throughout a considerable part of the year, and, as the young Rabbit may begin breeding at the age of three months, the rate of increase is very rapid.

The class Mammalia is divided into a number of orders, that to which the Rabbit belongs being called the Rodentia, and also including rats and mice, squirrels, beavers, porcupines, and many others. All these are vegetable feeders and are mostly of small size. They possess no canine teeth, and their incisors, which are adapted for gnawing, are never more than two in number in the lower jaw, and in most of them there are only two in the upper jaw also.

\section{PRACTICAL DIRECTIONS}

The specimen used should be over three months old. Place it in a sufficiently large jar or box with a close-fitting lid together with a piece of cotton-wool well soaked in chloroform, and leave it until a short time after all movements have ceased. A pair of bone-forceps (p. I2) will be required.

\section{A. External characters (see pp. 467-470).}

Microscopic preparations of the skin of a Mammal should be examined, and the hairs, and hair-sacs, and sebaceous glands (p. 468) noted. (For mode of preparation, see p. 136 ).

B. Skeleton. If more convenient, the detailed examination of the skeleton may be postponed until after the soft parts have been dissected.

The skeleton of a young rabbit, about sị weekss old, as well as that 
of an adult, should be obtained for examination : the former is the more important of the two for making out the individual bones, which should all be separated from one another by prolonged maceration in water, or by boiling for a short time. In the adult skeleton the bones are best kept in their natural connection. An additional skull should be prepared, and a longitudinal vertical section made of it with a fine saw ; but as it is difficult to do this very accurately and perfectly in the rabbit, owing to the delicacy of some of the bones, the skull of a young dog or cat may be used for the purpose.

Work through the account of the skeleton given on $\mathrm{pp}$. 470-488, as well as of the teeth on pp. 492-494, comparing the form, number and arrangement of the teeth in the herbivorous rabbit and the carnivorous dog or cat, noting the presence of canines in the two last mentioned. Sketch typical parts of the skeleton.

\section{Superficial dissection, and injection of the arteries.}

Fix the animal on its back on the dissecting-board by inserting large pins through the limbs. If you wish to inject the arteries, cut through the skin on the inner side of one of the thighs, reflect it, and with the seeker expose the femoral artery (p. 507); if your specimen is a small one, expose one of the carotid arteries (p. 506) instead, by carefully cutting through the skin along the middle ventral line of the neck. Pass a piece of thread round the artery thus laid bare and make a small slit in it with the fine scissors distally to the thread. Insert and tie in the cannula, and first inject about half a small syringe-full of strong formaline ( $I$ part formaline and 2 parts water), following this with the coloured starch injection-mass (p. 99), which will force the formaline into the capillaries and help to preserve the specimen : this method is of special advantage for the subsequent examination of the enteric canal, especially if it is not possible to get to the end of $\$ \mathrm{VI}, \mathrm{p} .530$, on the first day; the veins need not be specially injected, as they will be naturally injected with blood.

Make a median longitudinal incision through the skin in the sternal region, and continue the cut backwards to the pelvic symphysis and forwards to the mandibular symphysis; then dissect away the skin from the underlying muscles over the whole ventral surface, being careful not to injure any of the larger blood-vessels (e.g., the jugular veins in the neck). Note :-

I. The thin cutaneous muscle, part of which you have very likely 
removed together with the skin; and in the adult female, the mammary glands (p. 489): trace the main ducts of some of these to their apertures on the teats.

2. In the neck:- $a$, the brachea and larynx (p. 489), to see which and the following structures clearly the cervical portion of the cutaneous muscle should be dissected away and the underlying parts carefully separated with a seeker; $b$, the hyoid bone, situated in a mass of muscle just anterior to the larynx; $c$, the submaxillary glands (p. 495); and $d$, the large external jugular veins (p. 509).

3. In the thorax:-a, the stermum and xiphisternum; $b$, the small clavicles (p. 483); $c$, the pectoral musiles; $d$, the vertebral and sternal portions of the ribs and the external intercostal muscles; and $e$, the " blood-vessels and nerves going to the fore-limbs.

4. In the abdomen:- $-a$, the muscles of the abdominal wall, separated in the middle ventral line ljy a whitish, fibrous band; $b$, Poupart's ligament (p. 489); $c$, the blood-vessels and nerves passing beneath Poupart's ligament to the hind-limbs.

II. On the same day on which the animal is killed, remove the skin from the dorsal surface of the head and anterior part of the neck, and remove the muscles covering the anterior cervical vertebræ. A membrane between the skull and first vertebra will then be exposed : cut through this, and part of the spinal cord will be seen. With the bone-forceps rut away the arches of the first three or four vertebre and the roof of the skull-taking particular care in the auditory region-so as to expose the brain : note the olfactory lobes, cerebral hemispheres, cerebellum, and medulla oblongata; and also the dura mater and pia mater. Then cut through the spinal cord about a quarter of an inch beyond its junction with the brain, lever up the latter with the handle of a scalpel, ancl carefully cut through all the nerves arising from it and separate it from the cranial walls, working from behind forwards. Dissect away the olfactory lobes from their attachments, remove the entire brain and place it in 3 per cent. formaline.

The animal may be preserved from day to day by wrapping a cloth round it soaked in 3 per cent. formaline or strong methylated spirit, and placing it in a closed vessel.

\section{Dissection of the abdomen.}

I. Once more pin the animal down, ventral side uppermost, and make an incision along the middle line of the abdominal muscles from 
the xiphisternum to the pelvic symphysis, so as to open up the abdoninal cavity. From the anterior extremity of the incision make transverse cuts, and turn back the flaps of muscle. ${ }^{I}$ Note :-

I. $u$, The peritonimm; $b$, the diaphragm, with its muscles and central tendon; $c$, the pink lungs, seen through the latter: make a small aperture in the diaphragm on one side of the median line and note the collapse of the corresponding lung.

2. a, The liver, stomach, small intestine, cucum, colon, and rectum (pp. 495-499); $b$, the urinary bladder; $c$, the scrotal sacs in the male; and-by turning aside the intestines- $d$, the kidneys; and $e$, the ovaries and oviducts (varying much in size according to age) in the female.

3. $a$, The characters and relations of the lobes of the liver (compare Fig. 128), and the folds of peritoneum which attach the liver to the diaphragm ; $b$, the gall-bladder; $e$, the gullet, and its entrance into- $e$, the stomach (cardiac and pyloric portions); $f$, the spleen.

4. $a$, The parietal and visceral layers of the peritoneum and the various subdivisions of the mesentery; $b$, the $\mathrm{U}$-shaped duodenum, passing into the ileum, and the connection of the latter with the proximal end of $-c$, the cacum, at the distal end of which is the vermiform appendix, while proximally it passes into-d, the colon, continuous with $-f$, the rectum, which enters the pelvic cavity to open by the anus.

II. Turn over the stomach to the animal's right side and make out-

I. , The postcaval vein, passing from the pelvis forwards, near the ventral surface of the backbone, through a notch in the liver to the diaphragm; $b$, the dorsal aorta, running partly above, partly alongside, the postcaval; $c$, the caliac artery, given off from the aorta about an inch posterior to the diaphragm-trace its main branches; $d$, the anterior mesenteric artery, arising about half an inch further back than the coliac-trace its main branches.

2. $u$, The left kidney, with its artery, vein, and ureter; $b$, the yellowish left adrenal, anterior to the origin of the renal artery and vein ; $c$, the left ovary, Fallopian tube, and uterus in the female.

1 You will very probably notice a number of transparent, rounded vesicles, about $\frac{1}{4}$ inch in diameter, in the abdomen. These are tapeworms (phylum Platylelminthes, p. 397) in the encysted stage, which develop into the adult form when swallowed by a dog. 
3. $a$, The small posterior mesenteric arlery, leaving the aortal a short distance posteriorly to the left kidney and supplying the rectum; $b$, the posterior mesenteric vein, in the mesentery of the rectum.

III. Turn the intestines over to the animal's left side and spread out the duodenum, putting its mesentery slightly on the sketch but taking care not to rupture it, so as to make out-

I. $t$, The large anterior mesenteric vein, into which the posterior mesenteric vein opens; $b$, the pancreas and its duct, opening into the distal limb of the duodenal loop an inch or so beyond the bend.

2. a, The right kidney, partly overlapped by the caudate lobe of the liver, and its ureter; $b$, the right adrenal.

IV. Replace the intestines in their natural position; cut through the gullet close to the diaphragm, draw the stomach backwards, turn forwards the lobes of the liver, and dissect out the following structures (Fig. 127):-

I. The common bile-duct-made up of cystic and hepatic ducts, and its entrance into the cluodenum.

2. $a$, The large hepatic portal vein, running in the mesentery ventrally to the postcaval, made up by the union of the mesenteric, gastric, and splenic veins, and entering the liver, sending a branch to each lobe ; $b$, the transparent lymphatic vessels (lacteals) in the mesentery.

V. Tie two pieces of thread, about half-an-inch apart, round the portal vein just before it enters the liver (the hepatic artery and bile-duct may be included in the ligature); cut through the rectum just anterior to the pelvic cavity, and through the portal vein between the ligatures, as well as through the mesenteric attachments of the stomach and intestine, and remove them from the body entire. Unravel the intestine by cutting or tearing the mesentery-except that part of it in which the pancreas lies-and spread it out on the dissecting-board or in a large dissecting dish, arranging it so that the varicus subdivisions may easily be distinguished from one another. In addition to the relations of these, note :-

I. The sacculus rotundus (Fig. 125, s. r), and the characters of the ciecum, which proximally passes insensibly into the colon.

2. The length of the intestine as a whole (about fifteen or sixteen times that of the animal), and the relative lengths of the five divisicns,

Sketch the entire canal.

VI. Remove and cut open the stomach and parts of the small intestine, colon, and rectum : wash thoroughly, and exaimine under water. Pract. Zool.

M M 
Remore the carcum tugether with a small portion of the ileum and colon, wash it out by directing. a stream of water through it, distend with water (it is better to harden it first with formaline) and examine in a dish of water; with the scissors cut away a piece of the wall here and sre, between the constrictions and in the appendix. Note:-

I. The mucous membrane, muscular coat, and peritoneum.

2. The longitudinal ridges or ruge in the stomach, and the pyloric valve.

3. The aperture of the bile-duct, and the villi and Peyer's patches (p. 498) in the small intestine.

4. ", The Peyer's patches and intra-colic valve in the proximal part of the colon; $b$, the thick lymphoid tissue in the walls of the sacculus rotundus; $i$, the ileo-colic aperture ant valve.

5. The spiral valve of the crecun, and the lmyphoid tissze of the vermiform appendix.

6. The characters of the mucous membrane of the cæcum and large intestine.

(Microscopic sections of the small intestine-injected and uninjected, should also be examined, compared with Fig. 39, and the villi and intestinal glands noted.)

VII. Ligature the postcaval at the points where it enters and leaves the liver: remove the entire liver, noting as you do so the aorta, gullet, postcaval, hepatic veins, and phrenic zeins. Sketch the liver from the posterior surface. Then return to the examination of the abdomen, and make out (Fig. I30)-

1. The renal and spermatic (or ovarian) arteries and veins.

2. The caudal artery, arising from the dorsal side of the aorta.

3. The common iliac arteries, each of which gives off an ilio-lumbar branch and divides into an external and an internal iliac, the former becoming continuous, beyond Poupart's ligament, with the femoral. (The course of these arteries and of the corresponding veins can be more easily traced after the urinogenital organs have been removed-see $\$$ IX.)

4. The ilio-lunbbar veins, and the trifurcation of the postcaval into the cxternal iliacs and a median vein formed by the fusion of the two internal iliacs.

Sketch all these vessels later, after the removal of the urinogenital organs.

VIII. Dissect away the peritoneum and fat from the kidneys, ureters, and genelative organs, and trace each ureter from the hilus of the 
kidney backwards to the bladder, which, if contracted, may be inflated from the urinogenital aperture. Then make out-

A. In the male.

I. The penis, with the prepuce, and the aperture of the urinogenital caural, which latter is lined by the soft corpus spongiosum, and strengthened dorsally by the corpora cavernosa, which diverge proximally and are attached to the ischia.

2. The scrotal sacs, each communicating with the abdominal cavity by the ingrinal canal, through which the corresponding spermiduct and the spermatic artery and vein pass to the spermary, to see which and the epididymis the scrotal sac should be slit open along its ventral wall.

3. The course of the spermiducts.

$B$. In the female.

I. The vutva; and the clitoris, with its corpora cavernosa, on the ventral wall of the urinogenital canal.

2. The vagiza, uteri, and Fallopian tubes with their funnels.

3. The ovaries, studded with ovisacs.

IX. Dissect away the kidneys, ureters-and in the male the scrotal sacs, in the female the ovaries-from the surrounding parts : cut through the pelvic symphysis with a knife, and sever the attachment of the corpora cavernosa to the ischia. Remove the whole of the urinogenital organs, together with the posterior end of the rectum, from the body, and after noting $\S$ VII, 3 and 4, pin them out in a clissecting-dish, with the ventral side uppermost, taking care to preserve the natural relations of the parts. Dissect away all the superfluous connective tissue and fat, separate the rectum at its cut end from the urinogenital canal, and turn it on one side, noting the rectal and perineal glands. Make out, in addition to the parts referred to in $\$$ VIII :-

A. In the male. The uterus masculinus and prostate, and the spermiducts passing between the former and the bladder.

Sketch the entire dissection. Then slit open the uterus masculinus so as to see the openings of the spermiducts, remove the rectum and rectal glands, and make out the relations of the corpus spongiosum. Make a median incision along the whole length of the penis, beginning at the apex and cutting through the fibrous septum between the two corpora cavernosa: continue the incision forwards, so as to open the bladder along its ventral wall, and note and indicate on your sketch the opening of the ureters into the bladder, and the crescentic aperture of the uterus masculinus into the urinogenital canal, just at the anterior edge of a cushion-like fold. 
13. In the female. The wide urinogenilal canat with its vascular walls.

Sketch the entire dissection.

Make a median longitudinal incision through the urinogenital canal, and continue it forwards until the cavity of the bladder is exposed : slit open the vagina and one of the uteri and Fallopian tubes in the same way. Note and indicate on your sketch the openings of the ureters into the bladder, the connection of the neck of the bladder with the urinogenital canal, the large aperture in the latter leading into the vagina, and the thick-lipped aperture (os ateri), cummunicating between the vagina and thick-walled uterus, between which and its fellow is a vestigial septum, indicating the primarily paired character of the vagina. Insert a seeker into the cœlomic aperture of the Fallopian tube. If the uteri contains embryos they present a series of swellings; cut each swelling open from the ventral side, and note the foetus attached to a discoid placenta (p. 524); remove each fotus, together with its placenta, and preserve it in corrosive sublimate (p. I35). Microscopic sections of the ovary should be examined, and the hollow ovisacs noted, each consisting of follicular cells enclosing a minute ovum.

With a scalpel or razor cut across one of the kidneys through the hilus, parallel to the dorsal and ventral faces of the organ, and note the pelvis, and its prolongations - the calices, the urinary pyramid, and the cortical and medullary portions. Sketch. Examine also microscopic preparations of injected and uninjected kidney (compare p. I46).

$\mathrm{X}$. Dissect out the lumbo-sacral plexus in the pelvic cavity (p. 515), and then cut through the body just behind the diaphragm; the abdomen will be no longer required, but you should retain one of the hind-limbs if you wish to examine any of its muscles and joints (compare p. 64).

\section{E. Dissection of the thorax and neck,}

I. Dissect away the pectoral nuscles, cut through all the vertebral ribs of the left side, except the last five, at about a quarter of an inch from their junction with the sternal ribs: from the posterior end of the incision thus made, cut downwards (i.e., lowards the sternum) for alout an inch, and then forwards, through the sternal ribs. Turn forward the flap thus separated and carefully dissect it away from the underlying tissues at the anterior end, so as to detach it altogether without injuring the jugular and brachial reins. Note in the thoracic cavily thus laid open :-

I. The bericartium and heart, and the thymus. 
2. The collapsed lungs: make a small aperture in the trachea and inflate.

3. The paricial and visceral layers of the pleura and the mediastimum (Fig. I28). To make out the relations of these and of the mediastinal space more accurately, cut away a flap from the right wall of the thorax, as you have already done on the left side, leaving the sternum intact.

4. The relations of the pericardium, to see which cut through the posterior end of the sternum : separate the mediastinum from the dorsal surface of the bone, turn the latter forwards and remove it: then cut the pericardium open longitudinally, noting the pericardial fuid.

II. Dissect away the pericardium, the thymus, and any fat about the base of the heart which may obscure the vessels arising from it. Follow out these vessels to the head by clearing away the connective tissue, fat, $\&$ c., by which they are surrounded, but taking care not to injure any of the nerves of the neck. Make out :-

1. $a$, The left and right ventricles and auricles, and the ramifications of the coronary artery and vein; $b$, the two bronchi.

2. $a$, The pulmonary artery and its division into left and right trunks; $b$, the pulmonary veins (the course of which will be better seen at a later stage); $c$, the two precaval veins, each formed by the union of a subclavian, an external jugular, and a smaller internal jirgular; $d$, the thoracic portion of the postcaval vin.

3. $a$, The gullet (cervical and thoracic portions); $b$, the phrenic nerves (p. 515), and the thoracic portions of the vagi (Fig. 125); $c$, the arch of the aorta, continuous with the dorsal aorta, and the short ligament (vestige of the foetal ductus arteriosus) connecting the former with the pulmonary artery; $d$, the thoracic portions of the sympathetic nerves and their ganglia, lying on the heads of the ribs; $e$, the azygos vein (p. 509), best seen by turning the heart and lungs over to the left sicle of the animal.

4. $a$, The innominate artery, giving off the left and right common carotids and the right subclavian; $b$, the left subclavian artery; $c$, the division of each common carotid, at about the level of the anterior end of the larynx, into an external and an internal carotid.

Sketch the heart and origins of the main vessels.

III. Now dissect out certain of the nerves and other structures in the neck, as follows :-

1. The vagus (Fig. 125), running to the outer side of each common 
carotid : with the seeker carefully separate it from the carolid, and trace it forwards to the angle between the head and neck-at which point it enlarges to form a ganglion, and backwards to the thorax. Branches are given off to the larynx (anterior and posterior or recurrent laryngeal nerves) and to the heart (depressor) nerves, but the dissection of these may be omitted by the beginner.

2. The sympathetic (Fig. I25), running on the dorsal side of the carotid artery. It is most easily distinguished by seizing the carotid with the small forceps just at the junction of the head and neck and putting it slightly on the stretch : the vagus ganglion will then be seen on the outer side of the artery, and the more elongated anterior cervical ganglion of the sympathetic on its inner side: trace the sympathetic nerve from this ganglion backwards to the thorax, at the anterior end of which (close to the subclavian artery) it enlarges to form the posterior cervical ganglion, and then becomes continuous with the thoracic portion of the cord.

3. The hypoglossal nerve will be seen at about the level of the vagus ganglion, passing forwards to the tongue.

4. Note- $a$, the thyroid gland; $b$, the thyroid and cricoid cartilages of the larynx; $i$, the brachial and vertebral branches of the subclavian artery; and $d$, the bracliral plexus (p. 515).

IV. Remove the heart and lungs from the body, together with the posterior end of the trachea and recognisable portions of the aorta, precaval, and postcaval. Fasten out the organs under water with their ventral surface uppermost, and after making out the course of the pulmonary arteries and zeins, cut through them close to the lungs and separate the latter from the heart. Note:-

I. The two main lobes of each lung and the two aicessory lobes on the right side.

2. The cartilages of the trachea and bronchi; trace the bronchi for a short distance into the lungs. Sketch.

V. Cut away the outer walls of both auricles so as to expose their cavities, taking care not to injure the veins which enter them. Wash out the contained clots of blood, and note-

I. $u$, The appendix of each auricle, and the network of muscular bands in its walls; $b$, the septum aimicularum and fossa ovalis-best seen from the left side by holding the heart between your eyes and the light.

2. $a$, The anericulo-ventricular apertures; $b$, the apertures of the 
precaval and postcaval veins; c, the Eustachian vauve; a, the apertuse of the coronary vein within the tunnel-like opening of the left precaval; $e$, the apertures of the pulmonary aicins.

Then cut away both auricles so as to expose the bases of the ventricles, and remove all but about an eighth of an inch of the aorta ane pulmonary artery; pour water into the ventricles through the auriculoventricular apertures, and squeeze the ventricles, noting-

3. a, The bicuspid and tricuspid valves, and the semilumar values at the origins of the aorta and pulmonary artery respectively; $b$, the apertures of the coronary arteries just on the distal side of the aortic valves. Sketch.

Now remove the outer walls of both ventricles by making first a transverse incision along the base of each, and then, from its extremities, converging incisions nearly to the apex of the heart. Make out :-

4. The relative thickness of the walls of the right and left ventricles, the form of the septum ventriculorum, the cavities of the two ventricles, and the muscular ridges in their walls.

5. The flaps of the tricuspid and bicuspid values, and their tendinous cords and papillary muscles.

6. The apertures of the aorta and pulmonary artery into the left and right ventricle respectively.

Sketch.

\section{F. Dissection of the head.}

I. Carefully dissect the skin away from one side of the head; either side will do, but if you are using the head from which you have already removed the brain, choose the side on which the auditory region is least damaged: Notice the Meibomian glands (p. 5 18). Cut away, with bone forceps, the supraorbital process of the frontal, being careful not to injure any of the contents of the orbit. Note-

I. $a$, A large mass of muscle (masseter) covering the posterior half of the mandible, on which branches of the facial nerve will be seen; $b$, the thin, irregular parotid gland, at the base of the pinna, and the large infraorbital gland, lying mainly within the orbit below (p. 495).

2. The four recti and the two oblique muscles of the eye. Note the course of the superior oblique through its tendinons loop (p. 5I7).

3. The lachrymal and Harderian glands, situated in the postero. superior and antero-inferior regions of the orbit respectively.

II. Remove the eyeball by cutting through the muscles and optic nerve, 
noting the retractor bulbi muscle around the latter. (For the structure of the cyt, see p. 538.) Cut off the pinna, and clear away the muscles, $\$ c$, covering the tympanic bone: remove the entire tympanic and periotic bones (1) 475) in one piece, lay open the external auditory passage, and very carefully and gradually cut away the outer wall of both tubular and bulbous portions of the tympanic with the boneforceps. Note:-

1. The tympanic membrane and the handle of the malleus; and after cutting away the former so as not to injure the latter, and removing rather more of the bulla, make out-

2. 'The tympanic cavity; the malleus, incus, and stapes; the tensor tympani and stapedius muscles; and the aperture of the Eustachian tube. Remove the auditory ossicles, noting as you do so the fenestra ovalis and fenestra rolunda, and examine them under the low power of the microscope: by cutting through the periotic with the bone forceps, the position of the membranous labjrinth with the cochlea may be made out.

III. Dissect off the muscles covering the mandible on the side you are working ; detach its ascending portion from the muscles inserted on to its inner surface, remove it entire, cutting through the symphysis, and clear away the underlying muscles. Pass a probe from the cut end of the gullet forwards into the mouth : lay open the gullet along this, and pull the tongue downwards, so as to get a view into the interior of the mouth (Fig. 125). Note :-

1. $a$, The palate and velum palati; $b$, the tongue and its papille (p. 492); $c$, the pharynx; $d$, the glottis and epiglottis; $e$, the continuation of the pharynx above the velum palati (naso-pharynx), with which the internal nostrils communicate; $f$, the positions of the teeth (incisors and grinders); $g$, the apertures of the naso-palatine canals (p. 490).

Then remove the nasal, premaxilla, and maxilla of the same side, noting the olfactory nerves and the turbinals; and after passing a probe backwards from the external nostril into the nasal chamber as far as it will go, remove the turbinals on this side, so as to expose the cartilaginous nasal septum. Also cut away all the remaining bone on the same side of the middle line, slit up the naso-pharynx, and reduce your dissection to a neat longitudinal section, noting-

2. The cranial cavity, the roof of which will have been cut away if the brain has been removed. 
3. The aperture of the Eustaclian tube: pass a probe from it into the tympanic cavity.

4. The position of Jacobson's organ, on the ventral side of the nasal septum; and after cutting away the latter, the cthmo-twrbinals, maxillo. turbinals, and naso-turbinals of the other side.

Sketch the entire longitudinal section.

IV. Remove the larynx with the anterior part of the trachea. Make a longitudinal vertical section of it, keep one half entire, and from the other dissect away the muscles and mucous membrane so as to see the cartilages clearly. Examine first the cartilages and then the soft parts, noting the relations of the thyroid, cricoid, and arytenoid cartilages, the epiglottis, and the vocal cords. Sketch.

\section{G. The Brain.}

I. - External characters. Note :-

I. The medulla oblongata, with its dorsal and ventral fissures and the pons Varolii; the convoluted cerebellum, consisting of a central lobe and two lateral lobes and flocculi.

2. The optic lobes, seen on slightly separating the hemispheres and cerebellum; the crura cerebri.

3. The olfactory lobes and cerebral hemispheres (frontal, parietal, and temporal lobes)-by gently separating them, the corpus callosum will be seen-and just behind them is the pineal body; the diencephalon is covered above by the hemispheres, below it is visible and is continued into the infundibulum; the pituitary body is nearly always left behind in the brain-case when the brain is removed, and a small aperture is seen in the centre of the infundibulum where it was attached; the optic chiasma.

4. The origins of the nerves from the brain ; these cannot, of course, be plainly made out unless the nerves have been carefully cut through when removing the brain. Compare pp. 163-166, and note in addition the spinal accessory and hypoglossal.

Sketch the brain from above and from below.

II. Carefully remove successive slices from one of the hemispheres nearly parallel to its upper surface down to the level of the corpus callosum, noting the fibres of the latter and the relations of the grey and white matter in the hemispheres. Cut through the corpus callosum longitudinally, near the middle line, and remove sufficient of the hemisphere already dissected to expose the lateral ventricle 
and its extensions into the frontal, parietal, and temporal lobes. Note:-

1. The hippocanpus, the corpus striatum, the choroid plexus, and the seplum lucidum (p. 514): Then cut away the rest of the same hemisphere so as to expose-

2. $a$, The diencephalon and its (wo lateral halves (optic thalami) between which the third ventriile is enclosed; from its roof the stalk of the pineal body arises; $b$, the two pairs of optic lobes. Cut away the lateral half of the cerebellum of the same side, and note-

3. The valve of Vieussens, the fourth ventricle, and the tree-like appearance (arbor zitce) of the cerebellum in section.

III. Now reduce the whole brain to a longitudinal vertical section (Fig. 132), cutting it through with a sharp knife very slightly to that side of the median line which you have already dissected, so as to expose the median ventricles in section. Wake out, on the uninjured half, the relations of the third zientricle, iter, fourth ventricie, foramen of Monro, infundibulum, optic chiasma, corpus callosum and formix, lamina terminalis, anterior, middle, and posterior commissure, crura cerebri, valie of Vieussens, pons Varolii, Bic. Sketch.

IV. A series of thick transverse sections should be made through another thoroughly hardened brain, and the relations of the parts slready seen by dissection examined.

\section{The Eye.}

On account of its larger size, it is better to substitute the eye of an ox or sheep-which can be obtained fresh from a butcher-for that of the rabbit, Two specimens should be obtained, and the fat, muscles, and any portions of the eyelirds remaining attached cleared away. I. After noting the conjunctiala, cornea, sclerutic, optic nerve, iris, and pupil, insert the scissors into the margin of the cornea and cut round and remove it, so as to expose the aqueous chamber, noting the aqueous humour, as well as the iris, lens, and pupil (compare Fig. 57). Sketch.

Insert the handle of a scalpel between the iris and sclerotic and separate off the latter from the choroid around the outer half of the eyeball, first making four radial cuts in the sclerotic, beginning at its margin, at equal distances from one another. Turn back the four flaps thus made, and insert pins through them so as to fix "the eye firmly down under water with the iris uppermost : note the ciliary muscle around 
the outer margin of the iris, the choroid, and the ciliary zessels and nerves. Then make two radial cuts, a short distance from one another, through the iris, and turn back the portion between the cuts, noting the ciliary processes. Sketch.

Cut through the choroid and the parts cnclused by it, horizontally, around the line up to which it has been alrearly exposed, so as to separate the eye-ball into an inner and outer hemisphere : in doing so, the gelatinous vitreous humour must be cut with the scissors. Examine and sketch both sections, noting in the outer hemisphere-the retina, stopping short at the outer margin of the ciliary processes, and the lens with its capsule; and in the inner hemisphere-the retina with its blourlvessels, and the blind-spot (or point of entrance of the optic nerve). Then remove the retina and observe the choroid with its iridescent tapetum.

2. Cut your second specimen into vertical halves with the scissors, making the cut pass through the cornea, pupil, and optic nerve. As it is difficult to cut through the lens without clisturbing its relations, it should be carefully separated from one half, and left entire on the other half. Examine the relations of the parts once more, compare Fig. 57, and sketch.

\section{Dissection of typical flexor and extensor muscles and joints of the fore-limb.}

I. Expose the biceps (chief flexor of the fore-arm). Its origin is from the anterior edge of the glenoid cavity on the pectoral arch: it arises by a single long tendon, working in the bicipital groove of the humerus. It is spindle-shaped, consisting of a single belly, and is inserted on to the proximal end of the radius.

2. Expose the triceps (chief extensor of the fore-arm). It arises by three main heads from the pectoral arch and humerus, and is inserted on to the olecranon process of the ulna.

3. Remove the muscles from the shoulder-joint, and note the capsular ligament (p. 55). Cut through this so as to open the synovial capsule, and note the synovial membrane and fluid and the cartilaginous articular surfaces of the glenoid cavity and head of the humerus respectively : or, prepare as directed on p. 64 in the case of the hipjoint of the frog.

4. As an example of a muscle with a multiple irsertion, dissect out the extensor communis digitorum (dorsal). It arises from the distal 
end of the humerus, and at the distal end of the fore-arm divides into four tendons, which pass through the annular ligament to digits 2-5 and are inserted into the middle and distal phalanges. Note the sesamoid bones on the palmar sicte of the joints of the digits.

\section{J. Side dissection.}

Obtain another rabbit, and make a dissection from the side (compare Fig. 125) as directed in the case of the dogfish and frog (p. 464). Make out the structure and relations of all the principal organs once more, and sketch your dissection.

\section{K. Transverse sections.}

The examination of transverse sections is most easily and satisfactorily done by preparing microscopical sections of a foetus (p. 532) not more than a couple of inches in length, as clirected in the case of the young dogfish (p. 464) : a foetal rat or mouse will answer the purpose equally well.

With a sharp knife, cut transverse sections, about $\frac{1}{8}$ th inch thick, from the following regions : $a$, snout ; $b$, cranial region ; $c$, neck ; $d$, thorax ; and $\varepsilon$, abdomen. Stain and imbed, and then cut and mount one or two sections of each, examining them with a low power and making sketches.

Then put on the high power, and make out as much as possible of the histology of the various organs. 


\section{CHAPTER XI}

THE MINUTE STRUCTURE OF CELLS: CELL-DIVISION : STRUCTURE OF THE OVUM : SPERMATOGENESIS : MATURATION AND FERTILIZATION OF THE OVUM : SEGMENTATION OF THE OOSPERM : EFFECT OF FOOD-YOLK ON DEVELOPMENT : FORMATION OF THE CHIEF ORGANS OF THE ADULT VERTEBRATE, AND OF THE AMNION, ALLANTOIS, AND PLACENTA.

Structure of the Cell.-We have learnt in previous chapters that all organisms are formed essentially of one or more cells (compare pp. I ro and I 8 ); that the cell consists of protoplasm, and contains a mucleus consisting of chromatin and achromatin (p. 129), in which one or more nucleoli can usually be distinguished; and that cells multiply by a process of binary fission (p. 236). It will now be necessary to study the structure of a typical animal cell and of its mode of division in somewhat greater detail.

There seems to be a good deal of variation in the precise structure of various animal and plant-cells, but the more recent researches show that in the cell-body or protoplasm two constituents may be distinguished, a clear semi-fluid substance, traversed by a delicate sponge-work. Now under the microscope the whole cell is not seen at once but only an optical section of it - that is, all the parts which 
are in focus at one time (see e.g. p. 315): by altering the focus we view the object at successive depths, each view being practically a slice parallel to the lenses of the instrument. This being this case, protoplasm presents the microscopic appearance of a clear or slightly granular matrix traversed by a delicate network. In the epithelial cells of animals the protoplasm is bounded externally by a cell-membrane of extreme tenuity: in amoboid cells the ectoplasm or transparent, non-granular portion of the cell consists of clear protoplasm only, the granular endoplasm alone possessing the sponge-work.

It is quite possible that the reticular character of the cell may be merely the optical expression of an extensive but minute vacuolation, or may be due to the presence of innumerable minute granules developed in the protoplasm as products of metabolism.

The nucleus is spherical or oval in form, and is enclosed in a delicate nuclear membrane (Fig. $136, \mathrm{~A}, n u . m$ ). The achromatin is a homogeneous semi-fluid substance which forms the groundwork of the nucleus: it resembles the clear cell-protoplasm in its general characters, amongst other things in being almost unaffected by dyes. The chromatin (chr) takes the form of a network or sponge-work of very variable form, and is distinguished from all other constituents of the cell by its strong affinity for aniline and other dyes (compare Part I., Chapters VII and VIII). Frequently, as we have seen, one or more minute globular structures, the mucleoli $\left(n i^{\prime}\right)$, occur -in the nucleus either connected with the network or lying freely in its meshes: they also have a strong affinity for dyes although they often differ considerably from the chromatin in their microchemical reactions.

In the body of some cells and possibly of all there is 

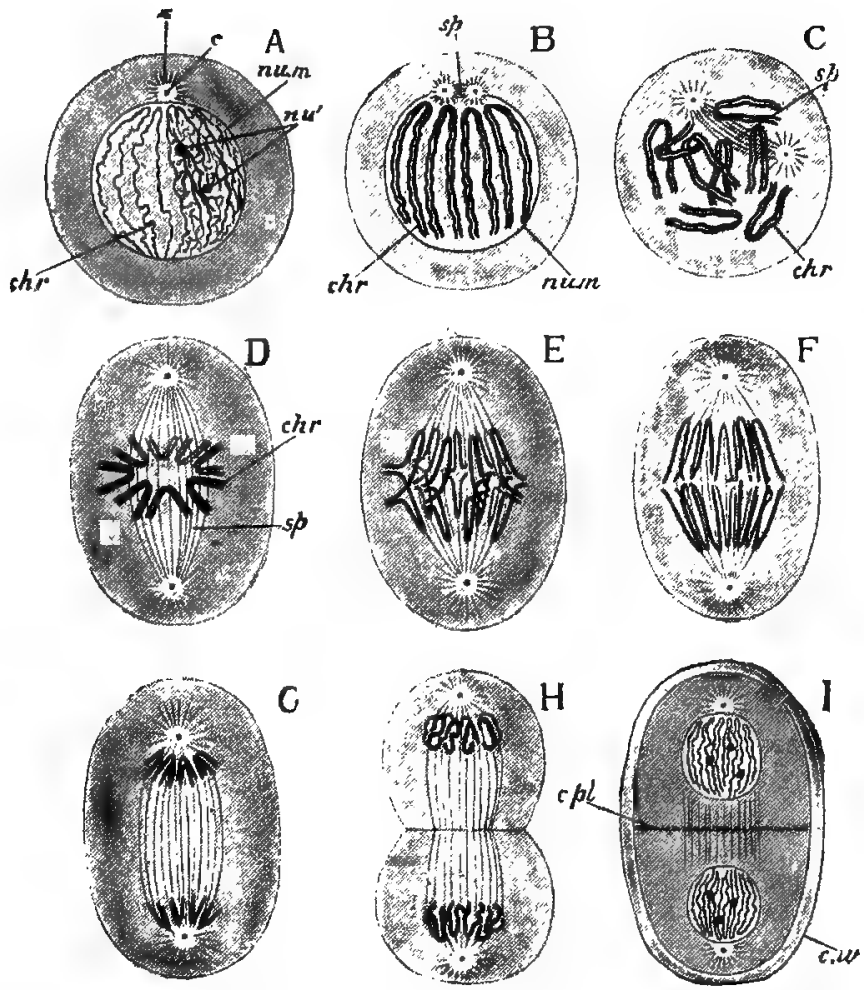

FIG. 136.-Diagrams illustrating the process of indirect cell-division or mitosis.

$A$, the resting cell ; $\mathbf{B}, \mathbf{C}, \mathbf{D}$, successive phases in the formation and arrangement of the chromatin-loops and of the nuclear spindle ; E, F, G, separation of the two sets of daughter chromosomes and their passage towards the poles of the spindle; $\mathbf{H}$, division of the cell-body and formation of the two nuclei; $I$, division of a plant-cell, showing the formation of the cell-wall $(c . w)$ by means of a cellplate $(c, p l)$

$c$. centrosome; chr. chromatin; $\| u^{\prime}$. nucleoli; $n u . m$. nuclear membrane; $s$. astrophere; sp. spindle. (From Parker's biology, altered from Flemming, Rabl, etc.)

found a globular body, surrounded by a radiating arrangement of the protoplasm and called the astrosphere $(s):$ it lies close to the nucleus, and contains a minute granule 
known as the central particle or centrosome (c). In many cases two astrospheres and centrosomes are found in each cell (B).

Cell-division. The precise changes which take place during the fission of a cell are, like the structure of the cell itself, subject to considerable variation. We will consider what may probably be taken as a typical case (Fig. 1 36 ).

First of all, the astrosphere, with its centrosome, divides (B), and the products of its division gradually separate from one another (C), ultimately passing to opposite poles of the nucleus (D). At the same time the network of chromatin divides into a number of separate filaments called chromosomes (B, chr), the number of which appears to be constant in any given species of animal or plant, although it may vary in different species from two to twenty-four or more. Soon after this the nuclear membrane and the nucleoli disappear (B, C), and the nucleus is seen to contain a spindleshaped body $(s p)$ formed of excessively delicate fibres which converge at each pole to the corresponding astrosphere. The precise origin of this nuclear spindle is uncertain: it may arise either from the nuclear matrix or, more probably, from the protoplasm of the cell : it is not affected by colouring matters.

At the same time each chromosome splits along its whole length so as to form two parallel rods or loops in close contact with one another (B); in this way the number of chromosomes is doubled, each one being now represented by a pair.

The divided chromosomes now pass to the equator of the spindle (D) and assume the form of more or less $\mathrm{V}$-shaped loops, which arrange themselves in a radiating manner so as to present a star-like figure when the cell is 
viewed in the direction of the long axis of the spindle. Everything is now ready for division, to which all the foregoing processes are preparatory.

The two chromosomes of each pair now gradually pass to opposite poles of the spindle (F, F), two distinct groups being thus produced (G) and each chromosome of each group being the twin of one in the other group. Perhaps the fibres of the spindle are the active agents in this process, the chromosomes being dragged in opposite directions by their contraction: on the other hand it is possible that the movement is due to the contractility of the chromosomes themselves.

After reaching the poles of the spindle the chromosomes of each group unite with one another to form a network $(\mathrm{H})$ around which a nuclear membrane finally makes its appearance (I). In this way two nuclei are produced within a single cell, the chromosomes of the danyhter-nuclet, as well as their attendant centrosomes, being formed by the binary fission of those of the mother-nucleus.

But pari passu with the process of nuclear division, fission of the cell-body is also going on. This takes place by a simple process of constriction $(\mathbf{H})$-in much the same way as a lump of clay or dough would divide if a loop of string were tied round its middle and then tightened.

In comparatively few cases the dividing nucleus instead of going through the complicated processes just described divides by simple constriction. We have therefore to distinguish between direct and indirect nuclear division. To the latter very elaborate method the name mitosis or karyokinesis is applied : direct division is then distinguished as amitotic.

In this connection the reader will not fail to note the extreme complexity of structure revealed in cells and their nuclei by the highest powers of the microscope. When the PKACT. ZOOL. 
constituent cells of the higher animals and plants were discovered, during the early years of the present century, by Schleiden and Schwann, they were looked upon as the ultima Thule of microscopic analysis. Now the demonstration of the cells themselves is an easy matter, the problem is to make out their ultimate constitution. What would be the result if we could get microscopes as superior to those of to-day as those of to-day are to the primitive instruments of eighty or ninety years ago, it is impossible even to conjecture. But of one thing we may feel confident-of the great strides which our knowledge of the constitution of living things is destined to make during the next half century.

Structure of the ovum. The striking general resemblance between the cells of the higher animals and entire unicellular organisms has been commented on as a very remarkable fact: there is another equally significant circumstance to which we must now advert.

All the higher animals begin life as an egg, which is either passed out of the body of the parent, as such, as in earthworms, crayfishes, frogs, birds, \&c. (oviparous forms), or undergoes development within the body of the parent, as in some dogfishes (p. 454) and nearly all mammals (viviparous forms).

The structure of an egg is, in essential respects, the same in all animals from the highest to the lowest (compare p. I95). It consists (Fig. I37) of a more or less globular mass of protoplasm, in which are deposited particles of a proteinaceous substance known as yolk-granules. Within the protoplasm is a large nucleus containing chromatin as well as one or more nucleoli-which are often known as serminal spots, the entire nucleus of the ovum being called 
the germinal vesicle. An investing cuticular membrane may or may not be present. In other words the egg, as we have already seen, is a cell.

The young or immature ova of all animals present this structure, but in many cases certain modifications are undergone before the egg is fully formed. loor instance, the protoplasm may throw out pseudopods, the egg becoming amœboid (p. 302); or, as mentioned above and as is usually the case, the surface of the protoplasm may secrete a cell-wall, often of considerable thickness, and known as the vitelline membrane (p. 196 and Fig. 137), which may be perforated at one pole by an aperture, the micropyle (p. 400). The most extraordinary modification takes place in some Vertebrata, such as dogfishes (p. 454) and birds. In a hen's egg,

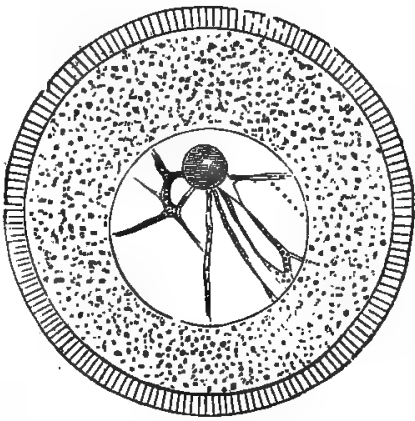

Fir. 137.-Ovum of a Sen-11rchin . (To.ro. tmenstes lizridus), showing the radiallystriated cell-wall (viteiline membrane), the protoplasm containing yolk granules (vitellus), the large hucleus (germinal vesicle) with its network of chromatin, and a large nucleolus (germinal spot). (From Balfour, after Hertwig.) for instance (Fig. I38), the yolk-granules increase immensely, swelling out the microscopic ovum until it becomes what we know as the "yolk" of the egg: around this layers of albumen or "white" are deposited by the glands of the oviduct and finally the shell-membrane and the shell. Hence we have to distinguish carefully in eggs of this character between the entire "egg" in the ordinary acceptation of the term, and the ovum or egg-cell. But complexities of this sort do not alter the fundamental fact that all the higher animals begin life as a single cell, or in other 
words, that multicellular animals, however large and complex they may be in their adult condition, originate as unicellular bodies of microscopic size; and the same is the case with plants.

Spermatogenesis and 0ogenesis. In the preceding chapters it has more than once been stated that sperms

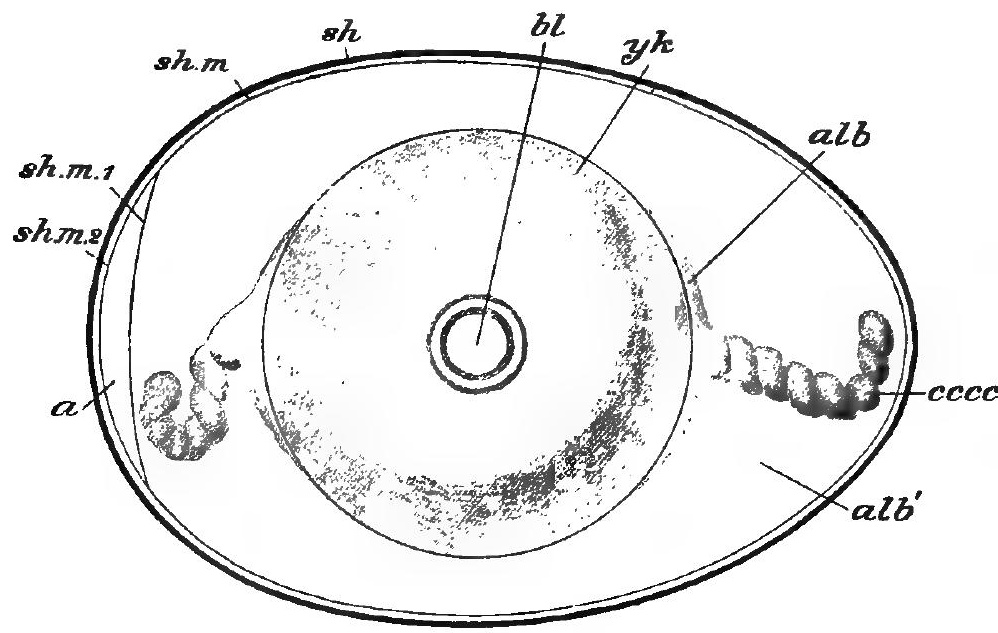

FIG. $3_{38}$. - Semi-diagrammatic view of the egg of the fowl at the beginning of incubation.

$a$. air-space; $a l b$. dense layet of albumen; $a l b^{\prime}$. more fluid albumen; $b l$. blastoderm; ch. chalaza, a twisted cord of the dense layer of albumen at either end of the egg, formed as the latter rotates down the oviduct; sh. shell; sh. $\|^{\prime}$. shellmembrane; sh.m. $I, s h . m .2$, its two layers separated to enclose air-cavity. (From Parker and Haswell's Zoology, after.Marshall, slightly altered.)

arise from ordinary undifferentiated cells in the spermary, and that ova are produced by the enlargement of similar cells in the ovary. Fertilization has also been described as the conjugation or fusion of ovum and sperm (compare 
p. 197). We have now to consider in greater detail what is known as to the precise mode of development of sperms (spermatogenesis) and of ova (oogenesis), as well as the exact steps of the process by which an oosperm or unicellular embryo is formed by the union of the two sexual elements.

Both ovary and spermary are at first composed of cells of the ordinary kind, the primitive sex-cells; and it is only by the further development of these that the sex of the gonad is determined.

In the spermary the sex-cells (Fig. I 39, A) undergo repeated fission, forming what are known as the sperm-mothercells (в). These have been found in several-instances to be distinguished by a peculiar condition of the nucleus. We saw (p. 544) that the number of chromosomes is constant in any given animal, though varying greatly in different species. In the formation of the sperm-mother-cells from the primitive sex-cells the number becomes doubled : in the case of the mole-cricket, for instance, shown in Fig. r 39, the ordinary cells of the body, including the primitive sex-cells, contain twelve chromosomes, while the spern-mother-cells contain twenty-four.

The sperm-mother-cell now divides (c), but instead of its chromosomes splitting in the ordinary way (p. 544, Fig r 36 ) half of their total number-in the present instance twelvepasses into each daughter cell: thus two cells are produced having the normal number of chromosomes. The process of division is immediately repeated in the same peculiar way (D), the result being that each sperm-mother-cell gives rise to a group of four cells having half the normal number of chromosomes-in the present instance six. The four cells thus produced are the immature sperms (E): in the majority of cases the protoplasm of each undergoes a great elongation, being converted into a long vibratile thread, the tail of 
the sperm $(F, G)$, while the nucleus becomes its more or less spindle-shaped head and the centrosome takes the form of a small intermediate piece at the junction of head and tail.

Thus the sperm or male gamete is a true cell, specially
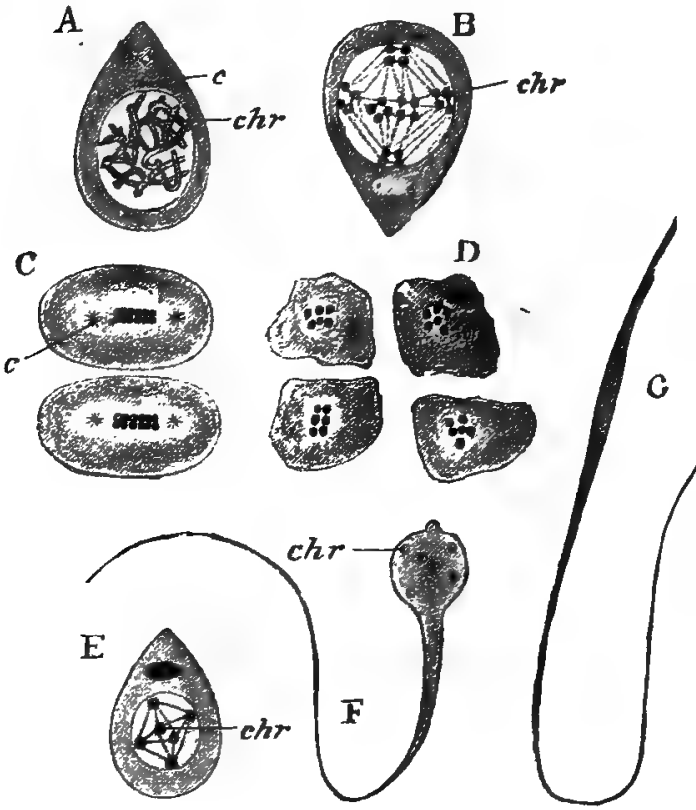

FIG. 139.-Spermatogenesis in the Mole-Cricket (Gryllotalpa).

A, primitive sex-cell, just preparatory to division, showing twelve chromosomes (chr); $c$. the centrosome. B, sperm-mother-cell, formed by the division of $A$, and containing twenty-four chromosomes; the centrosome has divided into two. C, the sperm-mother-cell has dirided into two by a reducing division, each daughter-cell containing twelve chromosomes. D, each daughter-cell has divided again in the same manner, a group of four sperm-cells being produced, each with six chromosomes. E, a single sperm-cell about to elongate to form a sperm. $F$, immature sperm ; the six chromosomes are still visible in the head. $G$, fully formed sperm. (From Parker's Biology, after vom Rath.)

modified in most cases for active movement. This actively motile, tailed form is, however, by no means essential : in 
some animals (e.g. Crayfish, p. 368) the sperms are nonmotile.

The peculiar variety of mitosis described above, by which the number of chromosomes in the sperm-mothercells is reduced by one-half, is known as a reducing division.

As already stated, the ova arise from primitive sex-cells, precisely resembling those which give rise to sperms. These divide and give rise to the egg-mother-cells in which, as in the sperm-mother-cells, the number of chromosomes is eventually doubled. The egg-mother-cells do not immediately undergo division, but remain passive and increase in size by the absorption of nutriment from surrounding parts : in this way each egg-mother-cell becomes an ovum. Sometimes this nutriment is simply taken in by diffusion or osmosis, in other cases the growing ovum actually ingests neighbouring cells after the manner of an Amoba. Thus in the developing egg the processes of constructive are vastly in excess of those of destructive metabolism.

We have seen (p. 249) that the products of destructive metabolism may take the form either of waste products which are got rid of, or of plastic products which are stored up as an integral part of the organism. In the developing egg, in addition to increase in the bulk of the protoplasm itself, a formation of plastic products usually goes on to an immense extent. In plants the stored-up materials may take the form of starch, of oil, or of proteid substance : in animals it consists, as mentioned above, of rounded or angular grains of proteid material, known as yolk-granules. These being deposited, like plums in a pudding, in the protoplasm, have the effect of rendering the fully-formed egg opaque, so that its structure can often be made out only in sections. 
Maturation of the ovum.- The fully-formed ovum as described on p. 546, is, however, incapable of being fertilized or of developing into an embryo: before it is ripe for conjugation with a sperm or able to undergo the first. stages of segmentation it has to go through a process known as maturation.

Maturation consists essentially in a twice-repeated process of cell-division. The nucleus (Fig. I40, A) loses its membrane, travels to the surface of the egg, and takes on the form of an ordinary nuclear spindle. Next the protoplasm grows out into a small projection or bud, into which one end of the spindle projects. Nuclear division then takes place, one of the daughter nuclei remaining in the bud (pol), the other in the ovum itself. Nuclear division is followed as usual by division of the protoplasm, and the bud becomes separated as a small cell distinguished as the first polar cell.

In some cases development from an unfertilized female gamete lakes place, the process-which is not uncommon among insects (c.g. the common little green plant-luuse or $A p h i s)$ and crustaceans (e.g. waterfleas)-being distinguished as parthenogenesis. It has been proved in many instances and is probably generally true that in such cases the egr begins to develop after the formation of the first polar cell. Thus in parthenogenetic ova it appears that maturation is completed by the separation of a single polar cell, after which the ovum contains the number of chromosomes normal to the species.

In the majority of cases, development takes place only after fertilization, and in these maturation is not complete until a second polar cell $(\mathrm{E}$, pol) has been formcd in the same manner as the first. The ovum has now lost a portion of its protoplasm together with three-fourths of its chromatin, half having passed into the first polar cell and half of what remained into the second: the remaining one- 

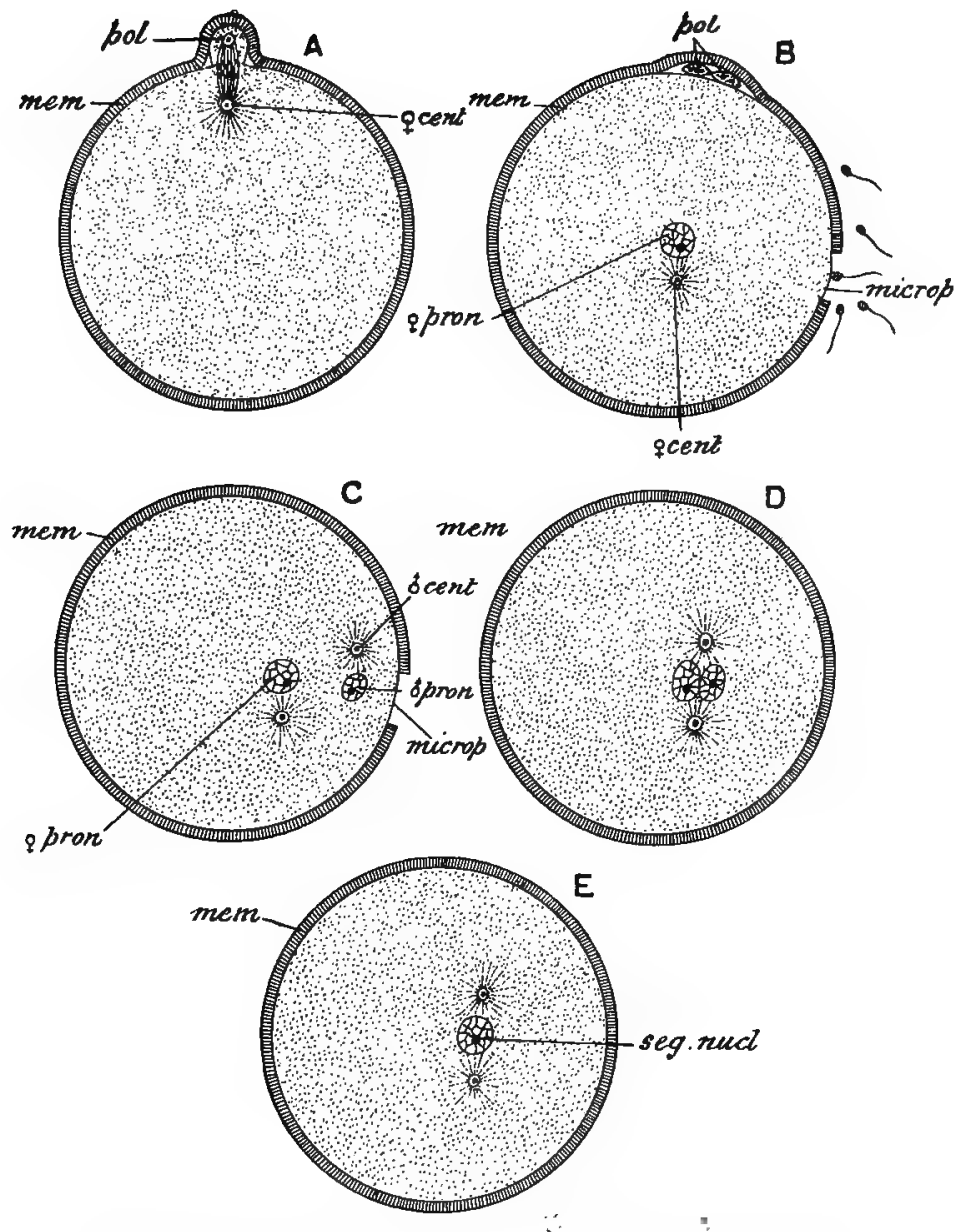

FiG. r40. The maturation and impregnation of the animal ovum (diagrammatic?. $\Lambda$, the ovum, surrounded by the vitelline membrane (mem), in the act of forming the first polar cell ( $\left.p_{0}\right)$; $q$ cent. centrosome. $\mathrm{B}$, both polar cells ( $p o l$ ) are formed, the female pronucleus ( 9 pron.) lies near the centre of the ovum, and one of the several sperms is shown making its way into the ovum at the mirropyle (microp). C, the head of the sperm has become the male pronucleus ( $\delta$ pron), its intermediate piece the male centrosome $(\delta c \theta n t)$; other structures as beforc. $\mathbf{D}$, the male and female pronuclei are in the act of conjugation. E, conjugation is complete, and the segmentation uucleus ( $s e g . n t u c l$ ) formed. (From Parker and Haswell's Zoology.) 
fourth of the chromatin becomes enclosed as a nucleus, which is distinguished as the female pronucleus ( $\mathrm{B}, q$ pron).

The formation of both polar cells takes place by a reducing division (p. $55 \mathrm{t}$ ); so that, while the immature ovum contains double the number of chromosomes found in the ordinary cells of the species, the mature ovum, like the sperm, contains only one-half the normal number.

In some animals the first polar body has been found to divide after separating from the egg. In such cases the egg-mother-cell or immature ovum gives rise to a group of four cells-the mature ovum and three polar-cells, just as the sperm-mother-cell gives rise to a group of four cells, all of which, however, become spërms (Fig. I 39).

Fertilization of the ovum.-Shortly after maturation, the ovum is fertilized by the conjugation with it of a single sperm. As we have found repeatedly, sperms are produced in vastly greater numbers than ova, and it often happens that a single egg is seen quite surrounded with sperms, all apparently about to conjugate with it (Fig. I40, B). It has, however, been found to be a general rule that only one of these actually conjugates: the others, like the drones in a hive, perish without fulfilling the one function they are fitted to perform.

The successful sperm (в) takes up a position at right angles to the surface of the egg and gradually passes through the micropyle (microp) or works its way through the vitelline membrane until its head lies within the eggprotoplasm. The tail is then lost, and the head, accompanied by the intermediate piere or centrosome, penetrating deeper into the protoplasm, takes on the form of a rounded body, the male pronucleus (c, opron).

The two pronuclei approach one another (D) and finally unite to form what is called the segmentation-nucleus ( $\mathrm{E}$, seg. nutcl), the single nucleus of what is not now the ovum but 
the oosperm-the impregnated egg or unicellular embryo (compare pp. 197 and 198 ).

The fertilizing process is thus seen to consist essentially in the union of two nuclear bodies, one contributed by the male gamete or sperm, the other by the female gamete or ovun. It follows from this that the essential nuclear matter or chromatin of the oosperm-often spoken of as the germplasm-is derived in equal proportions from each of the two parents. Morcover, as both male and female pronuclei contain only half the number of chromosomes found in the ordinary cells of the species, the union of the pronuclei results in the restoration of the normal number to the oosperm.

Fertilization being thus effected, the process of segmentattion or division of the oosperm takes place as described in previous chapters.

Different types of ova and their segmentation.-Before passing on to consider further details in the process of development of the oosperm, we must briefly refer to some differences already noted in the ova of different animals.

We have seen that in all cases the immature egg is a simple, minute cell, but that owing to the deposition of yolk-granules in its protoplasm, it may reach a comparatively large size (e.g. Crayfish, Dogfish, Bird). The presence of a greater or less amount of yolk in the ovum results, as we know, in very considerable differences as regards its mode of segmentation, as well as in its subsequent development. The minute eggs of Amphioxus and the Rabbit, for instance, which are each only ${ }_{10}^{1} \mathrm{~mm}$. (about $\frac{1}{2} \frac{1}{50}$ inch) in diameter, contain so comparatively small an amount of food-yolk as not to interfere materially with the process of segmentation: such ova are called alecithal. When the quantity of food yolk is relatively greater, it may 
become accumulated towards the centre of the egg, eventually leaving a layer of protoplasm comparatively free from yolk round the periphery (centrolecithal ova, e.g. Crayfish, Fig. 91); or, as in the case of telolecithal ova (Figs. 64, I 19 , and I38), the yolk-granules may become aggregated more at the lower than at the upper pole (Frog), until in the most extreme cases there is only a layer of yolkless protoplasm - the germinal disc (Dogfish, Bird)-lying at the upper pole of the egg.

As yolk is an inert substance, the more of it an egg contains the less actively can the latter divide, and the quantity may be relatively so great in some parts as to prevent segmentation in these parts altogether. We can therefore distinguish between holoblastic oosperms, which undergo entire segmentation (e.g., Hydra, Earthworm, Mussel, Amphioxus, Frog, Rabbit), and meroblastic oosperms, in which segmentation is limited to that part of the egg in which the protoplasm is comparatively free from yolk (e.g. Crayfish, Jogfish, Bird), this portion, after segmentation, being known as the blastoderm. In the centrolecithal ovum it is evident that the segmentation must be superficial or peripheral (p. 369), and in the meroblastic telolecitha ovum discoid or restricted to a small germinal disc at its upper pole (Figs. 119 and 138). In the case of holoblastic ova the segmenting cells or blastomeres may be equal, or nearly equal, in size (e.g., Amphioxus, Rabbit); or if the yolk is present in greater quantities towards the lower pole, unequal, (e.g. Earthworm, Frog).

The influence of the food-yolk in modifying the early processes of development is thus evidently very great, and in order to understand these processes in their simplest form it is necessary to select for our study an alecithal holoblastic egg, such as that of the lancelet. 
of the two resulting cells dividing again into two $(\mathrm{C}, \mathrm{D})$. This process is continued until a globular mass of cells or blastomeres is produced by the repeated division of the one cell which forms the starting point of the series. Owing to there being rather more yolk at the lower than at the upper pole, the lower cells are slightly larger than the upper, so that the segmentation is not quite equal $(\mathrm{E}-\mathrm{K})$. The embryo has now arrived at the polyplast or morula stage, and sections show that it is hollow, the blastomeres being arranged in a single layer around a central segmenta tion-cavity (p. 200): such a hollow polyplast is often known as a blastula $(K)$. The lower side of the blastula then becomes tucked in, or ini'aginated, the result being that the single-layered sphere is converted into a double-layered cup (Fig. I 42). This process can be sufficiently well imitated by pushing in one pole of a hollow india-rubber ball with the finger. The resulting embryonic stage is known as the gastrula (Fig. 142, C): its cavity is the primitive enteron or archenteron (p. 20I), and is bounded by the invaginated cells which now constitute the endoderm, the remaining cells forming the outer wall of the gastrula being the ectoderm (p. 202). The two layers are continuous at the aperture of the cup, the gastrula-mouth or blastopore. Between the ectoderm and endoderm is at first a space, the greatly diminished segmentation-cavity, which gradually becomes entirely obliterated, so that the ectoderm and endoderm are in contact (A.B). The general resemblance of the gastrula to a simplified Hydra, ${ }^{1}$ devoid of tentacles, will at once be apparent, and the stage in the development of the frog's egg re-

I It must, however, be remembered (pp. 303 and 313) that the ectoderm and endoderm of Hydroids are differentiated before the mouth is formed, so that the mouth does not correspond to the blasto. pore of the gastrula. 
presented in Fig. 64, $\mathrm{F}$, though much modified by the quantity of food-yolk, will be seen to correspond to the gastrula-stage. As in the frog, the blastopore soon closes, the mouth and anis being subsequently formed from the stomodrum and the proctodæum respcctively (p. 204).

The gastrula becomes elongated, flattened on one side,
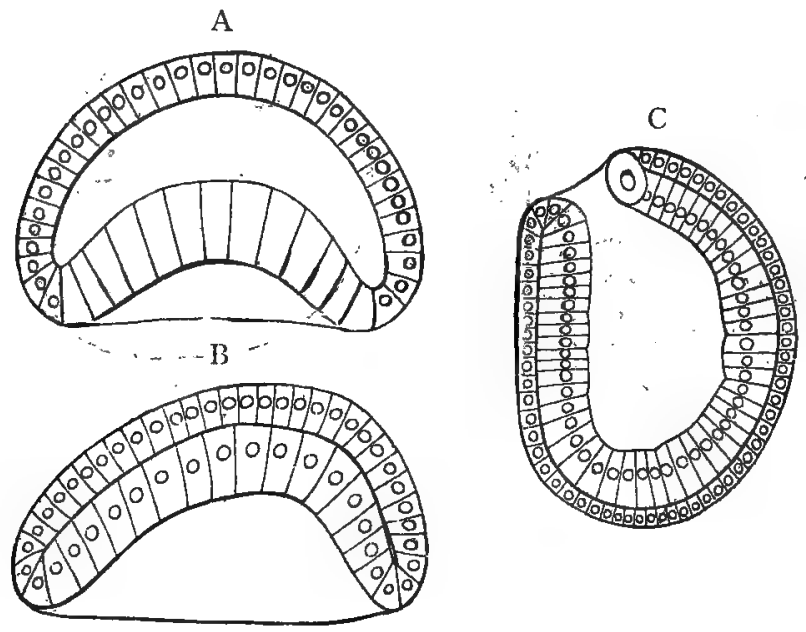

Fic. I $_{42 .}$ - Three stages in the formation of the gastrula of Amplioxns. In A, the nuclei of the endoderm have been omitted. (From Korschelt and Heider,
after Hatschek.)

and convex on the other. The flattened side corresponds to the dorsal surface of the adult, and the blastopore now comes to be situated, as in the frog-embryo (Fig. 64 $H, K)$, at the posterior end of the dorsal surface. A medullary plate and groove (Fig. I $43, m p$ ) are then formed, the central nervous system being developed in a manner essentially similar to that already described in the case of the tadpole (p. 202), except that the central canal of the medullary cord $(n)$ is formed after the 
plate has become separated from the outer ectoderm. In the mid-dorsal line a thickening of the endoderm (ch) soon becomes constricted off to form the notochord

A

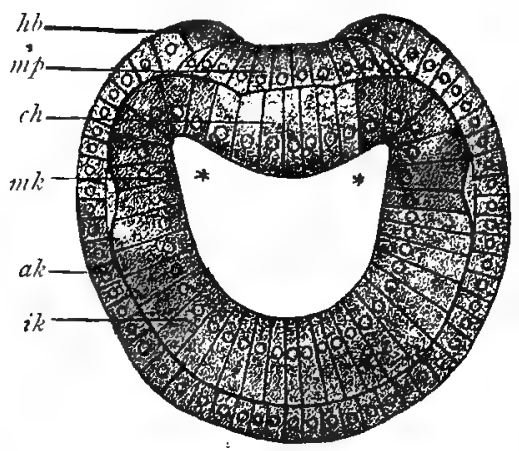

C

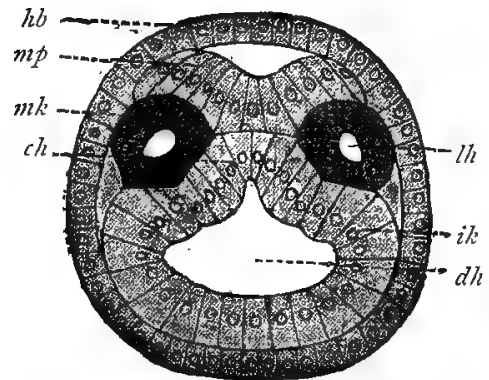

$B$

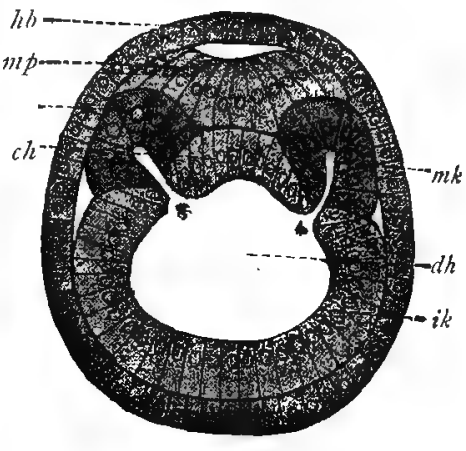

$\mathrm{D}$

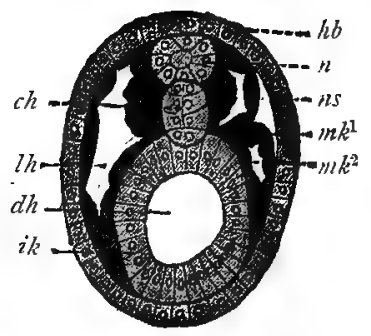

FIG. 143.-Four stages in the levelopment of the notochord, nervous system, and mesoderm of Amphioxus.

$a k$. ectoderm ; $c h$ notochord ; $d h$. cavity of auchenteron; $h b$. ridge of ectoderm growing over medullary plate; $i k$. endoderm ; th. ccelome ; $m k$. coelonic pouch ; $m k 1$. pariêtal layer of mesoderm; $m k^{2}$. visceral layer ; $m p$. medullary plate; $n$. medullary cord; $n s$. protovertebra. (From Korschelt and Heider, after Hatschek.)

(pp. 203, 404 and 425), and on either side of this a series of hollow endodermic pouches arise, arranged metamerically (Figs. 143, $m k$, and 144, $u s, m k$ ). The 
cavities of these, which subsequently give rise to the coelome $(h, u s h)$, are thus at first in free communication with the archenteron and are known as enteroceles; from their walls the mesoderm is derived. Subsequently the communications between the enteric and enterocolic cavities become
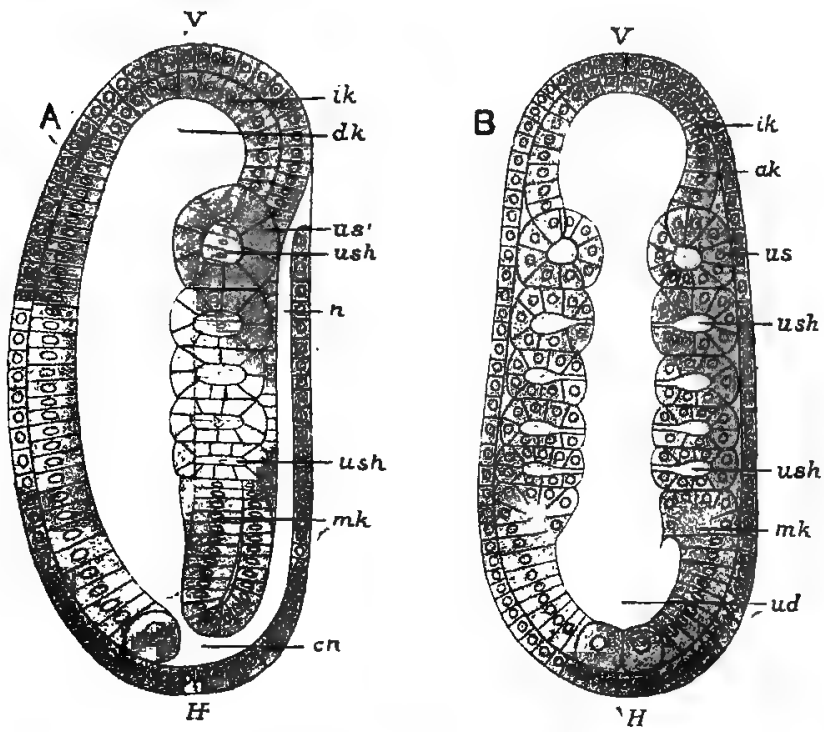

Fig. r44.-Embryo of Amphioxus.

$A$, in vertical section, slightly to the left of the middle line; $B$, in horizontal section, $a k$. ectoderm ; $c n$. neurenteric canal; $d k$, $w d$. archenteron; $i k$. endoderm; $n k$. mesodermal folds; $n$. medullary canal; $u s$. first coelonic pouch; ush . coelomic cavity; $V$. anterior ; H. posterior end. (From Korschelt and Heider, after Hatchek.)

closed, and the paired pouches gradually extend between the ectoderm and endoderm, both dorsally and ventrally (Fig. $\mathrm{r} 43, \mathrm{C}, \mathrm{D}$ ), their outer walls (parietal or somatic layer of the mesoderm, $m k^{1}$ ) being in contact with the ectoderm and forming with it the somatopleure or body-wall, and their inner walls (visceral or splanchnic layer of the mesoderm,

Prac'T, Zool. 
$\left.m k^{2}\right)$ in contact with the endoderm and with it forming the splanchnopleure or wall of the enteric tube (compare p. 203). Thus the body-wall and the enteric canal are separated by a cavity, the colome $(D, l h)$, which, much as in the adult Earthworm, is divided into a series of metamerically arranged portions: later on, however, the adjacent walls of these cœlomic sacs disappear, and the cœlome becomes a continuous cavity.

The embryo Amphioxus is hatched soon after reaching the gastrula-stage, when it moves about by means of cilia developed on the ectoderm cells and has to get its own living, having by this time used up its small reserve of yolk. It then passes through a complicated series of larval stages, gradually leading up to the adult form.

Early development of other types.-The presence of a greater amount of food-material in the egg renders it possible for the embryo to go on developing further than the gastrula-stage before being hatched, and as a general rule, the greater the relative quantity of yolk present in the ovum of an animal, the less clearly can a gastrula-stage be recognised.

In the earthworm and mussel the segmentation is entire, but unequal, and the larger, lower cells become invaginated to form the endoderm and archenteron while the smaller upper cells give rise to the ectoderm. In the earthworm the blastopore does not become closed, but gives rise to the mouth.

In the frog (p. 201) the archenteron arises by a split appearing amongst the yolk-cells, beginning at the edge of the blastopore and gradually extending forwards : the process is probably supplemented by a limited amount of invagination of the ectoderm. The archenteron is at first a very narrow cleft, but soon widens considerably (Fig. 64, $F, e n t)$ : for some time it does not actually communicate with the exterior, the blastopore $(b / p)$ being filled up by a yolk-plug $(y k . p)$. As the archenteron extends forwards, and the relatively small segment" 
ation-cavity (bl. co $l)$ gradually disappears, the edges of the; lower margin of the blastopore approach one another, and uniting in the median plane, give rise to a vertical groove, the primitive groove, as it is called.

In the centrolecithal cgg of the crayfish (Fïg. 91) a gastrula-stage is formed by invagination, but as the centre of the oosperm is filled with solicl yolk in the place of a segmentation-cavity containing fluid, the invagination only extends a short distance inwards, the archenteron

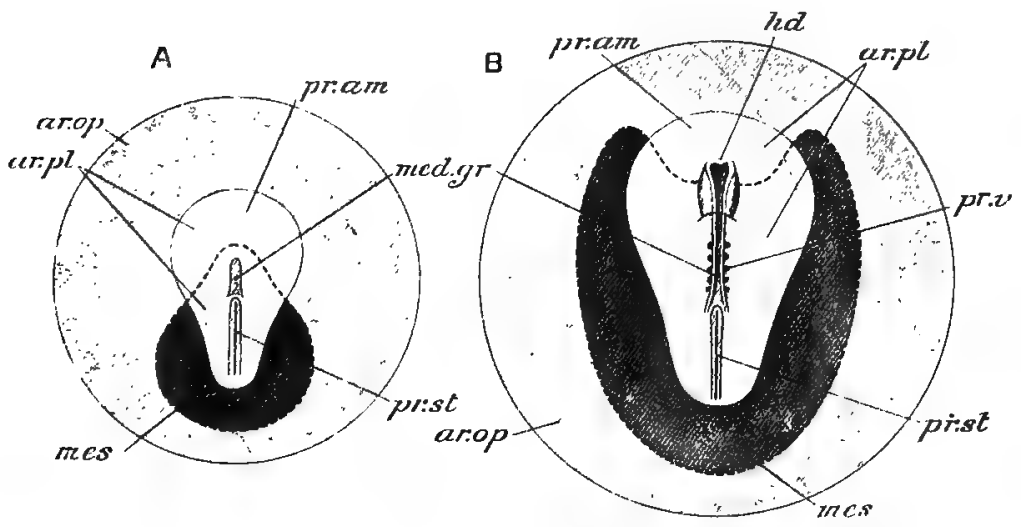

FIC. 145.-Two stages in the development of the: blastoderm of the chick, at about the twentieth and twenty-fourth hour of incubation respectively; diagrammatic. $a r$. op. area opaca; $a r$. $p l$. area pellucida; hd. head; med. gr. medullary groove; mes. mesoderm, indicated by dotted outline and deeper shade; pr,am, proamnion; $p r . s t$. primitive streak and groove: $p r . v$. mesodermal segments or protovertebræ. (From Marshall's Embryology, in part after Duval.)

being relatively very small and the ectoderm separated from the endoderm by the yolk.

The gastrula-stage is much less clearly distinguishable in the segmenting eggs of the dogfish and bircl (pp. 454 and 547), in which the relatively enormous mass of unsegmented yolk is, as in the crayfish, sufficient to nourish the embryo until it has reached a stage closely resembling the adult in almost every essential respect except size. $\Lambda$ blastopore can sometimes be recognised in such cases, but in the embryo of the common fowl it is only represented by a primitive groove (see above and Fig. $145 \mathrm{pr}$. st). The blastoderm soon becomes differ- 
entiated inte an utter ectuderm and an inner, lower layer of cells (compare Fig, 119), between which and the yolk the enteric cavity is formed: a segmentation-cavity is hardly recognisible. As the embryo develops, it becomes folded off from the yolk, which forms a yolk-sac on its ventral side (Figs. 120 and I54).

The minute egg of the rabbit and of most other Mammals, although alecithal and undergoing a holoblastic segmentation, has presumably

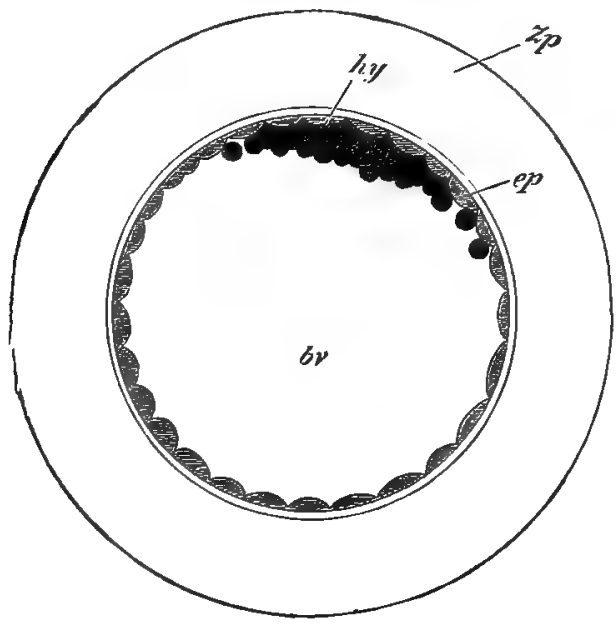

F1G. 146.-Oosperm of rabbit 70-go hours after impregnation.

tw. cavity of blastodermic vesicle (yolk-sac); cp. outer layer of cells (trophoblast); $h y$. inner mass of cells of the embryonic area; $Z \not p$. albuminous envelope. (From Balfour, after E. vall Beneden.)

been derived from a meroblastic type with abundant yolk like that of the bird, and some Mammals living in Australia at the present day still possess eggs of this type. In the higher Mamnalia the yolk has disappeared, as it is no longer needed, the embryo, as we have seen, being nourished by means of a placenta, which will be described presently. The early processes of development are therefore somewhat peculiar, and though the segmentation is holoblastic, the subsequent development is essentially similar to that of the bird, the embryo beginning to appear in a mass of rells (Fig $146, h y^{\prime}$ ) altached to 
the upper pole of a large blastodermic ressicle $\left(b z^{\prime}\right)$, representing the yolksac of a bird but containing a fluid instead of yolk' and being surrounded by a layer of cells known as the Trophohlast (ep).

In Amphioxus alone amongst the triploblastic animals described in this book, cloes the mesoderm arise as a series of enterocclic pouches : it is usually at first solid, and may be budded off from the endoderm, from the lip of the blastopore or primitive groove at the junction of the ectoderm and endoderm, or both endoderm and mesoderm may be differentiated at the same time from the lower layercells or yolk-cells (e.g. frog); or, finally, it may arise in all these ways (e.g. fowl, rabbit). The colome is formed by a split taking place in the mesoderm on cither side (Figs. 65 , mes, and Fig. $47 \%$, msd, som, spl), the split gradually extending with the extension of the mesoderm between the ectoderm and endoderm. Thus the coelome is formed, not as an enterocœle, but as a schizoccele.

In Vertebrates each mesoderm-band becomes differentiated into a dorsal portion, the vertebral plate, which soon loses its cœlomic space, and a ventral portion, the lateral plate, which is divided into parietal and visceral layers by the coelome (Figs. I43 D and 147 ). The vertebral plate undergoes metameric segmentation, becoming divided into a row of squarish masses, the mesodermal segments or protovertebra (pr.v), from the dorsal portions of which the muscular segments or myomeres are formed (p. 203), and from their ventral portions the vertebral column, the segmentation of which alternates with that of the myomeres.

Development of the chief organs in the Craniata (compare pp. 20I-210).--The nervous system, as well as the essential parts of the sensory organs are, as we have 
seen, in all cases formed from the ectoderm (pp. 202, 209 , and Figs. 64, 65, and 143 ); and in craniate Vertebrates the anterior end of the hollow medullary tube becomes dilated, forming three bulb-like swellings-the fore-brain (Fig. $\mathbf{1} 48, A, f . b)$, mid-brain ( $m . b)$, and hind-brain $(h . b)$. Soon a hollow outpushing grows forwards from the first vesicle

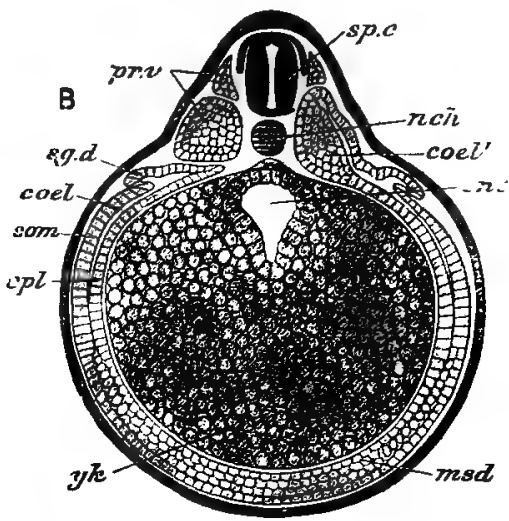

Fic. I47.-Transverse section of emilryo of frog.

$c \alpha t$. coelome; car $t^{\prime}$. prolongation of coelome into protovertebra (the reference line should end at the space); ent. mesenteron (archenteron); $n$ sd. mesoderm; $n c h$. notochord; pr. $v$. protovertebra; sg.d. pronephric duct; som. somatic layer of mesoderm; sp.c. spinal cord; spl. splanchnic layer of mesoderm; $y k$. yolk-cells. (From Parker and Haswell's Zoology, after Marshall.)

(B, prs. en), and the third gives off a similar hollow outgrowth $(c b l m)$ from its dorsal surface. The brain now consists of five divisions: the prosencephalon (prs. $c n$ ) and diencephalon (dien) derived from the fore-brain, with the pineal apparatus ( $p n . b, p n . e)$ and the infundibulum and pituitary body (inf.pty): the mid-brain or mesencephalon (m.b) which gives rise to the optic lobes and crura cerebri: and the epencephalon or cerebellum (cblm) and 


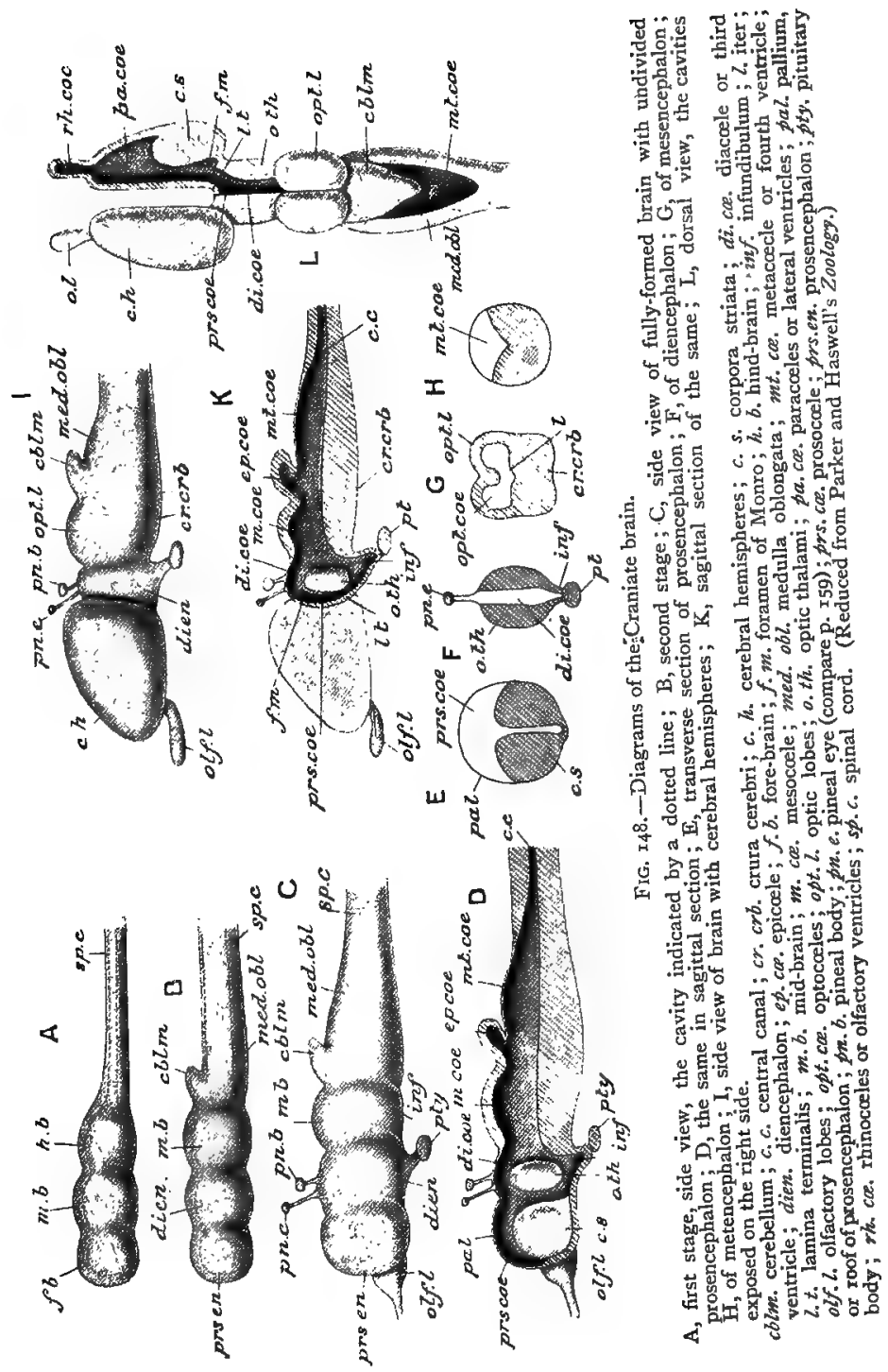


metencephalon or medulla oblongata (med. obl) derived from the hind-brain. The original cavity of the brain becomes correspondingly divided into a series of chambers or ventricles (compare Figs. 148 and 50), all communicating with one another, and called respectively the fore-ventricle or prosocale, third ventricle or diacale, mid-ventricle or mesocale (iter and optic ventricles or optocales), cerebellar ventricle or epiccele, and fourth ventricle or me. ${ }^{2}$ accele.

In some fishes (e.g. dogfish, Fig. II 5 ) the brain consists throughout life of these five divisions, but in most cases (Figs. 49 and 131), the prosencephalon grows out into paired lobes, the right and left cerebral hemispheres or parencephala (Figs. I48, I-L, c. h), each containing a cavity, the lateral ventricle or paracale (pa.coe) which communicates with the diacœle $(d i . c \infty)$ by a narrow passage, the foramen of Monro $(f . m)$. From the prosencephalon or the hemispheres are given off a pair of anterior prolongations, the olfactory lobes or rhinencephala (olf. $l$ ), each containing an olfactory ventricle or rhinocale $(r h, c \alpha)$.

In the preceding description the brain has been described as if its parts were in one horizontal plane; but, as a matter of fact, at a very early period of development the anterior part becomes bent down over the end of the notochord, so that the whole organ assumes a retortshape, the axis of the fore-brain being strongly inclined to that of the hind-brain. The bend is known as the cerebral flexure (Fig. I55) : it is really permanent, but as the hemispheres grow forwards parallel to the hind-brain and the floor of the mid-brain and hind-brain thickens, it becomes obscure and is not noticeable in the adult.

The ganglia of the dorsal roots of the spinal nerves are developed from a paired newal ridge formed close to the junction of the medullary plate and outer ectoderm, and the dorsal roots themselves appear as outgrowths from their ganglia (see Fig. I 47 , above $s p . c$ ): the ventral foots arise as direct outgrowths from the medullary cord. Certain of the cerebral nerves are developed in an essentially similar manner to 
the dorsal roots of the spinal nerves, while others arise as direct ventral outgrowths from the brain, like the ventral roots.

The olfactory organs arise as sac-like invaginations of the ectoderm, one on either side of the snout, and become enclosed by the cartilaginous olfactory capsules, developed, with the rest of the skeleton, from the mesoderm. The aperture of invagination gives rise to the external nostril,
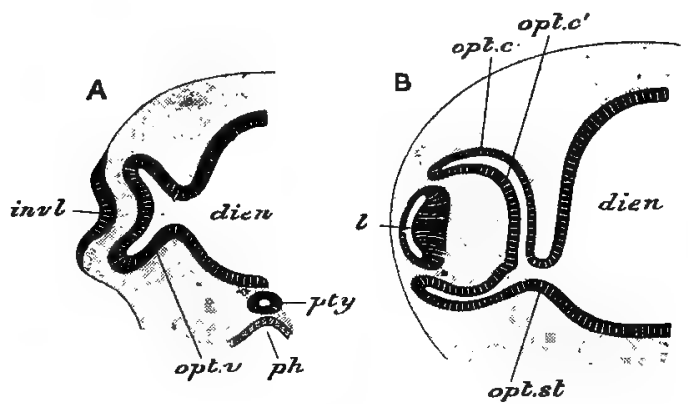

FıG. 149--Early (A) and later (B) stages in the development of the eye. dien. diencephalon; inv. l. invagination of ectoderm to form lens; 2 . lens; opt.c outer layer of optic cup; opt.c'. inner layer; opt. st. optic stalk; opt.v. optic vesicle ; $p h$. pharynx ; pty, pituitary body. (From Parker and Haswell's Zoology altered from Marshall.)

the internal nostrils (in air-breathing forms) being developed subsequently.

The mode of development of the paired eye of vertebrates is peculiar and characteristic.

At an early stage of development a hollow outgrowth-the optic vesicle (Fig. I49, A, opt. v) -is given off from each side of the fore-brain and extends towards the side of the head, where it meets with an in-pushing of the ectoderm (inv. $l$ ) which becomes thickened, and finally, separating from the ectoderm, forms a closed, spherical sac $(\mathrm{B}, \zeta)$ with a very 
small cavity and thick walls (compare Fig. 64, I, e). This body is the rudiment of the lens: as it enlarges it pushes against the optic vesicle and causes it to become invaginated $(B)$, the single-layered optic vesicle thus becomes converted into a two-layered optic cup (opt.c, opt. $\left.c^{\prime}\right)$, its cavity, originally continuous with the diacoele, becoming obliterated. Between the edge of the cup and the lens, on the ventral sicle, is a small space which gradually extends towards the stalk of the cup, and thus gives rise to a slit

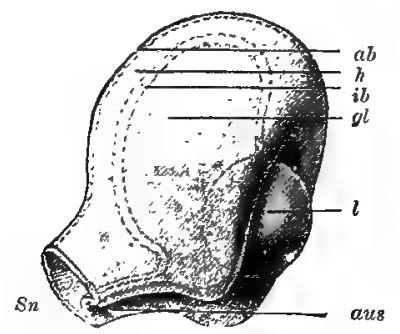

FIG. İ 50.-Plastic representation of the optic cup and lens.

$a b$. outer wall of optic cup; ans. choroid fissure; $g l$. cavity of optic cup; $h$. space between the two walls, which afterwards disappears ; $i b$. inner wall of optic cup; $l$, lens ; Sn. stalk of optic cup (rudiment of optic nerve), (After Hertwig.) in the wall of the latter: this choroid fissure (Fig. $\mathrm{I} 5 \mathrm{O}$, aus), as it is called, soon becomes closed by the union of its edges. The outer layer of the optic cup becomes the pigment-layer of the retina (p. I 83): from its inner layer the rest of that membraneincluding the rods and cones - -is formed. The stalk of the optic cup occupies, in the embryonic eye, the place of the optic nerve, but the actual fibres of the nerve are formed from the nerve-cells of the retina and grow inwards to the brain.

During the formation of the lens, mesoderm extends in between the ingrowth from which it arises and the external ectoderm; from this the main substance of the cornea and its inner or posterior epithelium are formed, the adjacent ectoderm becoming the external epithelium, i.e. that of the conjunctiva (p. I82). Mesoderm also makes its way into the optic cup, through the choroid fissure, and gives rise to the vitreous humour. Lastly, the mesoderm imme- 
diately surrounding the optic cup is differentiated to form the choroid, the iris, and the sclerotic.

Thus the eye of Vertebrates has a threefold origin: the sclerotic, choroid, iris, vitreous humour, and the greater part of the cornea are mesodermal: the lens and external epithelium of the cornea are derived from the ectoderm of the head: the retina and optic nerve are developed from a hollow pouch of the brain, and are therefore in their ultimate origin ectodermal. The sensory cells of the retina-the rods and cones-although not directly formed from the external ectoderm, as in Invertebrates, are ultimately traceable into the superficial layer of ectoderm since they are developed from, the inner layer of the optic vesicle, which is a prolongation of the inner layer of the brain, the latter being continuous, before the closure of the medullary groove, with the ectoderm coyering the general surface of the body (compare Fig. 149).

The organ of hearing, like that of smell, arises in the embryo as a paired invagination of the ectoderm in the region of the hind-brain, a shallow depression being formed (Figs. $64 \mathrm{~L}$ and $155, a u . s$ ) which deepens and becomes flask-shaped; and finally, as a rule, loses its connection with the external ectoderm, becoming a closed sac surrounded by mesoderm in which the cartilaginous auditory capsule is subsequently developed. At first simple, it soon becomes divided by a constriction into dorsal and ventral compartments, from the former of which arise the utriculus and semicircular canals, and from the latter the sacculus and cochlea.

The mode of development of the enteric canal has already been dealt with (pp. 204-210). The first traces of the liver and pancreas are seen as simple offshoots of the mesenteron (archenteron), which gradually become branched in a complicated manner, the numerous lobules being more or less closely connected together by mesoderm. The gill-pouches arise as paired outgrowths of the endoderm lining the "pharynx which come into contact with the 
uctoderm, the latter becoming perforated to form the external branchial apertures. Gill-clefts appear in the embryo of reptiles, birds, and mammals - animals in which gills are never developed (Fig. I 55); but they early disappear with the exception of the first cleft, corresponding with the spiracle of the dogfish, which gives rise in all Vertebrates above fishes to the tympano-eustachian passage (p. 433): the branchial skeleton, as we have seen, undergoes a corresponding reduction or modification (pp. 422 and 479). In air-breathing Vertebrates the lungs arise as a ventral outgrowth of the pharynx.

The circulatory organs are developed from the mesoderm, the heart arising in the visceral layer on the ventral side of the pharynx. It has at first the form of a straight tube, which soon becomes twisted into an S-shape and in which transverse constrictions are formed dividing it into the different chambers. The auricular and ventricular portions are each at first single, but from the Amphibia onwards the former subsequently becomes divided into two by a septum and the ventricle is similarly subdivided in birds and mammals. The modification of the arterial arches in the examples studied has already been described (pp. 435 and 5o6).

In the meroblastic eggs of the dogfish and bird the dorsal aorta, in addition to its other branches, gives rise to paired vitelline arteries: these vessels branch up over the extraembryonic part of the blastoderm (p. 578), which spreads over the yolk, and take an important share in the absorption of the latter by the embryo. From this area vasculosi (Fig. 154), the blood is returned by vitelline veins into vessels which eventually give rise to the hepatic portal veins. The other chief veins in all embryonic Craniates are, as in the dogfish, the jugulars and the cardinals. In all Vertebrates above the fishes, the cardinals became subsequently 
more or less entirely replaced functionally by the development of a postcaval (compare p. 440): the anterior part of one or both cardinals may, however, persist as the azygos vein or veins (e.g. Rabbit, p. 509).

Urinogenital organs. The excretory organ, speaking of craniate Vertebrates as a whole, consists of three parts, all paired and situated along the dorsal wall of the coelome: the fore-kidney or pronephros (Fig. 151 , A, p.nph), the mid kidney or mesonephros (ms. nph) and the hind-kidney or metanephros (mt. nph). Each of these is provided with a duct, the pro- (sg.d), meso- (msn.d), and metanephric (mt.n.d) ducts, which open into the cloaca. The gonads (gon) lie in the coelome suspended to its dorsal wall by a fold of peritoneum : they are developed as ridges covered by colomic epithelium (compare pp. I94-i 96 and 336).

The pronephros is nearly always functionless in the adult and often even in the embryo, and usually disappears altogether: in the young tadpole it acts as the sole excretory organ for some time. The mesonephros is usually the functional kidney in the lower Craniata, in which as a rule no metanephros is developed (see p. 576), and the mesonephric duct acts as a ureter, often in addition carrying off the seminal fluid of the male (e.g. frog). In the higher forms the mesonephros is replaced in its excretory function by the metanephros, the metanephric duct being the ureter (e.g. Rabbit).

The development of the kidney reveals a resemblance to the nephridia of worms which would hardly be suspected from its adult structure. The pronephros (Fig. I 5 I $\mathrm{A}, p$. $n p h)$ originates as two or threc coiled tubes formed from mesoderm in the body-wall at the anterior end of the colome; they are arranged metamerically and each opens 
into the cœlome by a ciliated funnel (nst). Obviously such tubes are nephridia (compare p. 33r); their chief peculiarity is that their outer ends do not open directly on to the exterior, but into a longitudinal tube, the pronephric duct $(s g . d)$, which passes backwards and discharges into the cloaca. It seems probable that this arrangement is to be explained by supposing that the nephridia originally opened externally into a longitudinal groove, which, by the apposition of its edges, was converted into a tube. All the nephridia of the pronephros open, by their ciliated funnels, into the narrow anterior end of the cœlome, into which projects a branch of the aorta ending in a single large glomerulus (p. I 46).

The pronephros soon degenerates, its nephridia losing their connection with the duct $(B)$, but in the meantime fresh nephridia appear in the segments posterior to the pronephros and together constitute the mesonephros or Wolffian body (ms. nph), from which the permanent kidney is formed in most of the lower Craniata (e.g. frog). The mesonephric nephridia open at one end into the duct $(s g . d)$, at the other, by ciliated funnels (nst), into the coelome; a short distance from the funnel each gives off a blind pouch which dilates at the end and forms a Malpighian capsule (m.c), and a branch from the aorta entering it gives rise to a glomerulus.

In some forms (e.g. Dogfish, p. 450) the pronephric duct now becomes divided by a longitudinal partition into two tubes: one retains its connection with the mesonephros and is now known as the mesonephric or Wolffian duct $(\mathrm{C}, m s . n . d)$ : the other, or Miullerian duct $(p, n . d)$, has no connection with the nephridia, but opens into the colome in the region of the vanishing pronephros, and assumes the functions of an oviduct in the female. In some Craniata the 

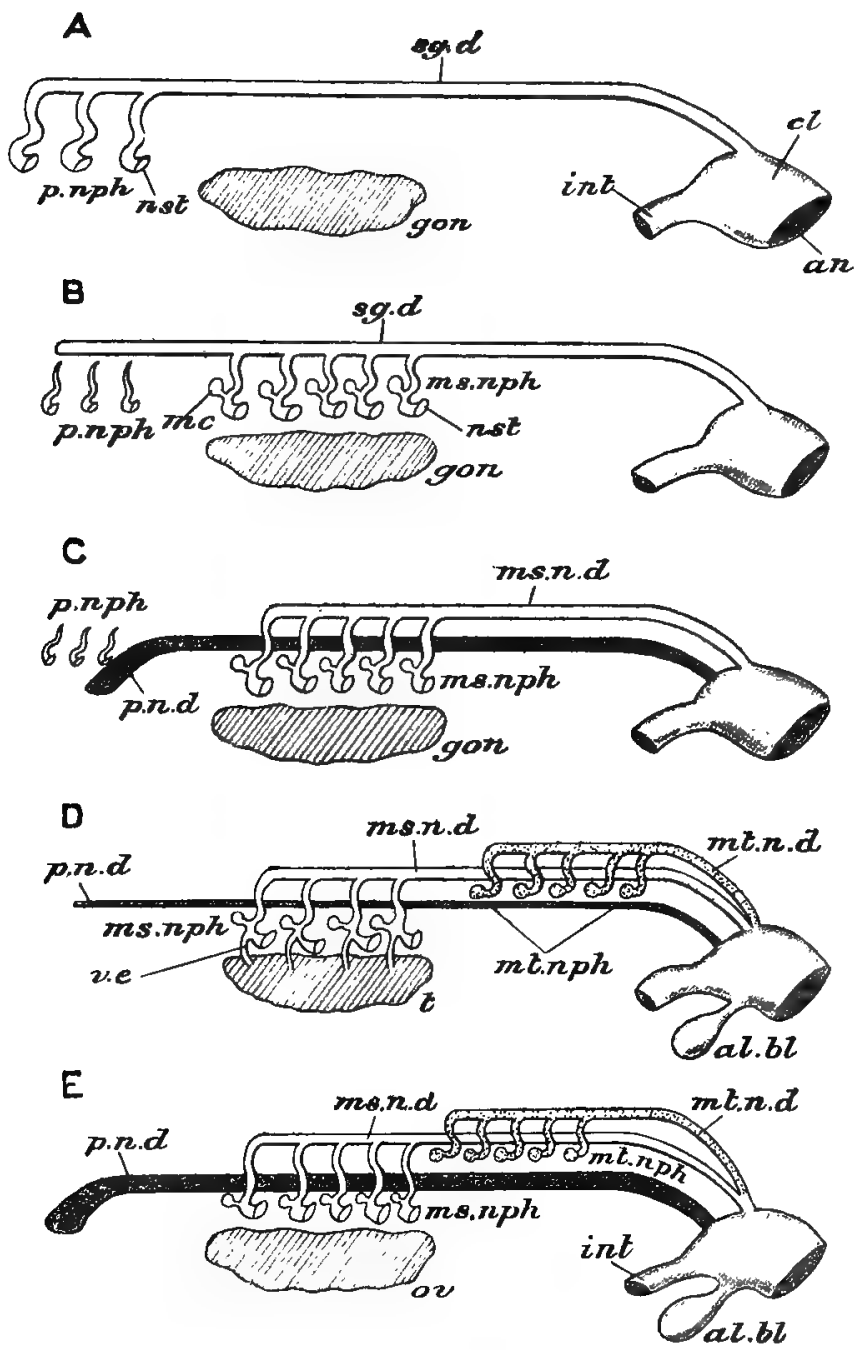

FIG. I5x.-Diagrams illustrating the development on the urinogenital; organs of

$A$, pronephros and its duct $B_{2}$ atrophy of pronephros, development of mesonephros ; C, differentiation of Wolfian and Miillerian ducts D, develcpment of metanephros, male type; $E$, female type.

al. bl. allantoic bladder; $a n$. anus ; $c l$. cloaca ; gon. gonad ; ' $i n t$. intestine; $m . c$ Malpighian capsule; $m s . n$. d. mesonephric (Wolffian) duct: $n s . n p h$. meso. 
nephros ; mt. n.d. metanephric duct; $n t . n p h$. metanephros ; sst. nephrostomes; $o$. ovary; t. n.d. Mällerian duct; $\not$. nph. pronephros; sg.d. pronephric duct; $t$. sperınary; $v . e$. efferent ducts. (From Parker and Haswell's Zoology).

Müllerian duct appears quite independently of the Wolffian duct; the latter is then simply the pronephric duct after the union with it of the mesonephric tubules.

In the higher Vertebrata (Reptiles, Birds, and Mammals) a diverticulum ( $\mathrm{D}, \mathrm{E}, m t . n . d)$ is given off from the posterior end of the Wolffian duct, which grows forwards and becomes connected with a series of posterior nephridia. In this way is formed a metanephros $(m t . n p h)$, which gives rise to the permanent kidney, and a metanephric duct (ml. n.d) which becomes the ureter. The Wolffian body ceases to discharge a renal function, becomes in the female a purely vestigial organ, and in the male gives rise to the epididymis (pp. 45 I and 52I), which receives the efferent ducts from the spermary and from which the Wolffian duct (spermiduct or vas deferens) arises.

The homology of the hinder part of the kidney in the dogfish (p. 45I) is differently interpreted by zoologists. It is usually considered as corresponding to part of the mesonephros, but in its differentiation from the anterior part of this organ and in the development of special ureters it resembles the metanephros of higher Vertebrates.

The majority of the muscles are developed, as we have seen (pp. 203 and 565) from the mesodermal segments others arising from the parietal and visceral layers of the mesoderm:

The first part of the endoskeleton to arise is the endodermic notochord (Pp. 203 and 560), in the mesoderm surrounding which cartilage appears and undergoes segmentation, giving rise to the vertebræ, the notochord becoming constricted by the ingrowing cartilage, and eventually disappearing more or less completely (compare pp. 425 and 565$)$ : it at first extends into the head as far as the 
pituitary body (Fig. $x_{5} 2 C$ ). The cranial cartilage does not become segmented, but gives rise to a pair of horizontal bars, the parachordals $(P E)$ : these are continued forwards, diverging around the pituitary body, as the trabeculoe cranii $(T r)$, and thus a support is formed for the developing brain. The two parachordals and trabeculæ then unite
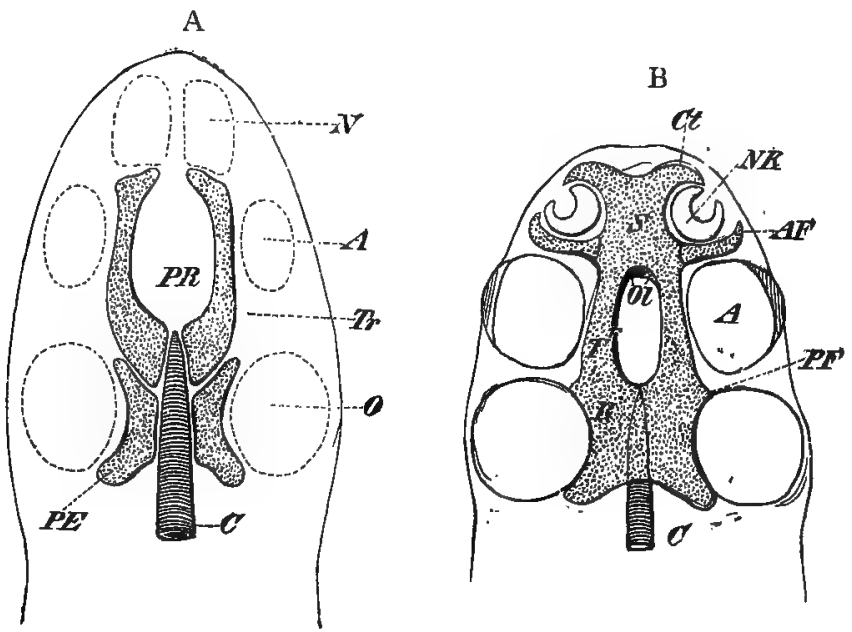

FtG. I52,-A and B, two stages in the development of the chondrocranium.

$A$. eye; $A F$. antorbital process; $B$. basal plate, formed from the parachordals; $C$. notochord; $C t$. anterior process of trabecula; $N, N k$. nose; $O$. ear; $O t$. position of foramina for olfactory nerves ; $P E$. parachordal cartilage ; $P F$. postorbital process; $P R$. pituitary space; $S$. nasal septum; $T$ r. trabecula. (From Wiedersheim's Anatomy.)

respectively with one another, and so form a firm floor (B) for the future brain-case, which is gradually developed by the floor growing up on either side and eventually meeting to a greater or less extent above the brain : there is never, however, a complete cartilaginous roof to the cranium, parts of which are only membranous and form the fontanelles (pp. 43 and 420). In the meantime Pract. Zoor. 
the cartilaginous sense-capsules are developed, the olfactory and auditory capsules uniting with the brain-case in front and behind respectively.

The visceral skeleton is formed as a series of cartilaginous bars within the visceral arches, the first of which forms the mandibular arch, the second the hyoid, and the others the branchial arches.

The limbs appear as small buds (Fig. I55) composed of ectoderm with a core of mesoderm, and their skeleton arises by the formation of cartilage at their bases, which extends inwards to form the arches, and outwards to form the skeleton of the free portions of the limbs.

As we have seen, the endoskeleton may remain more or less entirely cartilaginous in the adult (e.g. Dogfish), but in higher forms extensive processes of ossification set in, certain bones (cartilage-bones) replacing this cartilage to a greater or less extent, and others being formed in the surrounding connective-tissue (compare p. 43)

\section{Development of the Amnion, Allantois and Placenta.-} We must now consider some important and characteristic structures which are developed in the embryos of the higher Craniata (Reptiles, Birds, and Mammals), and known as embryonic membranes. Taking the chick as a convenient example, these are formed as follows.

The blastoderm gradually extends peripherally so as to cover the yolk, and thereby becomes divisible into an embryonic portion, from which the embryo is formed, and an extra-embryonic portion which invests the yolk-sac and takes no direct share in the formation of the embryo (Fig. I54). The extension of the ectoderm and endoderm takes places regularly and symmetrically, but the mesoderm, while extending equally in the lateral and posterior 
regions, grows forwards in the form of paired prolongations which afterwards unite, so that for a time there is an area of the blastoderm in front of the head of the embryo formed of ectoderm and endoderm only, and called the pro-amnion (Fig. I 45 pr. am).

Before the embryo has begun to be folded off from the yolk the rudiment of one of the two embryonic membranes, the amnion, has appeared. A crescentic amniotic fold (Fig. I 53, A, am. f) arises in front of the head-end of the embryo from the region of the pro-amnion: it consists at first of ectoderm only, the mesoderm not having yet spread into the pro-amnion. The fold is soon continued backwards along the sides of the body (B) and round the tail (A), but in these regions $\left(a m . f^{\prime}\right)$ it consists from the first of ectoderm plus the parietal layer of mesoderm, i.e., it is a fold of what may be called the embryonic body-wall or somatopleure (p. 56r). Its cavity is a prolongation of the space between the parietal and visceral layers of mesoderm, i.e., is an extension of the extra-embryonic cœlome.

The entire amniotic fold gradually closes in above (C), forming a double-layered dome over the embryo. Its inner layer, formed of ectoderm internally and mesoderm externally, is the amnion (am), the cavity of which becomes filled with a watery amniotic fluid, serving as a protective watercushion to the enclosed embryo. Its outer layer, formed of ectoderm externally and mesoderm internally, is the serous membrane (sr. $m$ ): this comes to lie just beneath the vitelline membrane, with which it subsequently fuses.

The second of the embryonic membranes, the allantois, is developed as an outpushing of the ventral wall of the mesenteron (archenteron) at its posterior end (C, all), and consists, therefore, of a layer of visceral mesoderm lined by endoderm. It has at first the form of a small, ovoid sac 


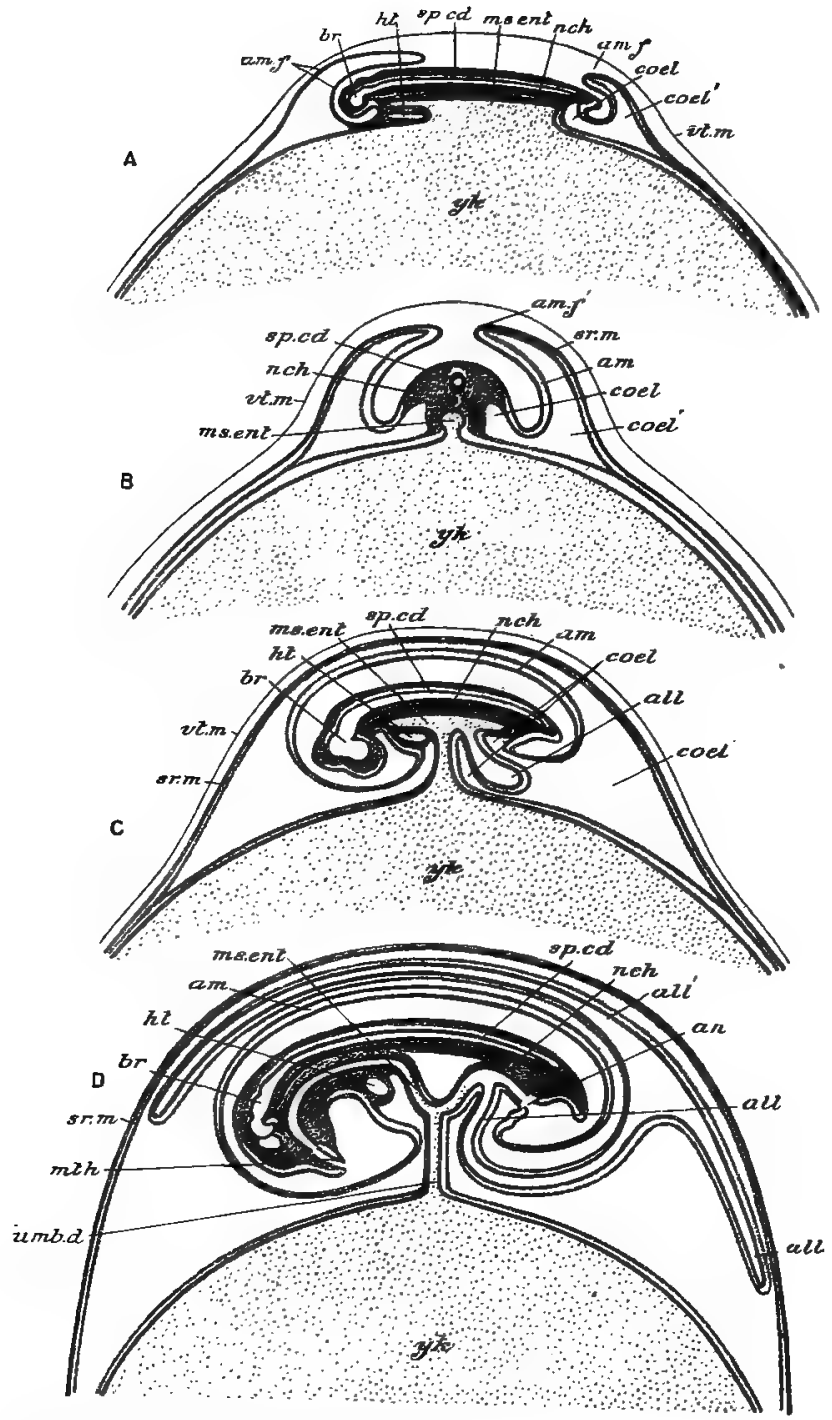

FIG. 153. -Diagrams illustrating the development of the foetal membranes of a bird. $A$, early stage in the formation of the amnion, longitudinal vertical section; $B$. 
slightly later stage, transverse section; C, stage with completed amnion and commencing allantois; $D$, stage in which the allantois has begun to envelop the embryo and yolk-sac. The ectoderm is represented by a blue, the endoderm by a red line; the mesoderm is grey.

all. allantois; all . the same growing round the embryo and yolk-sac; an. amnion; am. $f$. amniotic fold; an. anus; br. brain; col. colome; cal. extra-embryonic coelome; $h t$. heart; ms. ent. mesenteron; $m t h$. mouth; nch. notochord; sp.cd. spinal cord; $s r, m$. serous membrane; $u m b$. d. umbilical duct; $v t . m$. vitelline membrane ; $y k$. yolk-sac. (Reduced from Parker and Haswell's Zoology.)

having the precise anatomical relations of the urinary bladder of the Frog. It increases rapidly in size (Figs. I 55 and I 54, all), and makes its way, backwards and to the right, into the extra-embryonic coelome, between the amnion and the serous

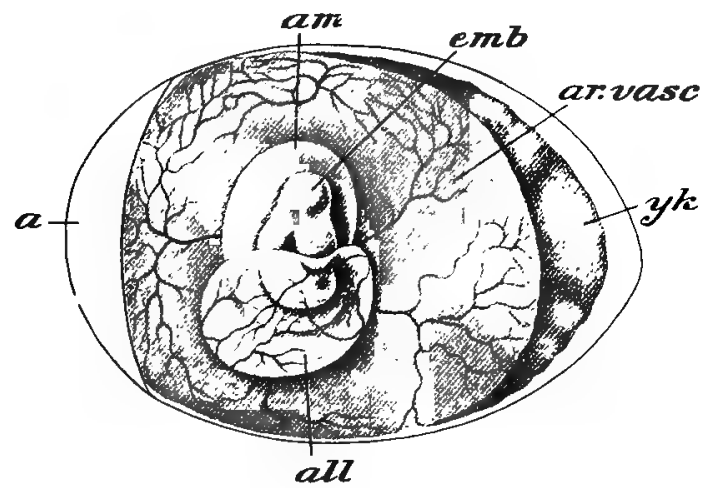

FIG. r54.-Egg of fowl, at the sixth day of incubation, with embryo and foeta appendages.

a. air-space ; all. allantois ; am. amnion; ar. vasc. area vasculosa ; ent. embryo; yk. yolk-sac. (From Parker and Haswell's Zoology, after Duval.)

membrane (Fig. 153, C, D). Arteries pass to it from the dorsal aorta, and its veins, joining with those from the yolk-sac, take the blood through the liver to the heart. Next, the distal end of the sac spreads itself out and extends all round the embryo and yolk-sac $(\mathrm{D}$, all' $)$, fusing, as it does so, with the serous and vitelline membranes, and so coming to lie immediately beneath the shell-membrane. It finally encloses the whole embryo and yolk-sac, together with the re- 
mains of the albumen, which has, by this time, been largely absorbed. The allantois serves as the embryonic respiratory organ, gaseous exchange readily taking place tbrough the

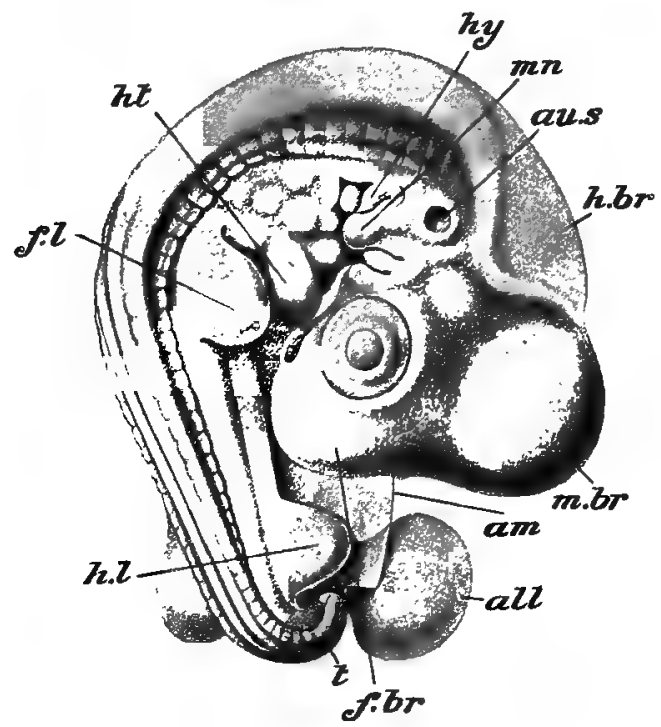

FIG. I55.-Chick at the fifth day of incubation.

all. allantois; am. cut edge of amnion; au. s. auditory sac; $f . b r$ fore-brain ; $f l$. fore-limb; $h . b r$. hind-brain; $h . l$. hind-limb; $h t$. heart; $h y$. byoid arch; $m . b r$. mid-brain ; $m n$. mandibular arch; $t$. tail. (From Parker and Haswell's Zoology, after Duval.)

porous shell; its cavity is an embryonic urinary bladder, excretory products being discharged into it from the kidneys.

At the end of incubation the embryo breaks the shell by means of a little horny elevation or caruncle at the end of the beak. By this time the remainder of the yolk-sac has been drawn into the cœlome, and the ventral body-walls have closed round it. On the shell being broken the allantois gradually shrivels up, respiratory movements begin, the 
aperture in the shell is enlarged, and the young bird is hatched and begins a free life. .

In the higher Mammalia the allantois takes on a further important function. The mode of development of the amnion and allantois in the Rabbit is similar to that described above in the case of the Bird. But the later. history of the allantois is widely different, owing to the modifications which it undergoes in order to take part in the formation of the placenta, the structure by means of which the foetus receives its nourishment from the walls of the uterus, with which the blastodermic vesicle (p. 565) becomes adherent. The foetal part of the placenta is formed from the outer layer of the amnion (serous membrane, Fig. I 56, sh) in a limited disc-shaped area where the distal portion of the allantois coalesces with it. The membrane thus formed (charion) develops vascular processes-the chorionic vill $(p l)$ - which are received into depressionsthe uterine crypts -in the mucous membrane of the dorsal wall of the uterus which constitutes the maternal portion of the placenta. The completed placenta with its villi is supplied with blood by the allantoic vessels, and the blood supply of the uterus is at the same time greatly increased: the fotal and maternal capillaries and sinuses are thus brought into intimate relation with one another in the placenta, and diffusion can take place between them, nutrient matter and oxygen diffusing from the blood of the mother into that of the foetus, while excretory substances-pass from the blood of the fœtus into that of the mother.

The discoidal placenta of the Rabbit is of the type termed deciduate, the villi of the placenta being so intimately connected with the uterine mucous membrane that a part of the latter comes away with it at birth in the decidua, or after- 
birth, which is attached to the newly-born young by the umbilical cord, consisting of the stalks, of the allantois (a) and flattened yolk-sac $(e d, f d)$ twisted together. The cord is gnawed through by the parent-rabbit, the blood-vessels being compressed in the process; and it soon shrivels up and comes away at the navel or umbilicus, which repre-

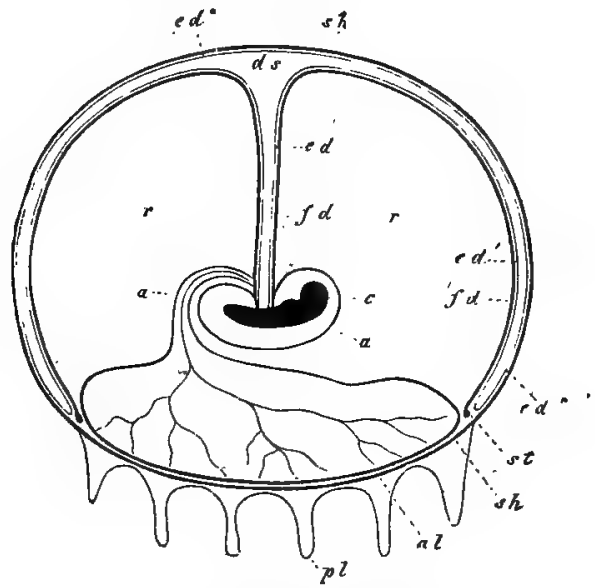

FIG. 156.-Diagrammatic longitudinal section of the fotus and embryonic membranes of a rabbit.

e. (on right) amnion ; $a$. (on left) stalk of allantois; $a l$. allantois with blood-vessels; $c$. embryo; $d s$. cavity of the flattened yolk-sac (blastodermic vesicle); $e d$. endodermal layer of yolk-sac; $e d^{\prime}$. inner portion, and $e d^{\prime \prime}$. outer portion of endoderm. lining the compressed cavity of the yolk-sac; $f a$. vascular layer of yolk-sac; f.l. placental villi ; $\%$ space filled with fluid between the amnion, the allantois, and the yolk-sac ; sh. serous membrane; st. margin of vascular area of yolk-sac, (From Balfour, after Bischoff.)

sents the point of connection between the foetus and the placenta. The intra-abdominal portion of the allantois is represented by a cord or ligament, the urachus, which connects the navel with the apex of the bladder, so that only. a small portion of the allantoic outgrowth, and not the whole of it as in the frog, persists in the adult. 


\section{PRACTICAL DIRECTIONS.}

A. Special methods are required to follow out the details of the structure and division of nuclei, but if you have not the opportunity of examining preparations illustrating these, a good deal may be made out as regards the chromatin and the behaviour of the chromosomes in the process of mitosis by the following simple method.

Obtain a young, gilled-larva or tadpole of the common newt (p. 218), kill, and place it in corrosive sublimate for about half an hour : wash thoroughly in water and transfer first into weak, and then into strong alcohol (p. I37). Stain entire, either with a solution of hematoxylize, which can be bought ready prepared, or alum-carmine, which you can prepare yourself by dissolving 200 grams of ammonia-alum in water, boiling the solution and adding carmine to excess, filtering, and diluting with three or four times its bulk of water; a drop or two of carbolic acid should be added to the solution.

Strip off small pieces of the skin of the stained preparation, and after putting them through weak and strong alcohol, transfer to absolute alcohol, then to turpentiue or oil of cloves, and mount in Canada balsam (p. 139). Examine, comparing Fig. 136 and pp. 54I-545, and sketch as many different stages as possible.

B. Observation of the details concerned in the process of maturation and fertilization of the ovum is too difficult for the beginner, but polar cells may easily be seen in the living, freshly-laid eggs of one of the common pond-snails (e.g. Limnaus stagmalis), in which some of the stages of segmentation can also be observed. Keep some of these snails in a glass vessel with water weeds, and notice that the eggs, when laid, are enclosed, a number together, in a common gelatinous envelope. Separate the eggs from one another with needle, and examine in water under the microscope. Note that the small egg is surrounded by fluid enclosed in a relatively large egg-case, and observe the minute polär cells at the periphery of the ovum.

C. A series of models of the development of Amphioxus, to be found in most mirseums; should be carefully examined (compare Figs I4II44).

D. In order to follow out the development of the chief organs in a 
Vertebrate animal, it is necessary to make a number of serial transverse sections.

For this purpose chick-embryos (see below) are, on the whole, the most convenient and satisfactory, but if you also wish to make sections of embryos of the frog, proceed as directed on p. 214.

Serial sections of embryos in different stages should be mounted on the same slide, after smearing it with collodion and oil of cloves (p. 139). It is a matter of some difficulty to make satisfactory sections of the early stages : the most important stages for the present purpose subsequently to segmentation are from the time when the embryo begins to become elongated up to hatching.

A number of fresh, impregnated fowl's eggs should be obtained and placed in an incubator at a temperature of from 37 to $40 \mathrm{C}$. or under a "broody" hen, first marking each with the date. One or two should be examined each day or oftener for the first four or five days of incubation. To expose the embryo, place the egg in a dish of warm water (temperature as above), in order that, after the first day, the beating of the heart and the circulation of the blood may not be stopped. With the forceps, tap the surface of the egg lying uppermost so as to break the shell into small pieces, which can then be removed: cut away sufficient of the shell-membrane with the scissors to expose the entire embryo and blastoderm. The early stages are difficult to observe, and the most important of those referred to below are from the end of the first to the third day of incubation. As the medullary groove only closes gradually from before backwards in the body-region, sections showing different stages in the development of the central nervous system may be obtained from the same embryo at these stages. Prepare as above.

I. Examine first an unincubated egg, as directed above, and make out ts structure (compare Fig. 138). (The blastoderm undergoes segmentation before the egg is laid.)

2. First day of incubation (18-20 hours). Examine with a lens and compare Fig I $45 \mathrm{~A}$. Then carefully cut round the blastoderm with fine scissors, float it off in the water, preserve, stain, and mount entire in Canada balsam. (The removal and preparation of the blastoderm at this stage is rather difficult, and sections of the next stage will illustrate the chief points equally well). 
3. End of first day (about 24 hours). Examine as before, com. paring Fig. I45 B. Sketch, and then preserve, stain, and eventually cut sections. Note the ectoderm, endoderm, mesoderm, medullary groove and its closure to form the medullary cord; the neural ridges (p. 568); the notochord; the tarietal (somatic) and visceral (splanchnic) layers of the mesoderm, and the calome (compare Figs. 64, 65, and 147). Sketch one or two typical sections.

4. Second day. Examine, prepare, cut sections, and sketch as before, noting the fore-, mid-, and hind-brain, the optic vesicles, lens, auditory pits, heart and vitelline vessels, and the head-fold of the amnion, as well as the increase in the number of mesodermal segments (compare Figs. 148,149 , and 153).

5. Third day. Examine, prepare, and sketch as before, noting, in addition to the points already referred to, the further gradual folding off of the embryo from the yolk (compare p. 454) and extension of the anmon and area vasculosa; the cerebral fexure; the visceral arches and clefts and the arterial arches; the mesonephric duct; and the further development of the eye and ear (compare Figs. 147, 148, 149, 150, 153 , and 155 ).

6. Fourth to sixth days. Observe the further development of the parts already seen and also the limb-bud's and the allantois (compare Figs. 153-1 55). Sketch.

1. Examine and compare a few later stages.

E. Obtain some advanced embryos of the rabbil (p. 542) or rat. and examine in situ in the uterus before removing and preserving them. Note the amnion and examine the piacenta and its connection with the uterine wall and with the fotus. Sketch. 

INDEX 



\section{INDEX}

(The numbers in italics refer to practical divections.)

A

A

bdomen, abdominal cavity, 20 , $347,418,467,489$

Abdominal pore, 416

Abiogenesis, 282

Acanthias, 415, 454

Acetabulum, 50, 486

Achromatin, I 29, 231, 54 I, 542

Acoustic spots, 188

Acrania, 406

Acromion, 483

Adaptation, 224

Adenoids, 498

Adrenal bodies, 145, 43r, 52 I

Agamobium, 314

Alimentary canal, see Enteric canal

Allantois, $579,583,587$

Allolobophora, see Earthworm.

Alternation of generations, 314

Alveoli of jaws, 493

Amnion, 579, 587

АМсвА: occurrence and general characters, 229 ; movements, 232 ; resting condition, 232; nutrition, 233; growth, 234; excretion, 235 ; respiration, 235 ; metabolism, 236 ; reproduction, 236 ; immortality, 236 ; conjugation, 237; death, 237 ; animal or plant? 255 ; practical directions, 238
Amœboid movements, I06, 23 I

Amphibia, 219, 403, 466

Amphicolous, 423

AmpHioxus, see Lancelet

Ampulla, of semicircular canals, 188

Ampulla, of sensory canals of the integument, 448

Anal segment, 319

Analogous, analogy, 217

Anatomy, 2 I7

Animals and Plants : comparison of typical forms, 255 ; discussion of doubtful forms, 255,257 ; boundaries artificial, $25^{8}$

Ankle, see Tarsus

Annulata, 220, 341

Anodonta, see Mussel

Anura, 219

Antenna, 352

Antennary gland, 36r

Antennule, 352

Anus, 6, 267, 276, 319, 357, 386, $4 \mathrm{I} 6,429,469,497$

Aorta, aortic arches, see Arteries.

Aperture, or apertures (see also Foranien, and under Nephridium, Kidney, Pores, Reproductive organs, \&cc.) auditory, Crayfish, 367 ; Rabbit, 47o, 472 ; cloacal, see anus ; exhalant and inhalant, $3^{82}$ 
Aphis, 552

Appendages, lateral, and their skeleton: Frog, 5, 28, 36, 48, 51 ; Crayfish, 346, 349 ; Dogfish, 417, 426; Rabbit, 484, 486 ; Development of in Vertebrates, 578 , 587

Aqueous chamber and humour, 183

Arachnoid fluid, 155

Archenteron, 201, $55^{8}$

Archicerebrum, 366

Area vasculosa, $572,581,587$

Arm, see Fore-limb

Arterial arches, 80, 436

Arteries: Frog, 27, 80; Crayfish, 362 ; Mussel, 392 ; Dogfish, 433438; Rabbit, 503-506; Vertebrate embryo, 572

Arthobranch, 36I

Arthropoda, 220, 346, 370.

Articular membrane, 347, 354

Articular processes, see Zygapophyses

Artificial selection, 227

Arytenoid cartilage, 144, 500

Ascaris, I 53

Asexual generation, see Agamobium

Asexual reproduction, see Fission, Budding, Spore

Assimilation, 149, 234, 300

Astacus, see Crayfish

Astragalus, 51, 487

Astrosphere, 543

Atlas vertebra, 481

Atrial pore, 405

Atrium, 404, 406

Atrophy, see Vestige

Auditory capsule, 39, 4I, 420, 475

Auditory organ: Frog, 45, I86; Cravfish, 367; Mussel, 394 ; Dog. fish, 449 ; Rabbit, 518 ; Development of, in Vertebrates, $57 \mathrm{I}$, 587

Auditory ossicles, 475, 519

Auricle, see Heart

Automatism, see Movements

Aves, 219, 403
Axial fibre : of Vorticella, 276; of Carchesium, 281

Axial parts, 4

Axis fibre, see Neuraxis

Axis vertebra, $4^{82}$

\section{B}

B SACTERIA, I52; structure, 257 ; nutrition, 257 ; animals or plants? 257 ; rapid multiplication, $28 \mathrm{z}$; practical directions, 260

Backbone, see Vertebral column

Basal cartilages of fins, or basalia, $425-427$

Bell of medusa, see Umbrella

Bilateral symmetry, 29I

Bile, 69, 43I

Bile-duct, 68, 431, 499

Bile-passages, 133

Binomial nomenclature, 215

Biogenesis, 282

Biology, I

Bird, development of, $563,572,576$, 578,587

Birds, see Aves

Bladder, see Gall-bladder and Urinary bladder

Blastocole, see Segmentation-cavity

Blastoderm, 369, 454, 563, 586

, embryonic and extraembryonic portions, 572 , 578

Blastodermic vesicle, $565,5^{84}$

Blastomere, 556

Blastopore, 201, 340, 369, 558, 562

Blastula, $55^{8}$

Blind spot, 539

Blood: Frog, 20, 78, 85; Earthworm, 329, 330 ; Crayfish, 365 ; Mussel, 392 ; Lancelet, 405 ; Dogfish, 442; Mammalia, 467

Blood-corpuscles: colourless, see Leucocytes ; red, IO5, 442

Blood-sinus, see Sinus

Blood-vessels: Frog, 78, 107; Earthroorm, 329 ; Crayfish, 362; 
Mussel, 392 ; Lancelet, 408; Dogfish, 433-442 ; Rabbit, 503

Body-cavity, see Coelome

Body of vertebra, see Vertebra

Body-segments, see Metamere

Bojanus, organ of, 390

Bone : cartilage and membrane, 43 , 472 ; nature of, 52 ; microscopical structure of, I I6

Bones, see Endoskeleton, Skull, Vertebra, Ribs, Sternum, and under individual bones of limbs

Botany, I

BoUGAINVILLEA : occurrence and general characters, 304; microscopic structure, 304; structure of Medusa, 307; nervons system, $31 \mathrm{I}$; organs of sight, 308,312 ; reproduction and development, $3 \mathrm{I2}$; alternation of generations, 3I4; practical directions, $3 I 7$

Brachial plexus, see Nerve-plexus

Brain: Frog, 28, 156, 202 ; Earthworm, 333; Crayfish, 365; Mussel, 392; Lancelet, 405, 408; Dogfish, 443; Rabbit, 501 ; development of, in Vertebrates, 202, 566,587

Brain-case, see Skull

Branchia, see Gill

Branchial apertures, arches, clefts, and septa: Tadpole, 204, 206; Lancelet, 405, 407 ; Dogfish, 4I6, $422,429,432$; development of, 571 .

Branchial rays, 422

Branchial vessels: Crayfish, 360, 363 ; Mussél, 392 ; Lancelet, 409 ; Dogfish; 433 ; Tadpole, 435

Breast-bone, see Sternum

Bronchus, 500

Buccal cavity, 16, 327, 429

Buccal groove, 262

Bud, budding, 301, 303, 3 I 3

Bufo, Bufonidæ, 2 I 8

Bulb, see Medulla oblongata

Bulbus aortæ, 89

Bulla, tympanic, 475

Byssus, 396

PrACT. Zoot.
C

æcum, 498

Calcaneum, 5I, 487

Calcar, 52

Canal : central, of spinal cord, 156 , 408, 443; naso-palatine, 490; neural, see Vertebral column; neurenteric, 203; radial and circular of Medusa, 307; semicircular of ear, see Auditory organ and Membranous labyrinth; sensory, of Dogfish, 416, 448; sternal, of Crayfish, 349 ; vertebrarterial, 48 I Canaliculi, see Bone

Canine teeth, 492

Capillaries, $95,363,435,438$

Capitular facet and capitulum, 48r 483

Carapace, 347

Carbohydrates, 72

Carbon dioxide, 66

Carchesium, 281, 288

Cardiac division, see Stomach.

'Carotid arch, see Arteries of Frog, Dogfish, and Rabbit

Carpus, 50,485

Cartilage, 20,35 , I I 5,4 I 9,470 ; calcified, 46, 48, 419

Caruncle, 582

Castings of earthworm, 318

Cell, I IO, 23I, 540; see under various types

Cell-colony, see Colony

Cell-differentiation, see Differentiation, and under development of various types

Cell-division, 544, 585

Cell-membrane or wall, 232, 244, 542

Cellulose, 244, 254, 405

Cement, 429, 493

Centrosome, 544

Centrum, see Vertebra

Cephalothorax, 347

Cerebellum: Frog, I57'; Dogfish, 443,513

Cerebral flexure, 568

Cerebral ganglion, see Brain 
Cerebral hemispheres: Frog, I59; Rabbit, 5II

Cerebral nerves, see Nerves

Cerebral vesicles, 566

Cerebro-pleural ganglion, 392

Cervical groove, 347

Chretopoda, 34I

Chalaza, 548

Change of function, 433

Chela, 352

Cheliped, 350

Chiạsma, optic, 164, 443, 513

Chick, see Bird

Chitin, 320, 347

Chlorophyll, 242, 251

Choroid, 183,5I7

Choroid-fissure, 570

Choroid-plexus, 157,512

Chromatin, 129, 23 I, 54I, 542

Chromatophores, 243, 299

Chromosomes, 544

Cilia, and ciliary movement, Iog, $242,250,265,273,275$

Cilia, absence of in Crayfish, 355

Ciliary folds, muscles, nerves, and vessels, I84, 5 I 7

Ciliata, Ciliate Infusoria, 26 I, 286

Circulation of blood, 86, 89, 329, $363,392,409,441, .503$

Circulatory organs, see Blooulvessels and Lymphafic system

Cirri, 406

Clasper, 417, 426, 453

Class, 219

Classification, $217,220,223,396,403$

Clavicle, 47,483

Clitellum, 319, 339

Clitoris, 524

Cloaca, 23, 386, 429

Cloacal aperture, see Anus

Cnidoblast, 297

Cnidocil, 297

Coagulation of blood, 78,107

Cochlea, 187,518

Cocoon, 339

Celenterata, 314

Colome, 20, 203, 314, 320, 325, $340,360,368,386,418$; Develop: ment of, $203,561,565$
Coelomata, 314

Colomic epithelium, 322, and see Epithelium

Coelomic fluid, 328

Collaterals, $17 \mathrm{I}$

Colon, 497

Colony, Colonial organisms, 28I

Columella, 45, r89

Commissures, see Blood-vessels and Nervous system ; of Brain, 515

Conchiolin, 384

Concrescence, 347

Condyle, occipital, see Skull

Cones of retina, 185

Conjugation, 197, 237, 268, 278

Conjunctiva, 182

Connectives, 333,365

Connective-tissue, 18, I 13

Contractility, nature of, I I 2

Conus arteriosus, 79, 88, 433

Coracoid, 47, 427

Coracoid process, 483

Cordylophora, 317

Cornea, I82, 366

Corpus adiposum, see Fat-body ; callosum, 5 II ; cavernosum and spongiosum, 523, 524; striatum, 5I4

Corpuscles, see Blood-corpuscles and Leucocytes

Cortex of Brain, 160, 511

Cortical layer, 264, 269, 273

Craniata, 406

Cranium, see Skull

CRAYFISH : general characters, 346 , 372 ;' limited number and concrescence of metameres, 346 ; appendages, $348,349,378$; exoskeleton, $347,349,355,372$, 379: muscular system, 355, 374, 375,377 ; enteric canal, 356, 375 ; gills, 360,373 ; kidney, $36 \mathrm{x}, 378$; blood-system, 361, 374, 375; nervous system, 365 , 377 ; senseorgans, 366,378 ; reproductive organs, 368, 375 ; development, 369,563

Creation, 221

Cribriform plate, 474 
Cricoid cartilage, 500

Crop, 327

Cross-fertilization, 339

Crura cerebri, I57, 443, 513, 566

Crustacea, 292, 37 I

Crystalline style, 386

Ctenidia, 383

lens, see Lens of Eye

Cutäneous glands: Frog, I29; l'abbit, 468

Cuticle : in unicellular animals, $25 \mathrm{I}$, 264,273 ; in multicellular animals, $306,320,321,355,383$

Cyst, see Cell-wall and Encystation

Cystic duct, see Bile-duct

\section{D}

Daughter cells and nuclei, 250, 254, 545

Death, II, I 52

Decalcifying, directions for, 137 ,

Decidua, 583

Decomposition, II, I5I ; and see Putrefaction

Degeneration, 406; and see Vestige

Dehydrating, directions for, 137

Dental formula, 494

Dental lamina and papilla, 429

Dentine. 417, 429, 493

Deric epithelium, see Epiderm

Derm, 128, 355, 417

Dermal teeth, 418

Descent, doctrine of, see Evolution

Development, meaning of the term, 9, 273, 280. For development of the various multicellular types, see under their names. Practical work, 212-214, 585

Dialyser, 73, 77

Diaphragm, 467, 489, 490

Diastema, 490

Diastole of heart, 90 ; of contractile vacuole, 266

Diencephalon, $157,443,512,566$

Differentiation, 204, 206, 237, 26 I

Diffuision, $73,551,583$

Digestion, 68; intra- and extracellular, 300,328
Digestive glands, 359,386 , and see Digestion, Enteric canal, Liver, Glands, Pancreas

Digestive system, see Enteric canal

Digits, 56, 485, 488

Dimorphic, Dimorphism, 250,310

Dicecious, 312,368

Diploblastic, 293, 304, 324

Directions for dissecting, $14 ;$ for drawing, 14; for killing, 31 , 239, $259,287,316,341,372,397$; 525 ; for preparing skeletons, $53,379,456,525$; for injecting blood-vessels, 99, 374, 379, $458,459,526$; for microscopic work, $I I Q$;' for histological and embryological work, 135,585

Disc of vorticella, 275

Dispersal, 272, 278, 396

Dissecting instruments, \&c., $I 2$

Distal, 6

Distribution of food-materials, 148

Divergence of character, 223

Division of physiological labour, 206, 237

DOGFISH : General characters, 415, 456 ; exoskeleton, 417, 456; endoskeleton, 419, 456 ; enteric canal, 428, 457, 459; gills, 432, $46 \mathrm{r}$; blood-system, 433, 458, 459,461 ; nervous system, 442, 462 ; kidneys, 450,459 ; reproductive organs, $45 \mathrm{I}, 459,460$; sense-organs, $448,462,463$; development, $453,563,572$

Dorsal, 6

Drum-membrane, see Tympanic membrane

Duct, see under names of individual ducts and glands

Ductus arteriosus, 533

Duodenum, 22, 497

Dura mater, I 55

E

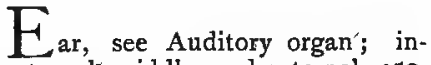
ternal; middle, and external, 450, $467,469,518,519$ 
EARTHWORM : general characters, 318, 34I ; metameric segmentation, 319; colome and enteric canal, $320,325,328,342,343$, 345 ; cell-layers, 321 , 345 ; bloodsystem, $328,342,344,345$; nephridia, 33I, 342, 344, 345; nervous system, 333,344 ; differentiation of organs and tissues, 335 ; reproduction and reproductive organs, $336,342,343$; development, 340,562

Ecdysis, 355

Echinodermata, 397

Ectoderm, 202, 209, 293, 304, 307, $340,369,454,55^{8}$

Ectoplasm, 23I

Efferent duct of spermary, 193, 338, $45^{\mathrm{I}}$

Egestion, 233

Egg of fowl, 547, 555, 586

Egg-cell, see Ovum

Egg-sac, 337

Elasmobranchii, 4I 5

Embryo, 9, 200; and see under various types

Embryology, 217

Embryonic membranes, 578, 587

Enulsification of fats, $75^{5}$

Enamel, 418, 429, 493

Enamel-organ, 429

Encystation, 232, 244, 254, 272, 279

Endoderm, 202, 210, 293, 298, 304, $307,340,369,454,558,565$

Endoderm-lamella, 309, 3 Io

Endolymph, 188

Endolymphatic duct, 187, 420, 449

Endoparasite, see Parasite

Endoplasm, 231

Endophragmal system, 349

Endopodite, $35^{\circ}$.

Endoskeleton : Frog, 16, 35, 205; Lancelet, 406; Dogfish, 4I9; Rabbit, 470; development of in Vertebrates, 576

Endostyle, 408

Energy, conversion of potential into kinetic, 235 ; source of, in chlorophyll-containing organisms, 248
Enteric canal: Frog, 23, 204; Earthworm, 320, 327 ; Crayfish, 356 ; Mussel, 386 ; Lancelet, 405, 408 ; Dogfish, 429 ; Rabbit, $490-$ 499 ; development of in Vertebrates, 204-210, $57 \mathrm{I}$

Enteric epithelium, 322, and see Epithelium

Enteroccele, 56I

Enteron or enteric cavity, 291, 304, 308,358

Epencephalon, 566

Epiderm : Frog, 128; Earthworm, 320,321 ; Crayfish, 355 ; Mussel, 385; Lancelet, 4II; Dogfish, 4I7; Rabbit, 468

Epididymis, 45I, 521, 576

Epiglottis, 495, 500

Epipharyngeal grocve, 408

Epiphysis of Vertebra, 480, 486

Epipodite, 352

Epistoma, 349

Epistylis, 281, 288

Epithelial cells: columnar, I07; ciliated, I09; glandular, I 30 et seq. ; squamous, Iro; stratified, 128

Epithelium, ro9, 31 I ; ccelomic, 322, and see Peritoneum; deric-see Epiderm; enteric, 322, and see Endoderm

Equivocal generation, see Abiogenésis

EUGLENA : 'occurrence and general characters, $25 \mathrm{I}$; movements, $25 \mathrm{I}$; structure, 25I; nutrition, 252 ; resting stage, 254 ; reproduction, 254; animal or plant? 255; practical directions, $=59$ ?

Eustachian tube or recess, 17, 189, 495,519

Eustachian valve, 506

Evolution : organic, 221, 285; of animals and plants, $25^{8}$

Excretion, 148, 150, 235

Excretory organs, see Kidney and Nephridium, and compare Con. tractile vacuole

Exopodite; 350 
Exoskeleton : cuticular, 306, 355, 383 ; dermal, 418, 429; epidermal, 466,468

Expiration, I'43

Extra-cellular digestion, 234

Eye: Frog, 4, 181 ; Crayfish, 366 ; Dogfish, 416, 449 ; Rabbit, 517 ; development in Vertebrates, 569

Eye : compound, 366

Eyelids, 5, 416, 469

Eye-muscles, see Muscles of eye

Eye-spots or ocelli : Euglena, 254; Medusa, 309, 312 ; Lancelet, 408 Eye-stalks, 349, 353

Fabellæ, 487
Fæces, 8
Fallopian tube, 523
Family, 21 8
Fascia, 59 I 8 8,
Fat-body, 25
Fats, 72
Femur, 51, 487
Fenestra ovalis, 46,

Fenestra rotunda, 476,518

Ferment, fermentation : amylolytic, 74 ; peptonizing or proteolytic, 74 ; putrefactive, 256,257

Fertilization, 197, 554, and see also Conjugation, and under development of various types

Fibrin, 107

Fibula, 5I, 487

Filum terminale, 155; 5 I 5

Fingers, see Digits

Fin-rays, 407, 4I4, 425, 426, 427; dermal, 426

Fins: Tadpole, 207 ; Lancelet, 404, 406 ; Dogfish, 417, 425

Fishes, see Pisces

Fission, 106, 198, 236, 250, 268, 272, 277; multiple, 254, 272

Fissures of spinal cord, 155

Fixing, directions for, ${ }_{3} 36$

Flagellata, Flagellate Infusoria, 261,286

Flagellum, 242, 251, 256, 298; of antenna and antennule, 353
Flocculus, 476, 513

Fœtus, 524

Follicle, ovarian, see Ovisac

Fontanelle, 43, 420

Foods, 67, 72

Foot, of mussel, 382 ; and see Pes

Foramen : lachirymal, 477 ;' intervertebral, $38,424,481$; magnum, $40,420,470$; obturator, 486 ; of Monro, I60, 514

Foramina for cerebral nerves, see Skull

Fore-brain, 202, 566

Fore-gut, $35^{8}$

Fore-limb or fin, see Appendages

Fornix, $5^{\text {I } 4}$

Fossa ovalis, 504

Fossils, 223

FROG : Preliminary account, 4; mouth cavity, I6; skin and muscles, $17,3 I$; abdomen and its contents, 20, 32 ; neural cavity and its contents, 27,33 ; structure of limbs, 28, 34 ; skeleton, 35,53 ; joints, 55,64 ; muscles, 57,64 ; enteric canal and digestion, 67,76 ; vascular system, 78,98 ; circulation of blood, 89 , 103 ; lymphatic system, 97,98 ; simple tissues, 104, $I 2 T$; compound tissues and glands, I26, 139 ; lungs and larynx, $14 \mathrm{I}, 152$; kidneys, 145, 153 ; structure and functions of nervous system, I 54, 175 ; sense-organs, $179, I 9 I$; reproductive organs, 193; 210 ; fertilization of eggs, 9, 197; development, 9, 198, $212,214,562$, 586 ; metamorphosis, I1, 206, 212 ; classification, 215; side dissection, 465 ; summary of characters, 466

Function, see Physiology

\section{G}

Tall-bladder, 22, 69, 431, 499

Gallus, see Bird

Gamete, 197, 268

Gamobium, 3I 4 
Ganglion, 163,167 ; and see Nerveganglia

Gastric glands, see Glands

Gastric juice, 71, 74, 132

Gastric mill, 358

Gastrolith, 359

Gastrula, $55^{8}$

Gastrocnemius, 59

Gemmation, see Budding

Generation, asexual, see Agamobium

Generation, sexual, see Gamobium alternation of, see Alternation of generations

Generative organs, see Reproductive organs

Genus, 215, 218

Germinal disc, 556

Germinal epithelium, I94, 196, 336

Germplasm, 555

Gestation, 525

Giant-fibres, 334

Gills : Tadpole, 10, 204, 207 ; Crayfish, 360; Mussel, 382, 386; Dogfish, 432, 455

Gill-arches and clefts, see Branchial apertures, arches, clefts and septa

Gill-cover, 347,360

Gill-rays, see Branchial rays.

Gizzard : Earthworm, 327 ; Cray. fish, $356,35^{8}$

Gland-cells: Hydra, 298, 299 ; Eàrthworm, 327, 339; and see Glands and Goblet-cells.

Glands : Cowper's, 523 ; digestive, see Enteric canal; gastric, I3I, 431, 497 ; green, 361 ; Harderian, 186,518 ; láchrymal, 518 ; mammary, $467,468,489$; Meibomian, 518 ; osophageal, 327 ; perineal, 469,523 ; prostate, 523 ; racemose, I35; rectal, 523 ; salivary, 494 ; and see kidney, liver, pancreas, \&c.

Glenoid cavity, 47

Glochidium, 396

Glomerulus, 146, 574

Glottis, I7, 495

Glycogen, I34
Goblet-cells, 109, I30, I3 I

Gonad, I93: and see Reproductive organs

Gonaduct, see Reproductive organs

Grey matter of spinal cord and brain, I 56, 160, I67

Growth, 236

Gryllotalpa, $55^{\circ}$

Gullet, 253, 265, 275; and see Enteric canal

\section{H}

$\mathrm{H}$ æmal arch and spine, 425

Hæmatochrome, 243

HeMATococcus : general eharacters, 240; rate of progression, 240; ciliary movements, 242, 250; colouring matter, 242: motile and stationary phases, 244 ; nutrition, 245 ; source of energy, 248; reproduction, 250; dimorphism, 250; animal or plant? 255; practical directions, 259

Hæmocyanin, 365

Hæmoglobin, 107, 330

Hairs, 466,468

Hallux, 487

Hand, see Manus

Hardening, directions for, 136

Head, 4, 347, 349,416, 468 .

Heart, 20, 79, 87, 362, 392, 433; 503 ; development of, 442,572 ; pulsation of, 90

Heat, evolution of, I5I

Hemibranch, 432

Hepatic ducts, see Bile duct

Hepatic cæcum, 408

Hepatic portal system, see Portal system

Hepato-pancreas, 359

Heredity, 225

Hermaphrodite, see Monocious

Heterogenesis, 284

Hibernation, 8

Higher (tripoblastic) animals, uniformity in general structure, 324

Hind-brain, 202, 566 
Hind-linb or fin, see Appendages

Hind-gut, 357

Hinge of lamellibranchiate shell, $3^{8} 3$

Hip-girdle, see Pelvic arch

Hippocampus, 5 I 4

Histological methods, I20, 135 , 585

Histology, IO4

Holobranch, 432

Holophytic nutrition, 247,253

Holozoic nutrition, 247,253

Homogenesis, , 284

Homology and homologous, 217, 310

Homology, serial, 39, 52

Host, $27 \mathrm{I}$

Humerus, 48, 484

Hybrids, 216

HYDRA : occurrence and general characters, 289; species, 29I ; movements, 29I ; mode of feeding, 292 ; microscopic structure, 292 ; digestion, 299 ; asexual, artificial, and sexual reproduction, 301 ; development, 303 ; practical directions, 314

Hydranth, 304

Hydroid polypes, 303

Hydrozoa, 3 I4

Hyoid, 40, 44, 422, 472, 479

Hyomandibular, 42i

Hypobranchial groove, see Endostyle

Hypostome, 289, 304, 308

\section{I}

Tleum, 22, 497

Ilium, 50,486

Imbedding, directions for, 137

Immortality, 236

Impregnation, see Fertilization

Incisors, 468, 492, 494

Income and expenditure, 148,236 , 245

Incubation, 586

Incus, see Auditory ossicles
Individual, 220, 28r

Individuation, $28 \mathrm{r}, 30 \mathrm{r}$

Infundibulum, of brain, I 59, 443; 513 , of lung, 5 Or

Infusoria, $26 \mathrm{I}, 286$

Ingesta and egesta, balance of, 236

Ingestion, 8, 233

Inguinal canal, $52 \mathrm{I}$

Injection of bloot-vessels, 99, 374, $399,458,459,46 r, 526$

Innominate bone, 5I, 486

Insertion of muscle, 60 .

Inspiration, I43

Integument, structure of, 127

Integumentary sense-organs, 4I4, $44^{8}$

Intercellular substance, I I 5, I I 7

Intern eural plate, 424

Interstitial cells, 293

Intervertebral discs, 480

Intervertebral foramina, see Foramen

Intervertebral substance, 424

Intracellular digestion, 234

Interrenals, $43 \mathrm{I}$

Intestine, 22 ; see its various sub: divisions and Enteric canal

Invagination, $55^{8}$

Iris, 5, 182, 571

Irritability, 60, 169, 232, 276

Ischium, 50, 486

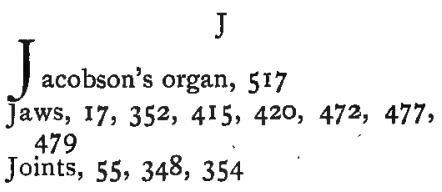

K

Karyokinesis, see Mitosis

Keber's organ, see Pericardial gland Kidney: Frog, 26, 145 ; Crayfish, $36 \mathrm{r}$; Mussel, 390 ; Dogfish, 450; Rabbit, 520; development of, in Vertebrates, 573 
L

\section{โabial palp, see Palp}

Labrum, 356

Lachrymal ducts and glands, see Naso-lachrymal ducts and glands

Lacunæ, see Bone

Lamellæ of gills (Mussel), 388

Lamellibranchiata, 396

Lamina terminalis, 514

LANCELET: general characters, 404 ; fins, 405; skeleton, 405 ; gill-slits and bars, 407 ; enteric canal, 408; blood-vessels, 408; nephridia, 408; nervous system, 408; gonads, 409 ; development, 409, 557; practical directions,

$410-413$

Larva, 9, 340, 370, 395, 396, 409

Laryngo-tracheal chamber, I4I

Larynx, 489, 500

Lateral line, 416,448

Legs, see Appendages

Lens of eye, $183,517,569$

LEPÚs, see Rabbit

Leucocyte, 105, 328

Life, origin of, 28I ; and see Biogenesis

Life-history, 8, 206 ; and see under various types

Ligaments, 55,57

Limbs, see Appendages

Lips, 468

Liver, 20, I33, 204, 359, 408, 43I, 499

Lobster, 346

Lumbricidæ, 34I

LUMBRICUS, see Earthworm

Lungs, IO, 22, I4I, 204, 208, 5OI

Lymnaus, 585

Lymph, 18

Lymph-hearts, 97, 98

Lymphatic glands, see Adenoids

Lymphatic system, 18, 97, 442

\section{M}

$\mathrm{M}$ alleus, see Auditory ossicles

Malpighian capsule, i 46,574

Mammalia, 219, 403, 466
Mammary glands, see Glands

Mandible, 16, 44, 352, 422, .479

Mantle, 38I

Mantle-cavity, 385

Manubrium : of Medusa, 307; of malleus, 519; of sternum, 483

Manus, 5, 5o, 468, 485

Marrow-cavity, 48

Matrix, see Intercellular substance

Maturation of ovum, $55^{2}$

Maxilla : Crayfish, $35^{2}$

Maxilliped, 350

Meckel's cartilage, 44, 422

Mediastinum, 501

Medulla oblongata, 157, 443, 513

Medullary : cord, folds, groove, and plate, 202, 559; sheath, I67 ; substance of Infusoria, 264, 269, 273

Medusa, 304, 307-312

Megagamete, 279

Meganucleus, 265, 273

Megazooid, 251, 279.

Membranous labyrinth, 186, 449, 518

Mesencephalon, see Mid-brain

Mesenteron, see Archenteron

Mesentery, 22, 431, 498

Mesoderm, 202, 203, 210, 340, 369, $454,559,565$; vertebral and lateral plates of, 565

Mesodermal segments, 565

Mesogloea, 293, 307

Mesonephros, 573, 574

Mețabolism, I49, 236

Metacarpus, 50, 485

Metamere, Metameric segmentation, 319, 346, 354

Metamorphosis, I I, 209, 280

Metanephros, 573,576

Metapleural fold, 406

Metatarsus, 51, 488

Metazoa, 286

Microgamete, 279

Micrometer, I2I

Micromillimetre $(\mu)=\frac{1}{1000}$ of a millimetre, or $25 \frac{1}{\delta 5 \sigma}$ of an inch

Micronucleus, 265, 275

Micropyle, 400, 547, 554

Microtome, $13^{8}$ 
Microzooid, 25I, 279

Mid-brain, 202

Mid-gut, 357

Milk-glands, see Glands

Milk-teeth, 493

Milt, see Spermatic fluid

Mitosis, 545,585 .

Molars, 492, 494

Mollusca, 220, 396

Monads: occurrence and general characters, 256 ; movements, 256 ; nutrition, 256 ; animals or plants ? 257; rapid multiplication, 282 ; practical directions, 260 .

Moncecious, 302

Morphology, 217

Morula, see Polyplast

Mother of pearl, see Nacre

Mounting sections, directions for, $13 \dot{9}$

Mouth, 4, I6, 253, 265, 275, 290, $307,319,356,386,408,415$, 428

Mouth-cavity, see Buccal cavity

Movement, spontaneous, voluntary, and involuntary, 7, I I, I I 2, $172,232,312,335,346 ; 417$;

$?$ and see under various types

Mucous membrane, I7

Mule, see Hybrid

Müllerian duct, 450,574

Multinucleate, 269, 369

Muscle-fibres: striped, II2, 355; unstriped, 'III' I3I, 'I33; and see under various types

Muscle-processes, 293, 299, 304

Muscles: Frog, I8, 57, 59, 63, 205; Crayfish, 321, 355; Mussel, 385 ; Rabbit, 489; and see Myomere

- Muscles : of eye, I86, 449, 517 ; of middle ear, 519

Muscles, papillary, 504

- Muscular contraction, 160, 276

Muscular impressions on shell, 384

Muscular layers of enteric canal,

70,75

Muscular system, see under various types ; development of, in Vertebrates, $203,565,576$.
Musçularis mucosæ, I 33

Mússei : general characters, $38 \mathrm{I}$, 397 ; mantle, shell, and foot 381 , 397.398; food-current, 382, 397 ; gills, 382 , 386, 398, 399, 400; muscles, 385,398 ; enteric canal, $386,390,40 T$; nephridia, 390 , 400 ; blood-system, 392, 399; nervous system, 392,400 ; senseorgans, 394, 40r; gonads,. 394, 402 ; development ard metamorphosis, $395,400,562$

Mustelus, 415,454

Myocomma, 4I 8

Myomere, 203, 355, 404, 418, 565

Myophan layer, 264

\section{N}

Vacre : nacreous layer, 384

Nasal organ, see Olfactory organ

Naso-lachrymal duct, $186,477,518$

Naso-palatine canals, see Canals

Naso-pharynx, 495

Natural History, 2

Natural selection, 226

Nauplius, 370

Neck, 468

Nemathelminthes, 397,457

Nematocyst, 295

Nephridiopore, 332

Nephridium, 146, 331, 361, 368, $390,404,406,408,450,574$

Nephrostome, I45, I46, 33 I, 574

Nerve-cells, 167,298

Nerve-collar, 333

Nerve-cord : ventral, 333,365

Nerve-fibre, 167

Nerve-foramina, $47^{8}$; and see Skull and Vertebral column

Nerve-ganglia, $162,163,164,167$, $333,365,366,392$

Nerve-plexus: brachial, I6I, 5 I5 ; cœliac, 516 ; sciatic or lumbosacral, 162, 515

Nerve-roots, I63, 409

Nerves : afferent and efferent, 166 , I75; cerebral, $163,445,515$, 568 ; of lateral line, 447 ; motor 
and sensory, $-162,169, .334$; sciatic, 62, 162, 515 ; spinal, I $60 ; 409,445,515,568$; sympathetic, $162,445,516$; vasomotor, I74; visceral, 366

Nervous impulse, 62,154

Nervous system: Frog, I 55 ; Hydra, 298; Bougainvillea, 3 II ; Earthworm, 333 ; Crayfish, 365 ; Mus. sel, 392; Lancelet, 404, 408; Dogfish, 442 ; Rabbit, 51 I ; development of, in Vertebrates, 565 , 587

Neural arch, spine, and canal, see

- Vertebra

Neural plate and process, 424

Neuraxis, I 67

Neurenteric canal, see Canal

Neurilemma, I67

Neuroccele, 443; and see Canal, central of spinal cord, and Ventricles of brain

Neuroglia, 169

Newt,'218

Nictitating membrane, 5, 469

Nodes of nerve-fibre, I67

Nose, see Olfactory organ

Nostrils, 5, 17, 180, 416, 448, 468, 470,517

Notochord, 203, 404, 425, 560, 576,587

Nuclear division, $544, ' 585$; indirect, 545

Nuclear membrane, 542 ; spindle, 544

Nucleolus, 109 et seq. ., 541, 542

Nucleus, ro6 t $t$ seq., I 29, 23I, 243 , $254,265,269,273$

Nucleus, conjugation-, 268; segmentation-, 554

Nutrition, 67 ; and see under various types

\section{belia, 317}

Occipital condyle, 'see Skutl

Ocellus, see Eye-spot

Oesophagus, see Gullet
Odontoblasts, 429

Odontoid process, 482

Olecranon, 49, 485

Olfactory capsule, $39,42,420,476$ " lobe, 160, 444, 5II, 514, 568

organs : Frog, 180; Cray.

fish, 367 ; Mussel, 394 ; Dogfish, 416, 448; Rabbit, 5 I I, 517; development of, in Vertebrates, 569

Olfactory pit, 408

Oligochæta, 34I

Ommatideum, 366

Ontogeny, 224

Oogenesi s, 196, 548

Oosperm, 198; and see under various types

Oosperm, holoblastic and meroblastic, 556

Opalina: occurrence and general characters, 269; structure and division of nuclei, 269 ; parasitic nutrition, 27I ; reproduction, $27 \mathrm{I}$; means of dispersal, 272 ; development, 254; praçtical directions, 287

Optic chiasma, see Chíasma; cup, 570 ; lobe, $157,443,513,566$; thalamus, 159 ; vesicle, 569

Oral cavity, see Buccal cavity

Oral hood, 406

Orbit, see Skull

Order, 218

Organ, 30, I 51, 238, 335

Organism, 23I

Origin of muscles, 60 of species, 225

Osmosis, see Diffusion

Osphradium, 394

Ossicles : of gizzard (Crayfish). 358 , 376 ; auditory (Rabbit), see Auditory ossicles

Ossification, 44

Ostiz: of heart, 362 ; of gills, 389

Otocyst, 394

Otoliths, 188

Ovary : Frog, 23, 193, 195: and see Reproductive organs

Oviduct, $25,196,337,368,453$, 523,574 
Ovisac, 195, 523

Ovum, 195, 546, 555 ; and see under various types.

Ovum : alecithal, centrolecithal, and telolecithal, $55^{6}$

Oxidation of protoplasm, 149,248

\section{$\mathrm{P}$}

D

Palate, 490

Pallial line, 384

Pallium, see Mantle

Palp : Crayfish, 353 ; Mussel, 386

Pancreas, 22, 70, 134, 204, 43I, 499

Pancreatic juice, jo

Papillæ of tongue, I8o, 492

Parachordal, 577

Paragnatha, 356

Paramylum, 25I

Paramacium: structure, 262 ; mode of feeding, 266 ; reproduction, 268; conjugation, 268 ; practical directions, 286

Parasite, parasitism, 33, 153, 269, 27I

Parietal layer of peritoneum, 26, 322 ; see also Mesoderm

Parthenogenesis, 552

\section{Patella, 487}

Pectoral arch, 20, 36, 46, 426, 483

", fin or limb, see Appendages

Pedal ganglion, 392

Pelecypoda, 396

Pelvic arch, 20, 36, 50, 426, 486 " fin or limb, see Appendages

Pelvis of kidney, $52 \mathrm{I}$

Penis, 470, 523

Pepsin, peptone, 74

Pericardial gland, 390

Pericardial sinus, 362

Pericardium, 20, 386, 418, 490, 503

Perichondrium, 55

Perilymph, 189

Perinæum, 469
Periosteum, 55

Periostracum, 384

Peristaltic movements, 75

Peristome, 275

Peristomium, 319

Peritoneum, 22, 26, 322, 418, 490

Pes, 5, 5I, 468, 488

Peyer's patches, 498

Phalanges, $50,5 \mathrm{I}, 485,488$

Pharynx, $17,327,405,408,429_{3}$ 495

Phylogeny, 223, 224

Phylum, 219

Physiology, I, 2 I 7

Pia mater, 155

Pigment-cells, I 28

Pigment-layer of retina, 185

Pigment-spot, 254

Pineal body, I 59, 443, 513, 566 .

Pineal eye, 159,567

Pinna, $467,469,519$

Pisces, 219, 403; general characters . of, 414

Pithing, directions for, $\mathrm{HO}_{3}$

Pituitary body, I 59, 443, 513, 566

Placenta, $467,524,583$

Planula, 313

Plasma, I04

Plastic products, $249,55 \mathrm{I}$

Platyhelminthes, 397

Pleopod, 350

Pleura, pleural membrane, 490

Pleurobranch, 36I

Pleuron, Crayfish, 347

Podobranch, 36r

Podomere, 349

Polar cells, 552, 585

Pollex, 485

Polychæta, 34I

Polymorphic, polymorphism, 3 ro

Polyplast, 200, $55^{8}$; and see under various types

Polystomum, 33

Pons Varolii, $5 \mathrm{I} 3$

Pores, dorsal, 325

Portal system: hepatic, $85,440,510$; renal, $.85,43^{8}$

Post-axial and pre-axial borders of $\operatorname{limb}, 484,486$ 
Poupart's ligament, 489

Premolars, 492, 494

Prepuce, 470

Preservative fluids, $I_{3}$

Primitive groove, 563

Prismatic layer, 384

Proamnion, 579

Processes of skull, $472^{\prime} \mathrm{et}$ 'seq.

Procclous, 36

Proctodxum, 204, 358, 43I

Pronephric duct, 573

Pronephros, 573

Pronucleus, male and female, 554

Prosencephalon, 444, 566

Prostate, see Gland

Prostomium, $3^{19}$

Proteids, 72

Protista, 257

Protococcus, see Hæmatococcus

Protoplasm, 106, 54I; and see Cell

Protopodite, 350

Protovertebra, 565

Protozoa, 220, 286

Proximal, 6

Psendobranch, 433

Pseudopod, ro6, 23I, 250, 298

Pterygiophores, 425-427

Pubis, 50, 486

Pulmonary artery and vein; see Arteries and Veins

Pupil, 5, I82

Putrefaction, 256, 257, 261, and see Decomposition

Pyloric division and valve, of stomach, see Stomach.

Pyrenoid, 243

\section{$\mathrm{R}$}

$\mathrm{R}$

ABBIT' : general characters, 467 , 525 ; skeleton, $470,52.5$; muscles and body wall, $488,526,527,532$, 535,539 ; cœlome, 489, 528,532 , digestive organs; 490, $528-530$; respiratory, and vocal organs, 489 , $500,533,534,537$; circulatory organs, 503, 527-535; nervous system, 5 I I, 527, 532-534, 537 ; sense - organs, $516, \quad 53^{6}-538$; urinogenital organs, 520, 528 532 ; development, $524,564,578$, 583,587

Racemose, I35

Radial canals, see Canals

Radial symmetry, 291

Radiolaria, symbiotic relations with Zooxanthella, 299

Radio-ulna, 49

Radius, 50, 484

Rana esculenta, 216

Rana TEMroraria, see Frog

Ranidæ, 218

Reagents, hardening, preserving, mounting, and staining, $I 35$

Recapitulation, theory of, $\mathbf{2 2 4}$

Rectal gland, 43I

Rectum, 23, 386, 43I, 497

Reducing division, 55 I, 554

Reflex action, I69, 3 I2

Regeneration, 336

Renal portal system, see Portal system,

Reproduction, 8, and see under. various types, and also Asexual. reproduction

Reproductive organs, 23, 25, 193, 302,3 I 2, 336, 368, 394, 409, 45I, 521,573

Reptilia, 219,403

Respiration, I4I, 144, 235, 330

Respiratory movements, 7, I42, 503

Retina, $183.184,570$

Retinula, 366

Rhabdome, 366

Rhinencephalon, 568

Rhizopoda, 286

Ribs, $424,468,483$

Rocks, sedimentary and ștratified, 223

Rodentia, 525

Rods and cones, 185

Rostrum, 349, 420

Rudiment, often used for Vestige (q.v.) 


\section{$\mathrm{S}$}

acculus, 187

Sacculus rotundus, 497, 498, 529

Sacrum, 482

Salamander, 2I 8

Salivary glands, see Glands

Saprophytic nutrition, 256, 257

Sarcolemma, I12

Scales, 4I 4,418

Scapula, $46,427,483$

Schizoccele, 565,587

Sclerite, 376

Sclerotic, 182, 5I 7; 57 I

Scrotal sac, 470,521

SCyllium, see Dogfish

Section-cutting, directions for, 126 , $\mathrm{I}_{38}$

Secretion, I30

Segment, see Metamere, Podomere Segmentation:cavity, 200, 558, 562 Segmentation of oosperm, I 98,200 , $212,303,313,340,369,585$

Segmentation, equal and unequal, 556 ; discoid, $55^{6}$

, superficial, 369,556

", metameric, see Metamere -nucleus, '554

Selection, natural and artificial, 226, 227

Self-fertilization, 339

Seminal funnel, $33^{8}$

$$
\text { vesicle, I94, 338, } 45 \text { I }
$$

Sense-organs and cells, I79, 308, $312,335,414,416,448,516$ Septet, of Earthworm, 325

Septum : lucidum, 5I4; nasal, 42, 476

Serous membrane of embryo, ' 579 , 583

Sesamoid bones, $485,487,488$

Seta, 320, 348, 367

Sex cells, primitive, 549

- Sexual characters, external, 7, 368, 417,469

Sexual generation, see Gamobium

Sexual organs, see Reproductive organs
Sexual reproduction, see under varions types

Shank, 5

Shell, 38I ; larval, 395

Shell of egg, 453.

Shell-gland of Mussel-embryo, 395 ; of Dogfish, 453

Shoulder-girdle, see Pectoral arch.

Sinus : blood, $360,362,364,438$, $44 \mathrm{I}$; lymph, 18,27 ; urinary and urinogenital 45 I ; venosus, 80,89

Siphon, inhalant and exhalant, 382

Skeleton, see Endo- and Exoskeleton

Skin, see Integument

Skull ; Frog, 16, 35, 39; Dogfish, 420 ; Rabbit, 470 ; development of, 576

Smell, organ of, see Olfactory organ

Snout, 4

Somatic layer of mesoderm, $56 \mathrm{I}$

Somatopleure, $56 \mathrm{I}$

- Spawn, 9

Species, 2I 5 et seq. ; origin of, 222, 225

Sperm, or spermatozooid, 194, 368, 549 ; and see under various types Spermary, 25, 193, 194; and see Reproductive organs

Spermatogenesis, 194, 548

Spermatophore, 368

Spermotheca, 338

Sperm-reservoir, $33^{8}$

Sperm-sac, 338, $45 \mathrm{I}$

Spinal cord, 28, I55, 443, 515

Spiracle, 4I6, 433

Splanchnic layer of mesoderm, 56 I

Splanchnopleure, 562

Spleen, 23, 98, 43r, 499

Spontaneous generation, see Abio. genesis

Spores, 254, 279

Stalk of Vorticella, 273, 276

Stapes, 46, 189, 473, 519

Starch, 72, 243

Sterilised infusions, 283

Sternebræe, 483

Sternum, I6, 48, 347, 483

Stimulus, "various kinds of, 62 
Stock, see Colony

Stomach, 22, 70, 357, 386, 429, 495

Stomodæum, 204, 358, 429, 43 I

Struggle for existence, 225

Substitution of organs, '209

Sucker, 203

Supporting lamella, see Mesoglœea

Suprarenals, see Adrenals

Suspensorum, 40, 422

Sutures, 50, 470

Swimmeret, see Pleopod

Symbiosis, 299

Sympathetic, see Nerves

Symphysis, 486

Syn-cerebrum, 366

Synovial capsule, 56

Systemic arch, $Y_{0}, 436,506$

Systole : of heart, 90 ; of contractile vacuole, 266

\section{$\mathrm{T}$}

$T$ actile organs, $179,368,394,5^{16}$

Tadpole, 9, 203 et seg.

Tail, 9, 209. 406, 416, 468

Tapetum, 539

Tarsus, 51,487

Taste-organs, 180, 492, 517

Teasing, directions for, 123

Teats, 468,489

Teeth, I $7,358,428,468,492$

Teleostomi, 4I 5

Telson, 347

Tendon, 59

Tentacles, 290, 291, 304

Tergum, 347

Testis, see Spermary .

Thalamencephalon, see Diencephalon

Thigh, 5

Thoracic duct, 5 II

Thorax, 347, 467, 489

Thread-cell, see Nematocyst

Thymus, 43I, 490, 503

Thyroid, 43I, 503

Thyroid cartilage, 500

Tibia, $5 \mathrm{I}, 487$
Tibio-fibula, $5 \mathbf{I}$

Toad, see Bufo

Toes, see Digits

Tissues, enumeration of, 3 I

Tongue, 8, 17, 429, 492

Tongue-cartilage or bone, see Hyoid

Tonsil, 490

Trachea, 489,500

Trabeculæ cranii, 577

Transverse process, see Vertebra

Trichocyst, 267

Triploblastic 306, 314, 324

Trochanter, 487

Trochosphere, 340

Trophoblast, 565

Trunk, 4, 416, 468

Trypsin, 74

Tubercle, and Tubercular facet, $48 \mathrm{I}$, 483

Tunicata, 406

Turbinals, 476, 517

Tympanic cavity, membrane, and ring, $5,45,189,433,475,519$

Typhlosole, $327,3^{86}$

\section{$\bigcup_{\ln a, 50,485}$}

Umbilical cord, 584

Umbilicus, 584

Umbo, 383

Umbrella, 307

Unicellular, 23I

Unio, see Mussel

Urachus, 584

Urea, 66, 147

Ureter, 26 ; and see under various types

Urethra, see Urinogenital canal

Uric acid, 66

Urinary bladder, 23, 36I, 390, 521

," tubules, see Nephridium

Urine, 8, 66, 147

Urinogenital aperture, 469

$\begin{array}{ll}, & \text { canal, 522, 523 } \\ \text { duct, I94 } & \text { organs, 193, 450, } 520\end{array}$


Urinogenital organs, development of, 573

Urodeles, 219

Uripod, 350

Urostyle, 35, 39

Uterine crypts, 583

Uterus, 523

Utriculus, 187

\section{V}

$\mathrm{V}$

acuole : contractile, 232,243 , 254, 265, 273; food-, 233, 266, 276

Vagina, 523

Valve : of Vieussens, 5 I2, 538 ; spiral 429,498 ; ileo-colic, 498

Valves : of heart, 88, 433, 504, 506; of shell, 381 ; of veins, 89

Variability, 225

Variation, individual, 216, 225

Variety, 225

Vascular system, see Blood-vessels, Arteries, Veins.

Vas deferens, see Spermiduct, Wolffian duct

Vasa efferentia, see efferent ducts

Veins: Frog, 19, 82 ; Crayfish, 363; Mussel, 392 ; Dogfish, 438 ; Rabbit, 504, 509 ; embryo Vertebrate, 572

Veliger, 396

Velum : of Medusa, 309 ; of Lancelet, 408

Velum palati, 490

Vena cava, see Veins

Vent, see Anus

Ventral, 6

Ventricle, see Heart

Ventricles of brain, I 57, 159, 408, $443,512-514,568$

Vermiform appendix, 498

Vertebrata, 2 I9; general characters of, 403

Vertebra and vertebral column, I6, $35,36,423,480,576$
Vertebrarterial canal, see Canal

Vessels; see Blood-vessels

Vestibule, see Urinogenital canal

Vestige, véstigial, I 59, $3^{61}$

Vibrissæe, 469

Villi : of intestine; 498 ; of chorion, 583

Viscera, abdominal, 20, 429, 495

Visceral arches and clefts, 419, 422,57 I, and see Branchial apertures

Visceral ganglion, 393

Visceral layer of peritoneum, 27, 323

Visceral mass, 385

Vitelline membrane, I96, 547, 579

Vitreous body of compound eye, 366

chamber and humour, 183

Vocal cords, 144, 500

, sacs, 218

VORTICKLLA : occurrence and general characters, 273; structure, 273; reproduction, 277; conjugation, 278 ; means of dispersal, 278; encystation, 279; spore-formation, 279:; metamorphosis, 230 ; practical directious, 287

Vulva, 47o, 523

\section{W}

W aste-products, 8, 66, 249

White matter of brain and spina cord, I 56, I 60

Wolffian body, see Epididymis

$$
\text { duct, } 450,574
$$

Work and waste, $66,148, .234$

Worms, 340

Wrist, see Carpus

\section{$\mathrm{X}$}

Xiphisternum, $48,4^{8} 3$ 


\section{Y}

Yellow cells : of Radiolaria, 299; of Earthworm, 323, 327, 332 Yolk, yolk-granules or spheres, 195 , 546 ; and see under various types

Yolk-cells, 200, 202

Yolk-plug, 20I

Yolk-sac, 454
Z

Cooid, 28I ; and see Megazooid and Microzooid Zoology, I

Zoophytes, see Hydroid polypes

Zooxanthella, 299

Zygapophysis; 36, $48 \mathrm{I}$

Zygoma: zygomatic arçh, 477, 478 .

Zygote, 197, 279

'THE END. 


\section{By Prof. T. J. PARKER and Prof. W. A. HASWELL.}

A MANUAL OF ZOOLOGY. By the late T. JefFery Parker, D.Sc., \&c., and William A. Haswele, M.A., D.Sc., \&c. With 300 Illustrations. Crewn 8vo, ros. 6 .

\section{A TEXT-BOOK OF ZOOLOGY. By}

T. JefFery Parker, D.Sc., F.R.S., Professor of Biology in the University of Otaga, Dunedin, N.Z., . and William A. Haswell, M.A., D.Sc., F.R.S., Professor of Biology in the University of Sydney, N.S.W. In 'Two Vols. With Illustrations. Medium 8vo, $36 s$. net.

NATURAL SCIENCE.-" "Parker and Haswell' has been looked forward to with expectation for some time. We welcome it gladly now that it appears; it should be in the hands of all students, and even teachers will find it of value... It is so well illustrated, so clearly printed, and generally good, that it will be found a useful addition to the student's and teacher's shelves."

\section{By Prof. T. JEFFERY PARKER.}

\section{A- COURSE OF INSTRUCTION IN} ZOOTOMY. Vertebrata. By Professor T. JefFerY

PARKER, F.R.S. With Illustrations. Crown 8 vo, $8 s .6 d$.

SATURDAY REVIEW._-"The admirable work, . . In large and well appointed morphological laboratories, this sort of help is supplied by a staff of demonstrators; but Mr. Parker has conferred an immense boon on teachers and students alike by enabling the latter *o dispense to a very great extent with the services of the former.

\section{ELEMENTARY PRACTICAL ZOOL-} OGY. By the lace Professor T. J. Parker, F.R.S., and Professor W. Newtor Parker. Illustrated. Crown 8vo.

\section{LESSON I N E L E M E N T A R Y}

BIOLOGY. By Professor T. Jefrery Parker, F.R.S.

Illustrated. Third Edition. Crown 8vo, ros. $6 d$.

NATURE.- "Prof. Jeffery Parker is to be congratulated on having produced a extremely well-written, well-considered, and original class-book."

\section{WILLIAM KITCHEN PARKER, F.R.S.}

A Short Memoir. By his son, T. JEFFery PARKer,

B.Sc., F.R.S. Crown 8 vo, $4 s$. net.

ATHENAEUM.- "Prof. Jeffery Parker is to be warmly congratulated on this. charming sketch of his beloved father's life. ... As a sketch it is, we think, perfect."

MACMILLAN AND CO., LTD., LONDON. 


\section{MACMILLAN \& CO.'S}

\section{BOOKS FOR STUDENTS OF ZOOLOGY.}

A TEXT-BOOK OF COMPARATIVE ANATOMY. By Dr. ARnold LAvg, Professor of Zoology in the University of Zirich. With Preface to the English Translation by Dr. ERNST HAECKel. Translated by Henry M. Bernard, M.A. Cantab., and Matilda Bernard. Vols. I. and II. (completing the Invertebrates). 8vo. r7s. net each.

LESSONS IN ELEMENTARY ANATOMY. By Șr'. George Mivart, F.R.S. Fcap. 8vo. 6s. $6 \pi$.

ELEMENTS OF THE COMPARATIVE ANATOMY OF VERTEBRATES. Adapted from the German of Professor WIEDERSheim. By Professor W. NEwTon PARKer. With Additions by the Author and Translator. 270 Woodcuts. Medium 8vo. S'Second Edition. J2s. 6d. net.

THE STRUCTURE OF MAN : AN INDEX TO HIS PAST HISTORY. ky Professor Wiedersheim. Translated by $\mathbf{H}$. and $\mathbf{M}$. BERnARD. The Translation, Edited and Annotated, and a Preface written by Professor G. B. Howes, F.R.S. With Ios Figures in the Text. 8vo. 8s. net.

AN INTRODUCTION'TO THE OSTEOLOGY OF $\overline{\mathrm{THE}}$ MAMMALIA. By Sir WILLIAM HEN RY F LOWER, F.R.S., F.R.C.S. Illustrated. Third Edition. Revised with the assistance of H.Ans GaDow, Ph.D. Crown 8 vo. ros. $6 d$.

ESSAYS ON MUSEUMS AND OTHER SUBJECTS CONNECTED WITH NATURAL HISTORY. By Sir WILliaM HENRY F LoWER, K.C. B., D.C.L., D.Sc. Demy 8vo. izs. net.

A IREATISE ON COMPARATIVE EMBRYOLOGY. By the late Professor F. M. Balfour, M.A., F.R.S. In two Vols. Second Edition. Medium 8vo. Vol. I. I8s. Vol. II. 21s.

THE ELEMENTS OF EMBRYOLOGY. By Sir MIChaEL Foster, K.C.B., M.D., F.R.S., and the late Professor F. M. Balfour, F.R.S.

r- Second Edition, revised. Edited by A. SEDGWICK, M.A., and W. HeApE, M.A. Illustrated. Crown 8vo. Ios. $6 d$.

THE VOYAGE OF THE CHALLENGER : THE ATLANTIC. By Sir C. Wyville Thomson, Knt., LL.D., D.Sc., Regius Professor of Natural History in the. University of Edinburgh. With Illustrations, Coloured Maps, Charts, etc, Two-Vols. 8vo. 45 s.

THE DEPTHS OF THE SEA. An Account of the Results of the Dredging Cruises of H.M.S.S. Lightning and Porcnpine, I8686g-7o. By Sir C. WYvih.e Thomson. With Illustrations, Maps, and Plans 8vo. 3Is. $6 d$.

ART ANATOMY OF ANIMALS. By ERNEST E. S. Thом PSoN. Illustrated. $4^{\text {to. }} 30 s$, net.

MACMILLAN AND CO., LTD., LONUON. 


\section{MACMILLAN \& CO*'S BOOKS FOR STUDENTS OF ZOOLOGY.}

AN ATLAS OF PRACTICAL ELEMENTARY BIOLOGY. By G. B. Howes, Assistant Professor of Zoology, Normal School of Science and Royal School of Mines. Second Edition. Lemy 4to. [Shortiy.

A COURSE OF ELEMENTARY INSTRUCTION IN PRACTICAL BIOLOGY. By T. H. Huxley, F.R.S., and H. N. Martin. Revised and extended by Prof, G. B. Howes and D. H. Szotr, Ph.D. Crown 8vo. Ios. $6 d$.

THE FOUNDATIONS OF ZOOLOGY. By W. K. BRooks, Ph.D., Ll.D. 8vo. ros. $6 a$. net.

MONOGRAPH OF THE BRITISH CICADÆ, OR TETTIGIDÆ. By G. B. BUCKToN. 2 vols. 8vo. 42s. net.

FISHES, IIVING AND FOSSIL. An Outline of their Forms and probable Relationships. By BASHFORD DEAN, Ph.D., Instructor in Biology, Columbia College, New York City. 8vo. Ios, $6 d$. net.

THE NATURAL HISTORY OF 'THE MARKETABLE MARINE FISHES of tile BRITISH ISLANDS. By J. T. CUNNINGHAM, M.A. With Preface by E. RAy Lankester, F.R.S. Illustrated. Medium 8vo. $7 s .6 d$. net.

ATLAS OF THE KARYOKINESIS OF THE OVUM. By Edmund B. WILson, Ph.D., Professor of Invertebrate Zoology in Columbia College, with the co-operation of EDWARD LEAMING, M.D., Instructor in Photography at the College of Jhysicians and Surgeons, Columbia College. 4to. I7s. net.

'THE CELL AND ITS DEVELOPMENT. By Prof. EDMUNB B. WILSON, Ph.D. 8vo. I4s. net.

A TEXT-BOOK OF ENTOMOLOGY, including the Anatomy, Physiology, Embryology and Metamorphoses of Insects, for use in Agricultural and Technical Schools and Colleges, as well as by the working Ento mologist. By Alpheus S. Packard, M D., Ph.D., Professor of Zoology and Geology, Brown University. Medium 8vo. r8s. net.

NATURAL HISTORY OF AQUATIC INSECTS. By L. C. M IAlL, F.R.S., Professor of Biology in the Yorkshire College, Leeds. Illustrated. Crown 8vo. $6 s$.

THE ORIGIN AND METAMORPHOSES OF INSECTS. By Sir Jorn Lubeock, F.R.S. With Illustrations. 3s. $6 d$.

[Nature Series.

LIFE HISTORIES OF AMERICAN INSECTS. By Clarence Moores Weed, D.Sc., Professor of Zoology and Entomology, New Hampshire College of Agriculture and the Mechanic Arts. With ar full-page Plates and many Figures in the Text. Crown 8vo. 6s.

CITIZEN BIRD. SCENES FROM BIRD-LIFE IN PLAIN ENGLISH. By M. O. Wright and E. Coues. Crown 8 vo. $6 s$.

FOUR-FOOTED AMERICANS AND THEIR KINBy Mabel Osgood Wrighr. Edited by Frank M. Chapman, Illustrated by Ernest Seton Thompson, Crown 8vo. 7s. $6 d$.

-

MACMILLAN AND CO., LTD., LONDON. 


\title{
The Cambrioge Nhatural Thistory.
}

\author{
EDITED BY
}

S. F. HARMER, M.A., ANd A. E. SHIPLEY, M.A.

In Ten Voilmes. 8vo. Price 17s. net each.

VOLUME I.

Protozoa, Marcus Hartor, M.A., Trinity College (Professor of Natural History in the Queen's College, Cork); Sponges, W. J. Sollas, Sc D., F.R.S., St. John's College (Professor of Geology in the University of Uxford); Jellyfish, Sea Anemones, \&e., S. J. Hickson, M.A, Downing College (Beyer Professor of Zoology in the Owens College, Manchester); Star-fish, SeaUrehins, \& e., $\mathrm{E}$ W. MACBRIDE, M.A., St. John's College (Professor of Zoology, M'Gill'University, Montreal).

VOLUME II.

Flatworms, \&e., F. W. Grambl., M.Sc. (Vict.), (Demonstrator and AssistantLecturer in Zoology in the Owens College, Manchester); Nemertines, Miss L. Sheldon, Newnham College; Thread-worms, \&c., A. E. SHipley, M.A., Christ's College; Rotifers, \&c., Marcus Hartog, M.A., Trinity College, D.Sc. (Lond.), (Professor of Natural History in the Queen's College, Cork); Polyehaet Worms, W. B. Benham, D.Sc. (Lond.), Hon. M.A. (Oxon.), Aldrichian Demonstrator of Comparative Anatomy in the University. of Oxford; Earthworms and Leeches, F. E. BEnDARD, M.A. (Oxon.); F.R.S. (Prosector to the Zoological Society); Gephyrea, A. E. SHipley, M.A., Christ's College ; Polyzoa, S. F. HAR.Mer, M.A., King's College.

VOLUME III.

[Ready.

Molluses, A. H. Cooke, M.A., King's College; Brachiopads (Recent), A. E. Shipley, M.A., Christ's College; Brachiopods (Fossil), F. R. C. REed, M.A., Trinity College.

VOLUME IV.

[Ready.

Spiders, Mites, \&c., C. WARBL'RTON, M.A., Christ's College (Zoologist to the Royal Agricultural Society); Scoppions, Trilobites, \&c., M. LAURIE, B A., King's College, D.Sc. (Edinb.), (Professor of Zoology in St. Mungo's College, Glasgow) ; Pyenogonids, \&c., D'ARCY W. Thompson, C.B., M.A., Trinity College (Professor of Zoology in University College, Dundee); Crustacea. W. F. R. Weldon, M.A., F.R.S., St. John's College (Jodrell Professor of Zoology in University College, London).

VOLUME $V$.

Peripatus, A. SEdGwick, M.A., F.R.S., Trinity College ; Centipedes, \&c., F. G. Sinclair, M.A., Trinity College; Inseets, Part I, D. Sharp, M.A., F.R.S.

VOLUME VI.

[Ready.

Inseets, Part II., Hymenoptera continued (Tubulifera and Aculeata), Coleoptera, Strepsiptera, Lepidoptera, Diptera, Aphaniptera, Thysanoptera, Hemiptera, Anoplura. David Sharp, M.A.Cantab., M.B.Edin., F.R.S.

VOLUME VII.

Belanoglossus, \&c., S. F. HARMER, Sc.D., F.R.S.. King's College ; Ascidians and Amphioxus, W. A. HERDMAN, D.Sc. (Lond.), F.R.S. (Professor of Natural History in University College, Liverponl); Físhes, T. W. BRIDGE, Sc.D., Trinity College (Professor of Zoology in the Mason University College, Birmingham).

VOLUME VIII.

Amphibia and Reptiles, H. GaDow, M.A., F.R.S., King's College.

VOLUME IX.

Birds, A. H. Evans, M.A., Clare College. With numerous Illustrations by G. E. LODGE.

VOLUME X.

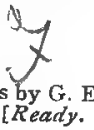
Mammals, F. E. Beddard, M.A. Oxon., F.R.S. (Prosector to the Zoological
Society).

MACMILLAN AND CO., LTD., LONDON. 



